\title{
Iron-Catalyzed Cross-Coupling of Arylboronic Acids with Unactivated N-Heterocycles and Quinones Under Microwave Heating
}

\author{
By \\ Keegan Start \\ A dissertation submitted to the School of Graduate Studies in partial fulfillment of the \\ requirements for the degree of Master of Science (M.Sc.) \\ Department of Chemistry \\ Memorial University of Newfoundland \\ St. John's, Newfoundland, Canada \\ March 25 $5^{\text {th }}, 2019$
}




\section{Abstract}

Traditional C-C cross-coupling reactions generally rely on the use of expensive and sometimes toxic metal catalysts, such as palladium or ruthenium. These methods generally require two functionalized reagents, which can increase reaction cost. In the interests of sustainability and reduced costs there has been considerable interest in catalysis using earth-abundant metals, such as iron, as well as in $\mathrm{C}-\mathrm{H}$ activation reactions. One example of a C-H activation reaction is the Minisci reaction, which is a radical reaction that couples $\mathrm{N}$ heteroarenes to a variety of organic species. Recently a variation of the Minisci reaction was discovered in which arylboronic acids, which are relatively cheap and easily available, are coupled to $N$-heteroarenes or quinones using either a silver or iron catalyst. This process occurs under air and at low temperatures, but requires long reaction times, has a somewhat limited scope, and is not regioselective. This thesis concerns the application of microwave heating to this reaction, in an attempt to mitigate these issues. Microwave heating has not been previously applied to the Minisci reaction, and in fact there are relatively few reports on radical reactions under microwave heating.

The reaction between pyrazine and arylboronic acids under microwave heating was first optimized. Ultimately, optimization led to similar product yields, but reactions that previously required $12-48 \mathrm{~h}$ could be conducted in $25 \mathrm{~min}$. The reaction scope was then examined. The scope of arylboronic acids when reacted with pyrazine was somewhat limited, as strong electron-withdrawing and electron-donating groups were not tolerated. 
Examination of the reaction mixtures revealed that two previously unobserved side reaction were taking place; first, a reaction in which the boronic acid moiety was replaced by a hydroxyl group, and second, $N$-heteroarene homo-coupling. The reaction scope with quinones was found to be similar to literature reports, but reactions still occurred at a much higher rate. The reaction was successfully extended to hydroquinones, but attempts to further expand scope to enones, imines, and other conjugated compounds were unsuccessful. Finally, the scope of reactions with $N$-heteroarenes was examined and was found to be similar to literature reports. The application of microwave heating did not significantly affect the regiochemical outcomes of the reaction. 


\section{Acknowledgments}

I would like to first thank my supervisor, Dr Chris Kozak. His support, his patience, and all the help and suggestions he has given me throughout my project have been invaluable. I would also like to thank Dr Francesca Kerton for her suggestions during group and committee meetings.

Thanks to the Green Chemistry and Catalysis Group for their help in and out of the lab. In particular I would like to thank Tim Anderson and Kori Andrea for showing me the ropes early in the project, for their advice and help in the lab, and for supplying several of the catalysts used in this thesis. I would also like to thank Juliana Vidal for her camaraderie and support, and for keeping things in the lab fun. In particular, I would like to thank her for demonstrating her fantastic dance moves. I cannot describe their glory in words, except to say that they are poetry in motion.

I would like to thank the staff at C-CART and in the Chemistry Department. Dr Celine Schneider provided NMR training and support. Mary Flinn, Rosalind Collins, and other staff members in the chemistry office have helped to navigate through the bureaucratic side of the process. In addition, I would like to thank Dr Liqin Chen, who graciously funded the Chen Graduate Scholarship, and NSERC for funding.

Finally, I would like to thank my family and in particular my girlfriend Kaylee. Their unwavering support throughout this project has been instrumental in getting me through with happiness, health, and sanity intact (relatively). Special thanks to Kaylee for 
patiently dealing with my complaints, doom-saying, distraction, and occasional bouts of workaholic fervor throughout the fits and starts of the project. Thanks to Kaylee again for proof-reading dozens and dozens of pages of what, to her, was probably largely incomprehensible techno-babble. 


\section{Table of Contents}

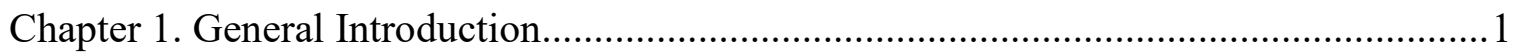

1.1 Synthesis of Heterobiaryls and Arylquinones via Radical C-H Bond Activation .....1

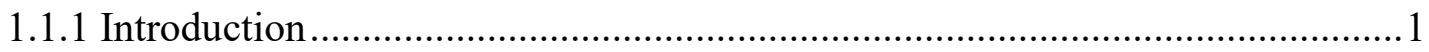

1.1.1.1 Introduction to Radical C-H Bond Activation.....................................1

1.1.1.2 $\quad N$-Heterobiaryls and Arylquinones ................................................

1.1.1.3 Radical Sources for Arylation Reactions............................................12

1.1.2 Synthesis of Biaryls and Arylquinones via Radical C-H Bond Functionalization

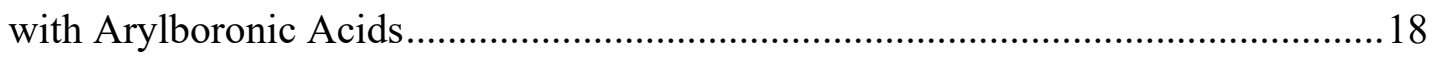

1.1.2.1 Development and Significance ..........................................................18

1.1.2.2 Mechanism of Reactions with Persulfates ............................................27

1.1.2.3 Scope and regioselectivity of reactions with $N$-heteroarenes ................31

1.1.2.4 Scope and Regioselectivity of Reactions with Quinones ......................35

1.1.2.5 Arylboronic Acid Scope ..................................................................37

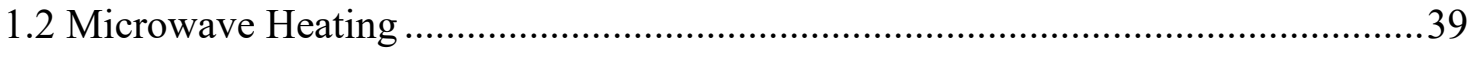

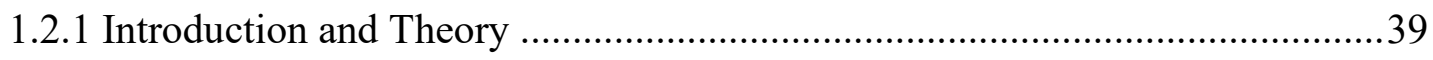

1.2.1.1 Introduction to Microwaves..................................................................39

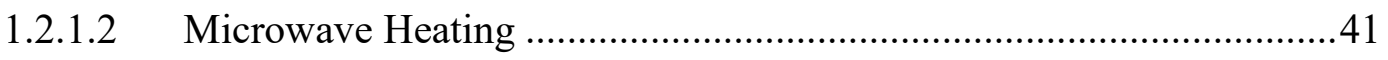


1.2.2.1 Bulk Thermal Effects and Microwave Specific Effects

1.2.2.2 Increases in Rate and Expanded Substrate Scope.................................46

1.2.2.3 Higher Yields and Cleaner Reaction Profiles .........................................49

1.2.2.4 Reduced Catalyst Loading .............................................................52

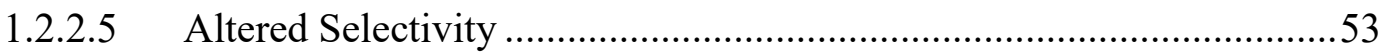

1.2.3 Organic Syntheses under Select Conditions ……………………....................55

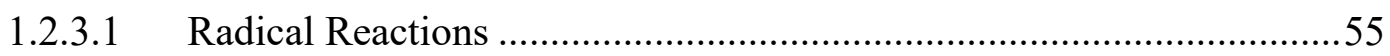

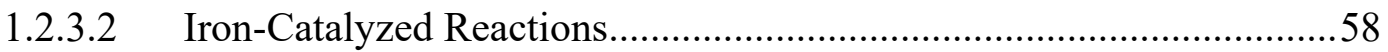

1.2.3.3 Biphasic Reactions..........................................................................64

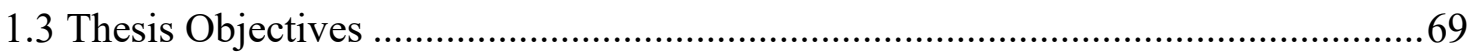

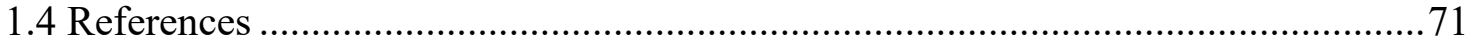

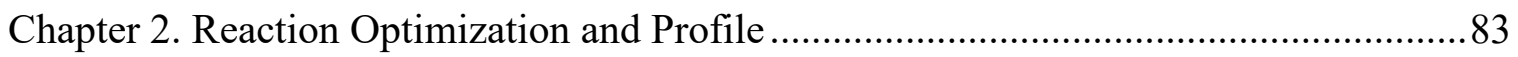

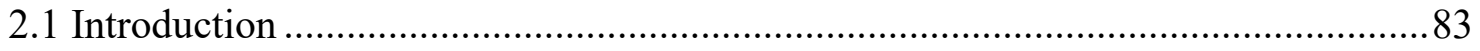

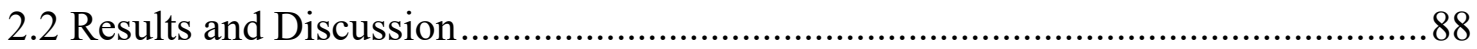

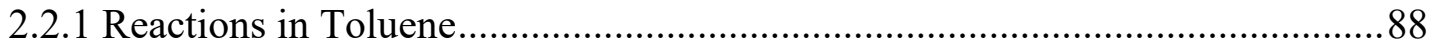

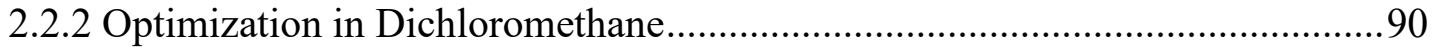

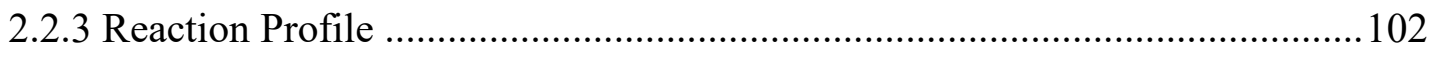

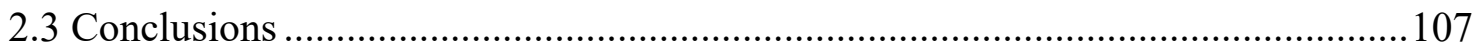




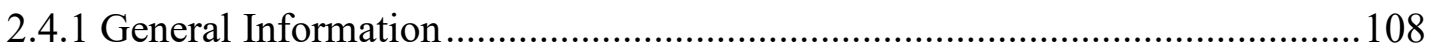

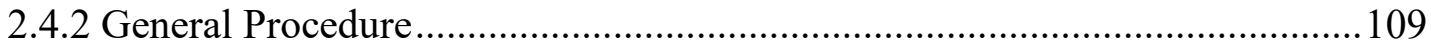

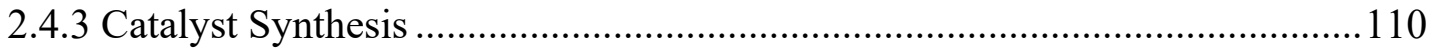

2.4.3.1 Citrate-stabilized Iron Nanoparticles ......................................... 110

2.4.3.2 Complex I ........................................................................... 111

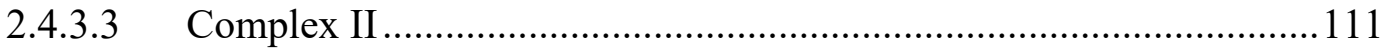

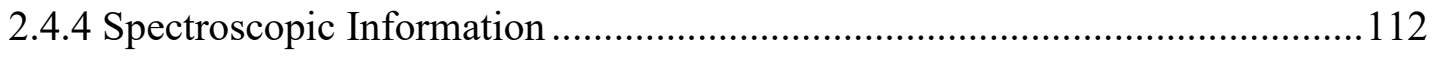

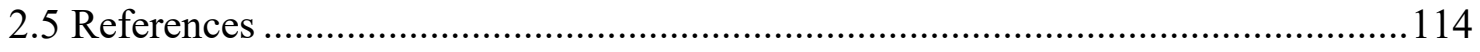

Chapter 3. Reaction Scope and Regioselectivity Under Microwave Heating .................118

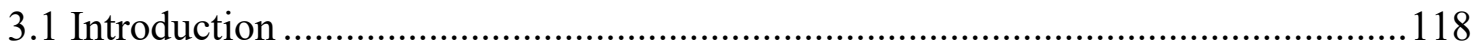

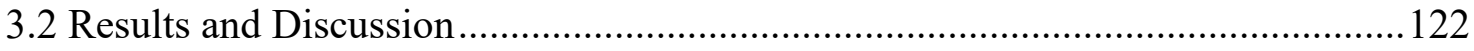

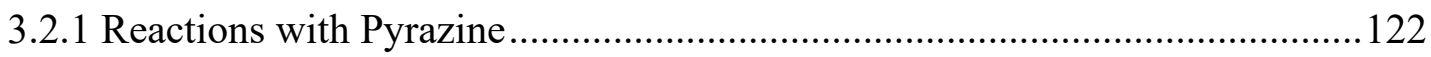

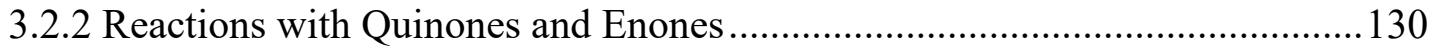

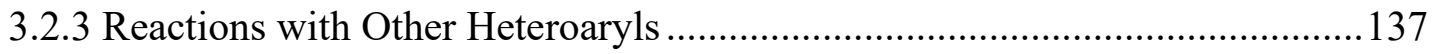

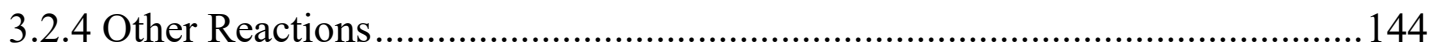

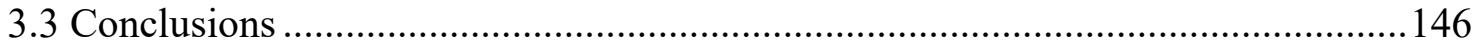

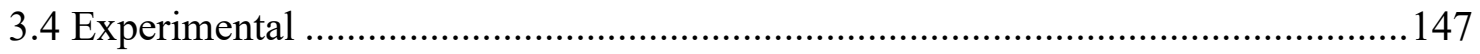

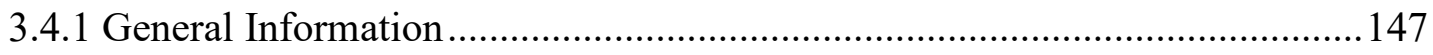


3.4.3.2 Spectroscopic Data of Phenylquinone Products

3.4.3.5 Spectroscopic Data of Products and Side Products of CDC Reactions 161

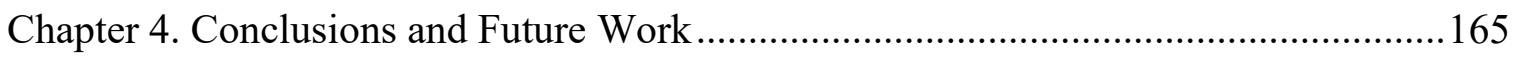

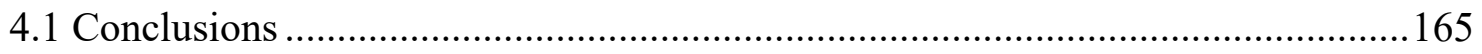

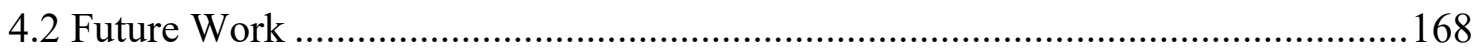

4.2.1 Future Work in This Reaction.....................................................................168

4.2.2 Microwave Heating in Other Reactions ......................................................170

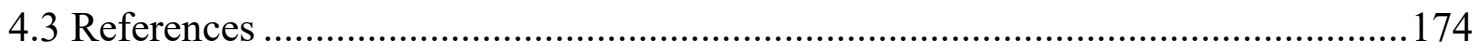

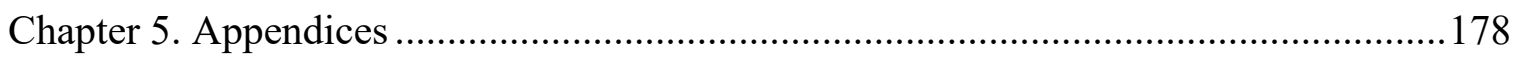




\section{List of Schemes}

Scheme 1.1 Synthesis of a product by traditional cross-coupling methods, a C-H bond activation, and a $\mathrm{CDC}$ reaction, showing the reduced number of reactions required by $\mathrm{C}-\mathrm{H}$ bond activation reactions.

Scheme 1.2 The Gomberg-Bachmann reaction, an early example of cross-coupling via radical $\mathrm{C}-\mathrm{H}$ bond activation. ${ }^{8}$

Scheme 1.3 An example of the Meerwein-type reaction, in which aryldiazonium salts are coupled to heteroarenes and olefins via a radical pathway. ${ }^{9}$

Scheme 1.4 Coupling of arylhydrazines to thiophenes and furans using manganese(III) acetate as an oxidant. ${ }^{46}$

Scheme 1.5 Coupling of pyridine with benzene using bromobenzene as an aryl radical precursor. $^{48}$

Scheme 1.6 Ruthenium-catalyzed coupling of aryliodonium salts with thiophenes, furans and pyrroles under photoirradiation. ${ }^{51}$

Scheme 1.7 Radical cross-coupling of arylboronic acids to benzene, thiophene and furan using manganese(III) acetate as an oxidant as reported by Demir and coworkers. ${ }^{55}$........20

Scheme 1.8 Conditions described by Baran and coworkers for the cross-coupling of $\mathrm{N}$ heteroarenes and quinones with arylboronic acids using potassium persulfate as a radical initiator. $^{18,53}$ .21 
Scheme 1.9 Top: Iron-catalyzed coupling of arylboronic acids with $N$-heteroarenes. ${ }^{19,20,63,64,66}$ Bottom: Iron-catalyzed coupling of arylboronic acids with quinones, ${ }^{19,20,62,64}$ hydroquinones, ${ }^{20,62,64}$ and phenols. ${ }^{40}$

Scheme 1.10 Metal and acid-free coupling of arylboronic acids to electron-deficient $N$-heteroarenes, as described by Singh and coworkers.

Scheme 1.11 The most commonly cited mechanism, first proposed by Baran and coworkers. $^{18}$

Scheme 1.12 A plausible mechanism originally suggested by Flowers and coworkers. ${ }^{68} 30$

Scheme 1.13 Regiochemistry of the cross-coupling of substituted and unsubstituted $\mathrm{N}$ heteroarenes in the persulfate initiated cross-coupling reaction with arylboronic acids. ...35

Scheme 1.14 Reagents that lead to the formation of arylquinone products in the persulfate initiated cross-coupling reaction with arylboronic acids.

Scheme 1.15 Intramolecular Diels-Alder reaction and hydrolysis of pyrazinones under microwave heating

Scheme 1.16 Alteration of reaction rate under microwave heating in the hydrogenation of alkenes .48

Scheme 1.17 C-F bond activation in a Suzuki-Miyaura coupling reaction under microwave heating

Scheme 1.18 Synthesis of cyanuric acid from urea under microwave heating .50 
Scheme 1.19 Suzuki coupling of 2-nitrophenylboronic acid and 4-bromoindole under microwave heating

Scheme 1.20 Comparison of microwave and conventional heating in the Suzuki coupling of a phenethylamine and 2-formylphenylboronic acid

Scheme 1.21 Suzuki and Heck reactions with extremely low catalyst loading under microwave heating. 53

Scheme 1.22 Alteration in selectivity in the alkylation of 1,2,4-triazole under microwave heating .54

Scheme 1.23 Alterations in chemoselectivity during the benzylation of 2-pyridone under microwave heating .55

Scheme 1.24 Radical coupling of aryl iodides and benzophenones under conventional and microwave heating

Scheme 1.25 Radical biaryl coupling of $N$-heteroarenes and aryl halides under conventional and microwave heating. .58

Scheme 1.26 Peroxide oxidation of 1-phenylethanol to acetophenone using microwave heating and homogeneous iron catalysts. Bottom left and centre: catalysts used in reference ${ }^{129}$. Bottom right: catalyst used in reference ${ }^{130}$

Scheme 1.27 Aziridination and amination reactions using a homogeneous iron catalyst under microwave heating.

Scheme 1.28 Aminocarbonylation of alkynes and amines under microwave heating using triiron dodecacarbonyl as a catalyst. 
Scheme 1.29 Rapid Hoffmann elimination under microwave heating using a differentially heated biphasic mixture

Scheme 1.30 Heck diarylation reaction, and subsequent hydrolysis in a biphasic mixture of dilute hydrochloric acid and toluene under microwave heating .66

Scheme 1.31 Oxidation of primary and secondary alcohols in a biphasic mixture of alcohol and aqueous hydrogen peroxide under microwave heating .67

Scheme 1.32 Epoxidation of olefins in a biphasic mixture of DCE and aqueous hydrogen peroxide under microwave heating .68

Scheme 1.33 Production of furfural or 5-hydroxymethylfurfural from biomass using microwave heating and a biphasic mixture

Scheme 2.1 General conditions of the cross-coupling of arylboronic acids with quinones or $N$-heteroaryls using potassium persulfate.

Scheme 2.2 General reaction mechanism for the cross-coupling of $N$-heteroaryls and quinones with arylboronic acids using silver or iron catalysts. .85

Scheme 2.3 Initial reaction conditions using a solvent mixture of toluene and water .89

Scheme 2.4 Optimized reaction conditions in a mixture of dichloromethane and water.

Scheme 2.5 Side reactions and side products observed in the GC-MS of the crude reaction mixture of pyrazine and 4-methylphenylboronic acid. 104 
Scheme 2.6 Proposed mechanism for the formation of 3 isomers of dimethylbiphenyl formed by the cross-coupling of 4-methylphenylboronic acid

Scheme 2.7 Proposed mechanism for the formation of 2-dichloromethylpyrazine and 2,2'bipyrazine. 106

Scheme 3.1 General conditions of the cross-coupling of arylboronic acids with quinones or $N$-heteroaryls using potassium persulfate.

Scheme 3.2 Yields of cross-coupling with pyrazine using a variety of arylboronic acids under standard conditions.

Scheme 3.3 Yields of cross-coupling with 1,4-benzoquinone using a variety of arylboronic acids under standard conditions.

Scheme 3.4 Yields of cross-coupling with 1,4-benzoquinone using a variety of arylboronic acids under standard conditions.

Scheme 3.5 Yields of cross-coupling of arylboronic acid with a variety of $N$-heterocycles under standard conditions..

Scheme 3.6 Iron-catalyzed radical cross-dehydrogenative coupling of toluene and 4 cyanopyridine under microwave heating.. 146

Scheme 4.1 Top: The two-electron direct Suzuki reaction described by $\mathrm{Yu}$ and coworkers $^{1,2}$. Bottom: A selection of macrocyclic polyamine (MCPA) ligands examined in the reaction. 166

Scheme 4.2 Proposed reaction to further examine the scope of the reaction with regards to substituted quinones and hydroquinones. 168 
Scheme 4.3 Proposed reaction to further examine the scope of the reaction with regards to substituted quinones and hydroquinones.

Scheme 4.4 Proposed homo-coupling or cross-coupling reaction of $N$-heteroarenes..... 172

Scheme 4.5 Top: Iron-catalyzed radical cross-coupling of aryl halides and unactivated arenes. Bottom: Radical iron-catalyzed dimerization of terminal alkynes. ...................174 


\section{List of Figures}

Figure 1.1 $N$-heteroarenes critical in the survival of all life. 5

Figure 1.2 $N$-heterobiarenes that are commonly used as dyes, semiconductors, ligands, and fibres. .8

Figure 1.3 Notable $N$-heterobiaryls in the agrochemical and pharmaceutical industries. ..9

Figure 1.4 Several common, basic quinones, which form the scaffold for the majority of more complex quinones.

Figure 1.5 Notable arylquinones from the pharmaceutical and dye industries.

Figure 1.6 Natural products, pharmaceuticals, and medicinally relevant scaffolds synthesized via radical $\mathrm{C}-\mathrm{H}$ activating cross-coupling between arylboronic acids and quinones, hydroquinones, or phenols. .26

Figure 1.7 Natural products, pharmaceuticals, and medicinally relevant scaffolds synthesized via radical C-H activating cross-coupling between arylboronic acids and $\mathrm{N}$ heterocycles.

Figure 1.8 Summary of reaction scope with respect to $N$-heteroarenes for persulfate initiated cross-coupling with arylboronic acids

Figure 1.9 Summary of reaction scope with respect to organoboron species for persulfate initiated cross-coupling with arylboronic acids

Figure 2.1 Gas chromatogram of the crude reaction mixture of the optimized reaction in toluene, showing products of toluene oxidation and homo-coupling 
Figure 2.2 Reaction yields under standardized conditions with different iron catalysts. All reactions were performed with $20 \%$ catalyst loading with respect to iron 100

Figure 2.3 Structures of the iron(III) amino-bis(phenolate) complexes I ${ }^{38}$ and II. ${ }^{39} \ldots . . .100$

Figure 2.4 Gas chromatogram of the crude reaction mixture of the optimized reaction in dichloromethane 105

Figure 3.1 Resonance structures of pyridine, demonstrating reduced electron density at the 2 and 4 positions.

Figure 3.2 GC traces of crude reaction mixtures after attempted cross-coupling between 4-chlorophenylboronic acid and pyrazine (top), and 4 (trifluoromethyl)phenylboronic acid and pyrazine (bottom)

Figure 3.3 GC traces of crude reaction mixtures after attempted cross-coupling between phenylboronic acid and 1,4-benzoquinone (top), and 4 (trifluoromethyl)phenylboronic acid and 1,4-benzoquinone (bottom) 


\section{List of Tables}

Table 1.1 Loss factors $(\tan \delta)$ of solvents and other relevant materials ${ }^{\mathrm{a}}$

Table 2.1 Optimization of acid amount and identity

Table 2.2 Optimization of reaction temperature.

Table 2.3 Optimization of reaction time.

Table 2.4 Optimization of reagent stoichiometry. .95

Table 2.5 Optimization of reaction concentration .96

Table 2.6 Screening of solvent mixtures. .98

Table 2.7 Comparison of reaction conditions and yields. 102

Table 3.1 Reoptimization of conditions for the persulfate-initiated cross-coupling of pyrazine and 4-chlorophenylboronic acid.

Table 3.2 Comparison of reaction conditions and yields of the radical coupling of 1,4benzoquinone and phenylboronic acid.

Table 3.3 Comparison of conditions, yields, and regiochemistry in the cross-coupling of phenylboronic acid and 4-cyanopyridine.

Table 3.4 Comparison of conditions, yields, and regiochemistry in the cross-coupling of phenylboronic acid and pyridine

Table 3.5 Comparison of conditions, yields, and regiochemistry in the cross-coupling of phenylboronic acid and quinoline 
Table 3.6 Comparison of conditions, yields, and regiochemistry in the cross-coupling of phenylboronic acid and pyridazine. . 


\section{List of Appendices}

Figure A1 ${ }^{1} \mathrm{H}$ NMR spectrum of 2-phenylpyrazine 178

Figure $\mathrm{A2}^{13} \mathrm{C}$ NMR spectrum of 2-phenylpyrazine

Figure A3 DEPT NMR spectrum of 2-phenylpyrazine 180

Figure A4 Gas chromatogram and mass spectrum of 2-phenylpyrazine

Figure $\mathbf{A 5}^{1} \mathrm{H}$ NMR spectrum of 2-(4-methylphenyl)pyrazine 182

Figure $\mathrm{A6}^{13} \mathrm{C}$ NMR spectrum of 2-(4-methylphenyl)pyrazine 183

Figure A7 DEPT NMR spectrum of 2-(4-methylphenyl)pyrazine 184

Figure A8 Gas chromatogram and mass spectrum of 2-(4-methylphenyl)pyrazine 185

Figure A9 ${ }^{1} \mathrm{H}$ NMR spectrum of 2-(3-methylphenyl)pyrazine 186

Figure A10 ${ }^{13} \mathrm{C}$ NMR spectrum of 2-(3-methylphenyl)pyrazine 187

Figure A11 DEPT NMR spectrum of 2-(3-methylphenyl)pyrazine 188

Figure A12 Gas chromatogram and mass spectrum of 2-(3-methylphenyl)pyrazine..

Figure A13 ${ }^{1} \mathrm{H}$ NMR spectrum of 2-(2-methylphenyl)pyrazine 190

Figure $\mathrm{A14}{ }^{13} \mathrm{C}$ NMR spectrum of 2-(2-methylphenyl)pyrazine 191

Figure A15 DEPT NMR spectrum of 2-(2-methylphenyl)pyrazine

Figure A16 Gas chromatogram and mass spectrum of 2-(2-methylphenyl)pyrazine

Figure A17 ${ }^{1} \mathrm{H}$ NMR spectrum of 2-(4-chlorophenyl)pyrazine 194 
Figure $\mathbf{A 1 8}{ }^{13} \mathrm{C}$ NMR spectrum of 2-(4-chlorophenyl)pyrazine 195

Figure A19 DEPT NMR spectrum of 2-(4-chlorophenyl)pyrazine 196

Figure A20 Gas chromatogram and mass spectrum of 2-(4-chlorophenyl)pyrazine

Figure A21 ${ }^{1} \mathrm{H}$ NMR spectrum of 2-(3-chlorophenyl)pyrazine 198

Figure A22 ${ }^{13} \mathrm{C}$ NMR spectrum of 2-(3-chlorophenyl)pyrazine 199

Figure A23 DEPT NMR spectrum of 2-(3-chlorophenyl)pyrazine .200

Figure A24 Gas chromatogram and mass spectrum of 2-(3-chlorophenyl)pyrazine ......201

Figure A25 ${ }^{1} \mathrm{H}$ NMR spectrum of 2-(2-chlorophenyl)pyrazine .202

Figure A26 ${ }^{13} \mathrm{C}$ NMR spectrum of 2-(2-chlorophenyl)pyrazine .203

Figure A27 DEPT NMR spectrum of 2-(2-chlorophenyl)pyrazine .204

Figure A28 Gas chromatogram and mass spectrum of 2-(2-chlorophenyl)pyrazine ......205

Figure A29 ${ }^{1} \mathrm{H}$ NMR spectrum of 2-(4-iodophenyl)pyrazine .206

Figure A30 ${ }^{13} \mathrm{C}$ NMR spectrum of 2-(4-iodophenyl)pyrazine .207

Figure A31 DEPT NMR spectrum of 2-(4-iodophenyl)pyrazine .208

Figure A32 Gas chromatogram and mass spectrum of 2-(4-iodophenyl)pyrazine .209

Figure A33 Gas chromatogram and mass spectrum of 2-(4-trifluoromethylphenyl)pyrazine .210

Figure A34 Gas chromatogram and mass spectrum of 2-(4-methoxyphenyl)pyrazine ..211 
Figure A35 Gas chromatogram of the crude reaction mixture of mesitylboronic acid and pyrazine (top). Mass spectrum of 2-mesitylpyrazine (bottom).

Figure A36 Gas chromatogram of the crude reaction mixture of 4-cyanophenylboronic acid and pyrazine (top). Mass spectrum of 2-(4-cyanophenyl)pyrazine (bottom). 213

Figure A37 Gas chromatogram of the crude reaction mixture of 4-formylboronic acid and pyrazine (top). Mass spectrum of 2-(4-formylphenyl)pyrazine (bottom). 214

Figure A38 ${ }^{1} \mathrm{H}$ NMR spectrum of 2-phenyl-1,4-benzoquinone 215

Figure A39 ${ }^{13} \mathrm{C}$ NMR spectrum of 2-phenyl-1,4-benzoquinone. 216

Figure A40 DEPT NMR spectrum of 2-phenyl-1,4-benzoquinone 217

Figure A41 Gas chromatogram and mass spectrum of 2-phenyl-1,4-benzoquinone......219

Figure A42 ${ }^{1} \mathrm{H}$ NMR spectrum of 2-(4-chlorophenyl)-1,4-benzoquinone .220

Figure $\mathrm{A} 43{ }^{13} \mathrm{C}$ NMR spectrum of 2-(4-chlorophenyl)-1,4-benzoquinone .221

Figure A44 DEPT NMR spectrum of 2-(4-chlorophenyl)-1,4-benzoquinone..... .222

Figure A45 Gas chromatogram and mass spectrum of 2-(4-chlorophenyl)-1,4-benzoquinone.

Figure A46 ${ }^{1} \mathrm{H}$ NMR spectrum of 2-(3-chlorophenyl)-1,4-benzoquinone .224

Figure A47 ${ }^{13} \mathrm{C}$ NMR spectrum of 2-(3-chlorophenyl)-1,4-benzoquinone .225

Figure A48 DEPT NMR spectrum of 2-(3-chlorophenyl)-1,4-benzoquinone. .226 
Figure A49 Gas chromatogram and mass spectrum of 2-(3-chlorophenyl)-1,4-benzoquinone.....

Figure A50 ${ }^{1} \mathrm{H}$ NMR spectrum of 2-(2-chlorophenyl)-1,4-benzoquinone.....................228

Figure A51 ${ }^{13} \mathrm{C}$ NMR spectrum of 2-(2-chlorophenyl)-1,4-benzoquinone ...................229

Figure A52 DEPT NMR spectrum of 2-(2-chlorophenyl)-1,4-benzoquinone...............230

Figure $\mathbf{A 5 3}$ Gas chromatogram and mass spectrum of 2-(2-chlorophenyl)-1,4-benzoquinone

Figure A54 ${ }^{1} \mathrm{H}$ NMR spectrum of 2-(4-methylphenyl)-1,4-benzoquinone....................232

Figure $\mathbf{A 5 5}{ }^{13} \mathrm{C}$ NMR spectrum of 2-(4-methylphenyl)-1,4-benzoquinone ...................233

Figure A56 DEPT NMR spectrum of 2-(4-methylphenyl)-1,4-benzoquinone..............234

Figure A 57 Gas chromatogram and mass spectrum of

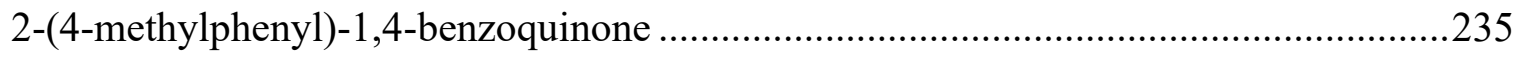

Figure A58 ${ }^{1} \mathrm{H}$ NMR spectrum of 2-(2-methylphenyl)-1,4-benzoquinone....................236

Figure A59 ${ }^{13} \mathrm{C}$ NMR spectrum of 2-(2-methylphenyl)-1,4-benzoquinone ..................237

Figure A60 DEPT NMR spectrum of 2-(2-methylphenyl)-1,4-benzoquinone..............238

Figure A61 Gas chromatogram and mass spectrum of

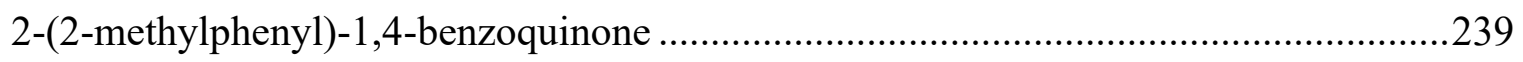

Figure A62 ${ }^{1} \mathrm{H}$ NMR spectrum of 2-mesityl-1,4-benzoquinone .................................240

Figure $\mathbf{A 6 3}{ }^{13} \mathrm{C}$ NMR spectrum of 2-mesityl-1,4-benzoquinone...............................241 
Figure A64 DEPT NMR spectrum of 2-mesityl-1,4-benzoquinone .242

Figure A65 Gas chromatogram and mass spectrum of 2-mesityl-1,4-benzoquinone.....243

Figure A66 ${ }^{1} \mathrm{H}$ NMR spectrum of 2-(4-trifluoromethylphenyl)-1,4-benzoquinone .......244

Figure A67 ${ }^{13} \mathrm{C}$ NMR spectrum of 2-(4-trifluoromethylphenyl)-1,4-benzoquinone ......245

Figure A68 DEPT NMR spectrum of 2-(4-trifluoromethylphenyl)-1,4-benzoquinone..246

Figure A69 Gas chromatogram and mass spectrum of

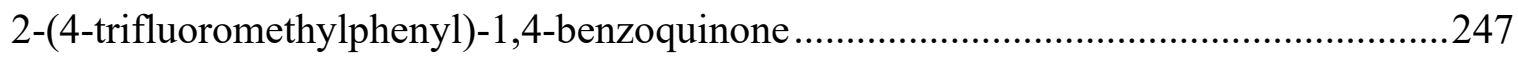

Figure A70 ${ }^{1} \mathrm{H}$ NMR spectrum of a mixture of 2,3-dimethyl-5-phenyl-1,4-benzoquinone and the starting product, 2,3-dimethyl-1,4-benzoquinone .248

Figure A71 ${ }^{13} \mathrm{C}$ NMR spectrum of a mixture of 2,3-dimethyl-5-phenyl-1,4-benzoquinone and 2,3-dimethyl-1,4-benzoquinone

Figure A72 DEPT NMR spectrum of a mixture of 2,3-dimethyl-5-phenyl-1,4-benzoquinone and 2,3-dimethyl-1,4-benzoquinone .............250

Figure A73 Gas chromatogram and mass spectrum of a mixture of 2,3-dimethyl-5-phenyl-1,4-benzoquinone and 2,3-dimethyl-1,4-benzoquinone .251

Figure A74 ${ }^{1} \mathrm{H}$ NMR spectrum of 2-phenyl-4-cyanopyridine .252

Figure A75 ${ }^{13} \mathrm{C}$ NMR spectrum of 2-phenyl-4-cyanopyridine .253

Figure A76 DEPT NMR spectrum of 2-phenyl-4-cyanopyridine. .254

Figure A77 Gas chromatogram and mass spectrum of 2-phenyl-4-cyanopyridine .255 
Figure A78 ${ }^{1} \mathrm{H}$ NMR spectrum of 3-phenyl-4-cyanopyridine .256

Figure A79 ${ }^{13} \mathrm{C}$ NMR spectrum of 3-phenyl-4-cyanopyridine 257

Figure A80 DEPT NMR spectrum of 3-phenyl-4-cyanopyridine. .258

Figure A81 Gas chromatogram and mass spectrum of 3-phenyl-4-cyanopyridine .259

Figure A82 ${ }^{1} \mathrm{H}$ NMR spectrum of 3-phenylpyridazine .260

Figure $\mathbf{A 8 3}{ }^{13} \mathrm{C}$ NMR spectrum of 3-phenylpyridazine. .261

Figure A84 DEPT NMR spectrum of 3-phenylpyridazine .262

Figure A85 Gas chromatogram and mass spectrum of 3-phenylpyridazine 263

Figure $\mathbf{A 8 6}{ }^{1} \mathrm{H}$ NMR spectrum of 4-phenylpyridazine .264

Figure $\mathbf{A 8 7}{ }^{13} \mathrm{C}$ NMR spectrum of 4-phenylpyridazine .265

Figure A88 DEPT NMR spectrum of 4-phenylpyridazine .266

Figure A89 Gas chromatogram and mass spectrum of 4-phenylpyridazine .267

Figure A90 ${ }^{1} \mathrm{H}$ NMR spectrum of 2-phenylquinoline .268

Figure A91 ${ }^{13} \mathrm{C}$ NMR spectrum of 2-phenylquinoline. .269

Figure A92 DEPT NMR 2-phenylquinoline .270

Figure A93 Gas chromatogram and mass spectrum of 2-phenylquinoline. .271

Figure A94 ${ }^{1} \mathrm{H}$ NMR spectrum of 4-phenylquinoline .272

Figure A95 ${ }^{13} \mathrm{C}$ NMR spectrum of 4-phenylquinoline.

Figure A96 DEPT NMR spectrum of 4-phenylquinoline .274 
Figure A97 Gas chromatogram and mass spectrum of 4-phenylquinoline. .275

Figure A98 ${ }^{1} \mathrm{H}$ NMR spectrum of 3-phenyllutidine .276

Figure A99 ${ }^{13} \mathrm{C}$ NMR spectrum of 3-phenyllutidine .277

Figure A100 DEPT NMR spectrum of 3-phenyllutidine. .278

Figure A101 Gas chromatogram and mass spectrum of 3-phenyllutidine. 279

Figure A102 ${ }^{1} \mathrm{H}$ NMR spectrum of 4-phenyllutidine .280

Figure $\mathbf{A 1 0 3}{ }^{13} \mathrm{C}$ NMR spectrum of 4-phenyllutidine .281

Figure A104 DEPT NMR spectrum of 4-phenyllutidine. 282

Figure A105 Gas chromatogram and mass spectrum of 4-phenyllutidine. .283

Figure A106 ${ }^{1} \mathrm{H}$ NMR spectrum of 2-phenylpyridine .284

Figure $\mathbf{A 1 0 7}{ }^{13} \mathrm{C}$ NMR spectrum of 2-phenylpyridine .285

Figure A108 DEPT NMR spectrum of 2-phenylpyridine .286

Figure A109 Gas chromatogram and mass spectrum of 2-phenylpyridine .287

Figure $\mathbf{A 1 1 0}{ }^{1} \mathrm{H}$ NMR spectrum of 4-phenylpyridine .288

Figure A111 ${ }^{13} \mathrm{C}$ NMR spectrum of 4-phenylpyridine .289

Figure A112 DEPT NMR spectrum of 4-phenylpyridine .290

Figure A113 Gas chromatogram and mass spectrum of 4-phenylpyridine .291

Figure A114 ${ }^{1} \mathrm{H}$ NMR spectrum of 1-methyl-2-phenylimidazole 292

Figure $\mathbf{A 1 1 5}{ }^{13} \mathrm{C}$ NMR spectrum of 1-methyl-2-phenylimidazole .293 
Figure A116 DEPT NMR spectrum of 1-methyl-2-phenylimidazole .294

Figure A117 Gas chromatogram and mass spectrum of 1-methyl-2-phenylimidazole ..295

Figure A118 Gas chromatogram of the raw reaction mixture of the reaction of 2-bromopyridine and phenylboronic acid (top). Mass spectrum of 2-bromophenylpyridine, isomer unknown (bottom). 296

Figure A119 Gas chromatogram of the crude reaction mixture of the reaction with 4-methlphenylboronic acid and pyrazine using toluene and water as solvent..... .298

Figure A120 Gas chromatogram of the crude reaction mixture of the reaction with 4-methylphenylboronic acid and pyrazine under optimized conditions. 300

Figure A121 Gas chromatogram of the crude reaction mixture of the reaction with 4-chlorophenylboronic acid and pyrazine under optimized conditions. 303

Figure A122 Gas chromatogram of the crude reaction mixture of the reaction with 4-trifluoromethylphenylboronic acid and pyrazine under optimized conditions. .305

Figure A123 Gas chromatogram of the crude reaction mixture of the reaction with phenylboronic acid and quinone under optimized conditions (top). Mass spectrum of 2-phenylquinone (bottom). .307

Figure A124 Gas chromatogram of the crude reaction mixture of the reaction with 4-trifluoromethlphenylboronic acid and quinone under optimized conditions (top).......309

Figure A125 Gas chromatogram of the crude reaction mixture of the reaction of quinone and toluene (top). Mass spectrum of the desired product, 2-benzyl-1,4-benzoquinone (bottom) 311 
Figure A126 Gas chromatogram of the crude reaction mixture of the reaction of

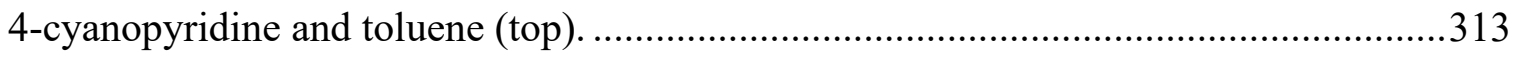




\title{
List of Abbreviations
}

\author{
acac - acetylacetonate \\ bmim - 1-butyl-3-methylimidazolium cation \\ CDC - Cross-dehydrogenative coupling \\ $\mathrm{CH}-$ conventional heating \\ DEPT - Distortionless enhancement by polarization transfer \\ EDG - electron donating group \\ EWG - electron withdrawing group \\ GC-MS - gas chromatography mass spectroscopy \\ $\mathrm{h}-$ hour \\ $\mathrm{m} / \mathrm{z}$ - mass-to-charge ratio \\ MCPA macrocyclic polyamine \\ $\min -$ minutes \\ $\mathrm{mL}-$ millilitre \\ mmol - millimole \\ mol - mole \\ MW - microwave \\ NMR - nuclear magnetic resonance
}


ox - oxalate

ppm - parts per million

$\mathrm{RT}$ - room temperature

TBAB - tetrabutylammonium bromide

${ }^{t} \mathrm{BuOOH}$ - tert-butyl hydrogen peroxide

TFA - trifluoroacetic acid

THF - tetrahydrofuran

TsOH - para-toluenesulfonic acid 


\section{Chapter 1. General Introduction}

\subsection{Synthesis of Heterobiaryls and Arylquinones via Radical C-H Bond Activation}

\subsubsection{Introduction}

\subsubsection{Introduction to Radical C-H Bond Activation}

$\mathrm{C}-\mathrm{H}$ bond activation refers to a reaction in which the typically relatively inert carbon-hydrogen bond is cleaved in order to form a C-X bond. ${ }^{1-3}$ Some $\mathrm{C}-\mathrm{H}$ bond activation reactions, such as alkane halogenations, are probably familiar to the majority of chemists, and are easily accomplished. Cross-coupling reactions that form C-C, C-N, or C-O bonds, on the other hand, are typically more challenging. Most commonly these reactions involve one reagent bearing a functional group that is cleaved during the reaction, and one reagent in which a $\mathrm{C}-\mathrm{H}$ bond is cleaved. ${ }^{1-5}$ Less common are cross-dehydrogenative-coupling (CDC) reactions, in which a $\mathrm{C}-\mathrm{H}$ bond is cleaved from each reagent. Both types of reaction have received a significant amount of attention due to their high atom and step economy, which in theory minimizes waste and the amount of time required to synthesize a given product (Scheme 1.1). ${ }^{1-5}$ This is because traditional cross-coupling methods usually require two functionalized reagents. For example, Suzuki coupling requires one reagent to have a boronic acid moiety, and the other a halide moiety. These groups must be installed before the Suzuki reaction can occur, meaning that at least two additional reactions are necessary before the desired Suzuki coupling. When relatively simple reagents are used, these 
reactions can be resource- and time-consuming, and may produce a significant amount of waste, but are usually synthetically simple and afford good yields. However, functionalization of complex or otherwise challenging starting materials may prove to be difficult, and constitute a significant drain on resources and time.

\section{CDC reaction}

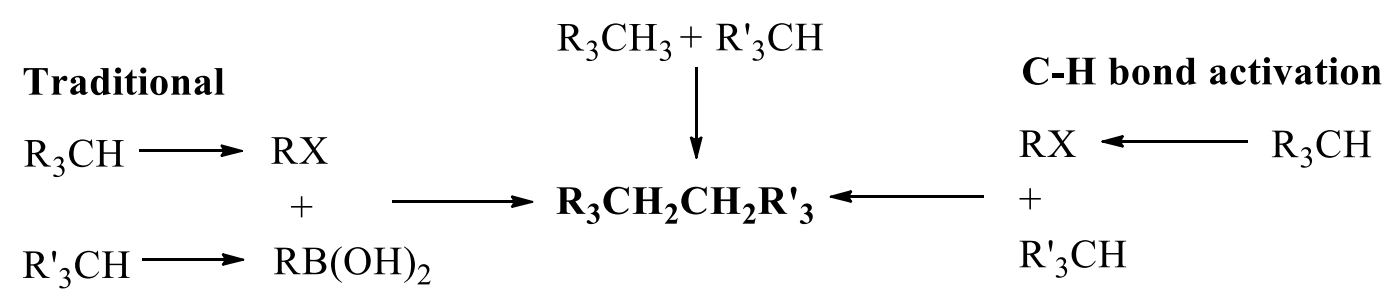

Scheme 1.1 Synthesis of a product by traditional cross-coupling methods, a C-H bond activation, and a $\mathrm{CDC}$ reaction, showing the reduced number of reactions required by $\mathrm{C}$ $\mathrm{H}$ bond activation reactions.

The majority of $\mathrm{C}-\mathrm{H}$ bond activation reactions are accomplished by organometallic, two-electron processes, in which a carbon-metal bond is formed. ${ }^{1-5}$ Palladium catalysts generally dominate the area, although rhodium, ruthenium, and iridium catalysts are also common. However, these metals are relatively expensive, and in several cases there are concerns about their toxicity; in addition, these catalysts often require complex and synthetically challenging ligands. ${ }^{1-5}$ There has therefore been significant interest in developing $\mathrm{C}-\mathrm{H}$ bond activation reactions that employ more cost-effective and less toxic metals such as copper, nickel, or iron, or even in developing catalyst-free processes. One method that has been investigated in order to accomplish this goal is radical $\mathrm{C}-\mathrm{H}$ bond 
activation. ${ }^{4,6,7}$ In these reactions, a radical is formed either by the cleavage of the C-H bond or the cleavage of a functional group, and coupling then occurs via a single-electron transfer pathway. These reactions often do not require the use of a precious metal catalyst, and in some cases do not require any catalyst. ${ }^{4,6,7}$

Descriptions of cross-coupling via radical C-H bond activation dates back as far as 1924, when Gomberg and Bachmann were able to couple an aryldiazonium salt with an unactivated arene via a radical intermediate (Scheme 1.2). ${ }^{8}$ While several other radical C$\mathrm{H}$ bond activating cross-coupling reactions were developed in the ensuing years, a great deal of research and development in this area has occurred in the past several decades. ${ }^{4,6,7}$ The number and scope of radical C-H bond activating cross-coupling reactions is now vast, and includes the coupling of alkanes, alkenes, alkynes, arenes, heteroarenes, alcohols, aldehydes, thiols, amines and more. ${ }^{4,6,7}$ The number of methods used to form radicals has also grown - currently, most radicals are formed in one of four ways; by cleavage of an unstable functional group; by irradiation with light; by the addition of an oxidant or reductant; or by the addition of a metal catalyst. Occasionally, radicals are also formed via electrochemistry. ${ }^{4,6,7}$

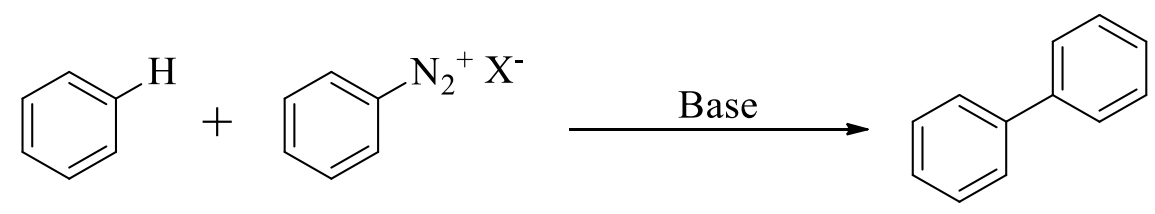

Scheme 1.2 The Gomberg-Bachmann reaction, an early example of cross-coupling via radical $\mathrm{C}-\mathrm{H}$ bond activation. ${ }^{8}$ 
Given the diversity and proliferation of radical C-H bond activating cross-coupling reactions, a review of the literature is beyond the scope of this thesis. For further information, the reader should refer to one of several excellent reviews on the subject. ${ }^{4,6,7}$ Instead, this chapter will focus exclusively on arylation and heteroarylation reactions. Even among this class of reactions, there exists a diverse array of radical sources, coupling partners, and reaction conditions..$^{9-11}$ The aim of the following sections is therefore not to provide a complete review of the available literature, but instead to highlight notable examples and examine their advantages and disadvantages.

\subsubsection{N-Heterobiaryls and Arylquinones}

$\mathrm{N}$-heteroarenes are aromatic compounds where one or more nitrogen atoms are included in the aromatic system. They are most commonly five- or six-membered rings, and may include one to four nitrogen atoms, and in some cases other heteroatoms, most notably oxygen and sulfur. ${ }^{12} \mathrm{~N}$-heteroarenes are aromatic, and are therefore usually somewhat more stable than analogous $\mathrm{N}$-heterocycles. Six-membered $\mathrm{N}$-heteroarenes differ from analogous aromatic hydrocarbons in that they are non-symmetric, possess a strong dipole, have an electron pair perpendicular to the aromatic pi orbitals, and are generally electron-deficient at the 2 and 4 positions due to inductive polarisation. ${ }^{12}$ The chemistry of five-membered $\mathrm{N}$-heteroarenes are more difficult to generalize, but often they possess a second nitrogen which has a lone pair that contributes to aromaticity, and the carbons are electron-rich. ${ }^{12} N$-heteroarenes are widely used as bases and ligands, and are important substructures in dyes, plastics, semiconductors, pharmaceuticals, and biochemistry. ${ }^{13-16} \mathrm{~N}$-heteroarenes are in fact vital in biochemistry, as they are found in 
many critical biological molecules, including the amino acids histidine and tryptophan; vitamins such as niacin (vitamin $\left.\mathrm{B}_{3}\right)$; $\mathrm{NADP}^{+}$and $\mathrm{NADPH}$, used in all cellular respiration; the heme macrocycle, responsible for oxygen transport in the blood; the purine bases adenine and guanine, found in DNA and RNA; and many more (Figure 1.1) ${ }^{17}$<smiles>N[C@@H](Cc1c[nH]c2ccccc12)C(=O)O</smiles>

Tryptophan

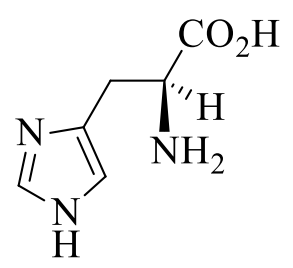

Histidine<smiles>NC(=O)c1cccnc1</smiles>

Niacin<smiles></smiles>

Heme<smiles>Nc1ncnc2[nH]cnc12</smiles>

Adenine<smiles>Nc1nc2[nH]cnc2c(=O)[nH]1</smiles>

Guanine

Figure 1.1 $N$-heteroarenes critical in the survival of all life.

$\mathrm{N}$-heterobiaryls are compounds that contain one or more separate aromatic moieties bound directly to an $N$-heteroarene; this does not include bicyclic structures such as quinoline or indole. Historically, $N$-heterobiaryls have been synthesize using aryl halides and heteroarylmetal compounds such as heteroarylzinc, heteroaryltin, heteroarylsilicon, 
and heteroaryl Grignard reagents. ${ }^{9-11,13,18-21}$ These reactions are still useful tools, but have fallen out of favor due to their cost and poor atom-economy. Suzuki reactions have been employed in the formation of $N$-heterobiaryls, but are limited due to difficulties in synthesizing $N$-heteroarene reagents with the appropriate regiochemistry, particularly given some $N$-heteroarenes sensitivity to acids and bases..$^{9-11,13,18-21}$ In addition, many $N$ heteroarenes are able to act as ligands on precious metal catalysts, interrupting the catalytic cycle and causing the formation of side products. There has been a considerable amount of interest in direct metal-mediated $\mathrm{C}-\mathrm{H}$ bond activating arylation of $N$-heteraorenes. ${ }^{9-11,13,18-}$ 21 The most common systems employ palladium, ruthenium, iron, and copper salts to couple $N$-heteroarenes with haloarenes. ${ }^{9-11,13,18-21}$ While these systems have better atomeconomy and may be more cost-efficient, they still often require complex ligands and harsh conditions. ${ }^{9-11,13,18-21}$ Finally, there has been considerable interest in developing C-H bond activating reaction using aryl radicals (see Sections 1.1.1.3 and 1.1.2).

Like $N$-heteroarenes in general, $N$-heterobiaryls are important as dyes, polymer building blocks, in electronic applications, in chemistry as ligands, and as pharmaceuticals. The most significant $N$-heterobiaryl dye is probably thioflavin, which is widely used as a histology stain in studies of protein aggregation (Figure 1.2). ${ }^{22}$ There are a number of other $N$-heterobiaryl dyes, but most find only niche applications. ${ }^{14} \mathrm{~N}$-heterobiaryls have been important polymer building blocks since at least the 1950s, when high-performance polybenzimidazole fibres were first discovered. ${ }^{23}$ Polybenzimidazole exhibits high thermal stability and toughness, and is still used in high-performance protective gear, including space suits. As electronics, $N$-heterobiaryls are important as conducting or semi-conducting 
polymers, mostly based on polypyrrole. ${ }^{23} \mathrm{~N}$-heterobiaryls are an extremely common class of ligand; significant examples include various bipyridine ligands, tripyridine ligands, and many porphyrin-based ligands. ${ }^{13,24}$ The most significant use of $N$-heterobiaryls, however, occurs in the pharmaceutical and agrochemical industries. In the agrochemical industry several herbicides are based on $N$-heterobiaryl moieties. ${ }^{23,25}$ Most notable are paraquat, one of the most widely utilized herbicides, and difenzoquat (Figure 1.3). ${ }^{23,25}$ In the pharmaceutical industry, $N$-heterobiarenes make up some of the most widely used pharmaceuticals. ${ }^{16,26-28}$ Notable $N$-heterobiaryl pharmaceuticals include atorvastatin (Lipitor), a cholesterol-reducing drug; celecoxib (Celebrex), a non-steroidal antiinflammatory; etoricoxib, used to treat arthritis and acute pain; zolpidem (Ambien), a sedative; imatinib, used to treat myeloid leukemia and GI tumours; enasidenib (Idhifa), used to treat myeloid leukemia; pyrimethamine (Daraprim), an antimalarial and antiparasitic; flutemetamol (Vizamyl) used as a PET scanning radiopharmaceutical for the diagnosis of Alzheimer's disease (Figure 1.3). ${ }^{16,26-28}$ 


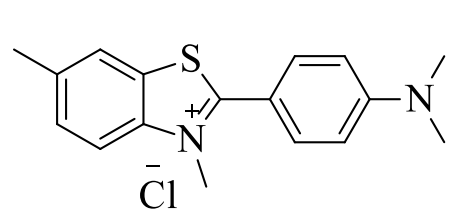

Thioflavin $\mathrm{T}$

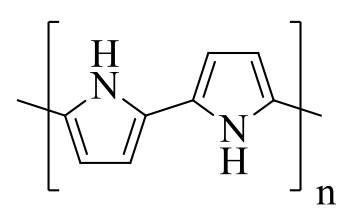

polypyrrole<smiles>c1ccc(-c2ccccn2)nc1</smiles>

2,2'-bipyridine

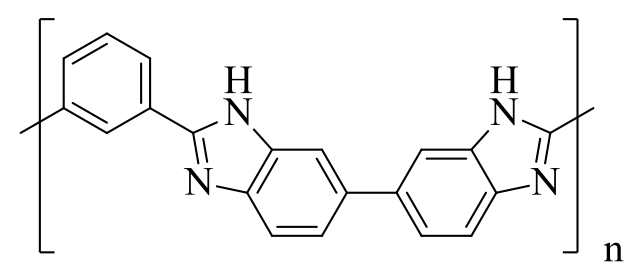

polybenzimidazole

Figure 1.2 $N$-heterobiarenes that are commonly used as dyes, semiconductors, ligands, and fibres. 
<smiles>C[n+]1ccc(-c2cc[n+](C)cc2)cc1</smiles>

Paraquat<smiles>Cc1ccc(-c2ncc(Cl)cc2-c2ccc(S(C)(=O)=O)cc2)cn1</smiles>

Etoricoxib<smiles>Cn1c(-c2ccccc2)cc(-c2ccccc2)[n+]1C</smiles>

Difenzoquat<smiles>Cc1ccc(-c2nc3ccc(C)cn3c2CC(=O)N(C)C)cc1</smiles>

Zolpidem<smiles>CNc1ccc(-c2nc3ccc(O)cc3s2)cc1F</smiles>

Flutemetamol<smiles>CC(C)c1c(C(=O)Nc2ccccc2)c(-c2ccccc2)c(-c2ccc(F)cc2)n1CC[C@H](O)C[C@H](O)CC(=O)O</smiles>

Atorvastatin<smiles>Cc1ccc(NC(=O)c2ccc(CN3CCN(C)CC3)cc2)cc1Nc1nccc(-c2cccnc2)n1</smiles>

Imatinib<smiles>Cc1ccc(-c2cc(C(F)(F)F)nn2-c2ccc(S(N)(=O)=O)cc2)cc1</smiles>

Celecoxib<smiles>CC(C)(O)CNc1nc(Nc2ccnc(C(F)(F)F)c2)nc(-c2cccc(C(F)(F)F)n2)n1</smiles>

Enasidenib

Figure 1.3 Notable $N$-heterobiaryls in the agrochemical and pharmaceutical industries. 
Quinones are a class of compounds that contain a cyclic, conjugated dione structure (Figure 1.4)..$^{29,30}$ Most commonly quinones are based on 1,2-benzoquinone or 1,4benzoquinone (sometimes referred to as simply benzoquinone or quinone). Chemically, quinones are multifunctional, exhibiting properties of alkenes and ketones. They also commonly serve as hydrogen acceptors and oxidants, as they can be reduced to the semiquinone anion or to hydroquinones. ${ }^{29,30}$ Quinones are ubiquitous in nature, and are found in plants, animals, fungi, and bacteria. ${ }^{29,31-33}$ Due to their role as an oxidant and electron transfer agent they play roles in cellular respiration, electron transport, cellular defence, and photosynthesis. Quinones have also been implicated in a variety of diseases, as they may be cytotoxic, and can cause or contribute to oxidative stress, neuronal degeneration, and certain cancers. ${ }^{29,31-33}$ Quinones are extremely common in pharmaceuticals, where they find widespread use as fungicides and anticancer, antimalarial, and antibacterial drugs. ${ }^{29,31-33}$ Quinones are also common in the dye industry, where they have been employed for thousands of years as pigments such as henna (lawsone) and the red pigment alizarin. ${ }^{34}$ More recently, quinone dyes have been used as food dyes, fluorophores and infrared-absorbing moieties, often for medical imaging applications. ${ }^{35-37}$ Finally, quinones are widely used in electrochemistry. 1,4-Benzoquinone forms part of the quinhydrone electrode which has been used to measure $\mathrm{pH}$ since $1921 .{ }^{38}$ Currently research is being conducted into the use of quinones in rechargeable batteries, phototransistors, and solar cells. ${ }^{39}$ 
<smiles>O=C1C=CC(=O)C=C1</smiles>

1,4-benzoquinone 1,2-benzoquinone<smiles>O=C1C=CC(=O)c2ccccc21</smiles>

1,4-naphthoquinone<smiles>O=C1c2ccccc2C(=O)c2ccccc21</smiles>

anthraquinone

Figure 1.4 Several common, basic quinones, which form the scaffold for the majority of more complex quinones.

Arylquinones constitute a small but important subsection of quinones. Arylquinones may be synthesized using traditional cross-coupling reactions such as the Suzuki reaction, but synthesis of the necessary halogenated quinone can be limited by chemo- and regioselectivity problems. ${ }^{40}$ Arylquinones may also be synthesized via $\mathrm{C}-\mathrm{H}$ arylation using precious metal catalysts and arylmercury, aryltin, and arylsilane compounds, or through the use of radical $\mathrm{C}-\mathrm{H}$ coupling with arylboron species using iron or silver catalysts (see Section 1.1.2.1). ${ }^{40}$ Arylquinone use in the dye industry is limited, although 2-pyrrolylnaphthoquinone derivatives may be used as hair dyes. ${ }^{36}$ In the pharmaceutical industry arylquinones, especially $N$-heteroarylquinones and the diarylated terphenyl quinones, are more common..$^{29,33,41}$ Notable examples include Betulinan A and B, which inhibit lipid peroxidation ${ }^{29}$; leucomelone, an antibacterial agent ${ }^{40}$; asterriquinones, which activate insulin receptors and possess antitumor properties ${ }^{33}$; diospyrin, which is used to treat tuberculosis and may possess anti-cancer properties ${ }^{33}$; and 2-(N-methylpyrrole)-1,4-naphthoquinone, a hair dye (Figure 1.5) ${ }^{36}$ 
<smiles>COC1=C(c2ccccc2)C(=O)C(OC)=C(c2ccccc2)C1=O</smiles>

Betulinan A<smiles>Cn1c(C2=C(O)C(=O)C(c3ccccc3)=C(O)C2=O)cc2ccccc21</smiles>

A synthetic asterridine<smiles>COC1=C(c2ccccc2)C(=O)C2=C(C1=O)c1ccccc1CO2</smiles>

Betulinan B<smiles>Cc1cc(O)c2c(c1)C(=O)C(c1c(C)cc3c(c1O)C(=O)C=CC3=O)=CC2=O</smiles>

Diospyrin<smiles>O=C1C(O)=C(c2ccc(O)c(O)c2)C(=O)C(O)=C1c1ccc(O)cc1</smiles>

Leucomelone<smiles>Cn1cccc1C1=CC(=O)c2ccccc2C1=O</smiles>

2-(N-methylpyrrole) -1,4-naphthoquinone

Figure 1.5 Notable arylquinones from the pharmaceutical and dye industries

\subsubsection{Radical Sources for Arylation Reactions}

Since aryl radicals are highly reactive and generally cannot be isolated, let alone stored, radical arylation reactions rely upon a number of aryl radical precursors. The purpose of this section is to provide a brief overview of some of the more notable precursors; for further information, readers should consult one of several excellent reviews. ${ }^{7,9,21,42,43}$ As described in Section 1.1.1.1, some of the earliest aryl radical reactions were carried out with aryldiazonium salts. ${ }^{8,9,21,42,43}$ Aryldiazonium salts form aryl radicals through homolytic cleavage of the $\mathrm{C}-\mathrm{N}$ bond, which can be induced through metal catalysis, electrochemistry, or photochemistry. ${ }^{8,9,21,42,43}$ However, electron-donating substituents on the aryl moiety stabilize the $\mathrm{C}-\mathrm{N}$ bond, and reactions with aryldiazonium salts bearing 
strong electron-donating groups often fail, or lead to poor yields. ${ }^{8,9,21,42,43}$ Aryldiazonium salts are highly reactive, and usually cannot be stored for long periods of time, although their reactivity can be reduced by employing larger, more complex anions..$^{9,42,44}$ In addition, in many cases aryldiazonium salts may explode if isolated. ${ }^{9,42,44}$ Instead, they are generally prepared immediately prior to use by the reaction of an aryl amine with sodium nitrite and hydrochloric acid, and kept in solution. Aryldiazonium salts high reactivity also often leads to a variety of side reactions, which ultimately result in lower yields. There are however some advantages to the reactivity of aryldiazonium salts, as reactions can generally be performed at room temperature, and proceed rapidly. ${ }^{8,9,21,42,43}$ In addition to the previously mentioned Gomberg-Bachmann reaction, aryldiazonium salts can also be coupled with olefins and heteroarenes in Meerwein-type reactions (Scheme 1.3)..$^{8,9,21,42,43}$ These reactions are usually catalyzed by copper salts, and reactions with heteroarenes are usually limited to electron-rich heteroarenes such as furans, thiophenes and protected pyrroles. In addition, it is often necessary to include a blocking group on the heteroarene to prevent diarylation..$^{8,9,21,42,43}$ While the mild conditions and rapid reactions that are possible with aryldiazonium salts are attractive, they are limited due to difficulties with storage, low yields, and a somewhat limited substrate scope. , $^{8,21,42,43}$ 


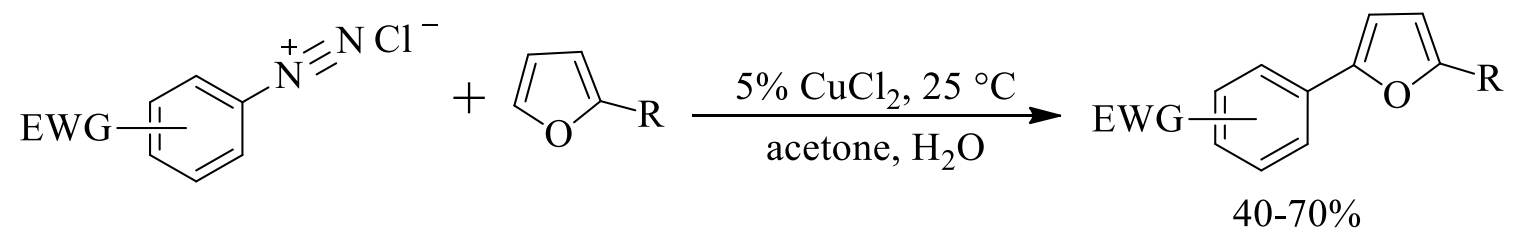

Scheme 1.3 An example of the Meerwein-type reaction, in which aryldiazonium salts are coupled to heteroarenes and olefins via a radical pathway. ${ }^{9}$

Arylhydrazines are another, closely related class of early aryl radical precursors. Arylhydrazines are in fact usually prepared by reacting aryldiazonium salts with sodium sulfite in the presence of a base. ${ }^{9,21}$ Unlike aryldiazonium salts, however, arylhydrazines are not explosive, and can be safely isolated and stored. However, many arylhydrazines are carcinogenic, genotoxic, and acutely toxic, causing contact dermatitis, hemolytic anemia, and liver damage. ${ }^{9,21}$ Arylhydrazines have been used as radical precursors for the coupling of benzene and other aromatic hydrocarbons, olefins, and heteroarenes. ${ }^{9,21,45-47}$ Reactions generally require stoichiometric amounts of oxidant; the oxidant causes the formation of an unstable diazine, which decomposes to form the aryl radical and nitrogen gas (Scheme 1.4). ${ }^{9,21,45-47}$ As with aryldiazonium salts, yields of reactions with arylhydrazines are often limited by the formation of side products or by diarylation. Reactions of arylhydrazines with heteroarenes are generally limited to electron-rich heterocycles and arylhydrazines with electron-withdrawing substituents. ${ }^{9,21,45-47}$ 


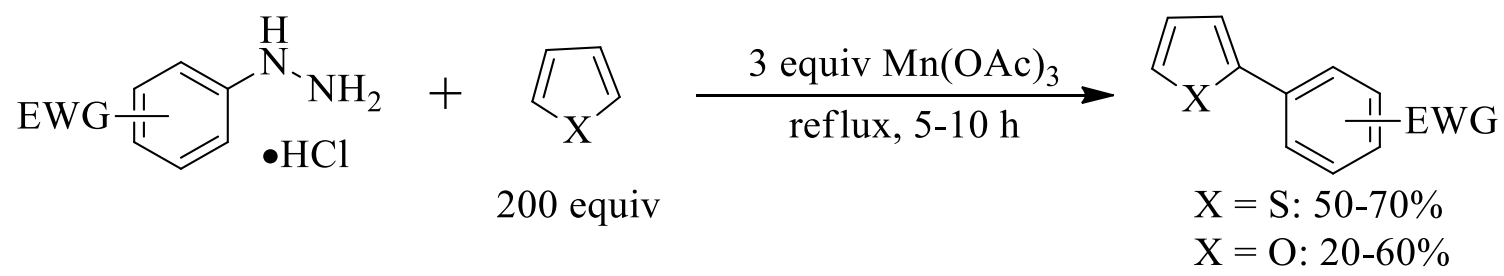

Scheme 1.4 Coupling of arylhydrazines to thiophenes and furans using manganese(III) acetate as an oxidant. ${ }^{46}$

Aromatic halides, particularly aryl bromides and aryl iodides, are widely used as aryl radical precursors..$^{7,9,21,48}$ They are able to form aryl radicals via irradiation with visible light, electrochemical reduction, or the use of reductants such as potassium tertbutoxide. , $9,21,48$ Aromatic halides are less reactive than arylhydrazines or aryldiazonium salts, and are therefore easier to store and are generally less toxic. On the other hand, aromatic halides often require harsh conditions to form aryl radicals, usually including several equivalents of strong base, a metal catalyst with relatively high catalyst loading, and temperatures of $100{ }^{\circ} \mathrm{C}$ or more (Scheme 1.5). ${ }^{7,9,21,48}$ Large excesses of arene are also usually required, as the harsh conditions cause side reactions. Aromatic halides have been coupled with benzene and its derivatives, electron-deficient heteroarenes such as pyridine, and electron-rich heteroarenes like thiophene and furan.,9,21,48 In reactions with heteroarenes a mixture of regioisomers are obtained; yields are generally highest at the ortho position, adjacent to the heteroatom, and diminish at each site moving away from the heteroatom (Scheme 1.5). ${ }^{7,9,21,48}$ 


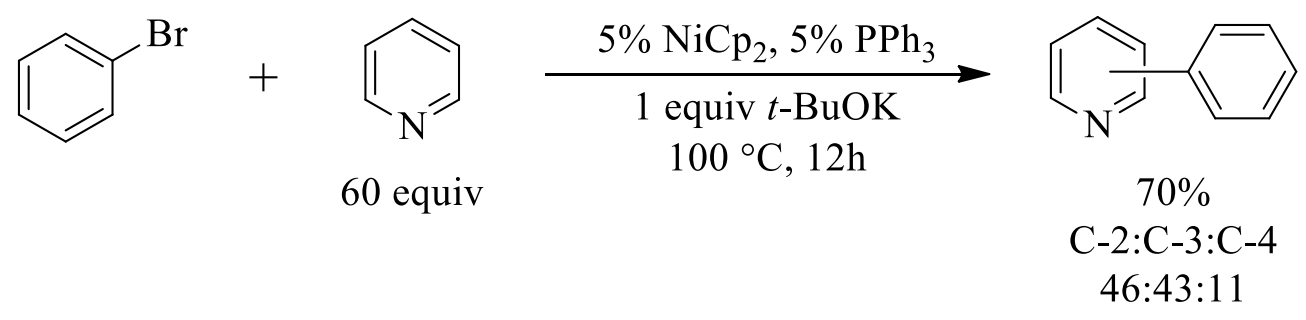

Scheme 1.5 Coupling of pyridine with benzene using bromobenzene as an aryl radical precursor. ${ }^{48}$

More recently, the use of hypervalent diaryliodonium salts as radical precursors have received considerable attention. ${ }^{9,21,49,50}$ These salts are more reactive than aromatic halides, allowing reactions to occur under more mild conditions, often at room temperature. They are light-sensitive, but can be stored if protected from light, and are relatively nontoxic. ${ }^{9,21,49,50}$ Diaryliodonium salts are commercially available, but are often relatively expensive; however, they can be synthesized fairly easy by treating the desired aryl iodide with meta-chloroperoxybenzoic acid ( $m$-CPBA) and a salt containing the desired anion. ${ }^{50}$ Aryl radicals are formed via the use of a reductant or by photoirradiation, and reactions can be performed with or without a transition-metal catalyst. ${ }^{9,21,49,50}$ Metal catalysts that have been used are generally precious metals such as ruthenium and palladium, although several copper-catalyzed reactions have been reported. One of the major drawbacks to the use of diaryliodonium salts is their low atom-economy, as only one aryl moiety forms an aryl radical, while the other aryl moiety forms an iodoarene. ${ }^{9,21,49,50}$ Metal-free and metalcatalyzed reactions have been reported with aromatic hydrocarbons, and electron-rich heteroarenes such as thiophenes, furans, pyrroles, and indoles although pyrroles and indoles must usually must have $N$-alkyl substituents to prevent side reactions. ${ }^{9,21,49,50}$ Reactions are 
often regioselective and result in higher yields than other aryl radical precursors. ${ }^{9,21,49,50}$ Scheme 1.6 presents a typical example of an aryl radical coupling with aryliodonium salts.

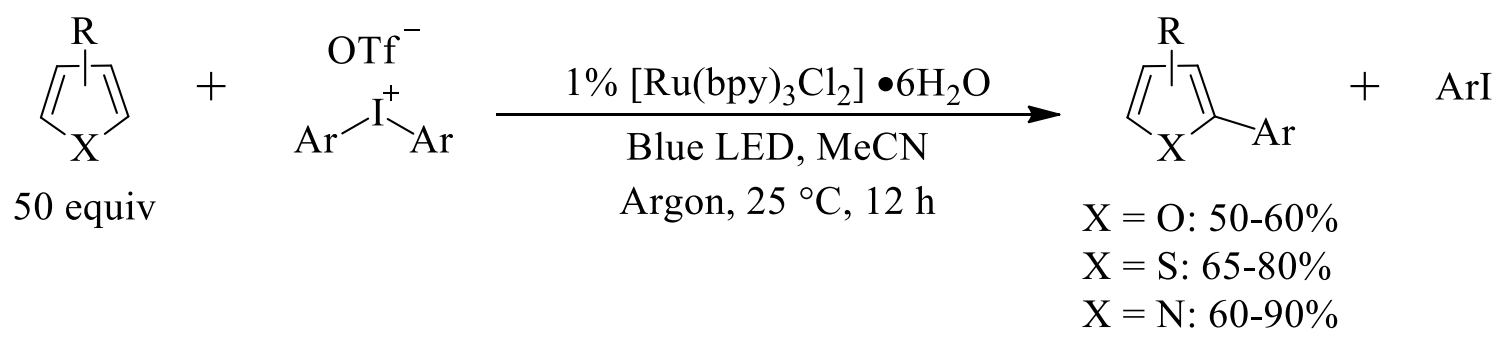

Scheme 1.6 Ruthenium-catalyzed coupling of aryliodonium salts with thiophenes, furans and pyrroles under photoirradiation. ${ }^{51}$

Finally, arylboronic acids and other arylboron compounds have received a significant amount of attention as aryl radical precursors over the last decade., $7,9,21,52$ Arylboronic acids are light, air, and moisture stable, and are generally non-toxic. ${ }^{9,18,53}$ They are also widely commercially available and in many cases inexpensive, in part due to their widespread use in the Suzuki reaction. ${ }^{9,18,53}$ In addition to arylboronic acids, aryltrifluoroborates and arylboronic acid esters have also been investigated as aryl radical precursors. ${ }^{7,9,21,52}$ Aryltrifluoroborates in particular have received attention because they are water-soluble, and therefore allow reactions to take place in aqueous media. Arylboron compounds generally require stoichiometric amounts of oxidant in order cleave the C-B bond and thereby form an aryl radical..$^{7,9,21,52}$ Reactions with arylboron compounds have been investigated with olefins, quinones, benzene and its derivatives, electron-rich heteroarenes such as furan and thiophene, but also with electron-deficient heteroarenes 
such as pyridine, quinoline, and other $N$-heteroarenes. ${ }^{7,9,21,52}$ The synthesis of biarenes and heterobiarenes via radical $\mathrm{C}-\mathrm{H}$ bond functionalization with arylboronic acids is examined fully in the following section.

\subsubsection{Synthesis of Biaryls and Arylquinones via Radical C-H Bond Functionalization with Arylboronic Acids}

\subsubsection{Development and Significance}

Interest in the use of arylboronic acids and other arylboron species, such as arylboronic acid esters and aryltrifluoroborates, as precursors to aryl radicals began due to their stability, commercial availability, low toxicity, easy synthesis, and widespread use in the Suzuki reaction. In addition, arylboron species had been shown to decompose into aryl radicals in the presence of an oxidant. ${ }^{54}$ The first report of a radical arylation reaction using an arylboron species was published in 2002, when Demir and coworkers reported the coupling of arylboronic acids with benzene, thiophene, and furan (Scheme 1.7) ${ }^{55}$ The reaction was performed at reflux, using benzene or heteroarene as a solvent, and required three equivalents of manganese acetate as an oxidant. The reaction was found to be tolerant to both electron-donating and electron-withdrawing substituents on the arylboronic acid, and were also tolerant of sterically hindered arylboronic acids. ${ }^{55}$ Reactions with benzene resulted in yields of $40 \%-90 \%$, higher than yields with thiophene (50\%-77\%), which gave better results than furan (19\%-67\%). A follow-up to this report was published in 2010 , when the same group reported synthesis of biaryls and heterobiaryls under microwave 
irradiation. ${ }^{56}$ In this report the group used a mixture of potassium permanganate and acetic acid as an oxidant, benzene as a solvent, and zeolite. The role of the zeolite was not explained or examined in the report, but presumably was meant to act as a heterogeneous catalyst, which would form a hotspot under microwave heating (see Section 1.2 for details). In addition, the temperature of the reaction mixture was not reported or monitored; instead, the group chose to report and optimize microwave power. In general, similar or slightly improved yields were reported under microwave heating. ${ }^{56}$ Curiously, however, reactions still required 20-30 min to reach completion, the same as reactions at reflux. In both of these reports yields were often limited by arylboronic acid side reactions, most commonly homo-coupling. ${ }^{55-57}$ Another report of arylboronic acid radical arylation under microwave heating was also published in 2010 by Guchhait and coworkers. ${ }^{57}$ They were able to perform reactions using a much smaller excess of arene - 10 equivalents instead of hundreds - in 7-10 min. They reported similar yields as Demir when coupling electron-rich or neutral arenes, and were able to extend the reaction scope to include electron-deficient arenes, such as pyridine, 4 -cyanopyridine, and quinoline, in moderate yields. ${ }^{57}$ This report was significant, as $\mathrm{N}$-heteroarenes are difficult targets for traditional Suzuki coupling due to difficulties in synthesizing the required starting materials and the $N$-heteroarenes' ability to act as ligands. They also noted that arylboronic acid homocoupling was significantly diminished under microwave heating. 


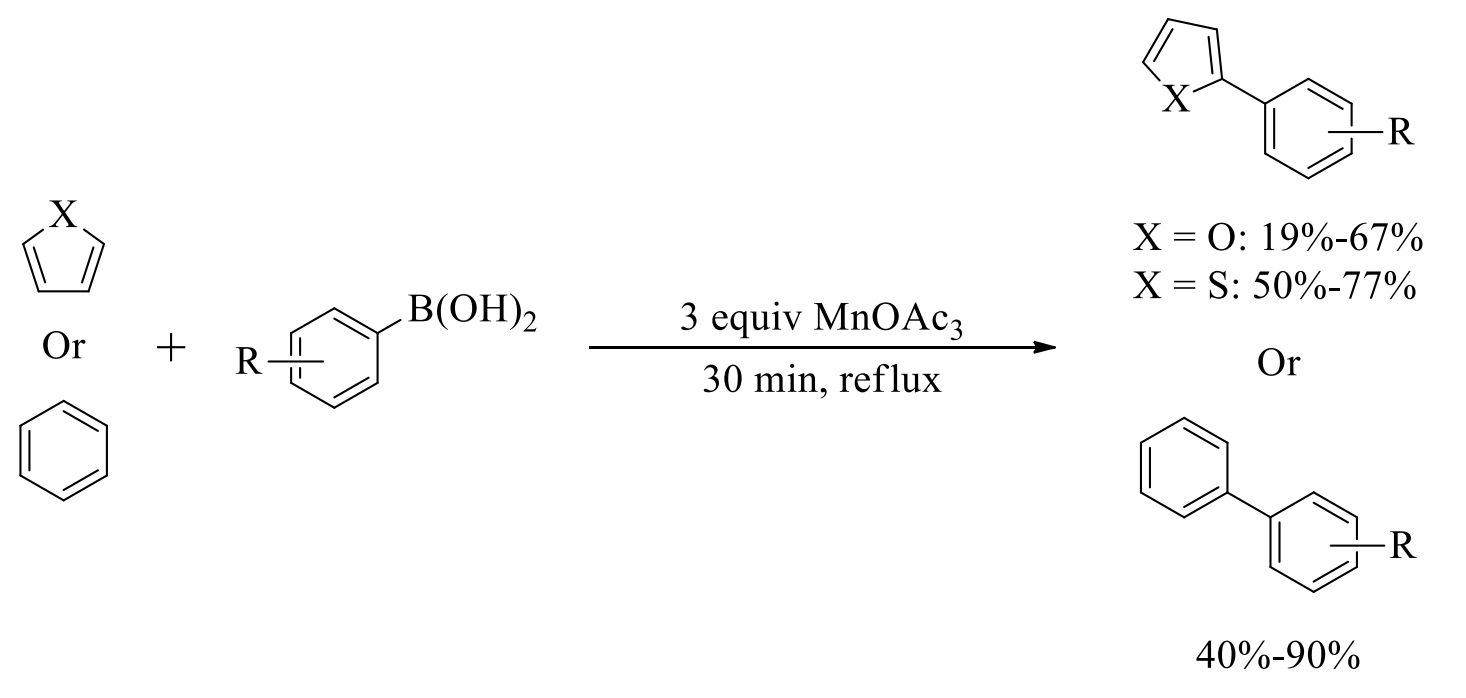

Scheme 1.7 Radical cross-coupling of arylboronic acids to benzene, thiophene and furan using manganese(III) acetate as an oxidant as reported by Demir and coworkers. ${ }^{55}$

In late 2010 and early 2011 Baran and coworkers published two reports describing the radical cross-coupling of arylboronic acids using potassium persulfate as an oxidant, as well as a report detailing radical cyclizations with arylboron species (Scheme 1.8). ${ }^{18,53,58}$ The first report described coupling with heteroarenes, primarily $N$-heteroarenes, ${ }^{18}$ while their second report described coupling with quinones. ${ }^{53}$ Both of these reports used very similar conditions; in both reports reactions were performed in a biphasic mixture of dichloromethane and water, using several equivalents of potassium persulfate and a catalytic amount of silver(I) nitrate. Reactions were also performed under air at room temperature, using only 1.5 equivalents of arylboronic acid, and required $3-24 \mathrm{~h}$ to reach completion in both reports. Reactions with $N$-heteroarenes bearing electron-withdrawing groups gave yields up to $92 \%$, while yields with unsubstituted $N$-heteroarenes ranged from $30 \%-96 \%{ }^{18}$ The reaction was also tolerant to a wide variety of arylboronic acids, 
including arylboronic acids bearing electron-donating and electron-withdrawing group. Notably, a number of regioisomers were detected when non-symmetrical $\mathrm{N}$-heteroarenes or quinones were coupled; in contrast, only one isomer was reported in most reactions using manganese(III) acetate as an oxidant. ${ }^{18,57}$ Reactions with quinones were tolerant to a wider variety of boronic acids, including strongly electron-deficient and electron-rich acids, and alkylboronic acids ${ }^{53}$ Reactions with aryltrifluoroborate salts or 'Molander salts' were also attempted and gave similar yields. Reactions with both $N$-heteroarenes and quinones were successful on the gram scale, and in the case of quinone reactions at this scale could be performed in water without an additional organic solvent. ${ }^{18,53}$

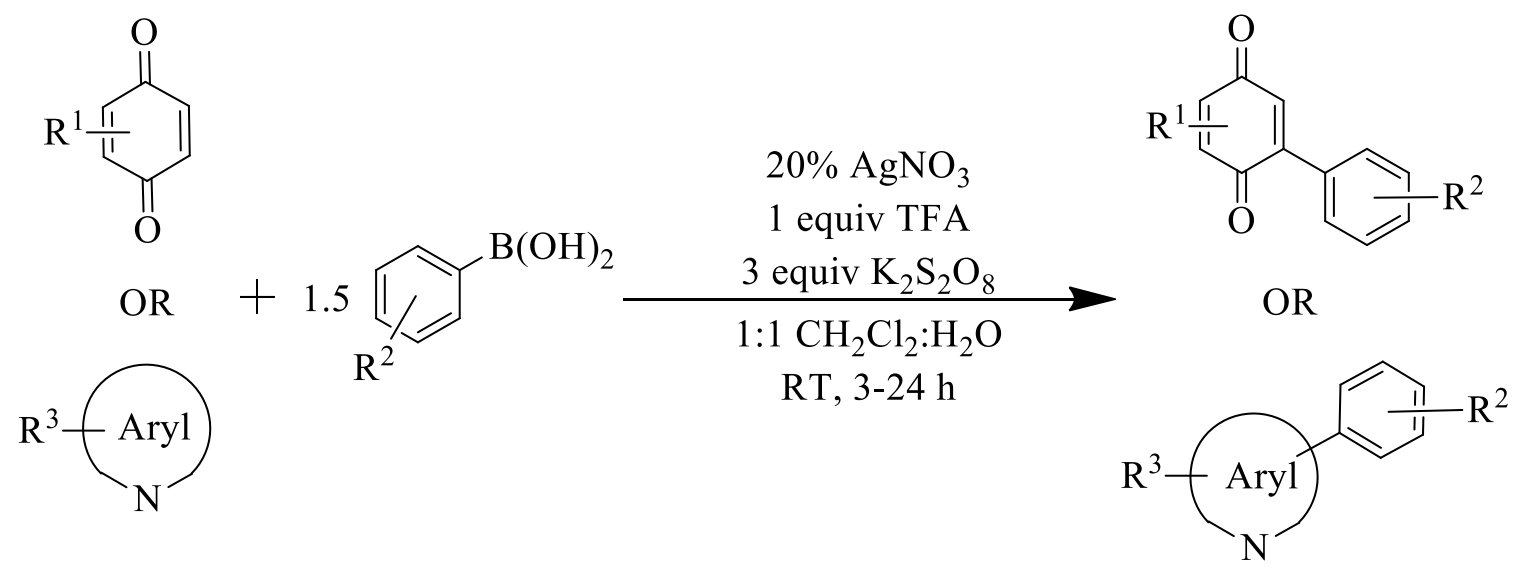

Scheme 1.8 Conditions described by Baran and coworkers for the cross-coupling of $N$ heteroarenes and quinones with arylboronic acids using potassium persulfate as a radical initiator. ${ }^{18,53}$

The reports by Baran and coworkers of radical arylboronic acid coupling to difficult and biologically relevant electron-deficient arenes and quinones under mild conditions 
sparked considerable interest, and several reports soon followed. Several reports attempted to extend the reaction scope, which is described in detail in Sections 1.1.2.3, 1.1.2.4, and 1.1.2.5. Other reports examined the role of the catalyst - some groups continued using silver catalysts, while others employed more cost-effective iron catalysts or were entirely catalyst-free. Silver catalyzed reactions were described by Mai and coworkers in 2011, when they reported the coupling of pyridine $N$-oxides with arylboronic acids under similar conditions to those in the Baran report. ${ }^{59}$ Pyridine $N$-oxides are strongly electron-deficient at the 2-position, and their use had therefore been reported to lead to higher yields and increased selectivity for the 2-position. These effects were observed by Mai and coworkers, who were able to increase arylboronic acid scope, and obtain products arylated exclusively at the 2-position. ${ }^{59}$ In 2013, a silver-catalyzed reaction was reported by Jain and coworkers, who were able to achieve regiospecific coupling with the amino acid L-histidines - the first report of a single-step regiospecific synthesis of 2-aryl-L-histidines. ${ }^{60}$ In $2018 \mathrm{Wu}$ and coworkers described the silver-catalyzed regiospecific arylation of $N$-methoxyquinoine-1ium tetrafluoroborate salts. As with $N$-oxides, these compounds are highly electrondeficient at the 2-position, and $\mathrm{Wu}$ and coworkers were therefore able to expand substrate scope, and achieve almost exclusive selectivity for the 2-position. ${ }^{61}$

The first iron-mediated reaction was reported by Yu and coworkers in $2012 .{ }^{20}$ They were able to achieve similar yields as Baran and coworkers with both quinones and $\mathrm{N}$ heteroarenes using 0.5 equiv of heterogeneous iron(II) sulfide, although the reactions were more sluggish, requiring $24-48 \mathrm{~h}$ to complete. In addition, they reported the first tandem oxidation and cross-coupling of hydroquinones, forming the arylquinone products with 
similar yields to reactions with quinones. ${ }^{20}$ Komeyama and coworkers also reported an iron-mediated reaction with both $N$-heteroarenes and quinones using 1 equiv of homogeneous iron(II) sulfate. ${ }^{62,63}$ However, they were able to use a catalytic amount of iron(II) sulfate when a reductant, such as ascorbic acid was present. ${ }^{62} \mathrm{~A}$ catalytic amount of iron(II) sulfate could also be used in reactions with hydroquinones, which themselves serve as reductants. Shortly after, Vishwakarma and coworkers also reported iron-catalyzed arylations of $N$-heteroarenes and quinones using iron(II) acetylacetonate and a phase transfer catalyst, tetrabutylammonium bromide (TBAB). ${ }^{19}$ Maiti and coworkers were also able to couple quinones, hydroquinones, $\mathrm{N}$-heteroarenes and $\mathrm{N}, \mathrm{S}$-heteroarenes using iron(III) nitrate as a catalyst. ${ }^{64}$ In later reports, Maiti and coworkers also described the generation of arylated quinones from hydroquinones and phenols, and the regioselective arylation of biologically relevant $N$-alkyl-2-pyridones. ${ }^{40,65}$ Finally, Huang and coworkers reported excellent yields with substituted pyridines using iron oxalate as a catalyst. ${ }^{66}$ In general, reactions using iron catalysts were found to proceed more slowly and sometimes required heating, but were able to expand substrate scope to include hydroquinones and phenols (Scheme 1.9; see Sections 1.1.2.3, 1.1.2.4, and 1.1.2.5 for more information). 

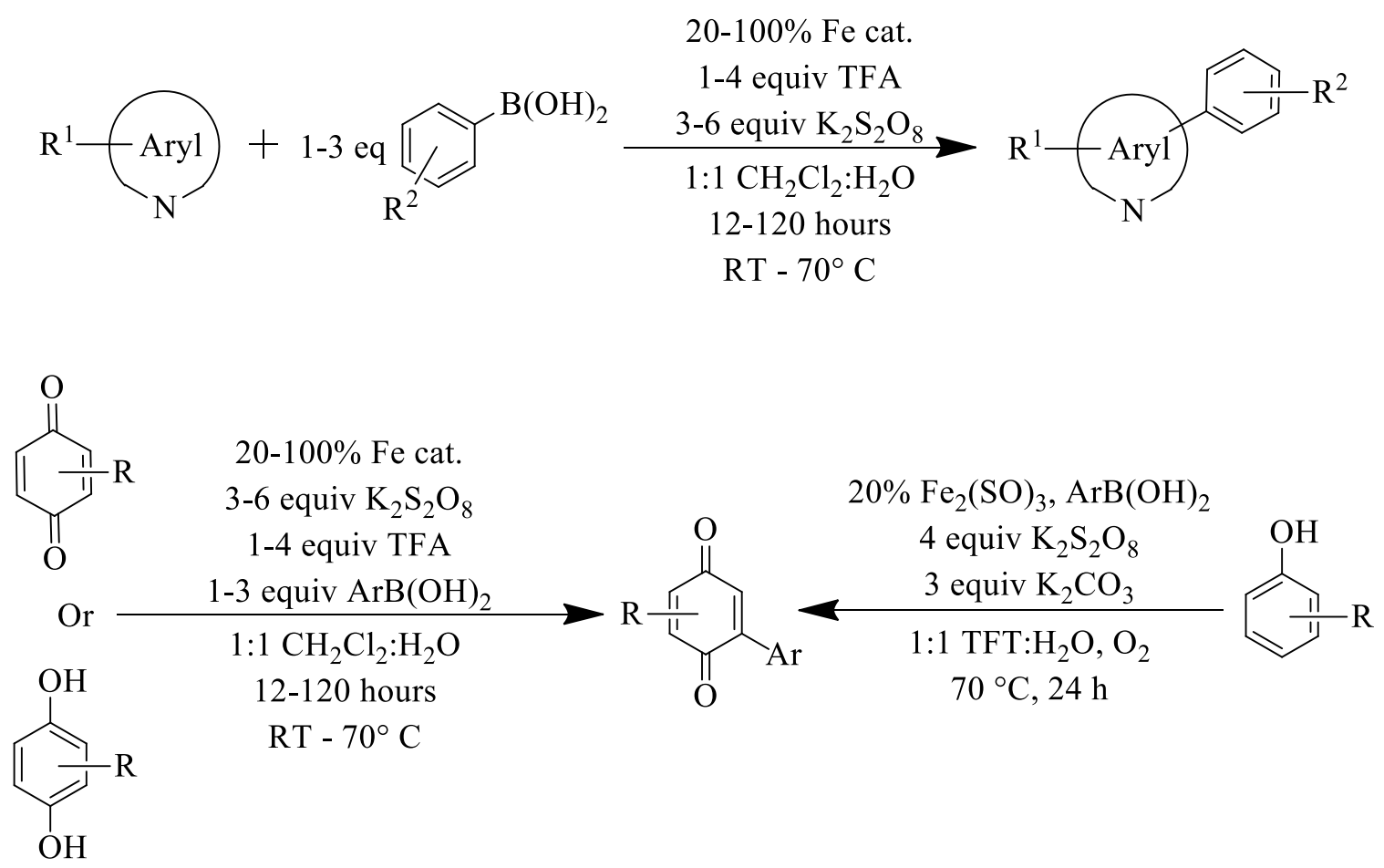

Scheme 1.9 Top: Iron-catalyzed coupling of arylboronic acids with $\mathrm{N}$-heteroarenes. ${ }^{19,20,63,64,66}$ Bottom: Iron-catalyzed coupling of arylboronic acids with quinones, ${ }^{19,20,62,64}$ hydroquinones, ${ }^{20,62,64}$ and phenols. ${ }^{40}$

Lastly, there has been one report of a metal-free reaction. Singh and coworkers reported the coupling of $N$-heteroarenes and arylboronic acids in the absence of a metal catalyst or acid using a persulfate oxidant (Scheme 1.10) ${ }^{67}$ Arylated products were obtained in moderate to good yields with more electron-deficient $N$-heteroarenes, but yields diminished with less electron-deficient heteroarenes. Arylboronic acid esters and aryltrifluoroborate salts were also examined, and gave moderately lower yields than arylboronic acids. ${ }^{67}$ While a metal and acid-free reaction is desirable, the reaction required heating to $160{ }^{\circ} \mathrm{C}$, and in most cases resulted in lower yields than most metal-catalyzed reactions. 


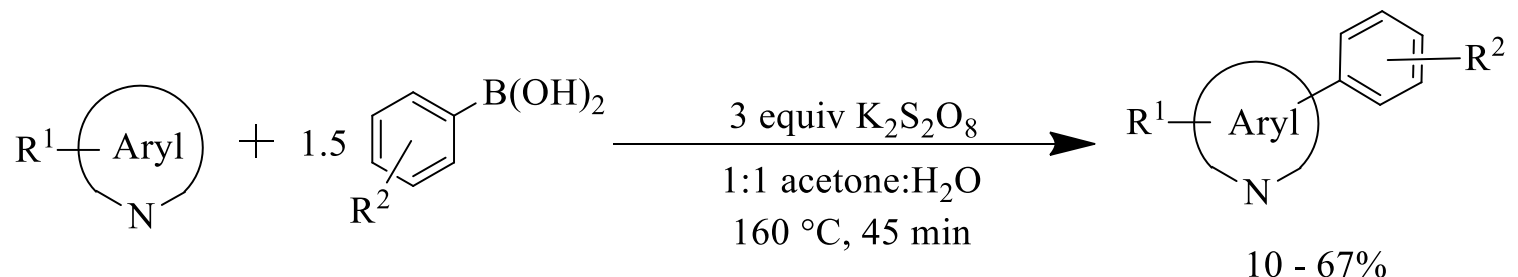

Scheme 1.10 Metal and acid-free coupling of arylboronic acids to electron-deficient $N$-heteroarenes, as described by Singh and coworkers.

Radical cross-coupling with arylboronic acids provides a simple, mild, and costeffective method for the arylation of electron-deficient heteroarenes and quinones without the need for precious metals catalysts. In addition, the starting materials employed are generally commercially available and shelf stable, and the reaction avoids the need to functionalize the $N$-heteroarene or quinone reagent. As described in Section 1.1.1.2, heterobiarenes and arylquinones are important in the pharmaceutical, agrochemical, electrochemical, and dye industries. There have been a number of syntheses of natural products or medically relevant compounds and scaffolds using this method in the academic literature (Figure 1.6 and Figure 1.7), which demonstrate the utility of the reaction in the synthesis of biologically active compounds. ${ }^{18,19,40,53,63,65}$ In addition, a number of these reports note that the reaction is tolerant to numerous functional groups, eliminating the need for time-consuming and wasteful protection/deprotection reactions. ${ }^{18,40,53,63}$ Despite these advantages, there have been no commercial products synthesized using this method. This may be simply because the technique is relatively new, or may be due to one of several disadvantages, most notably long reaction times and the amount of waste produced as a 
byproduct of the reaction. This waste includes products from the homo-coupling and protodeboronation of the arylboronic acid, but most significantly the waste from the several equivalents of potassium persulfate required as a radical initiator. These waste products are non-toxic and easy to dispose of on a bench scale, but may be more difficult to deal with if a significant scale-up is required.<smiles>C[C@]12CC[C@H]3c4ccc(C5=CC(=O)C=CC5=O)cc4CC[C@H]3[C@H]1CCC2=O</smiles>

estrone benzoquinone hybrid ${ }^{53}$<smiles>O=C1C(O)=C(c2ccc(O)c(O)c2)C(=O)C(O)=C1c1ccc(O)cc1</smiles>

Leucomelone ${ }^{40}$<smiles>CC(C)=CCC/C(C)=C/CC/C(C)=C/CC1=CC(=O)C=CC1=O</smiles>

2-farnesyl-1,4-benzoquinone ${ }^{53}$<smiles>COC1=C(c2ccccc2)C(=O)C(OC)=C(c2ccccc2)C1=O</smiles>

Betulinan $\mathrm{A}^{40}$

Figure 1.6 Natural products, pharmaceuticals, and medicinally relevant scaffolds synthesized via radical $\mathrm{C}-\mathrm{H}$ activating cross-coupling between arylboronic acids and quinones, hydroquinones, or phenols. 


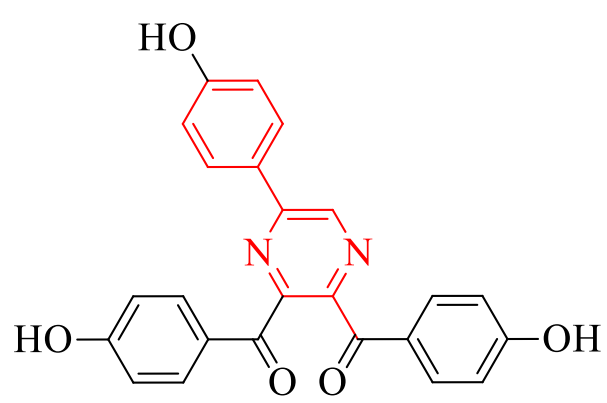

Botryllazine $\mathrm{A}^{19}$

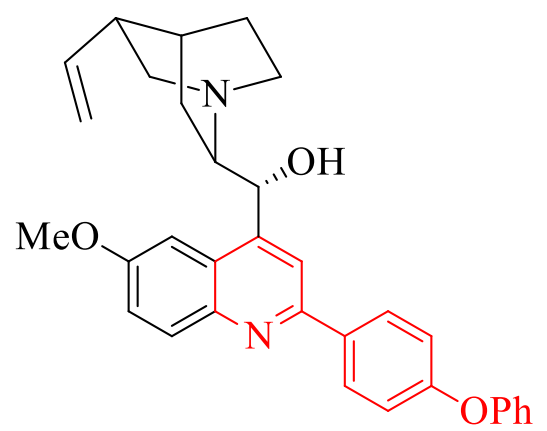

2-(4-phenoxyphenyl)quinine ${ }^{18}$<smiles>Nc1nccc(-c2c[nH]c3ncccc23)n1</smiles><smiles>CN1CCCC(c2ccccc2)C1=O</smiles><smiles>c1ccc2nc3oc4ccccc4c3cc2c1</smiles>

Meriolin $1^{67} \quad$ 1-methyl-3-phenylpiperidine-2-one ${ }^{65}$ benzofuro[2,3-b]quinoline ${ }^{65}$<smiles>NC(=O)c1cc(N2CCOCC2)cc(-c2ccc(Oc3ccc(F)cc3)cc2)n1</smiles>

Sodium channel inhibitor $7^{63}$<smiles>O=C(c1ccc(O)cc1)c1cncc(-c2ccc(O)cc2)n1</smiles>

Botryllazine $\mathrm{B}^{63}$

Figure 1.7 Natural products, pharmaceuticals, and medicinally relevant scaffolds synthesized via radical $\mathrm{C}-\mathrm{H}$ activating cross-coupling between arylboronic acids and $\mathrm{N}$ heterocycles.

\subsubsection{Mechanism of Reactions with Persulfates}

The general mechanism of arylboronic acid radial coupling with $N$-heteroarenes is agreed upon, although the details of the mechanism are not. Several publications have confirmed that the reaction is radical in nature through the use of a radical trap, generally 2,2,6,6-tetramethyl-1-piperidinyloxy (TEMPO) experiments. ${ }^{19,20,59,60,64}$ This compound 
reacts rapidly in the presence of radicals to form a relatively unreactive product. Upon addition to the reaction mixture, TEMPO retards or entirely halts the reaction, confirming the presence of radicals in the reaction cycle. ${ }^{19,20,59,60,64}$ It is also generally agreed that the original source of the radicals is the oxidation of the metal by the persulfate anion, which forms the oxidized metal species and a sulfate radical anion. ${ }^{18,20,40,62,64,68}$ This is consistent with literature reports on persulfate chemistry, which has been thoroughly investigated due to the widespread use in radical polymerization reactions. Arylation is thought to occur via nucleophilic attack of an aryl radical formed from the arylboronic acid, which is thought to be formed by the homolytic cleavage of the carbon-boron bond. ${ }^{18,20,40,62,64,68}$ This is strongly suggested by the fact that coupling is favored at electron-deficient sites on the $\mathrm{N}$ heteroarene, and occur exclusively at the site of the carbon-boron bond. Further evidence is provided by side reactions of the arylboronic acid, which can undergo homocoupling, hydroxylation, or protodeboronation, which also occur selectively at the site of the C-B bond. ${ }^{19,62,65,68}$

What remains unclear is the role of the metal catalyst and the identity of the species responsible for the formation of the aryl radical. There are two proposed mechanisms (Scheme 1.11). In their 2010 report Baran and coworkers suggested based on literature precedent that the persulfate anion disproportionated in the presence of silver(I), forming a sulfate dianion, a sulfate radical anion, and a silver(II) species. ${ }^{18}$ They then suggested that the sulfate radical anion reacted with the arylboronic acid to form an aryl radical, with the eventual formation of boric acid and another sulfate dianion. The aryl radical would then couple to a protonated $N$-heteroarene, which would then lose a hydrogen radical to form 
the final product and regenerate the silver(I) catalyst. ${ }^{18}$ Most literature reports suggest a similar mechanism, changing only the identity of the metal and the termination step, as in metal-mediated reactions the catalytic cycle is not completed. This pathway appears plausible, but is not supported by any in-depth studies of the reaction mechanism.

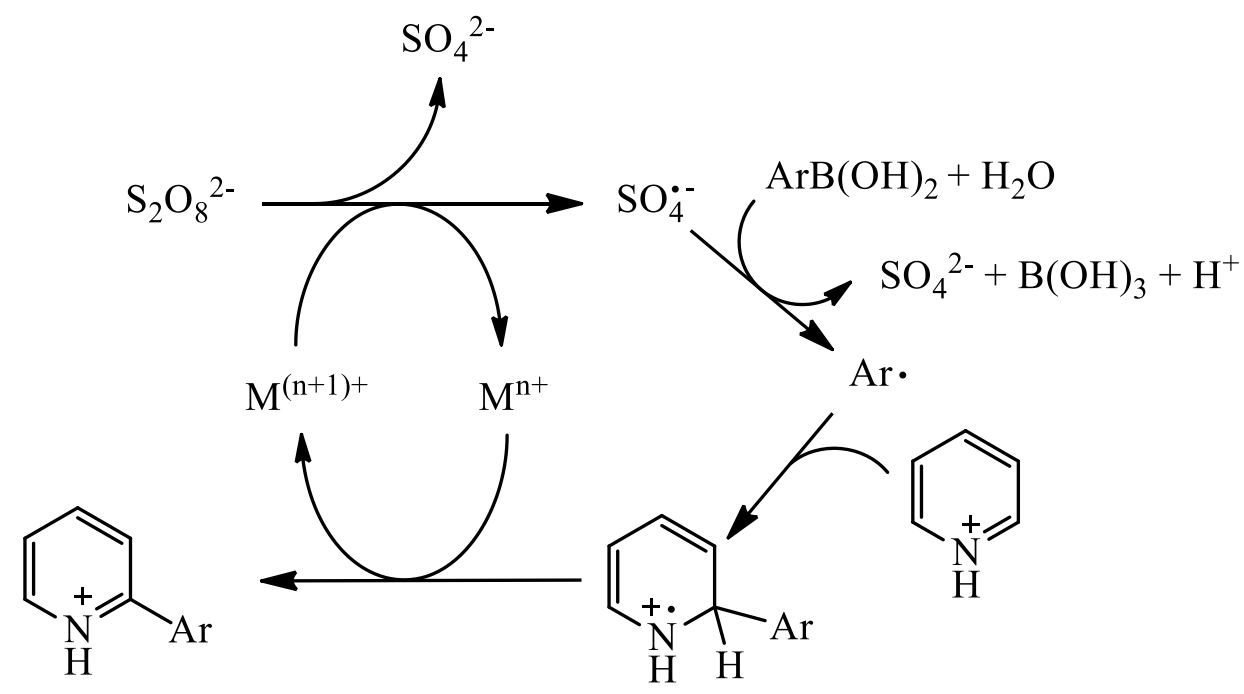

Scheme 1.11 The most commonly cited mechanism, first proposed by Baran and coworkers. ${ }^{18}$

However, several other reports, including the only report dedicated specifically to determining the reaction mechanism, suggested an alternative pathway (Scheme 1.12). ${ }^{64,68}$ These groups suggested that the initial step was the formation of a metal- $N$-heteroarene complex. The formation of a metal- $N$-heteroarene complex was first suggested based on unusual rate orders with respect to the $N$-heteroarene and arylboronic acid, and was further confirmed by proton NMR studies in $\mathrm{D}_{2} \mathrm{O}^{68}$ The metal would then be oxidized by the persulfate, then act as a reducing agent in order to cleave the carbon-boron bond and form 
the aryl radical, rather than having the persulfate radical anion cleave the carbon-boron bond. ${ }^{64,68}$ This proposal was supported by experiments employing allyl acetate, a common radical trap for persulfate radical anions. The inclusion of allyl acetate did not impact the yield, and in fact increased yields slightly, indicating that the persulfate radical anion was not directly involved in the reaction. ${ }^{68}$ The aryl radical would then couple to a protonated $\mathrm{N}$-heteroarene, with the final product being formed by the loss of a hydrogen radical. ${ }^{64,68}$. This mechanism is not proposed as often in the literature, but seems to be more wellsupported by experimental data.

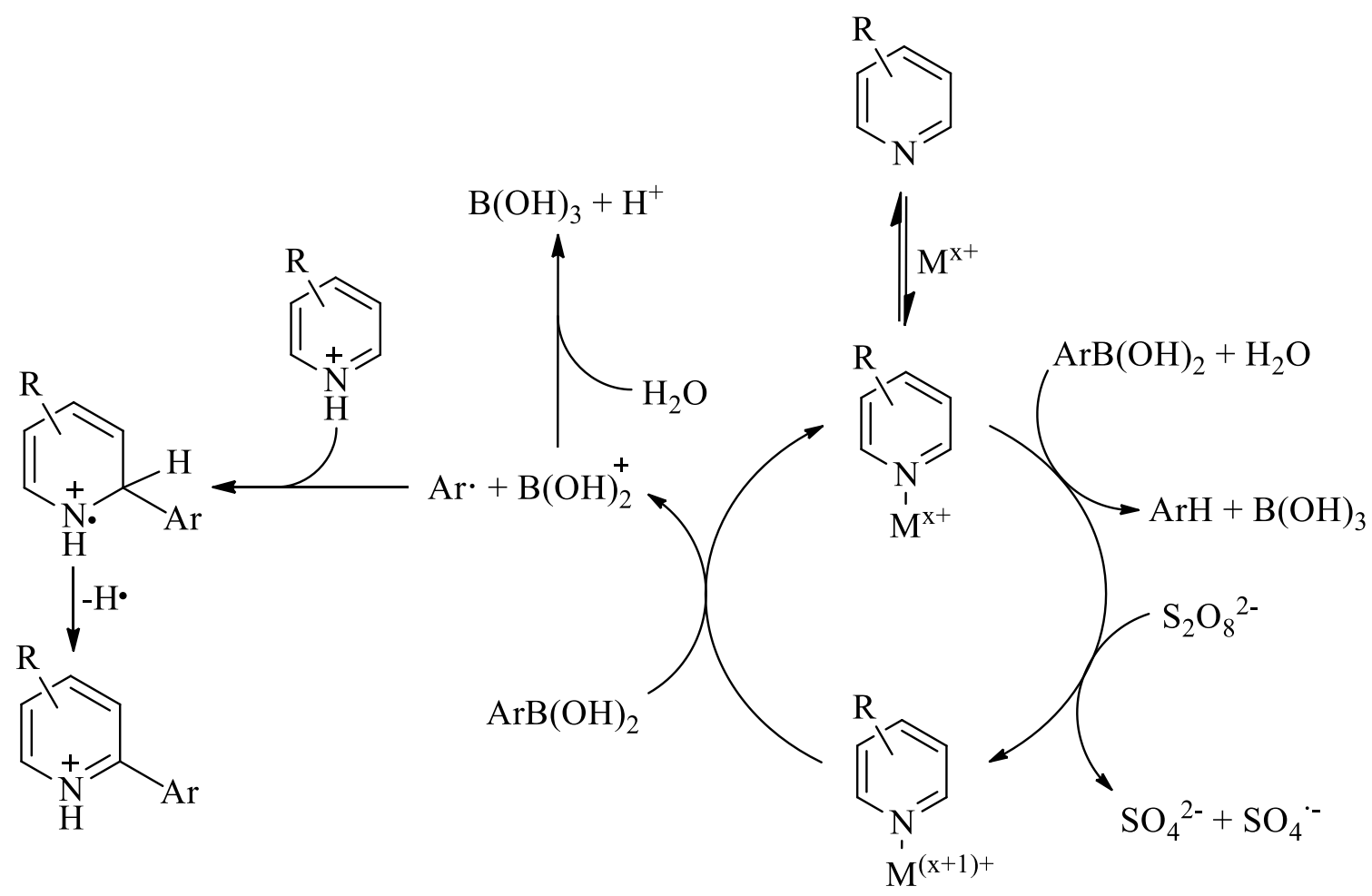

Scheme 1.12 A plausible mechanism originally suggested by Flowers and coworkers. ${ }^{68}$ 
Relatively little research has been conducted into the mechanism of quinone or hydroquinone arylation. In general, the mechanisms that have been proposed are similar to those proposed for $N$-heteroarenes. Some groups have suggested that coordination with the metal might occur ${ }^{40}$, increasing the electrophilic nature of the quinone, while others do not

suggest coordination. ${ }^{20,62}$ It is also unclear whether cleavage of the carbon-boron bond is caused by the sulfate radical anion or by the metal. Reactions with hydroquinones and phenols are thought to proceed via the same mechanism, with the phenol or hydroquinone being oxidized to quinone before arylation takes place. ${ }^{20,40,62}$

\subsubsection{Scope and regioselectivity of reactions with $N$-heteroarenes}

The scope of $\mathrm{N}$-heteroarene cross-coupling in the reaction has been examined several times, usually focussing on six-membered rings containing one or more nitrogen atom (Figure 1.8). ${ }^{18-20,63-67}$ Unactivated $N$-heteroarenes such as quinoline, pyridine, pyrazine, or pyrimidine have been cross-coupled in good yields, but better results are obtained when a strong electron-withdrawing group, such as a cyano or trifluoromethyl group, is present on the $N$-heteroaryl. ${ }^{18,20,63,64,66}$ Reactions with weakly electronwithdrawing halide substituents have been described with mixed results, with some groups reporting greatly diminished yields, and others reporting little difference between the halides and more strongly electron-withdrawing groups. ${ }^{18,20,63,64,66}$ Weakly electrondonating alkyl groups, most commonly methyl and tert-butyl groups, are also generally well tolerated, but stronger electron-donating groups, such as alkoxy groups, lead to a significant reduction in yield. ${ }^{18,20,63,64,66} \mathrm{~N}$-heteroarenes containing fused ring systems, as in the case of quinoline, quinoxaline, quinazoline or phthalazine, are generally well 
tolerated, and they can be further activated by electron-withdrawing groups. ${ }^{18,20,63,64,66}$ Arylation in these cases takes place exclusively on the ring containing the nitrogen atom. ${ }^{18,20,63,64,66}$ Finally, there is only one report of coupling with a non-aryl $N$-heterocycle, in which $N$-alkyl-2-pyridones were coupled in poor to moderate yields. ${ }^{65}$

As previously mentioned, by far the most common $N$-heteroaryl coupling partners are 6 membered rings containing one or more nitrogen atoms. However, there are a small number of reports that describe reactions with 5-membered rings containing one or more nitrogen atoms, most commonly an imidazole ring, which usually produce poor yields or no conversion to the desired coupled product (Figure 1.8). ${ }^{18,20,60,64}$ Yields are particularly poor if an $\mathrm{NH}$ bond is present in the ring, ${ }^{18,60}$ and there have been no reports of successful coupling with $N$-heteroarenes containing only a nitrogen with an $\mathrm{NH}$ bond. ${ }^{18,60}$ There are two reports of reactions of five-membered rings containing other heteroatoms. ${ }^{20,64} \mathrm{Yu}$ and coworkers reported moderate yields with sulfur-containing benzothiazole and 4,5-dimethylthiazole, but were not able to couple oxygen-containing benzoxazole. ${ }^{20}$ Maiti and coworkers also reported the coupling of 4-methylthiazole and benzothiazole, this time in good to excellent yields. ${ }^{64}$ Maiti's report appears to be the only report of a high-yielding reaction with a 5-membered ring. 


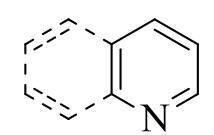

Good to excellent yields

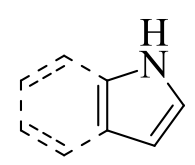

No product<smiles>CCCc1ccnc2ccccc12</smiles>

Improved yields<smiles>c1ccc2c(c1)NCN2</smiles>

Poor yields<smiles>O=C(O)c1ccnc2ccccc12</smiles>

Diminished yields<smiles>c1ccc2scnc2c1</smiles>

Poor to excellent yields

Figure 1.8 Summary of reaction scope with respect to $N$-heteroarenes for persulfate initiated cross-coupling with arylboronic acids.

Reactions with unsubstituted $N$-heteroarenes almost invariably generate a mixture of isomers (Scheme 1.13). ${ }^{18,20,63,64,66}$ Reactions with unsubstituted pyridine or quinoline, for example almost always generate a mixture of products at the 2 and 4 positions, with little or no product detected at the 3 position. ${ }^{18,20,63,64,66}$ This regioselectivity is typical for nucleophilic reactions with these compounds, and is due to reduced electron density at the 2 and 4 positions. However, reports on regioselectivity are often contradictory. For example, in their original paper, using a silver catalyst, Baran and coworkers cross-coupled pyridine and generated twice as much product with substitution at the 2 position than at the 4 position - the expected ratio given that there are twice as many 2 positions available for reaction. ${ }^{18}$ In contrast, Maiti and coworkers reported the opposite, with twice as much product at the 4 position compared to the 2 position. ${ }^{64}$ The cause of these differences in regioselectivity are not well understood, but may be due to differences in catalyst identity or reaction conditions. 
Attempts to control regioselectivity focus mainly on the inclusion of functional groups on the $\mathrm{N}$-heteroarene, typically at the 4 position, or on the use of aryl $\mathrm{N}$-oxides (Scheme 1.13). The inclusion of strong electron-withdrawing groups, which are known to be activating, at the 4-position leads primarily to the 2 product, with a much smaller amount of 3 product. ${ }^{18,20,64}$ The inclusion of other groups, most commonly alkyl groups, often leads exclusively to the 2 substituted product. ${ }^{18,20,64}$ There have also been several reports of reactions with substituents at the 2 or 3 positions of pyridine, which yielded a mixture of all 4 isomers, generally in poor yields. ${ }^{18,20,63}$ Substitution of quinoline at the 2 position yielded only the 4 position product, also in diminished yields ${ }^{64}$ Curiously, there are no reports of reactions with 2,6-disubstituted pyridine, which would presumably yield only the 4 product. An alternate method to control regiochemistry is the use of pyridine or quinoline $\mathrm{N}$-oxides, or $\mathrm{N}$-methoxyquinoline-1-ium tetrafluoroborate salts. ${ }^{59,61,69}$ Reactions with these starting materials have similar yields to unactivated $N$-heteroarenes, but are much more selective for the 2 position due to decreased electron density at this site, in many cases yielding only the 2 product. ${ }^{59,61,69}$ 
<smiles>c1ccc2ncccc2c1</smiles><smiles>[R]c1ccnc2ccccc12</smiles><smiles>[O-][n+]1cccc2ccccc21</smiles>

Ag or Fe salt $\underset{\mathrm{RT}-70{ }^{\circ} \mathrm{C}, 3-120 \mathrm{~h}}{\mathrm{ArB}(\mathrm{OH})_{2}, \mathrm{~K}_{2} \mathrm{~S}_{2} \mathrm{O}_{8}, \mathrm{TFA}}$<smiles>[Al]c1ccc2ccccc2n1</smiles>
Major<smiles>[R]c1cc([Al])nc2ccccc12</smiles>
Major<smiles>[R]c1c(C)cnc2ccccc12</smiles>

Scheme 1.13 Regiochemistry of the cross-coupling of substituted and unsubstituted $\mathrm{N}$ heteroarenes in the persulfate initiated cross-coupling reaction with arylboronic acids.

\subsubsection{Scope and Regioselectivity of Reactions with Quinones}

The scope of reactions with quinones has been repeatedly explored. ${ }^{19,20,53,62,64}$ Reactions with unsubstituted 1,4-benzoquinone generally give excellent yields. ${ }^{19,20,53,62,64}$ The inclusion of alkyl groups leads to a modest decline in yields, as does the inclusion of halides. ${ }^{20,53,62,64}$ Reactions with quinones containing more strongly electron-donating ether groups results in similar or slightly reduced yields when compared with unsubstituted 1,4-benzoquinone. ${ }^{20,53,62,64}$ No reactions have been reported with either stronger electrondonating or stronger electron-withdrawing groups. Results with 1,4-napthoquinone and derivatives are mixed, with reported yields ranging from $11 \%$ to $98 \% .^{20,53,62,64}$ There has 
also been one reaction reported with 1,2-napthoquinone, which was coupled in $48 \%$ yield. ${ }^{53}$ No other literature reports could be found in which a 1,2-quinone was successfully coupled.

There are also reports of the one-pot oxidation and homocoupling of hydroquinones, phenols, and 4-methoxyphenols, which yield the corresponding arylquinone (see Scheme 1.14). ${ }^{20,40,62,64}$ Curiously, such reactions have been reported only when using iron catalysts instead of silver catalysts. ${ }^{20,40,62,64}$ Reactions with hydroquinones give similar yields to reactions with quinones, while reactions with phenols and 4-methoxyphenols have somewhat poorer yields..$^{20,40,62,64}$ The effect of substituents appears to be similar to that described with quinones. ${ }^{20,40,62,64}$ There are no reports of reactions with cyclic or noncyclic enones, nor with enals or any other unsaturated species. The vast majority of quinones employed are symmetric, so regioselectivity is of less concern. When reactions are carried out with asymmetric quinones (as in mono-substituted quinones), all regioisomers are formed, generally in similar yields. ${ }^{53,62}$<smiles>[R]C1CC(=O)C=CC1=O</smiles><smiles>[R]c1ccc(O)c(OC)c1</smiles>

Fe salt, $\operatorname{ArB}(\mathrm{OH})_{2}$<smiles>[R]c1ccc(O)c(O)c1</smiles><smiles>[R]c1cccc(O)c1</smiles>

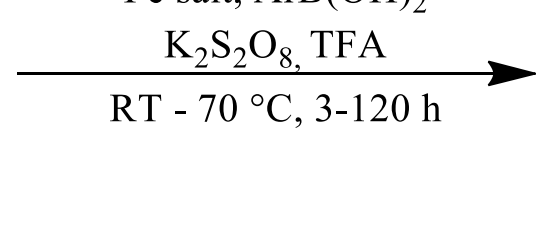<smiles>[Y17]C1=CC(=O)C([R])CC1=O</smiles>

Scheme 1.14 Reagents that lead to the formation of arylquinone products in the persulfate initiated cross-coupling reaction with arylboronic acids. 


\subsubsection{Arylboronic Acid Scope}

The scope of arylboronic acids has been explored many times in reactions with both quinones and $N$-heteroaryls, with somewhat contradictory results (Figure 1.9). ${ }^{18-20,40,53,62-}$ ${ }^{66}$ Nearly all reactions focus on the coupling of phenylboronic acid or its derivatives. In these cases, unsubstituted phenylboronic acid or arylboronic acids with weakly electrondonating alkyl substituents at the 3 or 4 positions are well tolerated. ${ }^{18-20,40,53,62-66}$ The presence of alkyl groups, or any substituent at the 2 position leads to mixed results. Several groups claim that there is little or no decrease in yield when substituents are present at the 2 position ${ }^{19,20,62,65}$, while others report significant reductions in yield, presumably due to steric hindrance. ${ }^{18,53,63,64}$ Literature reports on reactions with halide substituents are also contradictory, with some groups observing a large decline in yields ${ }^{64-66}$, and others finding little to no effect $19,20,40,62,63$, regardless of position. When using stronger electronwithdrawing groups such as cyano or trifluoromethyl groups, yields decline. However, some groups report moderate to good yields when using these substituents ${ }^{18,53,66}$, while others report very low yields or even no conversion. ${ }^{19,62,63}$ The presence of electrondonating groups, most commonly alkoxy groups, results in only a small decline in yields. ${ }^{18-}$ 20,40,53,62-66 Reactions with stronger electron-donating groups, such as hydroxyl or amine groups, have not been reported. In general, reactions with quinones appear to be tolerant to a wider array of substituted arylboronic acids than reactions with $N$-heteroaryls.

There are also reports of couplings with arylboronic acids that are not based on benzene, non-aromatic boronic acids, and other boron-containing groups (Figure 1.9). Two groups have reported the coupling of $N$-heteroarylboronic acids to $N$-heteroarenes or 
quinones, with all reactions returning low yields. ${ }^{19,69}$ An attempt with 2-thienylboronic acid gave only a trace amount of product. ${ }^{19}$ There has been one report of the coupling of 2furanylboronic acid to aryl $\mathrm{N}$-oxides under metal-free conditions. ${ }^{69}$ There are two reports of the coupling of trans-2-phenylvinylboronic acids to $\mathrm{N}$-heteroaryls at the terminal position, both in good yields. ${ }^{19,69}$ Attempts to couple phenylvinylboronic acids to quinones failed, and attempts to couple phenylethynylboronic acids led to $\mathrm{C}_{\mathrm{sp}}-\mathrm{C}_{\mathrm{sp}}$ coupling ${ }^{62}$ Finally, Baran and coworkers reported the coupling of several alkylboronic acids to 1,4-benzoquinone. ${ }^{53}$ This appears to be the only account of coupling with non-conjugated boronic acids. In addition, there are several accounts of coupling with other boron groups. There are four accounts of coupling with arylboronic acid pinacol esters. ${ }^{19,53,62,67}$ All accounts reported that the pinacol esters were more sluggish, and gave lower yields, than the corresponding arylboronic acid. ${ }^{19,53,62,67}$ There are also 5 reports of coupling with potassium aryltrifluoroborate salts (Molander salts), with both quinones and $\mathrm{N}$ heteroaryls. ${ }^{19,53,62,63,67}$ In all cases, the aryltrifluoroborate salt reacted with similar or slightly higher yield when compared to the corresponding arylboronic acids. ${ }^{19,53,62,63,67}$ 
<smiles>Oc1ccccc1</smiles>

Good to excellent yields<smiles>Oc1ccc(Br)cc1</smiles>

Conflicting reports<smiles>BrC=Cc1ccccc1</smiles>

Good yields: $\mathrm{N}$-arenes only<smiles>[R]c1ccc(Br)cc1</smiles>

Good to excellent yields<smiles>[R]c1cccc(Br)c1</smiles>

Good to excellent yields<smiles>Oc1ccncc1</smiles>

Low yields<smiles>[Y]c1ccccc1Br</smiles>

Good to excellent yields<smiles>[X]c1ccccc1Br</smiles>

Conflicting reports

$$
\mathrm{R}^{-\mathrm{B}(\mathrm{OH})_{2}}
$$

Moderate yields: Quinones only<smiles>[Hg]Oc1ccco1</smiles>

Good yields: $\mathrm{N}$-oxides only<smiles>CC1(C)OB(c2ccccc2)OC1(C)C</smiles>

Low yields

Figure 1.9 Summary of reaction scope with respect to organoboron species for persulfate initiated cross-coupling with arylboronic acids. In general excellent yields are $90 \%$ or above, good yields are $70-90 \%$, moderate yields are $40-70 \%$, and poor or low yields are $40 \%$ and below. These classifications are based on the majority of reports with common coupling partners; yields obviously also depend on the identity of the coupling partner.

\subsection{Microwave Heating}

\subsubsection{Introduction and Theory}

\subsubsection{Introduction to Microwaves}

The term microwave radiation refers to electro-magnetic radiation with a frequency of $300 \mathrm{MHz}$ to $300 \mathrm{GHz}$, corresponding to wavelengths of $1 \mathrm{~m}$ to $1 \mathrm{~mm} \cdot{ }^{70-75}$ Microwaves therefore have a lower frequency than infrared radiation, but a higher frequency than radio waves. Photons at $2.45 \mathrm{GHz}$, the frequency most often used for microwave heating, have 
an energy of approximately $0.01 \mathrm{meV} \cdot{ }^{73} \mathrm{In}$ comparison, Brownian motion at $200 \mathrm{~K}$ has an energy of approximately $17 \mathrm{meV}$, hydrogen bonds up to $440 \mathrm{meV}$, and a typical $\mathrm{C}-\mathrm{H}$ bond is approximately $4510 \mathrm{meV} \cdot{ }^{73}$ Microwave radiation is therefore non-ionizing, meaning that it does not possess enough energy to directly break molecular bonds and thereby create ions. ${ }^{70-75}$ Reactions under microwave heating therefore do not (in the vast majority of cases) rely on microwave radiation directly breaking or altering chemical bonds; microwaves energy is usually simply converted into thermal energy. ${ }^{70-75}$

Microwave radiation has extremely wide-ranging applications, from satellite communication to radar to medicine. ${ }^{71,73}$ In fact, the discovery of microwave heating in 1945 was due to heating observed in proximity to a high-powered radar station. ${ }^{71,73}$ In order to avoid interfering with other devices that employ microwave irradiation, the vast majority of commercially available microwave heating systems, including those most often used in chemistry, employ $2.45 \mathrm{GHz}(12.2 \mathrm{~cm})$ light. $^{70-75}$ Commercially available microwave heating units were available as early as 1947, and today microwave ovens are ubiquitous. ${ }^{71,73}$ The use of microwaves in organic chemistry began to gain popularity in 1986, when dramatic increases in rate were reported in reactions in domestic microwaves in independent reports by Gedye and Giguere. ${ }^{76,77}$ Microwave heating in chemistry was initially slow to be adopted due to safety concerns with domestic microwaves, but rapidly increased in popularity with the advent of microwaves designed for lab use beginning in the $1990 \mathrm{~s}^{70-75}$ Interest in microwave heating in chemistry, especially organic chemistry, increased rapidly throughout the 2000 s as it became clear that microwave heating offered a host of advantages, including diminished reaction times, higher yields, fewer side 
products, expanded substrate scope, diminished catalyst loading or reagent stoichiometry, and alterations in regiochemistry. ${ }^{70-75,78}$ Microwave heating has also received attention as a green technique. ${ }^{79}$ It is often a more efficient way of heating reaction mixtures, is often safer due to built-in safety features, and may allow reactions to occur with substrates that are safer or more environmentally friendly, but usually less reactive. ${ }^{78,79}$ Microwave heating has now been applied to a huge variety of disciplines, including inorganic chemistry, radical chemistry, polymer chemistry, electrochemistry, photochemistry, heterogeneous and homogeneous catalysis, protein and carbohydrate chemistry, natural product synthesis, gas-phase and solid-state chemistry, biomass valorization, and more. ${ }^{70-}$ $75,78,79$ The utility of microwave heating has also continued to expand due to the invention and commercialization of microwave systems that allow scale-up in continuous-flow or stop-flow reactors, and parallel synthesis. ${ }^{70-75,78,79}$

\subsubsection{Microwave Heating}

Microwave heating occurs through a process known as dielectric heating, which relies upon the material to absorb microwave energy and convert it into heat energy. ${ }^{70-75}$ This mechanism is different than conventional heating $(\mathrm{CH})$ methods, in which heat energy is transferred from one surface or object to another. Dielectric heating is caused by the electronic component of the electro-magnetic field, and can be further broken down into ionic conduction and dipolar polarization. ${ }^{70-75}$ Ionic conduction occurs when there are ions present in the reaction mixture. As the electric field oscillates, it exerts a force on the ions, causing them to move, and thereby generating heat. Dipolar polarization occurs when polar species are present in the reaction mixture..$^{70-75}$ The dipoles align when exposed to the 
electromagnetic field. Then, as the field oscillates, the dipoles attempt to realign themselves, in essence transferring rotational energy to the molecules. As the molecules collide, this rotational energy can be transferred into heat energy.$^{70-75}$ The magnitude of heating relies on the ability of the mixture to align itself with the applied field; if alignment with the field is rapid, or if the dipole does not have time to begin to realign, no heating can occur. The frequency of $2.45 \mathrm{GHz}$ used by almost all microwave heating systems usually falls between these extremes, allowing the material to begin to realign before oscillating. ${ }^{70-75}$

The efficiency with which a material is heated under microwave radiation is dependent on a number of factors, including the efficiency with which microwave radiation is absorbed, and the ability of the molecule to align with the electromagnetic field. ${ }^{70-75} \mathrm{In}$ most cases the behaviour of a material under microwave irradiation can be described by the dielectric loss tangent, $\tan \delta$. A $\tan \delta$ of 0 indicates that a material is completely microwave transparent, and cannot convert microwave energy to heat, while substances with a high $\tan \delta$ are able to efficiently and rapidly transform microwave radiation to heat. For practical purposes, materials with a $\tan \delta$ of above 0.5 are considered to be high microwave absorbers, while materials with a $\tan \delta$ of 0.1 or below are considered low microwave absorbers. Most solids have low $\tan \delta$ values (less than 0.1), as do most nonpolar liquids (Table 1.1). Polar solvents can range from low to high microwave absorbers, with tan $\delta$ values from 0.042 for dichloromethane to 1.35 for ethylene glycol..$^{70-74}$ Microwave heating of mixtures with low $\tan \delta$ values are still possible if polar or ionic materials can be dissolved. Tan $\delta$ values are not readily available for ionic liquids. However, microwave heating of ionic liquids is 
extremely efficient and rapid, and temperature increases of hundreds of degrees have been observed in a matter of seconds. ${ }^{72,80}$

Table 1.1 Loss factors $(\tan \delta)$ of solvents and other relevant materials ${ }^{\mathrm{a}}$.

\begin{tabular}{cccc}
\hline Material & Tan & Material & Tan $\boldsymbol{~}$ \\
\hline Ethylene Glycol & 1.350 & $1 \% \mathrm{NaCl}$ in water ${ }^{\mathrm{b}}$ & 0.3 \\
Ethanol & 0.941 & Distilled Water & 0.123 \\
DMSO & 0.825 & Dichloromethane & 0.042 \\
5\% NaCl in water & 0.8 & Hexane & 0.020 \\
Methanol & 0.659 & Borosilicate Glass & 0.0010 \\
1-Butanol & 0.571 & Fused Quartz & \\
& & & 0.00006 \\
\hline
\end{tabular}

${ }^{\mathrm{a}}$ Information from ${ }^{71}$. ${ }^{\mathrm{b}}$ Information from ${ }^{81}$. ${ }^{\mathrm{c}}$ Information from ${ }^{75}$.

\subsubsection{Effects of Microwave Heating}

\subsubsection{Bulk Thermal Effects and Microwave Specific Effects}

The effects of microwave heating can be broadly divided into three categories; thermal effects, microwave specific thermal effects, and non-thermal effects. Thermal effects are due to an increase in temperature of the bulk reaction mixture, and can be obtained using conventional heating methods; microwave specific thermal effects cannot be easily replicated by conventional heating methods, but are still thermal in nature; and non-thermal effects are effects of microwaves not related to an increase in thermal 
energy. ${ }^{70-75,82}$ The existence of non-thermal microwave effects remains controversial, and they are usually only invoked in very limited circumstances. ${ }^{70-75,82,83}$ For this reason, nonthermal microwave effects will not be explored further in this work.

The majority of observed rate increases in microwave heating are attributable to bulk thermal effects. ${ }^{70-75}$ Most microwave systems allow for reactions in sealed containers, allowing reactions to be superheated well above their boiling point, which is the maximum temperature employed in most syntheses with conventional heating methods. Applying the Arrhenius equation, $k=A e^{-E a / R T}$, we can see that reaction rate will increase exponentially as temperature increases. ${ }^{70-75,84}$ Even modest increases in temperature can therefore have a large effect on reaction rate. Baghurst and Mingos have reported, based on the Arrhenius equation, that a reaction that required 68 days at $27{ }^{\circ} \mathrm{C}$ will require $13.4 \mathrm{~h}$ at $77{ }^{\circ} \mathrm{C}, 11.4$ min at $127{ }^{\circ} \mathrm{C}$, and only 23 seconds at $177^{\circ} \mathrm{C} .{ }^{84}$ More generally, reaction times can be crudely estimated using the "rule of thumb" that an increase of $10{ }^{\circ} \mathrm{C}$ in temperature corresponds to a doubling of reaction rate. ${ }^{85}$

The unique mechanism of microwave heating may lead to a number of microwave specific effects. These effects are still thermal in nature, but may be difficult to attain using conventional heating methods. ${ }^{70-75}$ First, it has been proposed that microwave heating may lead to selective heating if some reaction components are much more strongly microwave absorbing. This could lead to the formation of heterogeneous or homogeneous hotspots, which would be much hotter than the bulk mixture; reactions occurring in these hotspots would therefore occur more rapidly than the Arrhenius equation would suggest. In some cases, it has been suggested that specific reagents in a homogeneous mixture may form a 
hotspot; such reagents are commonly referred to as molecular radiators. Since these effects are microscopic in scale, and usually occur in sealed vessels during microwave irradiation, they are difficult to study. The existence of heterogeneous hotspots is generally accepted, but the existence and effects of homogeneous microwave hotspots remains disputed..$^{70-75,82}$ A second microwave specific effect is the rapid heating of reaction mixtures, or "flash" microwave heating. ${ }^{70-75}$ Reaction mixtures that contain a strongly microwave-absorbing solvent or reagents (ie with a high $\tan \delta$ ) may reach the desired temperature within seconds; in ionic liquids, temperature increases of hundreds of degrees have been reported in as little as several seconds ${ }^{80}$ Finally, microwave heating may lead to the reduction or elimination of so-called "wall effects" ${ }^{70-75}$ In conventional heating, heat must be conducted through the walls of the vessel to the reaction mixture. A temperature gradient must therefore be present, with the walls of the reaction mixture exceeding the temperature of the reaction solution. It has been proposed that certain side reactions, particularly catalyst degradation, take place in the high-temperature region at or near the walls of the vessel. In contrast, since the vessel walls are usually nearly microwave transparent, microwaves heat the bulk reaction mixture directly while the vessel walls remain relatively cool, eliminating any wall effects.

The reason why certain reactions proceed differently under microwave heating is somewhat contentious and varies from reaction to reaction. During the early development of microwave heating in chemistry microwave specific effects and even non-thermal microwave effects were invoked in a large number of reactions. ${ }^{70-75}$ However, in 2009 and 2013 Kappe and coworkers pioneered the use of silicon carbide containers that were able 
to mimic the rapid superheating commonly observed under microwave conditions without exposing the reaction mixture to microwaves ${ }^{86,87}$ These experiments demonstrated that in the majority of cases it was the bulk thermal effects that led to observed improvements, not microwave specific effects. ${ }^{86,87}$ Microwave specific effects are still invoked in certain circumstances, but such claims are subject to a great deal more scrutiny. Circumstances in which microwave specific effects are commonly claimed include reactions with highly polar reagents in a nonpolar medium, in which molecular radiators may be formed; biphasic mixtures in which one phase has a higher $\tan \delta$, in which selective heating occurs; or heterogeneously catalyzed reactions, in which heterogeneous hotspots may form..$^{70-75}$

Microwave heating has become an extremely widespread technique in synthetic chemistry, especially in organic chemistry. Even within the domain of organic chemistry, or even homogeneously catalyzed organic chemistry, the literature is far too vast for any sort of systematic review in this limited space. Instead, the following sections will present select reactions that demonstrate alterations in reaction rate, yield, catalyst loading, and selectivity observed under microwave heating.

\subsubsection{Increases in Rate and Expanded Substrate Scope}

The most common effect observed under microwave heating is a rapid increase in rate. ${ }^{70-75}$ The increased rate of reactions under microwave heating is a major reason for the success and spread of microwave chemistry, as microwave heating has been widely applied as a cost and time-saving measure. ${ }^{70-75}$ Microwave heating is now routinely employed by many companies and groups in target screening and reaction optimization, as hundreds of reactions can be run in minutes on a parallel synthesis system. As described in Section 
1.2.2.1, increases in reaction rate under microwave heating are generally attributable to bulk thermal effects, as microwave heating allows rapid heating to temperatures above the boiling point of a solvent, but may in some cases be attributed to microwave specific thermal effects. $^{71-73,88,89}$ One example of the former is the intramolecular Diels-Alder reaction and subsequent hydrolysis of pyrazinones (Scheme 1.15). ${ }^{90}$ Under conventional heating, this reaction was performed via $24 \mathrm{~h}$ reflux in chlorobenzene, followed by an $18 \mathrm{~h}$ hydrolysis to form the final product, for a total reaction time of $42 \mathrm{~h} .{ }^{90,91}$ Under microwave heating the Diels-Alder reaction could be performed in $1 \mathrm{~h}$ in DCE at $170{ }^{\circ} \mathrm{C}$. However, when the reaction was doped with 1-butyl-3-methylimidazolium hexafluorophosphate (bmimPF $)$, an ionic liquid, the reaction could be performed at $190{ }^{\circ} \mathrm{C}$ in only $8 \mathrm{~min} .{ }^{90}$ Hydrolysis could then be performed simply by adding a small amount of water via syringe and heating the reaction to $130{ }^{\circ} \mathrm{C}$ for $5 \mathrm{~min}$. The use of microwave techniques therefore allowed a reaction that required $42 \mathrm{~h}$ in the literature to be performed in only $13 \mathrm{~min}$, nearly 200 times faster, with nearly identical product yield..$^{90}$

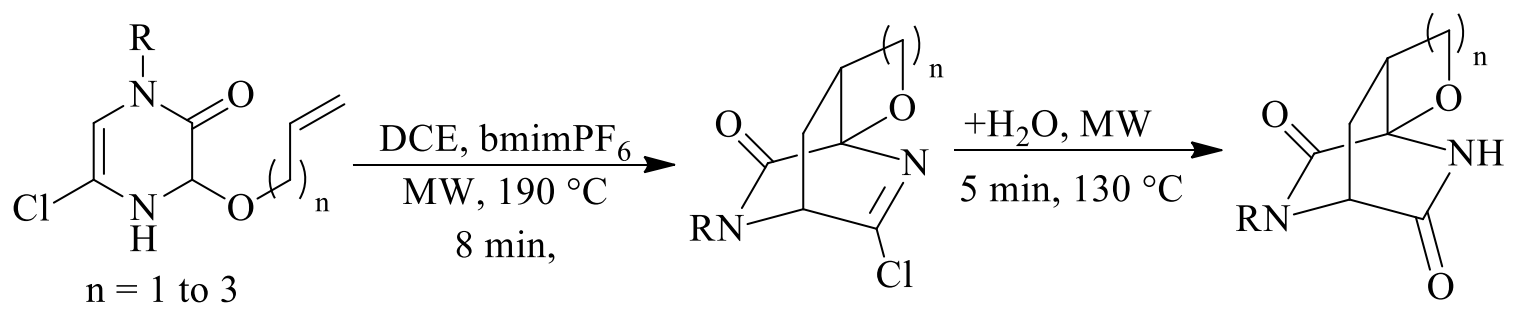

Scheme 1.15 Intramolecular Diels-Alder reaction and hydrolysis of pyrazinones under microwave heating 
Reactions in which increases in rate are attributed to microwave specific effects are less common. However, reactions with polar reagents in nonpolar solvents and reactions with heterogeneous catalysts often report increases in rate attributed to microwave hotspots or molecular radiators. Reactions with heterogeneous catalysts in particular often cause large increases in rate, and the formation of hotspots hundreds of degrees hotter than the bulk solution have been reported. A typical example is the hydrogenation of alkenes using palladium-on-carbon $(\mathrm{Pd} / \mathrm{C})$ as a heterogeneous catalyst (Scheme 1.16). ${ }^{92}$ Using microwave heating, these reactions reached yields of $99 \%$ in only $5 \mathrm{~min}$. Under conventional heating, a yield of only $55 \%$ was obtained under similar, strictly controlled conditions. $^{92}$

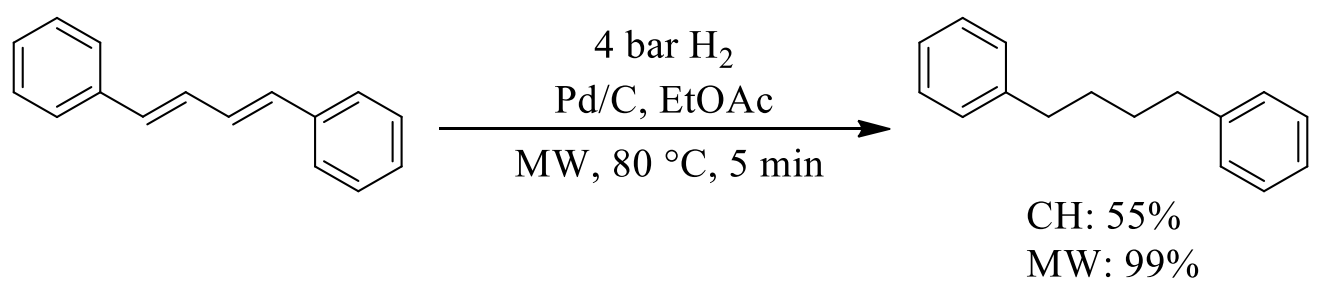

Scheme 1.16 Alteration of reaction rate under microwave heating in the hydrogenation of alkenes

Increased reaction rates frequently lead to the simple, rapid reaction of substrates that are sluggish, or do not react at all under conventional heating. This effect can be observed in the Suzuki reaction, which has been extensively studied under microwave heating. In 2004 Lidstrom and coworkers reported that challenging aryl chlorides could be rapidly coupled in good yields under microwave heating. ${ }^{93}$ A number of papers describing 
Suzuki coupling with aryl chlorides soon followed ${ }^{94}$ Further, Sanford and co-workers described a highly unusual Suzuki cross-coupling via C-F bond activation, as they were able to successfully couple a number of fluoroaryl reagents under microwave heating (Scheme 1.17). ${ }^{95}$ Similar expansion in substrate scope has been described in Kumada coupling, with aryl chlorides that reacted sluggishly under conventional heating reacting rapidly under microwave heating to attain higher yields. ${ }^{96}$

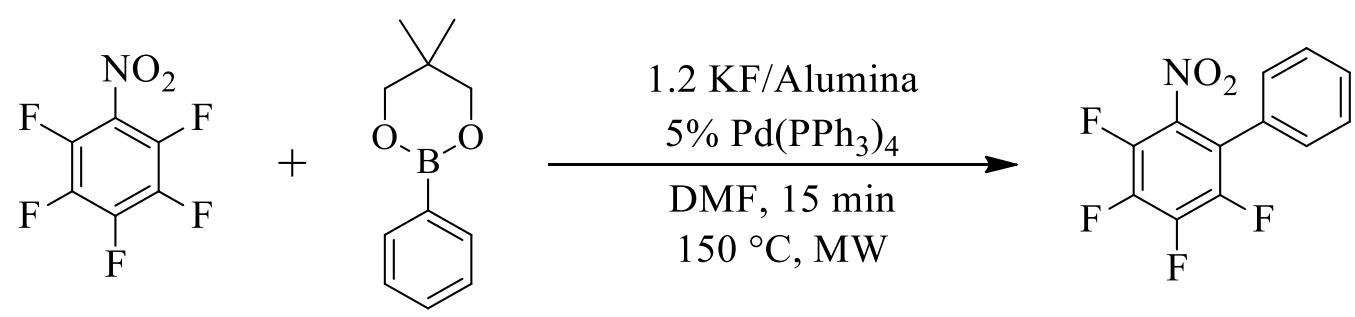

Scheme 1.17 C-F bond activation in a Suzuki-Miyaura coupling reaction under microwave heating

\subsubsection{Higher Yields and Cleaner Reaction Profiles}

Microwave heating may in some cases produce higher yields and cleaner reaction profiles than conventional heating; often, this allows smaller stoichiometric excesses to be used under microwave heating. ${ }^{70-75}$ Higher yields and cleaner reaction profiles are usually ascribed to effects such as rapid bulk heating of the reaction mixture and the absence of wall effects, as described in Section 1.2.2.1. One notable example is the reaction of urea to form cyanuric acid (Scheme 1.18) ${ }^{97}$ Under conventional heating the reaction is sluggish, and only low yields of the desired product are obtained, while a significant amount of 
ameline and amelide side products are obtained. Under microwave heating yields of $83 \%$ can be obtained by heating to the same temperature for $2 \mathrm{~min} .{ }^{97}$ The difference was revealed to be due to the formation of cyanuric acid on the walls of the reaction vessel, which formed an insulating layer and prevented efficient heat transfer. Increasing the temperature further under conventional heating resulted in decomposition of the cyanuric acid on the walls of the vessel. ${ }^{97}$<smiles>NC(N)=O</smiles>

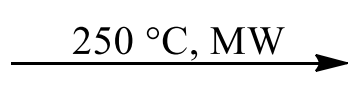<smiles>O=c1[nH]c(=O)[nH]c(=O)[nH]1</smiles>

$83 \%$

Scheme 1.18 Synthesis of cyanuric acid from urea under microwave heating

Van der Eycken and coworkers have reported increased yields and reduction of side products in several Suzuki reactions. They were able to successfully couple 2-nitrophenylboronic acid with a number of halogenated $N$-heteroarenes (Scheme 1.19). ${ }^{98}$ Under conventional heating, reactions with 2-nitrophenylboronic acid are susceptible to protodeboronation, and yields of the desired product are reduced as nitrobenzene is formed as the major product. $^{98}$ However, under microwave heating protodeboronation was suppressed, and the desired product was obtained in yields of $86 \%$. In another report, they directly compared the extremely challenging coupling of phenethylamine and 2-formylphenylboronic acid under microwave heating and conventional heating (Scheme 1.20). ${ }^{99}$ Under conventional heating they were able to attain a maximum yield of $22 \%$ after 
$14 \mathrm{~h}$ at $150{ }^{\circ} \mathrm{C}$. The majority of the 2 -formylphenylboronic acid was found to have undergone protodeboronation, producing benzaldehyde as the major product. Using microwave heating, a maximum yield of $84 \%$ was obtained at the same temperature in only 15 min. Benzaldehyde was still observed, but its formation was significantly diminished. ${ }^{99}$ The group was unable to fully explain the observed difference between microwave and conventional heating, but suggested microwave specific effects were involved. Similar activity was noted by the group during their later synthesis of buflavine analogues. ${ }^{100}$

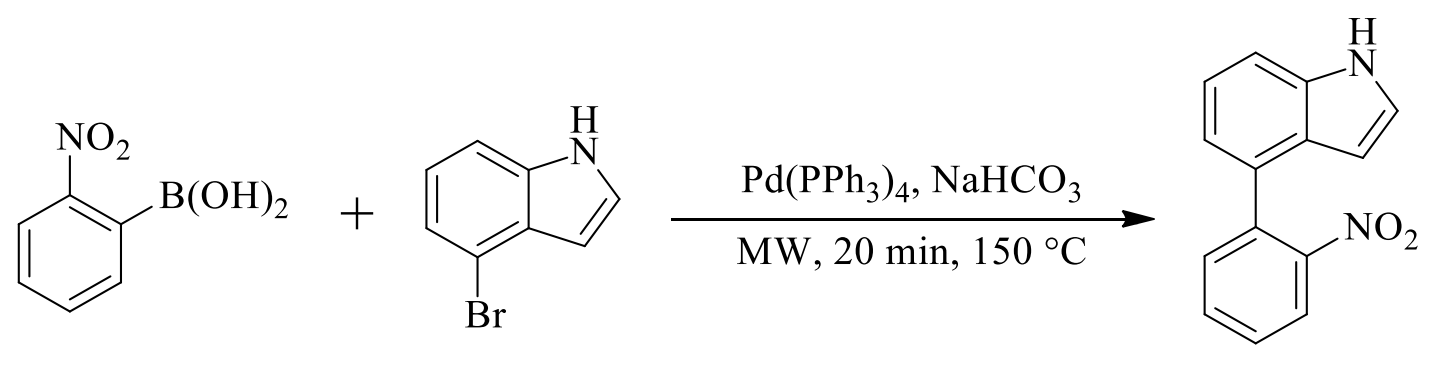

Scheme 1.19 Suzuki coupling of 2-nitrophenylboronic acid and 4-bromoindole under microwave heating<smiles>COc1cc(Br)c(CCNC(=O)OCc2ccccc2)cc1OC</smiles>

Scheme 1.20 Comparison of microwave and conventional heating in the Suzuki coupling of a phenethylamine and 2-formylphenylboronic acid 


\subsubsection{Reduced Catalyst Loading}

Microwave heating has been observed to allow reactions to proceed with lower catalyst loadings, or lower stoichiometric amounts of reagents. In 2003 Leadbeater and Marco reported what appeared to be a metal-free Suzuki reaction under microwave conditions - the first such reaction to be reported. ${ }^{101,102}$ They were able to successfully couple aryl iodides and bromides in an aqueous solution at $150{ }^{\circ} \mathrm{C}$ in only 5 min using only tetrabutylammonium bromide (TBAB) and sodium carbonate (Scheme 1.21). ${ }^{101,102}$ Using inductively coupled plasma-atomic absorption (ICP-AA) spectroscopy, the reaction mixture was determined to be palladium-free below $0.1 \mathrm{ppm}$, and free of any transition metal that had been previously used as a catalyst for Suzuki reactions below 1 ppm. ${ }^{101,102}$ Since there were no reports of catalysis with such low concentrations of metal catalyst, they concluded that the reaction was fully metal-free. However, in 2005 they re-examined the reaction using a more sensitive analytical technique, and determined that the reaction could be catalyzed by palladium impurities present in the sodium carbonate salt with a concentration as low as $50 \mathrm{ppb} .{ }^{103}$ This corresponded to a catalyst loading of 0.0000008 mol\%, with a turnover number of $1250000 .{ }^{103}$ This method was later extended to Heck reactions. ${ }^{104}$ Attempts were made to reproduce these reactions using conventional heating methods. However, it was discovered that the yield of the reaction using conventional heating was much lower until catalyst loading approached $2.5 \mathrm{ppm}$, at which point similar yields were observed. point similar yields were observed. point similar yields were observed. 


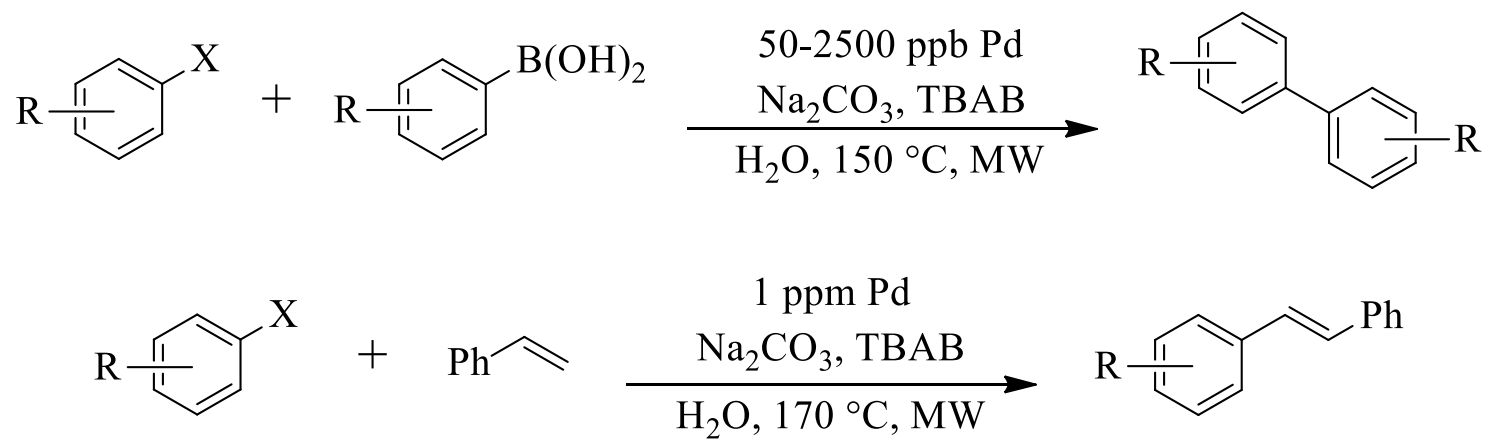

Scheme 1.21 Suzuki and Heck reactions with extremely low catalyst loading under microwave heating.

\subsubsection{Altered Selectivity}

There are a number of reports of reactions performed under microwave heating that yield products with different regio-, stereo-, enantio-, or chemoselectivity than reactions performed under conventional heating. ${ }^{89,91,105}$ These alterations are not generally wellunderstood, but are usually rationalized by examination of the thermodynamics of a given reaction and the various intermediates formed. ${ }^{89,91,105}$ Alterations to chemo- and regioselectivity appear to be particularly common in cases involving $N$-heterocycles as reagents. One early example of this phenomenon occurred in the alkylation of 1,2,4-triazole with benzyl chloride, which was found to proceed without base or solvent under microwave irradiation (Scheme 1.22). ${ }^{106}$ Under conventional heating methods this reaction afforded low yields $N$-1,4-disubstituted product. ${ }^{106}$ However, under microwave heating, a 70\% yield was obtained of $N$-1-substituted product. The exact mechanism behind this selectivity remains unclear, and the researchers invoked selective heating effects to explain the phenomeon. ${ }^{106}$ Similar work by Moneuse and coworkers in the phenacylation of $1,2,4-$ 
triazole showed similar selectivity when the reactions occurred solvent-free or in a nonpolar solvent. ${ }^{107}$ In polar solvents, however, conventional heating and microwave heating gave similar product distributions, supporting the conjecture that differences in selectivity were due to selective microwave heating. ${ }^{107}$ Modifications to selectivity were also observed during the benzylation of 2-pyridones under microwave heating, as it was discovered that selectivity could be altered under microwave heating (Scheme 1.23). ${ }^{108}$ At lower temperatures and microwave power the $\mathrm{N}$-alkylated product was formed. ${ }^{108}$ At higher temperatures and powers a mixture of 3-substituted, 5-substituted, and 3,5-disubstituted products were formed. Reactions under conventional heating afforded only the $\mathrm{N}$ substituted product. ${ }^{108}$

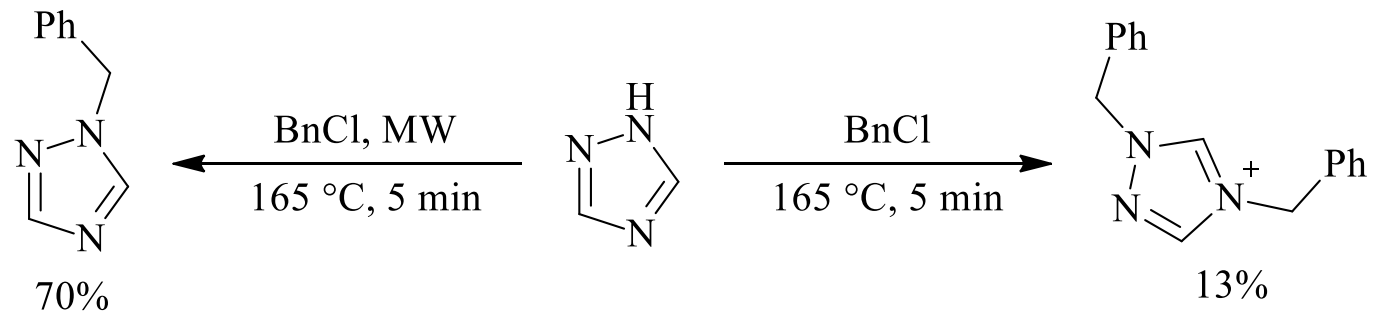

Scheme 1.22 Alteration in selectivity in the alkylation of 1,2,4-triazole under microwave heating 


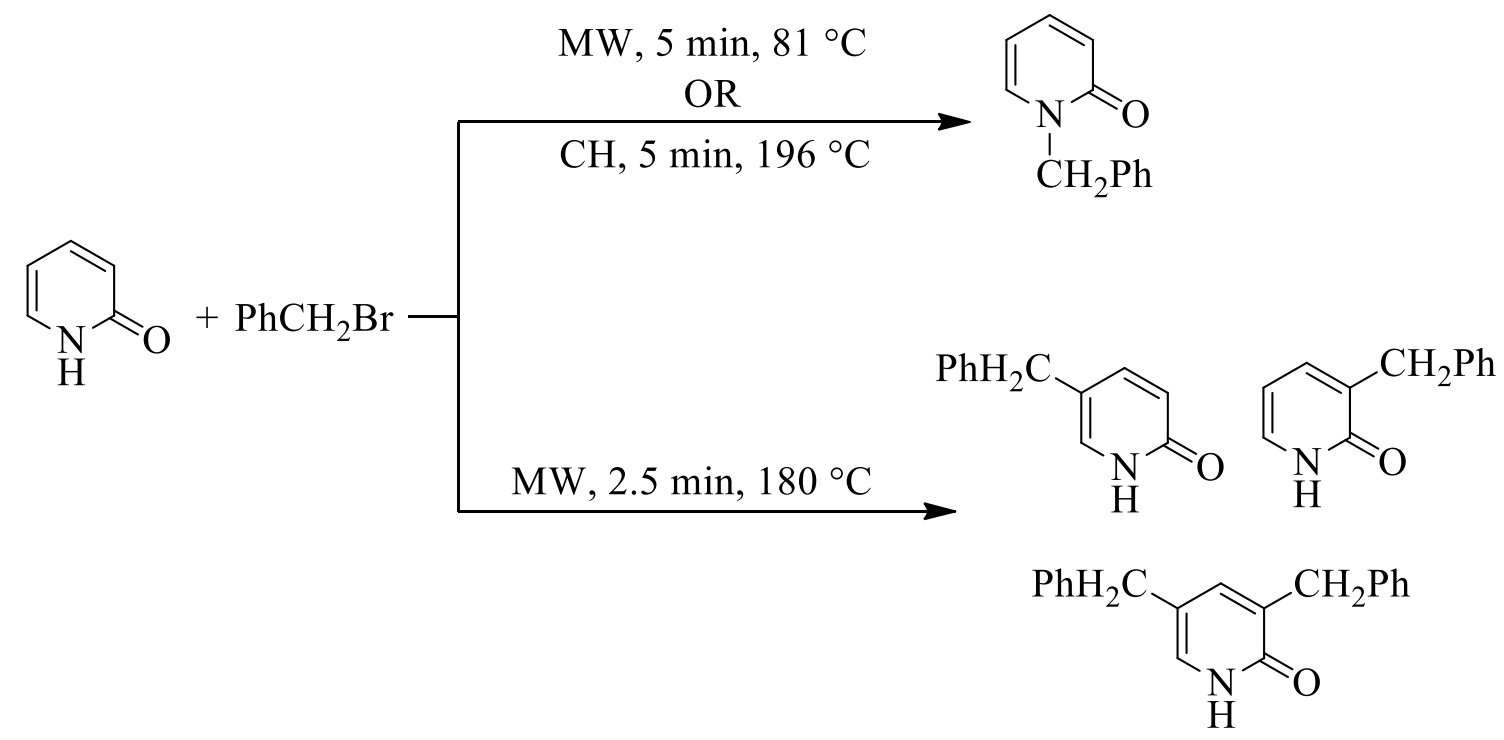

Scheme 1.23 Alterations in chemoselectivity during the benzylation of 2-pyridone under microwave heating

\subsubsection{Organic Syntheses under Select Conditions}

\subsubsection{Radical Reactions}

Despite the advantages demonstrated by microwave heating in conventional organic chemistry, the application of microwave heating to radical organic synthesis has been relatively limited. Microwave heating is often applied to radical polymerization reactions $^{109}$ and in the radical degradation of biomass ${ }^{110,111}$ or pollutants ${ }^{112-114}$, but reports of radical organic syntheses remain somewhat rare. This may be in part due to the remarkably late initial interest in these reactions, as the first radical reaction under microwave heating was not reported until $1991^{115}$, and the second was not reported until 1999. ${ }^{116}$ These early reactions, and radical reactions under microwave heating in general, show similar benefits to other reactions, such as diminished reaction times and cleaner 
reaction profiles. ${ }^{89,117}$ It has been suggested that this is due to the higher temperatures that can be easily achieved under microwave heating. Higher temperatures increase the rate of thermal dissociation in common initiators such as peroxide, persulfate or azo compounds. The higher temperatures also increase the rate of propagation steps. However, termination steps, which have very low activation energies in most cases, will see a smaller rate increase; therefore, longer chain reactions and better yields would be expected at higher temperatures.

The plurality of reported radical reactions under microwave synthesis are either radical oxidations, often using hydrogen peroxide, or intramolecular ring-forming reactions, generally involving the coupling of an alkene or alkyne to a carbon adjacent to a halide, or in some cases forming a C-N bond with an amine or imine ${ }^{89,117}$ These reactions generally proceed in a manner of minutes, with few side products formed. Cross-coupling reactions are much less common, although recently interest seems to have increased. Demonceau and coworkers have reported the coupling of carbon tetrachloride and chloroform to primary and secondary alkenes, and were able to reduce reaction times from $30 \mathrm{~h}$ under conventional heating at $85^{\circ} \mathrm{C}$, to only 10 min under microwave heating at 160 ${ }^{\circ} \mathrm{C} .{ }^{118}$ Horikoshi and coworkers were able to successfully couple iodocyclohexane with crotonophenone using $t$-butylhydroperoxide and triethylborane. ${ }^{119}$ Under microwave irradiation they were able achieve a yield of $93 \%$, while a yield of only $40 \%$ was achieved under conventional conditions. Peñéñory and coworkers reported a thermal $\mathrm{S}_{\mathrm{RN}} 1$ reaction in which aryl halides could be coupled with aryl or alkyl enolate anions in as little as 10 min. ${ }^{120}$ Under microwave heating they were able to obtain a yield of $55 \%$ when coupling 
iodobenzene and excess acetophenone; under the same conditions, they were only able to obtain a yield of $10 \%$ with conventional heating (Scheme 1.24). They attributed this difference to localized superheating, although they were unable to confirm this. Finally, there have been only three reports of radical biaryl coupling under microwave heating. Two were the manganese-mediated coupling of arylboronic acids with arenes and heteroarenes described in Section 1.1.2.1. The other is a report from 2008 from Itami and coworkers, who reported the coupling of $N$-heteroarenes and aryl iodides using $\mathrm{KO}^{t} \mathrm{Bu}$ in as little as 5 min using microwave heating (Scheme 1.25). ${ }^{121}$ Under microwave heating they reported yields as high as $98 \%$, while reactions using conventional heating required higher temperatures and longer reaction times, and still returned lower yields. Strangely, although this report generated significant interest, and has been examined using a variety of catalysts and other reagents, no other reports have utilized microwave heating in these reactions.

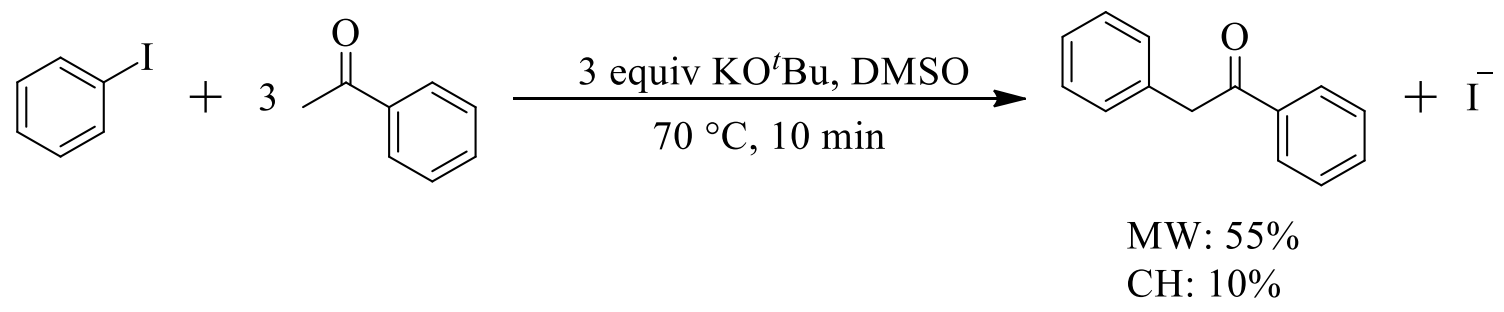

Scheme 1.24 Radical coupling of aryl iodides and benzophenones under conventional and microwave heating 


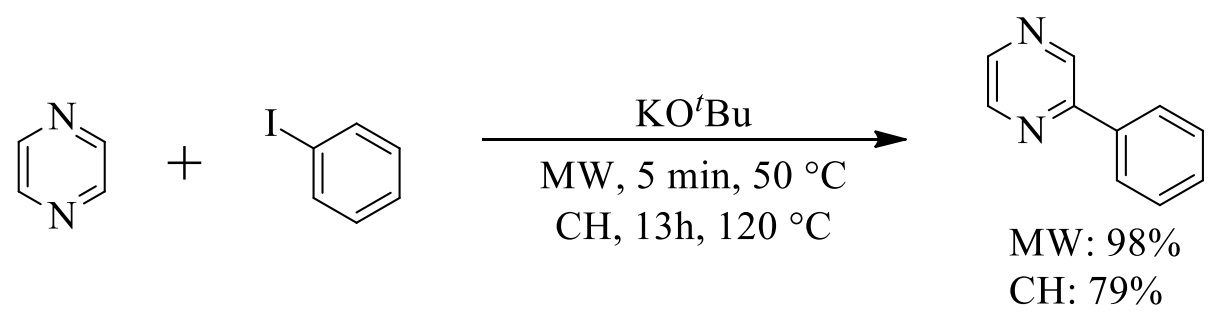

Scheme 1.25 Radical biaryl coupling of $N$-heteroarenes and aryl halides under conventional and microwave heating

Overall, radical reactions under microwave heating remain significantly understudied. The majority of studies that do exist focus on oxidation reactions and intramolecular cyclization reactions. The use of microwave heating in radical coupling reactions remains understudied despite the fact that microwave heating has proven to be beneficial in at least two such reactions.

\subsubsection{Iron-Catalyzed Reactions}

Iron-catalyzed reactions under microwave heating largely focus on heterogeneous catalysis, especially on the use of iron salts, iron nanoparticles or iron compounds supported on alumina, clay, or graphite. ${ }^{122-124}$ Iron nanoparticles have proven particularly attractive due to their relatively low cost and simple synthesis, their ability to form heterogeneous microwave hotspots, and in some cases their interaction with the microwaves magnetic field. Common applications of heterogeneous iron catalysts under microwave heating include the formation of carbon nanotubes or graphene sheets, oxidation or reduction reactions, and pyrolysis. ${ }^{122-124}$ Iron-catalyzed heterogeneous reactions have also been reported in continuous-flow and stop-flow reactors, and on larger scales. 
Iron-catalyzed homogeneous reactions under microwave heating, on the other hand, are remarkably rare. This is particularly surprising given the widespread utilization of microwave heating, and the widespread interest in the use of iron as a catalyst in an effort to perform more environmentally friendly chemistry. ${ }^{124-126}$ Further, noble-metal catalyzed cross-coupling reactions have been studied extensively under microwave conditions, and significant efforts have been made to replace these comparatively expensive and toxic metals with iron. It is remarkable then, that to the best of my knowledge there are no examples of homogeneous or heterogeneous iron-catalyzed Heck, Suzuki, Kumada or Sonogashira couplings under microwave heating. Research into iron-catalyzed homogeneous reactions under microwave heating are so rare that this section constitutes a review of the available literature.

Several homogeneous iron-catalyzed oxidation reactions have been reported. Santos and coworkers reported the iron-catalyzed Fenton oxidation of amoxicillin under microwave heating. ${ }^{127}$ They reported that under microwave heating amoxicillin could be completely degraded using low concentrations of iron catalyst in only 5 min, significantly improved from reactions under conventional heating. ${ }^{127}$ Antunes and coworkers reported the oxidation of cyclohexane using peroxides, catalyzed by tridentate pyridylamine complexes. ${ }^{128}$ They obtained a mixture of products including cyclohexanol, cyclohexanone, cyclohexyl hydroperoxide and adipic acid. Similarly, Pombeiro and coworkers reported oxidation of 1-phenylethanol and cyclohexane using pentadentate iron pyridyl amino and imino thioether complexes with peroxides and nitrogen-containing additives without additional solvent (Scheme 1.26). ${ }^{129}$ They were able to oxidize 1- 
phenylethanol to acetophenone in $74 \%$ yield in only $5 \mathrm{~min}$, for a turnover number of 369 and turnover frequency of $4430 \mathrm{~h}^{-1}$, comparable to the best iron catalysts. ${ }^{129}$ Pombeiro and coworkers then carried out another study describing the oxidation of alcohols with iron arylhydrazone of ethyl 2-cyanoacetate or formazan ligands. ${ }^{130}$ Reactions in those cases proved more sluggish, but reaction yields were higher, and the scope of alcohols that were coupled was wider.

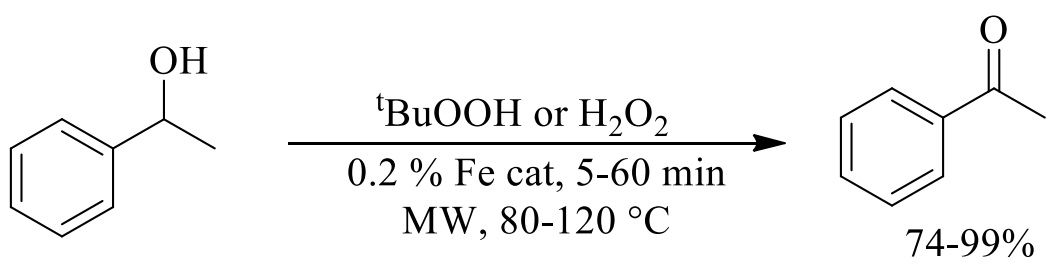

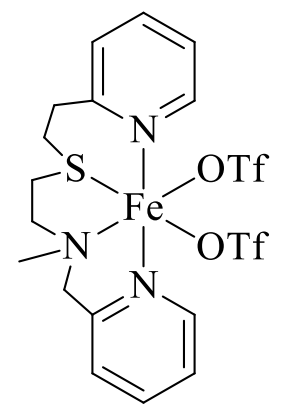

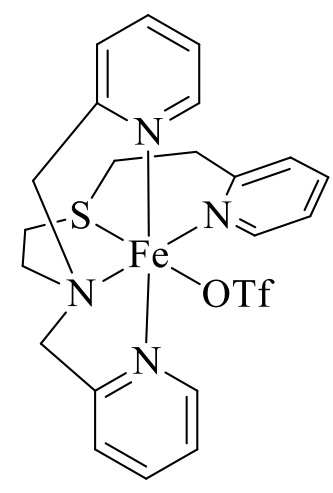

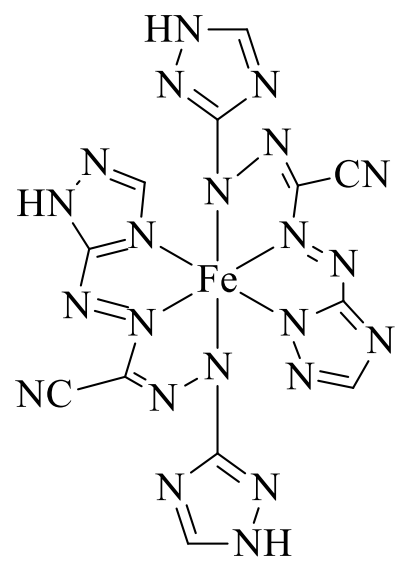

Scheme 1.26 Peroxide oxidation of 1-phenylethanol to acetophenone using microwave heating and homogeneous iron catalysts. Bottom left and centre: catalysts used in reference ${ }^{129}$. Bottom right: catalyst used in reference ${ }^{130}$.

A number of $\mathrm{C}-\mathrm{X}$ bond-forming reactions have been reported using homogeneous iron catalysts. Che and coworkers have reported the use of [ $\left.\mathrm{Fe}^{\mathrm{III}}\left(\mathrm{F}_{20}-\mathrm{tpp}\right) \mathrm{Cl}\right]$, a porphyrinato 
type complex, in the aziridination of styrenes and amination of saturated cyclic hydrocarbons and $\alpha$-methylstyrenes (Scheme 1.27). ${ }^{131}$ Under conventional heating, reactions often required 24 to $48 \mathrm{~h}$ to complete under reflux in 1,2-dichloroethane, which has a boiling point of $83.5^{\circ} \mathrm{C}$. Reactions under microwave heating were conducted at $100{ }^{\circ} \mathrm{C}$, and led to rate increases of as much as 16 times with similar yields. ${ }^{131} \mathrm{Che}$ and coworkers have also reported an intramolecular amination reaction with alkyl azides, which occurs via $\mathrm{C}-\mathrm{H}$ bond activation. ${ }^{132}$ They employed a catalyst bearing $N$-heterocyclic carbene and porphyrin ligands, which was able to selectively aminate the $\mathrm{C} 4$ or $\mathrm{C} 5$ position, creating pyrrolidines or piperidines. Under microwave heating the reaction rate increased, although yields declined somewhat. However, reactions under microwave heating were also found to be much more resistant to moisture and oxygen, and could be run under air without drying solvents. Reactions under conventional heating had to be run under argon, with rigorously dried solvents and reagents. ${ }^{132}$ Finally, Breeden and coworkers have described the microwave-assisted synthesis of diaryl sulfones from arylsulfonyls and arenes using iron (III) chloride. ${ }^{133}$ They were able to synthesize diaryl sulfones in yields as high as $86 \%$ in only $1 \mathrm{~min}$. They also compared the carbon dioxide emissions that would be caused by conventional and microwave heating and concluded that microwave heating would significantly reduce $\mathrm{CO}_{2}$ emissions to the rapid reaction times and greater energy efficiency observed under microwave heating. 


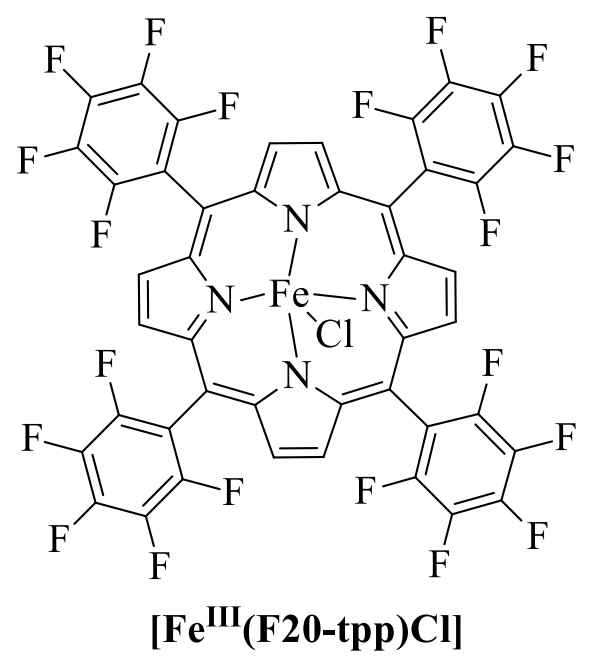<smiles>[R]NC(=C)c1ccccc1C1CN1[R]</smiles><smiles></smiles>

Scheme 1.27 Aziridination and amination reactions using a homogeneous iron catalyst under microwave heating.

A search of the literature revealed only three examples of $\mathrm{C}-\mathrm{C}$ bond-forming reactions using a homogeneous iron catalyst under microwave heating. Kang and coworkers have described an intramolecular didehydro-Diels-Alder reaction of styrene-ynes using a dual catalyst system of iron(III) chloride and iron(II) acetylacetonate. ${ }^{134}$ They were able to form a number of polyaromatic cycloadducts in 
excellent yields without the use of additional functional groups to control the reaction. $\mathrm{Fu}$ and coworkers have reported the alcoholysis of furfural alcohol into alkyl levulinates using iron(III) chloride. ${ }^{135}$ Reactions using microwave heating were able to reduce the reaction time from $2 \mathrm{~h}$ to $5 \mathrm{~min}$, with similar yields. Finally, Petricci and coworkers reported the aminocarbonylation of alkynes and amines using triiron dodecacarbonyl at low pressures of carbon monoxide (Scheme 1.28). ${ }^{136}$

$$
\mathrm{R}=+\mathrm{R}^{2} \mathrm{NH}_{2} \frac{1.3 \text { bar CO, } 0.1 \text { eq Fe }(\mathrm{CO})_{12}}{4 \mathrm{eq} \mathrm{Et}_{3} \mathrm{~N}, \mathrm{MW}, 90^{\circ} \mathrm{C}, 20 \mathrm{~min}} \longrightarrow \mathrm{R}_{\mathrm{H}}^{\sim} \mathrm{R}^{2}
$$

Scheme 1.28 Aminocarbonylation of alkynes and amines under microwave heating using triiron dodecacarbonyl as a catalyst.

Overall, the use of iron catalysts under microwave heating focuses largely on simple heterogeneous iron catalysts, perhaps due to the formation of heterogeneous hotspots. Homogeneous iron catalysts under microwave heating remains remarkably rare. This is particularly unusual given the growing interest in the use of iron as a replacement for noble metal catalysts, and the use of microwave chemistry and iron catalysis as green chemistry techniques. Iron catalysts have been reported in a number of reactions, including oxidation reactions and coupling reactions. Typically, the use of microwave heating in these cases results in lower reaction times and higher yields. There is therefore an opportunity for further exploration of iron catalyzed reactions under microwave heating. 


\subsubsection{Biphasic Reactions}

Microwave heating in liquid-liquid biphasic systems are uncommon. Reactions in such mixtures often exhibit differential heating of the two phases, especially if the two have significantly different $\tan \delta$ values. ${ }^{88,137,138}$ This can lead to altered reactivity but can also makes it difficult to accurately measure the temperature of the reaction mixture; since most microwave systems use an external IR sensor, the location of the sensor determines whether temperature is being measured in the upper phase, lower phase, or somewhere in between. For example, while heating a biphasic mixture of the ionic liquid mbimPF 6 and hexane temperature sensors nearer the top of the mixture, in the hexane phase, measured a temperature of only $70{ }^{\circ} \mathrm{C} .{ }^{138}$ Meanwhile, temperature sensor nearer the bottom of the mixture, in the ionic liquid phase, measured temperatures of up to $110{ }^{\circ} \mathrm{C}$. This difference was, however, in part due to the fact that no stirring was used in this process; efficient agitation of the reaction mixture is likely to reduce this temperature difference.

Biphasic mixtures are somewhat more common in areas outside of organic synthesis. For example, microwave heating has often been used in microwave-assisted extractions, often for the recovery and reuse of valuable chemicals. Takao and coworkers have reported the extraction of platinum-group metals from aqueous nitric acid to an ionic liquid $^{139}$. Without heating, or using conventional heating, 1 hour of shaking extracted $35 \%$, $68 \%$ and $90 \%$ extraction for $\mathrm{Ru}(\mathrm{III}), \mathrm{Rh}(\mathrm{III})$, and $\mathrm{Pd}(\mathrm{II})$ from the nitric acid layer to the ionic liquid, respectively. Under microwave heating all three metals could be more than $90 \%$ extracted in $200 \mathrm{~s}$ or less, which they attributed to superheating of the ionic liquid phase. Biphasic mixtures are also sometimes employed in polymer chemistry. In particular, 
microwave heating has often been applied to emulsion polymerization, where it can lead to the formation of extremely high-molecular-weight polymers. ${ }^{140}$

An early example of biphasic mixtures in organic synthesis was reported by Trainor and coworkers in $1995 .{ }^{141,142}$ They were able to use a mixture of water and chloroform under microwave irradiation to perform a rapid Hoffmann elimination due to the widely varying microwave absorbing abilities of the two solvents (Scheme 1.29). The elimination occurred in $1 \mathrm{~min}$ in the aqueous phase, at $110^{\circ} \mathrm{C}$; the product was then collected in the dichloromethane layer, which reached a temperature of only $48{ }^{\circ} \mathrm{C}$ in the sealed reaction vessel. Hallberg and coworkers reported the use of another biphasic mixture under microwave heating in 2001 (Scheme 1.30). ${ }^{143}$ They initially reported a Heck tri- or diarylation process, forming amine-substituted aryl vinyl ethers. They then sought to hydrolyse these products to the corresponding aldehydes. However, under conventional heating with a one-phase reaction low yields of the desired aldehydes were isolated due to extensive side reactions. In contrast, hydrolysis in a biphasic mixture of dilute hydrochloric acid and toluene under microwave heating resulted in high yields of the aldehyde in only 1 $2 \mathrm{~min}$. They suggested that the reaction occurred in the superheated acidic aqueous layer, and the aldehydes that were formed were then collected in the toluene phase, protecting them from further reaction. 
<smiles>C=CC(=O)c1ccc(OCC)cc1</smiles>

Scheme 1.29 Rapid Hoffmann elimination under microwave heating using a differentially heated biphasic mixture

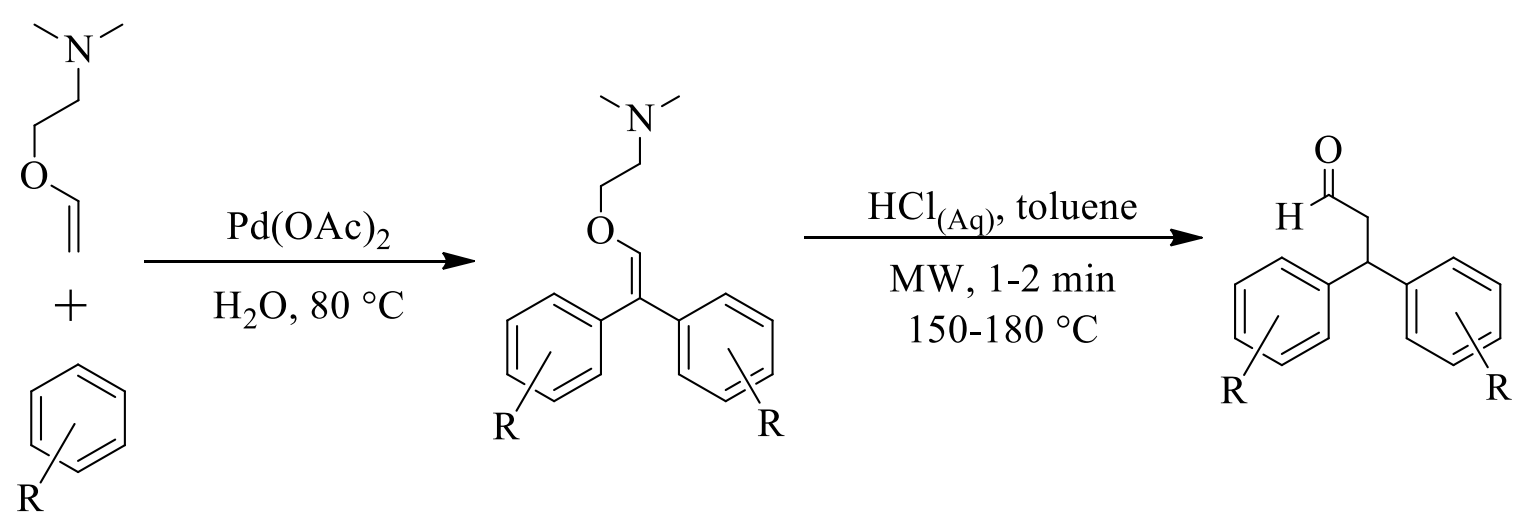

Scheme 1.30 Heck diarylation reaction, and subsequent hydrolysis in a biphasic mixture of dilute hydrochloric acid and toluene under microwave heating

A more recent example is the report by Kasprzyk and coworkers of the oxidation of primary and secondary alcohols to form carboxylic acids or ketones, and the epoxidation of olefins (Scheme 1.31 and Scheme 1.32). ${ }^{144}$ The oxidation of alcohols occurred using sodium tungstate as a catalyst and tetrabutylammonium hydrogen sulfate (TBAHS) as a phase transfer catalyst in a biphasic mixture of aqueous hydrogen peroxide and an alcohol. They reported yields ranging from 60 to $97 \%$ in the oxidation of alcohols, with secondary alcohols generally reaching higher yields than primary alcohols. They also reported that reactions under microwave heating were higher yielding than conventional heating, which gave a mixture of aldehyde and carboxylic acid products in reactions with primary alcohols. 
The presence of the phase transfer catalyst TBAHS was found to be vital to successful reactions. Epoxidation reactions were carried out with a variety of primary, secondary and tertiary olefins. Reactions took place in a mixture of dichloroethane (DCE) and aqueous hydrogen peroxide, with sodium tungstate, phosphoric acid and aliquat 336. Aliquat 336 is a quaternary ammonium salt which contains 3 octyl or decyl chains and one methyl chain and is commonly used as a phase transfer catalyst. Yields varied from 14 to $98 \%$. Reactions were most successful with simple primary and secondary arenes, while the use of substituted alkenes resulted in lower yields. Similar to the reactions with alcohols, yields were found to be higher under microwave heating, and the phase transfer catalyst aliquat 336 was found to be vital for successful reactions.

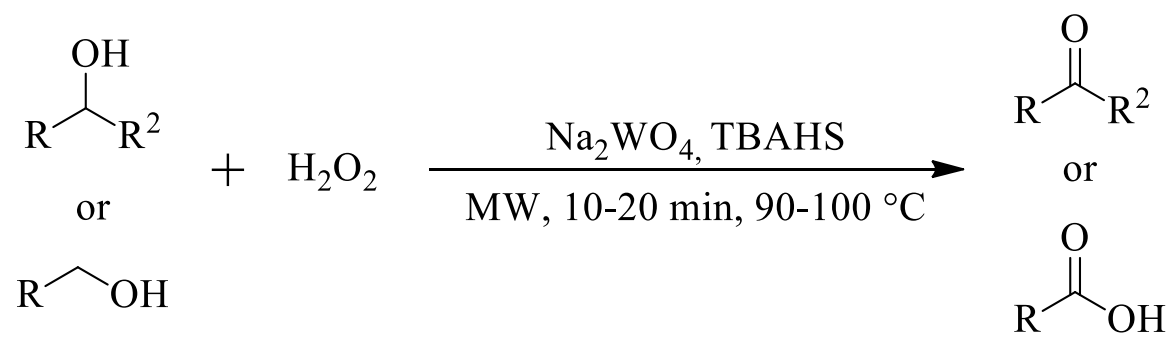

Scheme 1.31 Oxidation of primary and secondary alcohols in a biphasic mixture of alcohol and aqueous hydrogen peroxide under microwave heating 


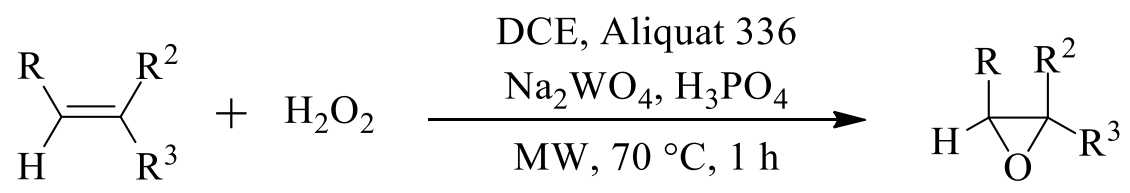

Scheme 1.32 Epoxidation of olefins in a biphasic mixture of DCE and aqueous hydrogen peroxide under microwave heating

Finally, since 2016 there have been a number of recent reports on the production of furfural or 5-hydroxymethylfurfural from biomass under microwave heating in a biphasic mixture (Scheme 1.33). ${ }^{145-149}$ All reported reactions were performed in a biphasic mixture consisting of dilute, aqueous acid and an organic layer of either acetonitrile or methyl isobutyl ketone. Reactions under microwave heating occurred rapidly compared to reactions using conventional heating, and resulted in the production of fewer side products, most notably limited formation of levulinic acid and humin. ${ }^{145-149}$ Depending on the starting material and conditions employed, yields ranged from 45 to $91 \%$ in 1 to $240 \mathrm{~min}$. Older reports were conducted on the gram scale or less. More recently, however, MartinezMerino and coworkers have reported a scale-up in a biorefinery, which was able to produce up to $2.5 \mathrm{~kg}$ of furfural from $50 \mathrm{~kg}$ of biomass, with an additional $12.6 \mathrm{~kg}$ of glucose formed. ${ }^{148}$ A detailed kinetic study was carried out by Appels and coworkers, and found that reaction rate was increased by a factor of 2.3 by microwave irradiation, even if the reactions under conventional heating were carried out in nearly identical conditions. ${ }^{146}$ Differential heating of the reaction phases did not significantly affect the reaction; instead, it was concluded that molecular radiators were formed at the $\mathrm{CH}_{2} \mathrm{OH}$ group of glucose and cellulose. A comparison was also made of energy efficiency under microwave and 
conventional heating; microwave heating was determined to be more efficient in all cases but were more favored by shorter reaction times.

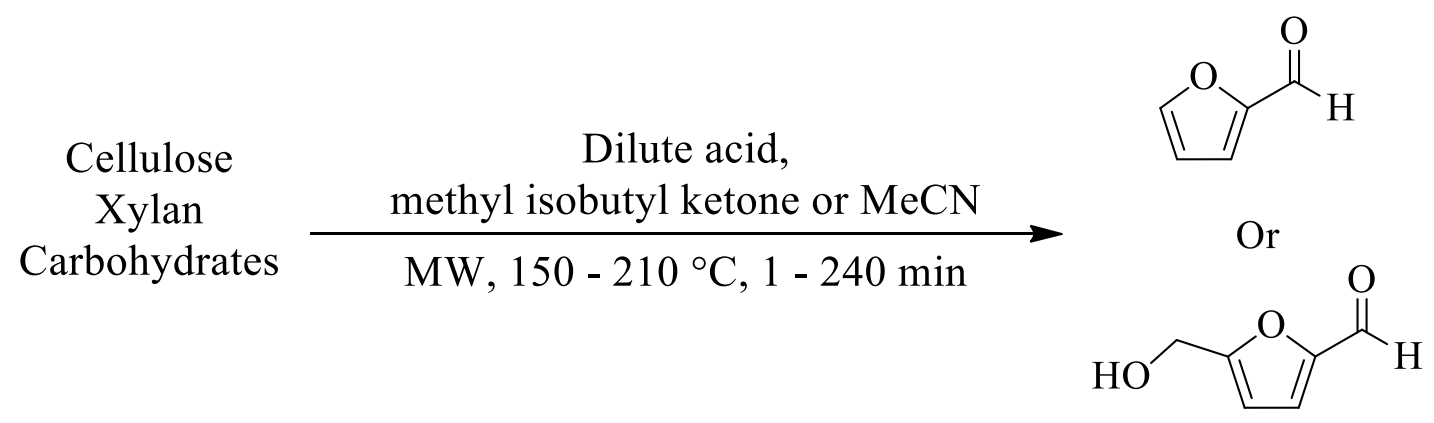

Scheme 1.33 Production of furfural or 5-hydroxymethylfurfural from biomass using microwave heating and a biphasic mixture

\subsection{Thesis Objectives}

As described in Section 1.1, radical $\mathrm{C}-\mathrm{H}$ bond activating reactions represent a valuable method of synthesizing heterobiaryl and arylquinone moieties, which are widely used in the agrochemical and pharmaceutical industries. One reaction that has received considerable attention since it was first reported in 2010 is the coupling of arylboronic acids with unactivated $N$-heterocycles and quinones (see Section 1.1.2). This reaction is advantageous due to the relatively mild conditions and the low cost of reagents. In particular, variants of the reaction using iron catalysts represent a cost-effective method for the formation of heterobiaryls or arylquinones, and also allow synthesis of arylquinones from hydroquinones and phenols. However, the reaction faces a number of challenges, including relatively long reaction times, poor regiochemical control, the need for several 
equivalents of oxidant, and limits to substrate scope. As described in Section 1.2, microwave heating of reaction mixtures can have several beneficial effects. These effects include increased reaction rates, increased yields, decreased yields of by-products, the ability to perform reactions with lower catalyst loadings or the use of fewer equivalents of reagents, and alterations to regio- and chemo-selectivity.

As noted in Section 1.1.2.1, some of the benefits of microwave heating were noted by Guchhait and coworkers when they employed microwave heating in a radical C-H bond arylation reaction using manganese(III) acetate as an oxidant and arylboronic acids as radical precursors. Despite this, microwave heating has not been applied to reactions using potassium persulfate as an oxidant. The purpose of this thesis, therefore, is to examine the iron-catalyzed radical $\mathrm{C}-\mathrm{H}$ bond arylation of $N$-heteroarenes and quinones using potassium persulfate as an oxidant under microwave heating, in an attempt to reduce reaction times, increase yields, lower catalyst loadings and the amount of oxidant required, and possibly effect the regioselectivity of the reaction. Chapter 2 presents reaction optimization under microwave heating, as well as the discovery of several unusual side products. Chapter 3 examines the scope and selectivity of the reaction under microwave heating with a variety of arylboronic acids, $N$-heteroarenes, and quinones, as well as attempts to extend the scope of the reaction. 


\subsection{References}

(1) Azman, A. M. Chapter 2: Radical-Mediated C-H Bond Activation, in C-H Bond Activation in Organic Synthesis; Li, J. J., Ed.; CRC Press, 2017.

(2) Yamaguchi, J.; Yamaguchi, A. D.; Itami, K. C-H bond functionalization: emerging synthetic tools for natural products and pharmaceuticals. Angewandte Chemie International Edition 2012, 51 (36), 8960-9009.

(3) Godula, K.; Sames, D. C-H Bond Functionalization in Complex Organic Synthesis. Science 2006, 312, 67-72.

(4) Yi, H.; Zhang, G.; Wang, H.; Huang, Z.; Wang, J.; Singh, A. K.; Lei, A. Recent Advances in Radical C-H Activation/Radical Cross-Coupling. Chemical Reviews 2017, 117 (13), 9016-9085.

(5) Li, C.-J. Cross-Dehydrogenative Coupling (CDC): Exploring C-C Bond Formations beyond Functional Group Transformations. Accounts of Chemical Research 2009, 42 (2), 335-344.

(6) Friestad, G. K. Chapter 2: Modern Developments in Aryl Radical Chemistry, in Radicals in Synthesis III; Heinrich, M. R.;Gansäuer, A., Eds.; Springer-Verlag, 2012; Vol. 320.

(7) Hammer, S. G.; Heinrich, M. R. Chapter 8: Carbon-Carbon Bond-Forming Reactions Involving Aryl Radicals, in Comprehensive Organic Synthesis II; Knockel, P.;Molander, G. A., Eds.; Elsevier, 2014; Vol. 4.

(8) Gomberg, M.; Backmann, W. E. The Synthesis of Biaryl Compounds by Means of the Diazo Reaction. Journal of the American Chemical Society 1924, 46, 23392343.

(9) Bonin, H.; Sauthier, M.; Felpin, F.-X. Transition Metal-Mediated Direct C-H Arylation of Heteroarenes Involving Aryl Radicals. Advanced Synthesis \& Catalysis 2014, 356 (4), 645-671.

(10) Bellina, F.; Rossi, R. Recent advances in the synthesis of (hetero)aryl-substituted heteroarenes via transition metal-catalysed direct (hetero)arylation of heteroarene $\mathrm{C}-\mathrm{H}$ bonds with aryl halides or pseudohalides, diaryliodonium salts, and potassium aryltrifluoroborates. Tetrahedron 2009, 65 (50), 10269-10310.

(11) Bellina, F.; Rossi, R.; Lessi, M.; Manzini, C.; Marianetti, G. Direct (Hetero)arylation Reactions of (Hetero)arenes as Tools for the Step- and AtomEconomical Synthesis of Biologically Active Unnatural Compounds Including Pharmaceutical Targets. Synthesis 2016, 48 (22), 3821-3862.

(12) Joule, J. A.; Mills, K. Chapter 1: Structures and Spectroscopic Properties of Aromatic Heterocycles, in Heteroclic Chemistry; Blackwell Publishing Ltd., 2010. 
(13) Joule, J. A.; Mills, K. Heterocyclic Chemistry; 5th ed.; Blackwell Publishing Ltd: Chichester, 2010.

(14) Murphree, S. S. Chapter 2: Heterocyclic Dyes: Preparation, Properties, and Applications, in Progress in Heterocyclic Chemistry; Gribble, G.;Joule, J. A., Eds.; Elsevier Ltd.: Amsterdam, 2011; Vol. 22.

(15) Hopkinson, M. N.; Richter, C.; Schedler, M.; Glorius, F. An overview of Nheterocyclic carbenes. Nature 2014, 510 (7506), 485-496.

(16) Kaur, K.; Jain, M.; Reddy, R. P.; Jain, R. Quinolines and structurally related heterocycles as antimalarials. European Journal of Medicinal Chemistry 2010, 45 (8), 3245-3264.

(17) Joule, J. A.; Mills, K. Chapter 32: Heterocycles in Biochemistry; Heterocyclic Natural Products, in Heterocyclic Chemistry; 5 ed.; Blackwell Publishing Ltd., 2010.

(18) Seiple, I. B.; Su, S.; Rodriguez, R. A.; Gianatassio, R.; Fujiwara, Y.; Sobel, A. L.; Baran, P. S. Direct C-H Arylation of Electron-Deficient Heterocycles with Arylboronic Acids. Journal of the American Chemical Society 2010, 132, 1319413196.

(19) Singh, P. P.; Aithagani, S. K.; Yadav, M.; Singh, V. P.; Vishwakarma, R. A. Ironcatalyzed cross-coupling of electron-deficient heterocycles and quinone with organoboron species via innate $\mathrm{C}-\mathrm{H}$ functionalization: application in total synthesis of pyrazine alkaloid botryllazine A. Journal of Organic Chemistry 2013, 78 (6), 2639-2648.

(20) Wang, J.; Wang, S.; Wang, G.; Zhang, J.; Yu, X. Q. Iron-mediated direct arylation with arylboronic acids through an aryl radical transfer pathway. Chemical Communications 2012, 48 (96), 11769-11771.

(21) Hofmann, J.; Heinrich, M. R. Recent developments in intermolecular radical arylations of arenes and heteroarenes. Tetrahedron Letters 2016, 57 (39), 43344340.

(22) Biancalana, M.; Koide, S. Molecular mechanism of Thioflavin-T binding to amyloid fibrils. Biochimica et Biophysica Acta 2010, 1804 (7), 1405-1412.

(23) Joule, J. A.; Mills, K. Chapter 31: Special Topics, in Heterocyclic Chemistry; 5 ed.; Blackwell Publishing Ltd, 2010.

(24) Swavey, S.; Brewer, K. J. Chapter 1.9: Polyatomic Bridging Ligands, in Comprehensive Coordination Chemistry II: From Biology to Nanotechnology; McCleverty, J. A.;Meyer, T. J.;Constable, E., Eds.; Elsevier Science, 2003; Vol. 1.

(25) Vats, S. Herbicides: History, Classification and Genetic Manipulation of Plants for Herbicide Resistance, in Sustainable Agriculture Reviews; Lichtfouse, E., Ed., 2015; Vol. 15. 
(26) Joule, J. A.; Mills, K. Chapter 33: Heterocycles in Medicine, in Heterocyclic Chemsitry; 5 ed.; Blackwell Publishing Ltd., 2010.

(27) Baumann, M.; Baxendale, I. R.; Ley, S. V.; Nikbin, N. An overview of the key routes to the best selling 5-membered ring heterocyclic pharmaceuticals. Beilstein Journal of Organic Chemistry 2011, 7, 442-495.

(28) Baumann, M.; Baxendale, I. R. An overview of the synthetic routes to the best selling drugs containing 6-membered heterocycles. Beilstein Journal of Organic Chemistry 2013, 9, 2265-2319.

(29) Dandawate, P. R.; Vyas, A. C.; Padhye, S. B.; Singh, M. W.; Baruah, J. B. Perspectives on Medicinal Properties of Benzoquinone Compounds. Mini-Reviews in Medicinal Chemistry 2010, 10, 436-454.

(30) Bolton, J. L.; Trush, M. A.; Penning, T. M.; Dryhurst, G.; Monks, T. J. Role of Quinones in Toxicology. Chemical Research in Toxicology 2000, 13 (3), 135-160.

(31) O'Brien, P. J. Molecular Mechanisms of Quinone Cytotoxicity. Chemico-Biological Interactions 1991, 80, 1-41.

(32) Monks, T. J.; Hanzlik, R. P.; Cohen, G. M.; Ross, D.; Graham, D. G. Quinone Chemistry and Toxicity. Toxicology and Applied Pharmacology 1992, 112, 2-16.

(33) Koyama, J. Anti-Infective Quinone Derivatives of Recent Patents. Recent Patents on Anti-Infective Drug Discovery 2006, 1 (1), 113-125.

(34) Melo, M. J. Chapter 1: History of Natural Dyes in the Ancient Mediterranean World, in Handbook of Natural Colorants; Bechtold, T.;Mussak, R., Eds.; John Wiley and Sons, Ltd.: Chichester, 2009.

(35) Bechtold, T. Chapter 10: Natural Colorants - Quinoid, Napththoquinoid and Anthraquinoid Dyes, in Handbook of Natural Colorants; Bechtold, T.;Mussak, R., Eds.; John Wiley and Sons, Ltd., 2009.

(36) Griffiths, J. In Ullmann's Encyclopedia of Industrial Chemistry Weinheim, 2006; Vol. 5.

(37) Dias, G. G.; King, A.; de Moliner, F.; Vendrell, M.; da Silva Junior, E. N. Quinonebased fluorophores for imaging biological processes. Chemical Society Reviews 2018, 47 (1), 12-27.

(38) Cullen, G. E.; Biilmann, E. The Use of the Quinhydrone Electrode for Hydrion Concentration Determination on Serum. Journal of Biological Chemistry 1925, 64, 727-738.

(39) Son, E. J.; Kim, J. H.; Kim, K.; Park, C. B. Quinone and its derivatives for energy harvesting and storage materials. Journal of Materials Chemistry A 2016, 4 (29), 11179-11202.

(40) Deb, A.; Agasti, S.; Saboo, T.; Maiti, D. Generation of Arylated Quinones by IronCatalyzed Oxidative Arylation of Phenols: Formal Synthesis of Phellodonin, 
Sarcodonin $\varepsilon$, Leucomelone and Betulinan A. Advanced Synthesis \& Catalysis 2014, 356 (4), 705-710.

(41) Thomson, R. J. Naturally Occurring Quinones IV; 4 ed.; Blackie Academic and Professional: London, 1997.

(42) Amaya, T.; Jin, Y.; Tobisu, M. Recent advances in Gomberg-Backmann biaryl synthesis. Tetrahedron Letters 2019, 60 (39), 151062.

(43) Hari, D. P.; Konig, B. The photocatalyzed Meerwein arylation: classic reaction of aryl diazonium salts in a new light. Angewandte Chemie International Edition 2013, 52 (18), 4734-4743.

(44) Klein, D. Chapter 23: Amines, in Organic Chemistry; 2 ed.; Kalkut, J.;Yee, J.;Armour, V.;Gee, L.;Swain, E., Eds.; John Wiley and Sons, Inc., 2015.

(45) Demir, A. S.; Reis, Ö.; Özgül-Karaaslan, E. Manganese(III) acetate-mediated oxidative coupling of phenylhydrazines with benzene: a novel method for biaryl coupling. Journal of the Chemical Society, Perkin Transactions 1 2001, (22), 30423045 .

(46) Demir, A. S.; Reis, Ö.; Emrullahoglue, M. Manganese(III) acetate-mediated oxidative coupling of phenylhydrazines with furan and thiophene: a novel method for hetero biaryl coupling. Tetrahedron 2002, 58, 8055-8058.

(47) Demir, A. S.; Findik, H. Potassium permanganate/carboxylic acid/organic solvent: a powerful reagent for enone oxidation and aryl coupling reactions. Tetrahedron 2008, 64 (27), 6196-6201.

(48) Kobayashi, O.; Uraguchi, D.; Yamakawa, T. Cp 2 Ni-KOt-Bu-BEt 3 (or $\mathrm{PPh}_{3}$ ) Catalyst System for Direct C-H Arylation of Benzene, Naphthalene, and Pyridine. Organic Letters 2009, 11 (12), 2679-2682.

(49) Wen, J.; Zhang, R. Y.; Chen, S. Y.; Zhang, J.; Yu, X. Q. Direct arylation of arene and N-heteroarenes with diaryliodonium salts without the use of transition metal catalyst. Journal of Organic Chemistry 2012, 77 (1), 766-771.

(50) Olofsson, B. Chapter 5: Arylation with Diaryliodonium Salts, in Hypervalent Iodine Chemistry; Wirth, T., Ed.; Springer International Publishing, 2016.

(51) Xiao, J.; Xue, D.; Liu, Y.-X.; Wang, J.-D.; Zhao, C.-J.; Zou, Q.-Z.; Wang, C. RoomTemperature Arylation of Arenes and Heteroarenes with Diaryl-iodonium Salts by Photoredox Catalysis. Synlett 2013, 24 (04), 507-513.

(52) Yan, G.; Yang, M.; Wu, X. Synthetic applications of arylboronic acid via an aryl radical transfer pathway. Organic \& Biomolecular Chemistry 2013, 11 (46), 79998008 .

(53) Fujiwara, Y.; Domingo, V.; Seiple, I. B.; Gianatassio, R.; Del Bel, M.; Baran, P. S. Practical $\mathrm{C}-\mathrm{H}$ functionalization of quinones with boronic acids. Journal of the American Chemical Society 2011, 133 (10), 3292-3295. 
(54) Riant, O.; Samuel, O.; Flessner, T.; Taudien, S.; Kagan, H. B. An Efficient Asymmetric Synthesis of 2-Substituted Ferrocenecarboxaldehydes. Journal of Organic Chemistry 1997, 62 (20), 6733-6745.

(55) Demir, A. S.; Reis, Ö.; Emrullahoglue, M. Generation of Aryl Radicals from Arylboronic Acids by Manganese(III) Acetate: Synthesis of Biaryls and Heterobiaryls. Journal of Organic Chemistry 2003, 68 (2), 578-580.

(56) Demir, A. S.; Findik, H.; Saygili, N.; Tuna Subasi, N. Manganese(III) acetatemediated synthesis of biaryls under microwave irradiation. Tetrahedron 2010, 66 (6), 1308-1312.

(57) Guchhait, S.; Kashyap, M.; Saraf, S. Direct C-H Bond Arylation of (Hetero)arenes with Aryl and Heteroarylboronic Acids. Synthesis 2010, 2010 (07), 1166-1170.

(58) Lockner, J. W.; Dixon, D. D.; Risgaard, R.; Baran, P. S. Practical Radical Cyclizations with Arylboronic Acids and Trifluoroborates. Organic Letters 2011, 13 (20), 5628-5631.

(59) Li, Z.; Mai, W.; Yuan, J.; Sun, G.; Qu, L. Silver-Catalyzed 2-Pyridyl Arylation of Pyridine N-Oxides with Arylboronic Acids at Room Temperature. Synlett 2011, 2012 (01), 145-149.

(60) Jain, R.; Mahindra, A. Regiospecific Direct C-H Arylation at the 2-Position of 1Histidine Using Arylboronic Acids. Synlett 2012, 23 (12), 1759-1764.

(61) Ren, X.; Han, S.; Gao, X.; Li, J.; Zou, D.; Wu, Y.; Wu, Y. Direct arylation for the synthesis of 2-arylquinolines from $\mathrm{N}$-methoxyquinoline-1-ium tetrafluoroborate salts and arylboronic acids. Tetrahedron Letters 2018, 59 (11), 1065-1068.

(62) Komeyama, K.; Kashihara, T.; Takaki, K. FeSO 4 -promoted direct arylation of benzoquinones with $\mathrm{ArB}(\mathrm{OH})_{2}$ or $\mathrm{ArBF}_{3} \mathrm{~K}$. Tetrahedron Letters 2013, 54 (9), 10841086.

(63) Komeyama, K.; Nagao, Y.; Abe, M.; Takaki, K. Scope and Limitation for FeSO ${ }_{4-}^{-}$ Mediated Direct Arylation of Heteroarenes with Arylboronic Acids and Its Synthetic Applications. Bulletin of the Chemical Society of Japan 2014, 87 (2), 301313.

(64) Deb, A.; Manna, S.; Maji, A.; Dutta, U.; Maiti, D. Iron-Catalyzed Direct C-H Arylation of Heterocycles and Quinones with Arylboronic Acids. European Journal of Organic Chemistry 2013, 2013 (24), 5251-5256.

(65) Modak, A.; Rana, S.; Maiti, D. Iron-Catalyzed Regioselective Direct Arylation at the C-3 Position of N-Alkyl-2-pyridone. Journal of Organic Chemistry 2015, 80 (1), 296-303.

(66) Huang, Y.; Guan, D.; Wang, L. Direct Arylation of Substituted Pyridines with Arylboronic Acids Catalyzed by Iron(II) Oxalate. Chinese Journal of Chemistry 2014, 32 (12), 1294-1298. 
(67) Thatikonda, T.; Singh, U.; Ambala, S.; Vishwakarma, R. A.; Singh, P. P. Metal free $\mathrm{C}-\mathrm{H}$ functionalization of diazines and related heteroarenes with organoboron species and its application in the synthesis of a CDK inhibitor, meriolin 1. Organic \& Biomolecular Chemistry 2016, 14 (18), 4312-4320.

(68) Patel, N. R.; Flowers, R. A., 2nd. Uncovering the mechanism of the $\operatorname{Ag}(\mathrm{I}) /$ persulfate-catalyzed cross-coupling reaction of arylboronic acids and heteroarenes. Journal of the American Chemical Society 2013, 135 (12), 46724675 .

(69) Bering, L.; Antonchick, A. P. Regioselective Metal-Free Cross-Coupling of Quinoline N-Oxides with Boronic Acids. Organic Letters 2015, 17 (12), 31343137.

(70) Larhed, M.; Moberg, C.; Hallberg, A. Microwave-Accelerated Homogeneous Catalysis in Organic Chemistry. Accounts of Chemical Research 2002, 35 (9), 717 727.

(71) Hayes, B. L. Microwave Synthesis: Chemistry at the Speed of Light; CEM Publishsing: Matthews, NC, 2002.

(72) Kappe, C. O. Controlled microwave heating in modern organic synthesis. Angewandte Chemie International Edition 2004, 43 (46), 6250-6284.

(73) Stuerga, D. Chapter 1: Microwave-Materials Interactions and Dielectric Properties: from Molecules and Macromolecules to Solids and Colloidal Suspensions, in Microwaves in Organic Syntehsis; 3 ed.; De La Hoz, A.;Loupy, A., Eds.; Wiley-VCH Verlag GmbH and Co. KGaA: Weinheim, Germany, 2013; Vol. 1.

(74) Schmink, J. R.; Leadbeater, N. E. Chapter 1: Microwave Heating as a Tool for Sustainable Chemistry: An Introduction, in Microwave Heating as a Tool for Sustainable Chemistry; 1 ed.; Leadbeater, N. E., Ed.; CRC Press: Boca Raton, Fl, 2011.

(75) Kappe, O. C.; Dallinger, D.; Murphree, S. S. Practical Microwave Synthesis for Organic Chemists: Strategies, Instruments and Protocols; Wiley-VCH Verlag $\mathrm{GmbH}$ and Co. KGaA: Weinheim, Germany, 2009.

(76) Gedye, R.; Smith, F.; Westawa, K.; Ali, H.; Baldisera, L.; Laberge, L.; Rousell, J. The Use of Microwave Ovens for Rapid Organic Synthesis. Tetrahedron Letters 1986, 27 (3), 279-282.

(77) Giguere, R. J.; Bray, T. L.; Duncan, S. M.; Majetich, G. Application of Commercial Microwave Ovens to Organic Synthesis. Tetrahedron Letters 1986, 27 (41), 49454948 .

(78) Stuerga, D.; Ondruschka, B.; Bonrath, W.; Pribetich, P.; Perreux, L.; Loupy, A.; Petit, A.; Diaz-Ortiz, A.; de la Hoz, A.; Carillo, J. R.et al. Microwaves in Organic Synthesis; 3 ed.; Wiley-VCH Verlag and Co. KGaA: Weinheim, Germany, 2012. 
(79) Schmink, J. R.; Leadbeater, N. E.; Stockland Jr., R. A.; Iannelli, M.; Cao, P.; Moseley, J. D.; McGowan, C. B.; Powell, G. L.; Suib, S. L.; Vanier, G. S. Microwave Heating as a Tool for Sustainable Chemistry; 1 ed.; CRC Press: Boca Raton, FL, 2017.

(80) Vallin, K. S. A.; Emilsson, P.; Larhed, M.; Hallberg, A. High-Speed Heck Reactions in Ionic Liquid with Controlled Microwave Heating. Journal of Organic Chemistry 2002, 67 (17), 6243-6246.

(81) Von Hippel, A. R. Dielectric Materials and Applications; MIT Press: Cambridge, Ma, 1954.

(82) Kappe, C. O.; Pieber, B.; Dallinger, D. Microwave effects in organic synthesis: myth or reality? Angewandte Chemie International Edition 2013, 52 (4), 10881094.

(83) Horikoshi, S.; Watanabe, T.; Narita, A.; Suzuki, Y.; Serpone, N. The electromagnetic wave energy effect(s) in microwave-assisted organic syntheses (MAOS). Scientific Reports 2018, 8 (1), 5151.

(84) Mingos, D. M. P.; Baghurst, D. R. Applications of microwave dielectric heating effects to synthetic problems in chemistry. Chemical Society Reviews 1991, 20 (1), $1-47$.

(85) Biotage Initiator Getting Started Guide. 2005, 1-20.

(86) Obermayer, D.; Gutmann, B.; Kappe, C. O. Microwave chemistry in silicon carbide reaction vials: separating thermal from nonthermal effects. Angewandte Chemie International Edition 2009, 48 (44), 8321-8324.

(87) Obermayer, D.; Damm, M.; Kappe, C. O. Simulating microwave chemistry in a resistance-heated autoclave made of semiconducting silicon carbide ceramic. Chemistry: a European Journal 2013, 19 (47), 15827-15830.

(88) Kappe, C. O.; Dallinger, D.; Murphree, S. S. Practical Microwave Synthesis for Organic Chemists: Strategies, Instruments and Protocols; Wiley-VCH Verlag $\mathrm{GmbH}$ an Co. KGaA: Weinheim, Germany, 2009.

(89) Caddick, S.; Fitzmaurice, R. Microwave enhanced synthesis. Tetrahedron 2009, 65 (17), 3325-3355.

(90) Van der Eycken, E.; Appukkuttan, P.; De Borggraeve, W.; Dehaen, W.; Dallinger, D.; Kappe, O. C. High-Speed Microwave-Promoted Hetero-Diels-Alder Reactions of 2(1,H)-pyrazinones in Ionic Liquid Doped Solvents. Journal of Organic Chemistry 2002, 67, 7904-7907.

(91) Oliver Kappe, C. Microwave dielectric heating in synthetic organic chemistry. Chemical Society Reviews 2008, 37 (6), 1127-1139.

(92) Vanier, G. Simple and Efficient Microwave-Assisted Hydrogenation Reactions at -Moderate Temperature and Pressure. Synlett 2007, 2007 (1), 0131-0135. 
(93) Bedford, R. B.; Butts, C. P.; Hurst, T. E.; Lidström, P. The Suzuki Coupling of Aryl Chlorides under Microwave Heating. Advanced Synthesis \& Catalysis 2004, 346 (13-15), 1627-1630.

(94) Russo, F.; Odell, L. R.; Olofsson, K.; Nilsson, P.; Larhed, M. Chapter 15: Microwave-Heated Transition Metal-Catalyzed Coupling Reactions, in Microwaves in Organic Synthesis; 3 ed.; De La Hoz, A.;Loupy, A., Eds.; WileyVCH Verlag GmbH and Co. KGaA: Weinheim, Germany, 2012.

(95) Cargill, M. R.; Sandford, G.; Tadeusiak, A. J.; Yufit, D. S.; Howard, J. A.; Kilickiran, P.; Nelles, G. Palladium-catalyzed C-F activation of polyfluoronitrobenzene derivatives in Suzuki-Miyaura coupling reactions. Journal of Organic Chemistry 2010, 75 (17), 5860-5866.

(96) Nilsson, P.; Gold, H.; Larhed, M. Microwave Irradiation as a High-Speed Tool for Activation of Sluggish Aryl Chlorides in Grignard Reactions. Synlett 2005, 2005 (10), 1596-1600.

(97) Berlan, J. Microwaves in Chemistry: Another Way of Heating Reaction Mixtures. Radiation Physics and Chemistry 1995, 45 (4), 581-589.

(98) Dehaen, W.; Appukkuttan, P.; Van der Eycken, E. Microwave-Enhanced Cadogan Cyclization: An Easy Access to the 2-Substituted Carbazoles and other Fused Heterocyclic Systems. Synlett 2004, 2005 (01), 127-133.

(99) Appukkuttan, P.; Orts, Amparo B.; Chandran, R. P.; Goeman, J. L.; Van der Eycken, J.; Dehaen, W.; Van der Eycken, E. Generation of a Small Library of Highly Electron-Rich 2-(Hetero)Aryl-Substituted Phenethylamines by the Suzuki-Miyaura Reaction: A Short Synthesis of an Apogalanthamine Analogue. European Journal of Organic Chemistry 2004, 2004 (15), 3277-3285.

(100) Appukkuttan, P.; Dehaen, W.; Van der Eycken, E. Microwave-Enhanced Synthesis of N-Shifted Buflavine Analgoues via a Suzuki-Ring-Closing Metathesis Protocol. Organic Letters 2005, 7 (13), 2723-2726.

(101) Leadbeater, N. E.; Marco, M. Transition-Metal-Free Suzuki-Type Coupling Reactions: Scope and Limitations of the Methodology. Journal of Organic Chemistry 2003, 68, 5660-5667.

(102) Leadbeater, N. E.; Marco, M. Transition-Metal-Free Suzuki-Type Coupling Reactions. Angewandte Chemie International Edition 2003, 42 (12), 1407-1409.

(103) Arvela, R. K.; Leadbeater, N. E.; Sangi, M. S.; Williams, V. A.; Granados, P.; Singer, R. D. A Reassessment of the Transition-Metal Free Suzuki-Type Coupling Methodology. Journal of Organic Chemistry 2005, 70, 161-168.

(104) Arvela, R. K.; Leadbeater, N. E. Microwave-Promoted Heck Coupling Using Ultralow Metal Catalyst Concentrations. Journal of Organic Chemistry 2005, 70, 1786-1790. 
(105) Diaz-Ortiz, A.; De La Hoz, A.; Carillo, J. R.; Herrero, M. A. Chapter 5: Selectivity Modifications Under Microwave Irradiation, in Microwaves in Organic Synthesis; 3 ed.; De La Hoz, A.;Loupy, A., Eds.; Wiley-VCH Verlag BmbH and Co. KGaA: Weinheim, Germany, 2012.

(106) Abenhaim, D.; Diez-Barra, E.; De la Hoz, A.; Loupy, A.; Sanchez-Migalion, A. Selective Alkylations of 1,2,4-triazole and Benzotriazole in the Absence of Solvent. Heterocycles 1994, 38 (4), 1793-1802.

(107) Loupy, A.; Perreux, L.; Liagre, M.; Burle, K.; Moneuse, M. Reactivity and Selctivity under Microwaves in Organic Chemistry: Relation with Medium Effects and Reaction Mechanisms. Pure and Applied Chemistry 2001, 71 (1), 161-166.

(108) Almena, I.; Diaz-Ortiz, A.; Diez-Barra, E.; De La Hoz, A.; Loupy, A. Solvent-Free Benzylations of 2-Pyridone, Regiospecific N- or C-Alkylation. Chemistry Letters 1996, 25 (5), 333-334.

(109) Bogdal, D.; Pisarek, U. Chapter 22: Polymer Chemistry Under Microwave Irradiation, in Microwaves in Organic Synthesis; 3 ed.; de La Hoz, A.;Loupy, A., Eds.; Wiley-VCH Verlag and Co. KGaA: Weinheim, Germany, 2012.

(110) Kostas, E. T.; Beneroso, D.; Robinson, J. P. The application of microwave heating in bioenergy: A review on the microwave pre-treatment and upgrading technologies for biomass. Renewable and Sustainable Energy Reviews 2017, 77, 12-27.

(111) Pan, J.; Fu, J.; Lu, X. Microwave-Assisted Oxidative Degradation of Lignin Model Compounds with Metal Salts. Energy \& Fuels 2015, 29 (7), 4503-4509.

(112) Xiao, H.; Sun, Q.; Shi, W.; Ma, J.; Lin, C.; Zhao, W.; Liu, X.; Qi, C. Degradation and dechlorination of pentachlorophenol by microwave-activated persulfate. Environmental Science and Pollution Research 2015, 22, 4670-4679.

(113) Hu, L.; Zhang, G.; Wang, Q.; Wang, X.; Wang, P. Effect of Microwave Heating on Persulfate Activation for Rapid Degradation and Mineralization of p-Nitrophenol. ACS Sustainable Chemistry \& Engineering 2019, 7 (13), 11662-11671.

(114) Nascimento, U. M.; Azevedo, E. B. Microwaves and their coupling to advanced oxidation processes: enhanced performance in pollutants degradation. Journal of Environmental Science and Health Part A: Toxic/Hazardous Substances and Environmental Engineering 2013, 48 (9), 1056-1072.

(115) Bose, A. K.; Manhas, M. S.; Ghosh, M.; Shah, M.; Raju, B. S.; Bari, S. S.; Newaz, S. N.; Banik, B. K.; Chaudhary, A. G.; Barakat, K. J. Microwave-Induced Organic Reaction Enhancement Chemistry. Journal of Organic Chemistry 1991, 56 (25), 6968-6970.

(116) Olofsson, K.; Kim, S.-Y.; Larhed, M.; Curran, D. P.; Hallberg, A. High-Speed, Highly Fluorous Organic Reactions. Journal of Organic Chemistry 1999, 64 (12), 4539-4541. 
(117) McBurney, R. T.; Portela-Cubillo, F.; Walton, J. C. Microwave assisted radical organic syntheses. RSC Advances 2012, 2 (4), 1264-1274.

(118) Borguet, Y.; Richel, A.; Delfosse, S.; Leclerc, A.; Delaude, L.; Demonceau, A. Microwave-enhanced ruthenium-catalysed atom transfer radical additions. Tetrahedron Letters 2007, 48 (36), 6334-6338.

(119) Horikoshi, S.; Tsuzuki, J.; Kajitani, M.; Abe, M.; Serpone, N. Microwave-enhanced radical reactions at ambient temperature : Part 3: Highly selective radical synthesis of 3-cyclohexyl-1-phenyl-1-butanone in a microwave double cylindrical cooled reactor. New Journal of Chemistry 2008, 32 (12), 2257.

(120) Soria-Castro, S. M.; Caminos, D. A.; Peñéñory, A. B. An expedient route to heterocycles through $\alpha$-arylation of ketones and arylamides by microwave induced thermal $\mathrm{S}_{\mathrm{RN}} 1$ reactions. RSC Advances 2014, 4 (34), 17490-17497.

(121) Yanagisawa, S. U., Kirika; Taniguchi, Tadashi; Itami, Kenichiro. Potassium tButoxide Alone can Promote the Biaryl Coupling of Electron-Deficient Nitrogen Heterocycles and Haloarenes. Organic Letters 2008, 10 (20), 4673-4676.

(122) Luque, R.; Balu, A. M.; Macqarrie, D. J. 18: Microwave-Assisted Heterogeneously Catalyzed Processes, in Microwaves in Organic Synthesis; 3 ed.; De La Hoz, A.;Loupy, A., Eds.; Wiley-VCH Verlag GmbH and Co. KGaA: Weinheim, Germany, 2012.

(123) Varma, R. S.; Baig, R. B. N. 10: Organic Synthesis Using Microwaves and Supported Reagents, in Microwaves in Organic Synthesis; 3 ed.; De La Hoz, A.;Loupy, A., Eds.; Wiley-VCH Verlag GmbH and Co. KGaA: Weinheim, Germany, 2012; Vol. 1.

(124) Bauer, I.; Knolker, H. J. Iron Catalysis in Organic Synthesis. Chemical Reviews 2015, 115 (9), 3170-3387.

(125) Bauer, E. B. Iron Catalysis: Historic Overview and Current Trends. Topics in Organometallic Chemistry 2015, 50, 1-18.

(126) Enthaler, S.; Junge, K.; Beller, M. Sustainable Metal Catalysis with Iron: From Rust to a Rising Star? Angewandte Chemie International Edition 2008, 47 (18), 3317 3321 .

(127) Homem, V.; Alves, A.; Santos, L. Microwave-assisted Fenton's oxidation of amoxicillin. Chemical Engineering Journal 2013, 220, 35-44.

(128) Carvalho, N. M. F.; Alvarez, H. M.; Horn, A.; Antunes, O. A. C. Influence of microwave irradiation in the cyclohexane oxidation catalyzed by $\mathrm{Fe}(\mathrm{III})$ complexes. Catalysis Today 2008, 133-135, 689-694.

(129) Fernandes, R. R.; Lasri, J.; Guedes da Silva, M. F. C.; da Silva, J. A. L.; Fraústo da Silva, J. J. R.; Pombeiro, A. J. L. Bis- and tris-pyridyl amino and imino thioether $\mathrm{Cu}$ and $\mathrm{Fe}$ complexes. Thermal and microwave-assisted peroxidative oxidations of 
1-phenylethanol and cyclohexane in the presence of various N-based additives. Journal of Molecular Catalysis A: Chemical 2011, 351, 100-111.

(130) Martins, N. M. R.; Mahmudov, K. T.; Guedes da Silva, M. F. C.; Martins, L. M. D. R. S.; Pombeiro, A. J. L. Copper(II) and iron(III) complexes with arylhydrazone of ethyl 2-cyanoacetate or formazan ligands as catalysts for oxidation of alcohols. New Journal of Chemistry 2016, 40 (12), 10071-10083.

(131) Liu, Y.; Che, C. M. [Fe $\left.{ }^{\mathrm{III}}\left(\mathrm{F}_{20}-\mathrm{tpp}\right) \mathrm{Cl}\right]$ is an effective catalyst for nitrene transfer reactions and amination of saturated hydrocarbons with sulfonyl and aryl azides as nitrogen source under thermal and microwave-assisted conditions. Chemistry: $a$ European Journal 2010, 16 (34), 10494-10501.

(132) Shing, K. P.; Liu, Y.; Cao, B.; Chang, X. Y.; You, T.; Che, C. M. N-Heterocyclic Carbene Iron(III) Porphyrin-Catalyzed Intramolecular $\mathrm{C}\left(\mathrm{sp}^{3}\right)-\mathrm{H}$ Amination of Alkyl Azides. Angewandte Chemie International Edition 2018, 57 (37), 1194711951.

(133) Cooke, M.; Clark, J.; Breeden, S. Lewis acid catalysed microwave-assisted synthesis of diaryl sulfones and comparison of associated carbon dioxide emissions. Journal of Molecular Catalysis A: Chemical 2009, 303 (1-2), 132-136.

(134) Mun, H. J.; Seong, E. Y.; Ahn, K. H.; Kang, E. J. Fe(II)/Fe(III)-Catalyzed Intramolecular Didehydro-Diels-Alder Reaction of Styrene-ynes. Journal of Organic Chemistry 2018, 83 (3), 1196-1203.

(135) Huang, Y.-B.; Yang, T.; Zhou, M.-C.; Pan, H.; Fu, Y. Microwave-assisted alcoholysis of furfural alcohol into alkyl levulinates catalyzed by metal salts. Green Chemistry 2016, 18 (6), 1516-1523.

(136) Pizzetti, M.; Russo, A.; Petricci, E. Microwave-assisted aminocarbonylation of ynamides by using catalytic $\left[\mathrm{Fe}_{3}(\mathrm{CO})_{12}\right]$ at low pressures of carbon monoxide. Chemistry: a European Journal 2011, 17 (16), 4523-4528.

(137) de la Hoz, A.; Diaz-Ortiz, A.; Gomez, M. V.; Prieto, P.; Migallon, A. S. Chapter 6: Elucidation of Microwave Effects: Methods, Theories, and Predictive Models, in Microwaves in Organic Synthesis; 3 ed.; de La Hoz, A.;Loupy, A., Eds.; WileyVCH Verlag and Co. KGaA: Weinheim, Germany, 2012.

(138) Kappe, C. O. How to measure reaction temperature in microwave-heated transformations. Chemical Society Reviews 2013, 42 (12), 4977-4990.

(139) Ikeda, S.; Mori, T.; Ikeda, Y.; Takao, K. Microwave-Assisted Solvent Extraction of Inert Platinum Group Metals from $\mathrm{HNO}_{3(\mathrm{aq})}$ to Betainium-Based Thermomorphic Ionic Liquid. ACS Sustainable Chemistry \& Engineering 2016, 4 (5), 2459-2463.

(140) Holtze, C.; Tauer, K. Surviving Radicals: Promises of a Microwave Effect on Miniemulsion Polymerization for Technical Processes. Macromolecular Rapid Communications 2007, 28 (4), 428-436. 
(141) Strauss, C. R.; Trainor, R. W. Developments in Microwave-Assisted Organic Chemistry. Australian Journal of Chemistry 1995, 48, 1665-1692.

(142) Trainor, R. W.; Strauss, C. R.; Raner, K. D. A New Microwave Reactor for Batchwise Organic Synthesis. Journal of Organic Chemistry 1995, 60 (8), 24562460 .

(143) Nilsson, P.; Larhed, M.; Hallberg, A. Highly Regioselective, Sequential, and Multiple Palladium-Catalyzed Arylations of Vinyl Ethers Carrying a Coordinating Auxiliary: An Example of a Heck Triarylation Process. Journal of the American Chemical Society 2001, 123 (34), 8217-8225.

(144) Bogdal, D.; Bednarz, S.; Łukasiewicz, M.; Kasprzyk, W. Intensification of oxidation and epoxidation reactions-Microwave vs. conventional heating. Chemical Engineering and Processing - Process Intensification 2018, 132, 208217.

(145) Peleteiro, S.; Raspolli Galletti, A. M.; Antonetti, C.; Santos, V.; Parajó, J. C. Manufacture of Furfural from Xylan-containing Biomass by Acidic Processing of Hemicellulose-Derived Saccharides in Biphasic Media Using Microwave Heating. Journal of Wood Chemistry and Technology 2018, 38 (3), 198-213.

(146) Sweygers, N.; Alewaters, N.; Dewil, R.; Appels, L. Microwave effects in the dilute acid hydrolysis of cellulose to 5-hydroxymethylfurfural. Scientific Reports 2018, 8 (1), 7719-7729.

(147) Wrigstedt, P.; Keskiväli, J.; Repo, T. Microwave-enhanced aqueous biphasic dehydration of carbohydrates to 5-hydroxymethylfurfural. RSC Advances 2016, 6 (23), 18973-18979.

(148) Cornejo, A.; Alegria-Dallo, I.; Garcia-Yoldi, I.; Sarobe, I.; Sanchez, D.; Otazu, E.; Funcia, I.; Gil, M. J.; Martinez-Merino, V. Pretreatment and enzymatic hydrolysis for the efficient production of glucose and furfural from wheat straw, pine and poplar chips. Bioresource Technology 2019, 288, 121583-121595.

(149) Delbecq, F.; Wang, Y.; Len, C. Various carbohydrate precursors dehydration to 5HMF in an acidic biphasic system under microwave heating using betaine as a cocatalyst. Molecular Catalysis 2017, 434, 80-85. 


\section{Chapter 2. Reaction Optimization and Profile}

\subsection{Introduction}

As discussed in Chapter 1, arylquinones and $N$-heterobiaryls are ubiquitous natural products that are of significant importance to the pharmaceutical and dye industries. ${ }^{1-8}$ However, conventional approaches to synthesizing these molecules face challenges due to the unique electronic properties of quinones, difficulties in synthesizing reagents with the appropriate regioselectivity, and reagents binding to and deactivating the catalysts. ${ }^{9,10}$ In addition, traditional methods often involve the use of expensive metals such as palladium or rhodium, and may require the use of complex, expensive ligands.

In 2010 and 2011, Baran and coworkers reported on a new method for the synthesis of these products. ${ }^{9,10}$ Using a silver catalyst and several equiv of the radical initiator potassium persulfate, they were able to cross-couple unactivated quinones and $\mathrm{N}$ heteroaryls with arylboronic acids (general reaction scheme shown in Scheme 2.1). ${ }^{9,10}$ Upon further investigation it was suggested that the sodium persulfate cleaved the boronic acid moiety forming an aryl radical that was then able to attack the desired coupling partner, with the catalyst being reduced by the resulting biaryl radical to form the final product (general reaction mechanism shown in Scheme 2.2). ${ }^{11}$ By directly functionalizing the C-H bond of the $N$-heteroaryls or quinones, they were able to avoid prefunctionalization steps required in other methods and make use of cheaper, commercially available reagents. ${ }^{9,10}$ This reaction required the use of only relatively cheap, commercially available reagents, 
was tolerant to a wide range of quinones, $N$-heteroaryls and arylboronic acids, and could be performed under air at mild conditions. ${ }^{9,10}$ As a result, the reports generated significant interest, and a number of other papers were soon published following up on this work. ${ }^{11-24}$

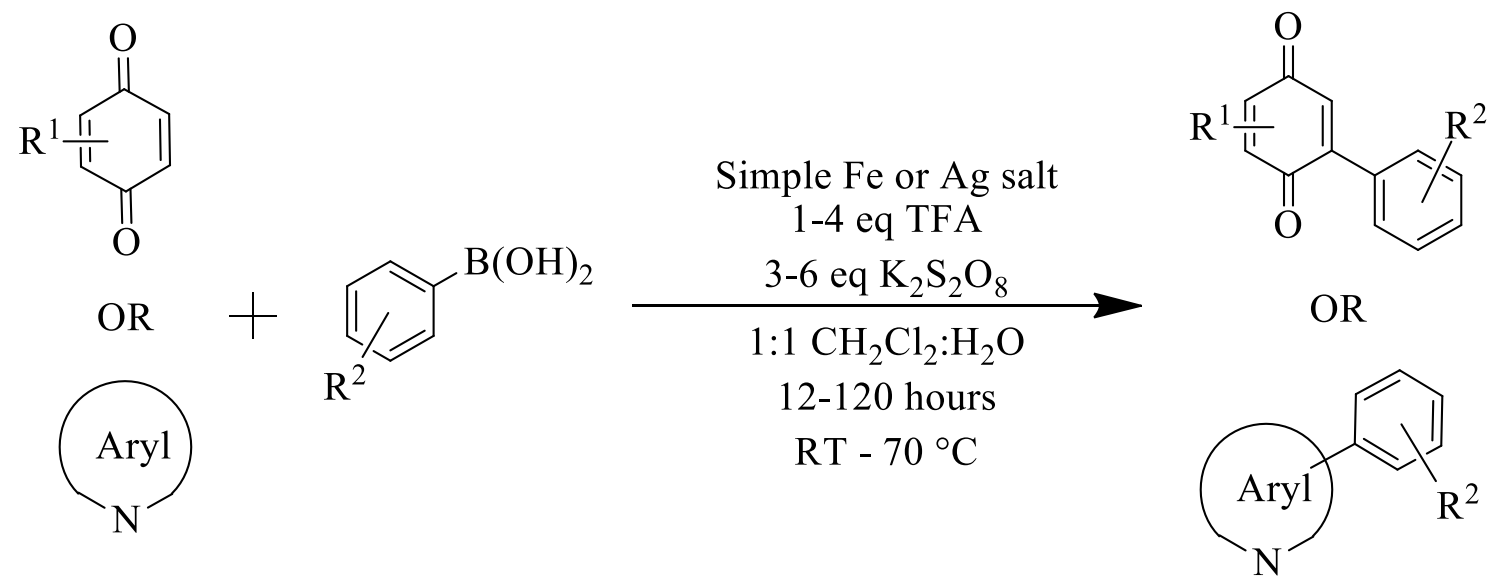

Scheme 2.1 General conditions of the cross-coupling of arylboronic acids with quinones or $N$-heteroaryls using potassium persulfate. 


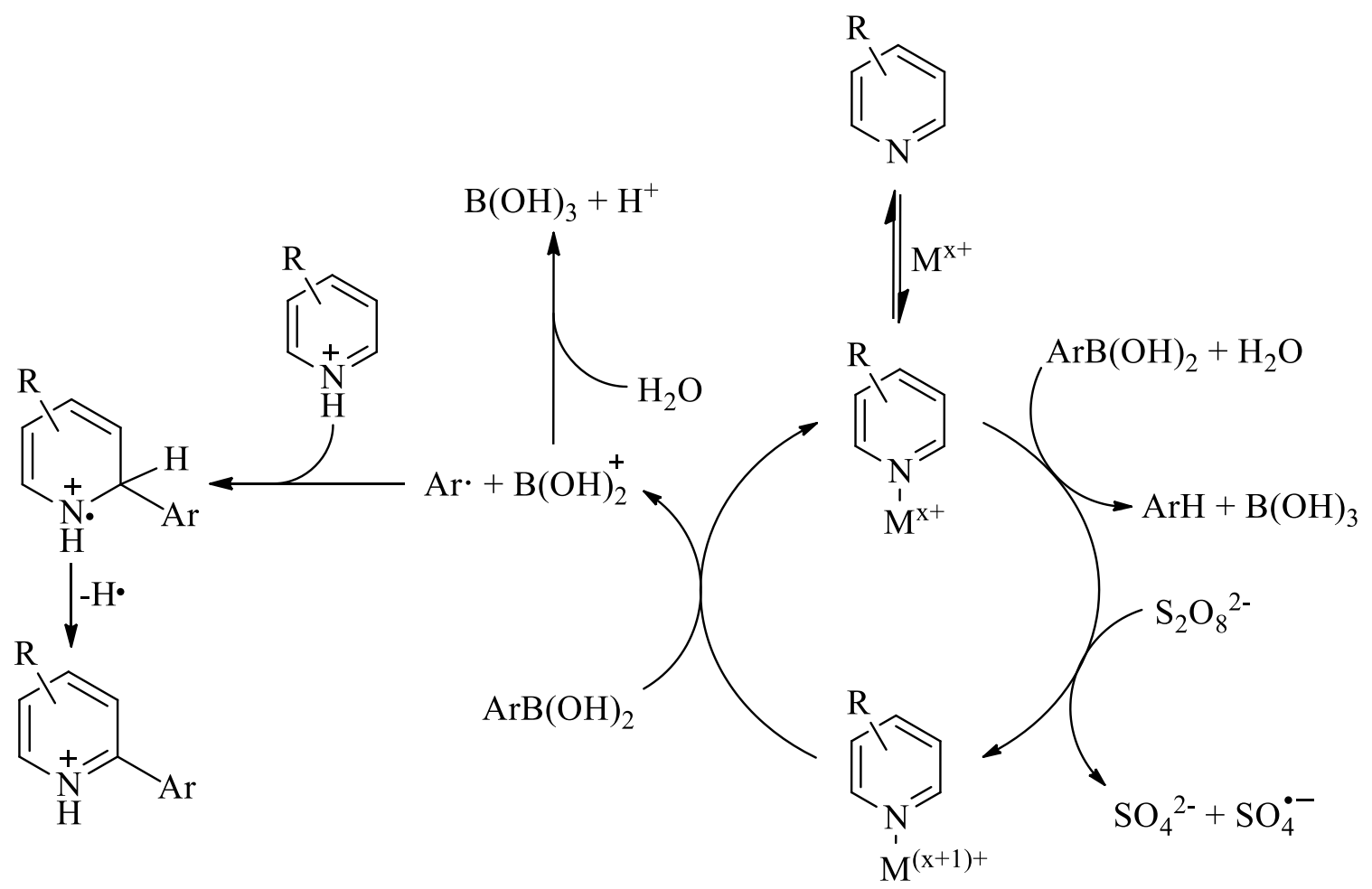

Scheme 2.2 General reaction mechanism for the cross-coupling of $N$-heteroaryls and quinones with arylboronic acids using silver or iron catalysts.

In 2012, Yu and coworkers reported the first use of a simple iron salt in the reaction system. ${ }^{12}$ Using insoluble iron(II) sulfide, they were able to successfully couple quinones and $N$-heteroaryls with a variety of arylboronic acids, often with higher yields than those reported using a silver catalyst. However, the reaction required an equiv of iron(II) sulfide, and took a minimum of $24 \mathrm{~h}$ to complete, compared to only $3 \mathrm{~h}$ using silver nitrate. Several other iron-mediated examples have been reported, using a variety of iron compounds. ${ }^{17,20}$ The first iron-catalyzed reaction was reported in 2013 and employed $\mathrm{Fe}(\mathrm{acac})_{2}$ as a catalyst and tetrabutylammonium bromide $(\mathrm{TBAB})$ as a phase transfer catalyst. ${ }^{18}$ Under similar conditions to those used by $\mathrm{Yu}$, this iron-catalyzed system was able to return similar yields 
in $12 \mathrm{~h}$. However, an attempt to reproduce this work was unsuccessful, and the yields obtained were similar to the amount of $\mathrm{Fe}(\mathrm{acac})_{2}$ added, suggesting the process may in fact have been iron-mediated, not iron-catalyzed. ${ }^{12,17,19,20}$ Regardless, a number of other ironcatalyzed reactions have been reported since, none of which employed a phase transfer catalyst. ${ }^{13,21,22}$ In addition, a number of natural products have been synthesized using this reaction. ${ }^{18,19,21,22}$

While these reactions constitute a significant advance over previous methods to generate arylquinones and $N$-heterobiaryls, both iron and silver-catalyzed reactions have several disadvantages. First, they all require several reagents in excess, including potassium persulfate (typically $2-4$ equiv), arylboronic acid (1.5 - 3 equiv), and trifluoroacetic acid (TFA, $1-3$ equiv), and all use relatively high catalyst loadings (typically 0.2 equiv). ${ }^{11-24}$ Second, several side-reactions are known to occur, most notably arylboronic acid homocoupling ${ }^{12,18-22}$ and bis-arylation..$^{910,12,14,19,25}$ Third, none are regioselective - when using quinones or $\mathrm{N}$-heteroaryls with more than one $\mathrm{sp}^{2}$ hybridized $\mathrm{C}-\mathrm{H}$ bond, a mixture of products is obtained. ${ }^{11-24}$ Finally, all reactions are sluggish, requiring at least $3 \mathrm{~h}$ to reach completion when using silver catalysts, and at least $12-24 \mathrm{~h}$ when using iron catalysts. ${ }^{11-24}$

Microwave heating is a widely used heating method in synthetic organic chemistry, first employed in 1986 by Gedye et al. and Giguere et al. ${ }^{26-30}$ Reactions are heated by electromagnetic radiation in the microwave region, typically with a wavelength of 2.45 $\mathrm{GHz} .{ }^{28-30}$ There are several effects associated with microwave heating that are not observed under traditional heating, known as microwave specific effects. ${ }^{28-31}$ First, microwave 
heating can be extremely rapid, with reports of temperature increases of up to several hundred degrees in seconds. Modern microwave instruments are also typically equipped with a cooling system that allows for rapid reaction cooling. ${ }^{28-31}$ Second, microwaves heat the bulk solution directly, rather than heating the vessel walls, then heating the reaction mixture through heat transfer with the vessel walls. This leads to more uniform heating of the solution, and prevents "wall effects", in which reactions may have taken place at the vessel wall, which is hotter than the bulk reaction mixture. ${ }^{28-31}$ Finally, hot spots may form in the microwave if certain reagents are better able to absorb microwave radiation than others. In particular, ionic compounds, especially solid ionic compounds, are better able to absorb microwave radiation than the bulk solution, and may therefore achieve temperatures up to several hundred degrees higher than the surrounding solution. ${ }^{28-31}$

Due to these microwave specific effects, microwave heating frequently offers several benefits over traditional heating methods. ${ }^{28-35}$ Most commonly, microwave heating is associated with an increase in rate. ${ }^{28-34}$ This increase is generally due to the ability to easily superheat solvents in the microwave but may also be caused by the formation of microwave hotspots. For the same reasons, reactions performed under microwave heating may have an expanded substrate scope, as reagents that are sluggish or unreactive under conventional heating may be efficiently reacted under microwave heating. ${ }^{28-32,34}$ Reactions performed under microwave heating have also been known to have a cleaner reaction profile, with fewer side products, and consequently reactions that require excess amounts of a reagent using traditional heating methods can be performed with only stoichiometric amounts of reagent under microwave heating. Reactions under microwave heating also 
frequently require lower catalyst loadings. ${ }^{28-32,35,36}$ These effects may be due to the finer control over reaction heating and cooling, more homogeneous heating, or the absence of wall effects. The use of microwave heating can also cause a change in chemo-, regio-, or enantio- selectivity. ${ }^{28-31,37}$ Microwave heating therefore offered a potential method to address the various disadvantages of the cross-coupling reaction discovered by Baran and coworkers.

\subsection{Results and Discussion}

\subsubsection{Reactions in Toluene}

Initial reactions were performed with pyrazine and 4-methylphenylboronic acid in a mixture of toluene and water in a procedure based upon that used by Deb et al. (Scheme 2.3). ${ }^{13}$ Toluene was chosen due to its previous use in the literature and relatively high boiling point, which would allow a large increase in temperature under microwave heating. These initial reactions gave the desired 2-(4-methylphenyl)pyrazine (1A) in 5\% yield. Further optimization of reaction parameters including time, temperature, concentration, and reagent stoichiometry increased yields substantially, to more than $60 \%$. However, during optimization a number of new products were detected that were not observed in the original reactions and had not been previously reported in the literature. These products included a then unidentified compound that proved difficult to separate from 1A. GC-MS was performed on the reaction mixture after extractions and drying and revealed the presence of a number of products stemming from toluene oxidation and homocoupling (Figure 2.1). The identified species included three isomers of dimethylbiphenyl, bibenzyl, and benzyl 
alcohol. Benzyl alcohol was found to be difficult to separate from the cross-coupled product. These products were confirmed to be at least partly due to the reaction of toluene when a reaction performed without 4-methylphenylboronic acid showed the same products. None of the desired $\mathbf{1 A}$ was observed during the reaction without 4-methylphenylboronic acid. It was therefore decided that dichloromethane, the solvent most commonly used in the literature, would be used in place of toluene to avoid the formation of side products.

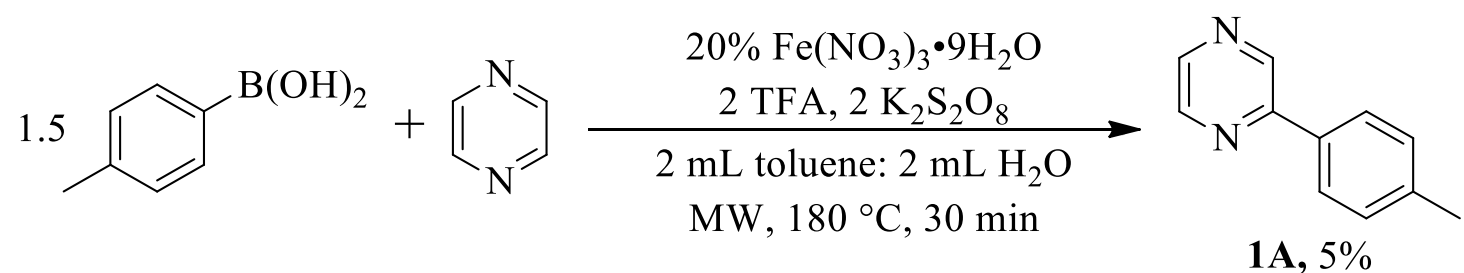

Scheme 2.3 Initial reaction conditions using a solvent mixture of toluene and water 


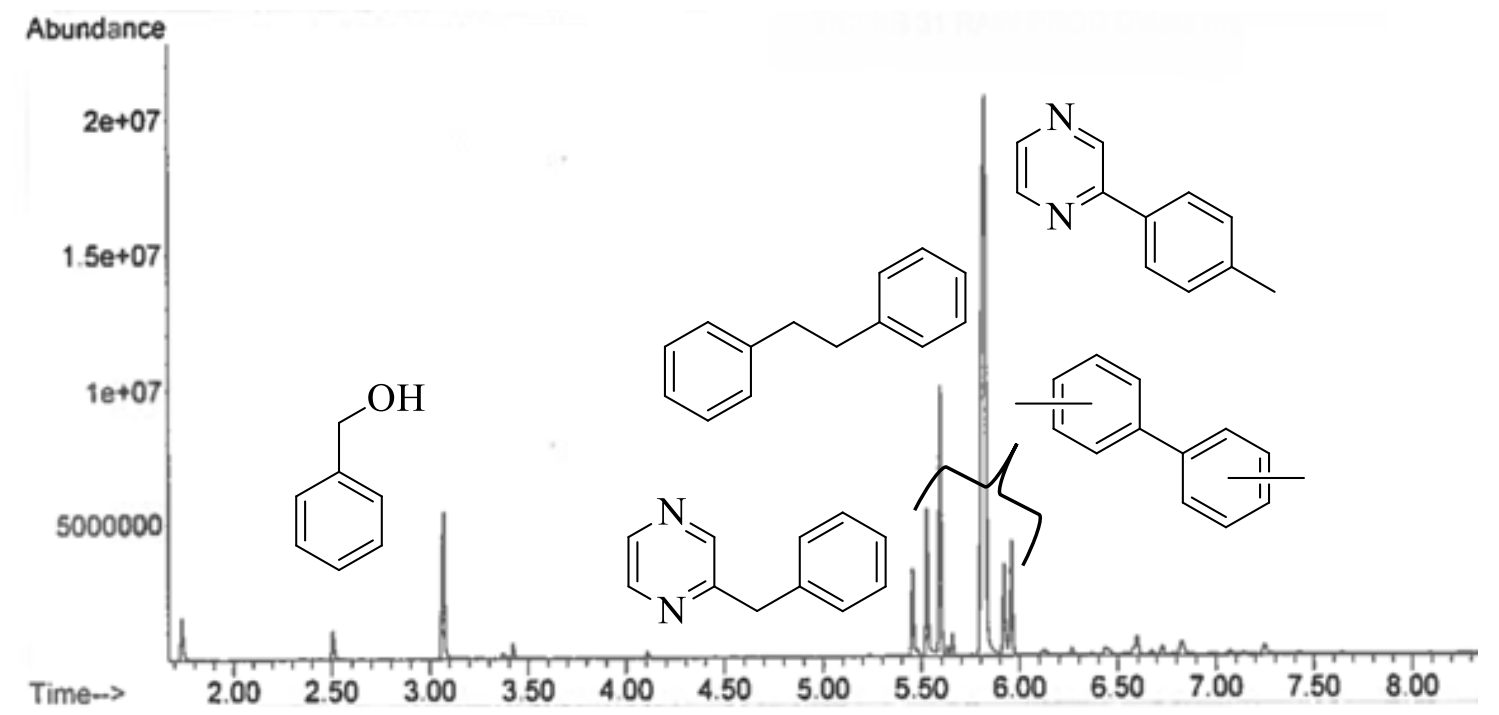

Figure 2.1 Gas chromatogram of the crude reaction mixture of the optimized reaction in toluene, showing products of toluene oxidation and homo-coupling. Benzyl alcohol: $\mathrm{R}_{\mathrm{t}}=$ 3.08 min. 2-benzylpyrazine: $R_{t}=5.45$ min. Bibenzyl: $R_{t}=5.59$ min. Dimethylbiphenyl isomers: $\mathrm{R}_{t}=5.54 \mathrm{~min}, 5.91 \mathrm{~min}, 5.97 \mathrm{~min}$. 2-(4-Methylphenyl)pyrazine: $\mathrm{R}_{t}=5.81 \mathrm{~min}$. For further information, consult the experimental and/or Figure A118.

\subsubsection{Optimization in Dichloromethane}

Initial reactions using dichloromethane were performed based on the optimized conditions in toluene; $5 \mathrm{~min}$ at $70{ }^{\circ} \mathrm{C}, 25 \mathrm{~min}$ at $180{ }^{\circ} \mathrm{C}$, using $0.75 \mathrm{mmol}$ of pyrazine, 4 equiv of $\mathrm{K}_{2} \mathrm{~S}_{2} \mathrm{O}_{8}, 2.0$ equiv of TFA, 0.2 equiv of $\mathrm{Fe}\left(\mathrm{NO}_{3}\right)_{3} \cdot 9\left(\mathrm{H}_{2} \mathrm{O}\right)$, and 1.5 equiv of 4methylphenylboronic acid in $8 \mathrm{~mL}$ each of dichloromethane and water. The maximum temperature was reduced to $130{ }^{\circ} \mathrm{C}$, which was the highest temperature that did not exceed the 20 bar pressure limit of the Biotage Initiator ${ }^{\mathrm{TM}}$ microwave reactor. These initial reactions gave a yield of $44 \%$, significantly lower than the yield using toluene. Reaction conditions were therefore reoptimized in the dichloromethane/water system using phenylboronic acid in an attempt to further increase reactions yields. 
Initial optimization concerned the amount and identity of acid (Table 2.1). While the role of acid in the reaction system is not well understood, it most likely serves to protonate the $N$-heteroarene and to prevent catalyst deactivation by preventing the formation of $\mathrm{Fe}(\mathrm{OH})_{2}$. Curiously, in toluene, adding TFA reduced the yield of $\mathbf{1 A}$; however, the amount of oxidized and homo-coupled toluene increased substantially when TFA was not present. In dichloromethane, the presence of 2 equiv of TFA (entry 3 ) slightly increased the yield, while limiting the amount of phenylboronic acid homo-coupling that occurred. This is somewhat different to most literature accounts, in which the absence of TFA lowers yields more dramatically. The use of 2 equiv of sulfuric, hydrochloric, and $p$ toluenesulfonic acid all resulted in decreased yields. The inclusion of 2 equiv of $\mathrm{K}_{2} \mathrm{CO}_{3}$ limited the reaction to a yield of only $9 \%$, and caused the formation of a black precipitate, most likely some form of iron oxide or iron hydroxide, immediately upon addition to the reaction mixture. 
Table 2.1 Optimization of acid amount and identity.

\begin{tabular}{cccc}
\hline Entry & Acid or Base & Equiv & Yield of 1B (\%) \\
\hline 1 & TFA & 0 & 44 \\
2 & TFA & 1 & 46 \\
3 & TFA & 2 & 50 \\
4 & TFA & 4 & 46 \\
5 & $\mathrm{H}_{2} \mathrm{SO}_{4}$ & 2 & 41 \\
6 & $\mathrm{HCl}$ & 2 & 40 \\
7 & $\mathrm{TsOH}_{8}$ & 2 & 20 \\
\hline & $\mathrm{K}_{2} \mathrm{CO}$ & 2 & 9 \\
\hline
\end{tabular}

All reactions were performed using $0.75 \mathrm{mmol}$ of pyrazine, 4 equiv of $\mathrm{K}_{2} \mathrm{~S}_{2} \mathrm{O}_{8}, 0.2$ equiv of $\mathrm{Fe}\left(\mathrm{NO}_{3}\right)_{3} \cdot 9\left(\mathrm{H}_{2} \mathrm{O}\right)$, and 1.5 equiv of phenylboronic acid in $8 \mathrm{~mL}$ of deionized water and $8 \mathrm{~mL}$ of dichloromethane. All reactions were heated to $70^{\circ} \mathrm{C}$ for $5 \mathrm{~min}$, then to $130^{\circ} \mathrm{C}$ for $25 \mathrm{~min}$.

The reaction temperature and reaction time were then optimized (Table 2.2 and Table 2.3). The highest yield was obtained at $70{ }^{\circ} \mathrm{C}$ (entry 3), similar to many literature reactions under conventional heating. ${ }^{13,21,22}$ Reactions at higher temperatures (entries 4-9) led to the formation of a black solid that coated the vial walls and floated at the solvent interface. This solid proved to be insoluble in most common solvents, and neither an NMR nor a GC-MS spectrum could be collected. The highest yield was obtained with a reaction 
time of $25 \mathrm{~min}$ (Table 2.3 , entry 4). The initial reaction rate was rapid, with a yield of 39\% obtained after only 1 min (entry 1), but then slowed dramatically, with yields plateauing at $25 \mathrm{~min}$.

Table 2.2 Optimization of reaction temperature.

\begin{tabular}{|c|c|c|}
\hline Entry & Temperature $\left({ }^{\circ} \mathrm{C}\right)$ & Yield of 1B (\%) \\
\hline 1 & 50 & 43 \\
\hline 2 & 60 & 53 \\
\hline 3 & 70 & 59 \\
\hline 4 & 80 & 50 \\
\hline 5 & 90 & 45 \\
\hline 6 & 100 & 36 \\
\hline 7 & 110 & 38 \\
\hline 8 & 120 & 37 \\
\hline 9 & 130 & 46 \\
\hline
\end{tabular}

All reactions were performed using $0.75 \mathrm{mmol}$ of pyrazine, 4 equiv of $\mathrm{K}_{2} \mathrm{~S}_{2} \mathrm{O}_{8}, 0.2$ equiv of $\mathrm{Fe}\left(\mathrm{NO}_{3}\right)_{3} \cdot 9\left(\mathrm{H}_{2} \mathrm{O}\right) 2$ equiv of TFA, and 1.5 equiv of phenylboronic acid in $8 \mathrm{~mL}$ of deionized water and $8 \mathrm{~mL}$ of dichloromethane. All reactions were heated to the desired temperature for $25 \mathrm{~min}$. 
Table 2.3 Optimization of reaction time.

\begin{tabular}{ccc}
\hline Entry & Time (min) & Yield of 1B (\%) \\
\hline 1 & 1 & 39 \\
2 & 5 & 50 \\
4 & 15 & 46 \\
5 & 25 & 59 \\
6 & 30 & 59 \\
7 & 40 & 58 \\
\hline
\end{tabular}

All reactions were performed using $0.75 \mathrm{mmol}$ of pyrazine, 4 equiv of $\mathrm{K}_{2} \mathrm{~S}_{2} \mathrm{O}_{8}, 0.2$ equiv of $\mathrm{Fe}\left(\mathrm{NO}_{3}\right)_{3} \cdot 9\left(\mathrm{H}_{2} \mathrm{O}\right) 2$ equiv of TFA, and 1.5 equiv of phenylboronic acid in $8 \mathrm{~mL}$ of deionized water and $8 \mathrm{~mL}$ of dichloromethane. All reactions were heated $70{ }^{\circ} \mathrm{C}$ for the desired time.

Reagent stoichiometry and catalyst loading were then optimized (Table 2.4). The highest yields were obtained when using 4 equiv of potassium persulfate, 1.5 equiv of phenylboronic acid, and 0.2 equiv of $\mathrm{Fe}\left(\mathrm{NO}_{3}\right)_{3} \cdot 9\left(\mathrm{H}_{2} \mathrm{O}\right)$ (entry 9), similar to conditions in the literature..$^{9,10,17-22}$ No reaction was observed when potassium persulfate was not present (entry 1 ), and only a small amount of product $(5 \%)$ was obtained when $\mathrm{Fe}\left(\mathrm{NO}_{3}\right)_{3} \cdot 9\left(\mathrm{H}_{2} \mathrm{O}\right)$ was not present (entry 6). Using only 1 equiv of phenylboronic acid (Table $\mathbf{2 . 5}$, entry 13) resulted in significantly decreased yields. Increasing the amount of potassium persulfate, phenylboronic acid, or $\mathrm{Fe}\left(\mathrm{NO}_{3}\right)_{3} \cdot 9\left(\mathrm{H}_{2} \mathrm{O}\right)$ resulted in slightly diminished yields (entries 5, 15,10 , and 11). 
Table 2.4 Optimization of reagent stoichiometry.

\begin{tabular}{ccccc}
\hline Entry & $\begin{array}{c}\mathrm{K}_{2} \mathrm{~S}_{2} \mathrm{O}_{8} \\
\text { (Equiv) }\end{array}$ & $\begin{array}{c}\mathrm{Fe}\left(\mathrm{NO}_{3}\right)_{3} \cdot 9\left(\mathrm{H}_{2} \mathrm{O}\right) \\
(\text { Equiv })\end{array}$ & $\begin{array}{c}4-\mathrm{MePhB}(\mathrm{OH})_{2} \\
(\text { Equiv })\end{array}$ & $\begin{array}{c}\text { Yield of } 1 \mathrm{~A} \\
(\%)\end{array}$ \\
\hline 1 & 0 & 0.2 & 1.5 & 0 \\
2 & 1 & 0.2 & 1.5 & 27 \\
3 & 2 & 0.2 & 1.5 & 43 \\
4 & 4 & 0.2 & 1.5 & 59 \\
5 & 6 & 0.2 & 1.5 & 45 \\
6 & 4 & 0 & 1.5 & 5 \\
7 & 4 & 0.05 & 1.5 & 51 \\
8 & 4 & 0.1 & 1.5 & 59 \\
10 & 4 & 0.5 & 1.5 & 50 \\
11 & 4 & 0.75 & 1.5 & 39 \\
12 & 4 & 0.2 & 1.5 & 25 \\
13 & 4 & 1 & 3.0 & 56 \\
\hline
\end{tabular}

All reactions were performed using $0.75 \mathrm{mmol}$ of pyrazine in $8 \mathrm{~mL}$ of deionized water and $8 \mathrm{~mL}$ of dichloromethane. All reactions were heated to $70{ }^{\circ} \mathrm{C}$ for $25 \mathrm{~min}$.

Finally, the reaction concentration was optimized (Table 2.5). Optimization of this parameter was undertaken for two reasons. First, because differences in reaction concentration could influence heat distribution under microwave heating. At higher 
concentrations, undissolved reagents or precipitates might be present. These solids would be much better absorbers of microwave radiation, and could cause the presence of microwave hotspots reaching temperatures as high as several hundred degrees. ${ }^{28,30,32}$ Secondly, reaction concentration could affect the rate of mass transfer from one solvent phase to another. Lower concentrations resulted in increased yields, and higher concentrations often had black solids coating the walls of the reaction vessel, most likely indicating the presence of hotspots at higher concentrations.

Table 2.5 Optimization of reaction concentration.

\begin{tabular}{ccccc}
\hline Entry & $\begin{array}{c}\text { Pyrazine } \\
(\mathrm{mmol})\end{array}$ & $\begin{array}{c}\text { Solvent } \\
\text { volume }(\mathrm{mL})\end{array}$ & $\begin{array}{c}\text { Concentration } \\
(\mathrm{mmol} \text { pyrazine/mL } \\
\text { solvent })\end{array}$ & $\begin{array}{c}\text { Yield of 1B } \\
(\%)\end{array}$ \\
\hline 1 & 0.75 & 15 & 0.05 & 70 \\
2 & 1.1 & 15 & 0.075 & 62 \\
3 & 0.50 & 5 & 0.10 & 62 \\
4 & 1.0 & 5 & 0.20 & 52 \\
\hline
\end{tabular}

All reactions were performed using 4 equiv of $\mathrm{K}_{2} \mathrm{~S}_{2} \mathrm{O}_{8}, 0.2$ equiv of $\mathrm{Fe}\left(\mathrm{NO}_{3}\right)_{3} \cdot 9\left(\mathrm{H}_{2} \mathrm{O}\right)$, and 1.5 equiv of phenylboronic acid in a biphasic mixture of dichloromethane and deionized water. All reactions were heated to $70{ }^{\circ} \mathrm{C}$ for $25 \mathrm{~min}$.

A number of other reaction conditions were tested. Changes in microwave power had no effect on the reaction. Several other oxidants were screened, including hydrogen 
peroxide, tert-butylperoxybenzoate, iron(II) perchlorate, and potassium chlorate, but all were unsuccessful. Surprisingly, even sodium persulfate, which is reported to give only moderately lower yields in the literature, was completely inactive in this case. ${ }^{17,21}$ Several other solvent mixtures were also screened (Table 2.6). Reactions carried out in a mixture of water and benzene or water and ethyl acetate provided slightly lower yields, while mixtures of acetone and tetrahydrofuran with water resulted in no yield of the desired product (entries 8,6,7, and 9). Reactions using mixtures of dimethyl sulfoxide, acetonitrile, and methanol with dichloromethane also gave no product (entries 3, 1, and 2). Dichloromethane by itself yielded no product (entry 5), while water by itself gave a modest yield (entry 9). In most cases where no yield is reported the potassium persulfate appeared poorly soluble, and a large amount of black solid was obtained after heating, possibly due to the superheating and decomposition of the solid persulfate. The use of tetrabutylammonium bromide as a phase transfer catalyst, reported elsewhere in the literature, did not improve yields. ${ }^{18}$ Similarly, the use of an $\mathrm{O}_{2}$ atmosphere in place of air did not affect the reaction. The use of an equiv of ascorbic acid or elemental sulfur, which have been reported to affect the redox cycle of iron, were both unsuccessful. ${ }^{17}$ 
Table 2.6 Screening of solvent mixtures.

\begin{tabular}{cccc}
\hline Entry & Solvent 1 & Solvent 2 & Yield of 1B (\%) \\
\hline 1 & Dichloromethane & Acetonitrile & 0 \\
2 & Dichloromethane & Methanol & 0 \\
3 & Dichloromethane & Dimethyl Sulfoxide & 0 \\
4 & Dichloromethane & Water & 70 \\
5 & Dichloromethane & NA ${ }^{\text {a }}$ & 0 \\
6 & Ethyl acetate & Water & 0 \\
7 & Acetone & Water & 0 \\
9 & Benzene & Water & 35 \\
\hline
\end{tabular}

All reactions were performed using $0.75 \mathrm{mmol}$ of pyrazine, 4 equiv of $\mathrm{K}_{2} \mathrm{~S}_{2} \mathrm{O}_{8}, 0.2$ equiv of $\mathrm{Fe}\left(\mathrm{NO}_{3}\right)_{3} \cdot 9\left(\mathrm{H}_{2} \mathrm{O}\right)$, and 1.5 equiv of phenylboronic acid in a $20 \mathrm{~mL}$ microwave vessel at a concentration of $0.050 \mathrm{mmol}$ pyrazine $/ \mathrm{mL}$ solvent. All reactions were heated to $70{ }^{\circ} \mathrm{C}$ for $25 \mathrm{~min}$. A 1:1 solvent ratio was used in all cases. ${ }^{a} \mathrm{NA}$ indicates that no additional solvent was used.

A variety of iron compounds were screened for their activity as catalysts, including common inorganic and metalorganic complexes, and two iron(III) amino-bis(phenolate) complexes (see Figure 2.2 and Figure 2.3). The majority were found to give good activity, with little difference in yield. This is unusual, as the majority of iron species used in the literature are completely inactive, or only provide minimal yields. ${ }^{17,18,21}$ Iron species with 
oxidation states of $0,+1,+2$, and +3 were all active, as were inorganic and metalorganic, and soluble and insoluble iron species. This most likely indicates that the iron compounds function as precatalysts, which are oxidized by the potassium persulfate to form an active species. Reactions with insoluble iron oxide and iron powder were successful, albeit with somewhat lower yields. This was somewhat surprising as these compounds are generally reported to be give little or no cross-coupled product. In general, simple iron salts were the most active. Iron chlorides, which are reported in the literature to be inactive, were amongst the most effective catalysts under microwave heating. ${ }^{17,18,21}$ The highest yields were obtained with $\mathrm{FeSO}_{4} \cdot 6 \mathrm{H}_{2} \mathrm{O}$, and it was therefore used for all subsequent reactions. 


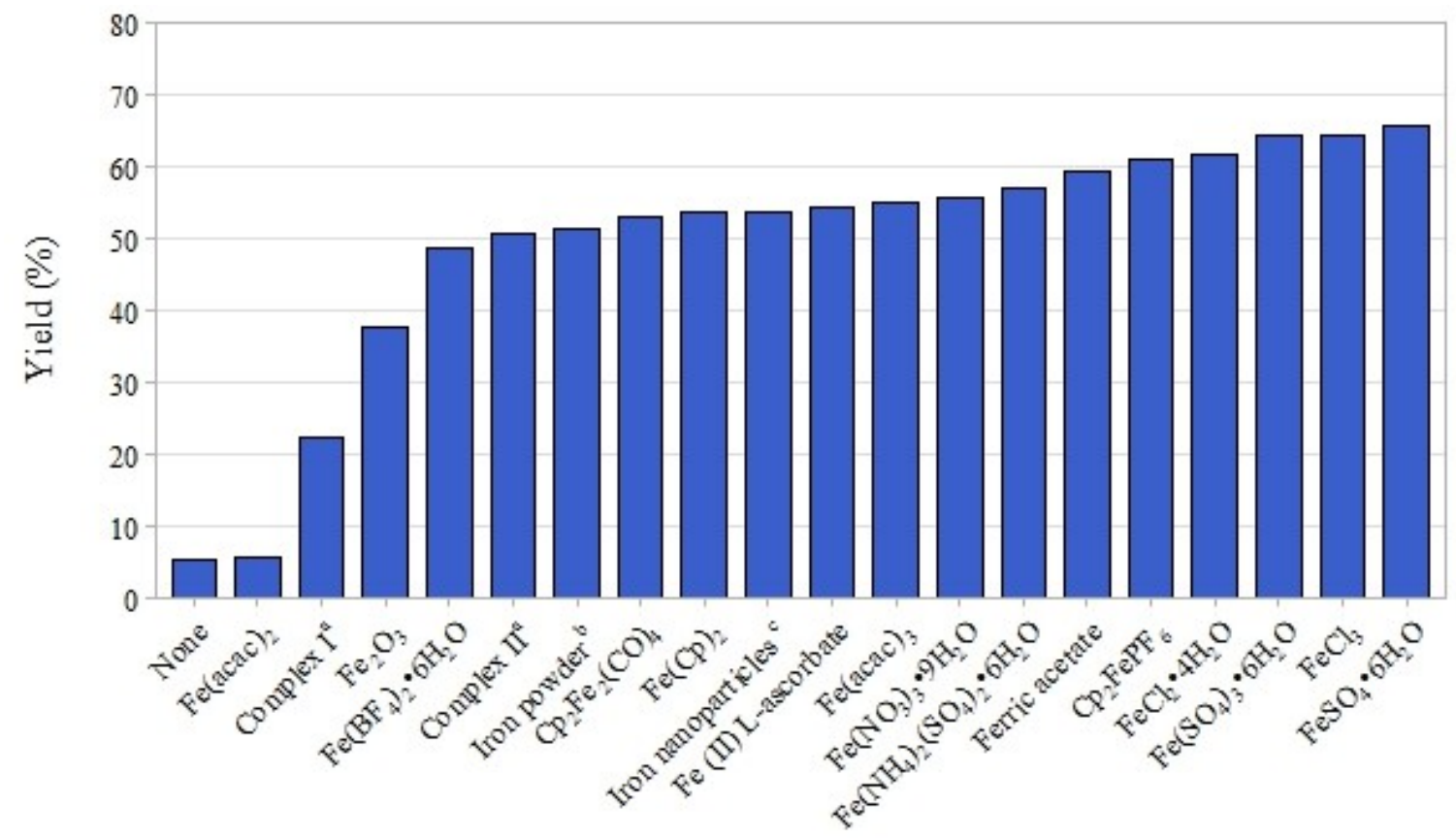

Figure 2.2 Reaction yields under standardized conditions with different iron catalysts. All reactions were performed with $20 \%$ catalyst loading with respect to iron. ${ }^{\text {a See Figure }}$ 2.3 for the structure of the iron(III) amino-bis(phenolate) complexes I and II. ${ }^{b} 40$ mesh iron powder. ${ }^{c} 20 \%$ loading by weight with respect to pyrazine. See the experimental for the synthesis and characterization of the citrate capped iron nanoparticles.<smiles></smiles>

I<smiles>CC(C)Cc1cc(C(C)C)cc2c1OC1(Br)N3CCCN(C2)P1(Br)(Br)c1c(CC(C)C)cc(CC(C)(C)C)cc1C3</smiles>

II

Figure 2.3 Structures of the iron(III) amino-bis(phenolate) complexes I ${ }^{38}$ and II. ${ }^{39}$ 
Overall, the optimized conditions consisted of $0.75 \mathrm{mmol}$ of pyrazine, 1.5 equiv of phenylboronic acid, 0.2 equiv of $\mathrm{FeSO}_{4} \cdot 6 \mathrm{H}_{2} \mathrm{O}, 2$ equiv of TFA, and 4 equiv of $\mathrm{K}_{2} \mathrm{~S}_{2} \mathrm{O}_{8}$ in 8 $\mathrm{mL}$ each of dichloromethane and deionized water, heated to $70{ }^{\circ} \mathrm{C}$ for $25 \mathrm{~min}$ under microwave heating, to give the desired cross-coupled product in $70 \%$ yield. Reactions with 4-methylphenylboronic acid gave a slightly higher yield of 72\% (Scheme 2.4). The required reaction stoichiometry is similar to literature reports, as is the overall yield. However, the reaction time is significantly lower under microwave heating (Table 2.7). Literature reports that used iron catalysts required a minimum of $12 \mathrm{~h}$ to reach completion, nearly 30 times longer than the $0.4 \mathrm{~h}(25 \mathrm{~min})$ required under microwave heating. Most papers reported lower yields, with the exception of Maiti and coworkers, who reported a similar yield, and Vishwakarma and coworkers, who reported a somewhat higher yield. ${ }^{13,18-}$ ${ }^{20}$ However, attempts to replicate Vishwakarma's results by Takaki and coworkers were unsuccessful, and they reported that the process appeared to be require stoichiometric amounts of iron salt. ${ }^{18,19}$ Literature reports that employed silver catalysts, which are generally more active, required a minimum of $6 \mathrm{~h}$ to reach completion, and in the case of pyrazine afforded poor yields. ${ }^{9}$

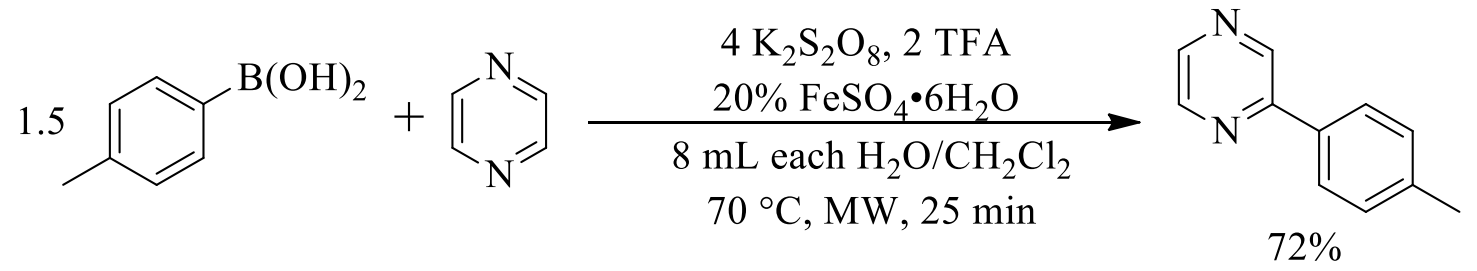

Scheme 2.4 Optimized reaction conditions in a mixture of dichloromethane and water. 
Table 2.7 Comparison of reaction conditions and yields.

\begin{tabular}{|c|c|c|c|c|c|}
\hline Entry & Source & Time (h) & $\begin{array}{c}\text { Temperature } \\
\left({ }^{\circ} \mathrm{C}\right)\end{array}$ & Catalyst & Yield (\%) \\
\hline 1 & This work & 0.4 & $70(\mathrm{MW})$ & $\mathrm{FeSO}_{4} \cdot 6 \mathrm{H}_{2} \mathrm{O}$ & 70 \\
\hline 2 & 20 & 48 & RT & $\mathrm{Fe}(\mathrm{ox})$ & 32 \\
\hline 3 & 18 & 12 & RT & $\mathrm{Fe}(\mathrm{acac})_{2}$ & 84 \\
\hline 4 & 18 & 12 & 50 & $\mathrm{Fe}(\mathrm{acac})_{2}$ & 82 \\
\hline 5 & 19 & 12 & $\mathrm{RT}$ & $\mathrm{Fe}(\mathrm{acac})_{2}$ & 25 \\
\hline 6 & 13 & 24 & 70 & $\mathrm{Fe}\left(\mathrm{NO}_{3}\right)_{3} \cdot 9\left(\mathrm{H}_{2} \mathrm{O}\right)$ & 70 \\
\hline 7 & 9 & 6 & $\mathrm{RT}$ & $\mathrm{AgNO}_{3}$ & 30 \\
\hline
\end{tabular}

\subsubsection{Reaction Profile}

In order to determine the fate of the pyrazine and 4-methylphenylboronic acid not involved in the formation of 2-(4-methylphenyl)pyrazine, GC-MS was performed on the crude reaction mixture of the optimized reaction. A large number of products were apparent, including the desired product, an unidentified isomer of doubly coupled product (2,6-(4-methylphenyl)pyrazine or 2,5-(4-methylphenyl)pyrazine), several isomers of

dimethylbiphenyl, p-cresol, 2,2'-bipyrazine, 4-methylphenylboronic acid, and 2-(dichloromethyl)pyrazine (see Figure 2.4 and Scheme 2.5). There are also several 
compounds that could not be identified. The formation of bis-coupled product was not unexpected, as they are commonly observed throughout the literature in small amounts. ${ }^{9} 10,12,14,19,25$ The presence of three unidentified isomers of dimethylbiphenyl, formed by the homocoupling of arylboronic acids, was unexpected. Arylboronic acid homocoupling has been reported several times, but is thought to result from the homocoupling of two aryl radicals, yielding only one isomer. ${ }^{12,18-22}$ There are no other reports of arylboronic acid homocoupling yielding multiple isomers. This suggests that homocoupling in this case may be proceeding via a different pathway, perhaps involving an aryl radical attacking an arylboronic acid (see Scheme 2.6), although it is not clear how the subsequent loss of the boronic acid moiety would occur. There is also a large 4methylphenol peak, the result of the replacement of the boronic acid moiety with a hydroxyl group, which was recovered in $11 \%$ yield. There is one previous report of the formation of phenol, however, in that case phenol was formed only if the reaction was performed with only phenylboronic acid, with no coupling partner present. ${ }^{18}$ Phenol is most likely formed by the generated aryl radical reacting with either a hydroxyl radical or a water molecule. Finally, there is a large 2,2'-bipyrazine peak and a much smaller 2(dichloromethyl)pyrazine peak. Neither of these products have been noted in the literature. Most likely these products are due to the formation of a pyrazine radical, which either homocouples to form 2,2'-bipyrazine, or reacts with dichloromethane to form 2-(dichloromethyl)pyrazine (see Scheme 2.7). 


\section{Coupling and bis-coupling}

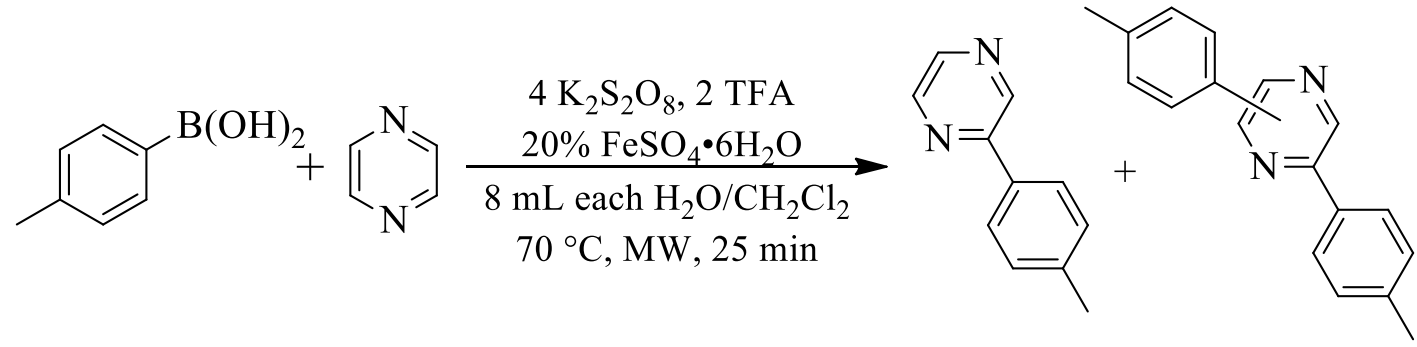

\section{Arylboronic acid side reactions}

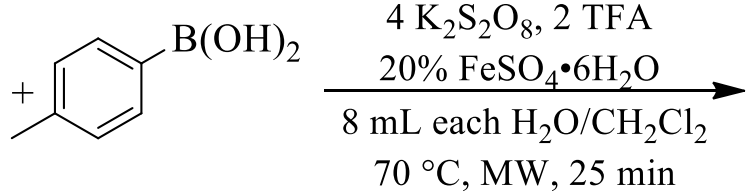

Pyrazine side reactions<smiles>Cc1ccc(O)cc1</smiles><smiles>Oc1ccc(-c2ccc(O)cc2)cc1</smiles>

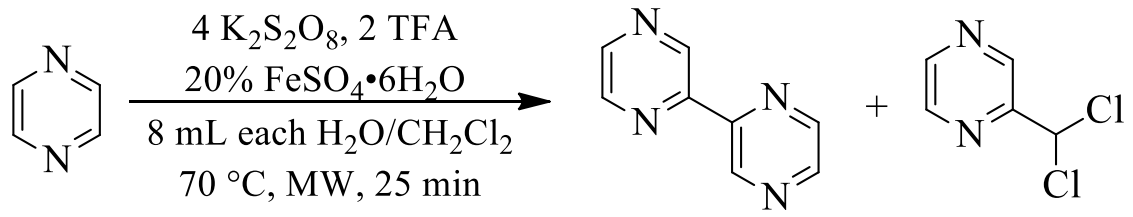

Scheme 2.5 Side reactions and side products observed in the GC-MS of the crude reaction mixture of pyrazine and 4-methylphenylboronic acid. 


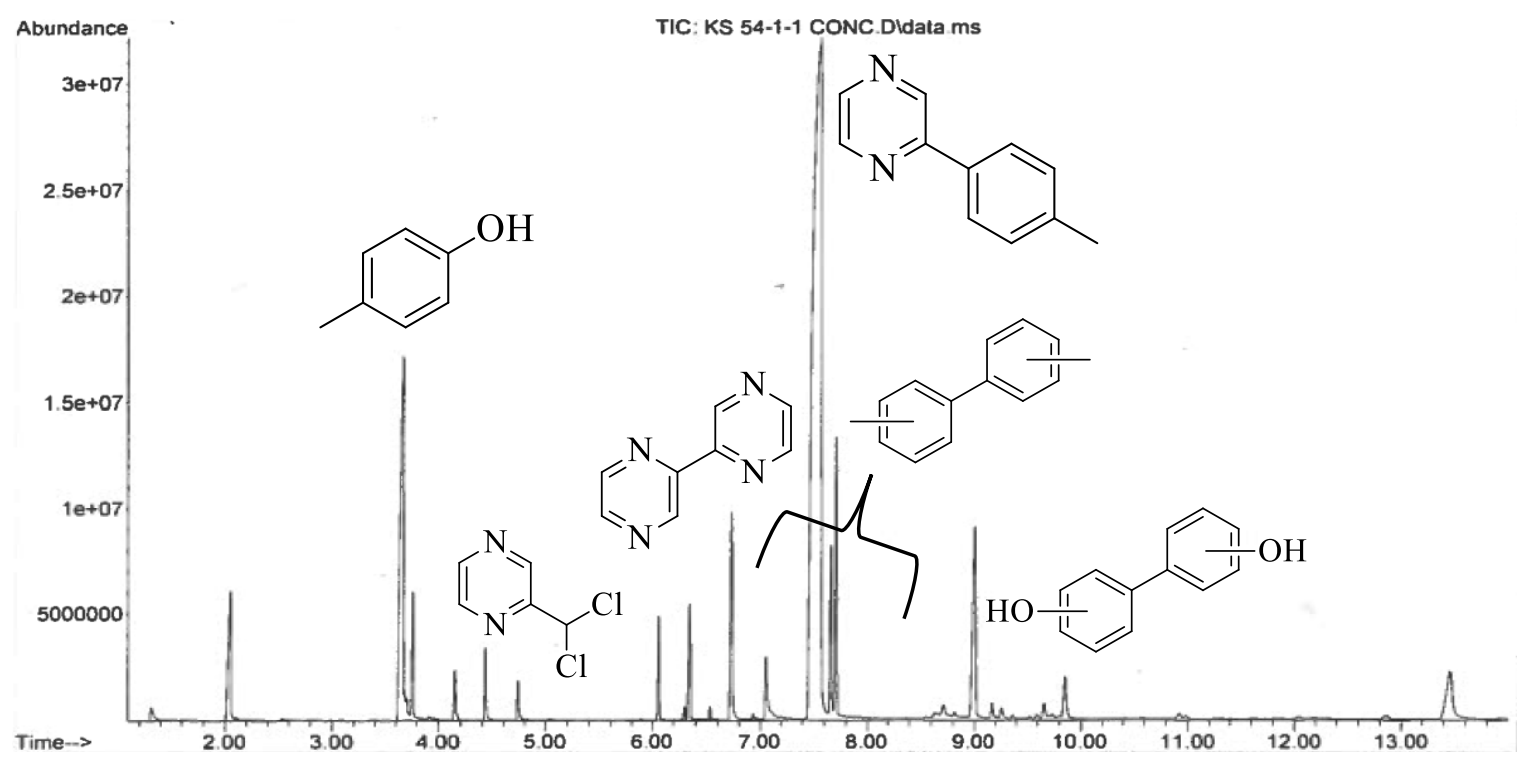

Figure 2.4 Gas chromatogram of the crude reaction mixture of the optimized reaction in dichloromethane. 4-Methylphenol: $\mathrm{R}_{\mathrm{t}}=3.66$. 2-dichloromethylpyrazine: $\mathrm{R}_{\mathrm{t}}=4.15$.

Bipyrazine: $\mathrm{R}_{\mathrm{t}}=6.72 \mathrm{~min}$. Dimethylbiphenyl isomers: $\mathrm{R}_{\mathrm{t}}=7.06 \mathrm{~min}, 7.66 \mathrm{~min}, 7.71 \mathrm{~min}$. Biphenyldimethanol, unknown isomer: $\mathrm{R}_{t}=9.00 \mathrm{~min}$. For further information, consult the experimental and/or Figure A119.

\section{Previous reports}

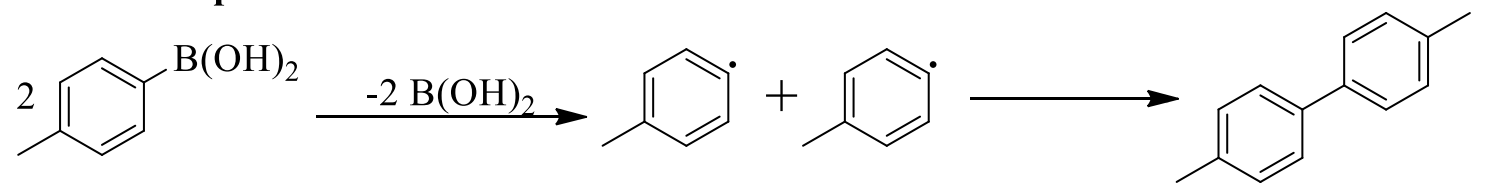

This work

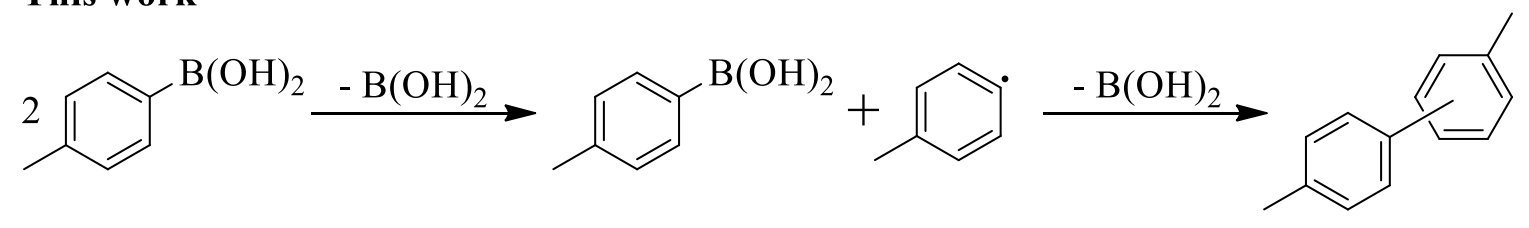

Scheme 2.6 Proposed mechanism for the formation of 3 isomers of dimethylbiphenyl formed by the cross-coupling of 4-methylphenylboronic acid 


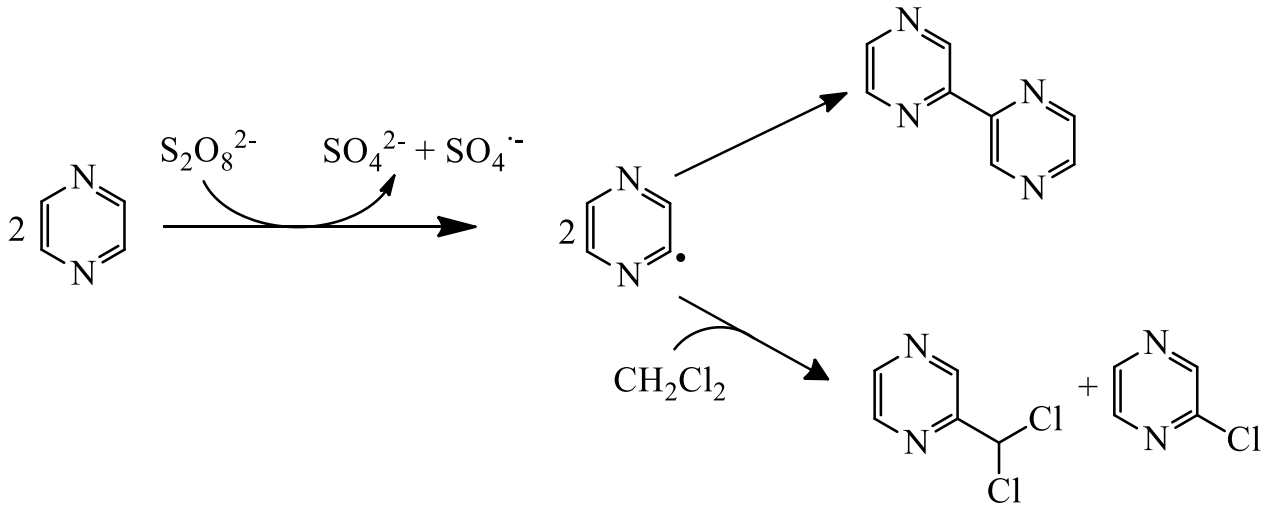

Scheme 2.7 Proposed mechanism for the formation of 2-dichloromethylpyrazine and 2,2'-bipyrazine.

Overall, there are an unusually large number of side products, although most are present in only small or trace amounts. Several of these side products have not been previously reported, suggesting a difference in reactivity between reactions under conventional heating and under microwave heating. This result is particularly unexpected given that microwave heating frequently results in the formation of fewer side products, in lower yields. These side products are most likely a result of microwave specific effects, since they are not observed in reactions under conventional heating at the same temperature. Most likely the abundance of side products is an effect of selective heating. It is possible that the aqueous layer, which is a much better microwave absorber, is being superheated above the temperature of the dichloromethane layer. The IR sensor built in to the microwave measures the temperature at approximately one third of the way up the vessel, so temperature readings reflect conditions at that location, which is covered by the dichloromethane layer. However, since the pressure reading never exceeded 2 bar, the temperature of the aquous layer must have remained at or below approximately $110{ }^{\circ} \mathrm{C} .{ }^{40}$ 
It is also possible that molecular radiators or heterogeneous hotspots are formed during the reaction, causing the side reactions. Molecular radiators could be formed by selective superheating of ionic compounds in the mixture, which more efficiently absorb microwave radiation via ionic conduction. The most likely molecular radiator would be the persulfate anion, but protonated pyrazine could also form a molecular radiator. The reaction mixture appears homogeneous to the naked eye after heating, although solids start to precipitate out as the reaction mixture cools. However, it is possible that small heterogeneous hotspots, invisible to the naked eye, are formed and superheated during irradiation.

\subsubsection{Conclusions}

The cross-coupling of arylboronic acids and pyrazine via direct $\mathrm{C}-\mathrm{H}$ functionalization was optimized under microwave heating. When toluene was used as a cosolvent, toluene homo-coupling and oxidation was observed. No cross-coupling of toluene with pyrazine was observed under these conditions. When using dichloromethane and water as solvents, arylboronic acid hydroxylation and pyrazine homo-coupling were observed for the first time. The optimized reaction conditions provided the desired product in $70 \%$ yield, similar to literature reports. However, the reaction occurred much more rapidly under microwave heating, requiring only $25 \mathrm{~min}$ to reach completion, while 3-24 h are required under conventional heating. A number of previously unreported side-products were observed, including homo-coupled pyrazine and hydroxylated arylboronic acid. The formation of these side products may be due to the formation of superheated hotspots in the microwave, with either the persulfate acting as a molecular radiator or heterogeneous 
hotspots forming during heating. The following chapters will discuss the effect of microwave heating on arylboronic acid, $N$-heteroaryl, and quinone substrate scope.

\subsection{Experimental}

\subsubsection{General Information}

Unless otherwise noted, reagents were purchased from commercial suppliers and used as received. All NMR spectra were obtained on a Bruker AVANCE III $300 \mathrm{MHz}$ NMR spectrometer. All spectra were recorded in ppm relative to an internal TMS standard. Unless otherwise noted, $\mathrm{CDCl}_{3}$ was used as a solvent. Data are reported in the following format: chemical shift, multiplicity $(\mathrm{s}=$ singlet, $\mathrm{d}=$ doublet, $\mathrm{t}=$ triplet, $\mathrm{dd}=$ doublet of doublets, $\mathrm{m}=$ multipet), coupling constant $(\mathrm{J}, \mathrm{Hz})$, and integration. All NMR spectra were processed using MestreNova software. Gas chromatography mass spectroscopy (GC-MS) data was recorded using an Agilent Technologies 7890 GC system coupled to an Agilent $5975 \mathrm{C}$ mass detector equipped with an Agilent HP-5ms column ( $30 \mathrm{~m} \times 250 \mu \mathrm{m} \times 0.25 \mu \mathrm{m}$, (5\%-Phenyl)-methylpolysiloxane). Samples were injected into a split-splitless inlet with a split ratio of $25: 1$, which was heated to $275^{\circ} \mathrm{C}$. The oven was held at $80^{\circ} \mathrm{C}$ for $2 \mathrm{~min}$, then the temperature was increased by $20{ }^{\circ} \mathrm{C}$ per minute until a maximum temperature of 200 ${ }^{\circ} \mathrm{C}$ was reached. The oven was maintained at this temperature for 4 min. A blank was performed in between samples: if products eluted near the end of the run or in the blank the hold time was increased, but the ramp conditions were not changed. The only exception to this occurs in Figure 2.1, as that data was obtained before a standard method had been 
developed. In that case the same conditions were used, except the temperature was increased by $30{ }^{\circ} \mathrm{C}$ per minute instead of $20{ }^{\circ} \mathrm{C}$ per minute. Unless otherwise noted all microwave reactions were performed in $20 \mathrm{~mL}$ Biotage microwave vials using a Biotage Initiator $^{\mathrm{TM}}$ microwave with expanded volume range (EXP). Column chromatography was performed using a Biotage Isolera One Flash Purification System, using Biotage SNAP 25 G cartridges. GC-MS information of trace products is reported, but could not be obtained. Similarly, in most cases GC-MS data is reported for side products, but in most cases NMR data was not collected. Where possible, the identity of products identified by GC-MS alone was confirmed by comparison to the literature, most notably the National Institute of Standards and Technology (NIST) Chemistry WebBook.

\subsubsection{General Procedure}

A $10-20 \mathrm{~mL}$ Biotage microwave vial with a magnetic stir bar was charged with $\mathrm{K}_{2} \mathrm{~S}_{2} \mathrm{O}_{8}$ (4 equiv), and $\mathrm{FeSO}_{4} \cdot 6 \mathrm{H}_{2} \mathrm{O}(0.2$ equiv). $8 \mathrm{~mL}$ each of water and dichloromethane were added, followed by trifluoroacetic acid (TFA, 2 equiv), the desired arylboronic acid ( 2 equiv), and pyrazine $(0.75 \mathrm{mmol})$. The vial was capped and crimped shut, then placed in the microwave, where it was pre-stirred for $1 \mathrm{~min}$ at $900 \mathrm{RPM}$. The vial was then heated to $70{ }^{\circ} \mathrm{C}$ using the high absorption level, and was heated with stirring for $25 \mathrm{~min}$. The vial was cooled using forced air to $50^{\circ} \mathrm{C}$, then removed from the microwave and uncapped.

The resulting biphasic mixture was placed in a separatory funnel, and the dichloromethane layer was collected. The remaining aqueous layer was then extracted with $3 \times 15 \mathrm{~mL}$ of dichloromethane, then neutralized using $20 \mathrm{~mL}$ of saturated sodium bicarbonate solution and extracted with an additional $2 \times 15 \mathrm{~mL}$ of dichloromethane. The 
combined dichloromethane extracts were then washed with $30 \mathrm{~mL}$ of saturated sodium bicarbonate solution, then concentrated in vacuo.

GC-MS data of the crude product mixture was collected if desired and the crude product was purified using flash chromatography with $25 \mathrm{G}$ Biotage SNAP columns containing Zeoprep 60 Eco / 40-63 $\mu \mathrm{m}$ silica, and an eluent mixture of ethyl acetate in hexanes (recorded individually for each compound in section 2.3.4). The identity and purity of the product was confirmed using GC-MS, ${ }^{1} \mathrm{H}$ NMR, ${ }^{13} \mathrm{C}$ NMR, and DEPT NMR.

\subsubsection{Catalyst Synthesis}

\subsubsection{Citrate-stabilized Iron Nanoparticles}

Citrate capped nanoparticles were synthesized based a procedure reported by Nigam et al. ${ }^{41} 4.44 \mathrm{~g}(27.3 \mathrm{mmol})$ of $\mathrm{FeCl}_{3}$ and $1.73 \mathrm{~g}$ (13.6 mmol) of $\mathrm{FeCl}_{2}$ were added to a $250 \mathrm{~mL}$ round-bottom flask, then dissolved in $80 \mathrm{~mL}$ nanopure water. The mixture was heated at $70{ }^{\circ} \mathrm{C}$ for $30 \mathrm{~min}$ with $1000 \mathrm{RPM}$ stirring under a nitrogen atmosphere. $20 \mathrm{~mL}$ of concentrated aqueous ammonia was then added via syringe, and the mixture was stirred for another $30 \mathrm{~min} .4 \mathrm{~mL}$ of an $0.5 \mathrm{~g} / \mathrm{mL}$ citric acid solution was added via syringe to stabilize the iron nanoparticles, and the mixture was heated at $90{ }^{\circ} \mathrm{C}$ for $60 \mathrm{~min}$. The reaction was cooled to room temperature, then the black solid was centrifuged and washed with $3 \times 50$ $\mathrm{mL}$ of nanopure water. The solid was dried overnight at $50{ }^{\circ} \mathrm{C}$ before characterization on XRD, DSL, and FTIR. The citrate capped particles were rod-shaped, with an average size distribution of $148.5 \mathrm{~nm} \pm 47.4 \mathrm{~nm}$. 


\subsubsection{Complex I}

The iron (III) amine-bis(phenolate) complex I was synthesized by a procedure reported by Chowdhury et al. ${ }^{38}$ The ligand was prepared by refluxing and stirring a solution of 1 equiv of 2-t-butyl-4-methylphenol, 0.5 equiv of tetrahydrofurfurylamine, and 1 equiv of $37 \%$ aqueous formaldehyde in methanol for $24 \mathrm{~h}$. After cooling, the reaction mixture formed two phases, and the upper phase was decanted. The oily bottom phase was collected and triturate with cold methanol to give a white powder. The methanol washings were combined with the upper phase and concentrated in vacuo until product precipitated from solution. A total yield of approximately $80 \%$ was obtained. The complex was prepared by dropwise addition of 1 equiv of a solution of $\mathrm{FeCl}_{3}$ in methanol to a methanol slurry of 1 equiv of the ligand at room temperature. The solution was neutralized using 2 equiv of $\mathrm{NEt}_{3}$ and stirred for $4 \mathrm{~h}$. The solution was then evaporated to dryness, extracted with toluene, filtered through Celite, and concentrated in vacuo to give the desired complex in $80 \%$ yield. The compound was characterized as in the literature.

\subsubsection{Complex II}

The iron (III) amine-bis(phenolate) complex II was prepared based on a report by Alhashmialameer et al. ${ }^{39}$ The ligand was prepared by refluxing and stirring a solution of 1 equiv of 2,4-di-t-butyl-phenol, 0.5 equiv of homopiperazine, and 1 equiv of $37 \%$ aqueous formaldehyde in methanol for $24 \mathrm{~h}$. After cooling, the white solid that had formed was collected and recrystallized from methanol and chloroform to form a white powder in $98 \%$ yield. The complex was prepared by dropwise addition of 1 equiv of a solution of $\mathrm{FeBr}_{3}$ in methanol to a methanol slurry of 1 equiv of the ligand at room temperature. The solution 
was neutralized using 2 equiv of $\mathrm{NEt}_{3}$ and stirred for $4 \mathrm{~h}$. The solution was then evaporated to dryness, extracted with toluene, filtered through Celite, and concentrated in vacuo to give the desired complex in $80 \%$ yield. The compound was characterized as in the literature.

\subsubsection{Spectroscopic Information}

\section{2-(4-Methylphenyl)pyrazine (1A)}

$\mathbf{1 A}$ was synthesized according to the general procedure and purified using an eluent mixture of $10 \%$ ethyl acetate in hexane. The purified product was a pale-yellow solid obtained in $72 \%$ yield. ${ }^{1} \mathrm{H}$ NMR $\left(300 \mathrm{MHz}, \mathrm{CDCl}_{3}\right) \delta 9.00(\mathrm{~d}, J=1.7 \mathrm{~Hz}, 1 \mathrm{H}), 8.60(\mathrm{dd}, J=2.5,1.6$ Hz, 1H), $8.47(\mathrm{~d}, J=2.6 \mathrm{~Hz}, 1 \mathrm{H}), 7.95-7.88(\mathrm{~m}, 2 \mathrm{H}), 7.31(\mathrm{~d}, J=8.1 \mathrm{~Hz}, 2 \mathrm{H}), 2.42$ (s, 3H). ${ }^{13} \mathrm{C} \mathrm{NMR}\left(75 \mathrm{MHz}, \mathrm{CDCl}_{3}\right) \delta 152.85,144.09,142.57,142.02,140.12,133.57,129.79$, 126.81, 21.36. DEPT NMR (75 MHz, $\left.\mathrm{CDCl}_{3}\right) \delta 152.86$ (down), 144.09, 142.58, 142.03, 140.13 (down), 133.57 (down), 129.80, 129.49, 129.00, 126.81, 21.36. GC-MS: $\mathrm{R}_{\mathrm{t}}=7.47$ min. m/z: $170.1\left(\mathrm{M}^{+}\right), 155.1\left(\mathrm{M}^{+}-15\right), 117.1\left(\mathrm{M}^{+}-53\right), 89.1\left(\mathrm{M}^{+}-81\right)$.

\section{2-Phenylpyrazine (1B)}

1B was synthesized according to the general procedure and purified using an eluent mixture of $10 \%$ ethyl acetate in hexane. The final product was a pale-yellow solid obtained in $70 \%$ yield. ${ }^{1} \mathrm{H}$ NMR (300 MHz, $\left.\mathrm{CDCl}_{3}\right) \delta 9.03(\mathrm{~d}, J=1.7 \mathrm{~Hz}, 1 \mathrm{H}), 8.62(\mathrm{dd}, J=2.5,1.5 \mathrm{~Hz}$, $1 \mathrm{H}), 8.50(\mathrm{~d}, J=2.6 \mathrm{~Hz}, 1 \mathrm{H}), 8.05-7.98(\mathrm{~m}, 2 \mathrm{H}), 7.54-7.45(\mathrm{~m}, 3 \mathrm{H}) .{ }^{13} \mathrm{C} \mathrm{NMR}(75 \mathrm{MHz}$, $\left.\mathrm{CDCl}_{3}\right) \delta 152.85,144.19,142.89,142.20,136.33,129.93,129.06,126.95$. GC-MS: $\mathrm{R}_{\mathrm{t}}=$ $5.32 \mathrm{~min} . \mathrm{m} / \mathrm{z}=156.1\left(\mathrm{M}^{+}\right), 129.1\left(\mathrm{M}^{+}-27\right), 103.1\left(\mathrm{M}^{+}-53\right), 76.1\left(\mathrm{M}^{+}-80\right)$. 


\section{4-Methylphenol}

4-Methylphenol was obtained as a side product during the synthesis of $\mathbf{1 A}$ as a white solid in $11 \%$ yield. $\mathrm{R}_{\mathrm{t}}=3.66 \mathrm{~min} . \mathrm{m} / \mathrm{z}=107.1\left(\mathrm{M}^{+}\right), 90.1\left(\mathrm{M}^{+}-17\right), 77.1\left(\mathrm{M}^{+}-30\right)$.

\section{2,2'-Bipyrazine}

2.2'-Bipyrazine was obtained as a side product during the synthesis of $\mathbf{1 A}$ as a red solid in $6 \%$ yield. $\mathrm{R}_{\mathrm{t}}=6.72 \mathrm{~min} . \mathrm{m} / \mathrm{z}=158.1,131.1,106.1,80.1$

\section{Dimethylbiphenyl (mixture of isomers)}

Three isomers of dimethylbiphenyl (unidentified) were obtained as side products during the synthesis of $\mathbf{1 A}$ as a white solid in $12 \%$ yield (with respect to 2-(4methylphenyl)pyrazine). Dimethylbiphenyl isomer: $R_{t}=7.66 \mathrm{~min} . \mathrm{m} / \mathrm{z}=182.1\left(\mathrm{M}^{+}\right)$, $167.1\left(\mathrm{M}^{+}-15\right), 152.1\left(\mathrm{M}^{+}-30\right), 139.1\left(\mathrm{M}^{+}-43\right), 128.1\left(\mathrm{M}^{+}-54\right), 115.1\left(\mathrm{M}^{+}-67\right), 89.1\left(\mathrm{M}^{+}-\right.$ 93). Dimethylbiphenyl isomer: $\mathrm{R}_{\mathrm{t}}=7.71 \mathrm{~min} . \mathrm{m} / \mathrm{z}=182.1\left(\mathrm{M}^{+}\right), 167.1\left(\mathrm{M}^{+}-15\right), 152.1$ $\left(\mathrm{M}^{+}\right.$-30), $139.1\left(\mathrm{M}^{+}-43\right), 128.1\left(\mathrm{M}^{+}-54\right), 115.1\left(\mathrm{M}^{+}-67\right), 89.1\left(\mathrm{M}^{+}-93\right)$. Dimethylbiphenyl isomer: $\mathrm{R}_{\mathrm{t}}=7.06 \mathrm{~min} . \mathrm{m} / \mathrm{z}=182.1\left(\mathrm{M}^{+}\right), 167.1\left(\mathrm{M}^{+}-15\right), 152.1\left(\mathrm{M}^{+}-30\right), 141.1\left(\mathrm{M}^{+}-41\right)$, $128.1\left(\mathrm{M}^{+}-54\right), 115.1\left(\mathrm{M}^{+}-67\right), 89.1\left(\mathrm{M}^{+}-93\right)$.

Benzyl alcohol: Benzyl alcohol: $\mathrm{R}_{\mathrm{t}}=3.08 \mathrm{~min} . \mathrm{m} / \mathrm{z}=108.1\left(\mathrm{M}^{+}\right), 107.1\left(\mathrm{M}^{+}-1\right), 90.1\left(\mathrm{M}^{+}-\right.$ 18), $79.1\left(\mathrm{M}^{+}-29\right), 77.1\left(\mathrm{M}^{+}-31\right)$.

2-dichloromethylpyrazine: $\mathrm{R}_{\mathrm{t}}=4.15 \mathrm{~min} . \mathrm{m} / \mathrm{z}=162.0\left(\mathrm{M}^{+}\right), 164.0\left(\mathrm{M}^{+}+2\right), 166.0\left(\mathrm{M}^{+}+4\right)$, $129.0\left(\mathrm{M}^{+}-33\right), 127.0\left(\mathrm{M}^{+}-35\right), 100.0\left(\mathrm{M}^{+}-62\right), 92.1\left(\mathrm{M}^{+}-70\right), 73.0\left(\mathrm{M}^{+}-89\right)$.

2-benzylpyrazine: $\mathrm{R}_{\mathrm{t}}=5.45 \mathrm{~min} . \mathrm{m} / \mathrm{z}=170.1\left(\mathrm{M}^{+}\right), 169.1\left(\mathrm{M}^{+}-1\right), 115.1\left(\mathrm{M}^{+}-55\right), 91.1$ $\left(\mathrm{M}^{+}-79\right), 65.1\left(\mathrm{M}^{+}-104\right)$. 
Bibenzyl: Bibenzyl: $\mathrm{R}_{\mathrm{t}}=5.59$ min. m/z: $182.1\left(\mathrm{M}^{+}\right), 104.1\left(\mathrm{M}^{+}-78\right), 91.1\left(\mathrm{M}^{+}-91\right), 77.1$ $\left(\mathrm{M}^{+}-105\right) .65 .1\left(\mathrm{M}^{+}-117\right)$.

\subsection{References}

(1) Baumann, M.; Baxendale, I. R. An overview of the synthetic routes to the best selling drugs containing 6-membered heterocycles. Beilstein Journal of Organic Chemistry 2013, 9, 2265-2319.

(2) Baumann, M.; Baxendale, I. R.; Ley, S. V.; Nikbin, N. An overview of the key routes to the best selling 5-membered ring heterocyclic pharmaceuticals. Beilstein Journal of Organic Chemistry 2011, 7, 442-495.

(3) Berg, S.; Bergh, M.; Hellberg, S.; Hogdin, K.; Lo-Alfredsson, Y.; Soderman, P.; von Berg, S.; Weigelt, T.; Ormo, M.; Xue, Y.et al. Discovery of novel potent and highly selective glycogen synthase kinase-3 $\beta$ (GSK3 $\beta$ ) inhibitors for Alzheimer's disease: design, synthesis, and characterization of pyrazines. Journal of Medicinal Chemistry 2012, 55 (21), 9107-9119.

(4) Koyama, J. Anti-Infective Quinone Derivatives of Recent Patents. Recent Patents on Anti-Infective Drug Discovery 2006, I (1), 113-125.

(5) Dandawate, P. R.; Vyas, A. C.; Padhye, S. B.; Singh, M. W.; Baruah, J. B. Perspectives on Medicinal Properties of Benzoquinone Compounds. Mini-Reviews in Medicinal Chemistry 2010, 10, 436-454.

(6) Thomson, R. J. Naturally Occurring Quinones IV; 4 ed.; Blackie Academic and Professional: London, 1997.

(7) Brown, E. G. Ring Nitrogen and Key Biomolecules: the biochemistry of $\mathrm{N}$ heterocycles; Kluwer Academic Publishers: Dordrecht, Netherlands, 1998.

(8) Murphree, S. S. Chapter 2: Heterocyclic Dyes: Preparation, Properties, and Applications, in Progress in Heterocyclic Chemistry; Gribble, G.;Joule, J. A., Eds.; Elsevier Ltd.: Amsterdam, 2011; Vol. 22.

(9) Seiple, I. B.; Su, S.; Rodriguez, R. A.; Gianatassio, R.; Fujiwara, Y.; Sobel, A. L.; Baran, P. S. Direct C-H Arylation of Electron-Deficient Heterocycles with Arylboronic Acids. Journal of the American Chemical Society 2010, 132, 1319413196.

(10) Fujiwara, Y.; Domingo, V.; Seiple, I. B.; Gianatassio, R.; Del Bel, M.; Baran, P. S. Practical $\mathrm{C}-\mathrm{H}$ functionalization of quinones with boronic acids. Journal of the American Chemical Society 2011, 133 (10), 3292-3295. 
(11) Patel, N. R.; Flowers, R. A., 2nd. Uncovering the mechanism of the $\operatorname{Ag}(\mathrm{I}) /$ persulfate-catalyzed cross-coupling reaction of arylboronic acids and heteroarenes. Journal of the American Chemical Society 2013, 135 (12), 46724675.

(12) Wang, J.; Wang, S.; Wang, G.; Zhang, J.; Yu, X. Q. Iron-mediated direct arylation with arylboronic acids through an aryl radical transfer pathway. Chemical Communications 2012, 48 (96), 11769-11771.

(13) Deb, A.; Manna, S.; Maji, A.; Dutta, U.; Maiti, D. Iron-Catalyzed Direct C-H Arylation of Heterocycles and Quinones with Arylboronic Acids. European Journal of Organic Chemistry 2013, 2013 (24), 5251-5256.

(14) Li, Z.; Mai, W.; Yuan, J.; Sun, G.; Qu, L. Silver-Catalyzed 2-Pyridyl Arylation of Pyridine N-Oxides with Arylboronic Acids at Room Temperature. Synlett 2011, 2012 (01), 145-149.

(15) Ren, X.; Han, S.; Gao, X.; Li, J.; Zou, D.; Wu, Y.; Wu, Y. Direct arylation for the synthesis of 2-arylquinolines from $\mathrm{N}$-methoxyquinoline-1-ium tetrafluoroborate salts and arylboronic acids. Tetrahedron Letters 2018, 59 (11), 1065-1068.

(16) Bering, L.; Antonchick, A. P. Regioselective Metal-Free Cross-Coupling of Quinoline N-Oxides with Boronic Acids. Organic Letters 2015, 17 (12), 31343137.

(17) Komeyama, K.; Kashihara, T.; Takaki, K. FeSO 4 -promoted direct arylation of benzoquinones with $\mathrm{ArB}(\mathrm{OH})_{2}$ or $\mathrm{ArBF}_{3} \mathrm{~K}$. Tetrahedron Letters 2013, 54 (9), 1084 1086.

(18) Singh, P. P.; Aithagani, S. K.; Yadav, M.; Singh, V. P.; Vishwakarma, R. A. Ironcatalyzed cross-coupling of electron-deficient heterocycles and quinone with organoboron species via innate $\mathrm{C}-\mathrm{H}$ functionalization: application in total synthesis of pyrazine alkaloid botryllazine A. Journal of Organic Chemistry 2013, 78 (6), 2639-2648.

(19) Komeyama, K.; Nagao, Y.; Abe, M.; Takaki, K. Scope and Limitation for FeSO4Mediated Direct Arylation of Heteroarenes with Arylboronic Acids and Its Synthetic Applications. Bulletin of the Chemical Society of Japan 2014, 87 (2), 301313.

(20) Huang, Y.; Guan, D.; Wang, L. Direct Arylation of Substituted Pyridines with Arylboronic Acids Catalyzed by Iron(II) Oxalate. Chinese Journal of Chemistry 2014, 32 (12), 1294-1298.

(21) Deb, A.; Agasti, S.; Saboo, T.; Maiti, D. Generation of Arylated Quinones by IronCatalyzed Oxidative Arylation of Phenols: Formal Synthesis of Phellodonin, Sarcodonin \&, Leucomelone and Betulinan A. Advanced Synthesis \& Catalysis 2014, 356 (4), 705-710. 
(22) Modak, A.; Rana, S.; Maiti, D. Iron-Catalyzed Regioselective Direct Arylation at the C-3 Position of N-Alkyl-2-pyridone. Journal of Organic Chemistry 2015, 80 (1), 296-303.

(23) Jain, R.; Mahindra, A. Regiospecific Direct C-H Arylation at the 2-Position of 1Histidine Using Arylboronic Acids. Synlett 2012, 23 (12), 1759-1764.

(24) Yan, G.; Yang, M.; Wu, X. Synthetic applications of arylboronic acid via an aryl radical transfer pathway. Organic \& Biomolecular Chemistry 2013, 11 (46), 79998008 .

(25) Thatikonda, T.; Singh, U.; Ambala, S.; Vishwakarma, R. A.; Singh, P. P. Metal free $\mathrm{C}-\mathrm{H}$ functionalization of diazines and related heteroarenes with organoboron species and its application in the synthesis of a CDK inhibitor, meriolin 1. Organic \& Biomolecular Chemistry 2016, 14 (18), 4312-4320.

(26) Gedye, R.; Smith, F.; Westawa, K.; Ali, H.; Baldisera, L.; Laberge, L.; Rousell, J. The Use of Microwave Ovens for Rapid Organic Synthesis. Tetrahedron Letters 1986, 27 (3), 279-282.

(27) Giguere, R. J.; Bray, T. L.; Duncan, S. M.; Majetich, G. Application of Commercial Microwave Ovens to Organic Synthesis. Tetrahedron Letters 1986, 27 (41), 49454948.

(28) Kappe, C. O. Controlled microwave heating in modern organic synthesis. Angewandte Chemie International Edition 2004, 43 (46), 6250-6284.

(29) Kappe, C. O.; Dallinger, D. Controlled microwave heating in modern organic synthesis: highlights from the 2004-2008 literature. Molecular Diversity 2009, 13, 71-193.

(30) Kappe, C. O.; Dallinger, D.; Murphree, S. S. Practical Microwave Synthesis for Organic Chemists: Strategies, Instruments and Protocols; Wiley-VCH Verlag $\mathrm{GmbH}$ an Co. KGaA: Weinheim, Germany, 2009.

(31) Kappe, C. O.; Pieber, B.; Dallinger, D. Microwave effects in organic synthesis: myth or reality? Angewandte Chemie International Edition 2013, 52 (4), 10881094.

(32) Caddick, S.; Fitzmaurice, R. Microwave enhanced synthesis. Tetrahedron 2009, 65 (17), 3325-3355.

(33) Walla, P.; Kappe, C. O. Microwave-assisted Negishi and Kumada cross-coupling reactions of aryl chlorides. Chemical Communications 2004, (5), 564-565.

(34) Nilsson, P.; Gold, H.; Larhed, M. Microwave Irradiation as a High-Speed Tool for Activation of Sluggish Aryl Chlorides in Grignard Reactions. Synlett 2005, 2005 (10), 1596-1600.

(35) Arvela, R. K.; Leadbeater, N. E. Microwave-Promoted Heck Coupling Using Ultralow Metal Catalyst Concentrations. Journal of Organic Chemistry 2005, 70, 1786-1790. 
(36) Arvela, R. K.; Leadbeater, N. E.; Sangi, M. S.; Williams, V. A.; Granados, P.; Singer, R. D. A Reassessment of the Transition-Metal Free Suzuki-Type Coupling Methodology. Journal of Organic Chemistry 2005, 70, 161-168.

(37) Diaz-Ortiz, A.; De La Hoz, A.; Carillo, J. R.; Herrero, M. A. Chapter 5: Selectivity Modification Under Microwave Irradiation, in Microwaves in Organic Synthesis; 3 ed.; De La Hoz, A.;Loupy, A., Eds.; Wiley-VCH Verlag GmbH and Co. KGaA: Weinheim, Germany, 2013.

(38) Chowdhury, R. R.; Crane, A. K.; Fowler, C.; Kwong, P.; Kozak, C. M. Iron(III) amine-bis(phenolate) complexes as catalysts for the coupling of alkyl halides with aryl Grignard reagents. Chemical Communications 2008, (1), 94-96.

(39) Alhashmialameer, D.; Collins, J.; Hattenhauer, K.; Kerton, F. M. Iron aminobis(phenolate) complexes for the formation of organic carbonates from $\mathrm{CO}_{2}$ and oxiranes. Catalysis Science \& Technology 2016, 6 (14), 5364-5373.

(40) Biotage Initiator Getting Started Guide. 2005, 1-20.

(41) Nigam, S.; Barick, K. C.; Bahadur, D. Development of citrate-stabilized $\mathrm{Fe}_{3} \mathrm{O}_{4}$ nanoparticles: Conjugation and release of doxorubicin for therapeutic applications. Journal of Magnetism and Magnetic Materials 2011, 323 (2), 237-243. 


\section{Chapter 3. Reaction Scope and Regioselectivity Under Microwave Heating}

\subsection{Introduction}

As previously described in chapters 1 and 2, in 2010 and 2011 Baran and coworkers reported a procedure for the direct C-C coupling of quinones and $\mathrm{N}$-heteroarenes with arylboronic acids. ${ }^{1,2}$ This reaction proceeded via a radical pathway, using a persulfate salt as an initiator and a simple silver salt as a catalyst, and provided an easier route to arylquinone or $N$-heterobiaryl compounds that are useful in the pharmaceutical and dye industries $^{1,2}$. This reaction was relatively low cost, as it employed inexpensive arylboronic acids and persulfate salts as reagents, inexpensive silver salts as catalysts, and did not require functionalized $N$-heteroarenes or quinones. ${ }^{1,2}$ In addition, reaction conditions were mild, with reactions taking place between room temperature and $70{ }^{\circ} \mathrm{C}$ under air. The reaction therefore garnered significant academic interest, with iron-catalyzed and metalfree variants described soon thereafter, as well as reports published examining the reaction mechanism. ${ }^{3-16}$ Further, several papers have explored the scope of the reaction with respect to quinones, $\mathrm{N}$-heteroarenes and arylboronic acids, as well as the regiochemistry of the reaction and accounts of attempts to influence or control regioselectivity (Scheme 3.1). ${ }^{3-16}$ 


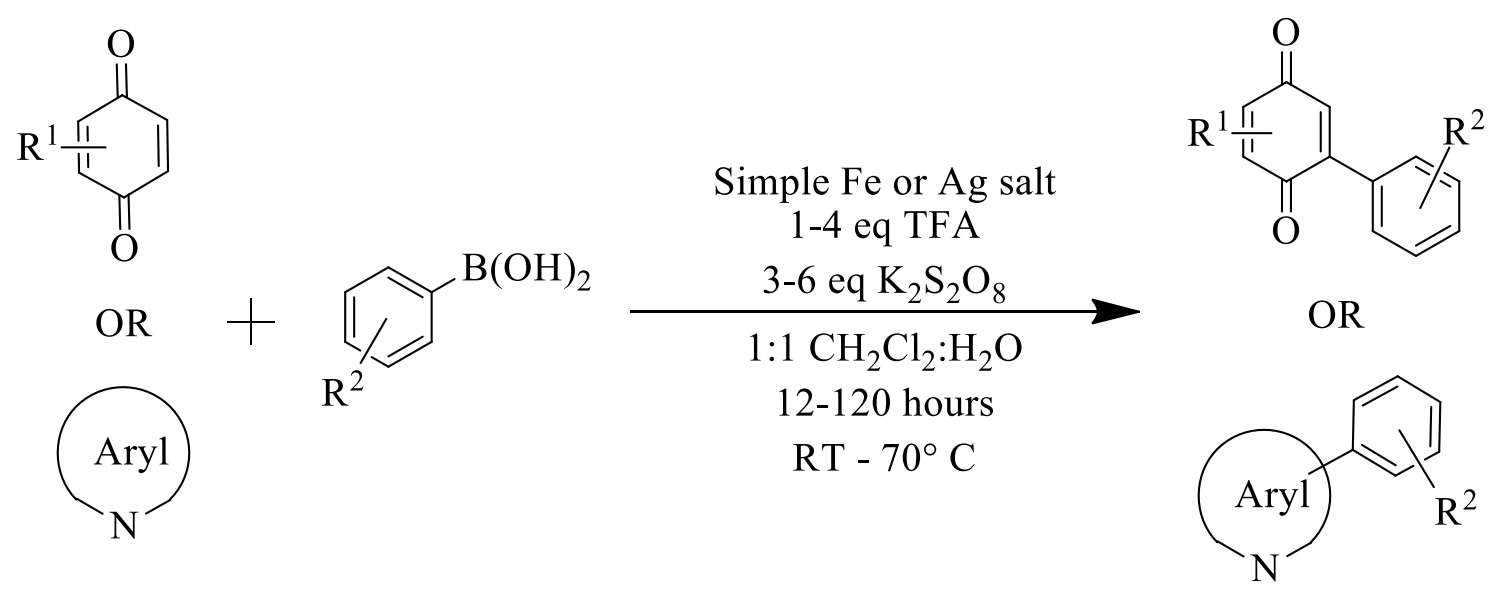

Scheme 3.1 General conditions of the cross-coupling of arylboronic acids with quinones or $N$-heteroaryls using potassium persulfate.

The scope of the reaction includes $N$-heteroarenes and quinones with arylboronic acids or aryltrifluoroborate salts, all of which can have a variety of functional groups. $\mathrm{N}$ heteroarene coupling is most successful with electron-deficient substrates; generally, reactions with $N$-heteroarenes bearing electron-withdrawing groups are most successful, followed by unsubstituted $N$-heteroarenes, with reactions with $N$-heteroarenes bearing electron-donating groups giving no conversion or low yields. ${ }^{1,3,6,8,15}$ This trend is explained by the proposed reaction mechanism, which suggests that the product forms when an aryl radical attacks the $N$-heteroarene. ${ }^{1,16}$ The presence of electron-withdrawing groups reduces electron-density on the $N$-heteroarene, making it more susceptible to attack. However, results with some substrates, such as halide or alkyl-substituted n-heteroarenes, are mixed, with some groups reporting good yields and others reporting little or no conversion. $1,3,6,8,15$ These differences may be due to the use of different substrates or different reaction conditions. Six-membered rings are also preferred, with five-membered $N$-heteroarenes 
generally giving poor yields. ${ }^{1,3,6,8,11,15}$ Reactions with quinones focus heavily on 1,4-benzoquinone and derivatives ${ }^{2,3,7,9,15}$ and are more tolerant to the presence of functional groups such as alkyls, halides, and alkoxy groups, which all give similar yields to

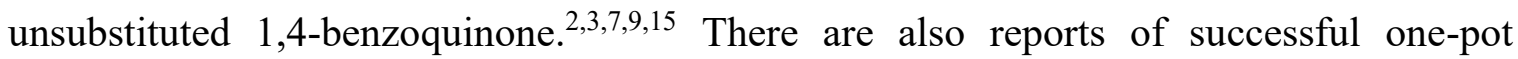
oxidation and coupling with hydroquinones, phenols, and methoxyphenols, usually in similar yields to reaction with quinones. ${ }^{3,4,7,15}$ However, there are no reports of reactions with other enones, with enals, or with other conjugated compounds.

The scope of reactions with arylboronic acids have been explored with quinones and $N$-heteroarenes and focuses on reaction with phenylboronic acid and its derivatives. ${ }^{1-}$ 9,15 Generally, the best results are observed with unsubstituted boronic acids, alkylsubstituted boronic acids, or alkoxy-substituted boronic acids. ${ }^{1-9,15}$ Strong electronwithdrawing groups lead to a decrease in yields, although the magnitude of the decrease varies. ${ }^{1-9,15}$ These results are also consistent with the proposed mechanism, as electrondonating groups improve the nucleophilicity of the aryl radical. Results with halidesubstituted boronic acids and boronic acids with substituents at the 2 position are mixed, with some groups claiming good yields and others reporting little or no conversion. ${ }^{1-9,15}$ Reactions have also been performed with aryltrifluoroborate salts, with similar results to reactions with the corresponding arylboronic acid..$^{2,7-10}$ An in-depth review of the scope of reactions with respect to $N$-heteroarenes, quinones, and arylboronic acids can be found in Section 1.1.2.

The regiochemistry of the reaction has been studied extensively, with several reports on attempts to control regiochemistry. These reports focus almost exclusively on 
$N$-heteroarenes, as quinones are very often symmetrical. When reactions are performed with asymmetric quinones, all isomers are produced, generally in similar yield to one another. ${ }^{2,7}$ Studies of regioselectivity in $N$-heteroarenes focus primarily on pyridine or quinoline and their derivatives. When they are unsubstituted, the 2 and 4 isomers are the major products due to reduced electron density at these position (see Figure 3.1). 1,3,6,8,15 However, the ratio of 2 to 4 product varies between reports, with some claiming a larger yield of the 2 product, and others a larger yield of the 4 product. ${ }^{1,3}$ A common method to control regiochemistry is to include a substituent at the 4 position. In these cases, the 2 product is preferred, although a small amount of 3 product may be formed. ${ }^{1,3,6,8,15}$ Another method to control regiochemistry is to use aryl- $N$-oxides. ${ }^{12-14}$ Reactions with these compounds occur almost exclusively at the 2 position, which has significantly reduced electron density. ${ }^{12-14}$ An in-depth review of the regiochemistry of reactions with $\mathrm{N}$ heteroarenes and quinones can be found in Sections 1.1.2.3 and 1.1.2.4.

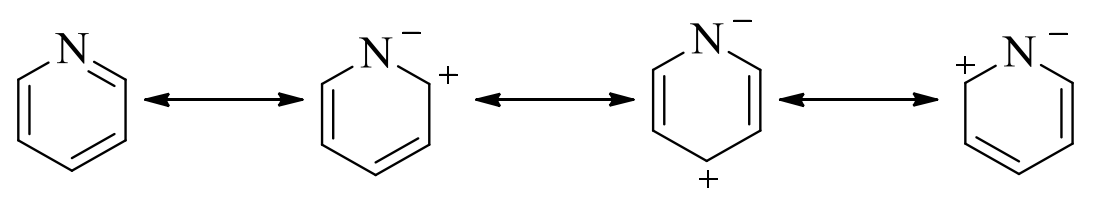

Figure 3.1 Resonance structures of pyridine, demonstrating reduced electron density at the 2 and 4 positions.

As described in greater detail in chapter 1.2, the manner in which microwave radiation heats reaction mixtures is different from that of conventional heating methods. ${ }^{17-}$ ${ }^{21}$ Conventional heating methods rely on thermal conduction, while microwave heating is 
caused by the absorption of microwave radiation, which can heat the bulk reaction mixture

directly. ${ }^{17-21}$ In addition, microwave heating is rapid, offers better control over heating conditions, and can heat certain substrates preferentially over others. ${ }^{17-21}$ These differences often lead to differences the outcomes of reactions under microwave heating from the outcomes of reactions under conventional heating. ${ }^{17-21}$ Commonly observed differences can include diminished reactions times ${ }^{17-23}$, increased yields ${ }^{17-24}$, a larger substrate scope ${ }^{17-24}$, and changes in regioselectivity. ${ }^{17-21,25}$ In the previous chapter, reaction conditions were optimized for the cross-coupling of arylboronic acids with pyrazine, an $N$-heteroarene. In this chapter a number of substrates that are challenging under conventional heating, such as $N$-heteroarenes bearing electron-donating groups, or arylboronic acids bearing sterically hindering or electron-withdrawing groups, are examined to determine whether microwave heating can be used to improve yields with. In addition, the effect of microwave heating on regiochemistry was examined.

\subsection{Results and Discussion}

\subsubsection{Reactions with Pyrazine}

Using the optimized conditions determined in Chapter $2(0.75 \mathrm{mmol}$ of pyrazine, 1.5 equiv of arylboronic acid, 0.2 equiv of $\mathrm{FeSO}_{4} \cdot 6 \mathrm{H}_{2} \mathrm{O}, 2$ equiv of TFA, and 4 equiv of $\mathrm{K}_{2} \mathrm{~S}_{2} \mathrm{O}_{8}$, in $8 \mathrm{~mL}$ each of $\mathrm{CH}_{2} \mathrm{Cl}_{2}$ and water, heated to $70{ }^{\circ} \mathrm{C}$ for 25 min under microwave heating), substrate scope was examined by screening arylboronic acids with a variety of functional groups with pyrazine, beginning with alkyl-substituted arylboronic acids. Reactions using phenylboronic acid and 3-methylphenylboronic acid gave similar yields to 4-methylphenylboronic acid (Scheme 3.2). 2-Methylphenylboronic acid gave a 
substantially lower yield, while only a trace of mesitylboronic acid product was detected (trace meaning that the product was detectable via GC-MS, but could not be isolated). These diminished yields are most likely due to steric hindrance. Previous literature reports concerning the effects of steric hindrance are mixed, with several groups using other $\mathrm{N}$ heterocycles reporting significant decreases in yield from the inclusion of a 2-methyl substituent ${ }^{1,3,8}$, and others reporting little or no effect. ${ }^{9,15}$ The only other literature report that examined substrate scope with pyrazine did not attempt a reaction using 2-methylphenylboronic acid, but saw only a slight decrease in yield (from $86 \%$ to $78 \%$ ) when using 2-ethylphenylboronic acid. ${ }^{9}$

Arylboronic acids with a variety of electron-withdrawing groups were then screened, beginning with the weakly electron-withdrawing halogens. In all cases yields were substantially diminished, with yields between $21 \%$ using 2-chlorophenylboronic acid and 39\% using 3-chlorophenylboronic acid. Literature reports are again mixed, with several groups reporting dramatically lower yields when a halide is present when using other $\mathrm{N}$ heterocycles ${ }^{1,3,15}$, while others report little or no difference. ${ }^{7,8}$ The only other group to examine substrate scope using pyrazine reported yields of $76 \%$ with 4-chlorophenylboronic acid, and $65 \%$ with 3 -chlorophenylboronic acid, only slightly lower than the $80 \%$ yield they reported with phenylboronic acid. ${ }^{9}$ Under microwave heating strongly electronwithdrawing groups at the para position, including cyano, trifluoromethyl, and formyl groups gave only trace yields, while the inclusion of a carboxyl group gave a $0 \%$ yield, meaning that no product could be detected in the GC-MS of the crude reaction mixture. The presence of stronger electron-withdrawing groups generally reduced yields in the 
literature as well. Several groups have reported successful reactions with trifluoromethyl or ester groups, albeit with diminished yields, while others report no conversion. ${ }^{1,3,6,8}$ One group has reported the successful cross-coupling of 4-trifluoromethylphenylboronic acid with pyrazine in $30 \%$ yield; this is the only successful example of cross-coupling between pyrazine and an arylboronic acid bearing a strongly electron-withdrawing group. ${ }^{69}$

Arylboronic acids with moderate or strong electron-donating groups were then screened. The moderately electron-donating 4-methoxycarbonyl group resulted in a trace yield, while strongly electron-donating hydroxy and amine groups returned a $0 \%$ yield. The 3-aminophenylboronic acid immediately began forming a black solid upon addition, which made up the majority of the product of the reaction. This is most likely due to the formation of a polymer with a polyaniline backbone, as the chemical formation of polyaniline may take place under similar conditions ${ }^{26}$ The reaction with 4-hydroxyphenylboronic acid also led to the formation of a black solid, this time after microwave irradiation. The black solid could not be identified, and GC-MS performed on the crude reaction mixture showed not only no product, but no starting material and none of the products from previously observed side reactions. Instead, the only compound observed was phenol. It is not clear whether phenol is formed simply by the removal of the boronic acid moiety, or by the loss of the hydroxyl group followed by the replacement of the boronic acid moiety with a hydroxyl group. The fate of the pyrazine also remains unclear, as no pyrazine and no compounds containing pyrazine could be detected. There are no reports in the literature of successful coupling with amine or hydroxy substituted arylboronic acids. 


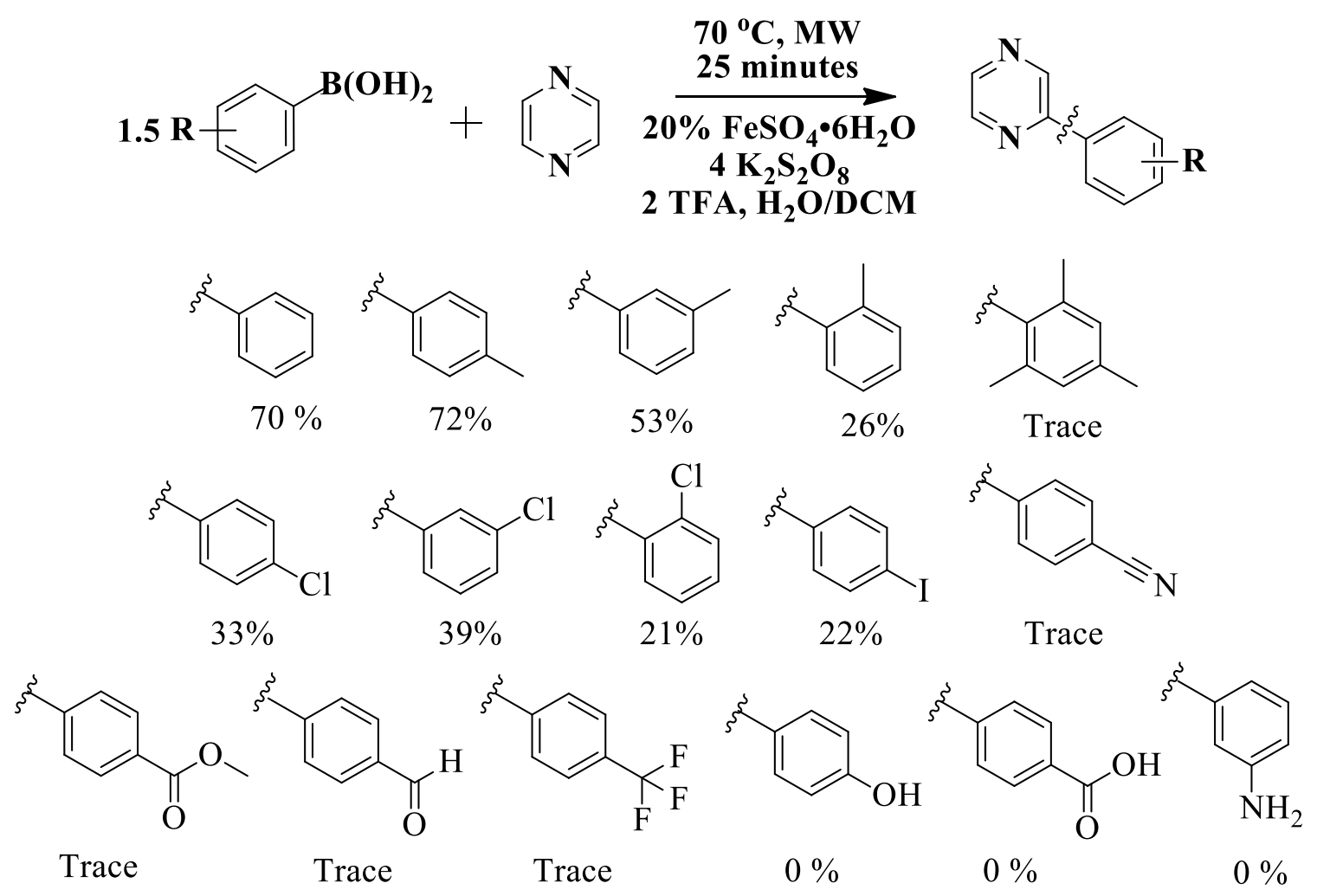

Scheme 3.2 Yields of cross-coupling with pyrazine using a variety of arylboronic acids under standard conditions. "Trace" indicates that product was visible in the GC-MS of the crude reaction mixture, but the product could not be isolated. $0 \%$ indicates that there was no product visible in the GC-MS of the crude reaction mixture.

Overall, although the literature is somewhat contradictory, substrate scope under microwave heating appears to be somewhat limited, with lower yields than most reports with halide substituents, and no yield with stronger electron-withdrawing or electrondonating substituents. This was surprising, given that an expanded substrate scope is often observed under microwave heating. In order to determine the cause of the reduced substrate scope, GC-MS was performed on the crude reaction mixtures. Typical examples, showing the GC trace from the crude reaction mixtures of reactions with 4-trifluoromethylphenylboronic acid and 4-chlorophenylboronic acid, are presented in 
Figure 3.2. Both show a similar result; while the intensity of the peaks from the cross-coupled and homo-coupled products are diminished, the peak that is associated with arylboronic acid hydroxylation has grown dramatically. There is only one other report of the formation of phenol side products, and in that case they were observed only when no coupling partner was added to the reaction mixture. ${ }^{9}$ Most groups report a mixture of arylboronic acid starting material and the corresponding homo-coupled products during low-yielding or unsuccessful reaction. The formation of phenol side-products in this case appears to indicate that the reaction of the phenylboronic acid with either water or a hydroxyl radical is preferred over the desired homo-coupling under microwave heating. It is not clear why this reaction is preferred, but it may be due to the formation of superheated molecular radiators or heterogeneous hotspots in the aqueous phase. 


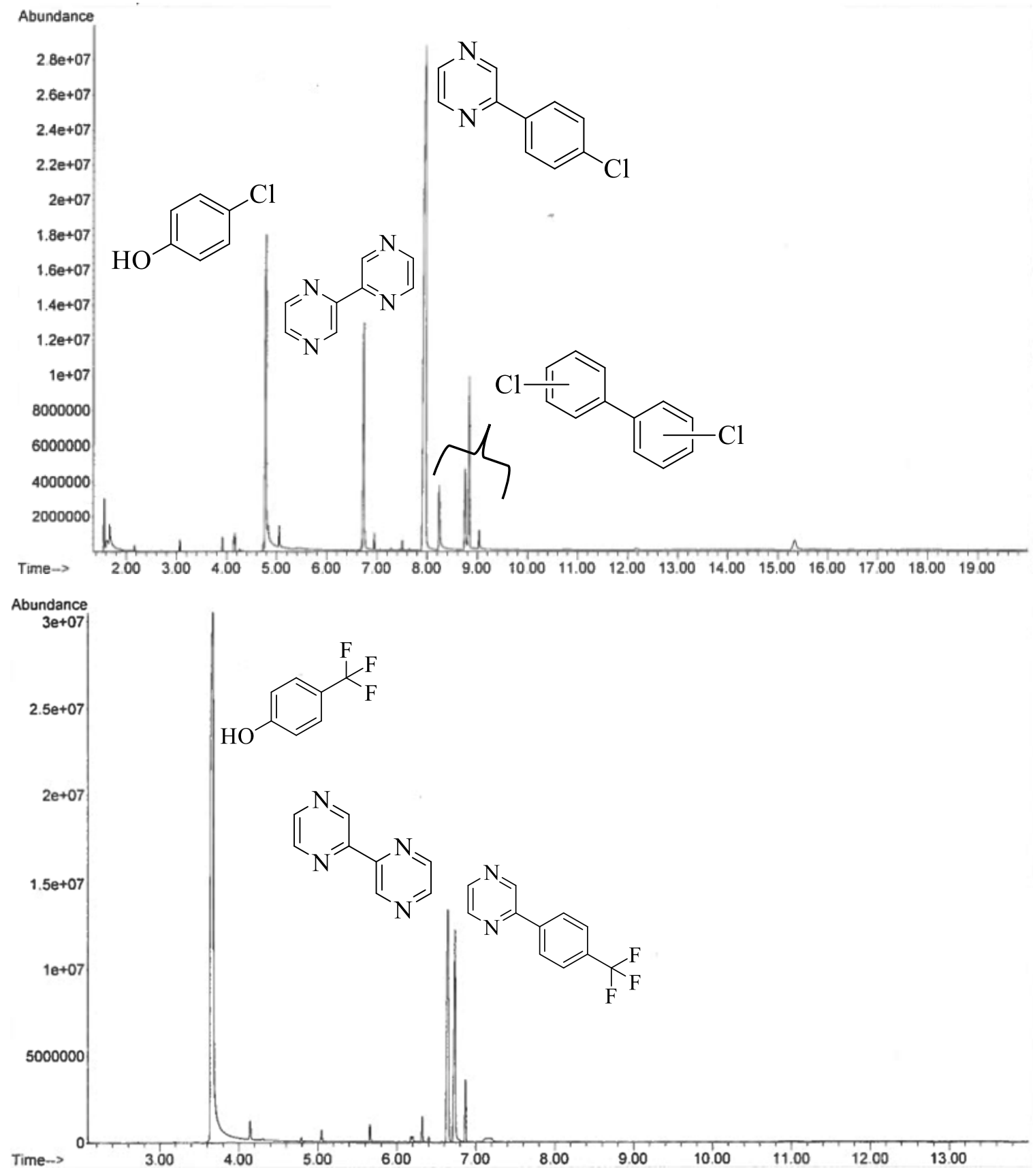

Figure 3.2 GC traces of crude reaction mixtures after attempted cross-coupling between 4-chlorophenylboronic acid and pyrazine (top), and 4 (trifluoromethyl)phenylboronic acid and pyrazine (bottom). 4-chlorophenol: $\mathrm{R}_{\mathrm{t}}=4.78 \mathrm{~min}$. Bipyrazine: $\mathrm{R}_{\mathrm{t}}=6.72 \mathrm{~min}$. 2-(4chlorophenyl)pyrazine: $\mathrm{R}_{\mathrm{t}}=8.00 \mathrm{~min}$. Isomers of $\mathrm{x}, \mathrm{x}^{\prime}$-dichlorobiphenyl: $\mathrm{R}_{\mathrm{t}}=8.24 \mathrm{~min}$, $8.75 \mathrm{~min}, 8.84 \mathrm{~min}$. 4-trifluorophenol: $\mathrm{R}_{\mathrm{t}}=3.67 \mathrm{~min}$. 2-(4-trifluoromethylphenyl)pyrazine: 
$\mathrm{R}_{\mathrm{t}}=6.63$ min. Bipyrazine: $\mathrm{R}_{\mathrm{t}}=6.72 \mathrm{~min}$. For further mass spectrometry information, consult Section 3.4.3 and/or Figure A120 and Figure A121.

Reoptimization was attempted with 4-chlorophenylboronic acid to determine if yields with functionalized arylboronic acids could be improved (Table 3.1). Reaction conditions including time, temperature, acid amount, and catalyst identity were examined. The use of $\mathrm{AgNO}_{3}$ and $\mathrm{CuSO}_{4}$ as catalysts decreased yields slightly, and a yield of $32 \%$ was obtained in both cases. The use of $\mathrm{BPh}_{3}$ and $\mathrm{Al}\left(\mathrm{NO}_{3}\right)_{3} \cdot 9 \mathrm{H}_{2} \mathrm{O}$ resulted in a larger decrease, to $16 \%$ and $24 \%$ respectively. Increasing reaction time from 25 to $50 \mathrm{~min}$ did not have a large effect on yield, while decreasing reaction time to 5 min resulted in only a $7 \%$ yield. Increasing the temperature to $120{ }^{\circ} \mathrm{C}$ decreased the yield to $26 \%$, while reducing it to $50{ }^{\circ} \mathrm{C}$ resulted in an even lower yield of only $13 \%$. Finally, increasing the amount of TFA to 4 equivalents resulted in a significantly decreased yield of only $21 \%$. 
Table 3.1 Reoptimization of conditions for the persulfate-initiated cross-coupling of pyrazine and 4-chlorophenylboronic acid.

\begin{tabular}{|c|c|c|c|c|c|}
\hline Entry & Catalyst & $\begin{array}{c}\text { Equiv of } \\
\text { TFA }\end{array}$ & Temperature $\left({ }^{\circ} \mathrm{C}\right)$ & $\begin{array}{l}\text { Time } \\
(\mathrm{min})\end{array}$ & $\begin{array}{c}\text { Yield } \\
(\%)\end{array}$ \\
\hline 1 & $\mathrm{FeSO}_{4} \cdot 6 \mathrm{H}_{2} \mathrm{O}$ & 2 & 70 & 25 & 39 \\
\hline $2^{\mathrm{a}}$ & $\mathrm{CuSO}_{4}$ & 2 & 70 & 25 & 32 \\
\hline $3^{a}$ & $\mathrm{Al}\left(\mathrm{NO}_{3}\right)_{3} \cdot 9 \mathrm{H}_{2} \mathrm{O}$ & 2 & 70 & 25 & 24 \\
\hline 4 & $\mathrm{BPh}_{3}$ & 2 & 70 & 25 & 16 \\
\hline 5 & $\mathrm{AgNO}_{3}$ & 2 & 70 & 25 & 32 \\
\hline $6^{\mathbf{a}}$ & $\mathrm{FeSO}_{4} \cdot 6 \mathrm{H}_{2} \mathrm{O}$ & 2 & 70 & 5 & 7 \\
\hline $7^{\mathbf{a}}$ & $\mathrm{FeSO}_{4} \cdot 6 \mathrm{H}_{2} \mathrm{O}$ & 2 & 70 & 50 & 38 \\
\hline 8 & $\mathrm{FeSO}_{4} \cdot 6 \mathrm{H}_{2} \mathrm{O}$ & 2 & 120 & 25 & 26 \\
\hline 9 & $\mathrm{FeSO}_{4} \cdot 6 \mathrm{H}_{2} \mathrm{O}$ & 2 & 50 & 25 & 13 \\
\hline 10 & $\mathrm{FeSO}_{4} \cdot 6 \mathrm{H}_{2} \mathrm{O}$ & 4 & 70 & 25 & 21 \\
\hline
\end{tabular}

Reactions were carried out as described in the general arylation procedure (See Section 3.4.2). Unless otherwise noted, the yields reported are of isolated product. ${ }^{\text {a }}$ Yield determined by ${ }^{1} \mathrm{H}$ NMR spectroscopy using an internal DMSO standard. 


\subsubsection{Reactions with Quinones and Enones}

The scope of the reaction under microwave heating was then examined with quinones. Initial reactions focused on the most basic quinone, 1,4-benzoquinone, and the scope of arylboronic acids (see Scheme 3.3). Reactions with phenylboronic acid yielded the desired 2-phenylquinone in $71 \%$ yield, comparable to the yields with pyrazine. This is

consistent with the literature, where yields range from $65 \%$ - 98\% (Table 3.2). ${ }^{2,3,7,9,15}$ Further, in the majority of cases with higher yields, spectroscopic yields are reported, not yields of isolated product. Amongst papers that reported the yield of isolated product, yields were between $65 \%$ and $91 \%$, with most reports between $70 \%$ and $90 \%$. Finally, the use of microwave heating remained significantly faster than reactions under conventional or no heating, with maximum yields still achieved after only $25 \mathrm{~min}$. In comparison, the minimum reaction time reported in the literature was $6 \mathrm{~h}$, an increase in rate of almost 15 times. Most reactions with iron catalysts required 12 - 24 h, 29 to 58 times slower than reactions under microwave heating. 
Table 3.2 Comparison of reaction conditions and yields of the radical coupling of 1,4benzoquinone and phenylboronic acid.

\begin{tabular}{cccccc}
\hline Entry & Source & Time (h) & $\begin{array}{c}\text { Temperature } \\
\left({ }^{\circ} \mathrm{C}\right)\end{array}$ & Catalyst & Yield (\%) \\
& & & & & \\
\hline 1 & This work & 0.4 & $70(\mathrm{MW})$ & $\mathrm{FeSO}_{4} \cdot 6 \mathrm{H}_{2} \mathrm{O}$ & 71 \\
2 & 2 & 6 & $\mathrm{RT}$ & $\mathrm{AgNO}_{3}$ & 91 \\
3 & 3 & 12 & 70 & $\mathrm{Fe}\left(\mathrm{NO}_{3}\right)_{3} \cdot 9\left(\mathrm{H}_{2} \mathrm{O}\right)$ & 89 \\
4 & 7 & 4 & 50 & $\mathrm{FeSO}_{4}$ & 65 \\
5 & 9 & 12 & $\mathrm{RT}$ & $\mathrm{Fe}(\mathrm{acac})_{2}$ & 90 \\
6 & 15 & 24 & $\mathrm{RT}$ & $\mathrm{FeS}$ & 82 \\
\hline
\end{tabular}

The inclusion of a weakly electron-donating methyl group at the 4 position decreased the yield to $56 \%$, while the more sterically hindered 2-methyl product was obtained in $41 \%$ yield. The inclusion of an electron-withdrawing chloride increased yield at the 4 position increased the yield to $76 \%$, but the inclusion of a chloride at the 3 and 2 position resulted in decreased yields of $49 \%$ and $45 \%$ respectively. Literature reports with alkyl-substituted phenylboronic acids are mixed, with some groups reporting similar yields with alkyl substituents ${ }^{3,7,9}$, and others reporting a modest decline. ${ }^{2}$ Altering the location of the alkyl group also led to mixed results; some groups reported signifcant declines with the alkyl group at the 2 or 3 position ${ }^{2,7}$, and others reported little difference or even improved 
yields. ${ }^{3,115}$ Yields with halide substituents are generally similar to those with unsubstituted phenylboronic acid. ${ }^{2,3,715}$ Literature reports on the effect of chloride position vary, with some reporting highest yields at the 3 position ${ }^{15}$, and others reporting the highest yield at the 2 position. ${ }^{7}$ The more strongly electron-withdrawing trifluoromethyl group lowered yields dramatically, to only $10 \%$, while the sterically hindered mesityl group resulted in a yield of $9 \%$. This is the only reported coupling with mesitylboronic acid, and the only succesful iron-catalyzed coupling with 4-trifluoromethylphenylboronic acid. Results with other sterically hindered arylboronic acids are mixed, with some groups reporting significant reductions in yield ${ }^{2,7}$ and others reporting little or no change. ${ }^{2,3}$ The only other iron-catalyzed reaction with 4-trifluoromethylphenylboronic acid was unsuccesful, and returned only starting material. ${ }^{7}$ Reactions reported in the literature with 3trifluoromethylphenylboronic acid, or arylboronic acids bearing other strong electronwithdrawing groups, generally have low yields, or are entirely unsuccessful. ${ }^{8,9}$ The presence of a strongly electron-donating hydroxyl group resulted in $0 \%$ yield, with no trace product detectable in the GC-MS of the crude reaction mixture. There are no reports of succesful coupling with arylboronic acids bearing hydroxyl groups. 
<smiles>O=C1C=CC(=O)C=C1</smiles>
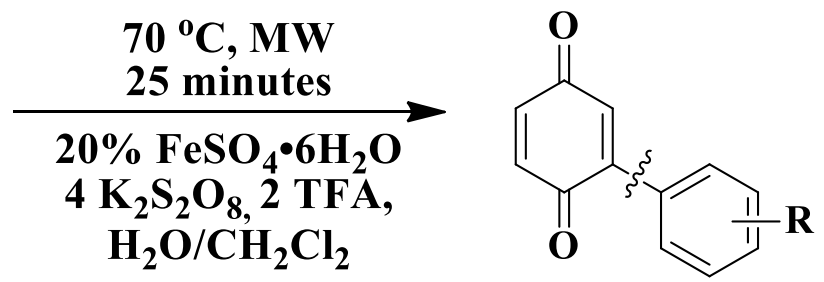<smiles>CCc1ccccc1</smiles>

$71 \%$<smiles>CCc1ccccc1Cl</smiles>

$45 \%$

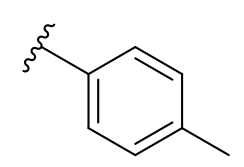

$58 \%$<smiles>CCc1c(C)cc(C)cc1C</smiles>

$9 \%$<smiles>CCc1ccccc1C</smiles>

$41 \%$<smiles>CCc1ccc(C(F)(F)F)cc1</smiles>

$10 \%$<smiles>CCc1ccc(Cl)cc1</smiles>

$76 \%$<smiles>Cc1ccc(O)cc1</smiles>

$0 \%$<smiles>CCc1cccc(Cl)c1</smiles>

$49 \%$

Scheme 3.3 Yields of cross-coupling with 1,4-benzoquinone using a variety of arylboronic acids under standard conditions.

The identity and abundance of side products was then examined via GC-MS of the crude reaction mixture (Figure 3.3). The side products in the reaction with 1,4-benzoquinone are similar to those observed with pyrazine, except that no quinone homo-coupling was observed. Instead, some unreacted 1,4-benzoquinone starting material remains. In addition, there is a relatively large amount of bis-coupled product, either 2,5-diphenyl-1,4-benzoquinone or 2,6-diphenyl-1,4-benzoquinone. Most groups report the presence of some bis-coupled product, although there are several reports in which no bis-coupling is reported. Curiously, although reports of boronic acid homo-coupling are common when using heteroarenes, there are no reports of homo-coupling when using quinones. This may be due to the relatively high yields obtained with most quinones, or 
due to limited examination of side products with quinones (most papers involving quinones are very short communications, and focus on reaction scope). The formation of phenol, most likely via reaction of the arylboronic acid with water or a hydroxyl radical, has not been previously observed. 


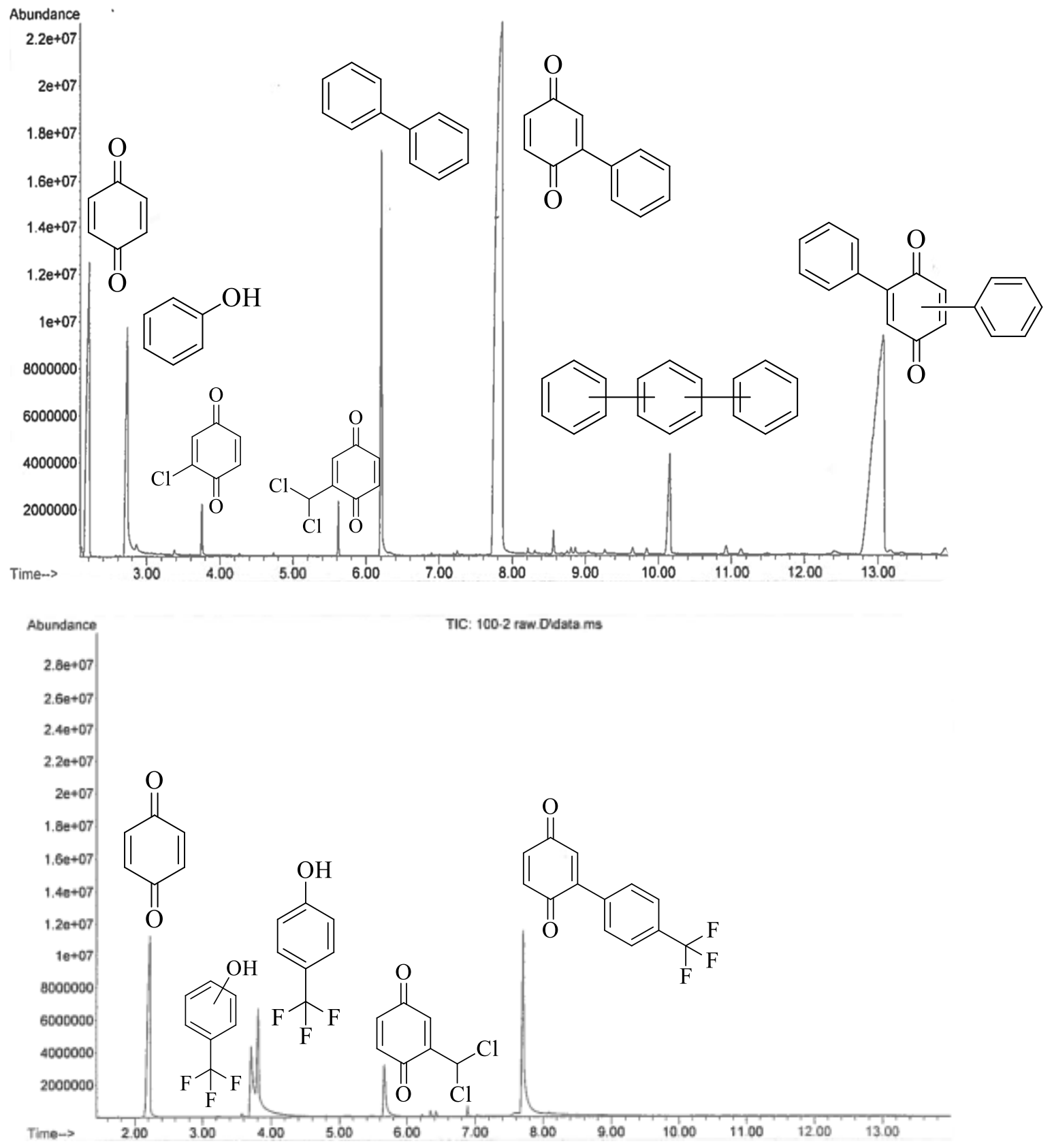

Figure 3.3 GC traces of crude reaction mixtures after attempted cross-coupling between phenylboronic acid and 1,4-benzoquinone (top), and 4 (trifluoromethyl)phenylboronic acid and 1,4-benzoquinone (bottom). Quinone: $R_{t}=2.22 \mathrm{~min}$. Phenol: $R_{t}=2.73 \mathrm{~min}$. 2-chloroquinone: $\mathrm{R}_{\mathrm{t}}=3.75 \mathrm{~min}$. 2-dichloromethylquinone: $\mathrm{R}_{\mathrm{t}}=5.67 \mathrm{~min}$.

Biphenyl: $R_{t}=6.21$ min. 2-phenyl-1,4-benzoquinone: $R_{t}=7.82$ min. Terphenyl (unknown isomer): $R_{t}=10.16$-13.09 min. 2,5-diphenylquinone or 2,6-diphenylquinone: $R_{t}=12.82$ 13.09 min. 4-trifluorophenol: $R_{t}=3.67$ min. Trifluorophenol, isomer unknown: $R_{t}=$ 3.82 min. 2-dichloromethylquinone: $\mathrm{R}_{\mathrm{t}}=5.67 \mathrm{~min}$. 2-(4-trifluoromethylphenyl)-1,4-

135 
benzoquinone: $R_{t}=7.70$ min. For further mass spectrometry information, consult Section 3.4.3 and/or figures Figure A122 and Figure A123.

The scope of the reaction with regards to hydroquinones, enones, phenols, and conjugated carboxylic acids was then investigated (Scheme 3.4). The one-pot oxidation and cross-coupling of 1,4-hydroquinone was successful, with only a small decline in yield when compared to reactions with 1,4-benzoquinone. This is somewhat unusual, as reactions with 1,4-hydroquinone usually gives similar or slightly higher yields than 1,4-benzoquinone. ${ }^{3,7,15}$ Reactions with 1,2-hydroquinone (catechol) were unsuccessful, perhaps due to the reduced stability of the 1,2-benzoquinone moiety. There are no reports of cross-coupling of 1,2-benzoquinone or catechol, although there is one report of crosscoupling with 1,2-naphthoquinone. ${ }^{2}$ Reactions with 2,3-dimethyl-1,4-hydroquinone were successful, but gave lower yields than the unsubstituted 1,4-hydroquinone. This is only the second reported reaction of a substituted hydroquinone. ${ }^{7}$ Reactions with phenol were performed using both TFA and with 3 equiv of $\mathrm{K}_{2} \mathrm{CO}_{3}$, as literature reports required a base for the reaction to proceed. ${ }^{4}$ In both cases, reactions failed under microwave heating and the phenol remained unreacted. Attempts to extend the reaction scope to other enones and a conjugated carboxylic acid were all unsuccessful, and all remained unreacted. There are no reports demonstrating the cross-coupling of these species under conventional heating. 
1.5<smiles>Oc1ccccc1</smiles>
Quinone
or Enone

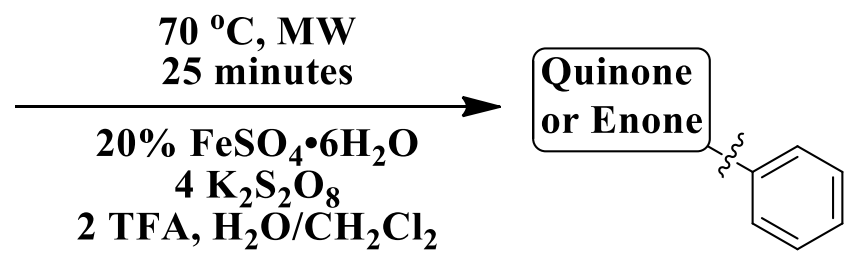<smiles>O=C1C=CC(=O)C=C1</smiles><smiles>Oc1ccc(O)cc1</smiles><smiles>Cc1c(O)ccc(O)c1C</smiles><smiles>Oc1ccccc1O</smiles><smiles>Oc1ccccc1</smiles>
$71 \%$ $61 \%$ $32 \%^{\mathrm{a}}$

$0 \%$

$0 \%$ b<smiles>O=C(/C=C/c1ccccc1)/C=C/c1ccccc1</smiles><smiles>CC(=O)/C=C/c1ccccc1</smiles><smiles>C/C=C/C(=O)O</smiles>

Scheme 3.4 Yields of cross-coupling with 1,4-benzoquinone using a variety of arylboronic acids under standard conditions. $0 \%$ indicates that product could not be isolated, and there was no product visible in the GC-MS of the crude reaction mixture. ${ }^{a}$ The product, 5-phenyl-2,3-dimethyl-1,4-benzoquinone, was collected as a mixture with 2,3-dimethyl-1,4-benzoquinone. Yield was determined via comparison of the integration of the respective methyl peaks in the ${ }^{1} \mathrm{H}$ NMR. ${ }^{b}$ Reactions performed with TFA and with 3 equiv of $\mathrm{K}_{2} \mathrm{CO}_{3}$.

\subsubsection{Reactions with Other Heteroaryls}

A variety of alternative $N$-heteroaryls were then screened in reactions with phenylboronic acid (Scheme 3.5), beginning with 4-cyanopyridine, also known as 4-pyridinecarbonitrile. 4-cyanopyridine was chosen because it is common in the literature due to the strongly electron-withdrawing cyano group, which appears to increase reactivity. The reaction still reached completion in only $25 \mathrm{~min}$, compared to 12 to $48 \mathrm{~h}$ 
reaction times reported in the literature (Table 3.3). A total yield of $64 \%$ was achieved, with $49 \%$ of the 2-phenyl product and the remainder 3-phenyl for a ratio of 2:3 product of 3.2. Most literature reports have somewhat higher yields, ranging from 68 to $92 \%$. Based on the GC-MS of the reaction mixture the lower yield is probably attributable to the formation of the phenol side-product. Oddly, $N$-heteroarene homo-coupling does not appear to occur in this case. In fact, no homocoupling was observed with any $N$ heteroarene other than pyrazine, including the pyrazine isomer pyridazine. Why this is the case is unclear. Reports on regiochemistry vary, as the ratio of 2 to 3 products varies from 2:1 to 5:1. Microwave heating therefore does not appear to alter the regiochemical outcome in this case. Preference for the 2 position is mostly due to its electron-deficient nature, and may be aided in this case by steric hindrance at the 3-position caused by the cyano group. 
1.5<smiles>Oc1ccccc1</smiles>
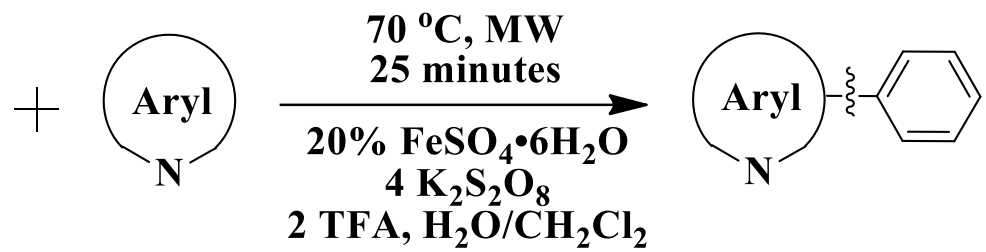<smiles></smiles>

2-Ph: $49 \%$

3-Ph: $15 \%$<smiles>c1ccc2ncccc2c1</smiles>

2-Ph: $41 \%$

4-Ph: $21 \%$<smiles>Cc1cccc(C)n1</smiles>

3-Ph: $12 \%$<smiles>c1ccncc1</smiles>

2-Ph: $43 \%$

4-Ph: $22 \%$<smiles>c1ccnnc1</smiles>

3-Ph: $32 \%$<smiles>CN(C)c1ccncc1</smiles><smiles>CCC(C)(C)C(C)(Br)Br</smiles><smiles>Brc1cccc(Br)n1</smiles><smiles>CC(=O)c1cccc(C(C)=O)n1</smiles><smiles>OCc1cccc(CO)n1</smiles><smiles></smiles><smiles>c1cn[nH]c1</smiles><smiles>Cc1cc(C)[nH]n1</smiles>

$0 \%$<smiles>c1c[nH]cn1</smiles><smiles>c1ccc2[nH]ccc2c1</smiles>

$0 \%$<smiles>Cn1ccnc1</smiles>

2-Ph: $26 \%$<smiles>c1ccc(-n2cccc2)cc1</smiles>

$0 \%$

Scheme 3.5 Yields of cross-coupling of arylboronic acid with a variety of $N$-heterocycles under standard conditions. "Trace" indicates that product was visible in the GC-MS of the crude reaction mixture, but the product could not be isolated. $0 \%$ indicates that there was no product visible in the GC-MS of the crude reaction mixture. ${ }^{\text {a }}$ Reaction performed without TFA. 
Table 3.3 Comparison of conditions, yields, and regiochemistry in the cross-coupling of phenylboronic acid and 4-cyanopyridine.

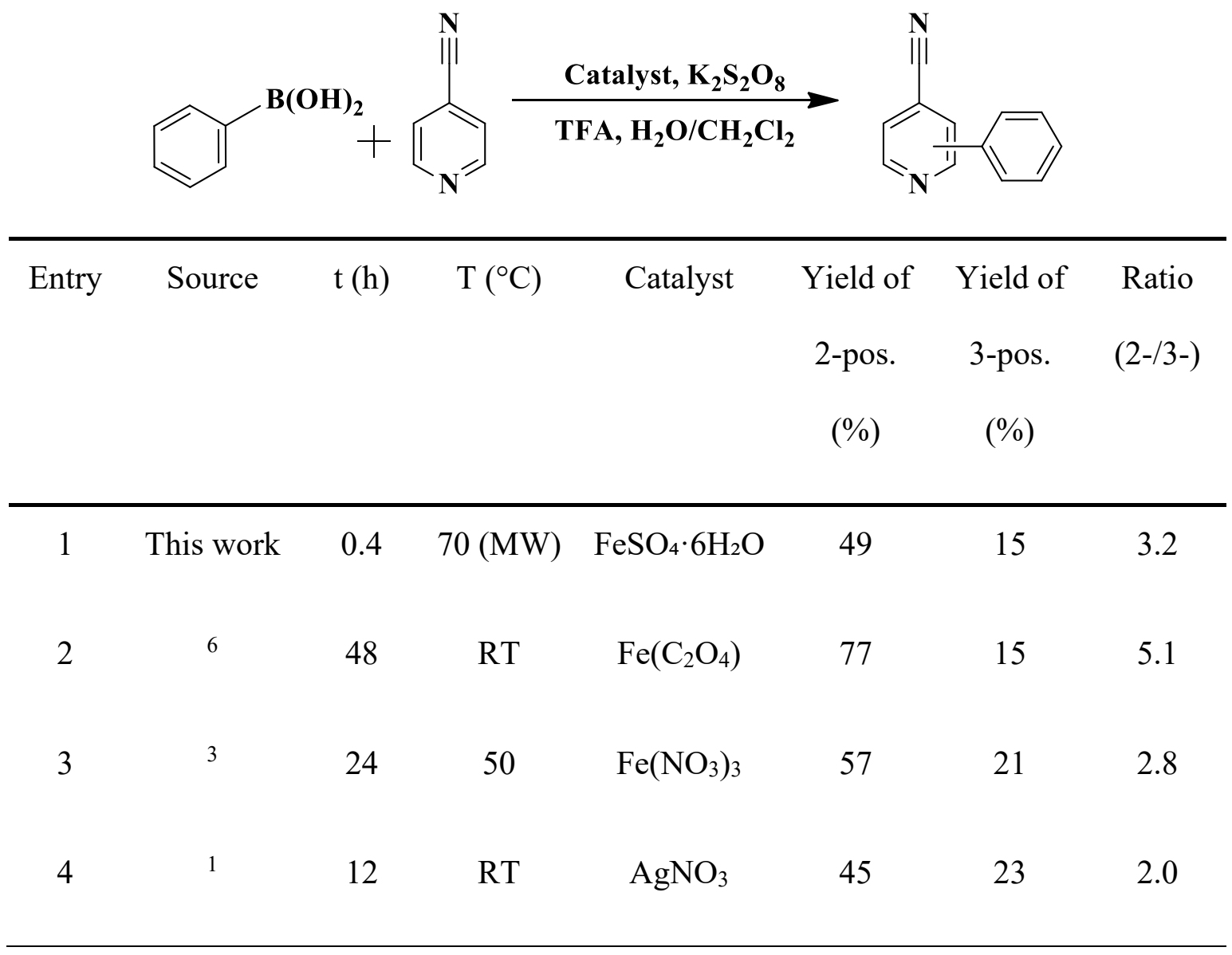

Reactions were then performed with pyridine (Table 3.4). As with 4-cyanophenylpyridine the reaction was completed within the standard $25 \mathrm{~min}$, with a total yield of $65 \%$. A mixture of 2 and 4-phenyl products were obtained, in $43 \%$ and $22 \%$ yield respectively. No 3 product was observed. The total reaction yield is among the higher yields reported in the literature, which range from $30-73 \%$. Again, the regiochemistry of the reaction was similar to other literature reports, with a ratio of $2: 1$ of 2 vs 4-position products, although one article did not mention the presence of any 4-phenyl product. This 
distribution is purely statistical, since there are twice as many 2-positions available to react as 4-positions.

Table 3.4 Comparison of conditions, yields, and regiochemistry in the cross-coupling of phenylboronic acid and pyridine.

\begin{tabular}{|c|c|c|c|c|c|c|c|}
\hline Entry & Source & $\mathrm{t}(\mathrm{h})$ & $\mathrm{T}\left({ }^{\circ} \mathrm{C}\right)$ & Catalyst & $\begin{array}{c}\text { Yield of } \\
\text { 2-pos. } \\
(\%)\end{array}$ & $\begin{array}{c}\text { Yield of } \\
\text { 4-pos. } \\
(\%)\end{array}$ & $\begin{array}{l}\text { Ratio } \\
(2-/ 4-)\end{array}$ \\
\hline 1 & This work & 0.4 & $70(\mathrm{MW})$ & $\mathrm{FeSO}_{4} \cdot 6 \mathrm{H}_{2} \mathrm{O}$ & 43 & 22 & 2.0 \\
\hline 2 & 6 & 48 & RT & $\mathrm{Fe}\left(\mathrm{C}_{2} \mathrm{O}_{4}\right)$ & 18 & 12 & 1.5 \\
\hline 3 & 3 & 24 & RT & $\mathrm{Fe}(\mathrm{acac})_{2}$ & 49 & 24 & 2.0 \\
\hline 4 & 3,9 & 12 & 50 & $\mathrm{Fe}(\mathrm{acac})_{2}$ & 45 & 0 & $\mathrm{~N} / \mathrm{A}$ \\
\hline 5 & 15 & 40 & RT & $\mathrm{FeS}$ & 20 & 14 & 1.4 \\
\hline 6 & 1 & 6 & RT & $\mathrm{AgNO}_{3}$ & 45 & 23 & 2.0 \\
\hline
\end{tabular}


Reactions performed with quinoline reached a total yield of $67 \%$, with $41 \% 2$ phenyl product and 21\% 4-phenyl product (Table 3.5). This is a somewhat higher yield than most literature reports, and also a higher ratio of 2-product to 4-product. Reactions with pyridazine reached a yield of $68 \%$, comparable to the only other literature report (Table 3.6). However, the regiochemical outcome was significantly different, as nearly equal amounts of 3-phenyl and 4-phenyl products were produced under microwave heating, whereas the literature reports a higher yield of 4-phenyl product. This difference may be due to the influence of microwave heating, but may also be due to the use of an iron catalyst, as the only literature report used a silver catalyst.

Table 3.5 Comparison of conditions, yields, and regiochemistry in the cross-coupling of phenylboronic acid and quinoline.

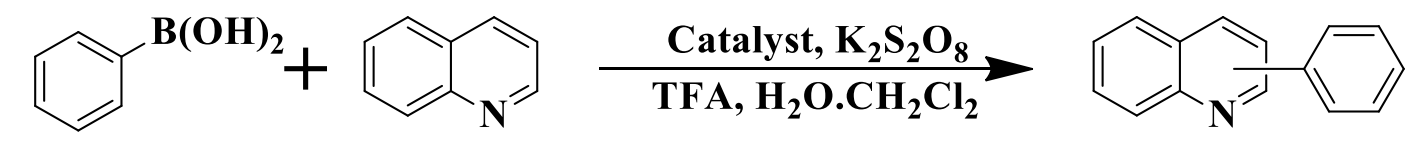

\begin{tabular}{|c|c|c|c|c|c|c|c|}
\hline Entry & Source & $\mathrm{t}(\mathrm{h})$ & $\mathrm{T}\left({ }^{\circ} \mathrm{C}\right)$ & Catalyst & $\begin{array}{c}\text { Yield of } \\
\text { 2-pos. } \\
(\%)\end{array}$ & $\begin{array}{c}\text { Yield of } \\
\text { 4-pos. } \\
(\%)\end{array}$ & $\begin{array}{l}\text { Ratio } \\
(4-/ 2-)\end{array}$ \\
\hline 1 & This work & 0.4 & $70(\mathrm{MW})$ & $\mathrm{FeSO}_{4} \cdot 6 \mathrm{H}_{2} \mathrm{O}$ & 41 & 21 & 2.0 \\
\hline 2 & 15 & 40 & RT & $\mathrm{FeS}$ & 29 & 21 & 1.4 \\
\hline 4 & 1 & 12 & RT & $\mathrm{AgNO}_{3}$ & 41 & 20 & 2.0 \\
\hline
\end{tabular}


Table 3.6 Comparison of conditions, yields, and regiochemistry in the cross-coupling of phenylboronic acid and pyridazine.

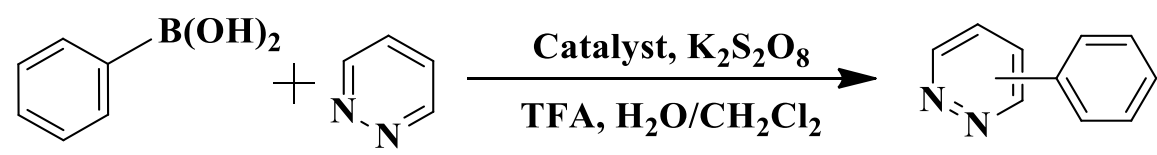

\begin{tabular}{|c|c|c|c|c|c|c|c|}
\hline Entry & Source & $\mathrm{t}(\mathrm{h})$ & $\mathrm{T}\left({ }^{\circ} \mathrm{C}\right)$ & Catalyst & $\begin{array}{c}\text { Yield of } \\
\text { 4-pos. } \\
(\%)\end{array}$ & $\begin{array}{c}\text { Yield of } \\
\text { 3-pos. } \\
(\%)\end{array}$ & $\begin{array}{l}\text { Ratio } \\
\text { (4-/3-) }\end{array}$ \\
\hline 1 & $\begin{array}{l}\text { This } \\
\text { work }\end{array}$ & 0.4 & $70(\mathrm{MW})$ & $\mathrm{FeSO}_{4} \cdot 6 \mathrm{H}_{2} \mathrm{O}$ & 36 & 32 & 1.1 \\
\hline 2 & 1 & 12 & $\mathrm{RT}$ & $\mathrm{AgNO}_{3}$ & 44 & 15 & 2.9 \\
\hline
\end{tabular}

The reaction scope was then examined with several other substituted pyridines (Scheme 3.5). Reactions with lutidine (2,6-dimethylpyridine) were successful, with a total yield of $45 \%$ (12\% 3-phenyl, 33\% 4-phenyl). To the best of my knowledge, this is the first reported reaction with a 2,6-substituted pyridine, although there have been reports of reaction with 2-substituted quinolines. ${ }^{3}$ Attempts to couple 2,6-dibromopyridine, 2,6-diacetylpyridine, and 2,6-pyridinedimethanol were unsuccessful, with the $\mathrm{N}$ heteroarene remaining unreacted. Reactions with 2-bromopyridine returned only a trace of product in this case. Reactions with dimethylaminopyridine (DMAP), which has a strong electron-donating group, were unsuccessful. Reactions with pyridine $N$-oxide were unsuccessful under acidic conditions, but returned a trace amount of product when no TFA 
was used. The majority of the product formed was at the 2-position, with a minority at the 4-position. In comparison, most literature reports observe only the formation of the 2position product.

Finally, reactions were attempted with a variety of 5-membered rings. Reactions with 5-membered rings with an $\mathrm{N}$-H bond, including imidazole, pyrazole, and pyrrole rings were unsuccessful, and the $N$-heteroarene remained unreacted. This is consistent with most literature reports, although there are some reports of low-yielding reactions with imidazole ${ }^{1}$ and L-histidine ${ }^{11}$ (which possesses an imidazole ring) using a silver catalyst. There have been no reports of successful coupling with five-membered rings with an $\mathrm{N}$-H bond using iron catalysts. Reactions with 1-methylimidazole were successful, albeit in low yield (23\%), while reactions with 1-phenylpyrrole were unsuccessful. All other iron-catalyzed reactions to five-membered rings have required a thiazole ring system.

\subsubsection{Other Reactions}

In addition to the previously described reactions with $N$-heteroarenes and quinones, several other substrates were examined, and several other reaction conditions. First, several reactions with reagents that were liquid or possessed a low boiling point were attempted with the reagent serving as the organic solvent in place of dichloromethane. It was speculated that the increased availability of coupling partner might lead to a reduction in arylboronic acid homo-coupling or hydroxylation, and therefore an increased yield. Species tested included pyridine, lutidine, quinoline, and thiophene. Unfortunately, no product was 
observed in any of these reactions. The reasons for the failure of these reactions are unclear but may be due to decreased solubility of arylboronic acids in these solvents, or a side reaction, as an unidentifiable black solid was often present after microwaving. There are no literature reports of reactions occurring under similar conditions.

Several attempts were also made to extend the reaction scope beyond quinones and $N$-heteroarenes. Reactions were attempted with pyrrole, a non-aromatic $N$-heterocycle, and $N$-benzylidenbenzylamine, a conjugated imine, but were unsuccessful. Reactions with the S-heteroarene thiophene yielded only a trace amount of product, while reactions with cyclohexane, cyclohexene, cyclohexanone, styrene, and benzene were completely unsuccessful. GC-MS of the crude reaction mixtures showed that the phenylboronic acid was being homo-coupled and hydroxylated, while the coupling partner remained unreacted. There are no reports of any of these reagents undergoing cross-coupling under similar conditions.

Finally, based on the reactivity of toluene observed in Chapter 2, in which toluene was homo-coupled at the benzylic position, attempts were made to determine if a crossdehydrogenative coupling (CDC) reaction was possible if the reaction was performed without arylboronic acid. Since this reaction with pyrazine had shown only minimal cross-coupling, initial reactions focussed on the reaction of toluene and quinone. However, instead of coupling with the toluene, quinone served as an oxidant, yielding a mixture of bibenzyl, benzaldehyde, benzyl alcohol, and benzoic acid. Reactions with 4-cyanopyridine were then attempted, but yielded a large number of trace products, including benzyl-4-cyanopyridine, benzoyl-4-cyanopyridine, methylphenyl-4-cyanopyridine, 
formylphenyl-4-cyanopyridine, and 3- and 2-phenyl-4-cyanopyridine, (Scheme 3.6). Attempts to optimize this reaction, including attempting the reaction under conventional heating, were not performed due to time constraints.
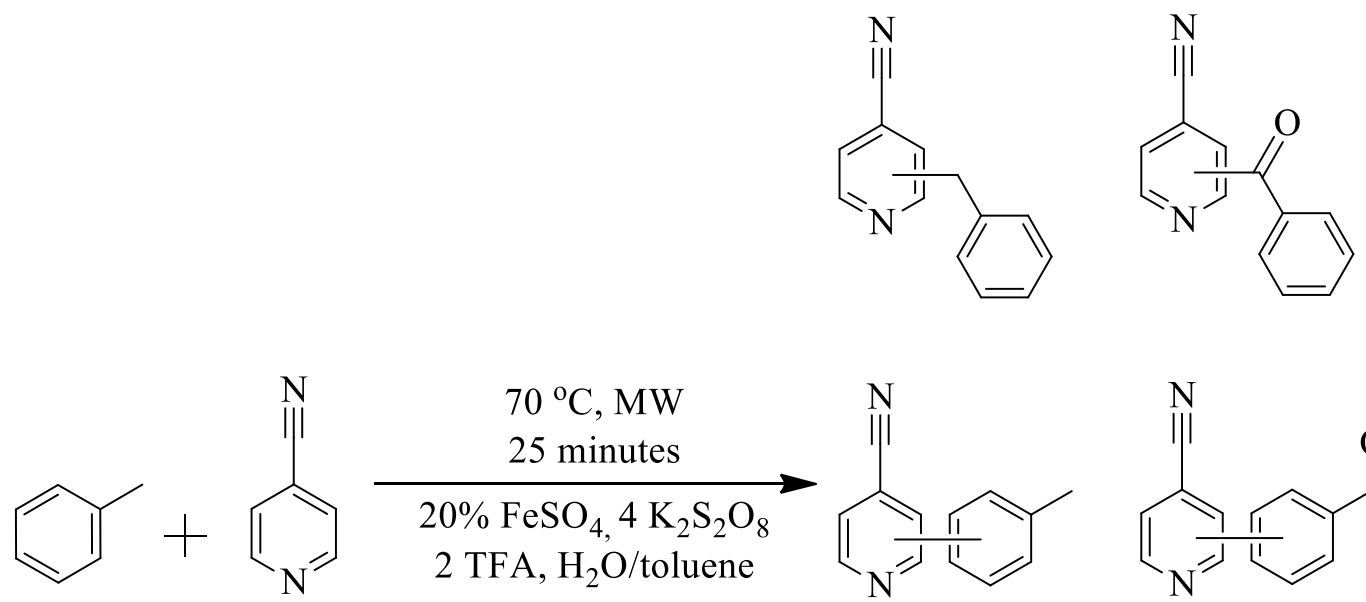<smiles>N#Cc1ccnc2ccc(C=O)cc12</smiles><smiles>N#Cc1ccnc(-c2ccccc2)c1</smiles><smiles>N#Cc1ccncc1-c1ccccc1</smiles>

Scheme 3.6 Iron-catalyzed radical cross-dehydrogenative coupling of toluene and 4 cyanopyridine under microwave heating. Consult Section 3.4.3.5 or Figure A126 for spectroscopic information.

\subsection{Conclusions}

The scope of the persulfate-initiated cross-coupling of $N$-heteroarenes or quinones with arylboronic acids was investigated under microwave heating. Arylboronic acid scope with $\mathrm{N}$-heteroarenes was found to be somewhat limited, as arylboronic acids bearing sterically hindering, electron-withdrawing, or electron-donating groups were coupled with only trace 
yields. The cause of these low yields was determined to be a side reaction in which arylboronic acids were hydroxylated to form the corresponding phenol. Additional attempts to reoptimize the reaction proved unsuccessful. This side reaction had only been observed previously when no coupling partner was present, and may be due to the formation of superheated molecular radiators or heterogeneous hotspots. Reaction scope with arylboronic acids was improved with quinone, but yields with stronger electron-donating, electron-withdrawing, or highly sterically hindered groups remained poor. Quinones and hydroquinones were coupled successfully, but attempts to extend the reaction scope to include phenols, other enones, and conjugated carboxylic acids failed. The scope of the reaction with other $N$-heteroarenes was examined. Broadly speaking, the scope proved to be similar to that under conventional heating, although this is the first report of successful coupling with 2,6-disubstituted pyridines, and reactions with $N$-oxides failed. Microwave heating did not appear to influence the regiochemistry of the reaction in most cases, as there were no major changes in regiochemistry except in the case of pyridazine and quinoline. It is unclear whether the difference in regiochemistry observed with pyridazine is due to the use of an iron catalyst instead of a silver catalyst, or due to microwave heating.

\subsection{Experimental}

\subsubsection{General Information}

Consult Section 2.3.1. 


\subsubsection{General Arylation Procedure}

Unless otherwise noted, reactions were performed and worked up as described in Section 2.3.2. In some cases, noted in the NMR descriptions, a Bruker AVANCE $500 \mathrm{MHz}$ NMR was used instead of a Bruker AVANCE $300 \mathrm{MHz}$ NMR. GC-MS information of trace products is reported, but NMR data could not be obtained due to the small amount of product obtained. Similarly, GC-MS data is reported for side products, but in most cases NMR data was not collected. Where possible, the identity of products identified by GCMS alone was confirmed by comparison to the literature, most notably the National Institute of Standards and Technology (NIST) Chemistry WebBook.

\subsubsection{Spectroscopic data}

\subsubsection{Spectroscopic Data of Phenylpyrazine Products}

2-Phenylpyrazine: Pale yellow solid, purified by flash chromatography using $10 \%$ ethyl acetate in hexane. ${ }^{1} \mathrm{H}$ NMR $\left(300 \mathrm{MHz}, \mathrm{CDCl}_{3}\right) \delta 9.03(\mathrm{~d}, J=1.7 \mathrm{~Hz}, 1 \mathrm{H}), 8.62(\mathrm{dd}, J=$ $2.5,1.5 \mathrm{~Hz}, 1 \mathrm{H}), 8.50(\mathrm{~d}, J=2.6 \mathrm{~Hz}, 1 \mathrm{H}), 8.05-7.98(\mathrm{~m}, 2 \mathrm{H}), 7.54-7.45(\mathrm{~m}, 3 \mathrm{H}) .{ }^{13} \mathrm{C}$ $\operatorname{NMR}\left(75 \mathrm{MHz}, \mathrm{CDCl}_{3}\right) \delta 152.85,144.19,142.89,142.20,136.33,129.93,129.06,126.95$. DEPT NMR (75 MHz, CDCl3) $\delta 144.18,142.90,142.22,129.92,129.05,126.95$. GC-MS: $\mathrm{R}_{\mathrm{t}}=5.32 \mathrm{~min} . \mathrm{m} / \mathrm{z}=156.1\left(\mathrm{M}^{+}\right), 129.1\left(\mathrm{M}^{+}-27\right), 103.1\left(\mathrm{M}^{+}-53\right), 76.1\left(\mathrm{M}^{+}-80\right)$.

2-(4-Methylphenyl)pyrazine: Pale yellow solid, purified by flash chromatography using $10 \%$ ethyl acetate in hexane. ${ }^{1} \mathrm{H}$ NMR $\left(300 \mathrm{MHz}, \mathrm{CDCl}_{3}\right) \delta 9.00(\mathrm{~d}, J=1.7 \mathrm{~Hz}, 1 \mathrm{H}), 8.60$ (dd, $J=2.5,1.6 \mathrm{~Hz}, 1 \mathrm{H}), 8.47(\mathrm{~d}, J=2.6 \mathrm{~Hz}, 1 \mathrm{H}), 7.95-7.88(\mathrm{~m}, 2 \mathrm{H}), 7.31(\mathrm{~d}, J=8.1 \mathrm{~Hz}$, 2H), $2.42(\mathrm{~s}, 3 \mathrm{H}) .{ }^{13} \mathrm{C} \mathrm{NMR}\left(75 \mathrm{MHz}, \mathrm{CDCl}_{3}\right) \delta 152.85,144.09,142.57,142.02,140.12$, 
133.57, 129.79, 126.81, 21.36. DEPT NMR (75 MHz, $\left.\mathrm{CDCl}_{3}\right) \delta 152.86$ (down), 144.09, $142.58,142.03,140.13$ (down), 133.57 (down), 129.80, 129.49, 129.00, 126.81, 21.36. GCMS: $\mathrm{R}_{\mathrm{t}}=7.47 \mathrm{~min} . \mathrm{m} / \mathrm{z}: 170.1\left(\mathrm{M}^{+}\right), 155.1\left(\mathrm{M}^{+}-15\right), 117.1\left(\mathrm{M}^{+}-53\right), 89.1\left(\mathrm{M}^{+}-81\right)$.

2-(3-Methylphenyl)pyrazine: Pale yellow solid, purified by flash chromatography using $10 \%$ ethyl acetate in hexane. ${ }^{1} \mathrm{H}$ NMR $\left(300 \mathrm{MHz}, \mathrm{CDCl}_{3}\right) \delta 9.01(\mathrm{~d}, J=1.7 \mathrm{~Hz}, 1 \mathrm{H}), 8.65$ $-8.54(\mathrm{~m}, 1 \mathrm{H}), 8.49(\mathrm{~d}, J=2.6 \mathrm{~Hz}, 1 \mathrm{H}), 7.88-7.74(\mathrm{~m}, 2 \mathrm{H}), 7.39$ (t, $J=7.6 \mathrm{~Hz}, 1 \mathrm{H}), 7.36$ $-7.24(\mathrm{~m}, 1 \mathrm{H}), 2.48-2.34(\mathrm{~m}, 3 \mathrm{H}) .{ }^{13} \mathrm{C} \mathrm{NMR}\left(75 \mathrm{MHz}, \mathrm{CDCl}_{3}\right) \delta 152.99,144.10,142.83$, 142.33, 138.81, 136.31, 130.70, 128.95, 127.64, 124.04, 21.52. DEPT NMR (75 MHz, $\left.\mathrm{CDCl}_{3}\right) \delta 152.99$ (down), 144.11, 142.83, 142.33, 138.82 (down), 136.31 (down), 130.70, 128.95, 127.64, 124.04, 21.52. GC-MS: $\mathrm{R}_{\mathrm{t}}=7.39 \mathrm{~min} . \mathrm{m} / \mathrm{z}: 170.1\left(\mathrm{M}^{+}\right), 155.1\left(\mathrm{M}^{+}-15\right)$, $117.1\left(\mathrm{M}^{+}-53\right), 89.1\left(\mathrm{M}^{+}-81\right)$.

2-(2-Methylphenyl)pyrazine: Pale yellow oil, purified by flash chromatography using $10 \%$ ethyl acetate in hexane. ${ }^{1} \mathrm{H}$ NMR $\left(500 \mathrm{MHz}, \mathrm{CDCl}_{3}\right) \delta 8.74(\mathrm{~s}, 1 \mathrm{H}), 8.69-8.63(\mathrm{~m}$, 1H), $8.57-8.47(\mathrm{~m}, 1 \mathrm{H}), 7.42(\mathrm{dd}, J=7.8,1.8 \mathrm{~Hz}, 1 \mathrm{H}), 7.38-7.29(\mathrm{~m}, 3 \mathrm{H}), 2.40(\mathrm{~s}, 3 \mathrm{H})$. ${ }^{13} \mathrm{C}$ NMR $\left(75 \mathrm{MHz}, \mathrm{CDCl}_{3}\right) \delta 155.71,145.12,143.87,142.46,136.75,136.31,131.11$, 129.83, 129.23, 126.19, 20.28. DEPT NMR (75 MHz, $\left.\mathrm{CDCl}_{3}\right) \delta 145.13,143.87,142.46$, 136.31 (down), 131.11, 129.83, 129.23, 126.19, 20.29. GC-MS: $\mathrm{R}_{\mathrm{t}}=7.39 \mathrm{~min} . \mathrm{m} / \mathrm{z}: 169.1$ $\left(\mathrm{M}^{+}\right), 155.1\left(\mathrm{M}^{+}-14\right), 117.1\left(\mathrm{M}^{+}-53\right), 89.1\left(\mathrm{M}^{+}-81\right)$.

2-(4-Chlorophenyl)pyrazine: Pale yellow solid. Purified by flash chromatography using $10 \%$ ethyl acetate in hexane. ${ }^{1} \mathrm{H}$ NMR $\left(300 \mathrm{MHz}, \mathrm{CDCl}_{3}\right) \delta 9.04-8.97(\mathrm{~m}, 1 \mathrm{H}), 8.66-$ $8.58(\mathrm{~m}, 1 \mathrm{H}), 8.52(\mathrm{~d}, J=2.6 \mathrm{~Hz}, 1 \mathrm{H}), 8.06-7.90(\mathrm{~m}, 2 \mathrm{H}), 7.53-7.40(\mathrm{~m}, 2 \mathrm{H}) .{ }^{13} \mathrm{C} \mathrm{NMR}$ (75 MHz, $\left.\mathrm{CDCl}_{3}\right) \delta 151.62,144.21,143.19,141.91,136.22,134.74,129.29,128.16$. DEPT 
NMR (75 MHz, $\left.\mathrm{CDCl}_{3}\right) \delta 151.62$ (down), 144.21, 143.19, 141.92, 136.23 (down), 134.74 (down), 129.29, 128.17. GC-MS: $\mathrm{R}_{\mathrm{t}}=8.00 \mathrm{~min} . \mathrm{m} / \mathrm{z}: 190.1\left(\mathrm{M}^{+}\right), 192.1\left(\mathrm{M}^{+}+2\right), 155.1\left(\mathrm{M}^{+}-\right.$ 35), $137.1\left(\mathrm{M}^{+}-53\right), 102.1\left(\mathrm{M}^{+}-88\right)$.

2-(3-Chlorophenyl)pyrazine: Pale yellow solid. Purified by flash chromatography using $10 \%$ ethyl acetate in hexane. ${ }^{1} \mathrm{H}$ NMR $\left(300 \mathrm{MHz}, \mathrm{CDCl}_{3}\right) \delta 9.02(\mathrm{~s}, 1 \mathrm{H}), 8.64(\mathrm{dd}, J=2.6$, $1.6 \mathrm{~Hz}, 1 \mathrm{H}), 8.55(\mathrm{~d}, J=2.6 \mathrm{~Hz}, 1 \mathrm{H}), 8.08-7.99(\mathrm{~m}, 1 \mathrm{H}), 7.95-7.82(\mathrm{~m}, 1 \mathrm{H}), 7.51-7.35$ (m, 2H). ${ }^{13} \mathrm{C}$ NMR $\left(75 \mathrm{MHz}, \mathrm{CDCl}_{3}\right) \delta 151.39,144.28,143.50,142.10,138.07,135.23$, 130.29, 129.94, 127.13, 124.90. DEPT NMR (75 MHz, $\left.\mathrm{CDCl}_{3}\right) \delta 151.39$ (down), 144.29, 143.50, 142.10, 138.07 (down), 135.25 (down), 135.24, 135.23, 130.30, 129.94, 127.13, 124.91. GC-MS: $\mathrm{R}_{\mathrm{t}}=7.97 \mathrm{~min} . \mathrm{m} / \mathrm{z}: 190.1\left(\mathrm{M}^{+}\right), 192.1\left(\mathrm{M}^{+}+2\right), 155.1\left(\mathrm{M}^{+}-35\right), 137.1\left(\mathrm{M}^{+}-\right.$ 53), $102.1\left(\mathrm{M}^{+}-88\right)$.

2-(2-Chlorophenyl)pyrazine: Pale yellow oil. Purified by flash chromatography using $10 \%$ ethyl acetate in hexane. ${ }^{1} \mathrm{H}$ NMR $\left(300 \mathrm{MHz}, \mathrm{CDCl}_{3}\right) \delta 8.99(\mathrm{~s}, 1 \mathrm{H}), 8.70(\mathrm{~s}, 1 \mathrm{H}), 8.59$ (s, 1H), $7.63-7.59(\mathrm{~m}, 1 \mathrm{H}), 7.54-7.50(\mathrm{~m}, 1 \mathrm{H}), 7.43-7.39(\mathrm{~m}, 2 \mathrm{H}) .{ }^{13} \mathrm{C} \mathrm{NMR}(75 \mathrm{MHz}$, $\left.\mathrm{CDCl}_{3}\right) \delta 152.73,145.86,144.24,143.11,135.97,132.36,131.71,130.56,130.33,127.31$. DEPT NMR (75 MHz, $\left.\mathrm{CDCl}_{3}\right) \delta$ 145.86, 144.24, 143.11, 135.97 (down), 132.35 (down), 131.71, 130.56, 130.33, 127.31. GC-MS: $\mathrm{R}_{\mathrm{t}}=7.54 \mathrm{~min} . \mathrm{m} / \mathrm{z}: 190.1\left(\mathrm{M}^{+}\right), 192.1\left(\mathrm{M}^{+}+2\right)$, $155.1\left(\mathrm{M}^{+}-35\right), 137.1\left(\mathrm{M}^{+}-53\right), 102.1\left(\mathrm{M}^{+}-88\right)$.

2-(4-Iodophenyl)pyrazine: White solid. Purified by flash chromatography using $15 \%$ ethyl acetate in hexane. ${ }^{1} \mathrm{H}$ NMR $\left(300 \mathrm{MHz}, \mathrm{CDCl}_{3}\right) \delta 9.01(\mathrm{~s}, 1 \mathrm{H}), 8.62(\mathrm{~s}, 1 \mathrm{H}), 8.53(\mathrm{~s}$, $1 \mathrm{H}), 7.85(\mathrm{~d}, J=8.5 \mathrm{~Hz}, 2 \mathrm{H}), 7.76(\mathrm{~d}, J=8.6 \mathrm{~Hz}, 2 \mathrm{H}) .{ }^{13} \mathrm{C} \mathrm{NMR}\left(75 \mathrm{MHz}, \mathrm{CDCl}_{3}\right) \delta$ 151.81, 144.25, 143.34, 141.85, 138.24, 135.80, 128.53, 96.60. DEPT NMR (75 MHz, 
$\left.\mathrm{CDCl}_{3}\right) \delta 151.82$ (down), 144.25, 143.34, 141.86, 138.24, 135.80 (down), 128.53, 96.61 (down). GC-MS: $\mathrm{R}_{\mathrm{t}}=9.64 \mathrm{~min} . \mathrm{m} / \mathrm{z}: 282.0\left(\mathrm{M}^{+}\right), 229.0\left(\mathrm{M}^{+}-53\right), 155.1\left(\mathrm{M}^{+}-127\right), 102.1$ $\left(\mathrm{M}^{+}-180\right)$.

2-(4-Trifluoromethylphenyl)pyrazine: GC-MS: $\mathrm{R}_{\mathrm{t}}=6.63 \mathrm{~min} . \mathrm{m} / \mathrm{z}: 224.1\left(\mathrm{M}^{+}\right), 205.1$ $\left(\mathrm{M}^{+}-19\right), 171.0\left(\mathrm{M}^{+}-53\right)$.

2-(4-Methoxycarbonylphenyl)pyrazine: GC-MS: $\mathrm{R}_{\mathrm{t}}=9.87 \mathrm{~min} . \mathrm{m} / \mathrm{z}=214.1\left(\mathrm{M}^{+}\right), 183.1$ $\left(\mathrm{M}^{+}-31\right), 155.1\left(\mathrm{M}^{+}-59\right), 130.0\left(\mathrm{M}^{+}-84\right), 102.0\left(\mathrm{M}^{+}-112\right), 75.0\left(\mathrm{M}^{+}-139\right)$.

2-Mesitylpyrazine: GC-MS: $\mathrm{R}_{\mathrm{t}}=7.84 \mathrm{~min} . \mathrm{m} / \mathrm{z}=198.1\left(\mathrm{M}^{+}+1\right), 197.1\left(\mathrm{M}^{+}\right), 182.1\left(\mathrm{M}^{+}-\right.$ 15), $168.1\left(\mathrm{M}^{+}-30\right), 144.1\left(\mathrm{M}^{+}-54\right), 130.1\left(\mathrm{M}^{+}-68\right), 115.1\left(\mathrm{M}^{+}-83\right), 91.1\left(\mathrm{M}^{+}-107\right)$.

2-(4-Cyanophenyl)pyrazine: GC-MS: $\mathrm{R}_{\mathrm{t}}=8.80 \mathrm{~min} . \mathrm{m} / \mathrm{z}=181.1\left(\mathrm{M}^{+}\right), 154.1\left(\mathrm{M}^{+}-27\right)$, $128.0\left(\mathrm{M}^{+}-53\right), 101.0\left(\mathrm{M}^{+}-80\right), 75.0\left(\mathrm{M}^{+}-106\right)$.

2-(4-Formylphenyl)pyrazine: GC-MS: $\mathrm{R}_{\mathrm{t}}=8.70 \mathrm{~min} . \mathrm{m} / \mathrm{z}=184.1\left(\mathrm{M}^{+}\right), 183.1\left(\mathrm{M}^{+}-1\right)$, 155.1 ( $\left.\mathrm{M}^{+}-29\right), 130.0\left(\mathrm{M}^{+}-53\right), 102.1\left(\mathrm{M}^{+}-82\right), 75.1\left(\mathrm{M}^{+}-109\right)$.

\subsubsection{Spectroscopic Data of Phenylquinone Products}

2-Phenyl-1,4-benzoquinone: Bright orange solid. Purified by flash chromatography using $2 \%$ ethyl acetate in hexanes. ${ }^{1} \mathrm{H}$ NMR $\left(300 \mathrm{MHz}, \mathrm{CDCl}_{3}\right) \delta 7.49-7.41(\mathrm{~m}, 5 \mathrm{H}), 6.92-$ $6.74(\mathrm{~m}, 3 \mathrm{H}) .{ }^{13} \mathrm{C}$ NMR $\left(75 \mathrm{MHz}, \mathrm{CDCl}_{3}\right) \delta 187.58,186.60,145.93,137.05,136.24$, $132.68,132.62,130.12,129.25,128.54$. DEPT NMR (75 MHz, $\left.\mathrm{CDCl}_{3}\right) \delta 187.58$ (down), 186.60 (down), 145.93 (down), 137.05, 136.24, 132.68, 130.12, 129.25, 128.54. GC-MS: $\mathrm{R}_{\mathrm{t}}=7.82$ min. $\mathrm{m} / \mathrm{z}: 184.1\left(\mathrm{M}^{+}\right), 156.1\left(\mathrm{M}^{+}-28\right), 128.1\left(\mathrm{M}^{+}-56\right), 102.1\left(\mathrm{M}^{+}-82\right), 82.1\left(\mathrm{M}^{+}-\right.$ 102). 
2-(4-Chlorophenyl)-1,4-benzoquinone: Bright orange solid. Purified by flash chromatography using $2 \%$ ethyl acetate in hexanes. ${ }^{1} \mathrm{H}$ NMR $\left(300 \mathrm{MHz}, \mathrm{CDCl}_{3}\right) \delta 7.42(\mathrm{~s}$, 4H), $6.86-6.82(\mathrm{~m}, 3 \mathrm{H}) .{ }^{13} \mathrm{C} \mathrm{NMR}\left(75 \mathrm{MHz}, \mathrm{CDCl}_{3}\right) \delta 187.31,186.29,144.72,137.01$, 136.54, 136.33, 132.65, 131.00, 130.56, 128.86. DEPT NMR (75 MHz, $\left.\mathrm{CDCl}_{3}\right) \delta 187.31$ (down), 186.29 (down), 144.71 (down), 137.01, 136.54 (down), 136.33, 132.65, 131.00 (down), 130.56, 128.85. GC-MS: $\mathrm{R}_{\mathrm{t}}=9.05 \mathrm{~min} . \mathrm{m} / \mathrm{z}: 218.0\left(\mathrm{M}^{+}\right), 220.0\left(\mathrm{M}^{+}+2\right), 183.0\left(\mathrm{M}^{+}-\right.$ 35), $155.0\left(\mathrm{M}^{+}-63\right), 136.0\left(\mathrm{M}^{+}-82\right)$.

2-(3-Chlorophenyl)-1,4-benzoquinone: Bright yellow solid. Purified by flash chromatography using $2 \%$ ethyl acetate in hexanes. ${ }^{1} \mathrm{H}$ NMR $\left(500 \mathrm{MHz}, \mathrm{CDCl}_{3}\right) \delta 7.44(\mathrm{t}$, $J=1.9 \mathrm{~Hz}, 1 \mathrm{H}), 7.39(\mathrm{dt}, \mathrm{J}=7.3,2.1 \mathrm{~Hz}, 1 \mathrm{H}), 7.37-7.29(\mathrm{~m}, 2 \mathrm{H}), 6.89-6.71(\mathrm{~m}, 3 \mathrm{H})$. ${ }^{13} \mathrm{C}$ NMR $(75 \mathrm{MHz}, \mathrm{CDCl} 3) \delta 187.22,186.05,144.55,136.99,136.32,134.50,134.29$, 133.16, 130.10, 129.80, 129.25, 127.38. DEPT NMR (75 MHz, CDCl3) $\delta 187.22$ (down), 186.05 (down), 144.55 (down), 136.99, 136.32, 134.49 (down), 134.29 (down), 133.15, 130.10, 129.80, 129.25, 127.38. GC-MS: $\mathrm{R}_{\mathrm{t}}=9.08 \mathrm{~min} . \mathrm{m} / \mathrm{z}: 218.0\left(\mathrm{M}^{+}\right), 220.0\left(\mathrm{M}^{+}+2\right)$, $183.0\left(\mathrm{M}^{+}-35\right), 155.0\left(\mathrm{M}^{+}-63\right), 136.0\left(\mathrm{M}^{+}-82\right), 101.1\left(\mathrm{M}^{+}-117\right), 82.0\left(\mathrm{M}^{+}-136\right)$.

2-(2-Chlorophenyl)-1,4-benzoquinone: Bright orange solid. Purified by flash chromatography using $2 \%$ ethyl acetate in hexanes. ${ }^{1} \mathrm{H} \mathrm{NMR}\left(500 \mathrm{MHz}, \mathrm{CDCl}_{3}\right) \delta 7.43$ $(\mathrm{dd}, \mathrm{J}=8.0,1.4 \mathrm{~Hz}, 1 \mathrm{H}), 7.35(\mathrm{td}, \mathrm{J}=7.7,1.8 \mathrm{~Hz}, 1 \mathrm{H}), 7.30(\mathrm{td}, \mathrm{J}=7.5,1.4 \mathrm{~Hz}, 1 \mathrm{H}), 7.18$ $(\mathrm{dd}, \mathrm{J}=7.6,1.8 \mathrm{~Hz}, 1 \mathrm{H}), 6.91-6.80(\mathrm{~m}, 2 \mathrm{H}), 6.76(\mathrm{~d}, \mathrm{~J}=2.4 \mathrm{~Hz}, 1 \mathrm{H}) .{ }^{13} \mathrm{C} \mathrm{NMR}(75 \mathrm{MHz}$, $\mathrm{CDCl} 3) \delta 187.25,185.06,146.13,136.82,136.41,135.02,133.09,132.43,130.75,130.65$, 129.84, 126.83. DEPT NMR (75 MHz, CDCl3) $\delta 187.25$ (down), 185.06 (down), 146.13 (down), 136.82, 136.41, 135.02, 133.09 (down), 132.42 (down), 130.75, 130.65, 129.84, 
126.83. $\mathrm{R}_{\mathrm{t}}=8.46 \mathrm{~min} . \mathrm{m} / \mathrm{z}: 218.0\left(\mathrm{M}^{+}\right), 220.0\left(\mathrm{M}^{+}+2\right), 183.0\left(\mathrm{M}^{+}-35\right), 155.0\left(\mathrm{M}^{+}-63\right), 136.0$ $\left(\mathrm{M}^{+}-82\right), 101.1\left(\mathrm{M}^{+}-117\right), 82.0\left(\mathrm{M}^{+}-136\right)$.

2-(4-Methylphenyl)-1,4-benzoquinone: Bright orange solid. Purified by flash chromatography using $2 \%$ ethyl acetate in hexanes. ${ }^{1} \mathrm{H}$ NMR $\left(300 \mathrm{MHz}, \mathrm{CDCl}_{3}\right) \delta 7.39(\mathrm{~d}$, $J=8.3 \mathrm{~Hz}, 2 \mathrm{H}), 7.29-7.21(\mathrm{~m}, 2 \mathrm{H}), 6.89-6.76(\mathrm{~m}, 3 \mathrm{H}), 2.40(\mathrm{~s}, 3 \mathrm{H}) .{ }^{13} \mathrm{C} \mathrm{NMR}(75 \mathrm{MHz}$, $\left.\mathrm{CDCl}_{3}\right) \delta 187.66,186.82,145.85,140.57,137.05,136.22,132.01,129.82,129.32,129.19$, 21.39. DEPT NMR (75 MHz, $\left.\mathrm{CDCl}_{3}\right) \delta 187.66$ (down), 186.82 (down), 145.84 (down), 140.56 (down), 137.05, 136.22, 132.01, 129.81 (down), 129.31, 129.19, 21.38. GC-MS: $R_{t}$ $=8.57 \mathrm{~min} . \mathrm{m} / \mathrm{z}: 198.1\left(\mathrm{M}^{+}\right), 183.1\left(\mathrm{M}^{+}-15\right), 170.1\left(\mathrm{M}^{+}-28\right), 155.1\left(\mathrm{M}^{+}-43\right), 141.1\left(\mathrm{M}^{+}-57\right)$, $115.1\left(\mathrm{M}^{+}-68\right), 82.0\left(\mathrm{M}^{+}-116\right)$.

2-(2-Methylphenyl)-1,4-benzoquinone: Dark orange oil. Purified by flash chromatography using $2 \%$ ethyl acetate in hexanes. ${ }^{1} \mathrm{H}$ NMR (500 MHz, $\left.\mathrm{CDCl}_{3}\right) \delta 7.31$ (td, $\mathrm{J}=7.5,1.5 \mathrm{~Hz}, 1 \mathrm{H}), 7.25-7.19(\mathrm{~m}, 2 \mathrm{H}), 7.07(\mathrm{dd}, \mathrm{J}=7.7,1.4 \mathrm{~Hz}, 1 \mathrm{H}), 6.88-6.77(\mathrm{~m}$, 2H), $6.69(\mathrm{~d}, \mathrm{~J}=2.4 \mathrm{~Hz}, 1 \mathrm{H}), 2.16(\mathrm{~s}, 3 \mathrm{H}) .{ }^{13} \mathrm{C} \mathrm{NMR}(75 \mathrm{MHz}, \mathrm{CDCl} 3) \delta$ 187.65, 186.17, $148.44,136.85,136.36,136.13,134.52,133.08,130.41,129.49,129.25,125.83,20.36$. DEPT NMR (75 MHz, CDCl3) $\delta 187.65$ (down), 186.16 (down), 148.44 (down), 136.85, 136.35, 136.12 (down), 134.52, 133.07 (down), 130.41, 129.49, 129.25, 125.83, 20.36. $\mathrm{R}_{\mathrm{t}}$ =7.97 $\mathrm{min} . \mathrm{m} / \mathrm{z}: 198.1\left(\mathrm{M}^{+}\right), 183.1\left(\mathrm{M}^{+}-15\right), 181.1\left(\mathrm{M}^{+}-17\right), 169.1\left(\mathrm{M}^{+}-29\right), 153.1\left(\mathrm{M}^{+}-45\right)$, $141.1\left(\mathrm{M}^{+}-57\right), 115.1\left(\mathrm{M}^{+}-83\right), 82.1\left(\mathrm{M}^{+}-116\right)$.

2-(4-Trifluoromethylphenyl)-1,4-benzoquinone: Waxy yellow solid, collected using flash chromatography with $1 \%$ ethyl acetate in hexane as eluent. ${ }^{1} \mathrm{H}$ NMR $(300 \mathrm{MHz}$, $\left.\mathrm{CDCl}_{3}\right) \delta 7.74-7.57(\mathrm{~m}, 4 \mathrm{H}), 6.96-6.82(\mathrm{~m}, 3 \mathrm{H}) .{ }^{13} \mathrm{C} \mathrm{NMR}(75 \mathrm{MHz}, \mathrm{CDCl}) \delta$ 187.11, 
$185.97,144.71,136.99,136.45,133.60,129.62,125.54,125.49,125.44,125.39,77.44$, 77.01, 76.59. DEPT NMR (75 MHz, CDCl3) $\delta 136.99,136.44,133.60,129.62,125.54$, 125.49, 125.44, 125.39. GC-MS: $\mathrm{R}_{\mathrm{t}}=7.70 \mathrm{~min} . \mathrm{m} / \mathrm{z}: 252.1\left(\mathrm{M}^{+}\right), 233.0\left(\mathrm{M}^{+}-19\right), 224.1$ ( $\left.\mathrm{M}^{+}-28\right), 196.1\left(\mathrm{M}^{+}-56\right), 183.1\left(\mathrm{M}^{+}-69\right), 170.0\left(\mathrm{M}^{+}-82\right), 151.0\left(\mathrm{M}^{+}-99\right), 120.0\left(\mathrm{M}^{+}-132\right)$ $82.0\left(\mathrm{M}^{+}-170\right)$.

2-Mesityl-1,4-benzoquinone: Waxy yellow solid, collected using flash chromatography with $1 \%$ ethyl acetate in hexane as eluent. ${ }^{1} \mathrm{H}$ NMR $\left(300 \mathrm{MHz}, \mathrm{CDCl}_{3}\right) \delta 6.92-6.85(\mathrm{~m}$, 4H), $6.78-6.65(\mathrm{~m}, 1 \mathrm{H}), 2.31(\mathrm{~s}, 3 \mathrm{H}), 2.07(\mathrm{~s}, 6 \mathrm{H}) .{ }^{13} \mathrm{C} \mathrm{NMR}(75 \mathrm{MHz}, \mathrm{CDCl} 3) \delta$ 187.58, $186.39,148.30,138.65,136.91,136.56,135.55,135.45,133.10,128.39,20.29$. DEPT NMR (75 MHz, CDCl3) $\delta 187.59$ (down), 186.39 (down), 148.29 (down), 138.66 (down), 136.91, 136.56, 135.55, 135.45 (down), 133.10, 128.40, 21.10, 20.29. GC-MS: $\mathrm{R}_{\mathrm{t}}=8.88$ min. m/z: $226.1\left(\mathrm{M}^{+}\right), 211.1\left(\mathrm{M}^{+}-15\right), 198.1\left(\mathrm{M}^{+}-28\right), 183.1\left(\mathrm{M}^{+}-43\right), 169.1\left(\mathrm{M}^{+}-57\right), 155.1$ $\left(\mathrm{M}^{+}-71\right), 143.1\left(\mathrm{M}^{+}-83\right), 128.1\left(\mathrm{M}^{+}-96\right), 115.1\left(\mathrm{M}^{+}-111\right)$.

2,3-Dimethyl-5-phenyl-1,4-benzoquinone: Translucent yellow liquid, collected as a mixture with 2,3-dimethyl-1,4-benzoquinone using flash chromatography with $1 \%$ ethyl acetate in hexane as eluent. Spectroscopic yield determined via comparison of the integration of the respective methyl peaks. ${ }^{1} \mathrm{H}$ NMR $\left(300 \mathrm{MHz}, \mathrm{CDCl}_{3}\right) \delta 7.54-7.34(\mathrm{~m}$, 4H), $6.71(\mathrm{~s}, 1 \mathrm{H}), 2.09(\mathrm{dt}, \mathrm{J}=7.2,1.3 \mathrm{~Hz}, 6 \mathrm{H}) .{ }^{13} \mathrm{C} \mathrm{NMR}(75 \mathrm{MHz}, \mathrm{CDCl} 3) \delta$ 187.51, $186.66,145.71,141.23,140.98,140.74,136.23,132.43,129.71,128.36,12.69,12.17$. DEPT NMR (75 MHz, CDCl3) $\delta 187.51$ (down), 186.65 (down), 145.70 (down), 141.22 (down), 140.97 (down), 140.73 (down), 136.23, 132.43, 129.71, 128.36, 12.69, 12.17. GC- 
MS: $\mathrm{R}_{\mathrm{t}}=9.33 \mathrm{~min} . \mathrm{m} / \mathrm{z}: 212.1\left(\mathrm{M}^{+}\right), 197.1\left(\mathrm{M}^{+}-15\right), 183.1\left(\mathrm{M}^{+}-29\right), 169.1\left(\mathrm{M}^{+}-43\right), 155.1$ $\left(\mathrm{M}^{+}-57\right), 141.1\left(\mathrm{M}^{+}-61\right), 102.1\left(\mathrm{M}^{+}-110\right)$.

2,3-Dimethyl-1,4-benzoquinone: Translucent yellow liquid, collected as a mixture with 2,3-dimethyl-5-phenyl-1,4-benzoquinone using flash chromatography with $1 \%$ ethyl acetate in hexane as eluent. Spectroscopic yield determined via comparison of the integration of the respective methyl peaks. ${ }^{1} \mathrm{H}$ NMR $\left(300 \mathrm{MHz}, \mathrm{CDCl}_{3}\right) \delta 6.80(\mathrm{~s}, 2 \mathrm{H}), 2.03$ (s, 6H). ${ }^{13} \mathrm{C}$ NMR $(75 \mathrm{MHz}, \mathrm{CDCl} 3) \delta 187.34,133.30,129.22,12.14$. DEPT NMR (75 MHz, CDCl3) $\delta 187.34$ (down), 133.29 (down), 129.22, 12.14. GC-MS: $136.1\left(\mathrm{M}^{+}\right), 108.1$ $\left(\mathrm{M}^{+}-28\right), 107.1\left(\mathrm{M}^{+}-29\right), 90.1\left(\mathrm{M}^{+}-46\right), 82.0\left(\mathrm{M}^{+}-54\right), 79.1\left(\mathrm{M}^{+}-57\right), 54.1\left(\mathrm{M}^{+}-79\right)$.

\subsubsection{Spectroscopic Data of Other Phenyl- $\mathrm{N}$-Heteroarenes}

2-Phenyl-4-cyanopyridine: White solid. Purified by flash chromatography using 5\% ethyl acetate in hexanes. ${ }^{1} \mathrm{H}$ NMR $\left(300 \mathrm{MHz}, \mathrm{CDCl}_{3}\right) \delta 8.86(\mathrm{~d}, J=5.0,1.0 \mathrm{~Hz}, 1 \mathrm{H}), 8.04-7.96$ (m, 2H), $7.94(\mathrm{~s}, 1 \mathrm{H}), 7.59-7.47(\mathrm{~m}, 3 \mathrm{H}), 7.44(\mathrm{dd}, J=5.0,1.5 \mathrm{~Hz}, 1 \mathrm{H}) .{ }^{13} \mathrm{C}$ NMR $(75$ $\left.\mathrm{MHz}, \mathrm{CDCl}_{3}\right) \delta 158.77,150.63,137.33,130.22,129.08,126.98,123.14,122.03,121.22$, 116.72. DEPT NMR (75 MHz, $\left.\mathrm{CDCl}_{3}\right) \delta 158.77$ (down), 150.63, 137.33 (down), 130.22, 129.08, 126.97, 123.14, 122.03, 121.22 (down), 116.72 (down). GC-MS: $\mathrm{R}_{\mathrm{t}}=8.22 \mathrm{~min}$. m/z: $180.1\left(\mathrm{M}^{+}\right), 153.1\left(\mathrm{M}^{+}-27\right), 126.1\left(\mathrm{M}^{+}-54\right), 103.1\left(\mathrm{M}^{+}-77\right), 76.1\left(\mathrm{M}^{+}-104\right)$.

3-Phenyl-4-cyanopyridine: White solid. Purified by flash chromatography using 5\% ethyl acetate in hexanes. ${ }^{1} \mathrm{H}$ NMR $\left(300 \mathrm{MHz}, \mathrm{CDCl}_{3}\right) \delta 8.88(\mathrm{~s}, 1 \mathrm{H}), 8.76(\mathrm{~d}, J=4.2 \mathrm{~Hz}, 1 \mathrm{H})$, $7.64-7.50(\mathrm{~m}, 6 \mathrm{H}) .{ }^{13} \mathrm{C} \mathrm{NMR}\left(75 \mathrm{MHz}, \mathrm{CDCl}_{3}\right) \delta 150.99,148.71,138.73,134.44,129.56$, 129.15, 128.80, 126.06, 118.82, 116.33. DEPT NMR (75 MHz, $\left.\mathrm{CDCl}_{3}\right) \delta 150.99,148.71$, 134.45 (down), 129.56, 129.15, 128.80, 126.06, 118.83 (down), 116.32 (down). GC-MS: 
$\mathrm{R}_{\mathrm{t}}=7.82$ min. $\mathrm{m} / \mathrm{z}: 180.1\left(\mathrm{M}^{+}\right), 153.1\left(\mathrm{M}^{+}-27\right), 126.1\left(\mathrm{M}^{+}-54\right), 103.1\left(\mathrm{M}^{+}-77\right), 76.1\left(\mathrm{M}^{+}-\right.$ 104).

3-Phenylpyridazine: Dark red solid, collected using $100 \%$ ethyl acetate as eluent. ${ }^{1} \mathrm{H}$ NMR (300 MHz, $\left.\mathrm{CDCl}_{3}\right) \delta 9.16(\mathrm{dd}, \mathrm{J}=4.9,1.6 \mathrm{~Hz}, 1 \mathrm{H}), 8.14-8.04(\mathrm{~m}, 2 \mathrm{H}), 7.87$ (dd, J $=8.6,1.7 \mathrm{~Hz}, 1 \mathrm{H}), 7.60-7.47(\mathrm{~m}, 4 \mathrm{H}) .{ }^{13} \mathrm{C}$ NMR $(75 \mathrm{MHz}, \mathrm{CDCl} 3) \delta 159.51,150.01$, 136.38, 130.12, 129.06, 127.14, 126.79, 123.91. DEPT NMR (75 MHz, CDCl3) $\delta 150.01$, 130.12, 129.05, 127.14, 126.79, 123.91. GC-MS: $\mathrm{R}_{\mathrm{t}}=7.91 \mathrm{~min} . \mathrm{m} / \mathrm{z}: 156.1\left(\mathrm{M}^{+}\right), 128.1$ $\left(\mathrm{M}^{+}-28\right), 102.1\left(\mathrm{M}^{+}-54\right), 76.1\left(\mathrm{M}^{+}-70\right), 63.1\left(\mathrm{M}^{+}-93\right), 51.1\left(\mathrm{M}^{+}-103\right)$.

4-Phenylpyridazine: Dark red solid, collected using $100 \%$ ethyl acetate as eluent. ${ }^{1} \mathrm{H}$ NMR (300 MHz, $\left.\mathrm{CDCl}_{3}\right) \delta 9.50-9.42(\mathrm{~m}, 1 \mathrm{H}), 9.22(\mathrm{~d}, \mathrm{~J}=5.3 \mathrm{~Hz}, 1 \mathrm{H}), 7.70-7.64(\mathrm{~m}$, 3H), $7.57-7.50(\mathrm{~m}, 3 \mathrm{H}) .{ }^{13} \mathrm{C} \mathrm{NMR}(75 \mathrm{MHz}, \mathrm{CDCl} 3) \delta 151.38,149.91,138.55$ (down), 134.46 (down), 130.16, 129.55, 127.08, 123.29. GC-MS: $\mathrm{R}_{\mathrm{t}}=8.08 . \mathrm{m} / \mathrm{z}: 156.1\left(\mathrm{M}^{+}\right), 128.1$ $\left(\mathrm{M}^{+}-28\right), 102.1\left(\mathrm{M}^{+}-54\right), 76.1\left(\mathrm{M}^{+}-80\right), 63.1\left(\mathrm{M}^{+}-93\right), 51.1\left(\mathrm{M}^{+}-105\right)$.

2-Phenylquinoline: White solid, eluted using 10\% ethyl acetate in hexane. ${ }^{1} \mathrm{H}$ NMR (300 $\left.\mathrm{MHz}, \mathrm{CDCl}_{3}\right) \delta 8.23-8.13(\mathrm{~m}, 4 \mathrm{H}), 7.86(\mathrm{~d}, \mathrm{~J}=8.7 \mathrm{~Hz}, 1 \mathrm{H}), 7.81(\mathrm{dd}, \mathrm{J}=8.1,1.5 \mathrm{~Hz}$, 1H), 7.72 (ddd, $\mathrm{J}=8.5,6.8,1.5 \mathrm{~Hz}, 1 \mathrm{H}), 7.58-7.41(\mathrm{~m}, 4 \mathrm{H}) .{ }^{13} \mathrm{C} \mathrm{NMR}(75 \mathrm{MHz}, \mathrm{CDCl} 3)$ $\delta 157.38,148.31,139.72,136.79,129.77,129.67,129.34,128.87,127.59,127.48,127.20$, 126.30, 119.03. DEPT NMR (75 MHz, CDCl3) $\delta 157.38,148.31,139.71,136.79,129.77$, $129.67,129.59,129.33,128.87,127.59,127.48,127.20,126.30,119.03$. GC-MS: $R_{t}=$ 11.09. m/z: $205.1\left(\mathrm{M}^{+}\right), 204.1\left(\mathrm{M}^{+}-1\right), 176.1\left(\mathrm{M}^{+}-29\right), 151.1\left(\mathrm{M}^{+}-54\right), 102.2\left(\mathrm{M}^{+}-103\right), 88.1$ ( $\left.\mathrm{M}^{+}-117\right), 76.1\left(\mathrm{M}^{+}-129\right)$. 
4-Phenylquinoline: Yellow oil, collected using $10 \%$ ethyl acetate in hexane. ${ }^{1} \mathrm{H}$ NMR (300 $\left.\mathrm{MHz}_{\mathrm{CDCl}}\right) \delta 8.96(\mathrm{dd}, \mathrm{J}=4.2,1.8 \mathrm{~Hz}, 1 \mathrm{H}), 8.21(\mathrm{dd}, \mathrm{J}=8.3,1.8 \mathrm{~Hz}, 1 \mathrm{H}), 7.83(\mathrm{dd}, \mathrm{J}=$ 8.1, 1.6 Hz, 1H), $7.72(\mathrm{dd}, \mathrm{J}=1.8,0.9 \mathrm{~Hz}, 1 \mathrm{H}), 7.61(\mathrm{dd}, \mathrm{J}=8.1,7.2 \mathrm{~Hz}, 1 \mathrm{H}), 7.50(\mathrm{tt}, \mathrm{J}=$ 6.7, $1.0 \mathrm{~Hz}, 2 \mathrm{H}), 7.45-7.37(\mathrm{~m}, 2 \mathrm{H}) .{ }^{13} \mathrm{C} \mathrm{NMR}(75 \mathrm{MHz}, \mathrm{CDCl} 3) \delta 150.28,146.06$, $140.93,139.56,136.24,130.61,130.33,128.74,128.01,127.54,127.38,126.27,120.99$. DEPT NMR (75 MHz, CDCl3) $\delta 150.28,136.24,130.61,130.33,128.01,127.54,127.38$, 126.27, 120.99. GC-MS: $\mathrm{R}_{\mathrm{t}}=10.07 . \mathrm{m} / \mathrm{z}: 205.1\left(\mathrm{M}^{+}\right), 204.1\left(\mathrm{M}^{+}-1\right), 176.1\left(\mathrm{M}^{+}-29\right), 151.1$ $\left(\mathrm{M}^{+}-54\right), 102.0\left(\mathrm{M}^{+}-103\right), 88.1\left(\mathrm{M}^{+}-117\right), 76.1\left(\mathrm{M}^{+}-129\right)$.

3-Phenyllutidine: Thick yellow oil, collected using 10\% ethyl acetate in hexane as eluent. ${ }^{1} \mathrm{H}$ NMR $\left(500 \mathrm{MHz}, \mathrm{CDCl}_{3}\right) \delta 7.40(\mathrm{dd}, \mathrm{J}=7.8,2.3 \mathrm{~Hz}, 3 \mathrm{H}), 7.38-7.31(\mathrm{~m}, 1 \mathrm{H}), 7.32-$ $7.26(\mathrm{~m}, 2 \mathrm{H}), 7.03(\mathrm{~d}, \mathrm{~J}=7.7 \mathrm{~Hz}, 1 \mathrm{H}), 2.56(\mathrm{~s}, 3 \mathrm{H}), 2.47(\mathrm{~s}, 3 \mathrm{H}) .{ }^{13} \mathrm{C}$ NMR $(75 \mathrm{MHz}$, $\mathrm{CDCl} 3) \delta 156.47,154.92,140.06,137.71,134.04,129.12,128.35,127.26,120.61,24.16$, 23.23. DEPT NMR (75 MHz, CDCl3) $\delta 137.71,129.12,128.35,127.26,120.62,24.15$, 23.23. GC-MS: $\mathrm{R}_{\mathrm{t}}=7.12 . \mathrm{m} / \mathrm{z}: 183.2\left(\mathrm{M}^{+}\right), 182.2\left(\mathrm{M}^{+}-1\right), 167.1\left(\mathrm{M}^{+}-16\right), 141.1\left(\mathrm{M}^{+}-42\right)$, $115.1\left(\mathrm{M}^{+}-68\right), 102.1\left(\mathrm{M}^{+}-81\right), 90.6\left(\mathrm{M}^{+}-92\right), 77.1\left(\mathrm{M}^{+}-106\right)$.

4-Phenyllutidine: Thick yellow oil, collected using 20\% ethyl acetate in hexane as eluent. ${ }^{1} \mathrm{H}$ NMR (300 MHz, $\left.\mathrm{CDCl}_{3}\right) \delta 7.63-7.57(\mathrm{~m}, 2 \mathrm{H}), 7.49-7.36(\mathrm{~m}, 3 \mathrm{H}), 7.17(\mathrm{~s}, 2 \mathrm{H}), 2.58$ (s, 6H). ${ }^{13} \mathrm{C}$ NMR (75 MHz, CDCl3) $\delta 158.14,149.07,138.73,128.94,128.73,127.01$, 118.39, 77.46, 77.04, 76.62, 24.55. DEPT NMR (75 MHz, CDCl3) $\delta 158.14$ (down), 149.07 (down), 138.73 (down), 128.94, 128.73, 127.01, 118.39, 77.46, 77.04, 76.61, 24.55. GC-MS: $\mathrm{R}_{\mathrm{t}}=7.62 . \mathrm{m} / \mathrm{z}: 183.2\left(\mathrm{M}^{+}\right), 167.1\left(\mathrm{M}^{+}-16\right), 153.1\left(\mathrm{M}^{+}-30\right), 141.1\left(\mathrm{M}^{+}-42\right), 128.1$ $\left(\mathrm{M}^{+}-55\right), 115.1\left(\mathrm{M}^{+}-68\right), 102.1\left(\mathrm{M}^{+}-81\right), 90.6\left(\mathrm{M}^{+}-92\right), 77.1\left(\mathrm{M}^{+}-106\right)$. 
2-Phenylpyridine: Yellow oil, collected using 10\% ethyl acetate in hexane. ${ }^{1} \mathrm{H}$ NMR (300 $\left.\mathrm{MHz}, \mathrm{CDCl}_{3}\right) \delta 8.69(\mathrm{dt}, \mathrm{J}=4.9,1.4 \mathrm{~Hz}, 1 \mathrm{H}), 8.07-7.91(\mathrm{~m}, 2 \mathrm{H}), 7.79-7.64(\mathrm{~m}, 2 \mathrm{H})$, $7.51-7.41(\mathrm{~m}, 3 \mathrm{H}), 7.28-7.15(\mathrm{~m}, 1 \mathrm{H}) .{ }^{13} \mathrm{C} \mathrm{NMR}(75 \mathrm{MHz}, \mathrm{CDCl} 3) \delta 157.48,149.69$, $139.42,136.75,128.97,128.77,126.93,122.11,120.58$. DEPT NMR (75 MHz, CDCl3) $\delta$ $149.69,136.75,128.97,128.77,126.93,122.11,120.58$. GC-MS: $\mathrm{R}_{\mathrm{t}}=6.80 . \mathrm{m} / \mathrm{z}: 155.1$ $\left(\mathrm{M}^{+}\right), 154.1\left(\mathrm{M}^{+}-1\right), 127.1\left(\mathrm{M}^{+}-28\right), 102.1\left(\mathrm{M}^{+}-53\right), 77.1\left(\mathrm{M}^{+}-78\right), 63.1\left(\mathrm{M}^{+}-92\right), 51.1\left(\mathrm{M}^{+}-\right.$ 104).

4-Phenylpyridine: Reddish solid, collected using $10 \%$ ethyl acetate in hexane. ${ }^{1} \mathrm{H}$ NMR $\left(500 \mathrm{MHz}, \mathrm{CDCl}_{3}\right) \delta 8.64(\mathrm{~d}, \mathrm{~J}=5.3 \mathrm{~Hz}, 2 \mathrm{H}), 7.62(\mathrm{~d}, \mathrm{~J}=7.5 \mathrm{~Hz}, 2 \mathrm{H}), 7.52-7.42(\mathrm{~m}, 5 \mathrm{H})$. ${ }^{13} \mathrm{C}$ NMR $(75 \mathrm{MHz}, \mathrm{CDCl} 3) \delta 150.00,148.57,138.06,129.15,127.03,121.73$. DEPT NMR $(75 \mathrm{MHz}, \mathrm{CDCl} 3) \delta 150.00,129.15,127.02,121.73 . \mathrm{GC}-\mathrm{MS}: \mathrm{R}_{\mathrm{t}}=6.89 . \mathrm{m} / \mathrm{z}: 155.1\left(\mathrm{M}^{+}\right)$, 154.1 ( $\left.\mathrm{M}^{+}-1\right), 140.1\left(\mathrm{M}^{+}-15\right), 127.1\left(\mathrm{M}^{+}-28\right), 115.1\left(\mathrm{M}^{+}-40\right), 102.1\left(\mathrm{M}^{+}-53\right), 77.1\left(\mathrm{M}^{+}-78\right)$, $63.1\left(\mathrm{M}^{+}-92\right), 51.1\left(\mathrm{M}^{+}-104\right)$.

1-Methyl-2-phenylimidazole: Dark red-brown solid, collected using 100\% ethyl acetate as eluent. ${ }^{1} \mathrm{H}$ NMR $\left(300 \mathrm{MHz}, \mathrm{CDCl}_{3}\right) \delta 7.89-7.74(\mathrm{~m}, 1 \mathrm{H}), 7.58(\mathrm{dq}, \mathrm{J}=6.2,2.4 \mathrm{~Hz}$, 2H), $7.45-7.40(\mathrm{~m}, 2 \mathrm{H}), 7.15(\mathrm{~d}, \mathrm{~J}=1.3 \mathrm{~Hz}, 1 \mathrm{H}), 6.96(\mathrm{~d}, \mathrm{~J}=1.3 \mathrm{~Hz}, 1 \mathrm{H}), 3.70(\mathrm{~s}, 3 \mathrm{H})$. ${ }^{13} \mathrm{C}$ NMR $(75 \mathrm{MHz}, \mathrm{CDCl} 3) \delta 147.67,133.71,130.09,128.83,128.51,127.95,122.13$, 77.50, 77.08, 76.66, 34.46. DEPT NMR (75 MHz, CDCl3) $\delta$ 133.71, 128.83, 128.51, 127.94, 122.13, 34.46. GC-MS: $\mathrm{R}_{\mathrm{t}}=$ 7.53. $\mathrm{m} / \mathrm{z}: 158.1\left(\mathrm{M}^{+}\right), 157.1\left(\mathrm{M}^{+}-1\right), 142.1\left(\mathrm{M}^{+}-16\right)$, $130.1\left(\mathrm{M}^{+}-28\right), 116.1\left(\mathrm{M}^{+}-42\right), 104.1\left(\mathrm{M}^{+}-42\right), 89.1\left(\mathrm{M}^{+}-69\right), 77.1\left(\mathrm{M}^{+}-81\right), 63.1\left(\mathrm{M}^{+}-95\right)$, $54.1\left(\mathrm{M}^{+}-104\right)$. 
2-Bromophenylpyridine (isomer unknown): GC-MS: $\mathrm{R}_{\mathrm{t}}=6.53-7.55 . \mathrm{m} / \mathrm{z}: 234.1\left(\mathrm{M}^{+}\right)$, $233.1\left(\mathrm{M}^{+}-1\right), 205.1\left(\mathrm{M}^{+}-29\right), 191.1\left(\mathrm{M}^{+}-43\right), 131.1\left(\mathrm{M}^{+}-103\right), 128.1\left(\mathrm{M}^{+}-106\right), 103.1\left(\mathrm{M}^{+}-\right.$ 131), $91.1\left(\mathrm{M}^{+}-143\right), 77.1\left(\mathrm{M}^{+}-157\right), 51.1\left(\mathrm{M}^{+}-183\right)$.

\subsubsection{Spectroscopic Data of Side Products}

4-Chlorophenol: GC-MS: $\mathrm{R}_{\mathrm{t}}=4.78 \mathrm{~min} . \mathrm{m} / \mathrm{z}: 128.1\left(\mathrm{M}^{+}\right), 130.1\left(\mathrm{M}^{+}+2\right), 100.0\left(\mathrm{M}^{+}-28\right)$, $93.1\left(\mathrm{M}^{+}-35\right), 65.1\left(\mathrm{M}^{+}-63\right)$.

Bipyrazine: GC-MS: $\mathrm{R}_{\mathrm{t}}=6.72 \mathrm{~min} . \mathrm{m} / \mathrm{z}=158.1\left(\mathrm{M}^{+}\right), 131.1\left(\mathrm{M}^{+}-27\right), 106.1\left(\mathrm{M}^{+}-52\right), 80.1$ $\left(\mathrm{M}^{+}-78\right)$.

Isomers of $\mathbf{x ,}, \mathbf{x}^{\prime}$-dichlorobiphenyl. Isomer 1: GC-MS: $\mathrm{R}_{\mathrm{t}}=8.24 \mathrm{~min} . \mathrm{m} / \mathrm{z}: 222.0\left(\mathrm{M}^{+}\right)$, $224.0\left(\mathrm{M}^{+}+2\right), 226.0\left(\mathrm{M}^{+}+4\right), 188.0\left(\mathrm{M}^{+}-34\right), 187.1\left(\mathrm{M}^{+}-35\right), 186.1\left(\mathrm{M}^{+}-36\right), 152.1\left(\mathrm{M}^{+}-\right.$ 35). Isomer 2: GC-MS: $\mathrm{R}_{\mathrm{t}}=8.75 \mathrm{~min} . \mathrm{m} / \mathrm{z}: 222.0\left(\mathrm{M}^{+}\right), 224.0\left(\mathrm{M}^{+}+2\right), 226.0\left(\mathrm{M}^{+}+4\right)$, 188.0 ( $\left.\mathrm{M}^{+}-34\right), 187.1\left(\mathrm{M}^{+}-35\right), 186.1\left(\mathrm{M}^{+}-36\right), 152.1\left(\mathrm{M}^{+}-35\right)$. Isomer 3: GC-MS: $\mathrm{R}_{\mathrm{t}}=8.84$ min. $\mathrm{m} / \mathrm{z}: 222.0\left(\mathrm{M}^{+}\right), 224.0\left(\mathrm{M}^{+}+2\right), 226.0\left(\mathrm{M}^{+}+4\right), 188.0\left(\mathrm{M}^{+}-34\right), 187.1\left(\mathrm{M}^{+}-35\right), 186.1$ $\left(\mathrm{M}^{+}-36\right), 152.1\left(\mathrm{M}^{+}-35\right)$.

Biphenyl: GC-MS: $\mathrm{R}_{\mathrm{t}}=6.21 \mathrm{~min} . \mathrm{m} / \mathrm{z}: 154.1\left(\mathrm{M}^{+}\right), 139.1\left(\mathrm{M}^{+}-15\right), 128.1\left(\mathrm{M}^{+}-26\right), 115.1$ $\left(\mathrm{M}^{+}-39\right), 76.1\left(\mathrm{M}^{+}-78\right)$.

Phenol: GC-MS: $\mathrm{R}_{\mathrm{t}}=2.73 \mathrm{~min} . \mathrm{m} / \mathrm{z}$ : $94.1\left(\mathrm{M}^{+}\right), 66.1\left(\mathrm{M}^{+}-28\right), 55.1\left(\mathrm{M}^{+}-39\right)$.

Quinone: GC-MS: $\mathrm{R}_{\mathrm{t}}=2.22 \mathrm{~min} . \mathrm{m} / \mathrm{z}: 108.0\left(\mathrm{M}^{+}\right), 82.0\left(\mathrm{M}^{+}-26\right), 80.0\left(\mathrm{M}^{+}-28\right), 54.0\left(\mathrm{M}^{+}-\right.$ 54). 
2-Dichloromethylquinone: GC-MS: $\mathrm{R}_{\mathrm{t}}=5.67 \mathrm{~min} . \mathrm{m} / \mathrm{z}: 190.0\left(\mathrm{M}^{+}\right), 192.0\left(\mathrm{M}^{+}+2\right), 194.0$ $\left(\mathrm{M}^{+}+4\right), 166.0\left(\mathrm{M}^{+}-24\right), 164.0\left(\mathrm{M}^{+}-26\right), 162.1\left(\mathrm{M}^{+}-28\right), 155.1\left(\mathrm{M}^{+}-35\right), 128.0\left(\mathrm{M}^{+}-62\right)$ $126.0\left(\mathrm{M}^{+}-64\right), 101.0\left(\mathrm{M}^{+}-89\right), 99.0\left(\mathrm{M}^{+}-91\right)$.

2-Chloroquinone: GC-MS: $\mathrm{R}_{\mathrm{t}}=3.75 \mathrm{~min} . \mathrm{m} / \mathrm{z}: 142.0\left(\mathrm{M}^{+}\right), 144.0\left(\mathrm{M}^{+}+2\right), 116.0\left(\mathrm{M}^{+}-26\right)$, $114.0\left(\mathrm{M}^{+}-28\right), 107.0\left(\mathrm{M}^{+}-35\right), 88.0\left(\mathrm{M}^{+}-54\right), 82.0\left(\mathrm{M}^{+}-60\right), 60.0\left(\mathrm{M}^{+}-82\right)$.

2,5-Diphenylquinone or 2,6-diphenylquinone: GC-MS: $R_{t}=12.82-13.09 \mathrm{~min} . \mathrm{m} / \mathrm{z}$ : $260.1\left(\mathrm{M}^{+}\right), 231.1\left(\mathrm{M}^{+}-29\right), 215.1\left(\mathrm{M}^{+}-45\right), 203.1\left(\mathrm{M}^{+}-57\right), 178.1\left(\mathrm{M}^{+}-82\right), 176.1\left(\mathrm{M}^{+}-84\right)$ $152.1\left(\mathrm{M}^{+}-108\right), 102.1\left(\mathrm{M}^{+}-158\right)$.

4-Trifluorophenol: GC-MS: $\mathrm{R}_{\mathrm{t}}=3.67 \mathrm{~min} . \mathrm{m} / \mathrm{z}$ : $162.1\left(\mathrm{M}^{+}\right), 143.1\left(\mathrm{M}^{+}-19\right), 133.1\left(\mathrm{M}^{+}-\right.$ 29), $114.1\left(\mathrm{M}^{+}-48\right), 112.1\left(\mathrm{M}^{+}-50\right)$.

Trifluorophenol, isomer unknown: GC-MS: $\mathrm{R}_{\mathrm{t}}=3.82 \mathrm{~min} . \mathrm{m} / \mathrm{z}: 162.1\left(\mathrm{M}^{+}\right), 144.0\left(\mathrm{M}^{+}-\right.$ 18), $142.0\left(\mathrm{M}^{+}-20\right), 116.0\left(\mathrm{M}^{+}-46\right), 114.0\left(\mathrm{M}^{+}-48\right), 88.0\left(\mathrm{M}^{+}-74\right), 82.0\left(\mathrm{M}^{+}-80\right), 60.0\left(\mathrm{M}^{+}-\right.$ 98).

Unknown isomers of phenylquinoline. Isomer 1: GC-MS: $\mathrm{R}_{\mathrm{t}}=9.94 . \mathrm{m} / \mathrm{z}: 205.1\left(\mathrm{M}^{+}\right)$, $204.1\left(\mathrm{M}^{+}-1\right), 176.1\left(\mathrm{M}^{+}-29\right), 151.1\left(\mathrm{M}^{+}-54\right), 102.0\left(\mathrm{M}^{+}-103\right), 88.1\left(\mathrm{M}^{+}-117\right), 76.1\left(\mathrm{M}^{+}-\right.$ 129). Isomer 2: GC-MS: $\mathrm{R}_{\mathrm{t}}=10.26 . \mathrm{m} / \mathrm{z}: 205.1\left(\mathrm{M}^{+}\right), 204.1\left(\mathrm{M}^{+}-1\right), 176.1\left(\mathrm{M}^{+}-29\right), 151.1$ $\left(\mathrm{M}^{+}\right.$-54), $102.0\left(\mathrm{M}^{+}-103\right), 88.1\left(\mathrm{M}^{+}-117\right), 76.1\left(\mathrm{M}^{+}-129\right)$. Isomer 3: GC-MS: $\mathrm{R}_{\mathrm{t}}=10.43$. m/z: $205.1\left(\mathrm{M}^{+}\right), 204.1\left(\mathrm{M}^{+}-1\right), 176.1\left(\mathrm{M}^{+}-29\right), 151.1\left(\mathrm{M}^{+}-54\right), 102.0\left(\mathrm{M}^{+}-103\right), 88.1\left(\mathrm{M}^{+}-\right.$ 117), $76.1\left(\mathrm{M}^{+}-129\right)$. Isomer 4: GC-MS: $\mathrm{R}_{\mathrm{t}}=11.57 . \mathrm{m} / \mathrm{z}: 205.1\left(\mathrm{M}^{+}\right), 204.1\left(\mathrm{M}^{+}-1\right), 176.1$ $\left(\mathrm{M}^{+}-29\right), 151.1\left(\mathrm{M}^{+}-54\right), 102.0\left(\mathrm{M}^{+}-103\right), 88.1\left(\mathrm{M}^{+}-117\right), 76.1\left(\mathrm{M}^{+}-129\right)$. 


\subsubsection{Spectroscopic Data of Products and Side Products of CDC Reactions}

2-Benzyl-1,4-benzoquinone: GC-MS: $\mathrm{R}_{\mathrm{t}}=8.07 \mathrm{~min} . \mathrm{m} / \mathrm{z}: 198.1\left(\mathrm{M}^{+}\right), 181.1\left(\mathrm{M}^{+}-17\right)$, 169.1 ( $\left.\mathrm{M}^{+}-29\right), 152.1\left(\mathrm{M}^{+}-46\right), 141.1\left(\mathrm{M}^{+}-57\right), 115.1\left(\mathrm{M}^{+}-83\right), 91.1\left(\mathrm{M}^{+}-107\right) 77.1\left(\mathrm{M}^{+}-\right.$ 121).

Bibenzyl: GC-MS: $\mathrm{R}_{\mathrm{t}}=7.16 \mathrm{~min} . \mathrm{m} / \mathrm{z}: 182.1\left(\mathrm{M}^{+}\right), 91.1\left(\mathrm{M}^{+}-1\right), 104.1\left(\mathrm{M}^{+}-78\right), 91.1\left(\mathrm{M}^{+}-\right.$ 91), $77.1\left(\mathrm{M}^{+}-115\right) .65 .1\left(\mathrm{M}^{+}-117\right)$.

Benzaldehyde: GC-MS: $\mathrm{R}_{\mathrm{t}}=2.57 \mathrm{~min} . \mathrm{m} / \mathrm{z}: 106.0\left(\mathrm{M}^{+}\right), 105.0\left(\mathrm{M}^{+}-1\right), 77.1\left(\mathrm{M}^{+}-29\right), 51.0$ $\left(\mathrm{M}^{+}-55\right)$.

Toluene: GC-MS: $\mathrm{R}_{\mathrm{t}}=1.30 \mathrm{~min} . \mathrm{m} / \mathrm{z}: 92.1\left(\mathrm{M}^{+}\right), 91.1\left(\mathrm{M}^{+}-1\right), 77.1\left(\mathrm{M}^{+}-15\right), 65.1\left(\mathrm{M}^{+}-27\right)$ $51.1\left(\mathrm{M}^{+}-40\right)$.

4-Cyanopyridine: GC-MS: $\mathrm{R}_{\mathrm{t}}=2.76 \mathrm{~min} . \mathrm{m} / \mathrm{z}: 104.1\left(\mathrm{M}^{+}\right), 77.1\left(\mathrm{M}^{+}-27\right), 64.1\left(\mathrm{M}^{+}-40\right)$, $50.1\left(\mathrm{M}^{+}-54\right)$.

2-Phenyl-4-cyanopyridine: See Section 3.4.3.3.

3-Phenyl-4-cyanopyridine: See Section 3.4.3.3.

(4-Methylphenyl)-4-cyanopyridine, isomer unknown: GC-MS: $\mathrm{R}_{\mathrm{t}}=8.35 \mathrm{~min} . \mathrm{m} / \mathrm{z}$ : 194.1 $\left(\mathrm{M}^{+}\right), 179.1\left(\mathrm{M}^{+}-15\right), 166.1\left(\mathrm{M}^{+}-28\right), 139.1\left(\mathrm{M}^{+}-55\right), 115.1\left(\mathrm{M}^{+}-79\right), 91.1\left(\mathrm{M}^{+}-103\right)$.

Benzyl-4-cyanopyridine, isomer unknown: GC-MS: $\mathrm{R}_{\mathrm{t}}=8.29 \mathrm{~min} . \mathrm{m} / \mathrm{z}: 194.1\left(\mathrm{M}^{+}\right)$, $193.1\left(\mathrm{M}^{+}-15\right), 168.1\left(\mathrm{M}^{+}-26\right), 139.1\left(\mathrm{M}^{+}-55\right), 115.1\left(\mathrm{M}^{+}-79\right), 91.1\left(\mathrm{M}^{+}-103\right)$.

(4-Formylphenyl)-4-cyanopyridine, isomer unknown: GC-MS: $R_{t}=9.65 \mathrm{~min} . \mathrm{m} / \mathrm{z}$ : $208.1\left(\mathrm{M}^{+}\right), 207.1\left(\mathrm{M}^{+}-1\right), 180.1\left(\mathrm{M}^{+}-28\right), 105.1\left(\mathrm{M}^{+}-103\right), 77.1\left(\mathrm{M}^{+}-131\right)$. 
Benzoyl-4-cyanopyridine, isomer unknown: GC-MS: $\mathrm{R}_{\mathrm{t}}=9.50 \mathrm{~min} . \mathrm{m} / \mathrm{z}: 208.1\left(\mathrm{M}^{+}\right)$, $181.1\left(\mathrm{M}^{+}-27\right), 131.0\left(\mathrm{M}^{+}-77\right), 105.1\left(\mathrm{M}^{+}-103\right), 103.0\left(\mathrm{M}^{+}-105\right), 77.1\left(\mathrm{M}^{+}-131\right)$.

\subsection{References}

(1) Seiple, I. B.; Su, S.; Rodriguez, R. A.; Gianatassio, R.; Fujiwara, Y.; Sobel, A. L.; Baran, P. S. Direct C-H Arylation of Electron-Deficient Heterocycles with Arylboronic Acids. Journal of the American Chemical Society 2010, 132, 1319413196.

(2) Fujiwara, Y.; Domingo, V.; Seiple, I. B.; Gianatassio, R.; Del Bel, M.; Baran, P. S. Practical $\mathrm{C}-\mathrm{H}$ functionalization of quinones with boronic acids. Journal of the American Chemical Society 2011, 133 (10), 3292-3295.

(3) Deb, A.; Manna, S.; Maji, A.; Dutta, U.; Maiti, D. Iron-Catalyzed Direct C-H Arylation of Heterocycles and Quinones with Arylboronic Acids. European Journal of Organic Chemistry 2013, 2013 (24), 5251-5256.

(4) Deb, A.; Agasti, S.; Saboo, T.; Maiti, D. Generation of Arylated Quinones by IronCatalyzed Oxidative Arylation of Phenols: Formal Synthesis of Phellodonin, Sarcodonin \&, Leucomelone and Betulinan A. Advanced Synthesis \& Catalysis 2014, 356 (4), 705-710.

(5) Modak, A.; Rana, S.; Maiti, D. Iron-Catalyzed Regioselective Direct Arylation at the C-3 Position of N-Alkyl-2-pyridone. Journal of Organic Chemistry 2015, 80 (1), 296-303.

(6) Huang, Y.; Guan, D.; Wang, L. Direct Arylation of Substituted Pyridines with Arylboronic Acids Catalyzed by Iron(II) Oxalate. Chinese Journal of Chemistry 2014, 32 (12), 1294-1298.

(7) Komeyama, K.; Kashihara, T.; Takaki, K. FeSO 4 -promoted direct arylation of benzoquinones with $\mathrm{ArB}(\mathrm{OH})_{2}$ or $\mathrm{ArBF}_{3} \mathrm{~K}$. Tetrahedron Letters 2013, 54 (9), 10841086.

(8) Komeyama, K.; Nagao, Y.; Abe, M.; Takaki, K. Scope and Limitation for FeSO ${ }_{4}$ Mediated Direct Arylation of Heteroarenes with Arylboronic Acids and Its Synthetic Applications. Bulletin of the Chemical Society of Japan 2014, 87 (2), 301313.

(9) Singh, P. P.; Aithagani, S. K.; Yadav, M.; Singh, V. P.; Vishwakarma, R. A. Ironcatalyzed cross-coupling of electron-deficient heterocycles and quinone with organoboron species via innate $\mathrm{C}-\mathrm{H}$ functionalization: application in total synthesis 
of pyrazine alkaloid botryllazine A. Journal of Organic Chemistry 2013, 78 (6), 2639-2648.

(10) Thatikonda, T.; Singh, U.; Ambala, S.; Vishwakarma, R. A.; Singh, P. P. Metal free $\mathrm{C}-\mathrm{H}$ functionalization of diazines and related heteroarenes with organoboron species and its application in the synthesis of a CDK inhibitor, meriolin 1. Organic \& Biomolecular Chemistry 2016, 14 (18), 4312-4320.

(11) Jain, R.; Mahindra, A. Regiospecific Direct C-H Arylation at the 2-Position of 1Histidine Using Arylboronic Acids. Synlett 2012, 23 (12), 1759-1764.

(12) Bering, L.; Antonchick, A. P. Regioselective Metal-Free Cross-Coupling of Quinoline N-Oxides with Boronic Acids. Organic Letters 2015, 17 (12), 31343137.

(13) Ren, X.; Han, S.; Gao, X.; Li, J.; Zou, D.; Wu, Y.; Wu, Y. Direct arylation for the synthesis of 2-arylquinolines from $\mathrm{N}$-methoxyquinoline-1-ium tetrafluoroborate salts and arylboronic acids. Tetrahedron Letters 2018, 59 (11), 1065-1068.

(14) Li, Z.; Mai, W.; Yuan, J.; Sun, G.; Qu, L. Silver-Catalyzed 2-Pyridyl Arylation of Pyridine N-Oxides with Arylboronic Acids at Room Temperature. Synlett 2011, 2012 (01), 145-149.

(15) Wang, J.; Wang, S.; Wang, G.; Zhang, J.; Yu, X. Q. Iron-mediated direct arylation with arylboronic acids through an aryl radical transfer pathway. Chemical Communications 2012, 48 (96), 11769-11771.

(16) Patel, N. R.; Flowers, R. A., 2nd. Uncovering the mechanism of the $\operatorname{Ag}(\mathrm{I}) /$ persulfate-catalyzed cross-coupling reaction of arylboronic acids and heteroarenes. Journal of the American Chemical Society 2013, 135 (12), 46724675 .

(17) Kappe, C. O. Controlled microwave heating in modern organic synthesis. Angewandte Chemie International Edition 2004, 43 (46), 6250-6284.

(18) Kappe, C. O.; Dallinger, D. Controlled microwave heating in modern organic synthesis: highlights from the 2004-2008 literature. Molecular Diversity 2009, 13, 71-193.

(19) Kappe, C. O.; Dallinger, D.; Murphree, S. S. Practical Microwave Synthesis for Organic Chemists: Strategies, Instruments and Protocols; Wiley-VCH Verlag GmbH an Co. KGaA: Weinheim, Germany, 2009.

(20) Kappe, C. O.; Pieber, B.; Dallinger, D. Microwave effects in organic synthesis: myth or reality? Angewandte Chemie International Edition 2013, 52 (4), 10881094.

(21) Caddick, S.; Fitzmaurice, R. Microwave enhanced synthesis. Tetrahedron 2009, 65 (17), 3325-3355. 
(22) Nilsson, P.; Gold, H.; Larhed, M. Microwave Irradiation as a High-Speed Tool for Activation of Sluggish Aryl Chlorides in Grignard Reactions. Synlett 2005, 2005 (10), 1596-1600.

(23) Walla, P.; Kappe, C. O. Microwave-assisted Negishi and Kumada cross-coupling reactions of aryl chlorides. Chemical Communications 2004, (5), 564-565.

(24) Arvela, R. K.; Leadbeater, N. E. Microwave-Promoted Heck Coupling Using Ultralow Metal Catalyst Concentrations. Journal of Organic Chemistry 2005, 70, 1786-1790.

(25) Diaz-Ortiz, A.; De La Hoz, A.; Carillo, J. R.; Herrero, M. A. Chapter 5: Selectivity Modification Under Microwave Irradiation, in Microwaves in Organic Synthesis; 3 ed.; De La Hoz, A.;Loupy, A., Eds.; Wiley-VCH Verlag GmbH and Co. KGaA: Weinheim, Germany, 2013.

(26) Boeva, Z. A.; Sergeyev, V. G. Polyaniline: Synthesis, properties, and application. Polymer Science Series C 2014, 56 (1), 144-153. 


\section{Chapter 4. Conclusions and Future Work}

\subsection{Conclusions}

In the early stages of this Master's project, it was proposed to examine the direct Suzuki reaction - the two-electron $\mathrm{C}-\mathrm{H}$ coupling of arylboronic acids to arenes and $\mathrm{N}$ heteroarenes, first reported by $\mathrm{Yu}$ and coworkers (Scheme 4.1) $)^{1,2}$, under microwave heating. Interest in this system stemmed from long-standing interest in green chemistry and a desire to examine iron-catalyzed $\mathrm{C}-\mathrm{H}$ bond activation reactions. The desire was to increase the rate of reaction, expand substrate scope, examine the effects of a variety of ligands, and perhaps apply amine-bisphenolate ligands that are widely used in the Kozak group to the system. However, all reactions attempted with this system under microwave heating failed, with not even a trace of product being observed. A previous literature search had found reports of radical, iron-catalyzed, persulfate-initiated reactions using the same substrate. Given that we had already purchased reagents, and our attempts with the twoelectron system was unsuccessful, it was decided to abandon a familiar catalytic and organometallic approach and attempt these radical, ligand-free reactions under microwave heating. 

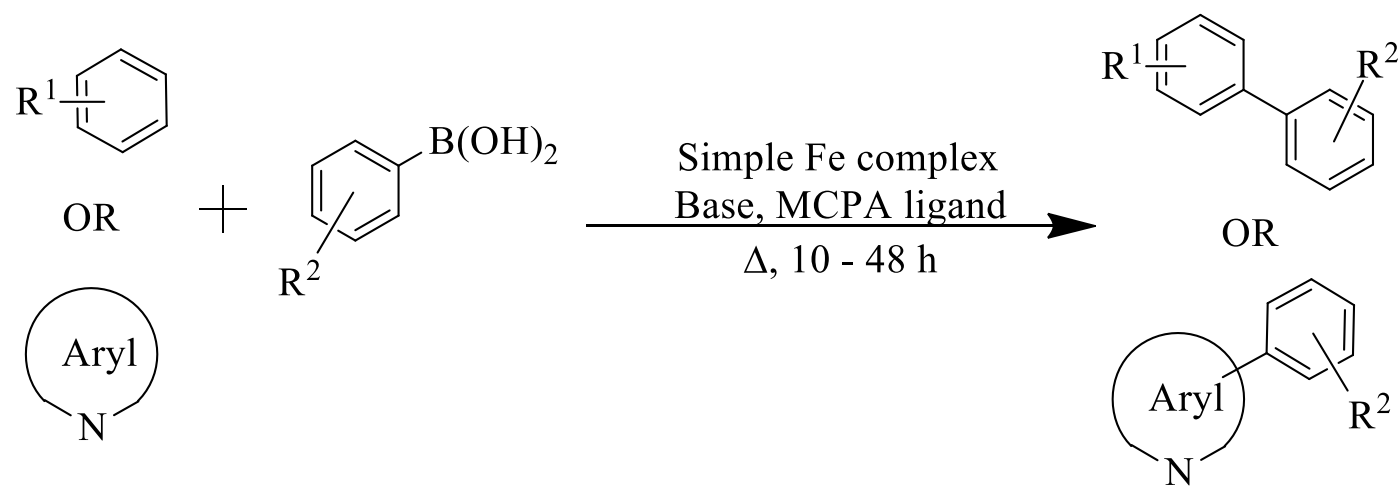

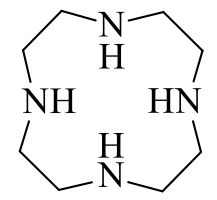

L1

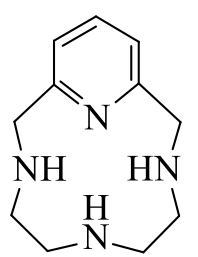

L2

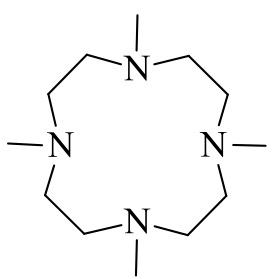

L3

Scheme 4.1 Top: The two-electron direct Suzuki reaction described by $\mathrm{Yu}$ and coworkers $^{1,2}$. Bottom: A selection of macrocyclic polyamine (MCPA) ligands examined in the reaction.

This decision immediately bore fruit, as initial reactions based on the work of Maiti and coworkers returned a yield of approximately $5 \% .^{3}$ The reaction was therefore optimized in a mixture of toluene and water, eventually reaching yields similar to those in the literature, as described in Chapter 2.2.1. However, toluene underwent several side-reactions under these optimized conditions, and some of these products proved difficult to separate from our desired product. It therefore became necessary to switch solvents to a mixture of dichloromethane and water, and reoptimized the reaction conditions. This work makes up the bulk of Chapter 2.2. After optimization of reaction conditions including solvent, time, temperature, microwave power, catalyst identity, catalyst loading, reagent stoichiometry, 
concentration, and the presence of phase transfer catalysts or other additives, a maximum yield of $70 \%$ was reached. This yield was similar to other literature reports, but was reached in only $25 \mathrm{~min}$, between 14 and 115 times faster than literature reports (See Table 2.7). In addition, the crude reaction mixture was examined with GC-MS, revealing the presence of several previously unreported side products, including hydroxylated arylboronic acid and cross-coupled pyrazine. It was proposed that these side reactions may be due to the specific heating of the aqueous layer above the $70{ }^{\circ} \mathrm{C}$ measured by the external IR sensor, or possibly due to the formation of molecular radiators or heterogeneous hotspots, both of which heat certain reaction components far above the bulk reaction temperature.

The scope of the reaction was then examined with respect to arylboronic acids, quinones, hydroquinones, and $N$-heterocycles. Contrary to our expectations and desires, the use of microwave heating did not result in an expanded substrate scope. The scope of reactions with arylboronic acids was limited by the formation of the phenol by-product when stronger electron-donating or electron-withdrawing groups were present. The scope of reactions with quinones and hydroquinones appears to be similar to the literature; attempts to extend the reaction to enones and phenols failed. The scope of reactions with other $N$-heterocycles also appears to be similar to the literature, and regiochemistry did not appear to be affected by microwave heating. Attempts to extend reaction scope to S-heteroarenes, ketones, and unactivated arenes proved unsuccessful, as did attempts at performing the reaction neat. 


\subsection{Future Work}

\subsubsection{Future Work in This Reaction}

There are several areas or discoveries presented in this thesis that may merit further investigation, ranging from simple expansions of scope to the development of side reactions. The first area would be to further investigate reactions with functionalized quinones and hydroquinones (Scheme 4.2). Several papers present cross-coupling reactions with benzoquinone bearing a variety of functional groups, including alkyl, halide, alkoxy, and cyano groups, as well as naphthoquinones. These functionalized benzoquinone moieties may have wider applications and be more synthetically useful than unfunctionalized benzoquinone. These reactions were not explored in this thesis due to time constraints. Additionally, this thesis is only the second report of the oxidation and coupling of a substituted hydroquinone. ${ }^{4}$ It is unclear whether previous reports that successfully coupled 1,4-hydroquinone did not extend the reaction scope because they were unable to, or simply because of lack of interest. In any event, this area remains underdeveloped, and further reactions with substituted hydroquinones may be worth pursuing.<smiles>[R]c1ccc(O)c(O)c1</smiles>

OR<smiles>[R][R]1cccc(=O)cc1</smiles>

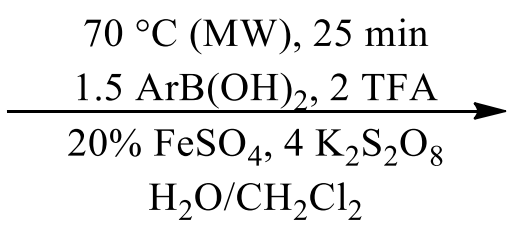<smiles>[Y8]C1=CC(=O)C([R])CC1=O</smiles>

Scheme 4.2 Proposed reaction to further examine the scope of the reaction with regards to substituted quinones and hydroquinones. 
Second, further studies may wish to examine the use of aryltrifluoroborate salts in place of arylboronic acids. A number of previous studies have examined the use of aryltrifluoroborate salts and found that they have similar activity to arylboronic acids. ${ }^{4-8}$ However, aryltrifluoroborate salts are water-soluble, and there are reports of successful reactions with aryltrifluoroborate salts in the absence of an organic solvent. ${ }^{5}$ Under conventional heating methods, this is unlikely to make much of a difference. However, under microwave heating, this could have several effects. First, if the aryltrifluoroborate salts are fully solubilized in water, the proposed formation of heterogeneous hotspots might be avoided. Secondly, this would remove mass transfer considerations from the reaction, and allow all reagents to interact directly.

Large-scale reactions might yield positive results for a similar reason. First, there are several reports that these reactions under conventional heating attain higher yields at larger scales. ${ }^{5,69}$ Second, there are reports that reactions with certain reagents at higher scales may be done without an additional organic solvent; this would, again, remove any mass transfer considerations from the reaction..$^{5}$ Third, and perhaps most significantly, large-scale reactions would allow a reduction in reaction concentration. If, as we suspect, side reactions are occurring due to the presence of heterogeneous hot-spots, lowering the reaction concentration may reduce the yield and variety of side products formed and increase yields of the desired product. In addition, if reactions performed at lower concentrations, or in aqueous solution, curtail the formation of the phenol product, it may be possible to further expand the scope of the reaction with respect to arylboronic acids, $\mathrm{N}$ heteroarenes, and perhaps even other compounds. 
Finally, any future work conducted using a biphasic mixture of dichloromethane and water, or a similar mixture of organic and aqueous phases, may wish to further examine the method of heating in the reaction mixture. Biphasic mixtures in microwave heating are relatively rare, and temperatures can be difficult to measure in such cases. In the Biotage Initiator microwave used in this work, temperature is measured via a single external IR sensor located approximately one-third of the way up the vial. ${ }^{10}$ The recorded temperature of $70{ }^{\circ} \mathrm{C}$ is therefore most likely a measure of the temperature of the denser dichloromethane layer, or perhaps a measurement of the temperature at or near the interface. Despite the vigorous stirring applied, there is likely to be a significant temperature gradient between the two phases, as water is significantly better at absorbing microwave energy than dichloromethane. ${ }^{11}$ Regrettably, measuring this temperature gradient can be quite difficult, as the vial must be fully ensconced in the microwave cavity, and is being heated above atmospheric pressure. Unfortunately, most thermocouples cannot be used, as they effect the way in which the reaction is heated, and may not be reliable when exposed to microwave irradiation. ${ }^{10}$ Most commonly, internal fibre-optic sensors are used to measure the reaction temperature. ${ }^{10}$ Unfortunately, fibre-optic sensors are quite expensive, and may be difficult or impossible to use with the Biotage Initiator microwave, which closes a "blast hatch" over the top of the vial before irradiating a sample for safety reasons.

\subsubsection{Microwave Heating in Other Reactions}

Several of the side reactions reported in this thesis might warrant further investigation. The homo-coupling of arylboronic acids has been documented extensively, 
as it is a common side product of Suzuki reactions, and probably does not warrant further investigation. ${ }^{12-14}$ However, it may be possible to cross-couple two different arylboronic acids if they have substituents with varying electronic properties (Scheme 4.3), and this reaction may be of interest. The oxidation of boronic acids to the corresponding phenol has been reported several times, and has been performed under catalyst-free, and more environmentally benign, conditions. ${ }^{15-19}$ However, these reactions generally require basic conditions; this appears to be the first report of arylboronic acid oxidation to phenols under acidic conditions. The development of reaction under acidic conditions may be of interest. By far the most interesting side-reaction, however, is the previously unobserved homocoupling of pyrazine. Nitrogen bi-heteroarenes are common in biological systems, and more extensive conjugated heteroarenes are common in biological systems, electrochemistry, and the dye industry. There have recently been two reports of a CDC homo-coupling or cross-coupling of unactivated $N$-heteroarenes. ${ }^{20,21}$ One report required a palladium catalyst ${ }^{20}$, the other an iridium catalyst ${ }^{21}$; both reactions required high temperatures $\left(125-150{ }^{\circ} \mathrm{C}\right)$, long reaction times (16 -30 h), and nitrogen atmospheres. There do not appear to be any reports of iron-catalyzed homo-coupling or cross-coupling of unactivated $N$-heteroarenes. However, it is currently unclear why other $N$-heteroarenes, including pyridazine, an isomer of pyrazine, did not undergo homo-coupling. Future studies may wish to optimize the reaction to favor homo-coupling, attempt to homo-couple other $\mathrm{N}$-heteroarenes under these new conditions, or even attempt to cross-couple $\mathrm{N}$-heteroarenes with different electronic properties (Scheme 4.4). 


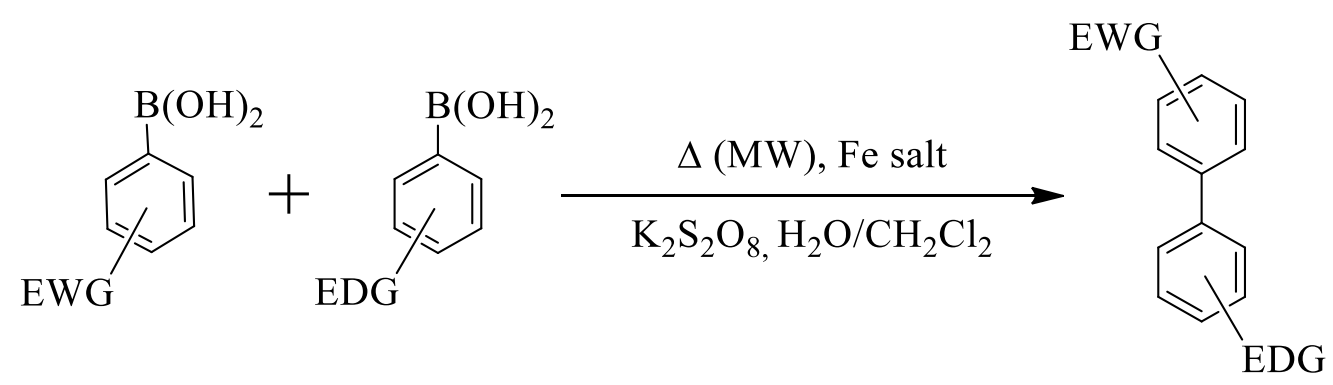

Scheme 4.3 Proposed reaction to further examine the scope of the reaction with regards to substituted quinones and hydroquinones.

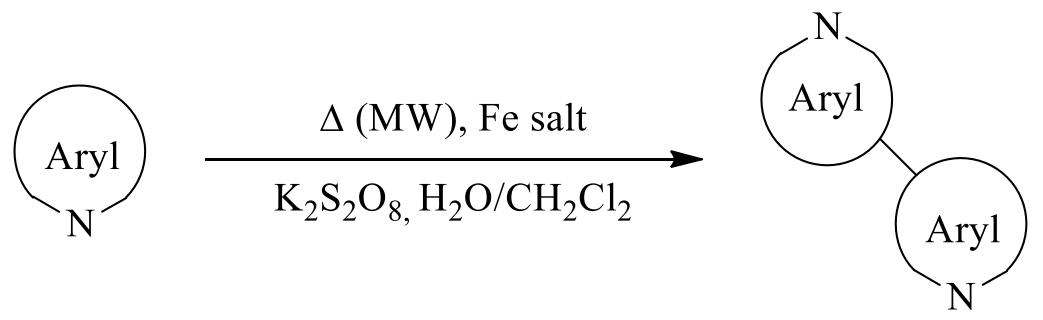

Scheme 4.4 Proposed homo-coupling or cross-coupling reaction of $N$-heteroarenes.

Finally, as described in Section 3.2.4, attempts to perform a CDC reaction between toluene and quinones or $N$-heteroaryls were attempted. Attempts with quinones failed, as quinone acts as an oxidant, returning a mixture of bibenzyl, benzyl alcohol, benzaldehyde, and benzoic acid. However, reactions with 4-cyanopyridine did return a trace amount of product coupled at the benzylic position (Scheme 3.6). This reaction was not optimized due to a lack of time. Further attempts to optimize this reaction, including examining other catalyst systems and oxidizing agents, should be attempted. In addition, reactions may be more successful with a reagent other than toluene. It might therefore be worth attempting 
reactions with reagents that are substituted at the benzylic position, such as diphenylmethane, vinylbenzene, benzyl alcohol, or benzaldehyde.

More generally, this thesis has demonstrated the utility of microwave heating in iron-catalyzed radical $\mathrm{C}-\mathrm{H}$ activating cross-coupling reactions. There are a several similar reactions that might benefit from exploration under microwave heating. For example, there have been several descriptions of iron-catalyzed or metal-free cross-coupling of unactivated arenes with aryl halides, forming biaryl species (Scheme 4.5). ${ }^{22-25}$ These reactions require relatively harsh conditions (strong organic base, $80-180{ }^{\circ} \mathrm{C}$, under nitrogen), are often limited to aryl iodides or aryl bromides, and require long reaction times, often 24 or more $h .{ }^{22-25}$ Another possible reaction of interest might be the dimerization of terminal alkynes, which require similar conditions and can sometimes yield a mixture of products (Scheme 4.5). ${ }^{26,27}$ There are also a number of iron-catalyzed radical amination reactions that may be worth investigation under microwave heating. ${ }^{28}$ Finally, it may be worth investigating the "holy grail" of iron-catalyzed reactions - an iron-catalyzed biaryl Suzuki reaction. There has been considerable research into iron-catalyzed Suzuki reactions, and there are several examples of reactions with alkyl halides. ${ }^{29-33}$ Only very recently has the first biaryl iron-catalyzed Suzuki reaction been reported. ${ }^{34,35}$ However, such reactions usually required harsh bases, magnesium bromide salt, pinacolborane or pinacolboronate esters, and often long reaction times. ${ }^{29-35}$ To the best of my knowledge, these reactions have not been attempted under microwave heating. 


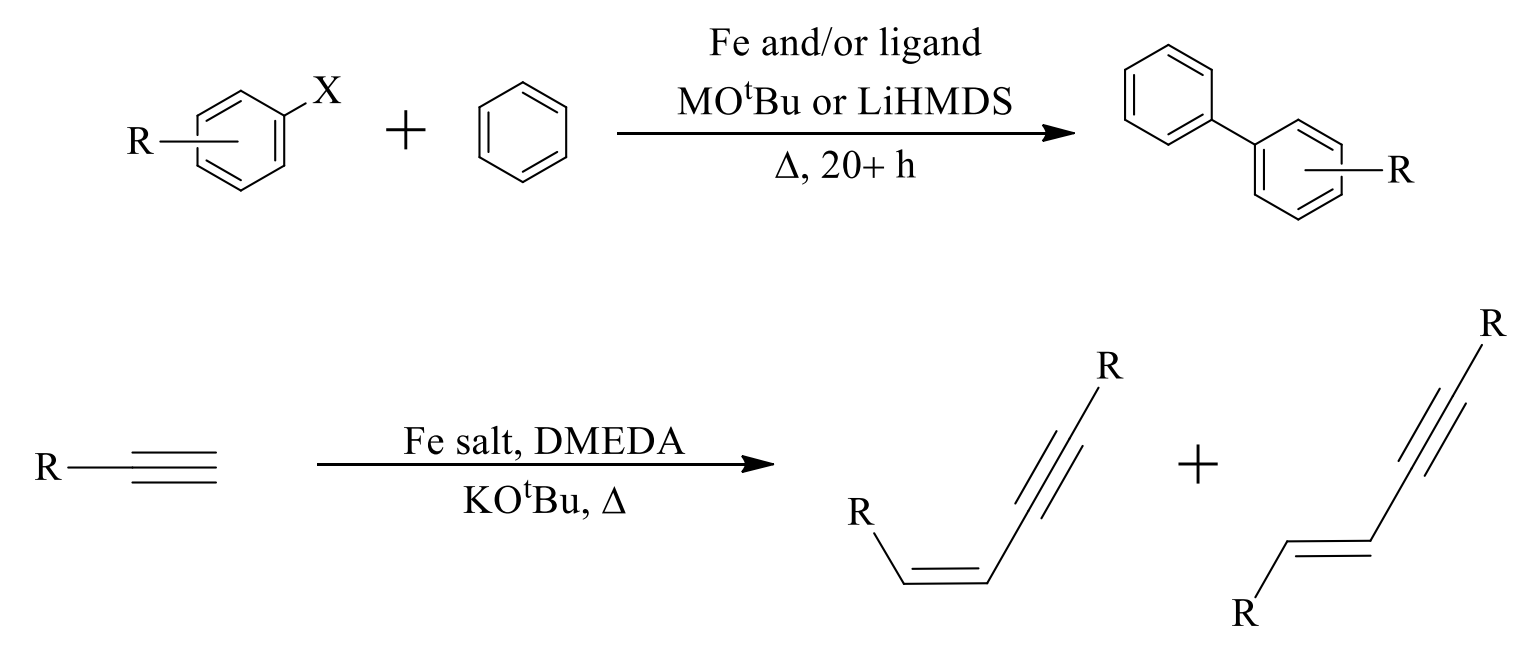

Scheme 4.5 Top: Iron-catalyzed radical cross-coupling of aryl halides and unactivated arenes. Bottom: Radical iron-catalyzed dimerization of terminal alkynes.

\subsection{References}

(1) Wen, J.; Zhang, J.; Chen, S. Y.; Li, J.; Yu, X. Q. Iron-mediated direct arylation of unactivated arenes. Angewandte Chemie International Edition 2008, 47 (46), 88978900 .

(2) Jun Wen, S. Q., Li-Fang Ma, Liang Dong, Ji Zhang, Shan-Shan Liu, Yi-Shu Duan, Shan-Yong Chen, Cheng-Wei Hu, Xiao-Qi Yu. Iron-Mediated Direct SuzukiMiyaura Reaction: A New Method for the ortho-Arylation of Pyrrole and Pyridine. Organic Letters 2010, 12 (12), 2694-2697.

(3) Deb, A.; Manna, S.; Maji, A.; Dutta, U.; Maiti, D. Iron-Catalyzed Direct C-H Arylation of Heterocycles and Quinones with Arylboronic Acids. European Journal of Organic Chemistry 2013, 2013 (24), 5251-5256.

(4) Komeyama, K.; Kashihara, T.; Takaki, K. FeSO 4 -promoted direct arylation of benzoquinones with $\mathrm{ArB}(\mathrm{OH})_{2}$ or $\mathrm{ArBF}_{3} \mathrm{~K}$. Tetrahedron Letters 2013, 54 (9), 1084 1086.

(5) Fujiwara, Y.; Domingo, V.; Seiple, I. B.; Gianatassio, R.; Del Bel, M.; Baran, P. S. Practical $\mathrm{C}-\mathrm{H}$ functionalization of quinones with boronic acids. Journal of the American Chemical Society 2011, 133 (10), 3292-3295.

(6) Komeyama, K.; Nagao, Y.; Abe, M.; Takaki, K. Scope and Limitation for FeSO ${ }_{4}$ Mediated Direct Arylation of Heteroarenes with Arylboronic Acids and Its 
Synthetic Applications. Bulletin of the Chemical Society of Japan 2014, 87 (2), 301313.

(7) Thatikonda, T.; Singh, U.; Ambala, S.; Vishwakarma, R. A.; Singh, P. P. Metal free $\mathrm{C}-\mathrm{H}$ functionalization of diazines and related heteroarenes with organoboron species and its application in the synthesis of a CDK inhibitor, meriolin 1. Organic \& Biomolecular Chemistry 2016, 14 (18), 4312-4320.

(8) Singh, P. P.; Aithagani, S. K.; Yadav, M.; Singh, V. P.; Vishwakarma, R. A. Ironcatalyzed cross-coupling of electron-deficient heterocycles and quinone with organoboron species via innate $\mathrm{C}-\mathrm{H}$ functionalization: application in total synthesis of pyrazine alkaloid botryllazine A. Journal of Organic Chemistry 2013, 78 (6), 2639-2648.

(9) Seiple, I. B.; Su, S.; Rodriguez, R. A.; Gianatassio, R.; Fujiwara, Y.; Sobel, A. L.; Baran, P. S. Direct C-H Arylation of Electron-Deficient Heterocycles with Arylboronic Acids. Journal of the American Chemical Society 2010, 132, 1319413196.

(10) Kappe, C. O. How to measure reaction temperature in microwave-heated transformations. Chemical Society Reviews 2013, 42 (12), 4977-4990.

(11) Kappe, C. O. Controlled microwave heating in modern organic synthesis. Angewandte Chemie International Edition 2004, 43 (46), 6250-6284.

(12) Adamo, C.; Amatore, C.; Ciofini, I.; Jutand, A.; Lakmini, H. Mechanism of the Palladium-Catalyzed Homocoupling of Arylboronic Acids: Key Involvement of a Palladium Peroxo Complex. Journal of the American Chemical Society 2006, 128, 6829-6836.

(13) Parrish, J. P.; Jung, Y. C.; Floyd, R. J.; Jung, K. W. Oxidative dimerization: Pd(II) catalysis in the presence of oxygen using aqueous media. Tetrahedron Letters $\mathbf{2 0 0 2}$, 43, 7899-7902.

(14) Smith, K. A.; Campi, E. M.; Jackson, R. W.; Marcuccio, S.; Naeslund, C. G. M.; Deacon, G. B. High Yields of Symmetrical Biaryls from Palladium Catalysed Homocoupling of Arylboronic Acids under Mild Conditions. Synlett 1997, 1, 131132.

(15) Cammidge, A. N.; Goddard, V. H. M.; Schubert, C. P. J.; Gopee, H.; Hughes, D. L.; Gonzalez-Lucas, D. Unexpected Phenol Production from Arylboronic Acids under Palladium-Free Conditions; Organocatalyzed Air Oxidation. Organic Letters 2011, 13 (22), 6034-6037.

(16) Webb, K. S.; Levy, D. A Facile Oxidation of Boronic Acids and Boronic Esters. Tetrahedron Letters 1995, 36 (29), 5117-5118.

(17) Zhu, C.; Wang, R.; Falck, J. R. Mild and rapid hydroxylation of aryl/heteroaryl boronic acids and boronate esters with N-oxides. Organic Letters 2012, 14 (13), 3494-3497. 
(18) Molloy, J. J.; Clohessy, T. A.; Irving, C.; Anderson, N. A.; Lloyd-Jones, G. C.; Watson, A. J. B. Chemoselective oxidation of aryl organoboron systems enabled by boronic acid-selective phase transfer. Chemical Science 2017, 8 (2), 1551-1559.

(19) Wagh, R. B.; Nagarkar, J. M. Facile and effective approach for oxidation of boronic acids. Tetrahedron Letters 2017, 58 (48), 4572-4575.

(20) Chen, X. W.; Zhao, H.; Xiong, B.; Jiang, H. F.; Dixneuf, P. H.; Zhang, M. Selective synthesis of nitrogen bi-heteroarenes by a hydrogen transfer-mediated direct $\alpha, \beta-$ coupling reaction. Organic \& Biomolecular Chemistry 2017, 15 (29), 6093-6097.

(21) Chen, C.; Chen, X.; Zhao, H.; Jiang, H.; Zhang, M. Direct Access to Nitrogen Biheteroarenes via Iridium-Catalyzed Hydrogen-Evolution Cross-Coupling Reaction. Organic Letters 2017, 19 (13), 3390-3393.

(22) Huang, Y.; Moret, M.-E.; Klein Gebbink, R. J. M. Iron-Mediated Direct Arylation of Unactivated Arenes in Air. European Journal of Organic Chemistry 2014, 2014 (18), 3788-3793.

(23) Liu, W. C., Hao; Zhang, Hua; Zhang, Heng; Chung, Kin Ho; He, Chuan; Wang, Haibo; Kwong, Fuk Yee; Lei, Aiwen. Organocatalysis in Cross-Coupling: DMEDA-Catalyzed Direct C-H Arylation of Unactivated Benzene. Journal of the American Chemical Society 2010, 132 (47), 16737-16740.

(24) Liu, W.; Cao, H.; Lei, A. Iron-catalyzed direct arylation of unactivated arenes with aryl halides. Angewandte Chemie International Edition 2010, 49 (11), 2004-2008.

(25) Vallee, F. M., James J; Charette, Andre B. Iron-Catalyzed Direct Arylation through an Aryl Radical Transfer Pathway. Journal of the American Chemical Society 2010, $132(5), 1514-1516$.

(26) Liang, Q.; Osten, K. M.; Song, D. Iron-Catalyzed gem-Specific Dimerization of Terminal Alkynes. Angewandte Chemie International Edition 2017, 56 (22), 6317 6320 .

(27) Midya, G. C.; Paladhi, S.; Dhara, K.; Dash, J. Iron catalyzed highly regioselective dimerization of terminal aryl alkynes. Chemical Communications 2011, 47 (23), 6698-6700.

(28) Ghorai, S. K.; Gopalsamuthiram, V. G.; Jawalekar, A. M.; Patre, R. E.; Pal, S. Iron catalyzed C N bond formation. Tetrahedron 2017, 73 (14), 1769-1794.

(29) Bedford, R. B.; Brenner, P. B.; Carter, E.; Carvell, T. W.; Cogswell, P. M.; Gallagher, T.; Harvey, J. N.; Murphy, D. M.; Neeve, E. C.; Nunn, J.et al. Expedient iron-catalyzed coupling of alkyl, benzyl and allyl halides with arylboronic esters. Chemistry: a European Journal 2014, 20 (26), 7935-7938.

(30) Nakajima, S.; Takaya, H.; Nakamura, M. Iron-catalyzed Methylation of Arylboron Compounds with Iodomethane. Chemistry Letters 2017, 46 (5), 711-714.

(31) Daifuku, S. L.; Kneebone, J. L.; Snyder, B. E.; Neidig, M. L. Iron(II) Active Species in Iron-Bisphosphine Catalyzed Kumada and Suzuki-Miyaura Cross-Couplings of 
Phenyl Nucleophiles and Secondary Alkyl Halides. Journal of the American Chemical Society 2015, 137 (35), 11432-11444.

(32) Bedford, R.; Gallagher, T.; Pye, D.; Savage, W. Towards Iron-Catalysed Suzuki Biaryl Cross-Coupling: Unusual Reactivity of 2-Halobenzyl Halides. Synthesis 2015, 47 (12), 1761-1765.

(33) Iwamoto, T.; Okuzono, C.; Adak, L.; Jin, M.; Nakamura, M. Iron-catalysed enantioselective Suzuki-Miyaura coupling of racemic alkyl bromides. Chemical Communications 2019, 55 (8), 1128-1131.

(34) Wu, G.; Jacobi von Wangelin, A. Iron-catalysed Suzuki biaryl couplings. Nature Catalysis 2018, 1 (6), 377-378.

(35) O'Brien, H. M.; Manzotti, M.; Abrams, R. D.; Elorriaga, D.; Sparkes, H. A.; Davis, S. A.; Bedford, R. B. Iron-catalysed substrate-directed Suzuki biaryl crosscoupling. Nature Catalysis 2018, 1 (6), 429-437. 


\section{Chapter 5. Appendices}

Figure A1 ${ }^{1} \mathrm{H}$ NMR spectrum of 2-phenylpyrazine

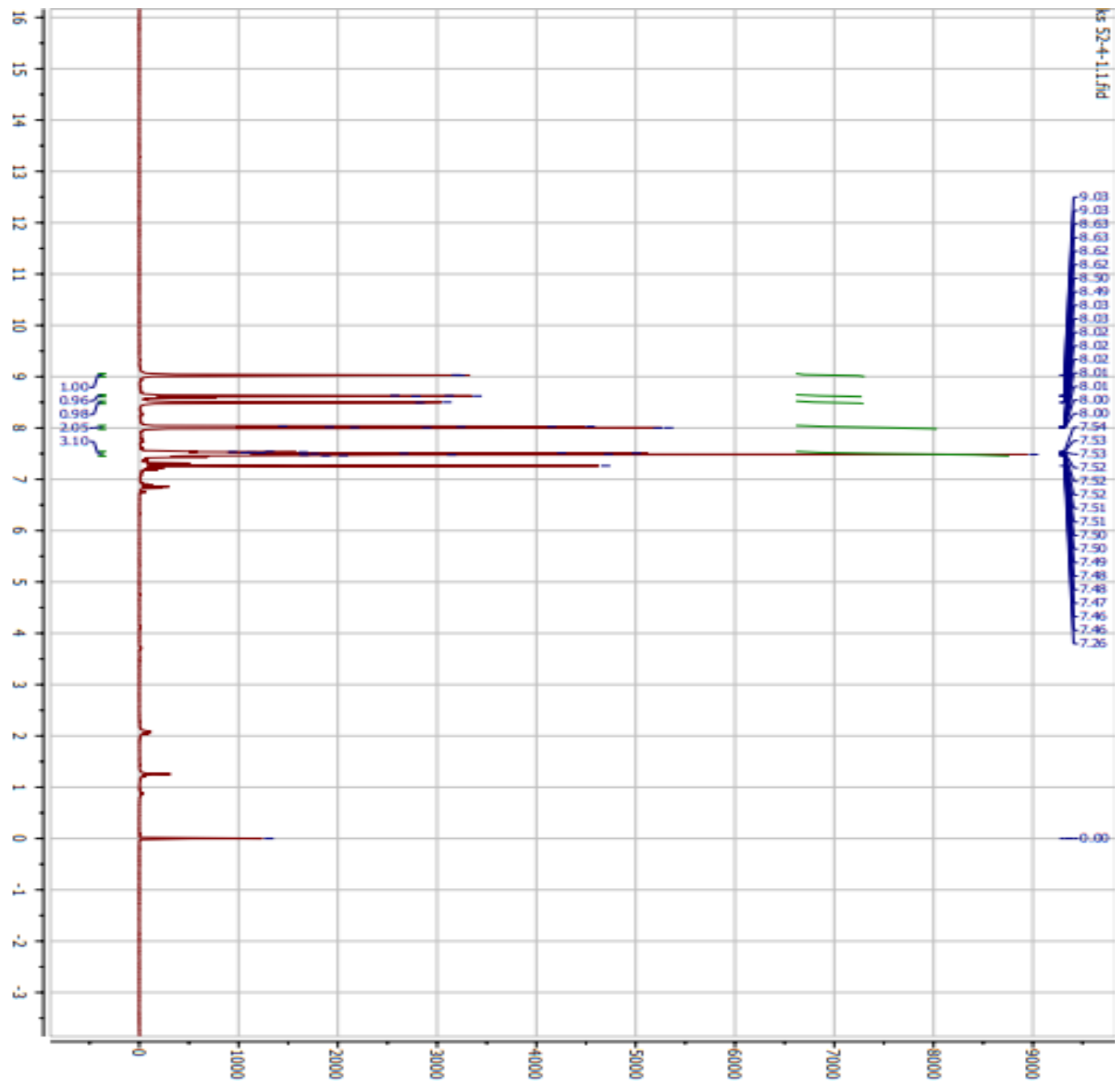


Figure A2 ${ }^{13} \mathrm{C}$ NMR spectrum of 2-phenylpyrazine

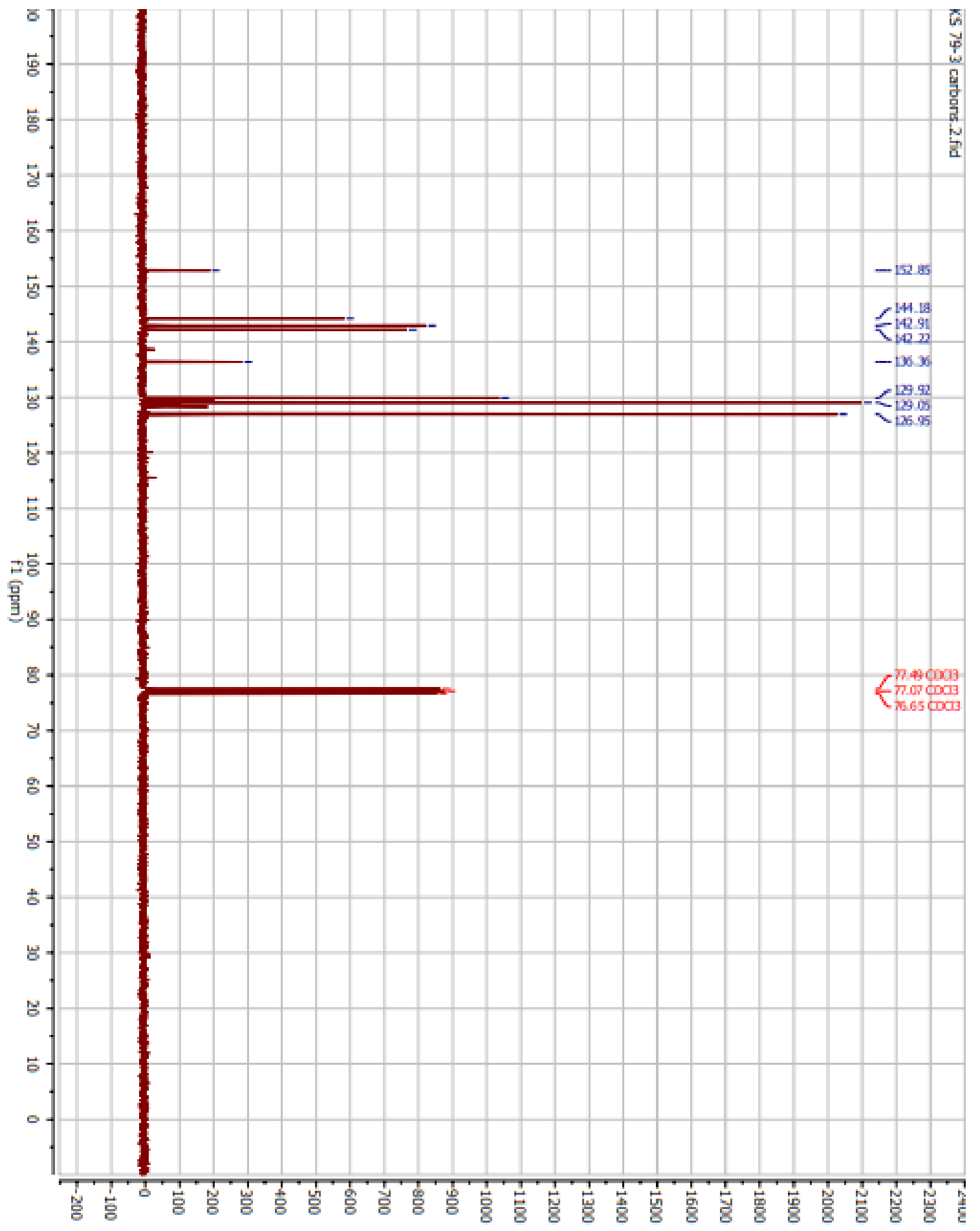


Figure A3 DEPT NMR spectrum of 2-phenylpyrazine

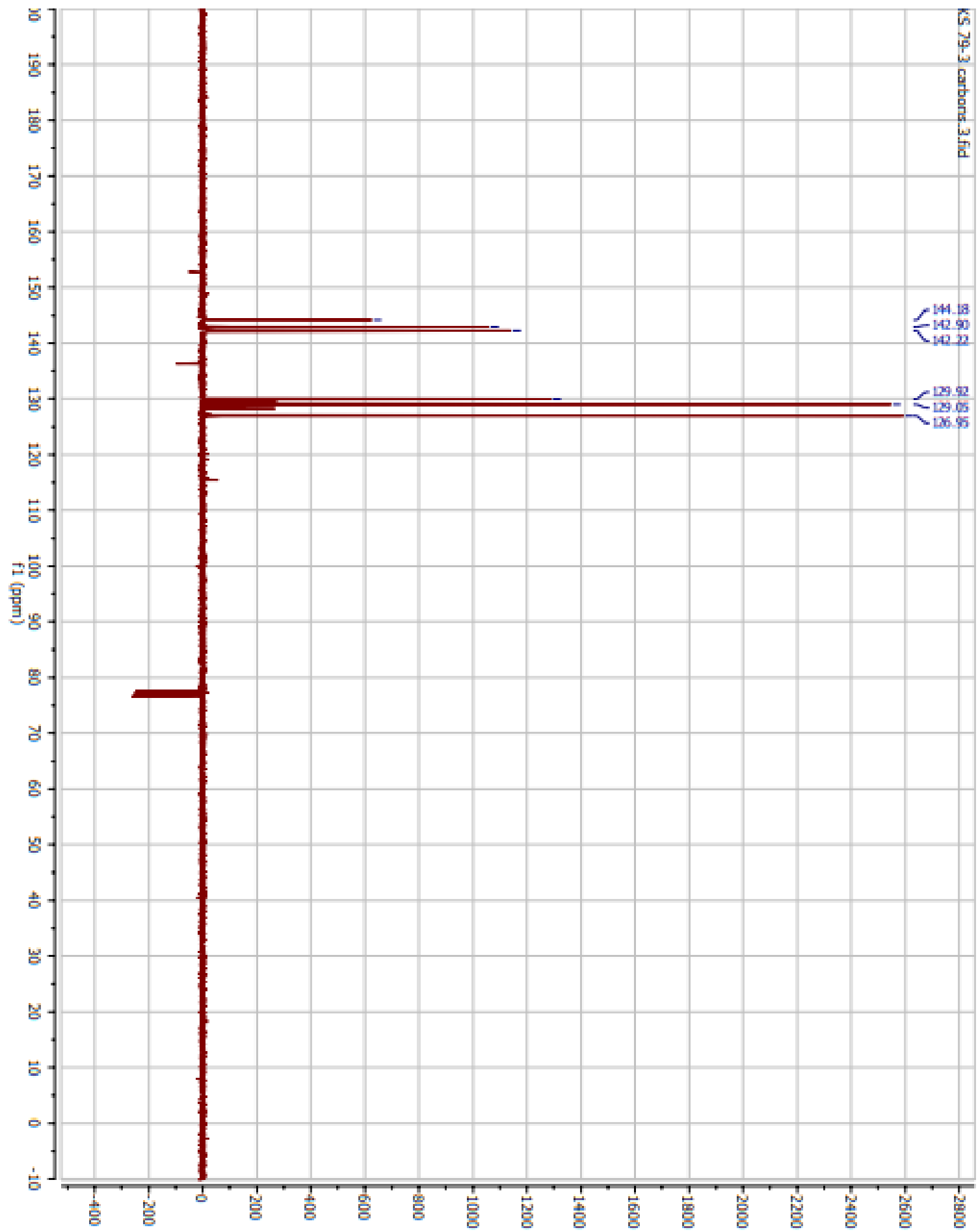


Figure A4 Gas chromatogram and mass spectrum of 2-phenylpyrazine

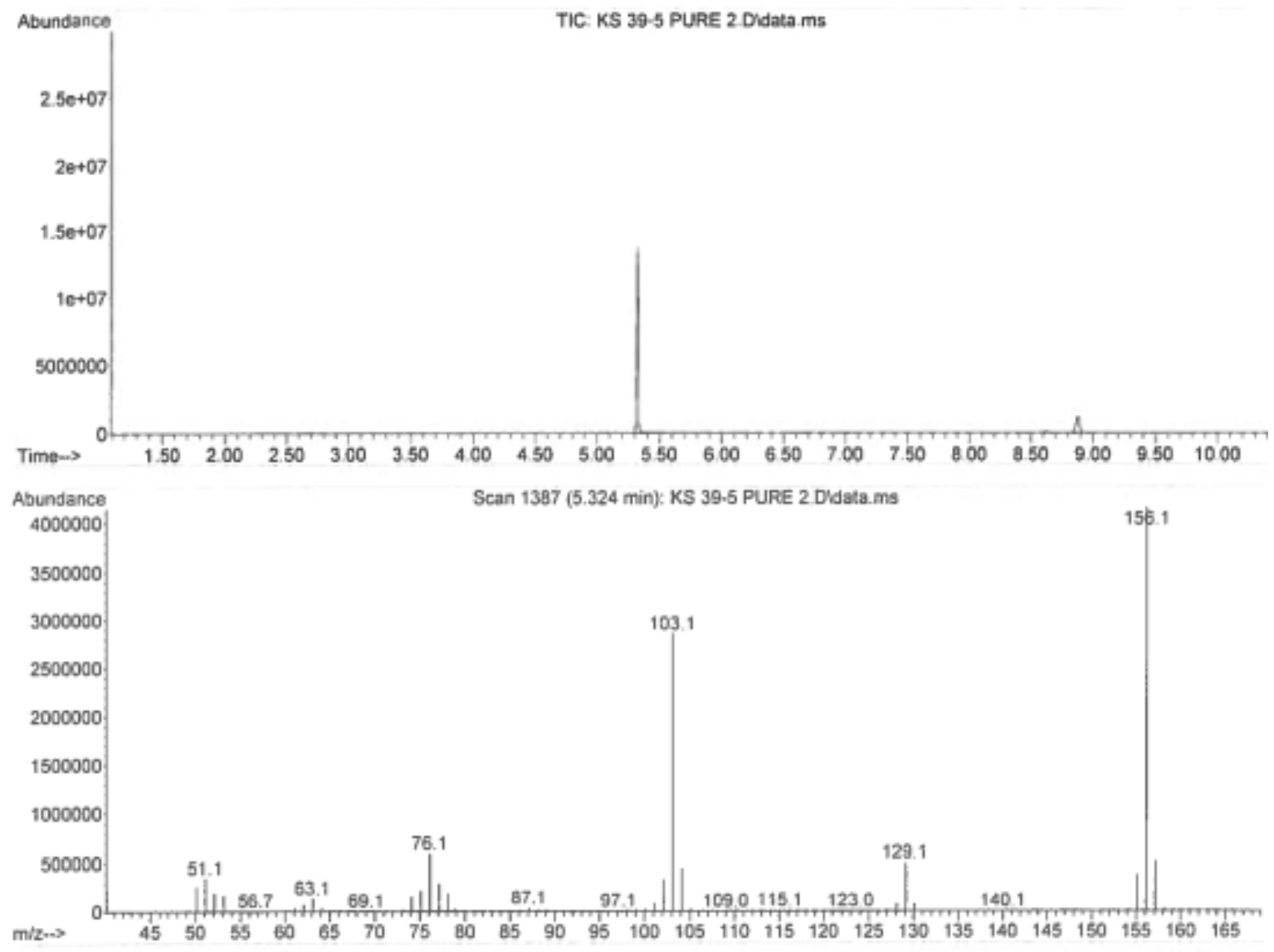


Figure A5 ${ }^{1} \mathrm{H}$ NMR spectrum of 2-(4-methylphenyl)pyrazine

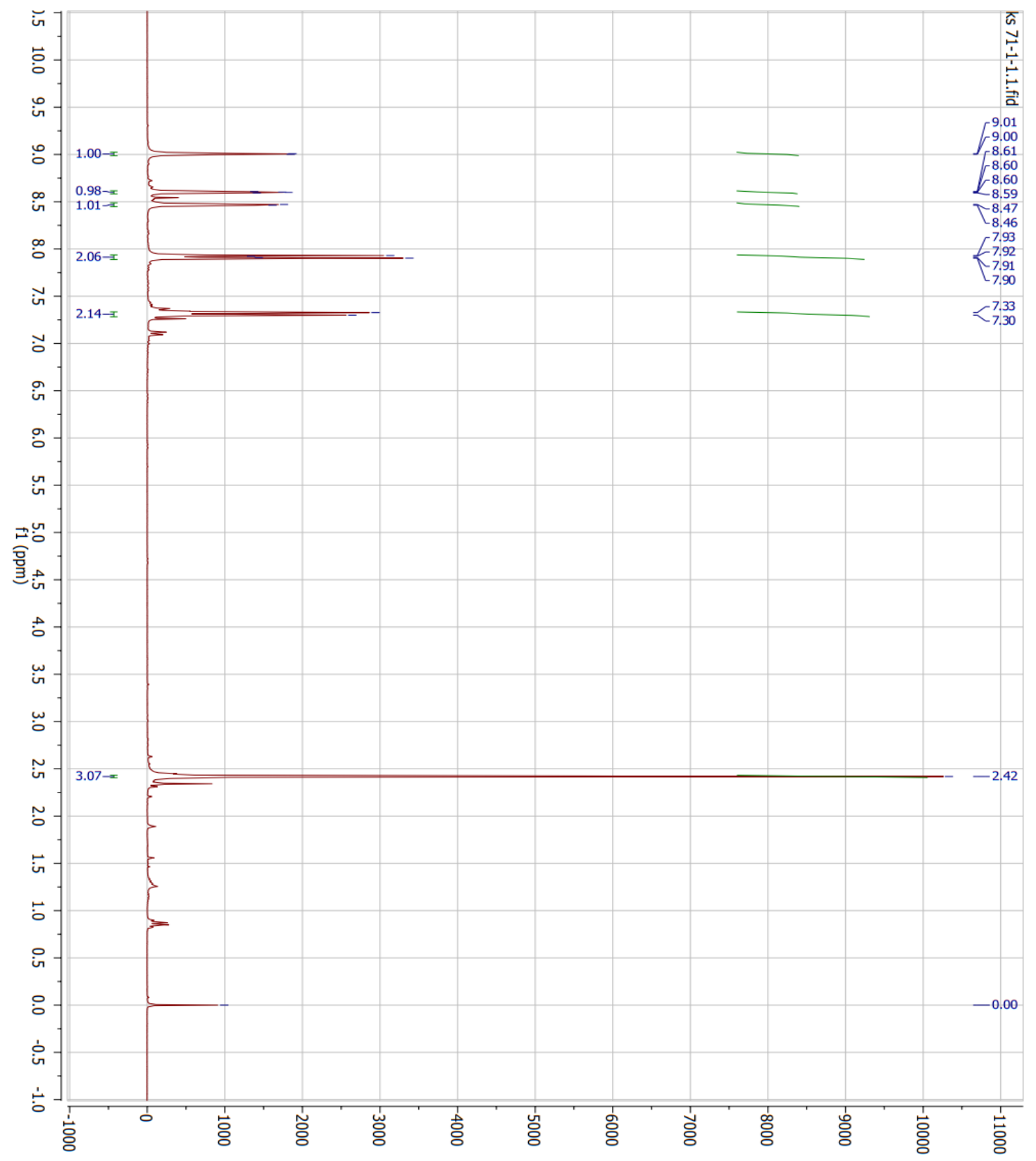


Figure $\mathbf{A 6}{ }^{13} \mathrm{C}$ NMR spectrum of 2-(4-methylphenyl)pyrazine

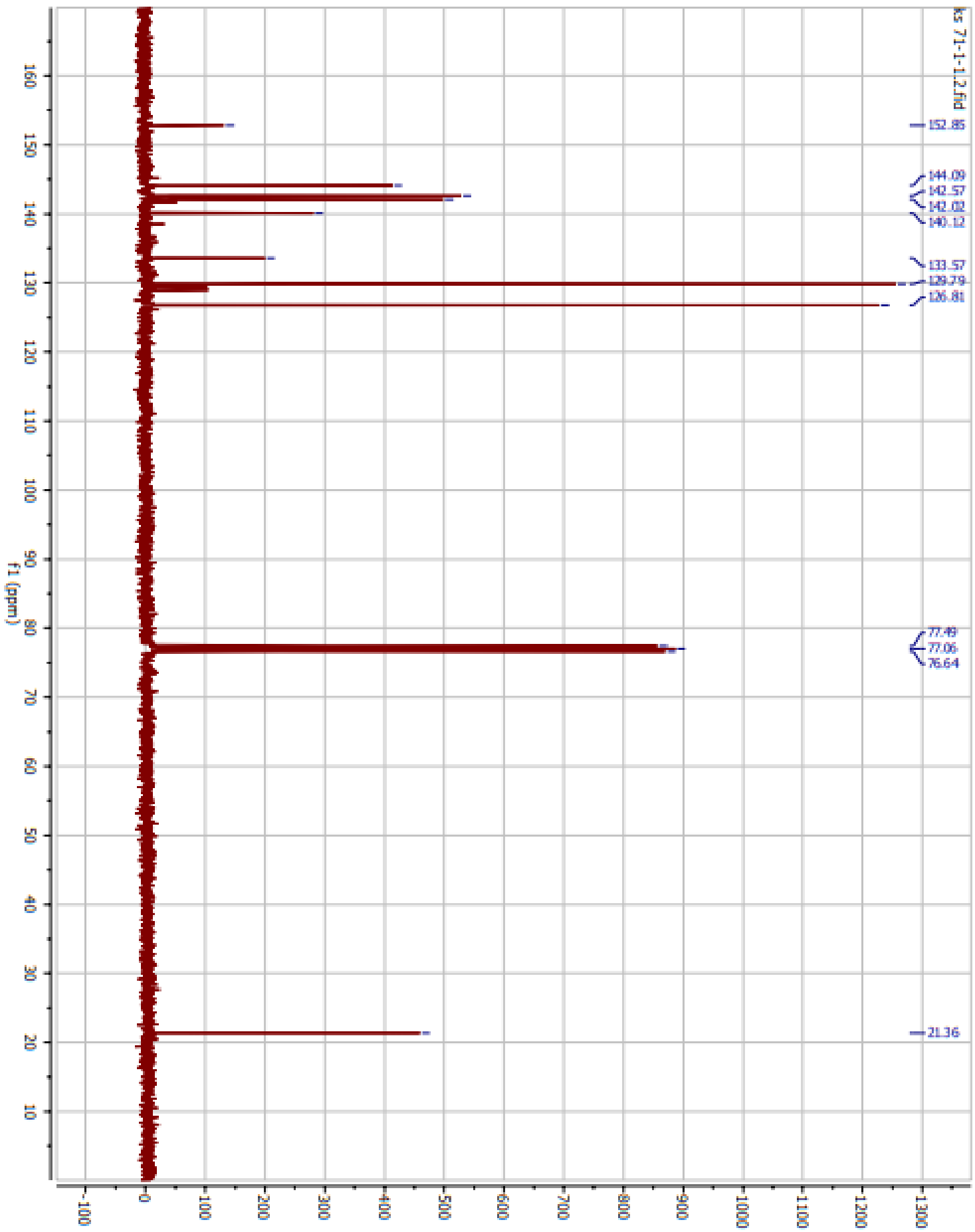


Figure A7 DEPT NMR spectrum of 2-(4-methylphenyl)pyrazine

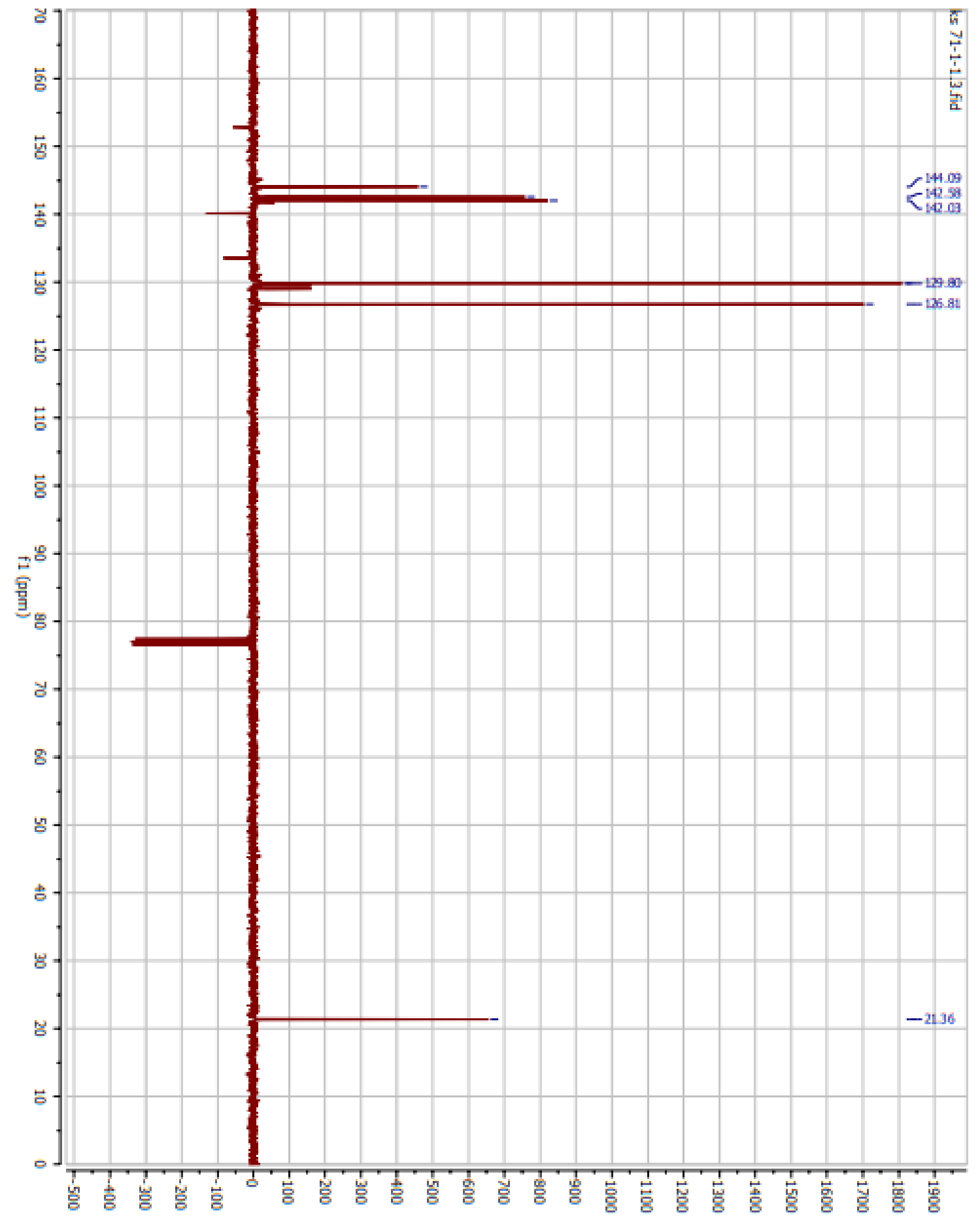


Figure A8 Gas chromatogram and mass spectrum of 2-(4-methylphenyl)pyrazine

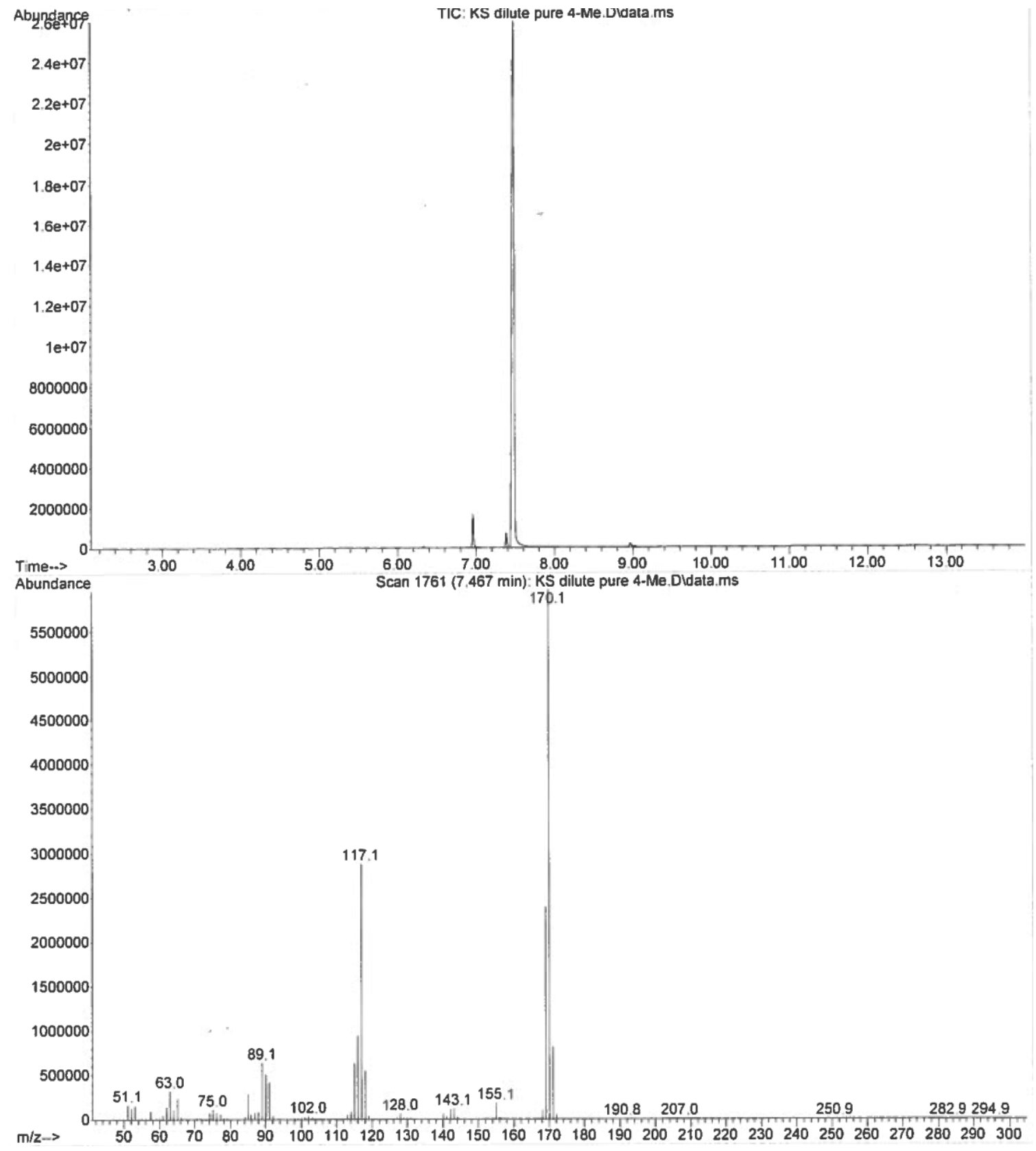


Figure A9 ${ }^{1} \mathrm{H}$ NMR spectrum of 2-(3-methylphenyl)pyrazine

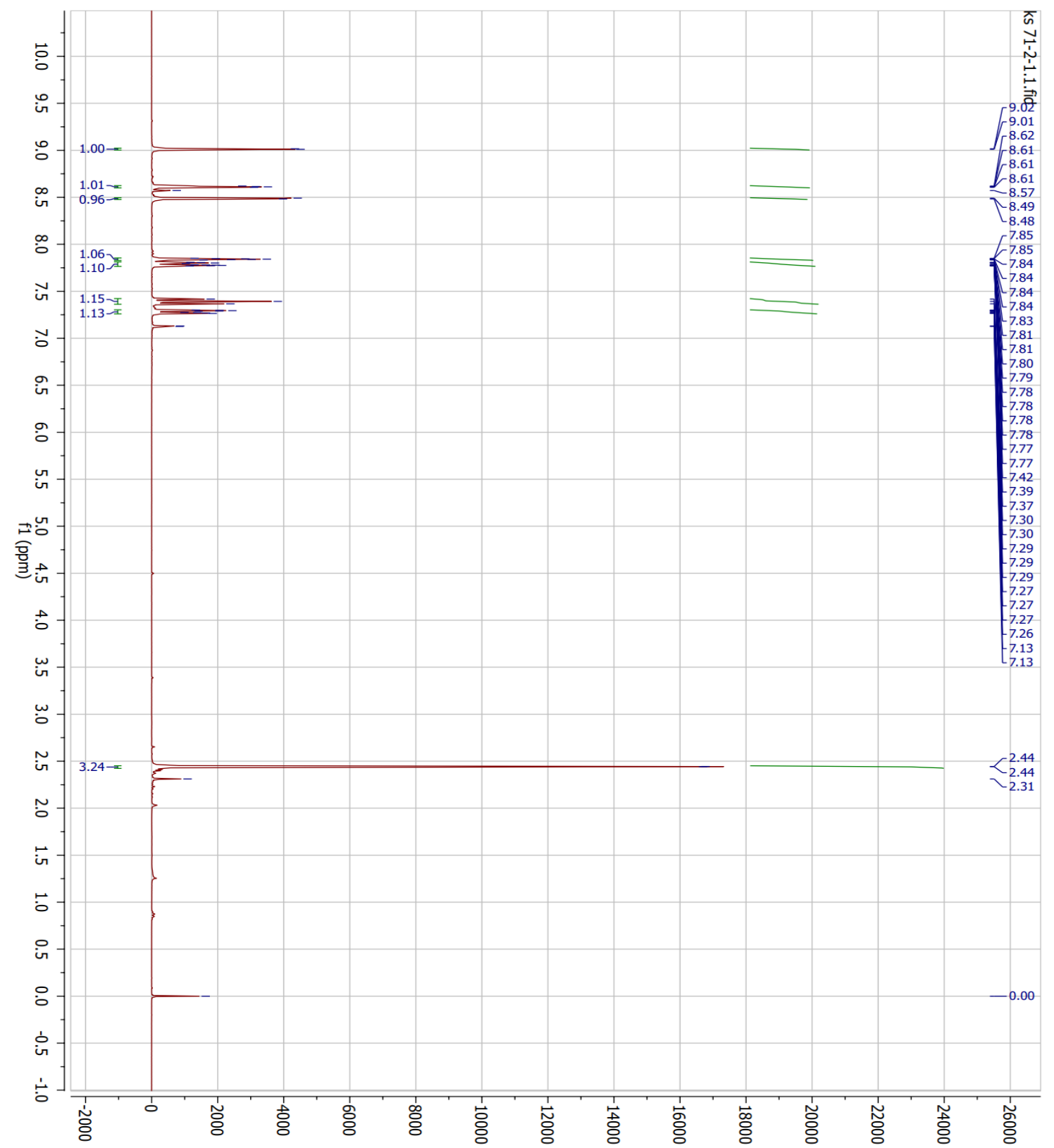


Figure A10 ${ }^{13} \mathrm{C}$ NMR spectrum of 2-(3-methylphenyl)pyrazine

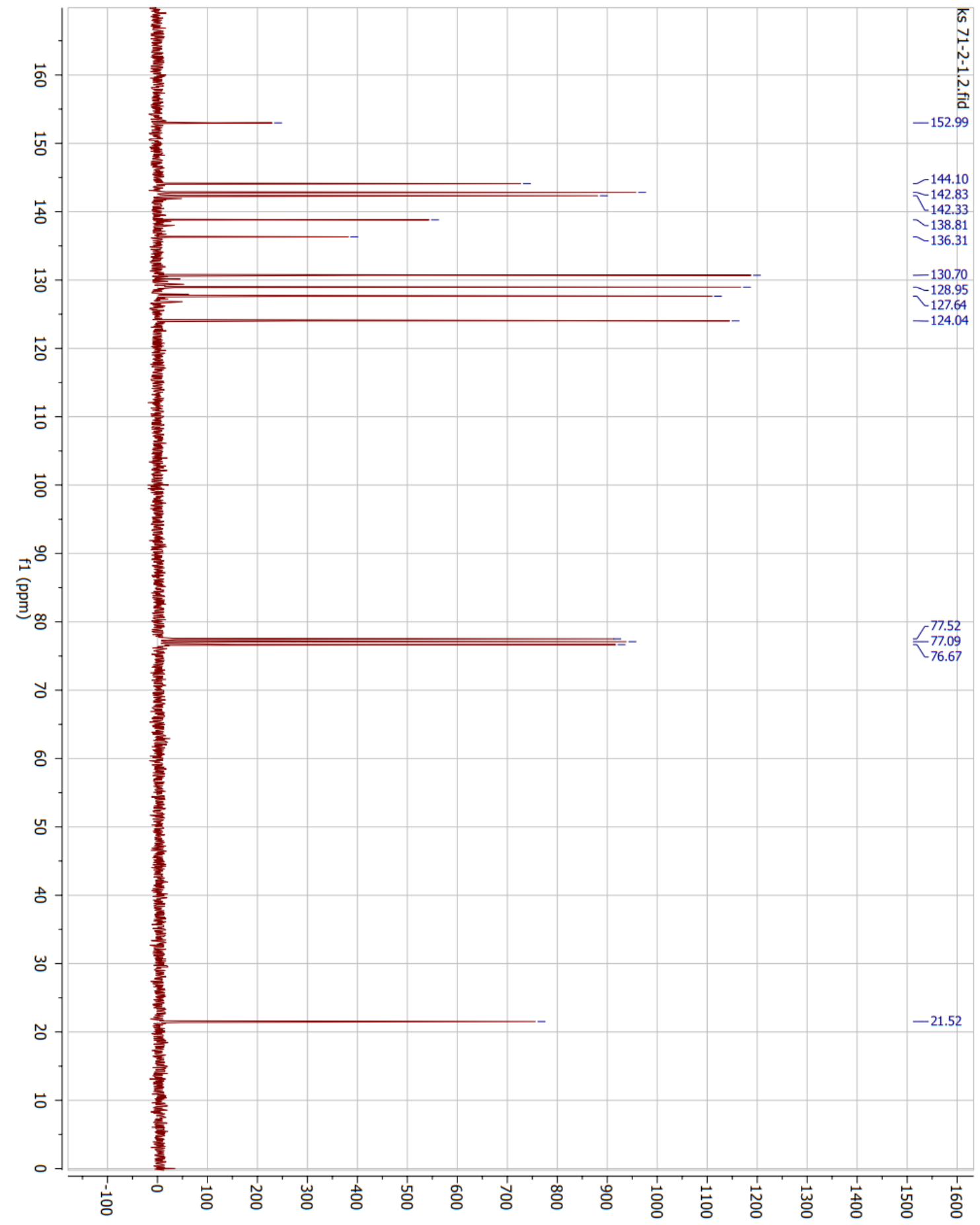


Figure A11 DEPT NMR spectrum of 2-(3-methylphenyl)pyrazine

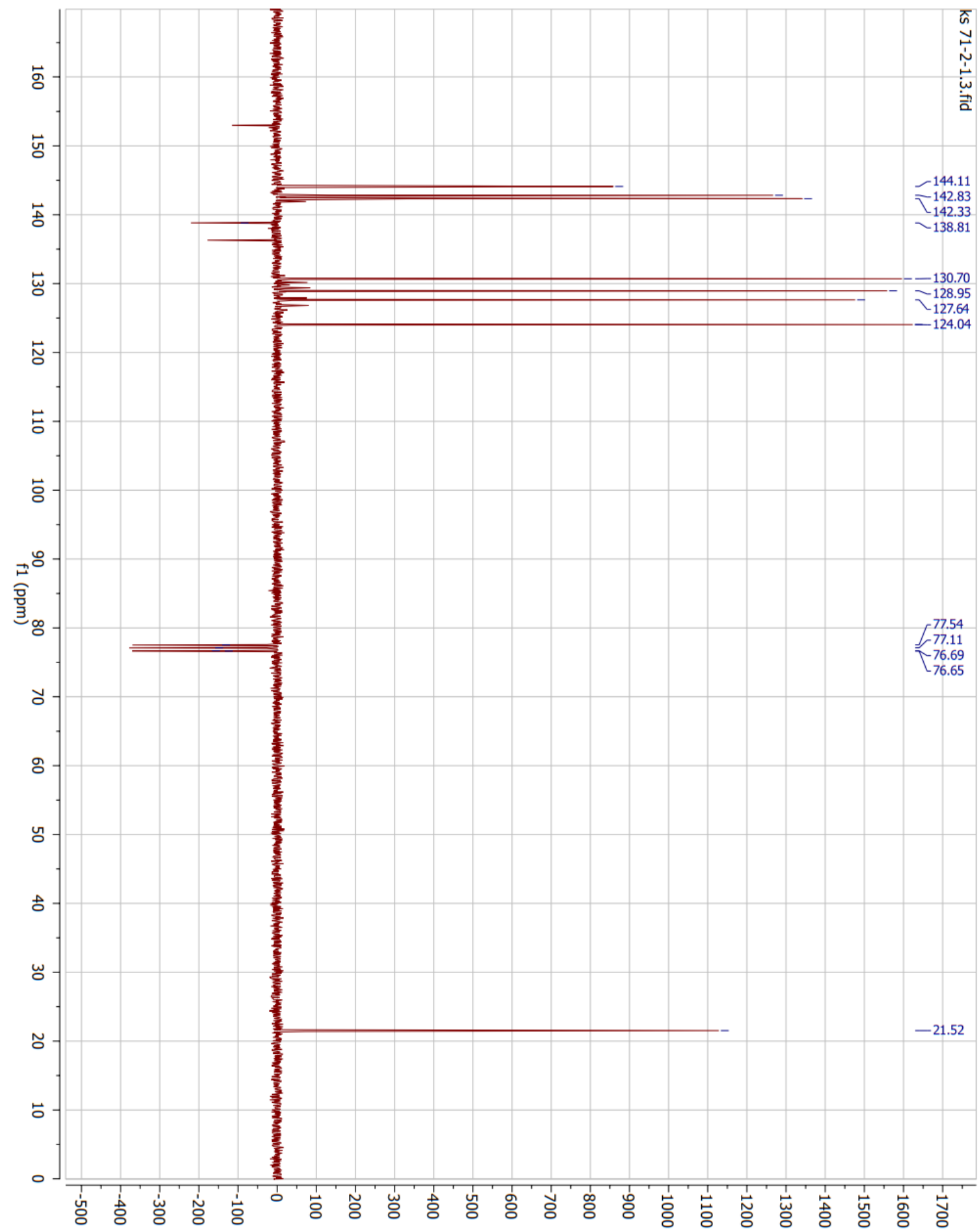


Figure A12 Gas chromatogram and mass spectrum of 2-(3-methylphenyl)pyrazine

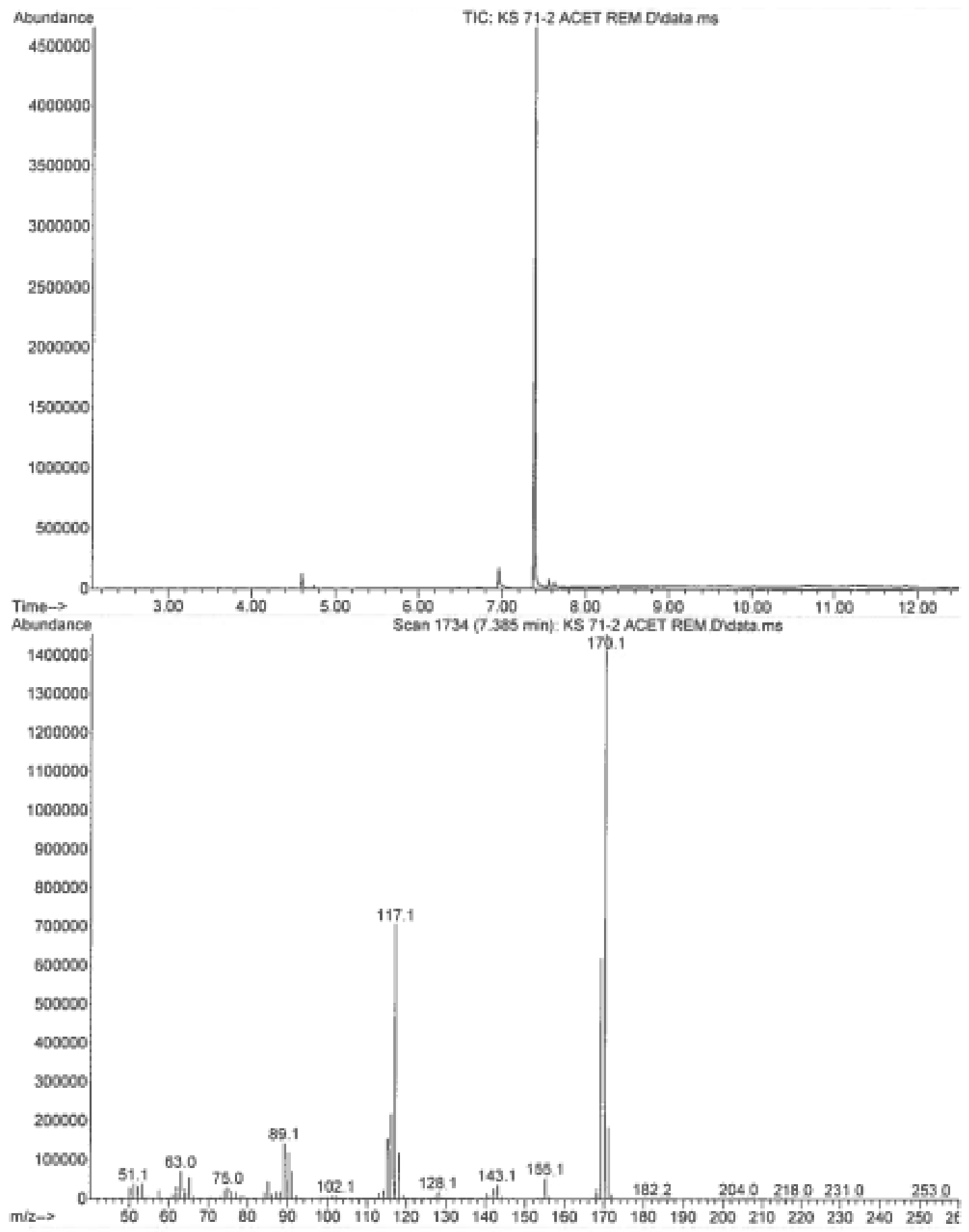


Figure A13 ${ }^{1} \mathrm{H}$ NMR spectrum of 2-(2-methylphenyl)pyrazine

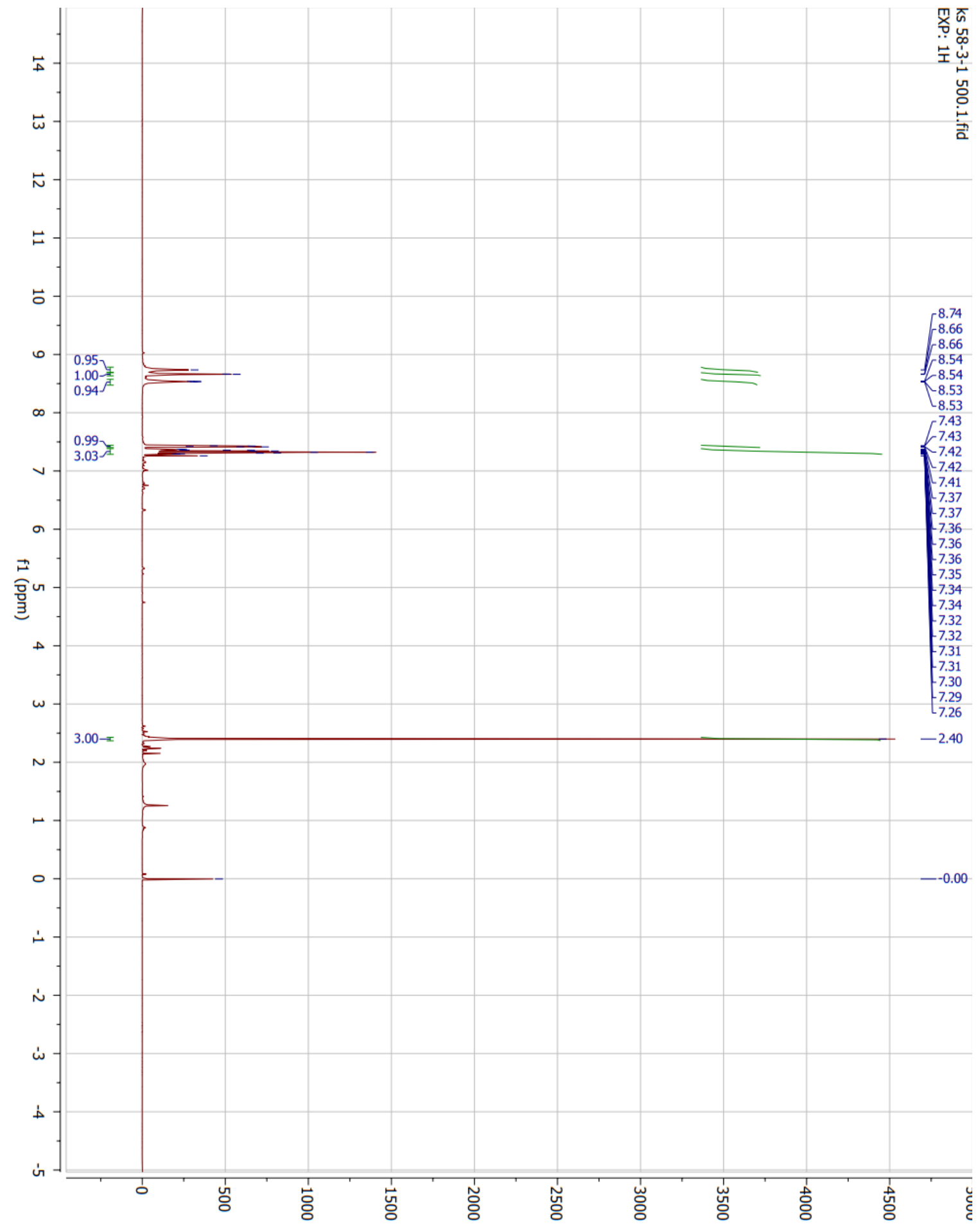


Figure A14 ${ }^{13} \mathrm{C}$ NMR spectrum of 2-(2-methylphenyl)pyrazine

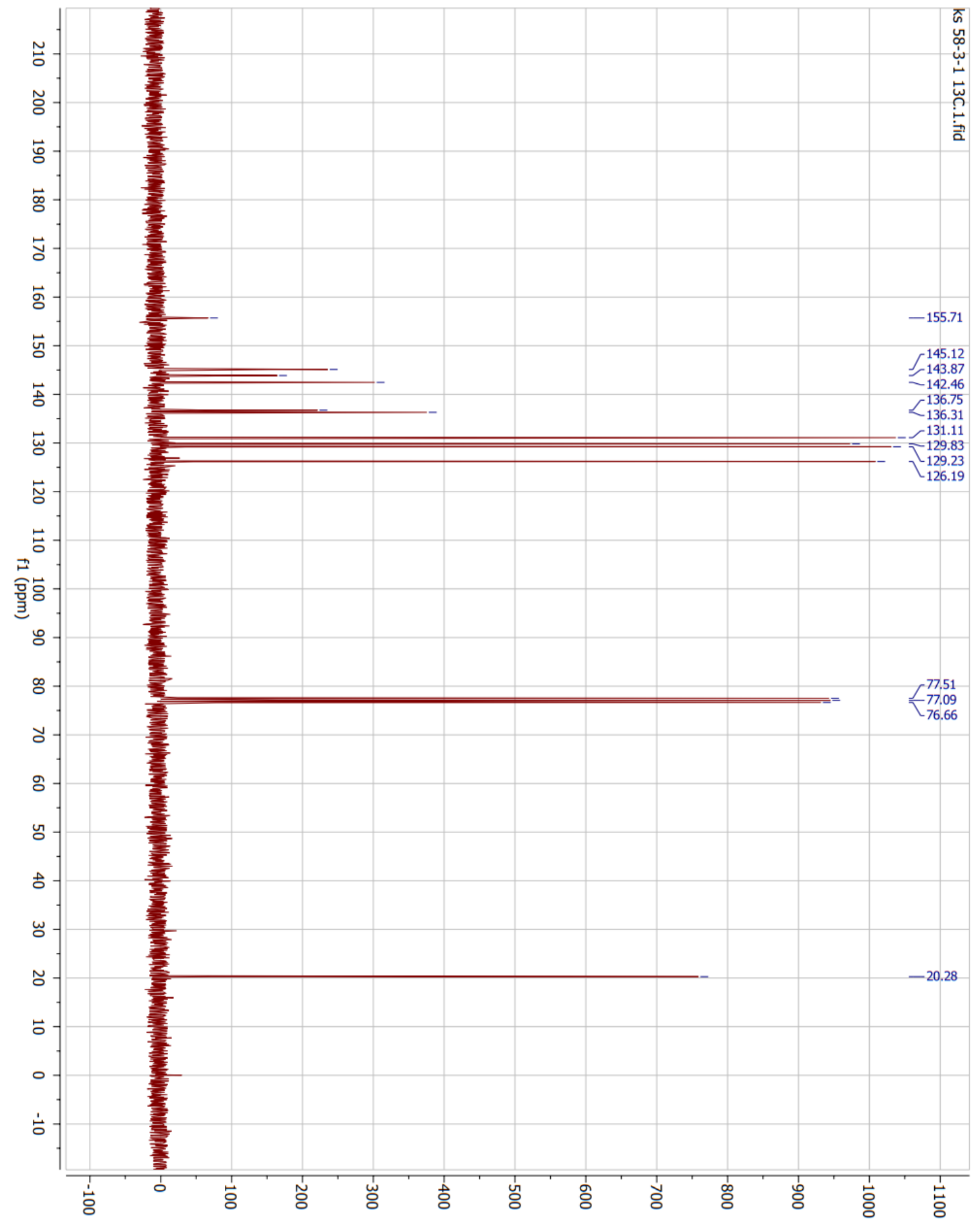


Figure A15 DEPT NMR spectrum of 2-(2-methylphenyl)pyrazine

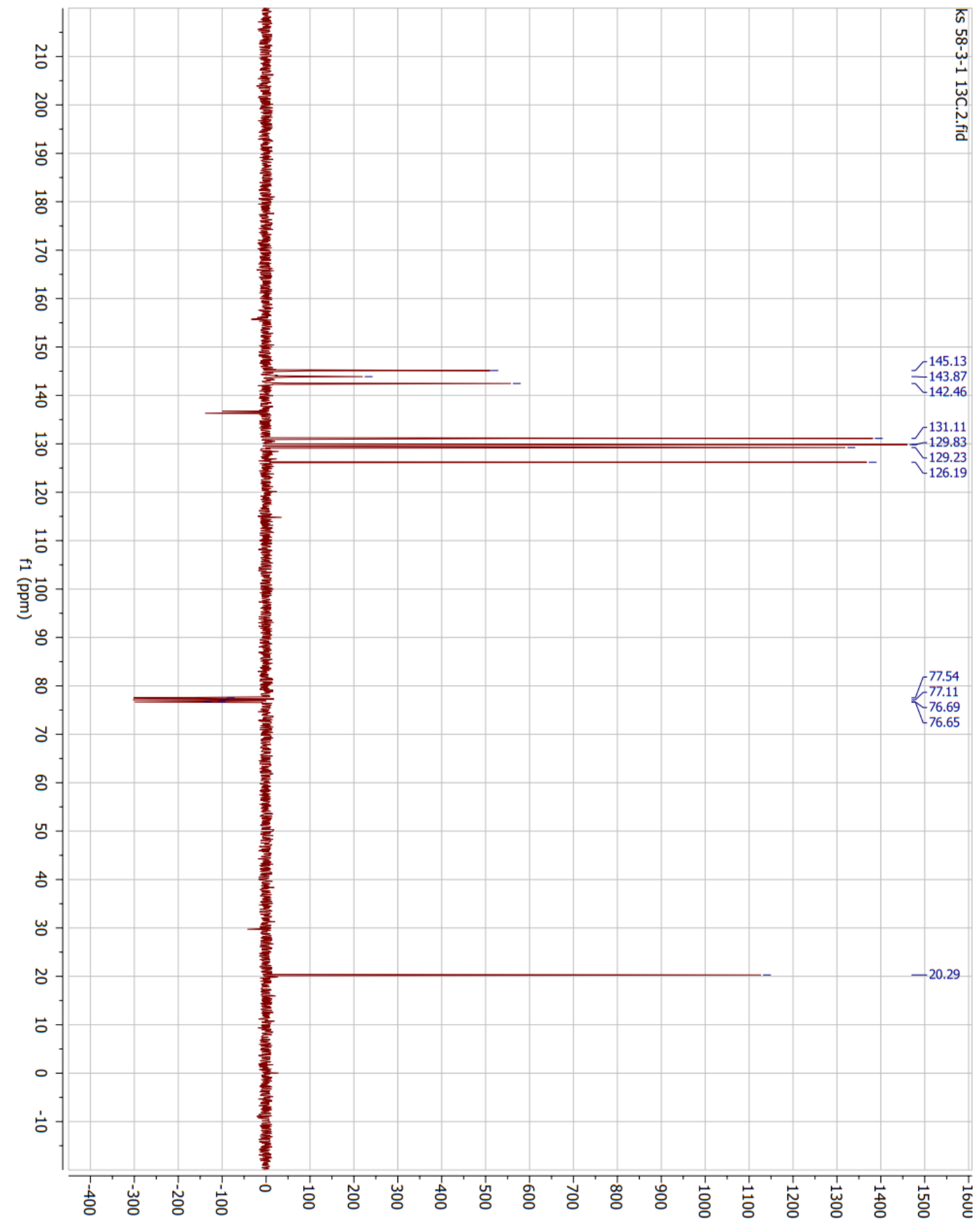


Figure A16 Gas chromatogram and mass spectrum of 2-(2-methylphenyl)pyrazine

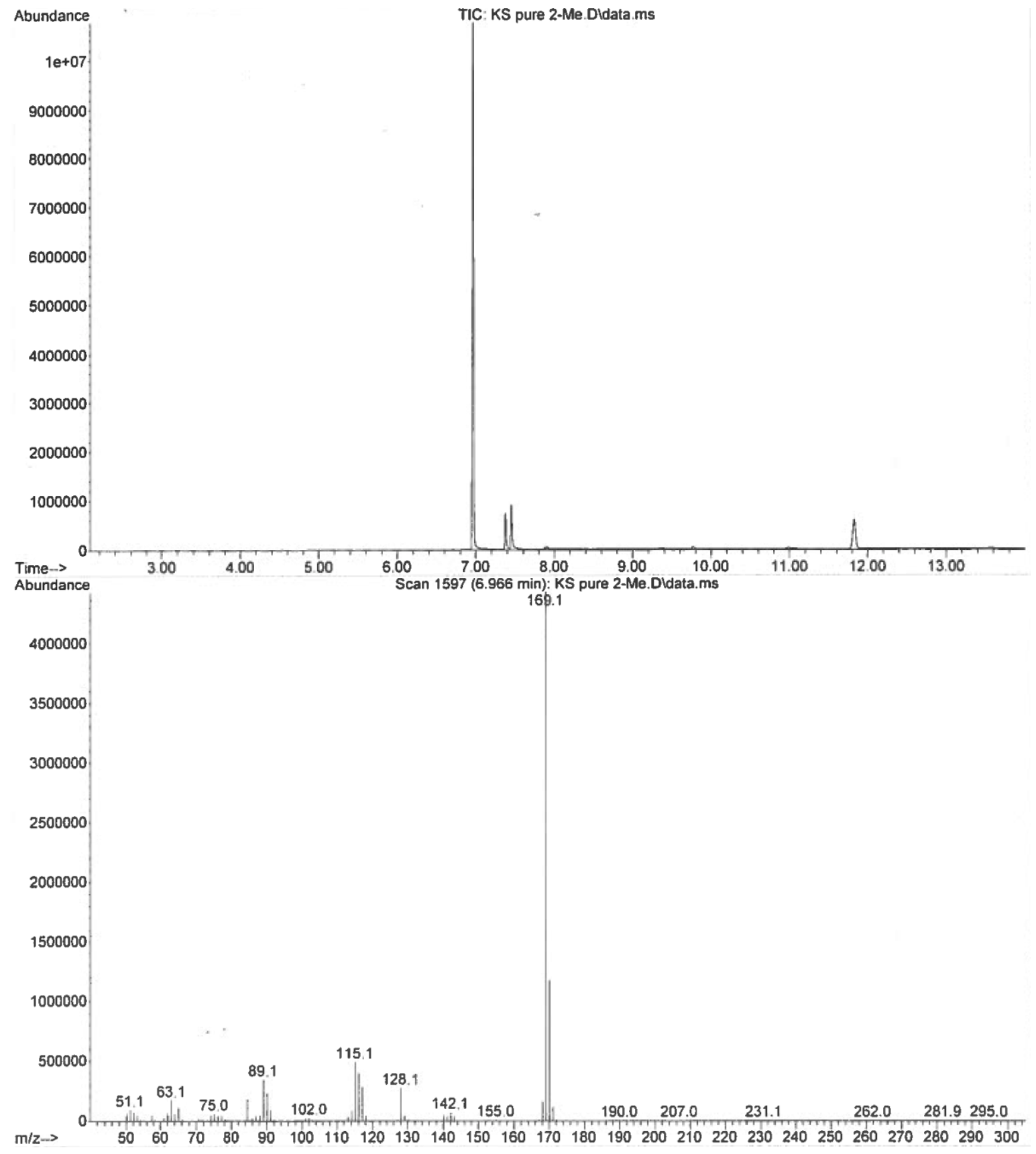


Figure A17 ${ }^{1} \mathrm{H}$ NMR spectrum of 2-(4-chlorophenyl)pyrazine

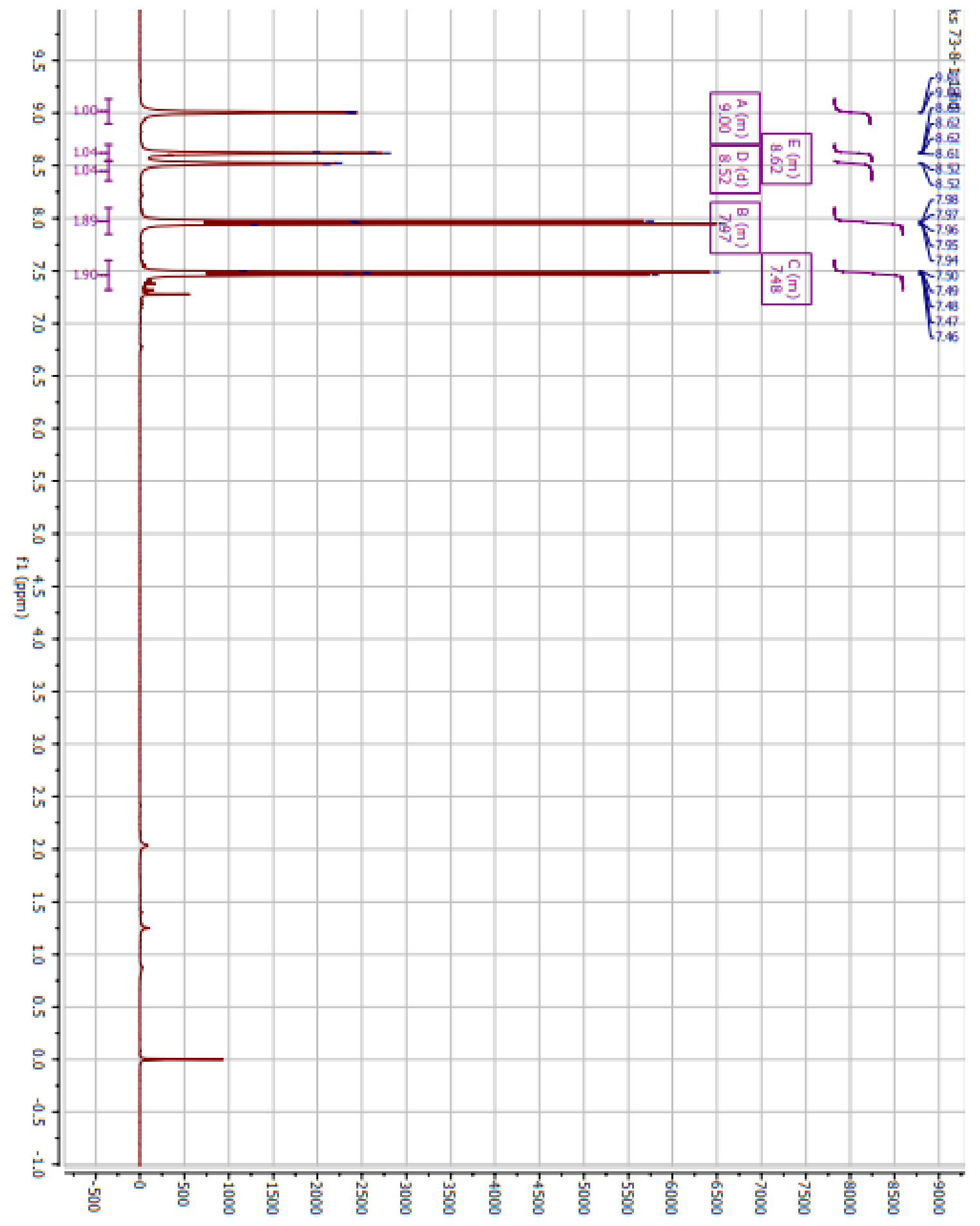


Figure A18 ${ }^{13} \mathrm{C}$ NMR spectrum of 2-(4-chlorophenyl)pyrazine

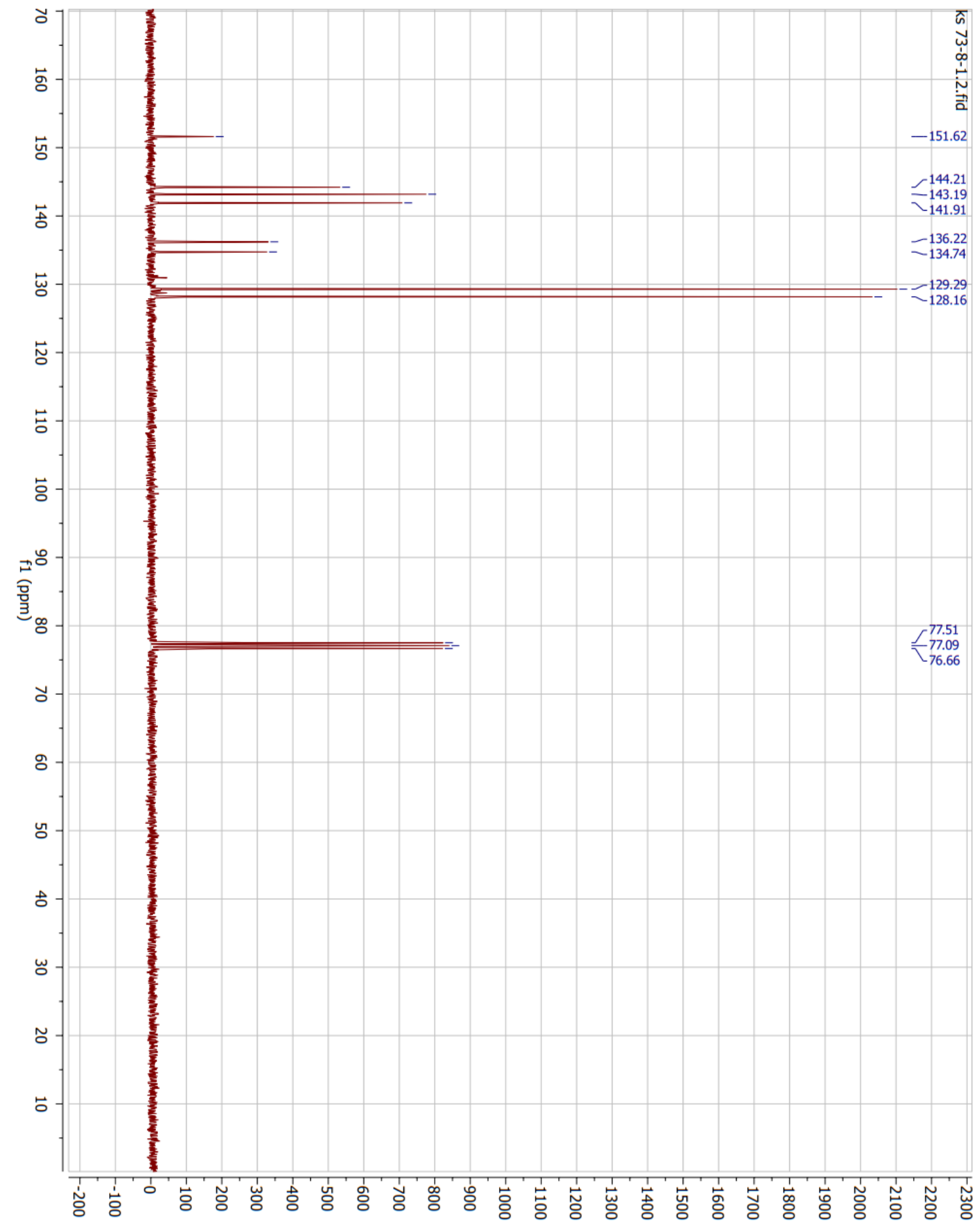


Figure A19 DEPT NMR spectrum of 2-(4-chlorophenyl)pyrazine

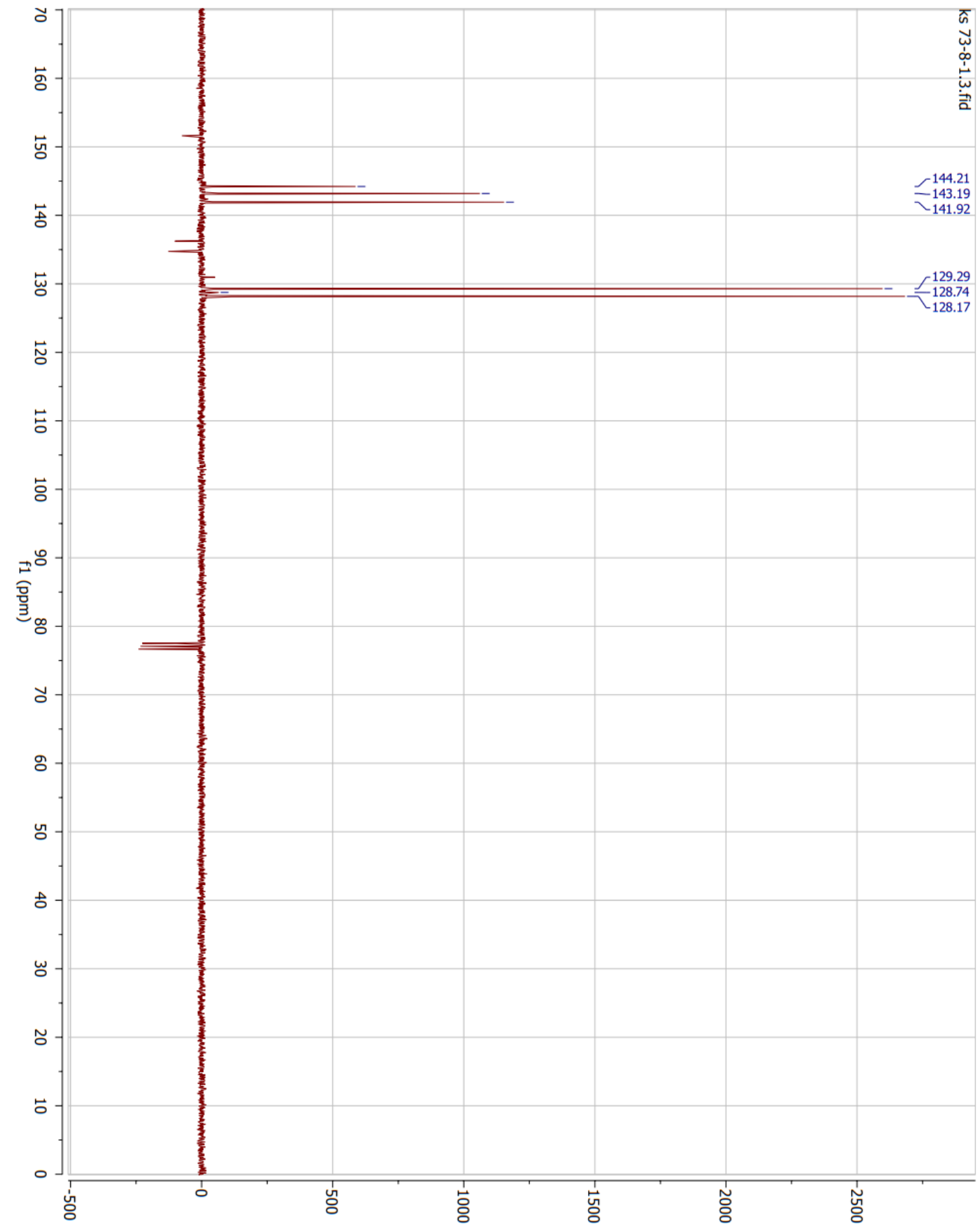


Figure A20 Gas chromatogram and mass spectrum of 2-(4-chlorophenyl)pyrazine

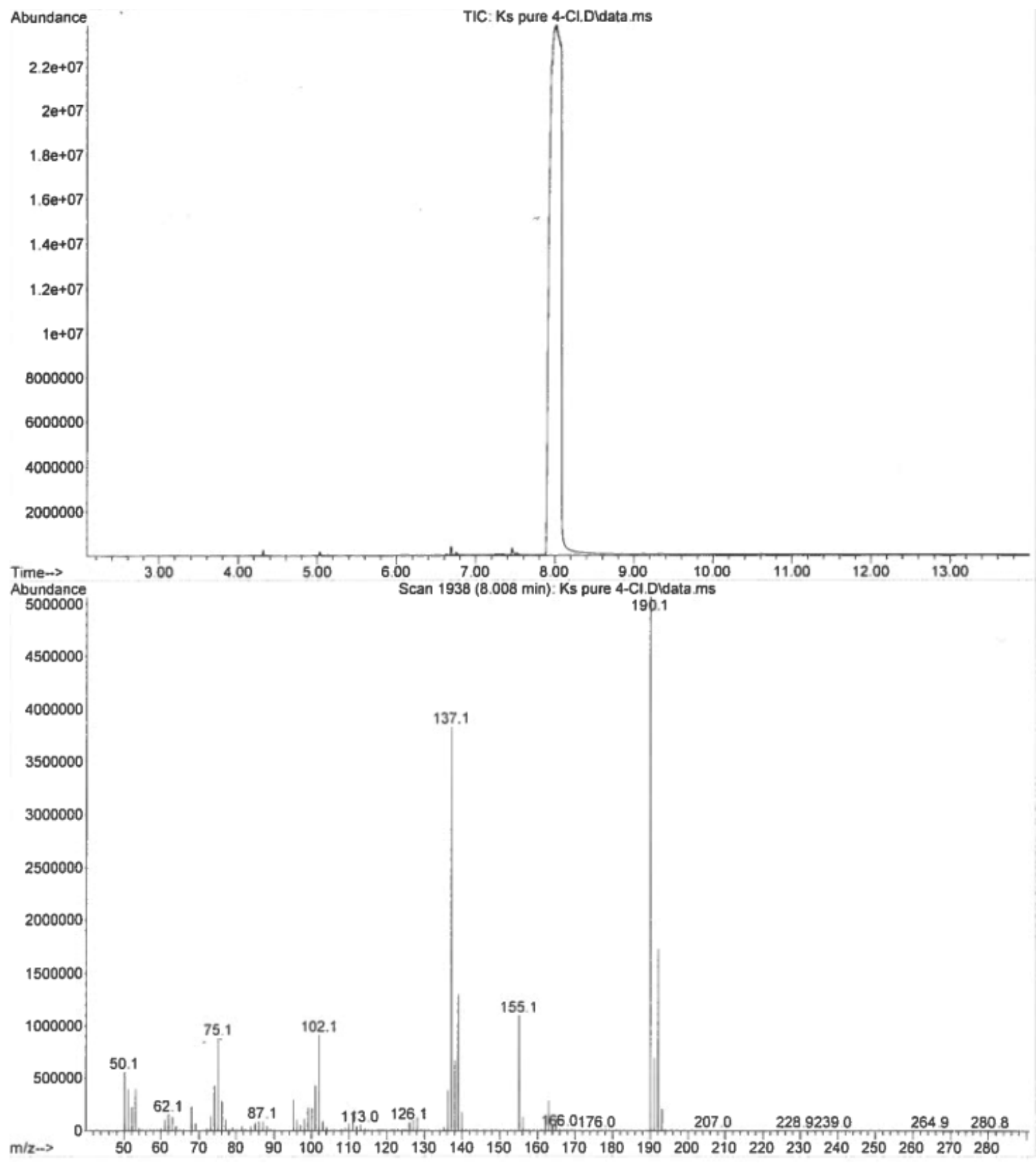


Figure A21 ${ }^{1} \mathrm{H}$ NMR spectrum of 2-(3-chlorophenyl)pyrazine

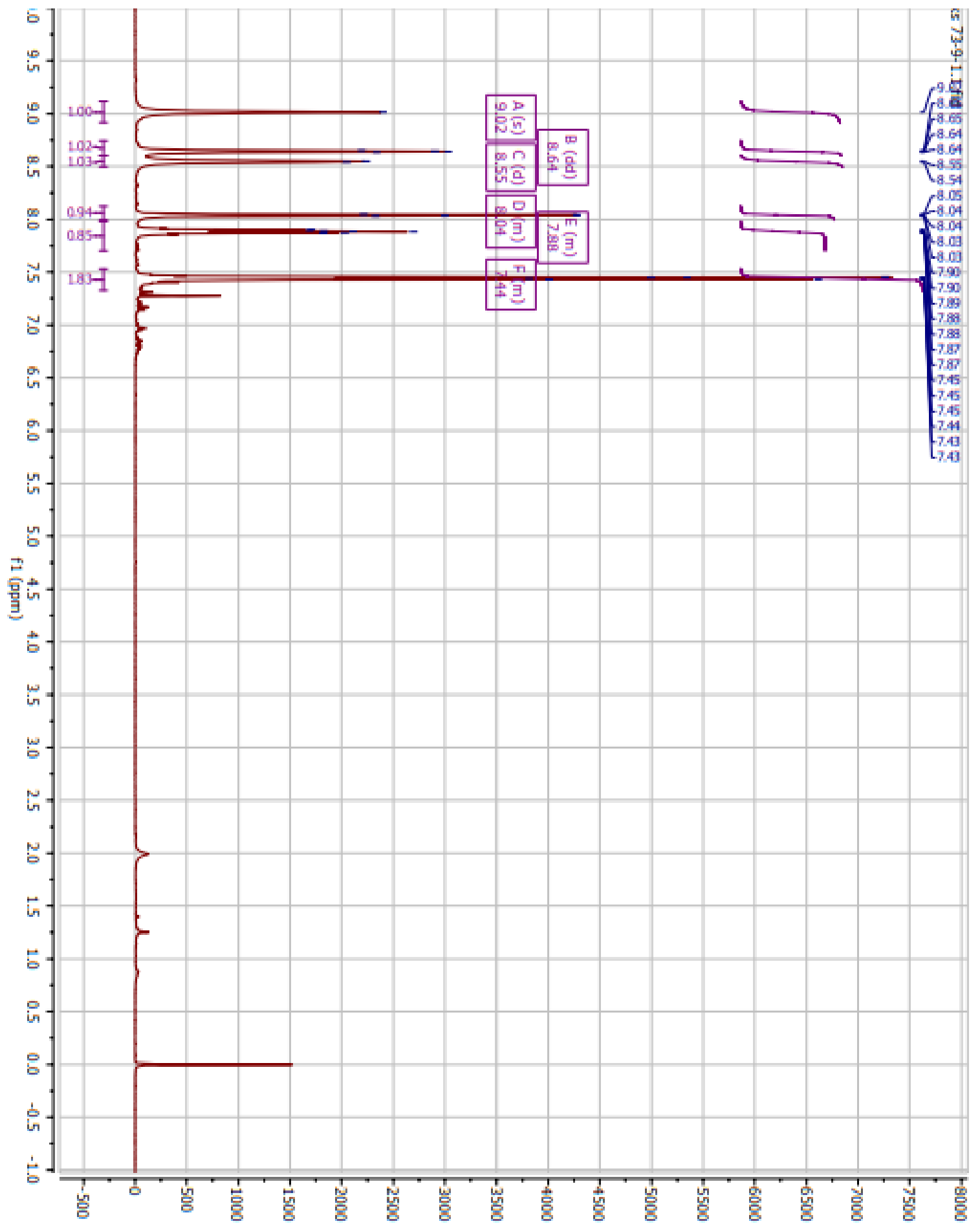


Figure A22 ${ }^{13} \mathrm{C}$ NMR spectrum of 2-(3-chlorophenyl)pyrazine

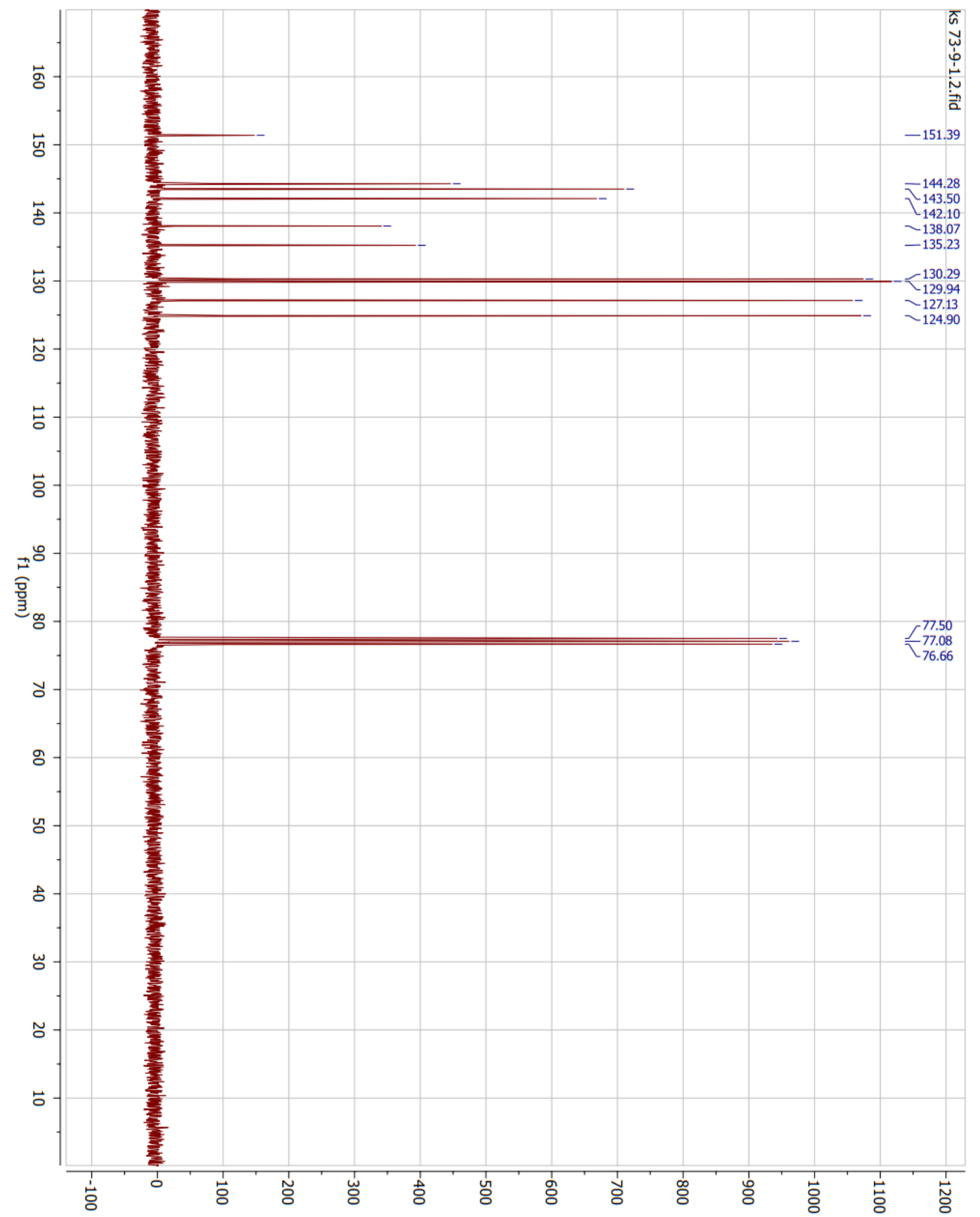


Figure A23 DEPT NMR spectrum of 2-(3-chlorophenyl)pyrazine

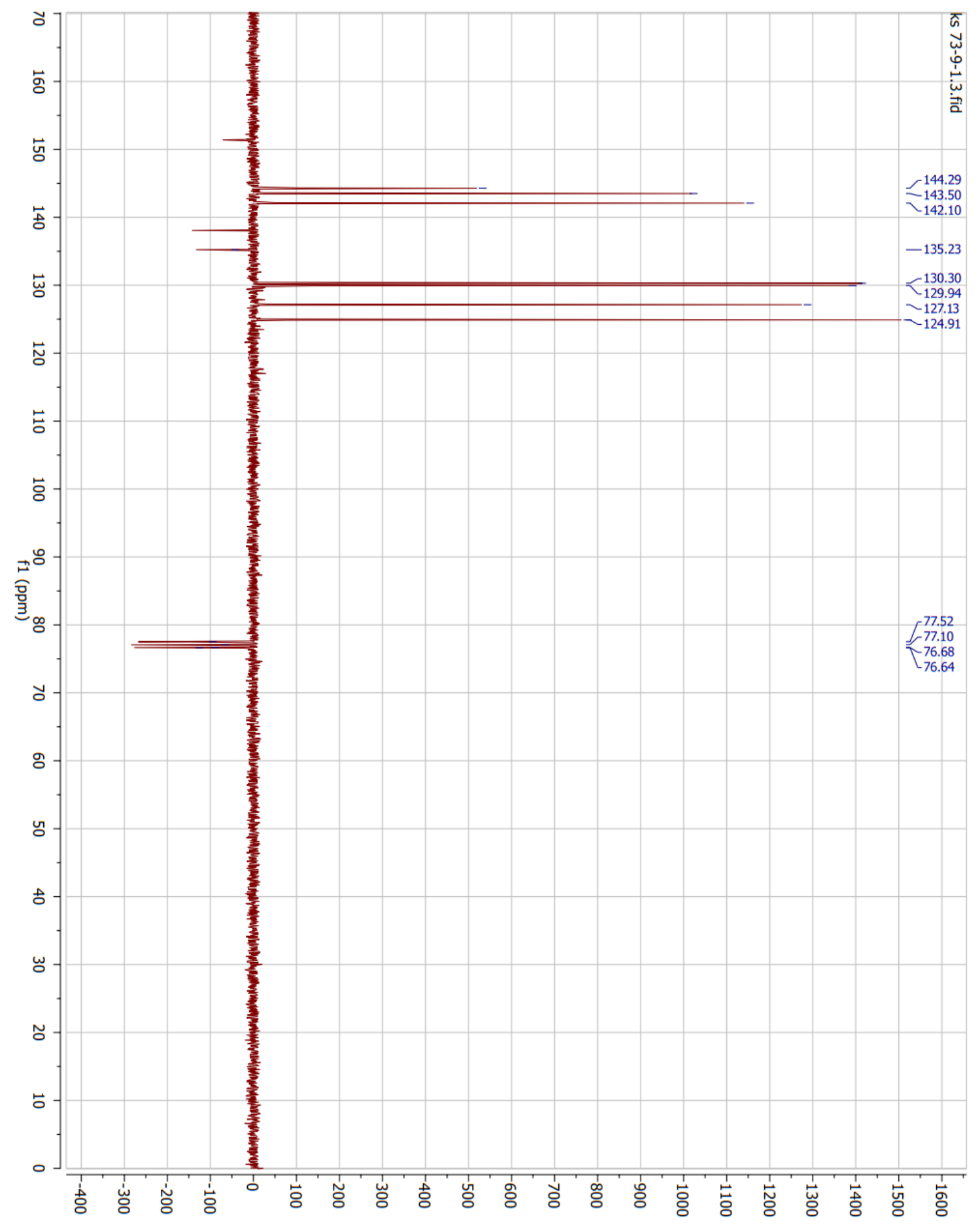


Figure A24 Gas chromatogram and mass spectrum of 2-(3-chlorophenyl)pyrazine

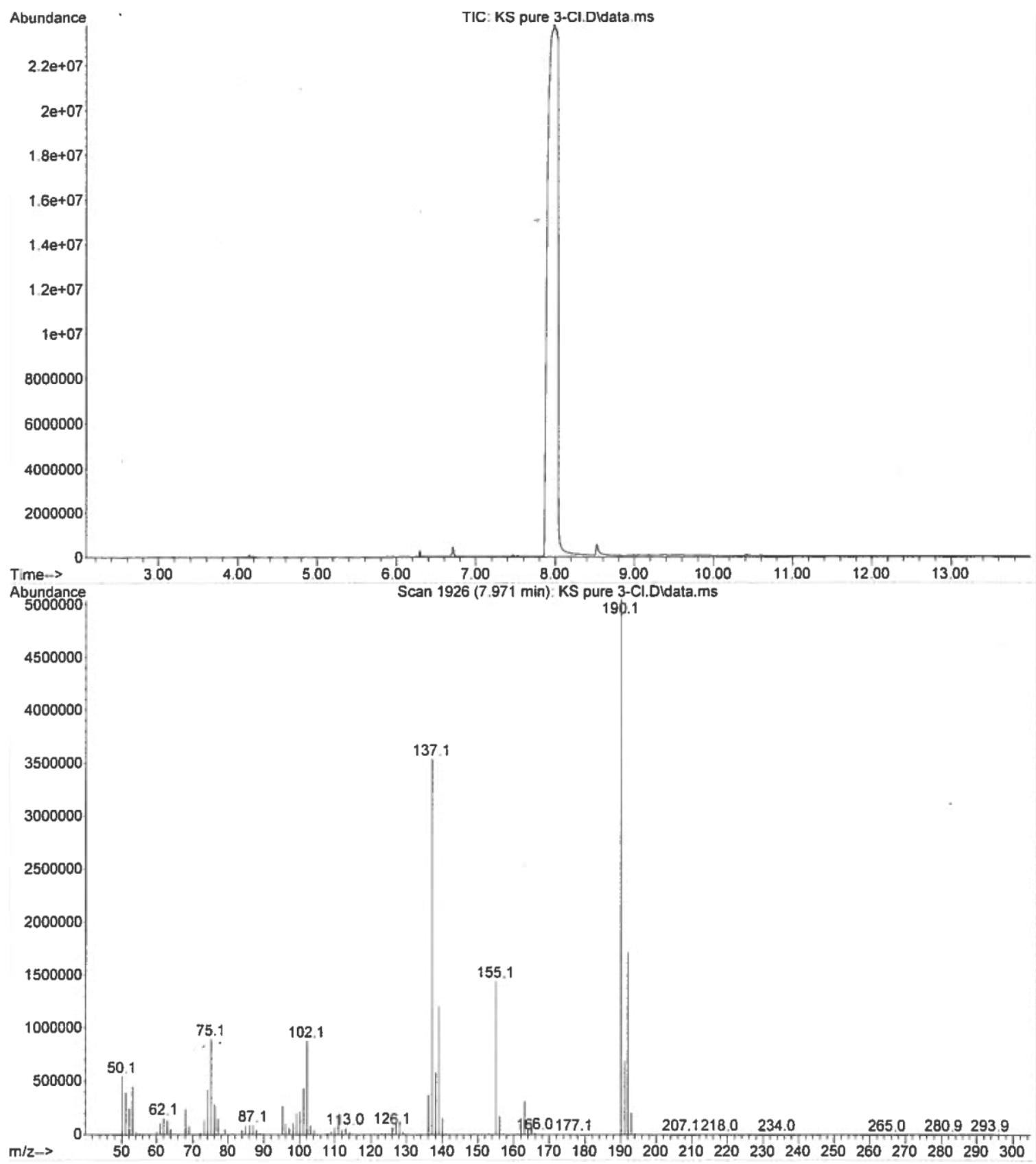


Figure A25 ${ }^{1} \mathrm{H}$ NMR spectrum of 2-(2-chlorophenyl)pyrazine

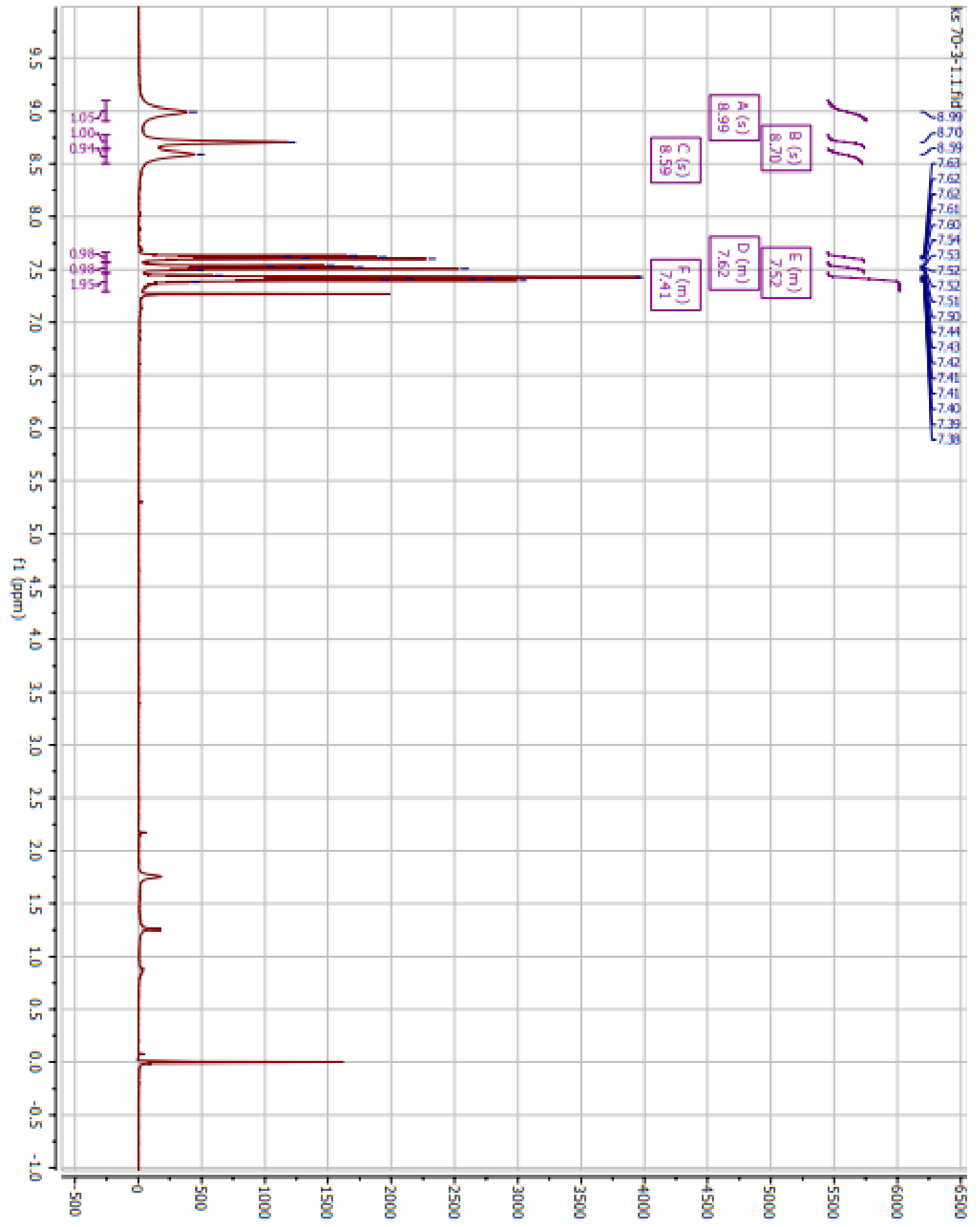


Figure A26 ${ }^{13} \mathrm{C}$ NMR spectrum of 2-(2-chlorophenyl)pyrazine

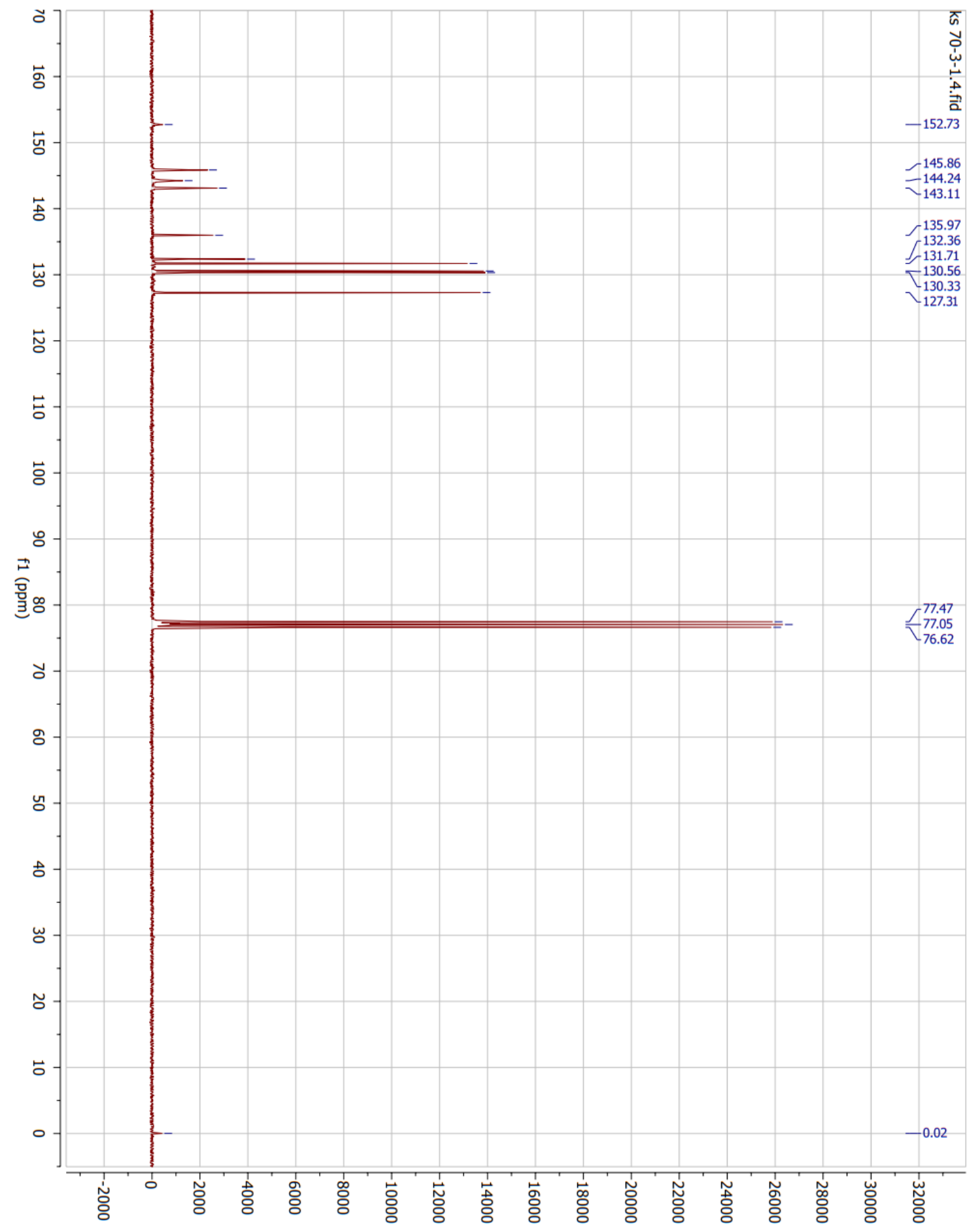


Figure A27 DEPT NMR spectrum of 2-(2-chlorophenyl)pyrazine

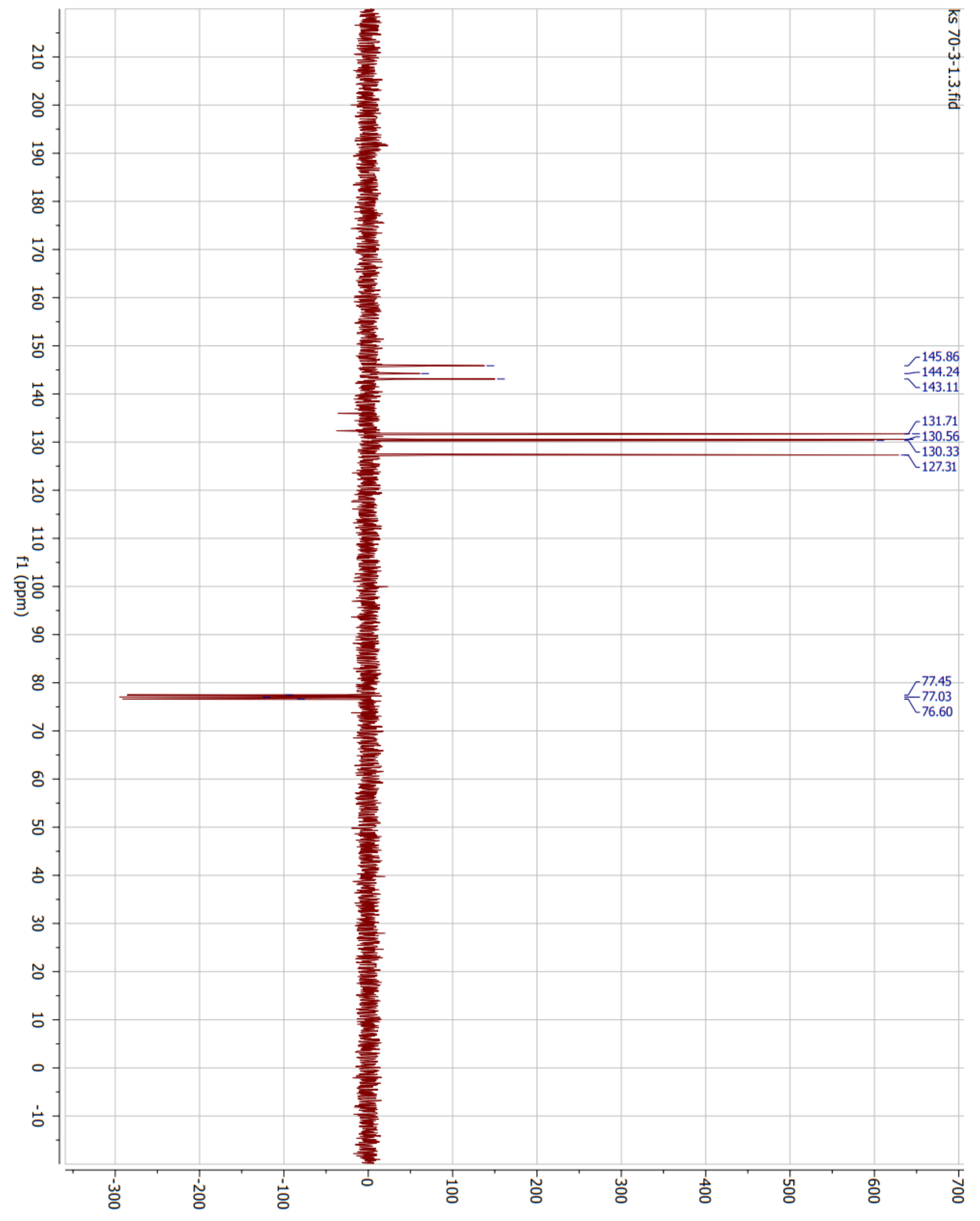


Figure A28 Gas chromatogram and mass spectrum of 2-(2-chlorophenyl)pyrazine

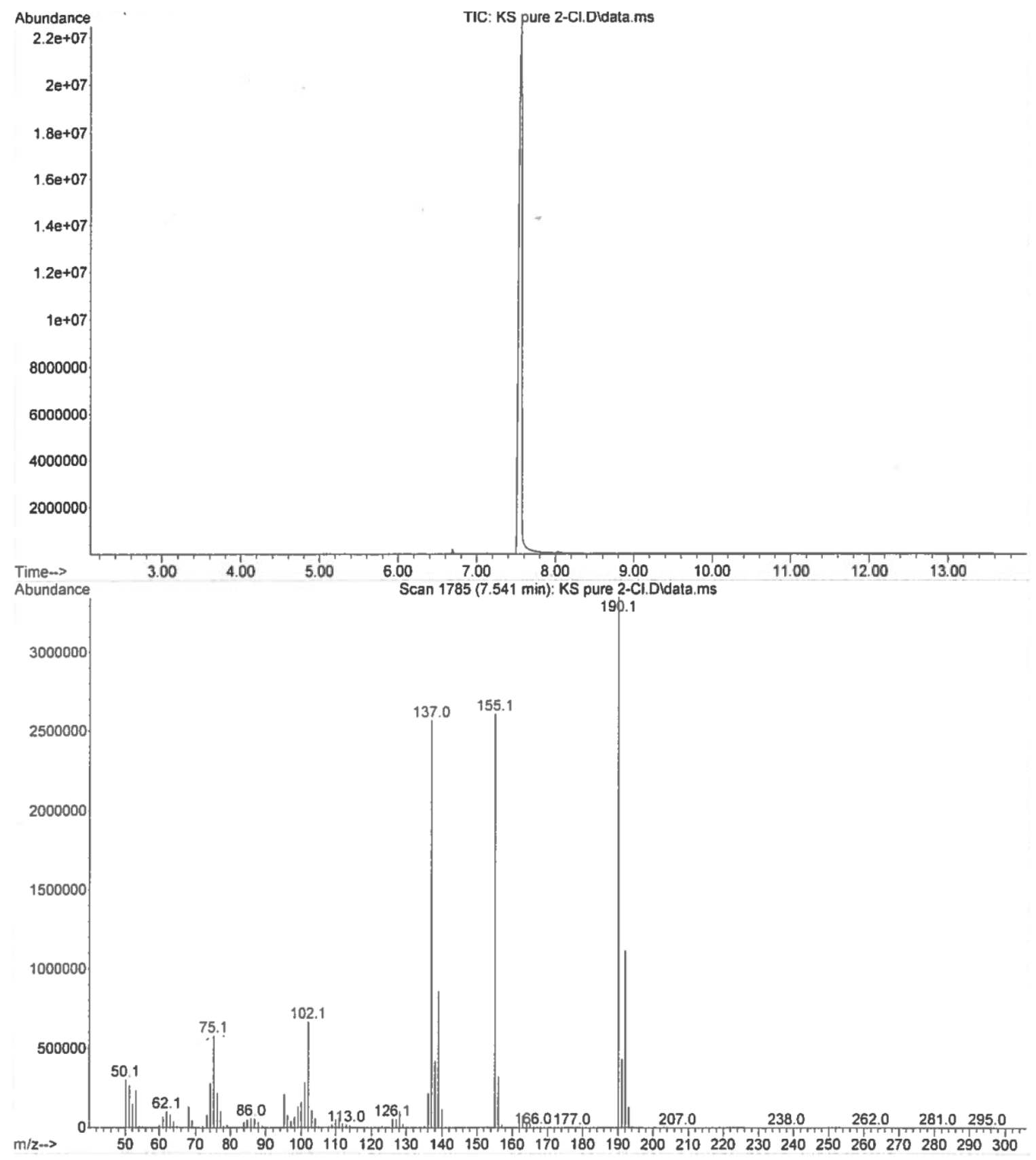


Figure A29 ${ }^{1} \mathrm{H}$ NMR spectrum of 2-(4-iodophenyl)pyrazine

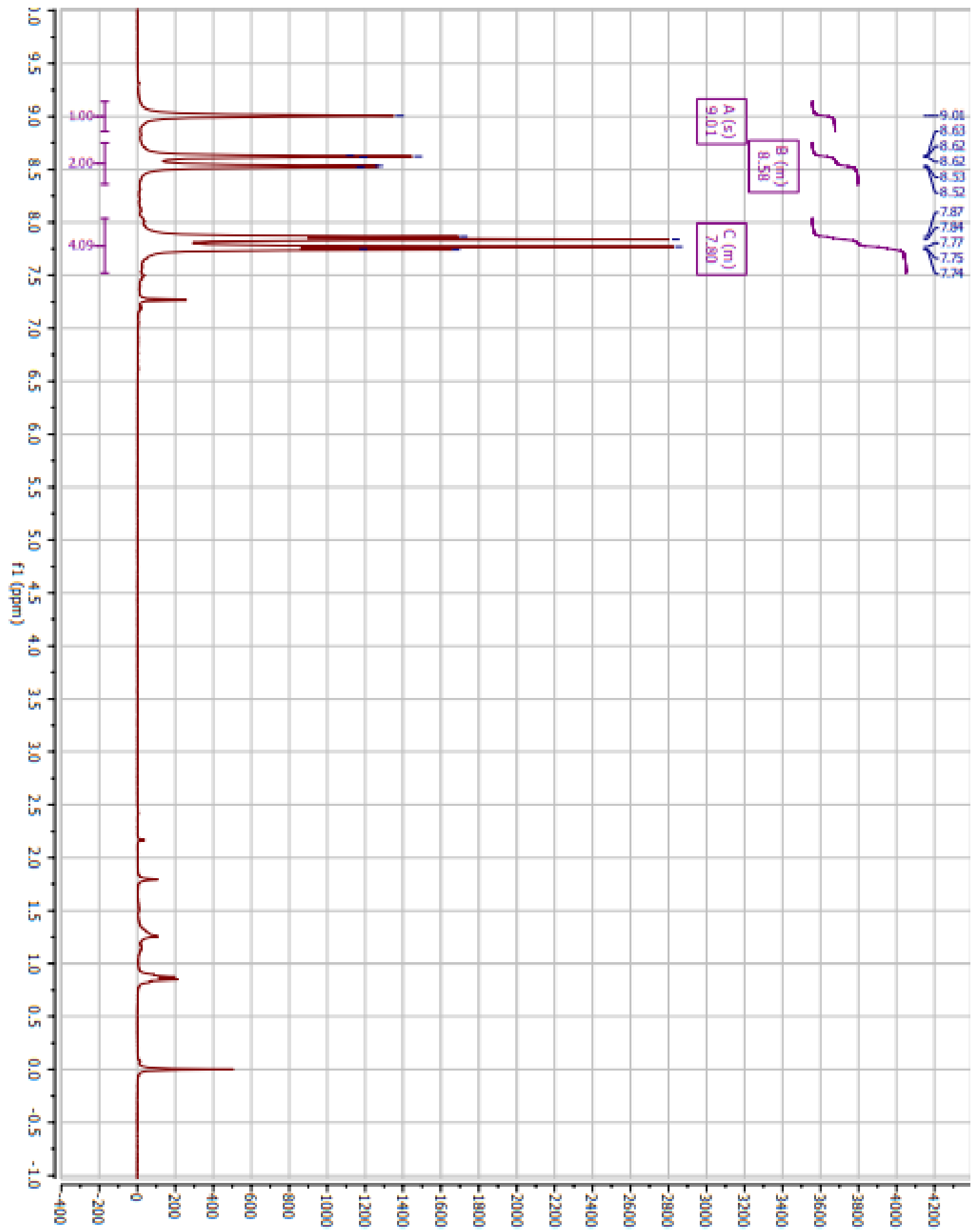


Figure A30 ${ }^{13} \mathrm{C}$ NMR spectrum of 2-(4-iodophenyl)pyrazine

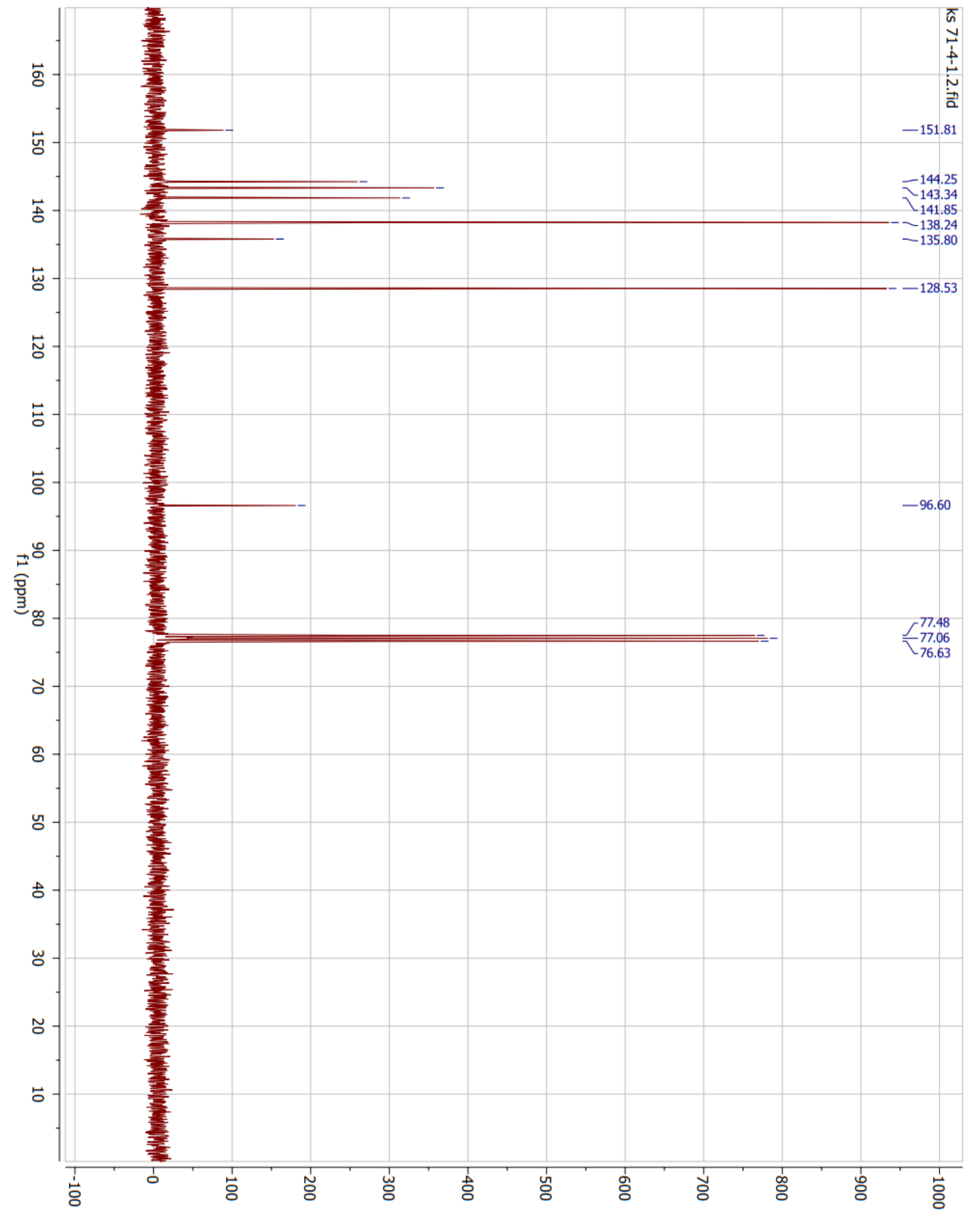


Figure A31 DEPT NMR spectrum of 2-(4-iodophenyl)pyrazine

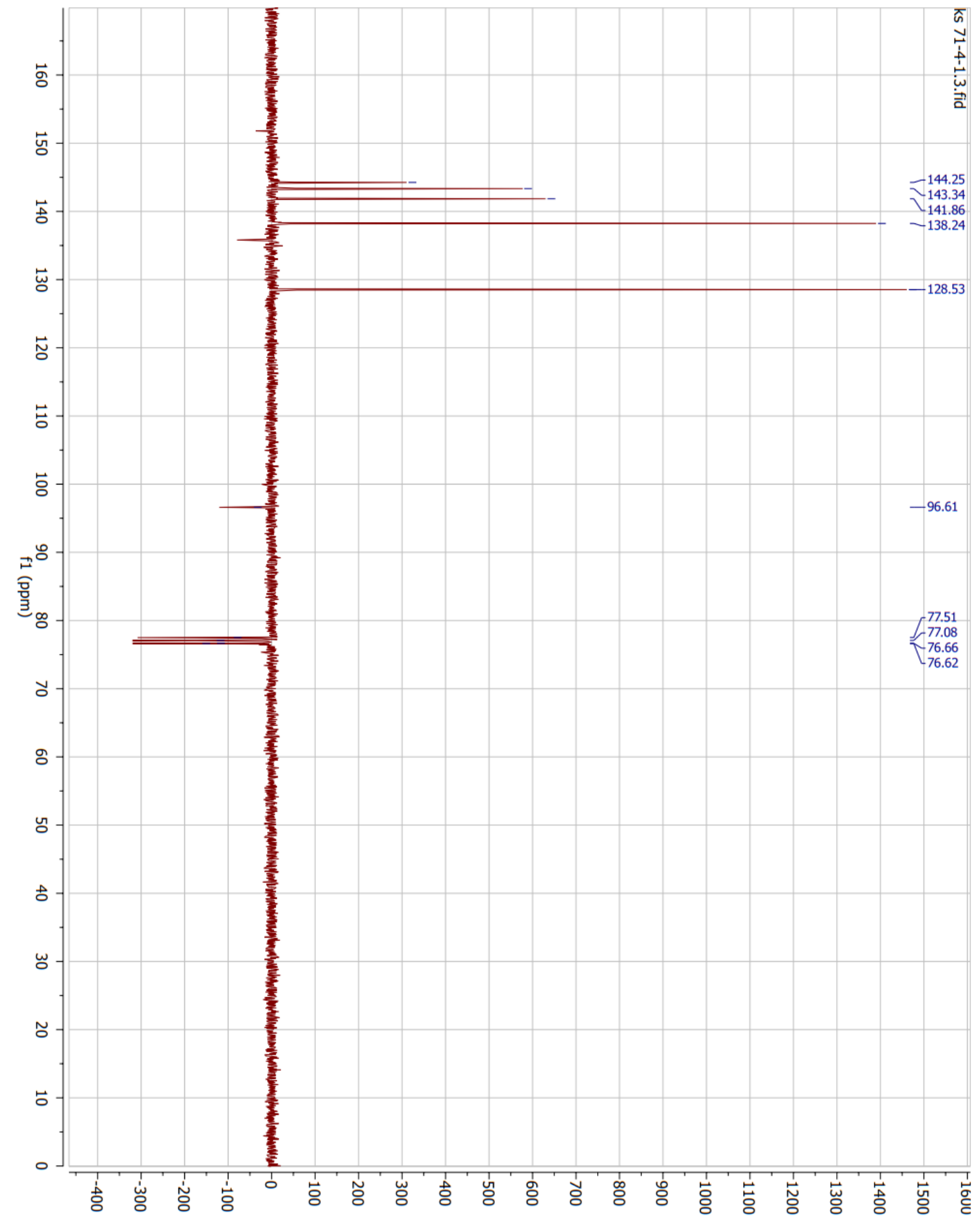


Figure A32 Gas chromatogram and mass spectrum of 2-(4-iodophenyl)pyrazine

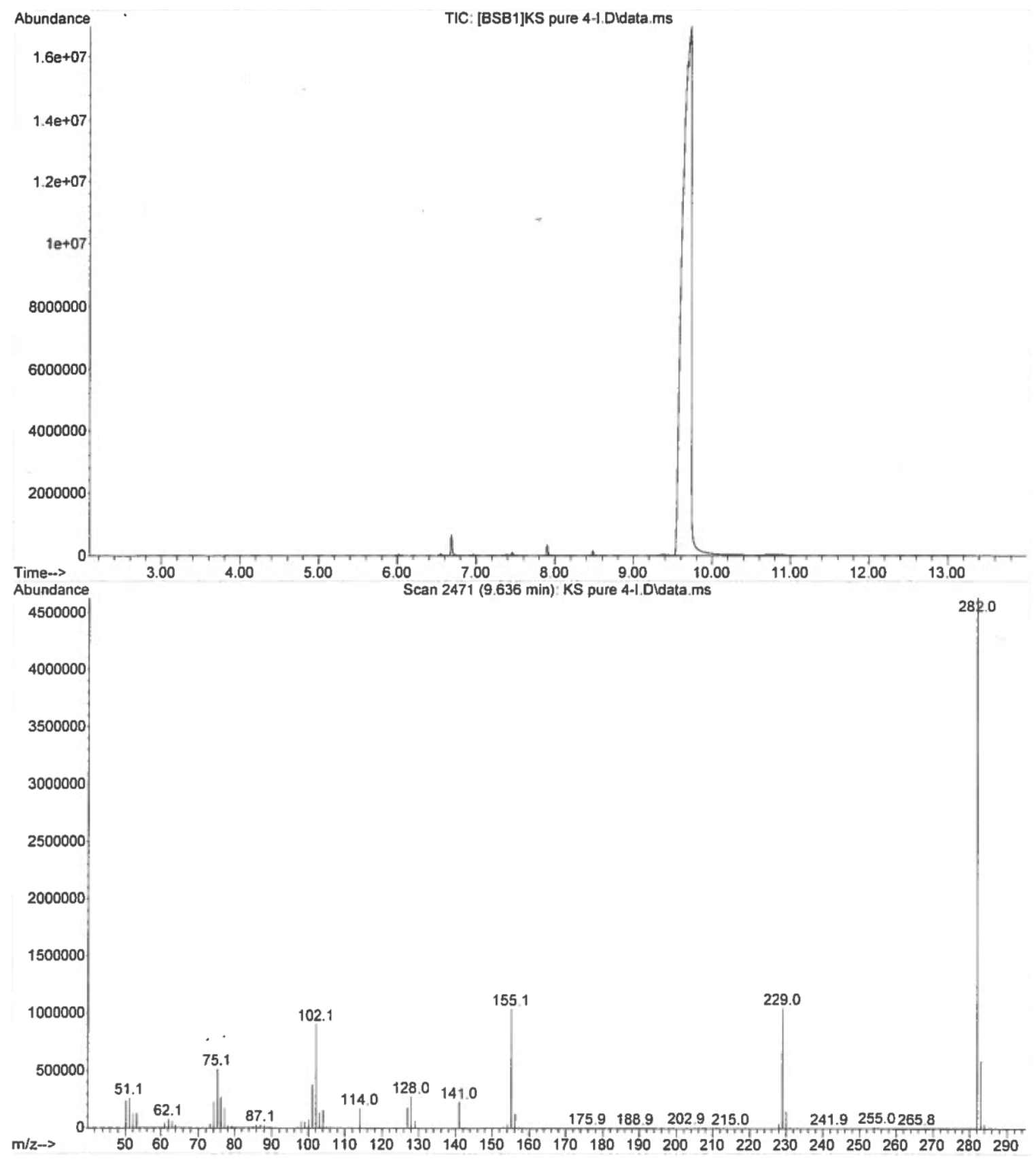


Figure A33 Gas chromatogram and mass spectrum of 2-(4-trifluoromethylphenyl)pyrazine

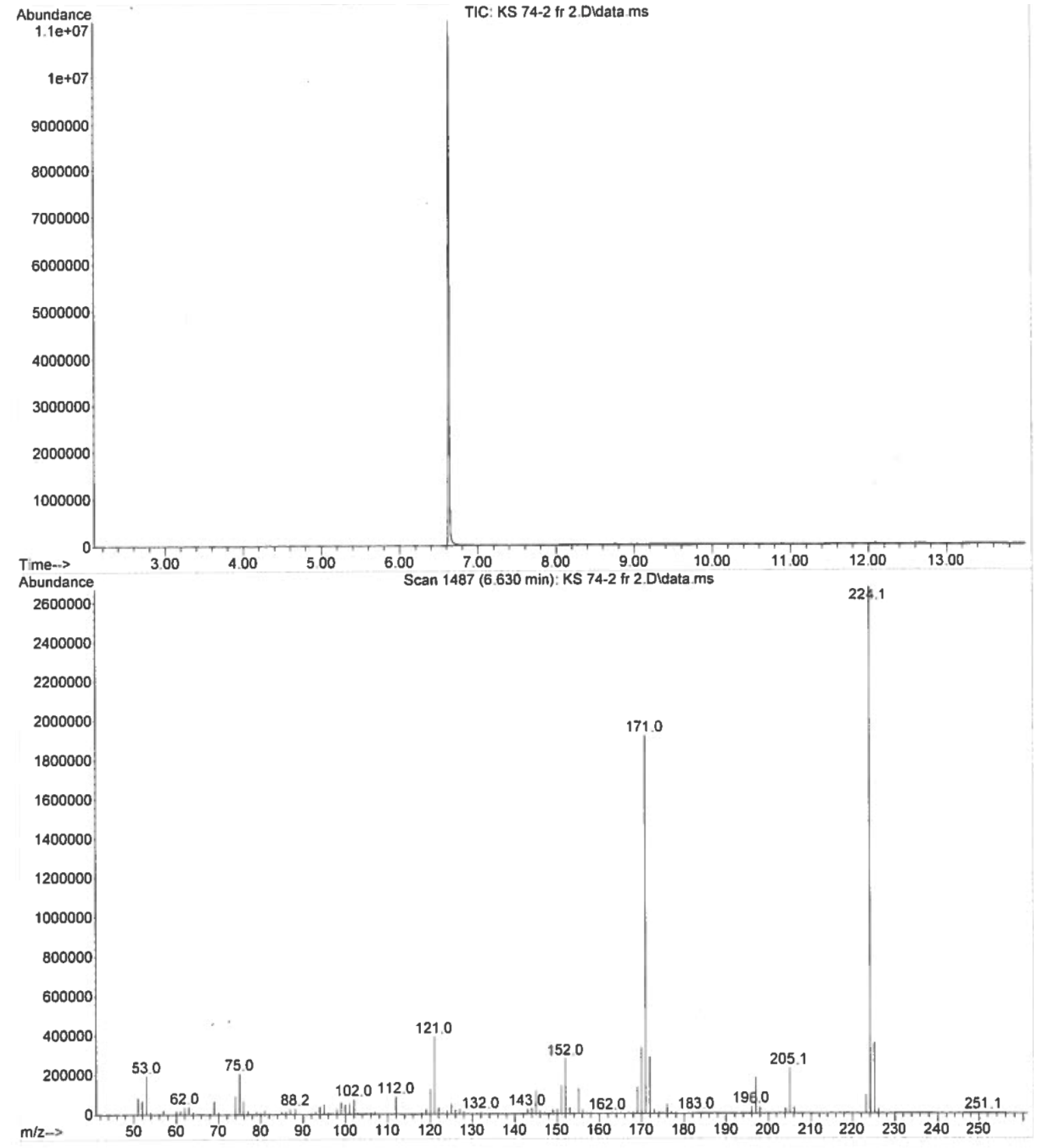


Figure A34 Gas chromatogram and mass spectrum of 2-(4-methoxyphenyl)pyrazine

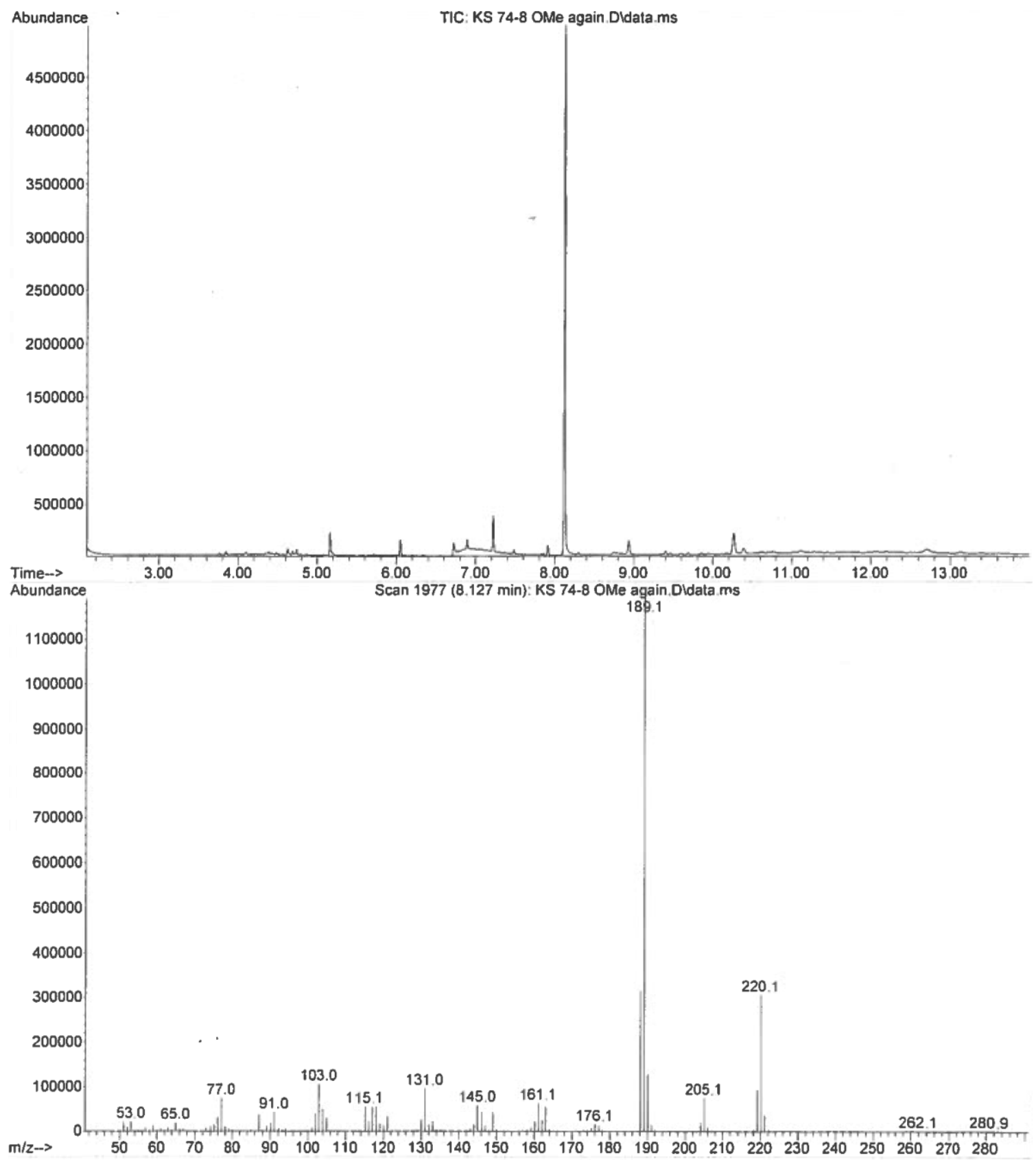


Figure A35 Gas chromatogram of the crude reaction mixture of mesitylboronic acid and pyrazine (top). Mass spectrum of 2-mesitylpyrazine (bottom).

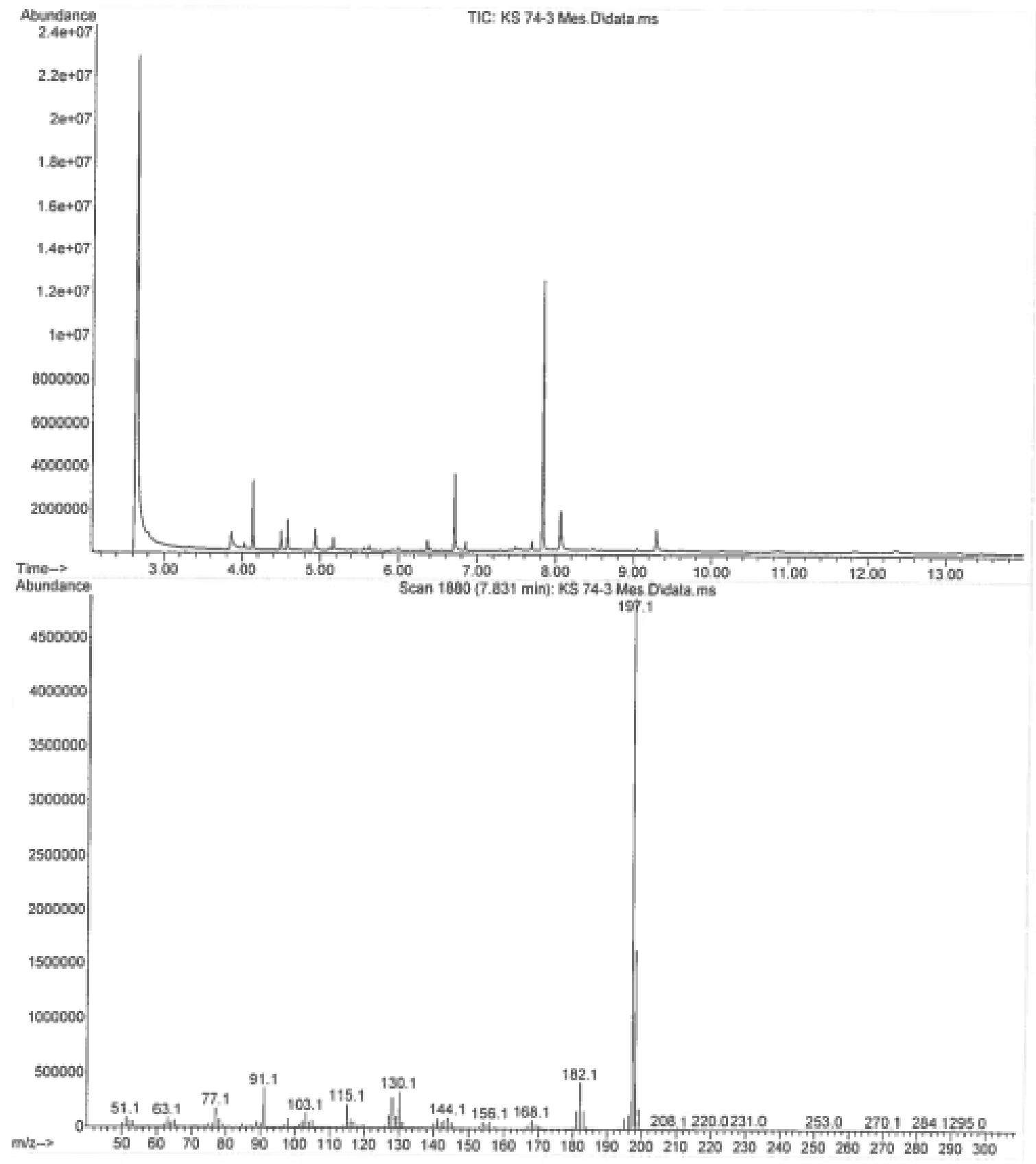


Figure A36 Gas chromatogram of the crude reaction mixture of 4-cyanophenylboronic acid and pyrazine (top). Mass spectrum of 2-(4-cyanophenyl)pyrazine (bottom).

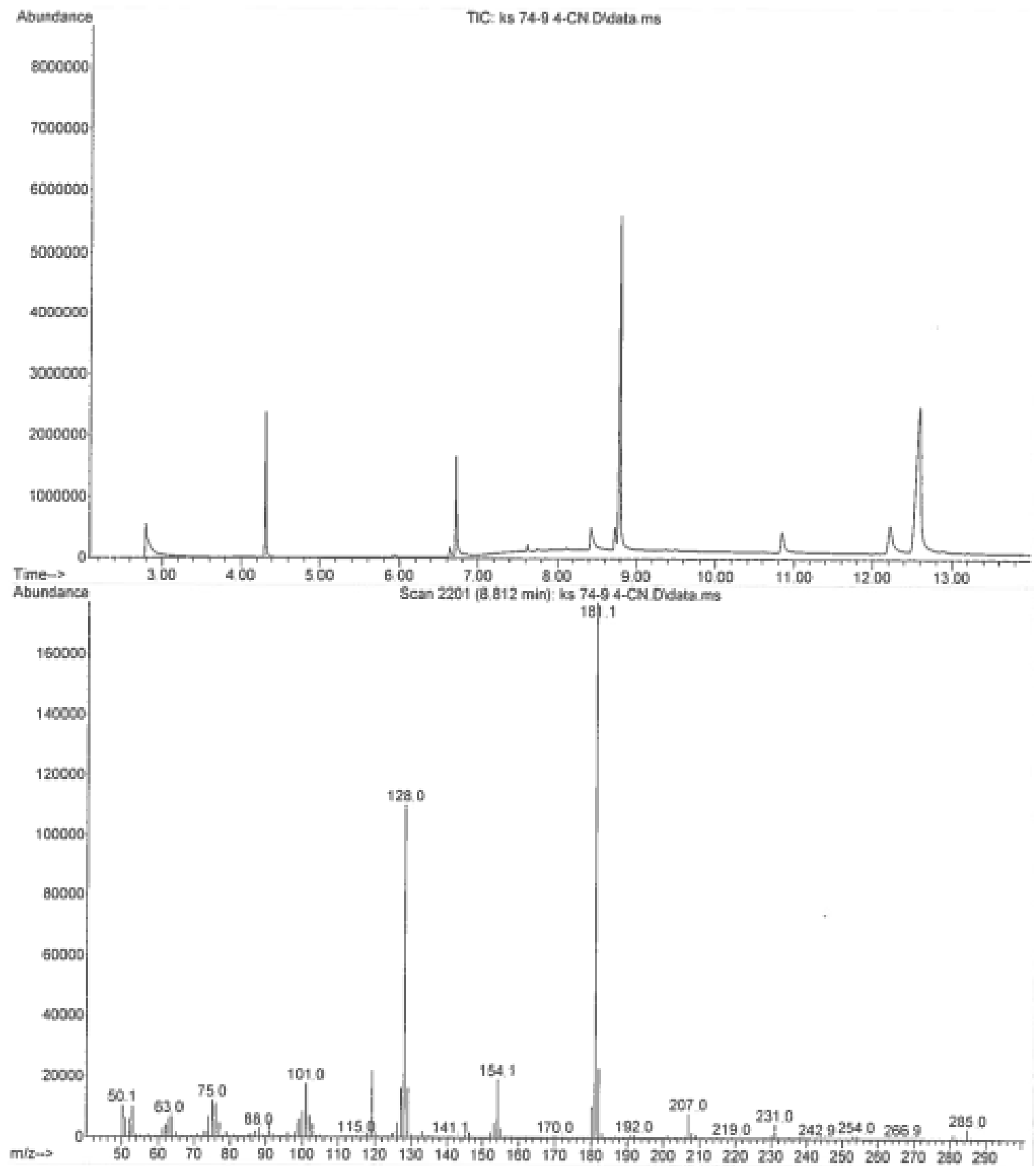


Figure A37 Gas chromatogram of the crude reaction mixture of 4-formylboronic acid and pyrazine (top). Mass spectrum of 2-(4-formylphenyl)pyrazine (bottom).

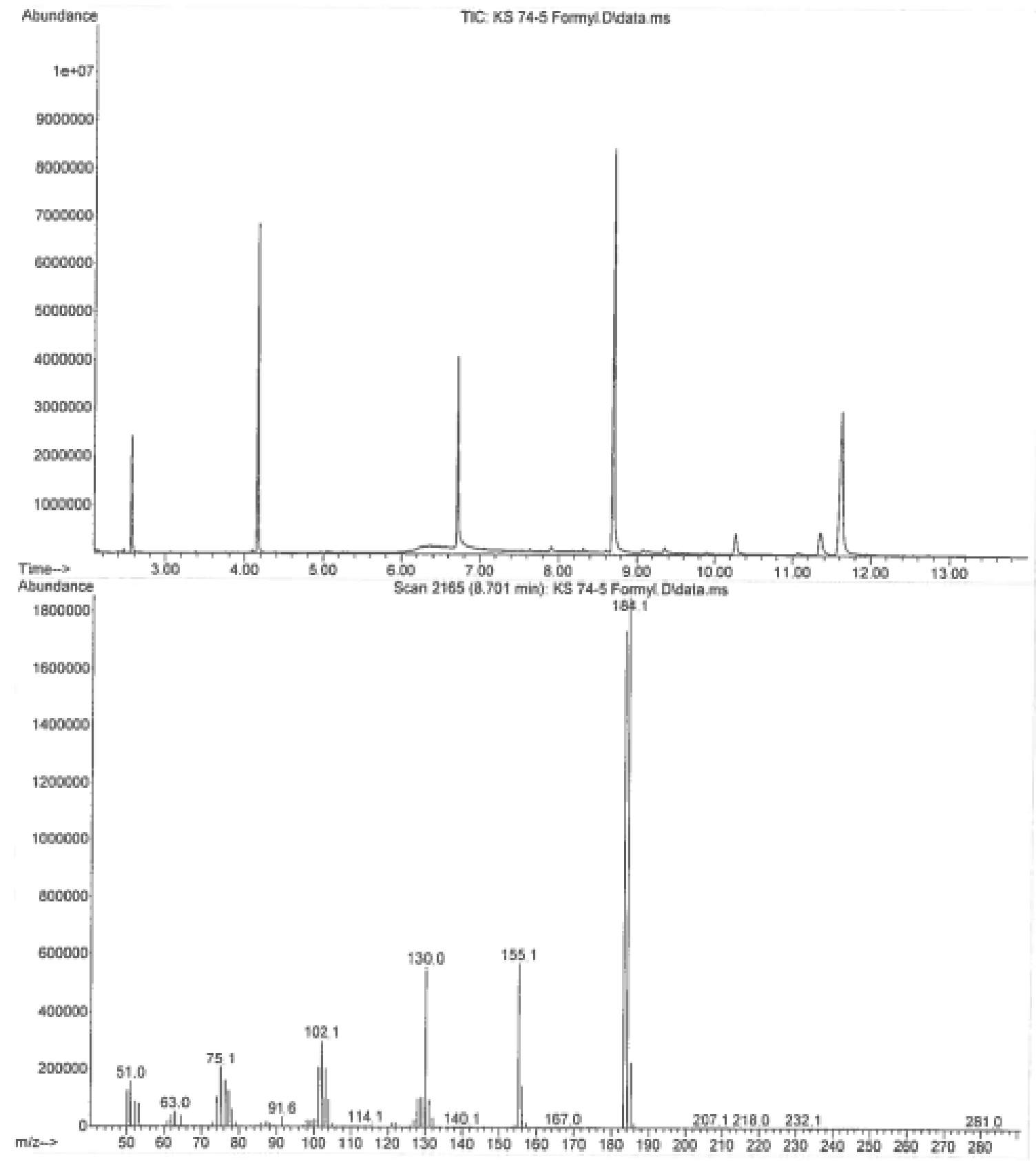


Figure A38 ${ }^{1} \mathrm{H}$ NMR spectrum of 2-phenyl-1,4-benzoquinone

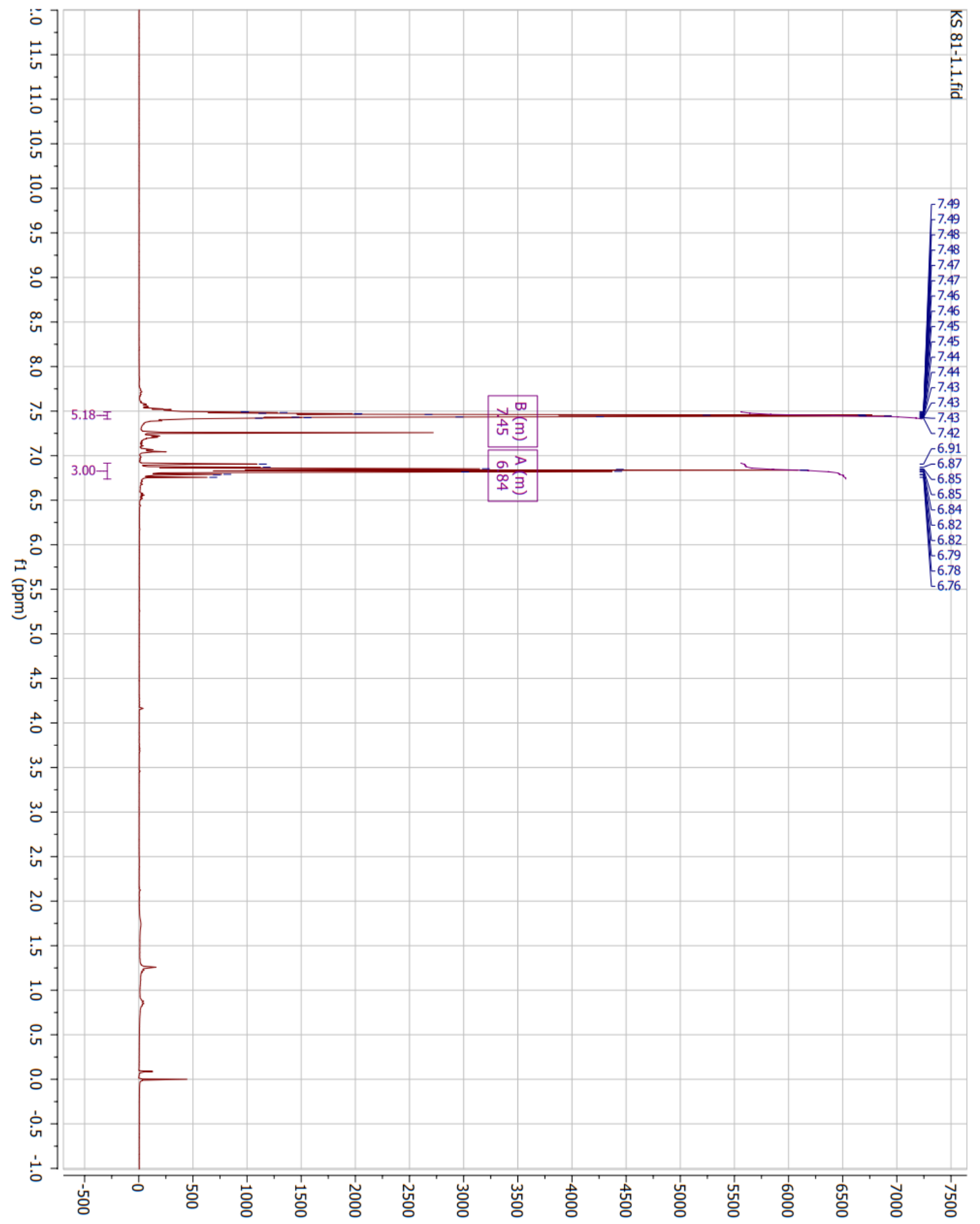


Figure A39 ${ }^{13} \mathrm{C}$ NMR spectrum of 2-phenyl-1,4-benzoquinone

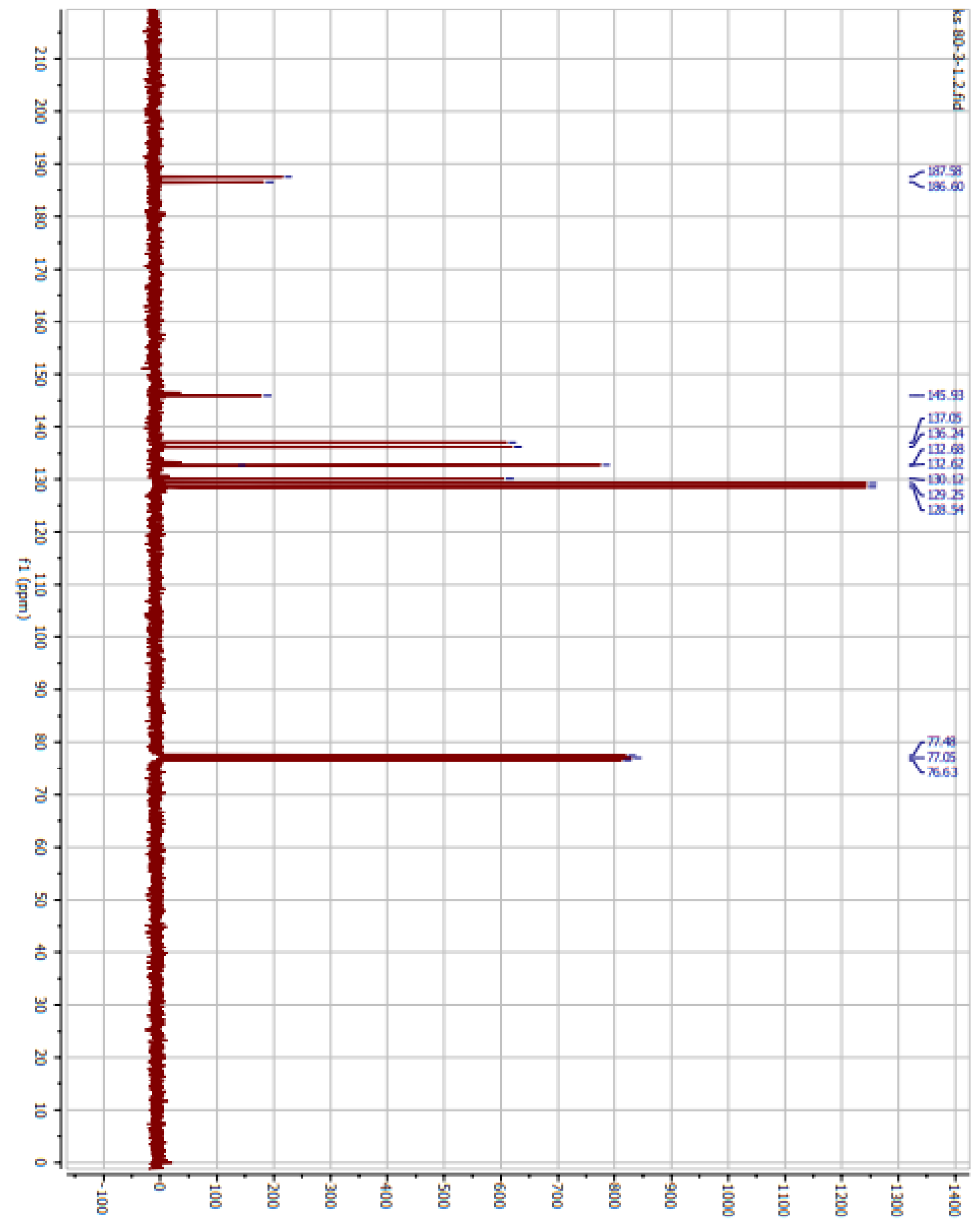


Figure A40 DEPT NMR spectrum of 2-phenyl-1,4-benzoquinone

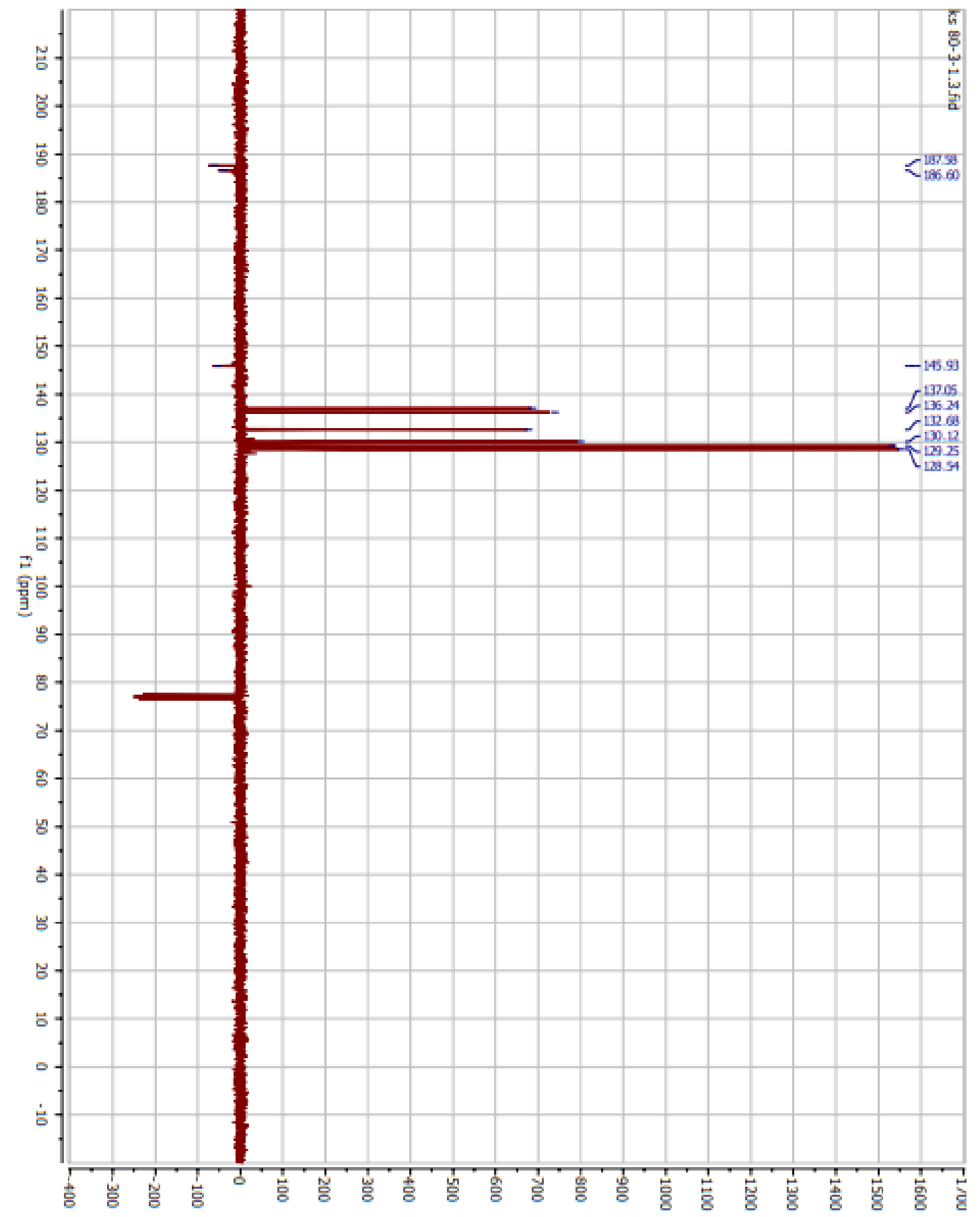




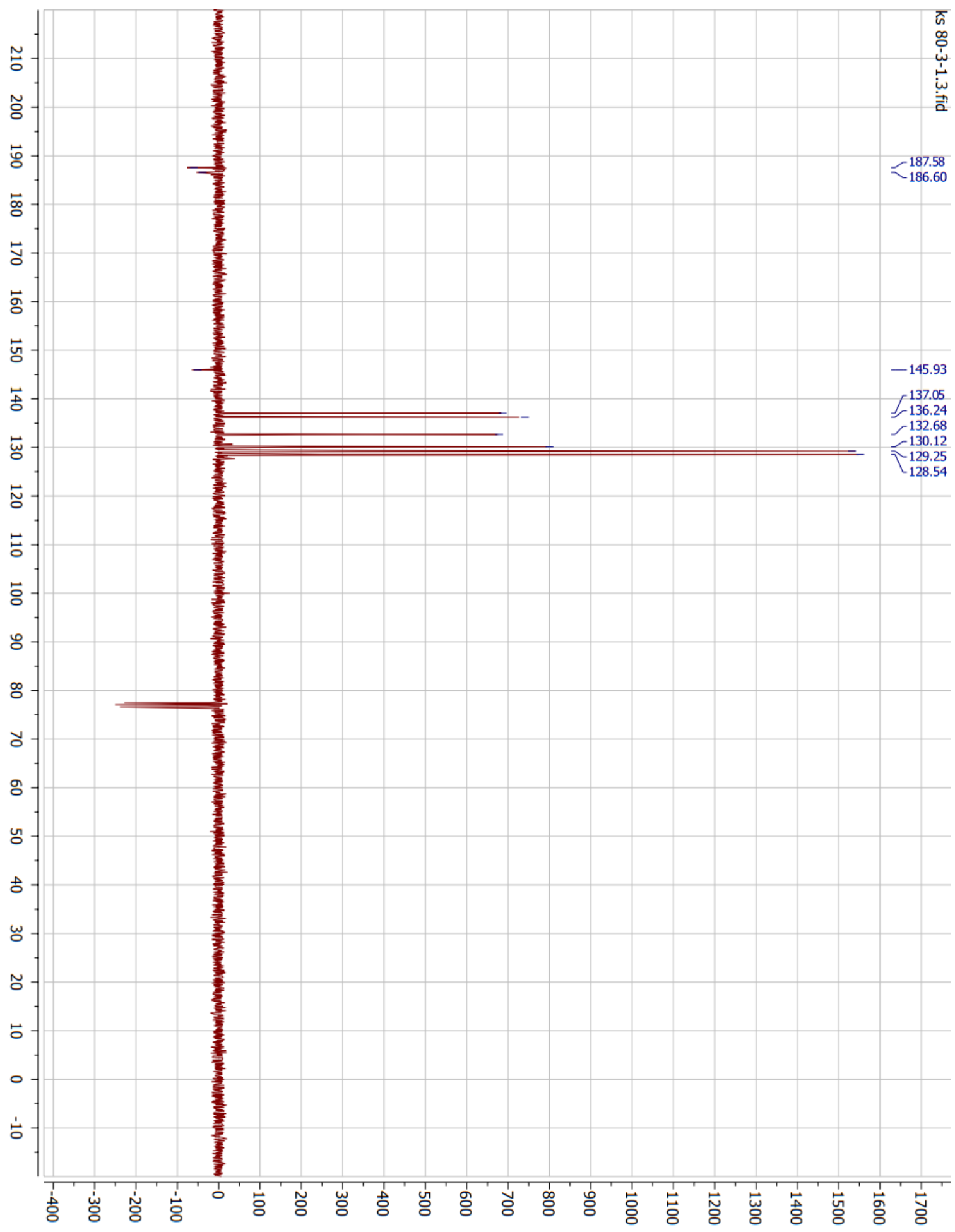


Figure A41 Gas chromatogram and mass spectrum of 2-phenyl-1,4-benzoquinone

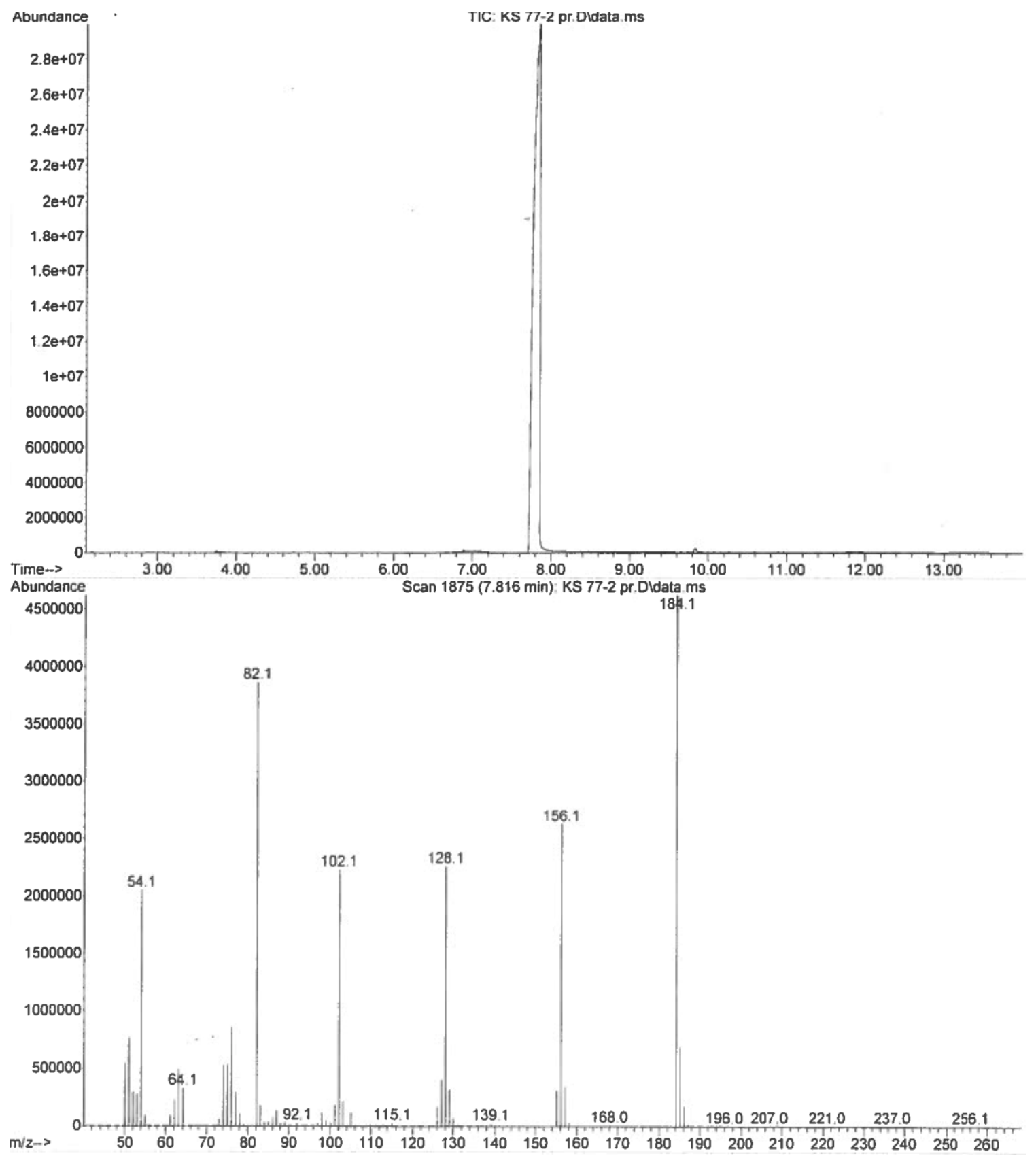


Figure A42 ${ }^{1} \mathrm{H}$ NMR spectrum of 2-(4-chlorophenyl)-1,4-benzoquinone

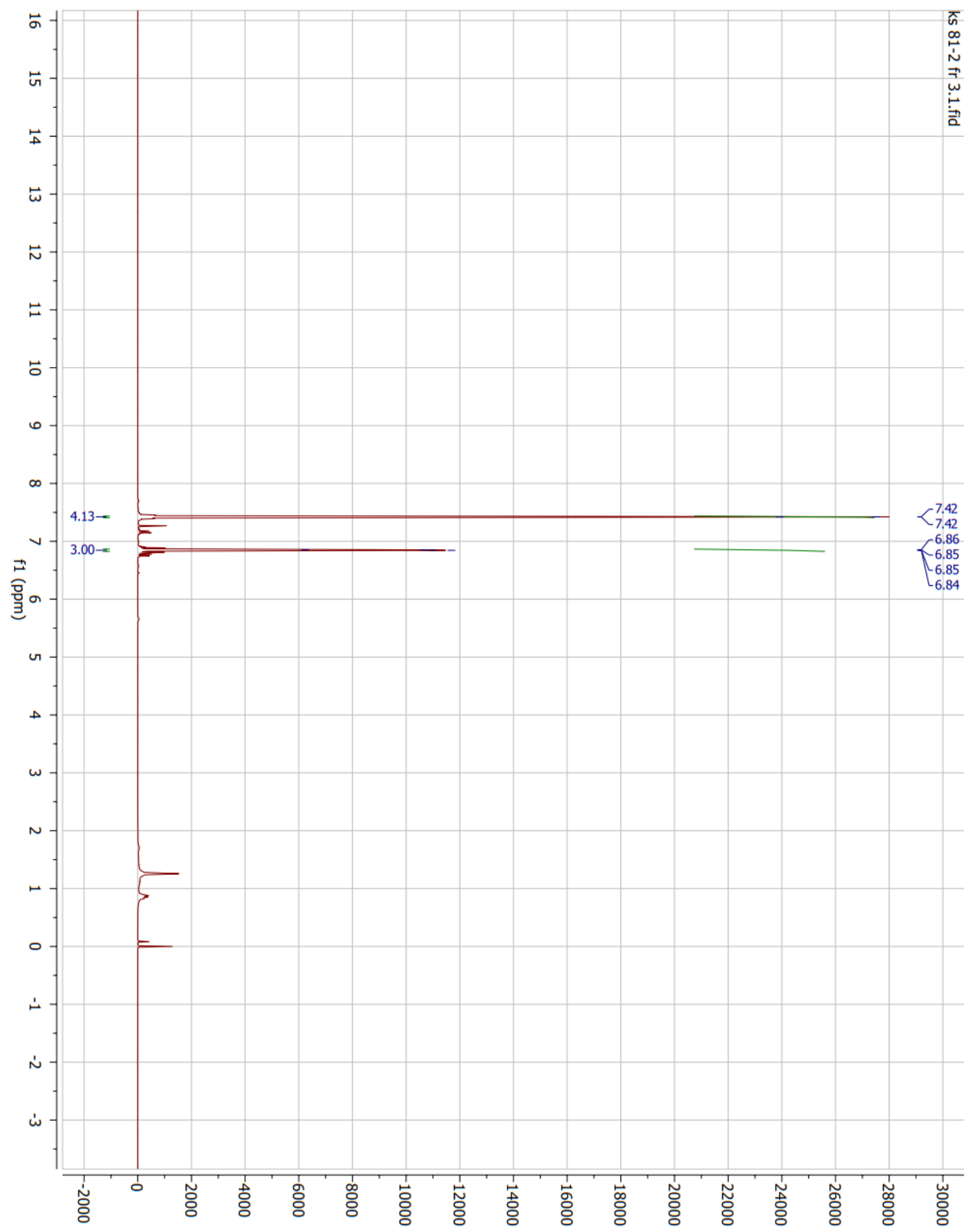


Figure A43 ${ }^{13} \mathrm{C}$ NMR spectrum of 2-(4-chlorophenyl)-1,4-benzoquinone

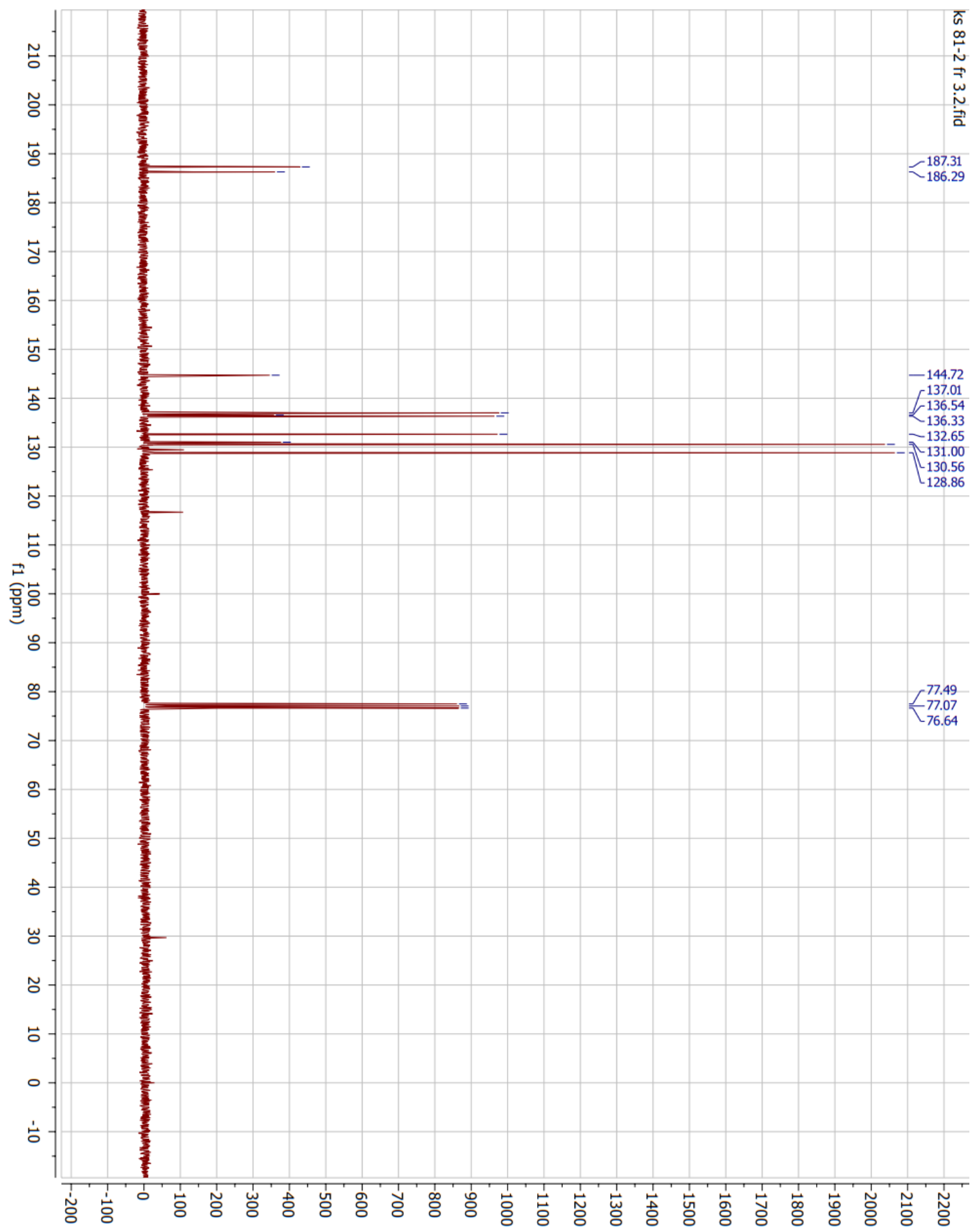


Figure A44 DEPT NMR spectrum of 2-(4-chlorophenyl)-1,4-benzoquinone

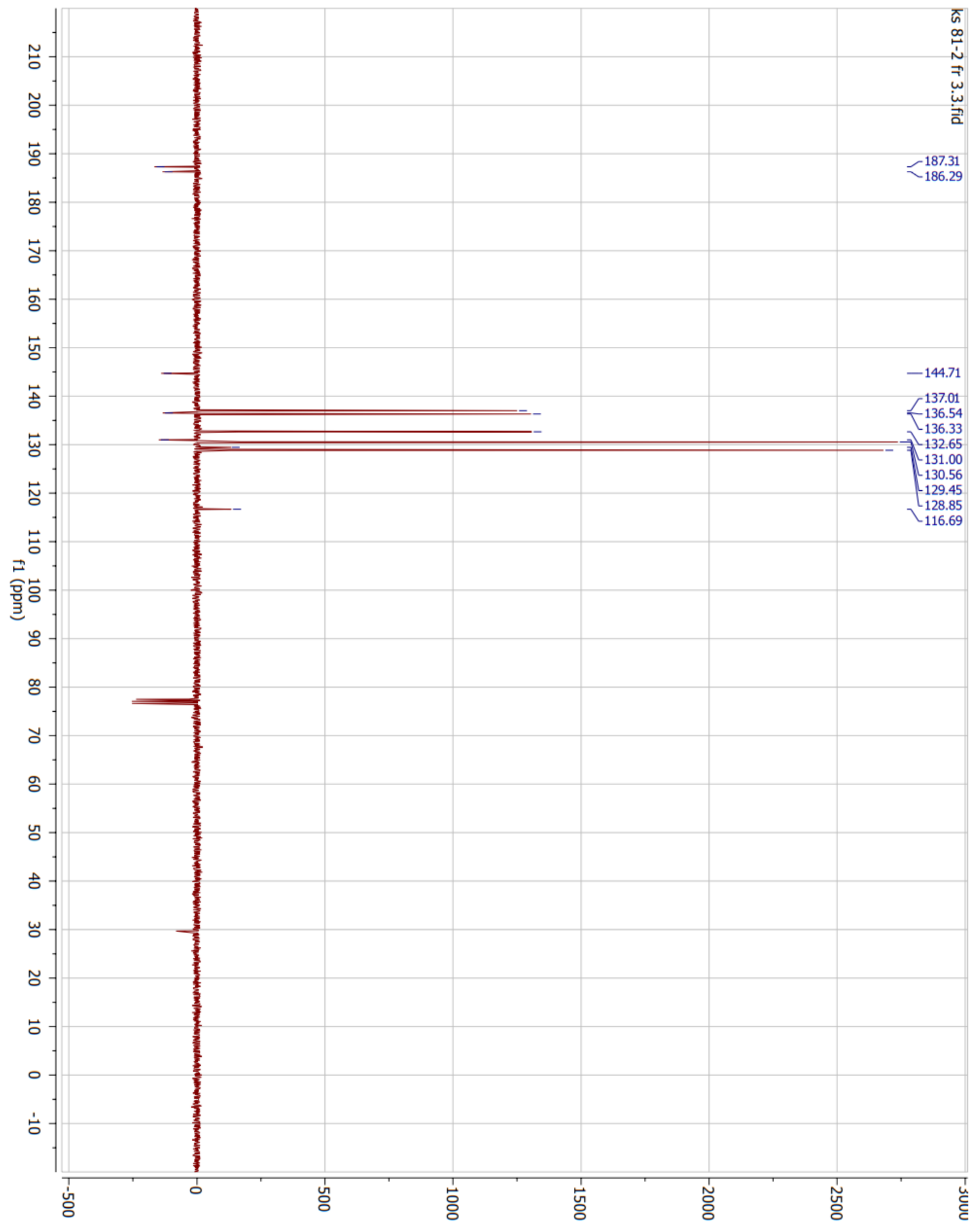


Figure A45 Gas chromatogram and mass spectrum of 2-(4-chlorophenyl)-1,4-benzoquinone

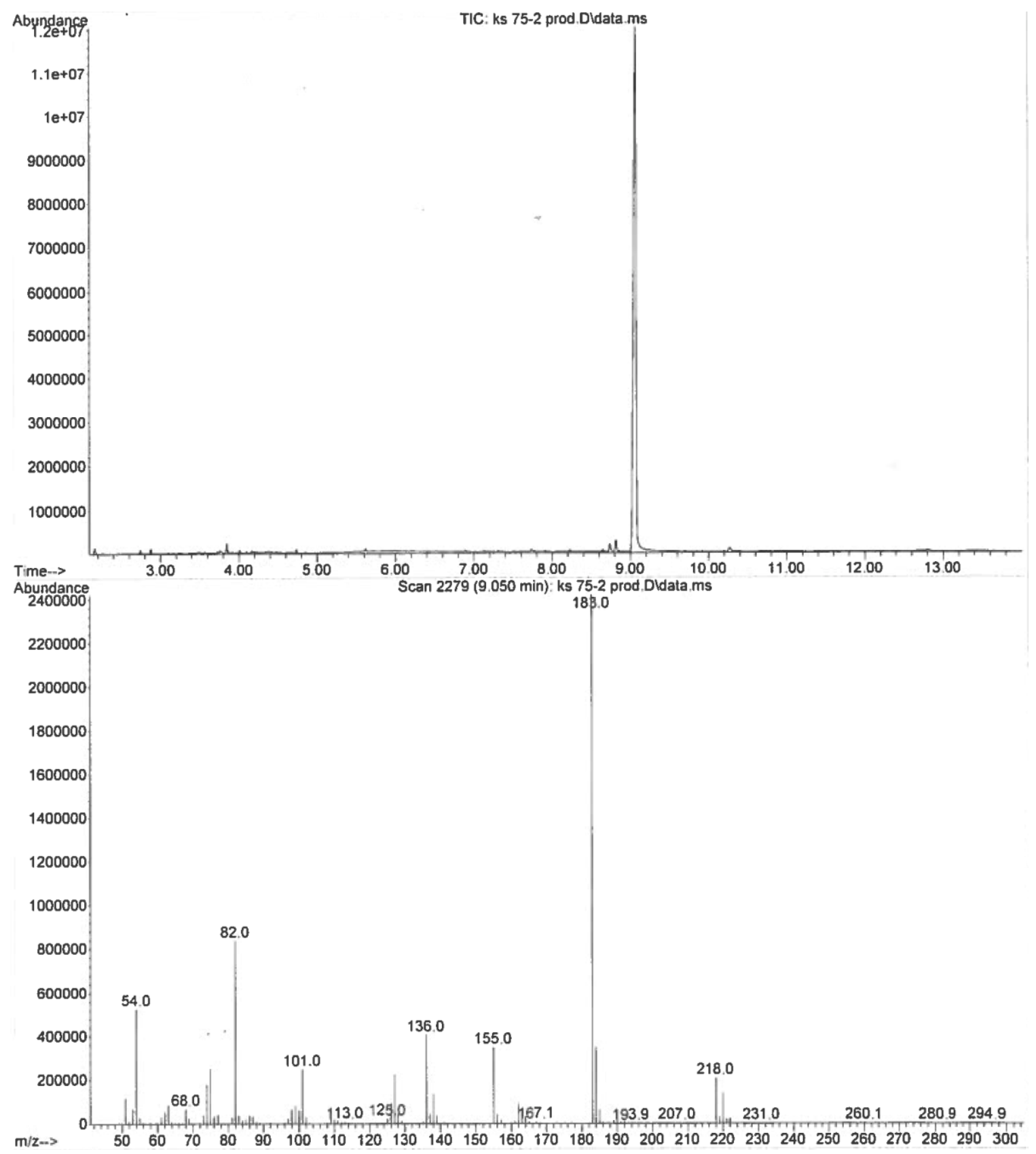


Figure A46 ${ }^{1} \mathrm{H}$ NMR spectrum of 2-(3-chlorophenyl)-1,4-benzoquinone

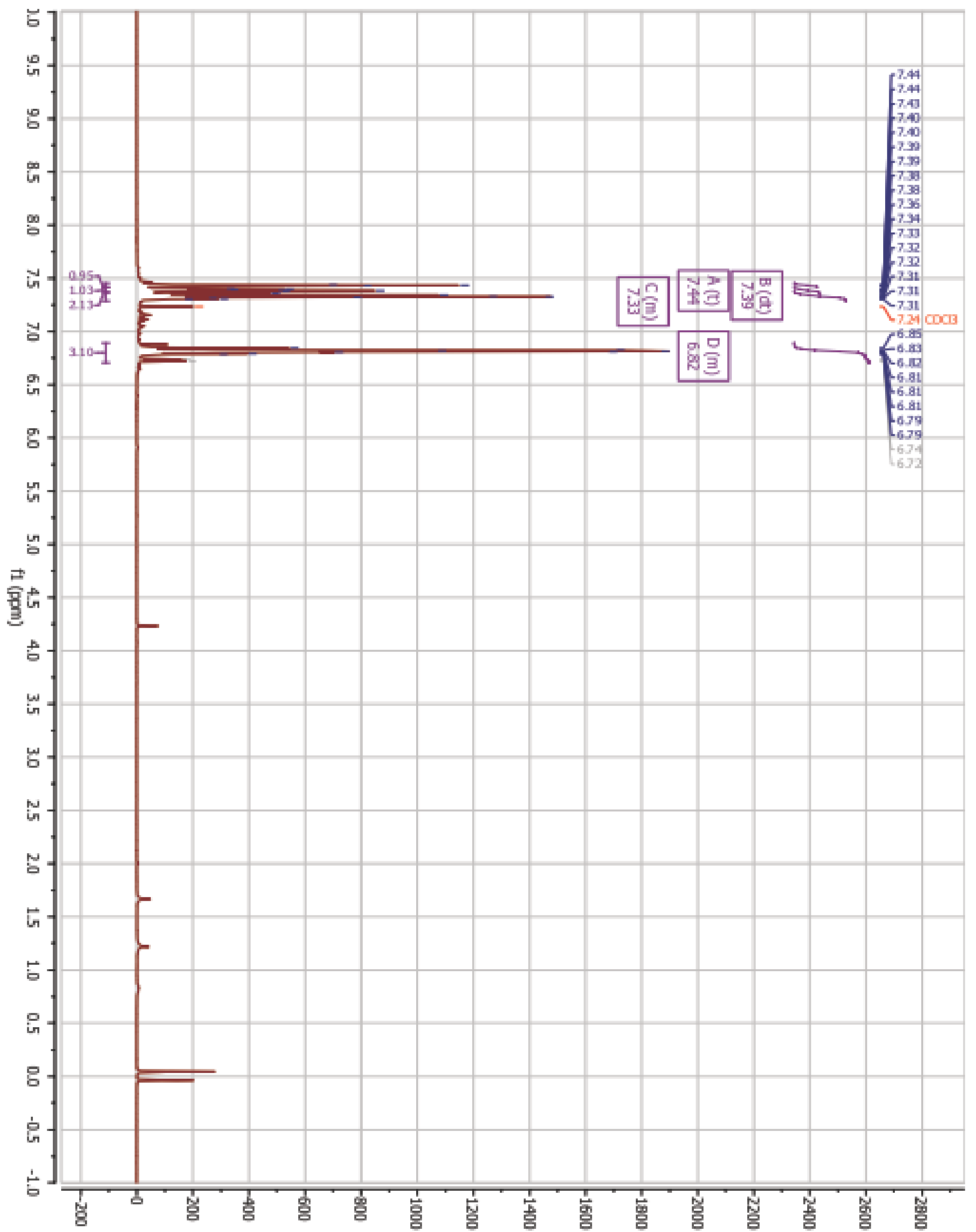


Figure A47 ${ }^{13} \mathrm{C}$ NMR spectrum of 2-(3-chlorophenyl)-1,4-benzoquinone

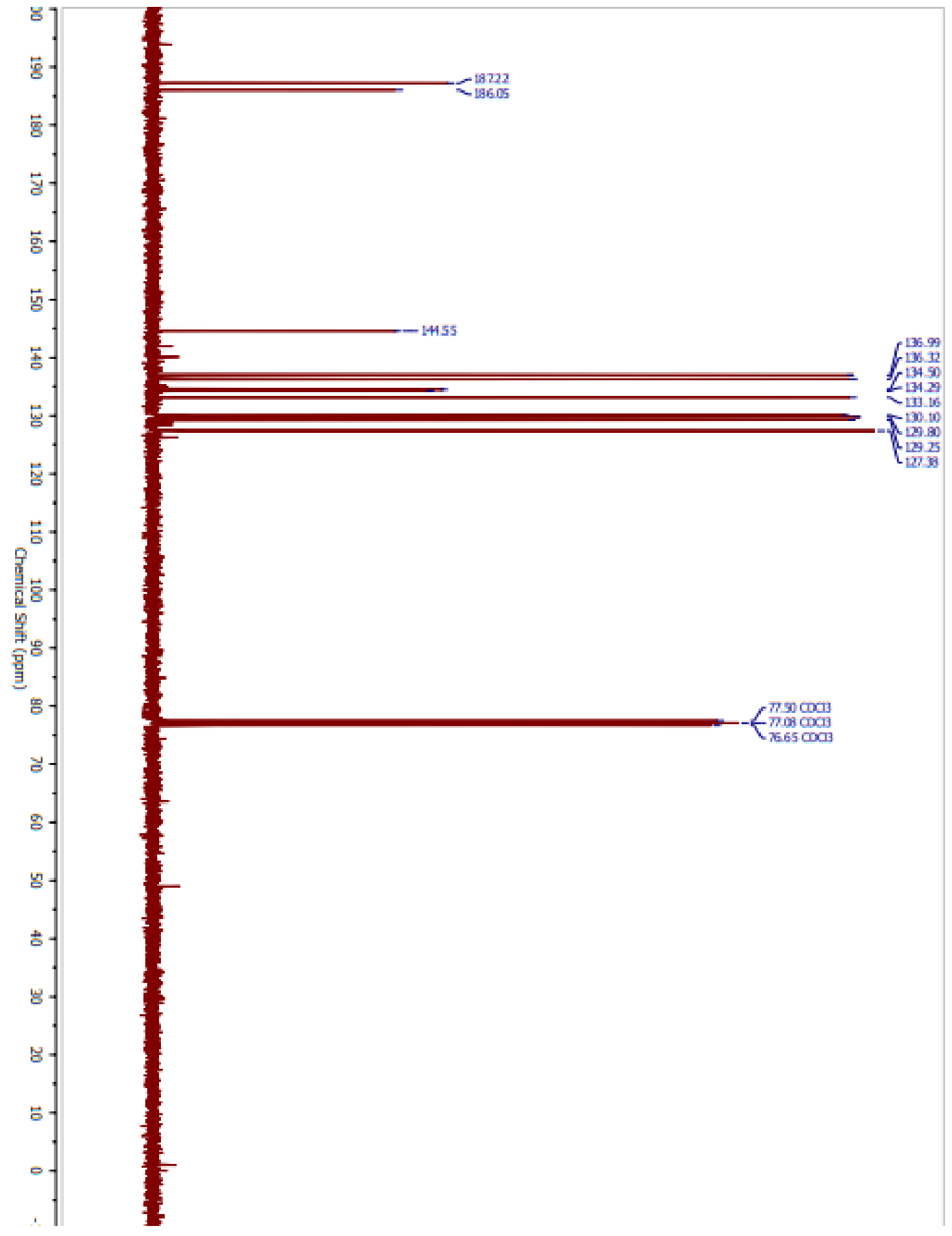


Figure A48 DEPT NMR spectrum of 2-(3-chlorophenyl)-1,4-benzoquinone

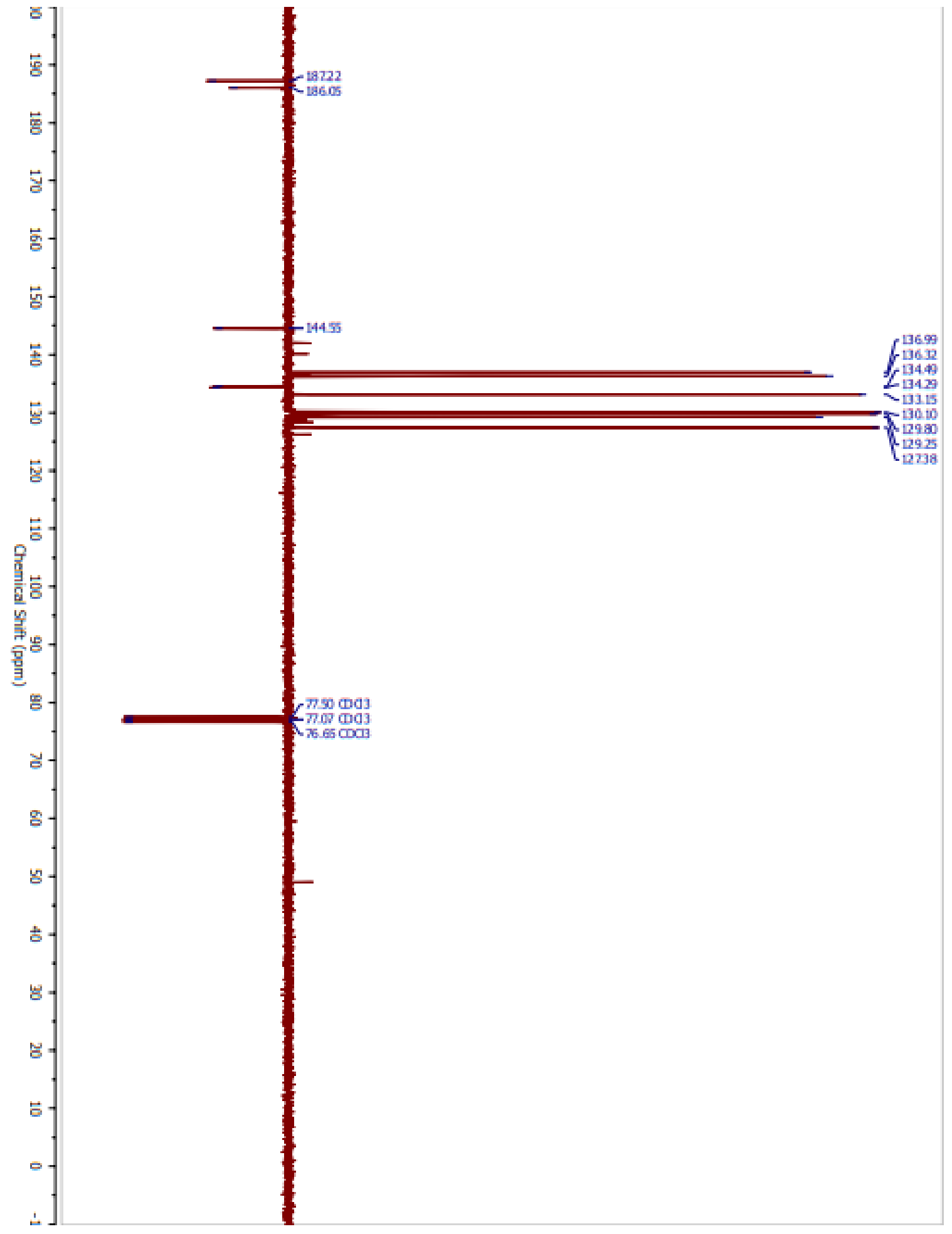


Figure A49 Gas chromatogram and mass spectrum of 2-(3-chlorophenyl)-1,4-benzoquinone

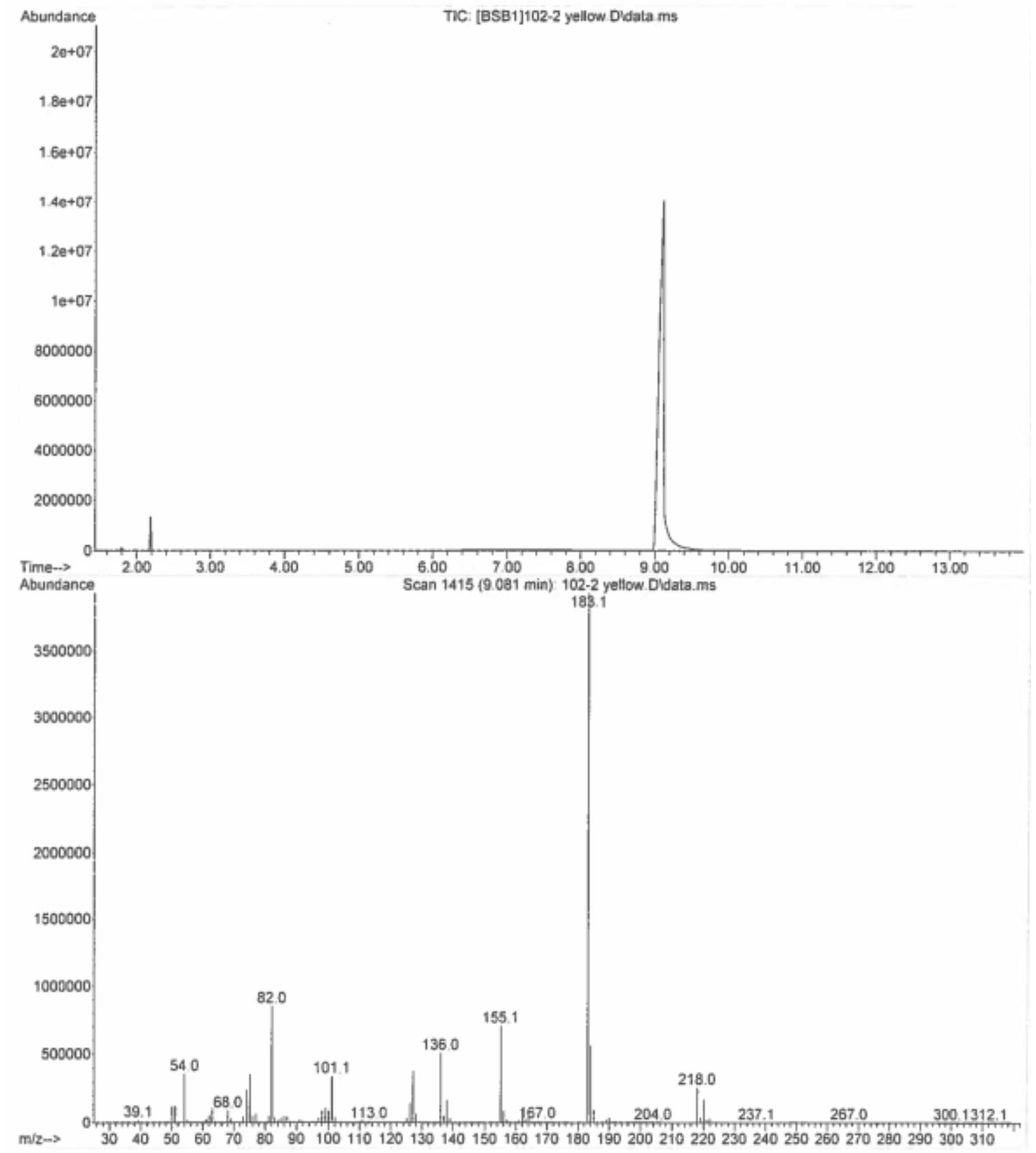


Figure A50 ${ }^{1} \mathrm{H}$ NMR spectrum of 2-(2-chlorophenyl)-1,4-benzoquinone

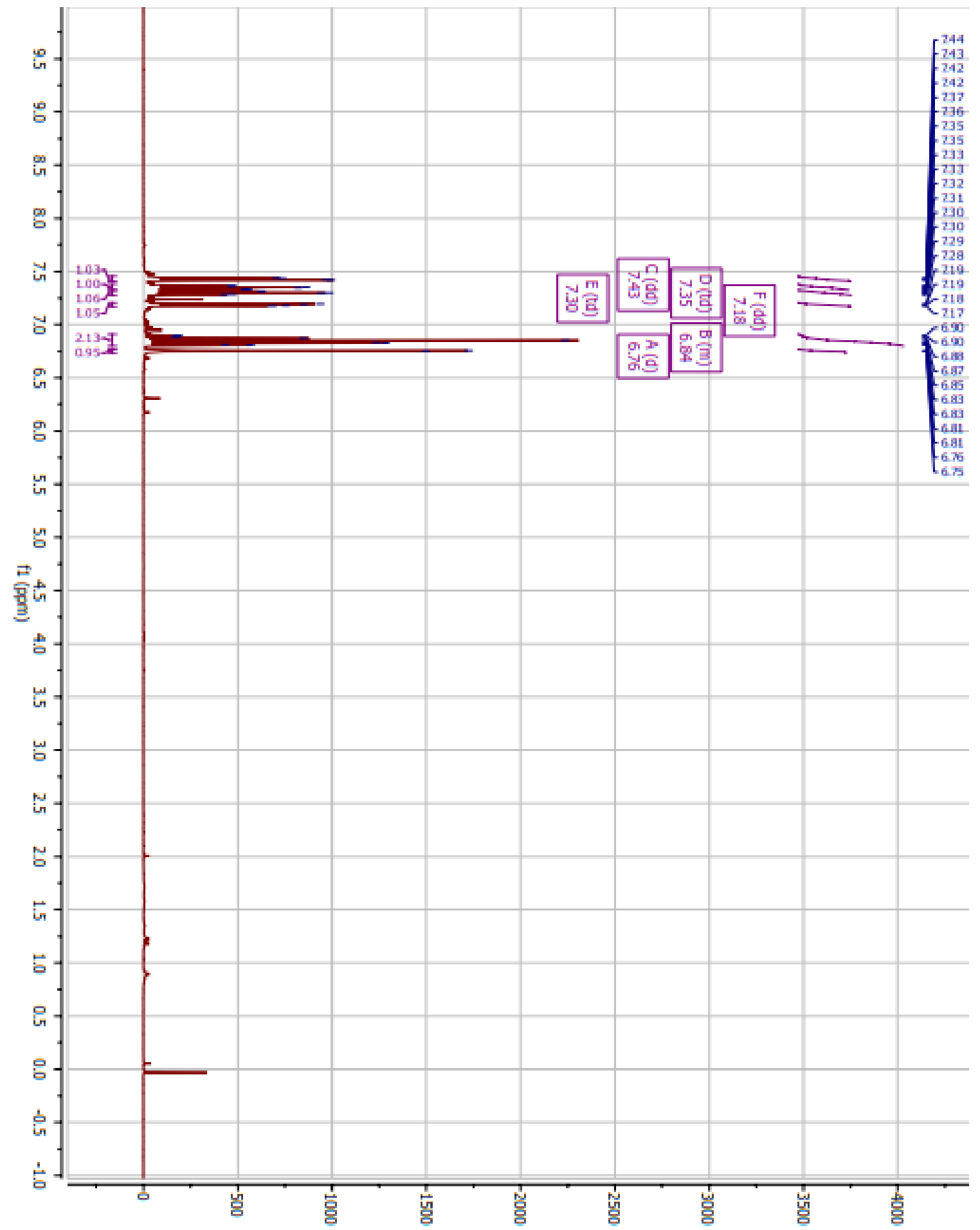


Figure A51 ${ }^{13} \mathrm{C}$ NMR spectrum of 2-(2-chlorophenyl)-1,4-benzoquinone

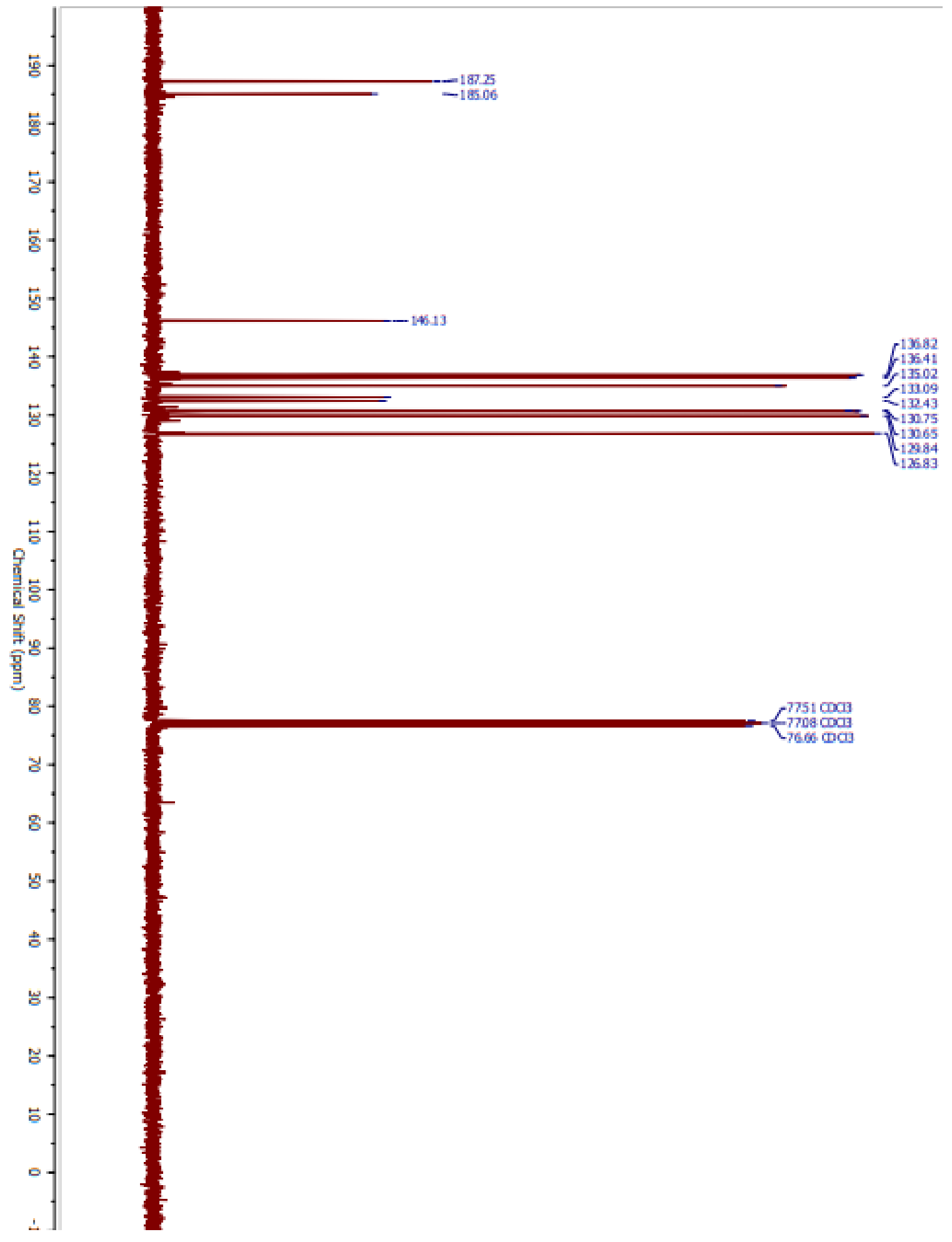


Figure A52 DEPT NMR spectrum of 2-(2-chlorophenyl)-1,4-benzoquinone

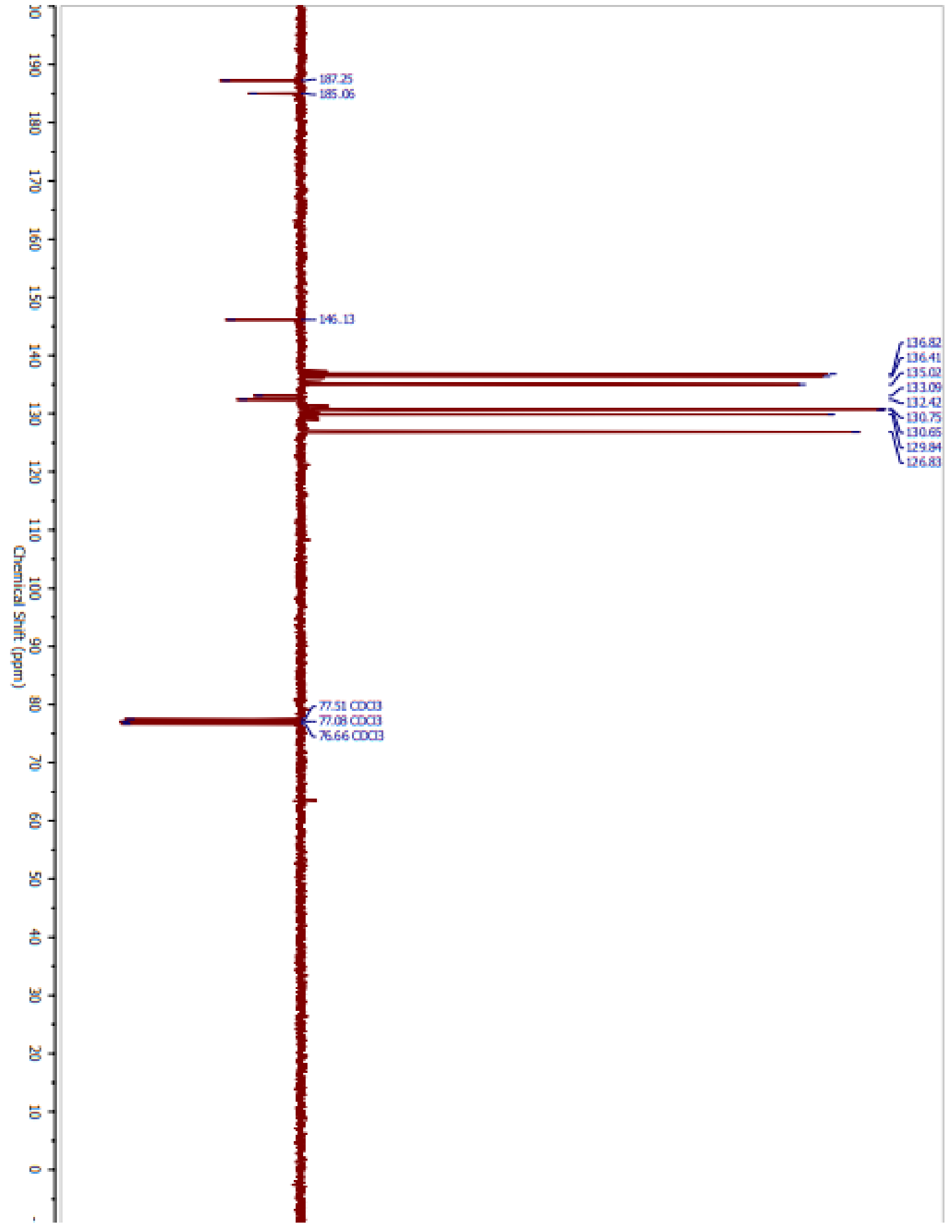


Figure A53 Gas chromatogram and mass spectrum of 2-(2-chlorophenyl)-1,4-benzoquinone

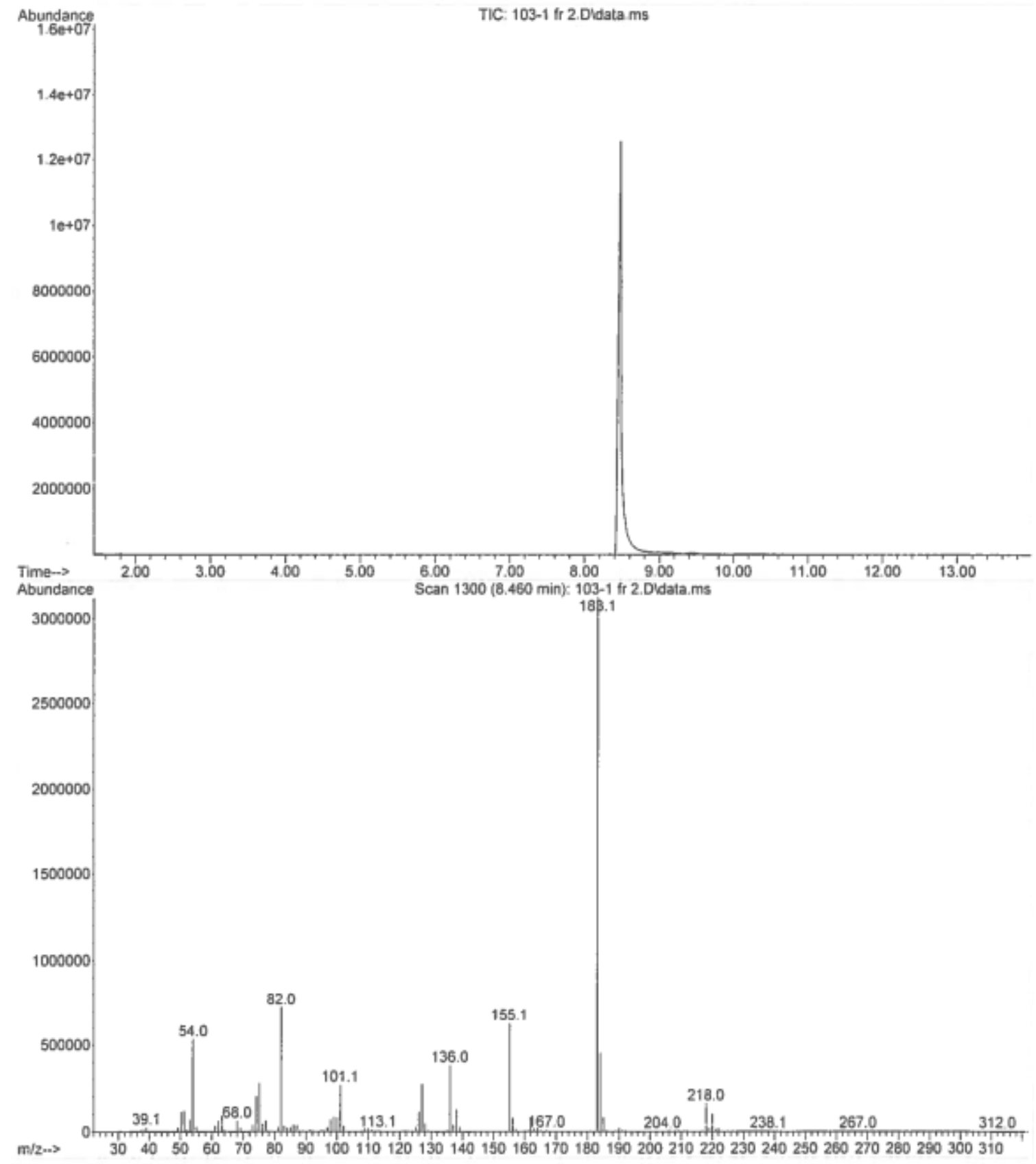


Figure A54 ${ }^{1} \mathrm{H}$ NMR spectrum of 2-(4-methylphenyl)-1,4-benzoquinone

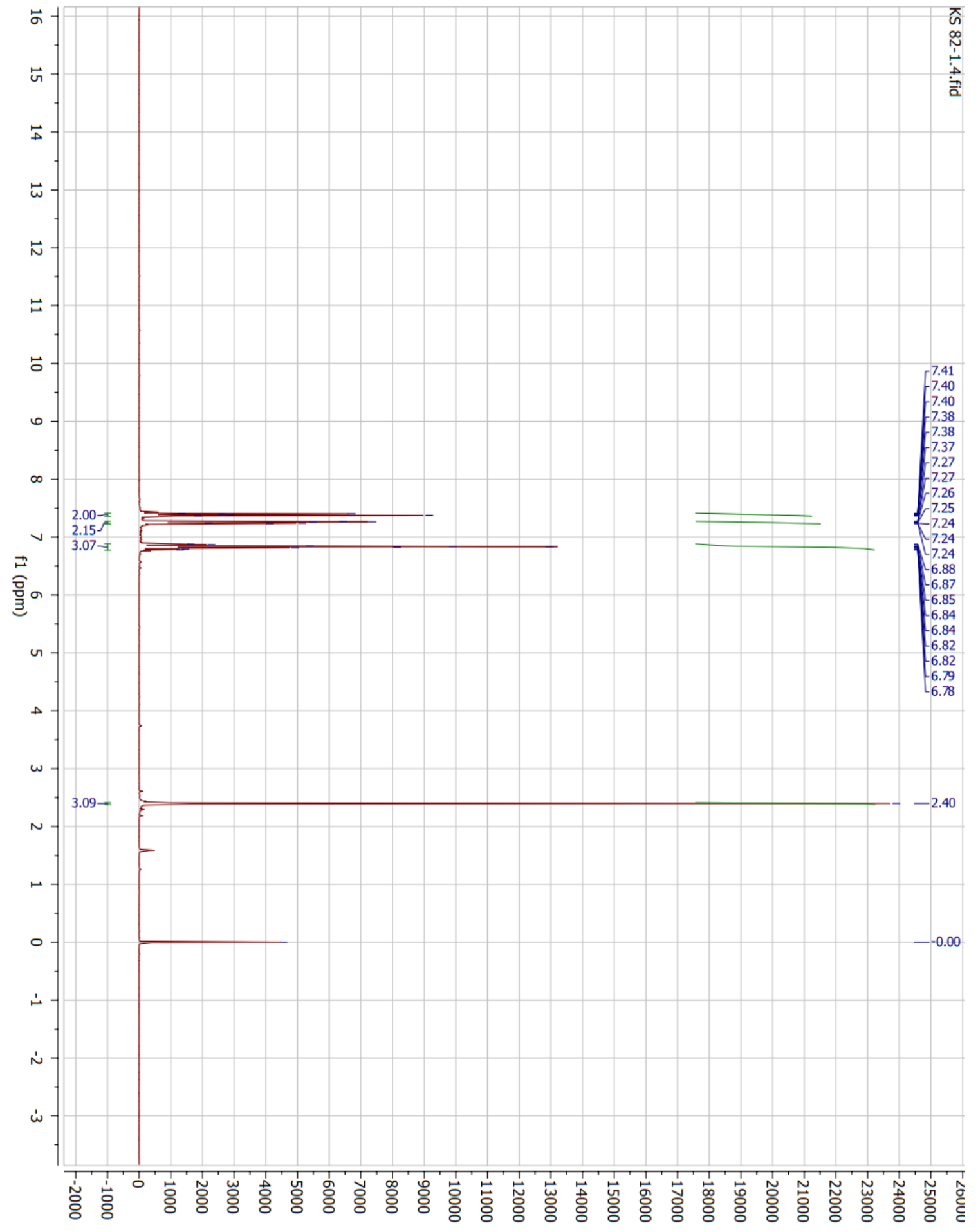


Figure A55 ${ }^{13} \mathrm{C}$ NMR spectrum of 2-(4-methylphenyl)-1,4-benzoquinone

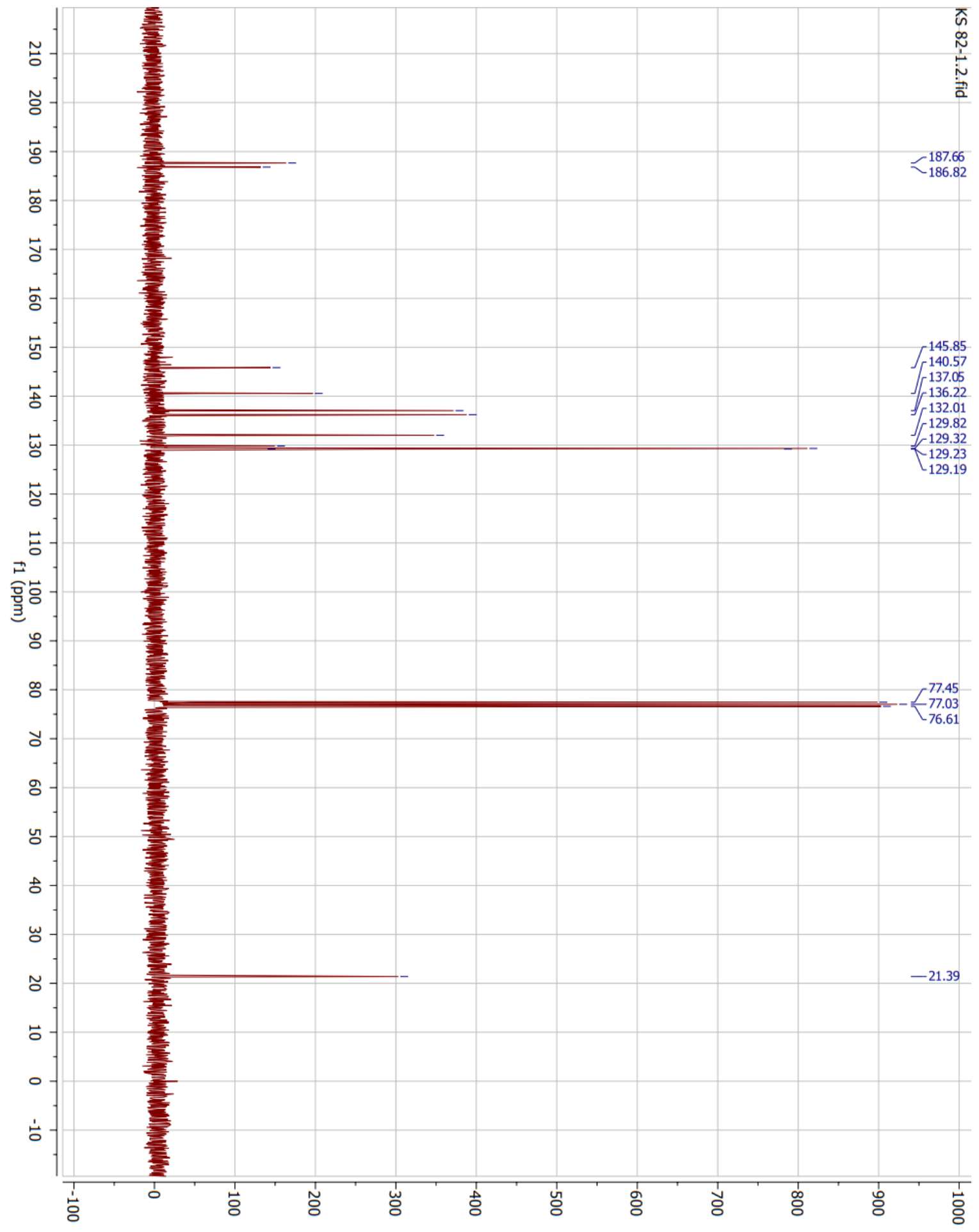


Figure A56 DEPT NMR spectrum of 2-(4-methylphenyl)-1,4-benzoquinone

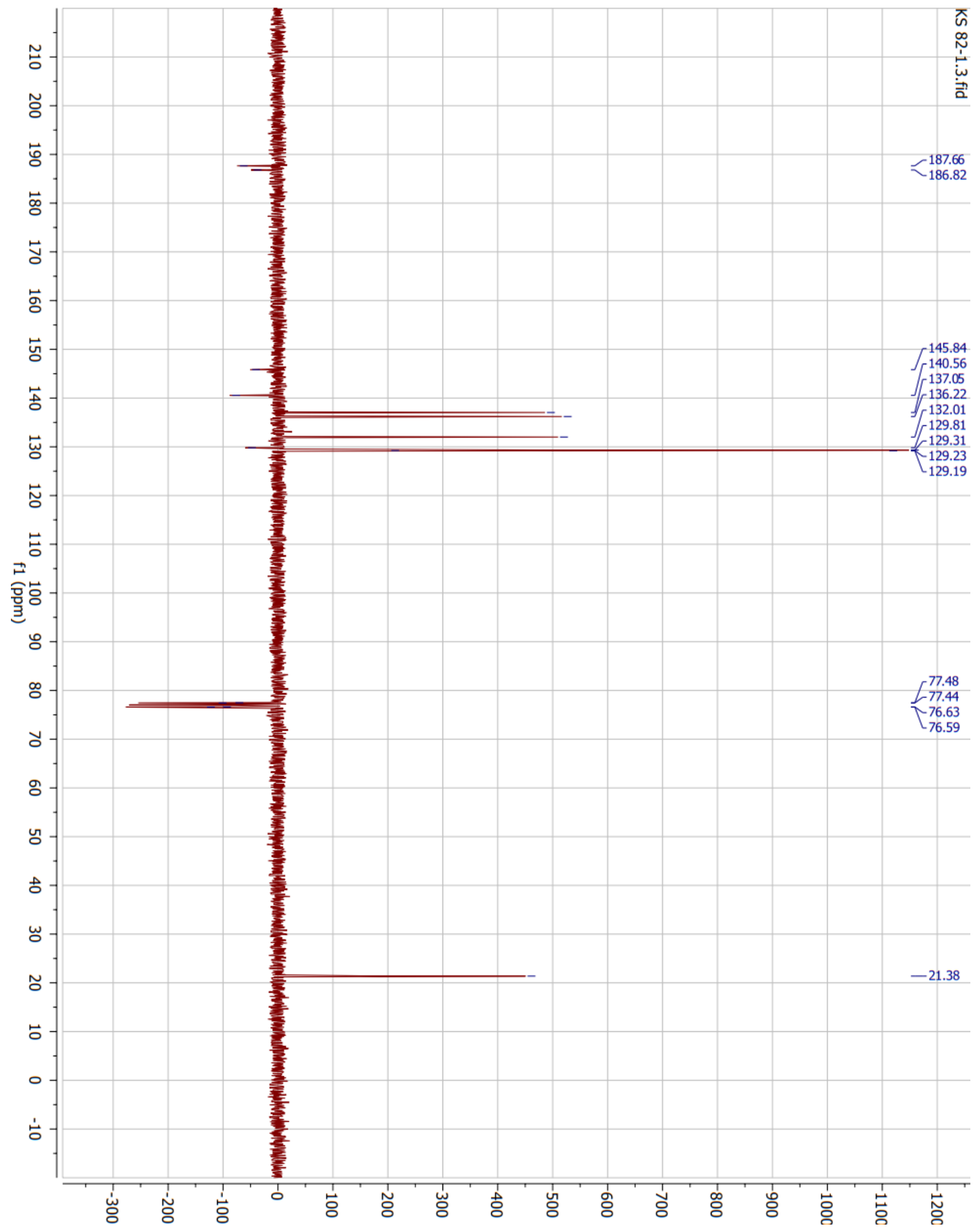


Figure A 57 Gas chromatogram and mass spectrum of 2-(4-methylphenyl)-1,4-benzoquinone

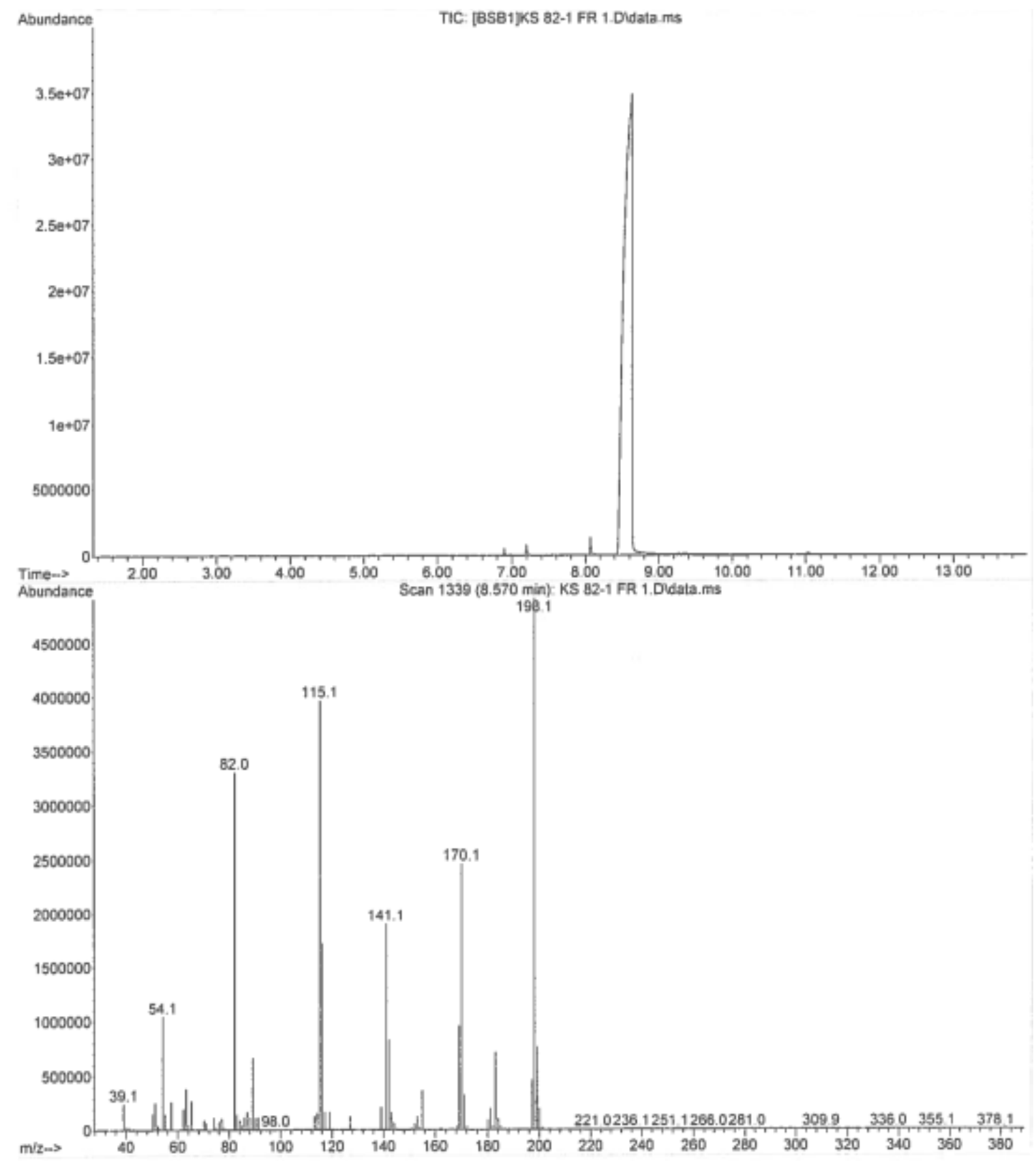


Figure A58 ${ }^{1} \mathrm{H}$ NMR spectrum of 2-(2-methylphenyl)-1,4-benzoquinone

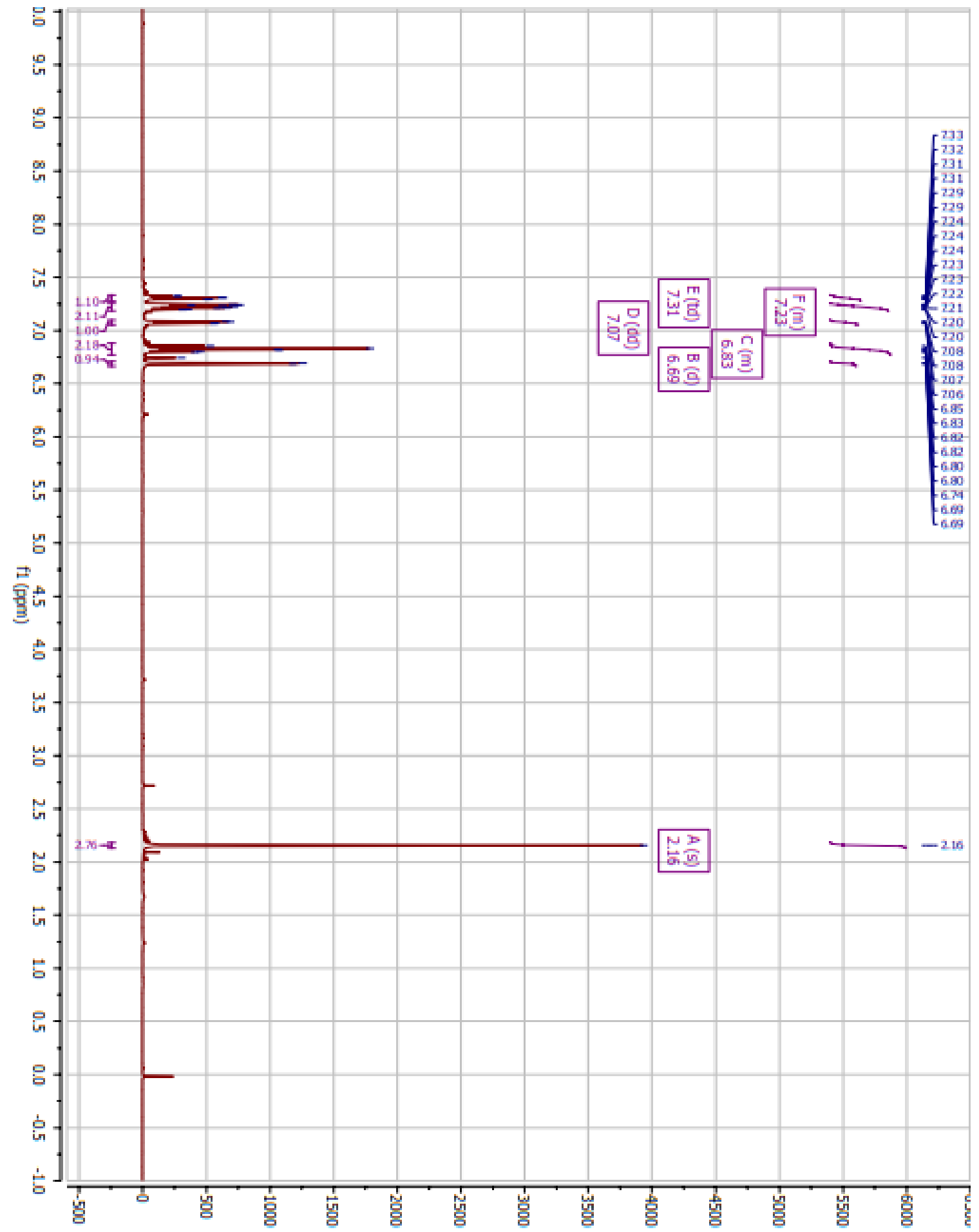


Figure A59 ${ }^{13} \mathrm{C}$ NMR spectrum of 2-(2-methylphenyl)-1,4-benzoquinone

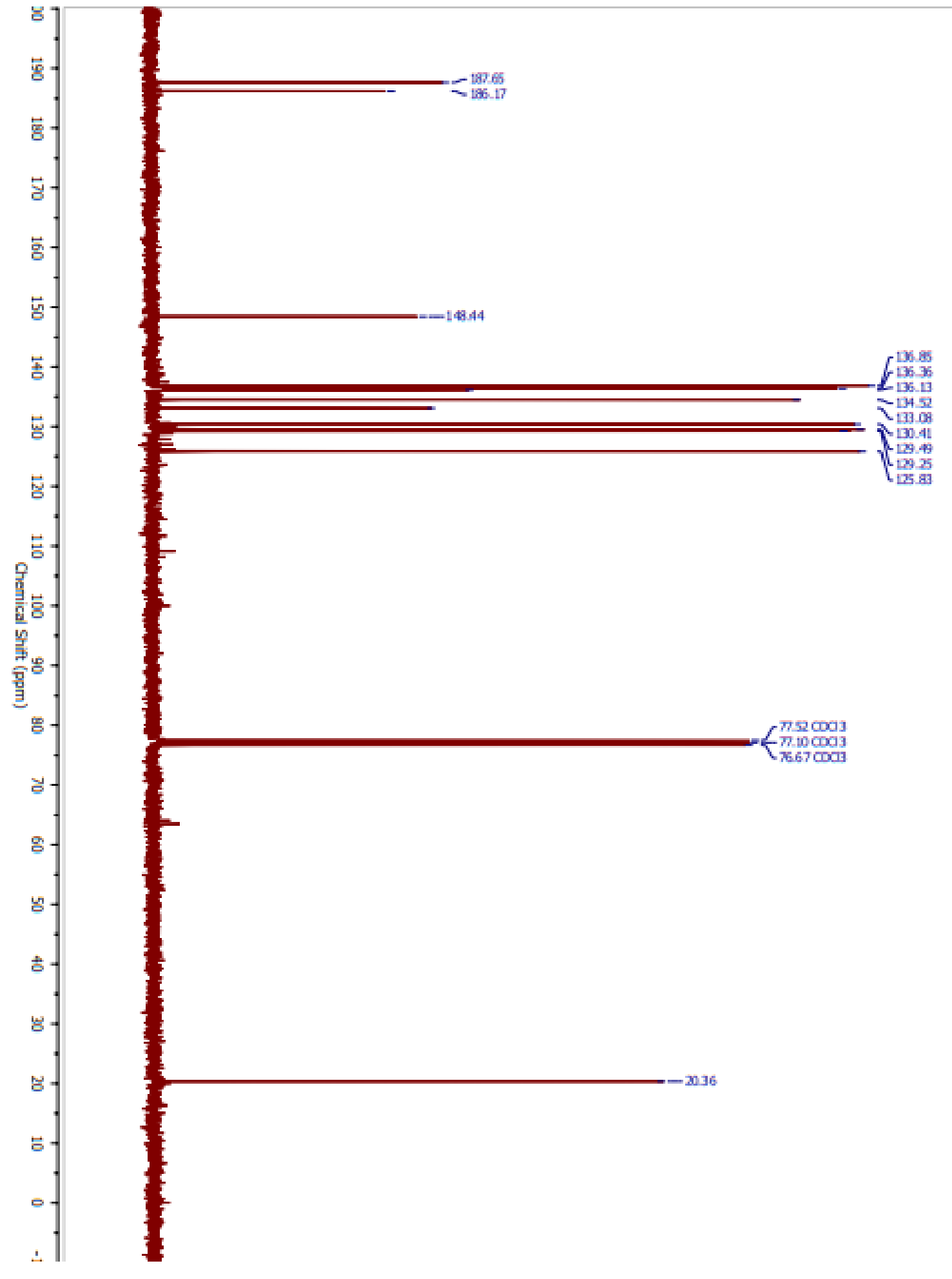


Figure A60 DEPT NMR spectrum of 2-(2-methylphenyl)-1,4-benzoquinone

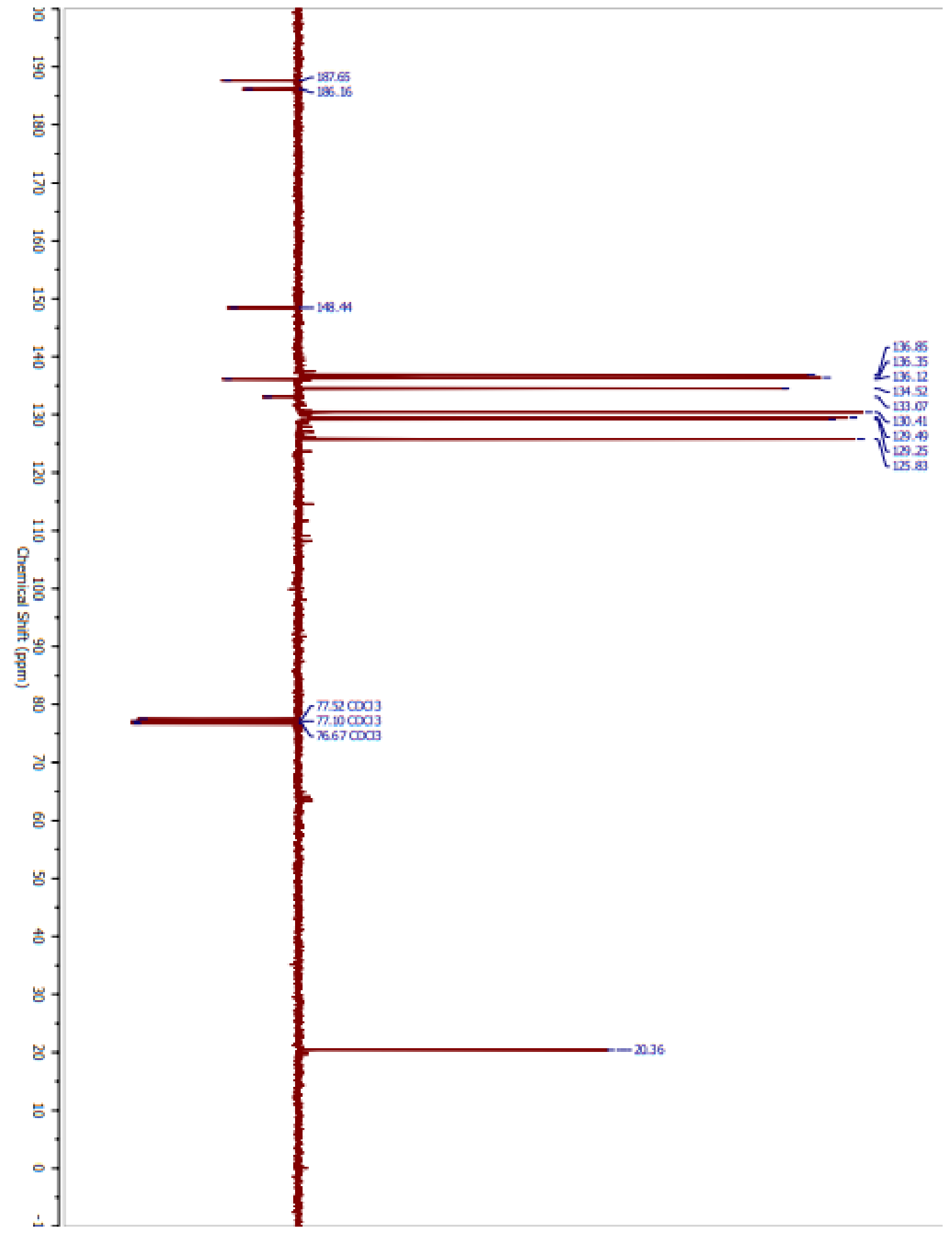


Figure A61 Gas chromatogram and mass spectrum of 2-(2-methylphenyl)-1,4-benzoquinone

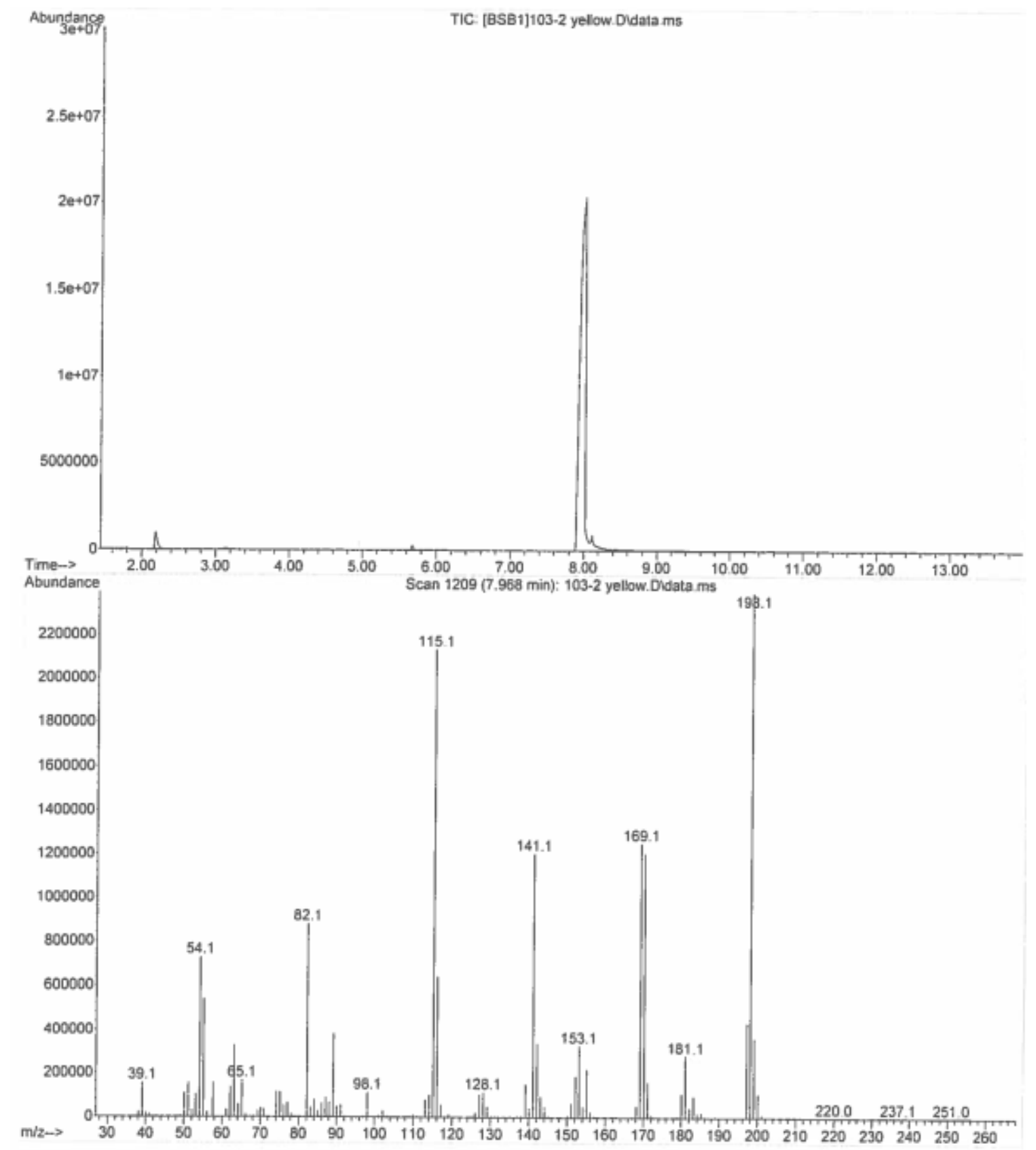


Figure A62 ${ }^{1} \mathrm{H}$ NMR spectrum of 2-mesityl-1,4-benzoquinone

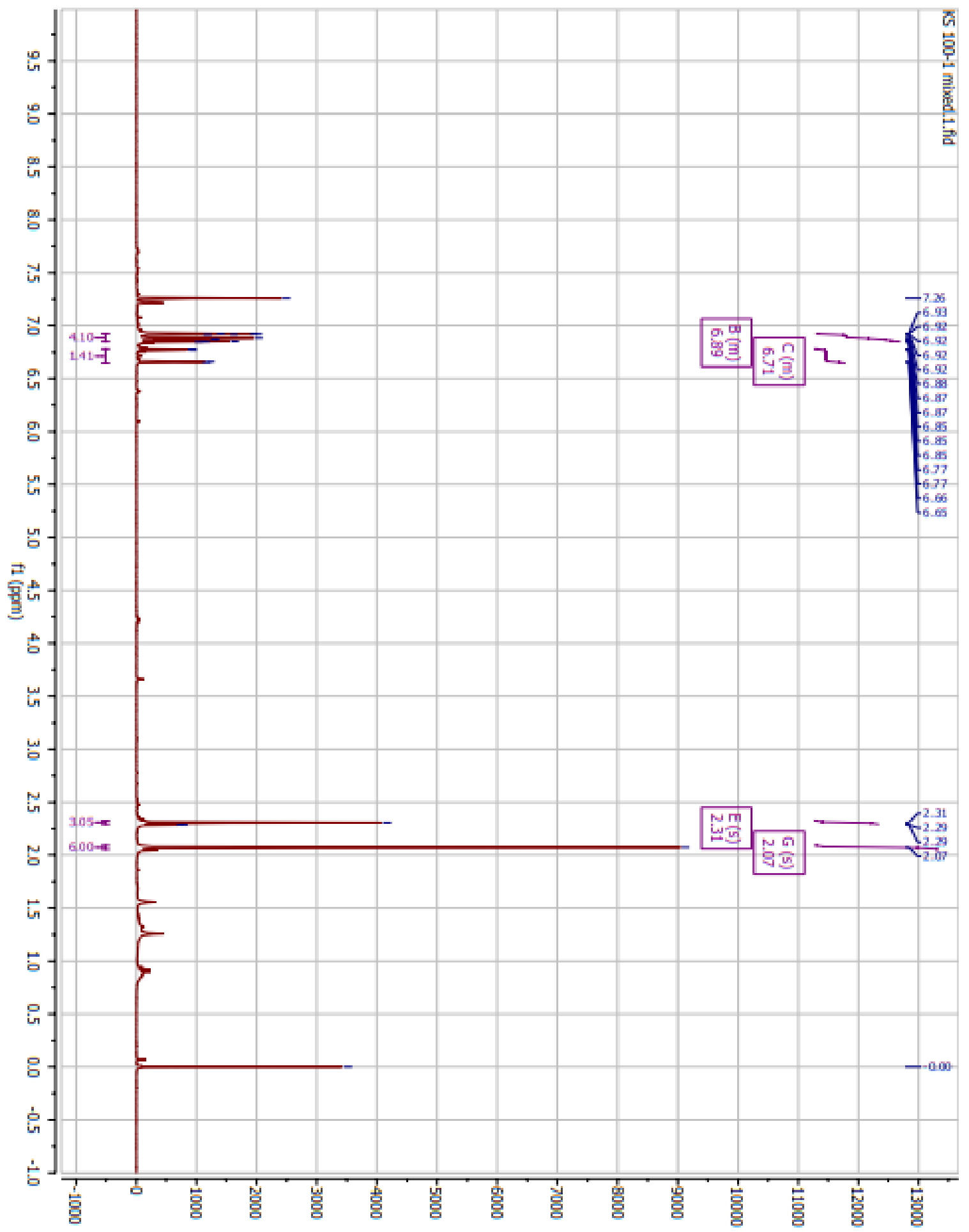


Figure A63 ${ }^{13} \mathrm{C}$ NMR spectrum of 2-mesityl-1,4-benzoquinone

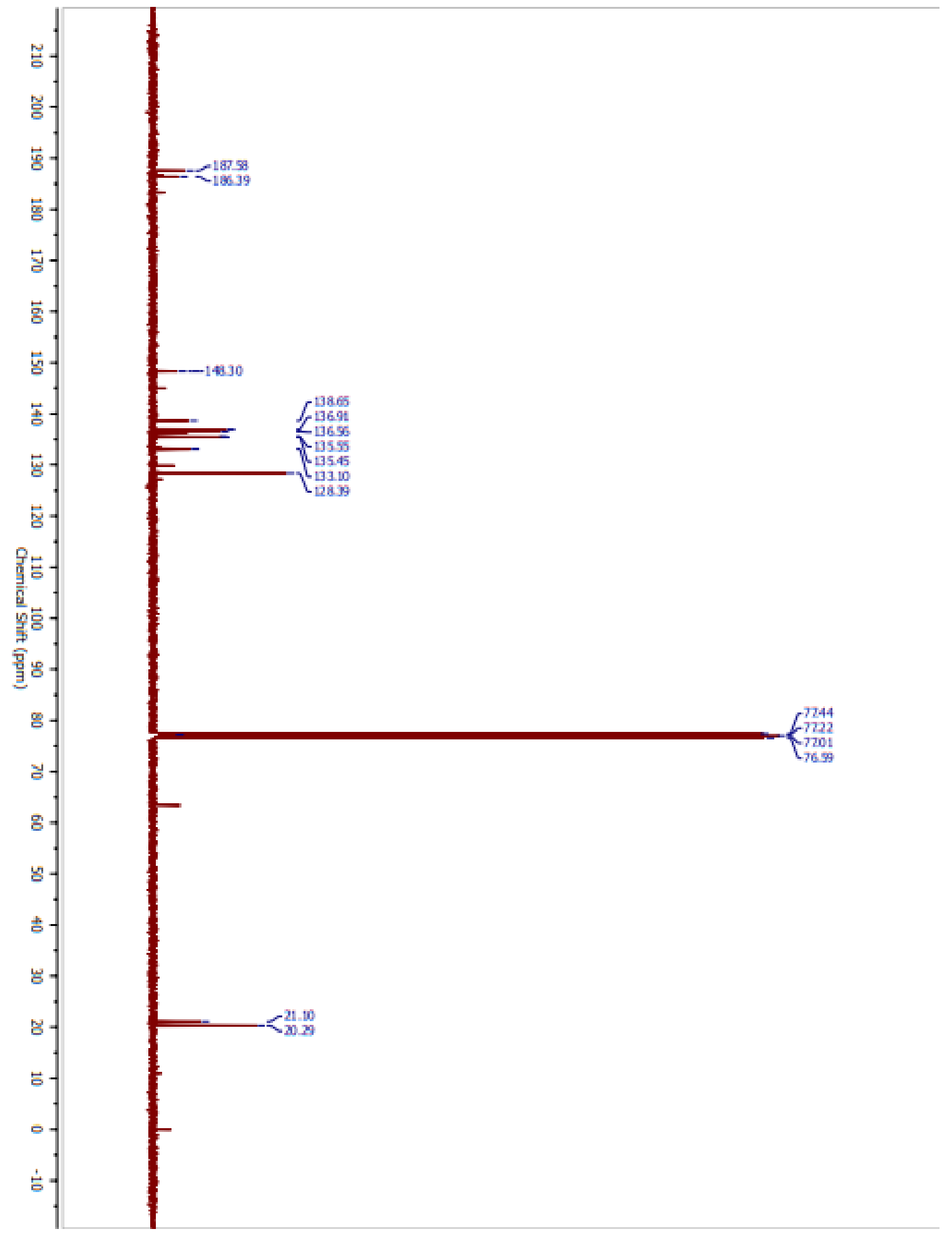




$$
F
$$


Figure A65 Gas chromatogram and mass spectrum of 2-mesityl-1,4-benzoquinone

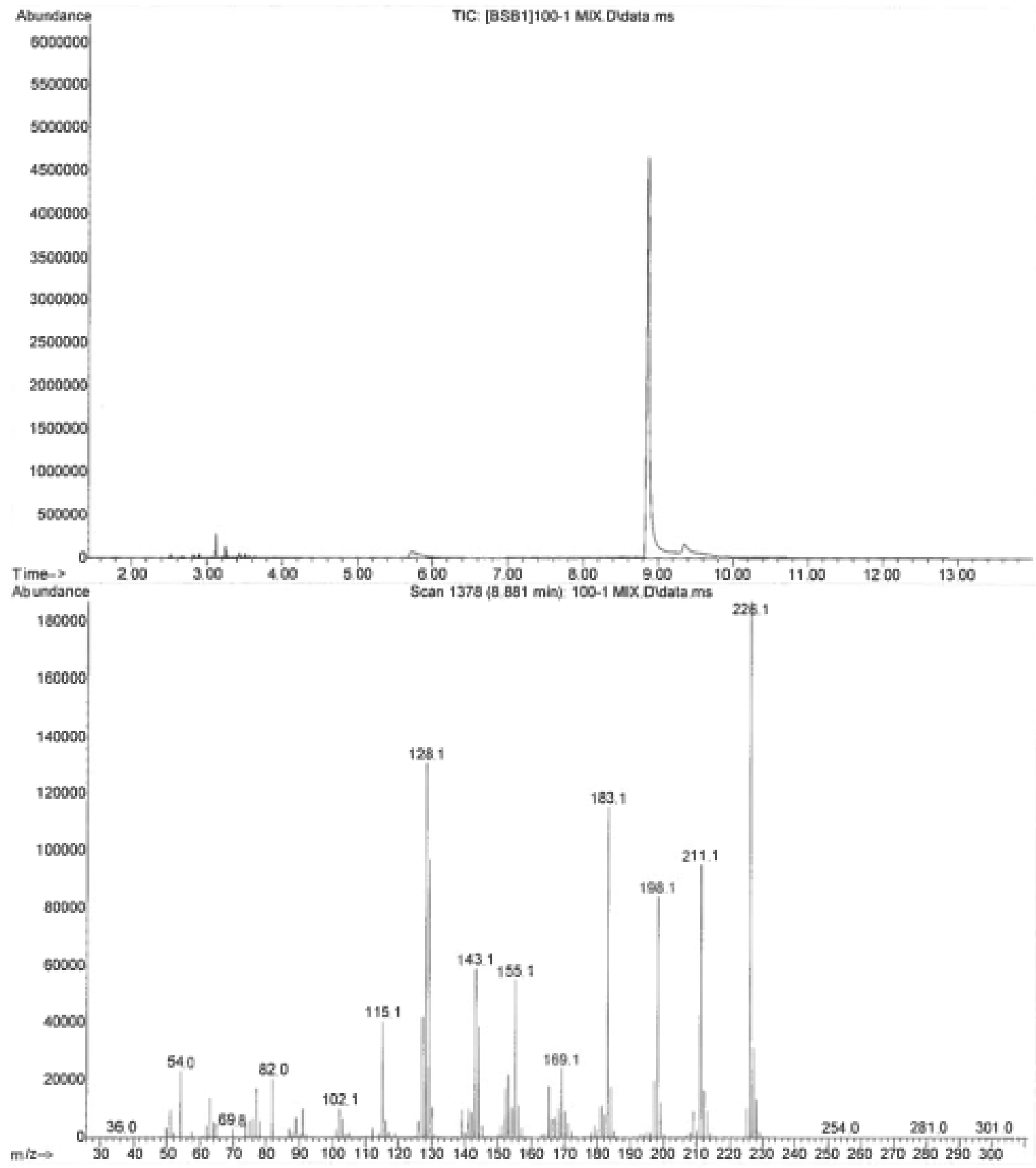


Figure A66 ${ }^{1} \mathrm{H}$ NMR spectrum of 2-(4-trifluoromethylphenyl)-1,4-benzoquinone

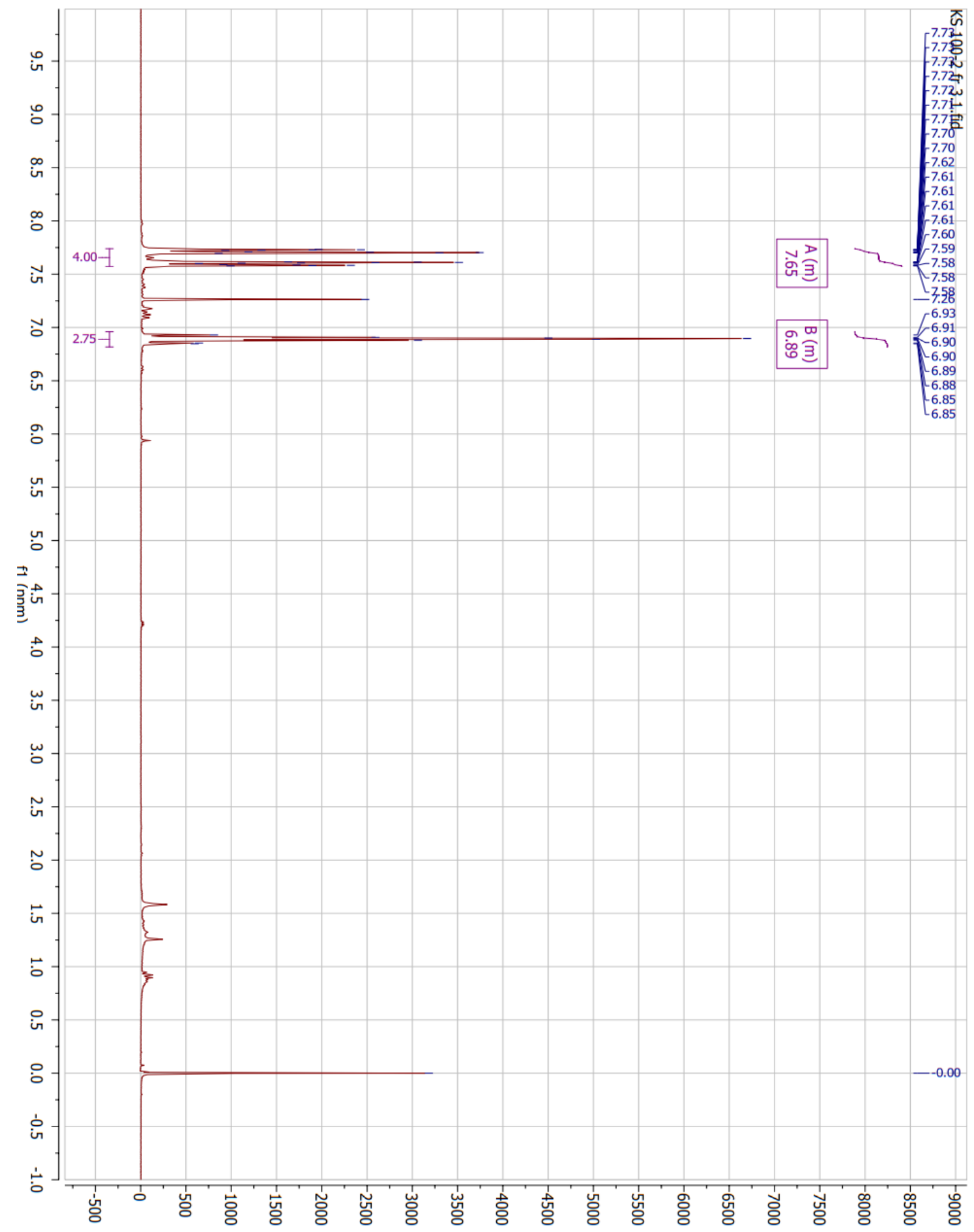


Figure A67 ${ }^{13} \mathrm{C}$ NMR spectrum of 2-(4-trifluoromethylphenyl)-1,4-benzoquinone

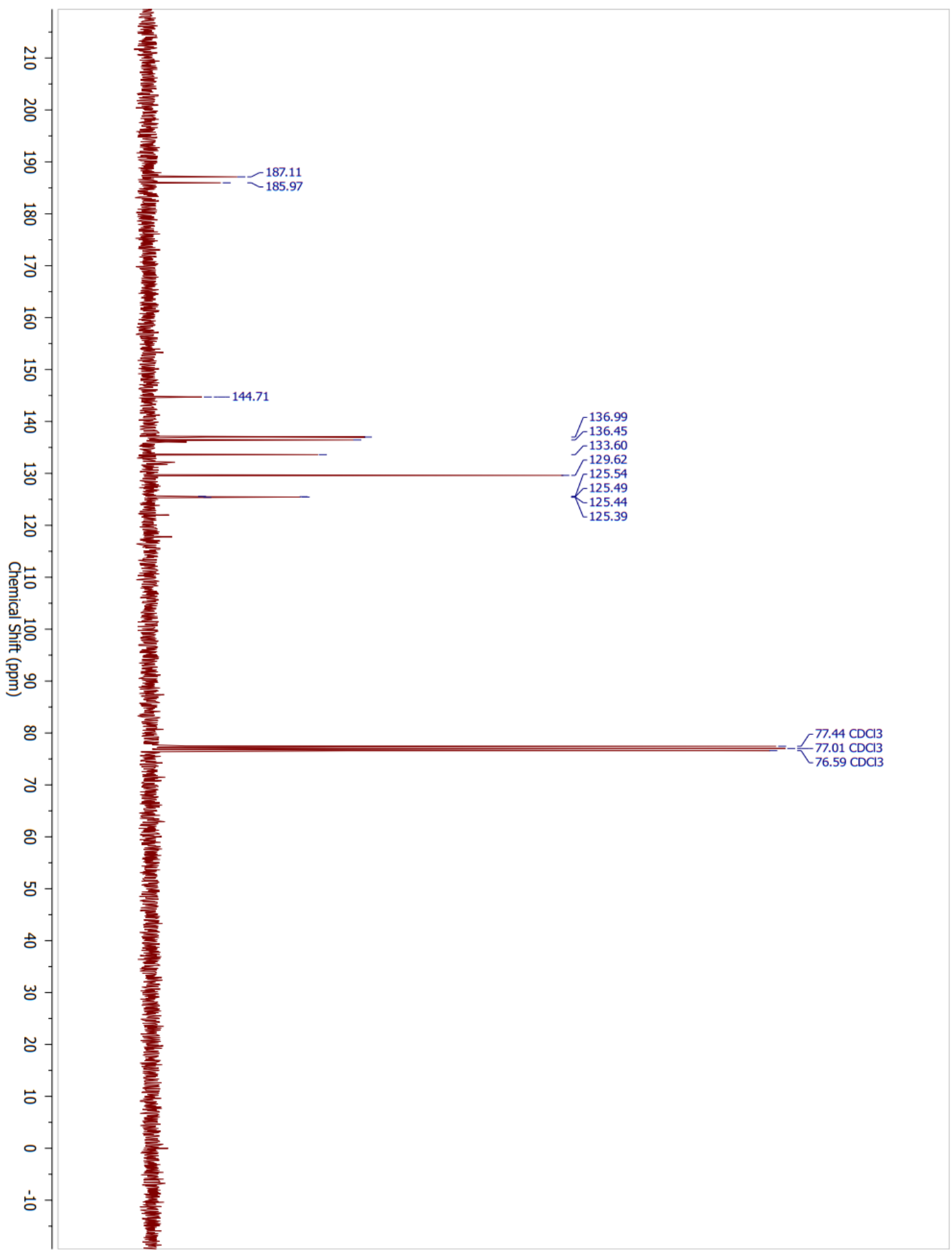


Figure A68 DEPT NMR spectrum of 2-(4-trifluoromethylphenyl)-1,4-benzoquinone

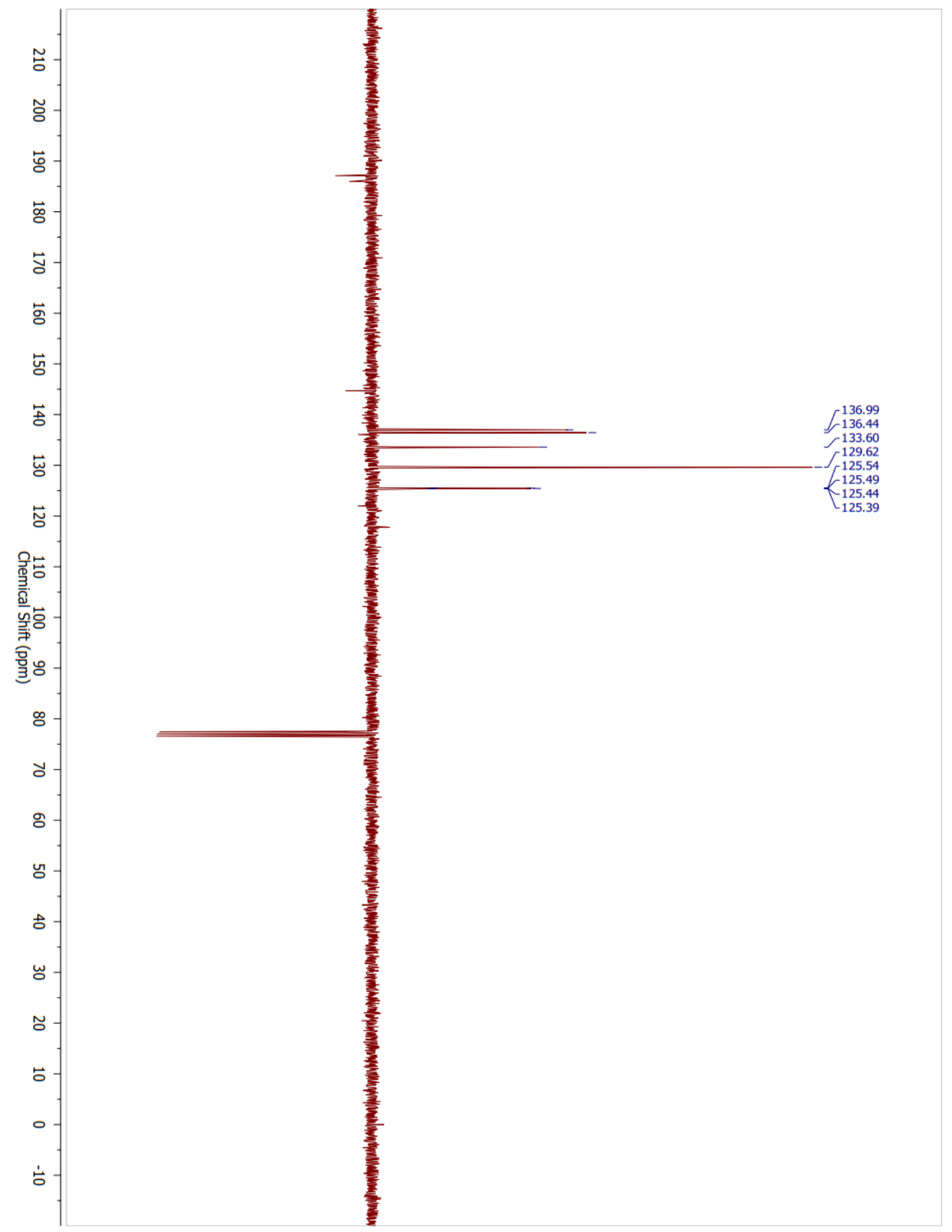


Figure A69 Gas chromatogram and mass spectrum of 2-(4-trifluoromethylphenyl)-1,4-benzoquinone

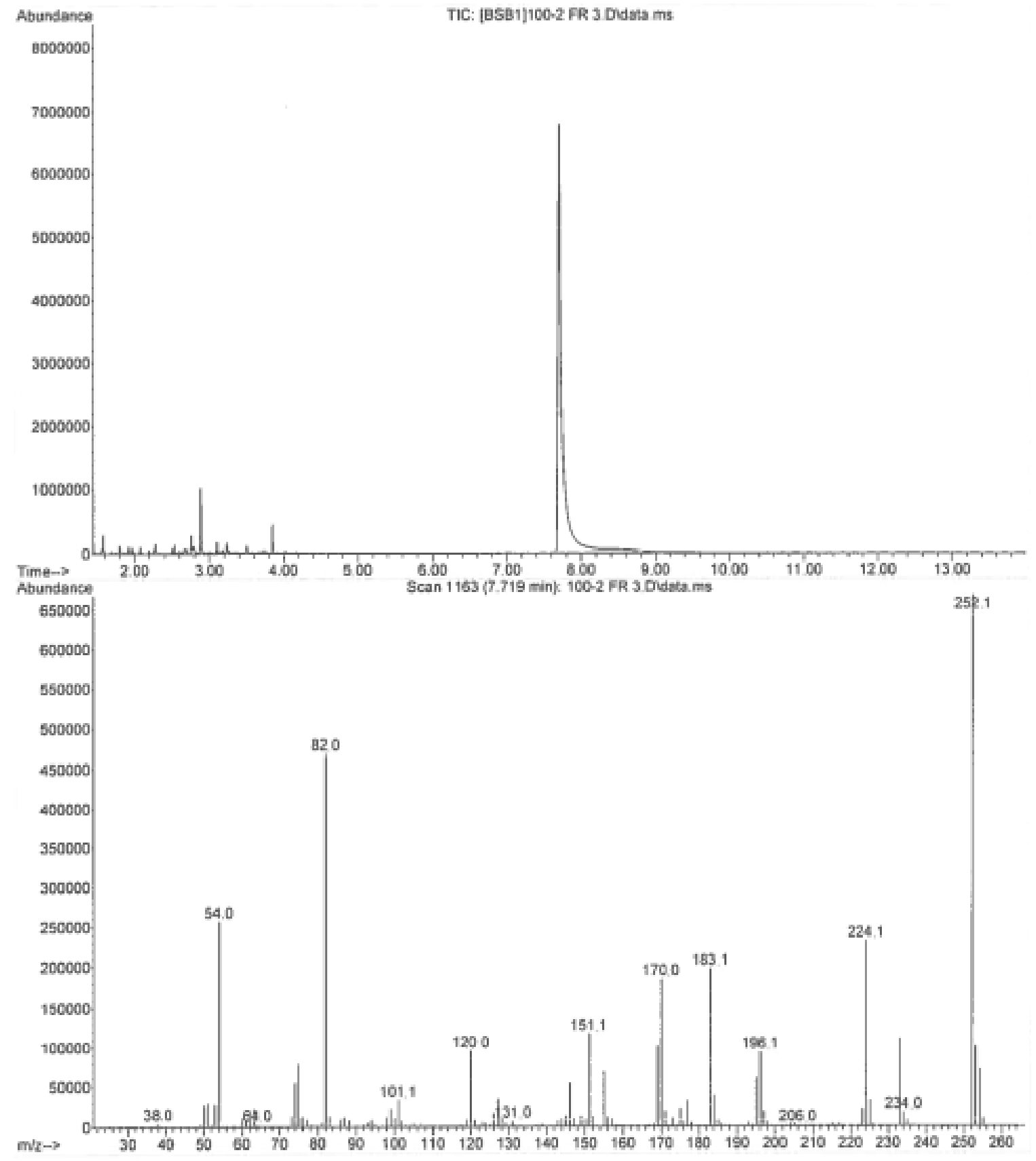


Figure A70 ${ }^{1} \mathrm{H}$ NMR spectrum of a mixture of 2,3-dimethyl-5-phenyl-1,4-benzoquinone and the starting product, 2,3-dimethyl-1,4-benzoquinone

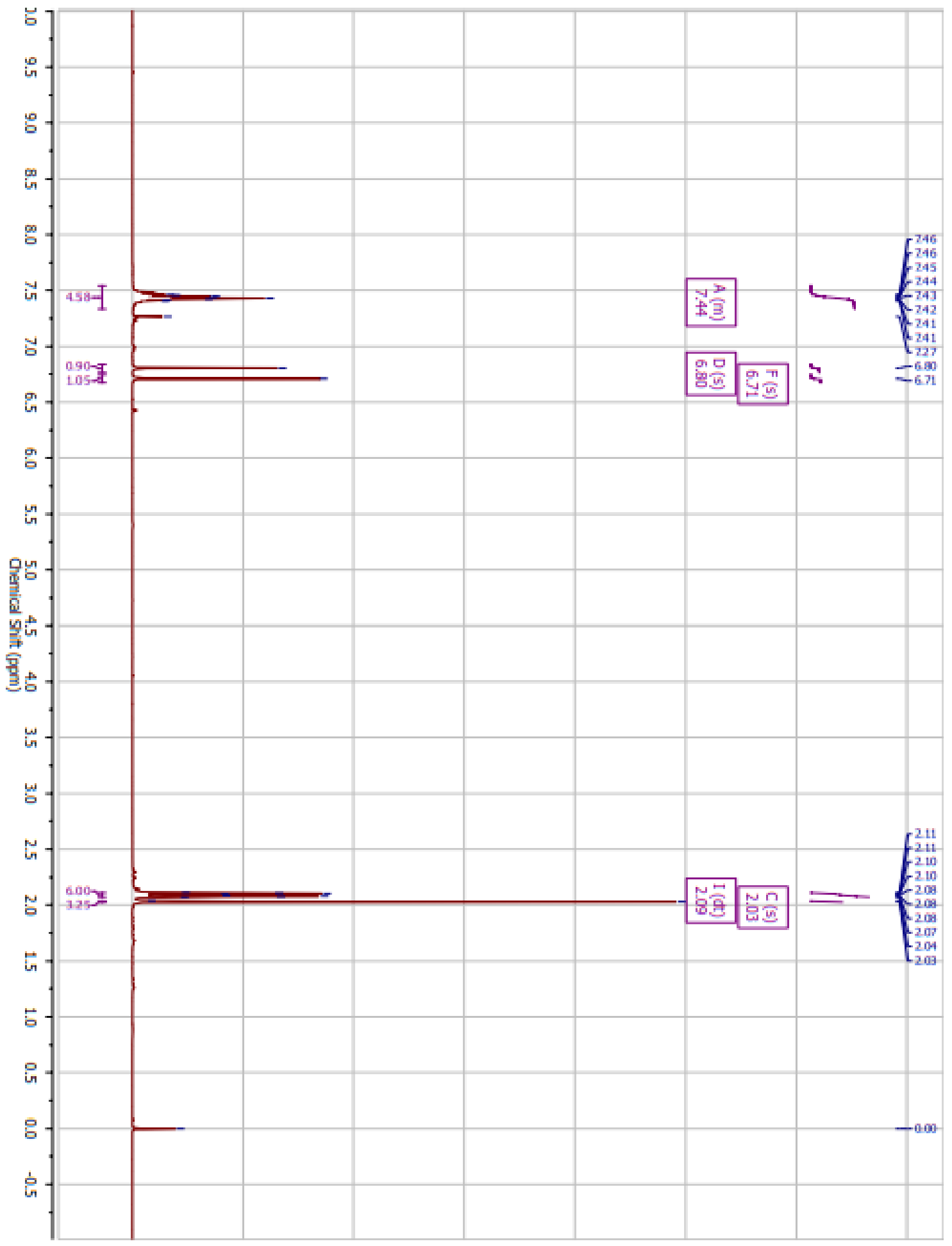


Figure A71 ${ }^{13} \mathrm{C}$ NMR spectrum of a mixture of 2,3-dimethyl-5-phenyl-1,4-benzoquinone and 2,3-dimethyl-1,4-benzoquinone

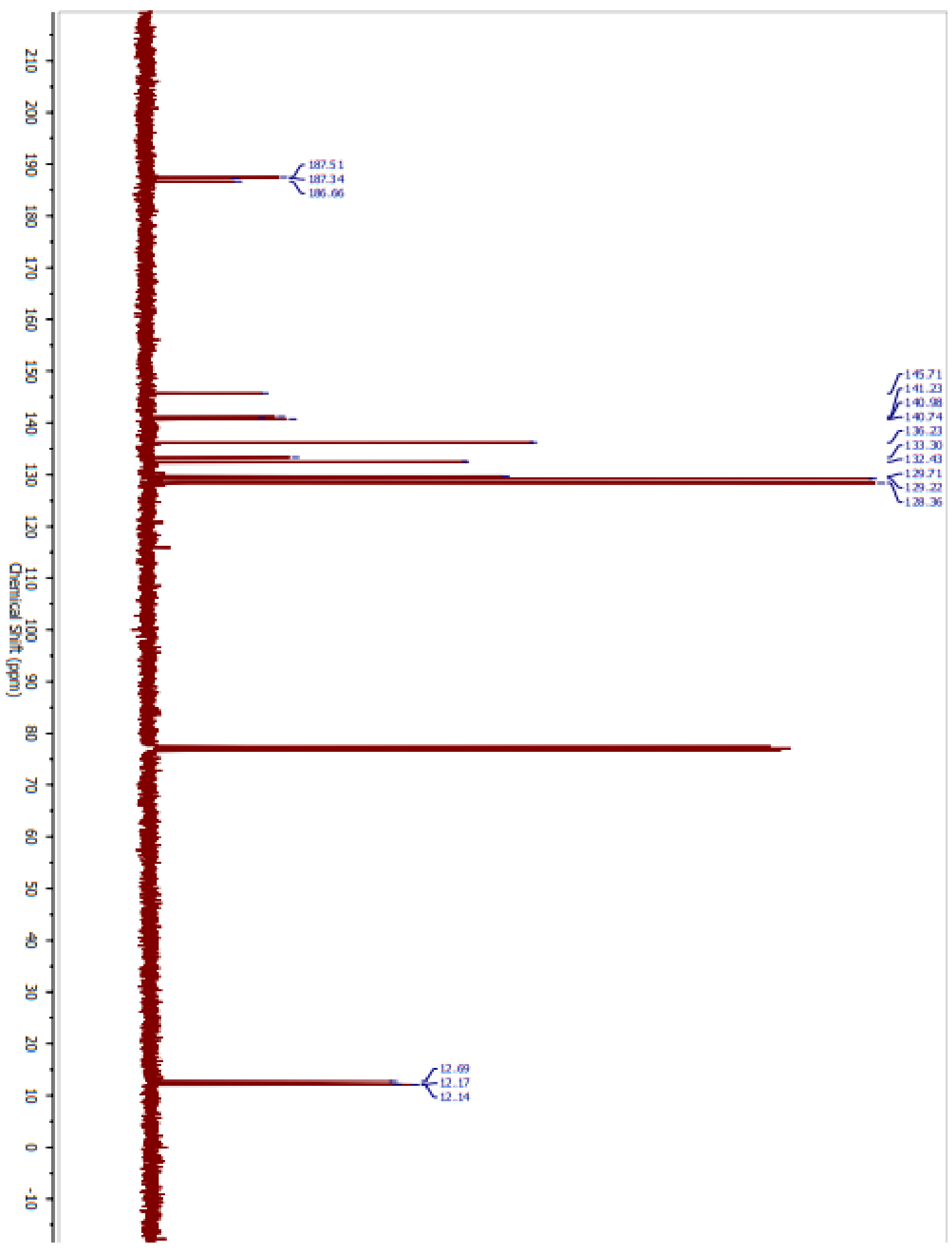


Figure A72 DEPT NMR spectrum of a mixture of

2,3-dimethyl-5-phenyl-1,4-benzoquinone and 2,3-dimethyl-1,4-benzoquinone

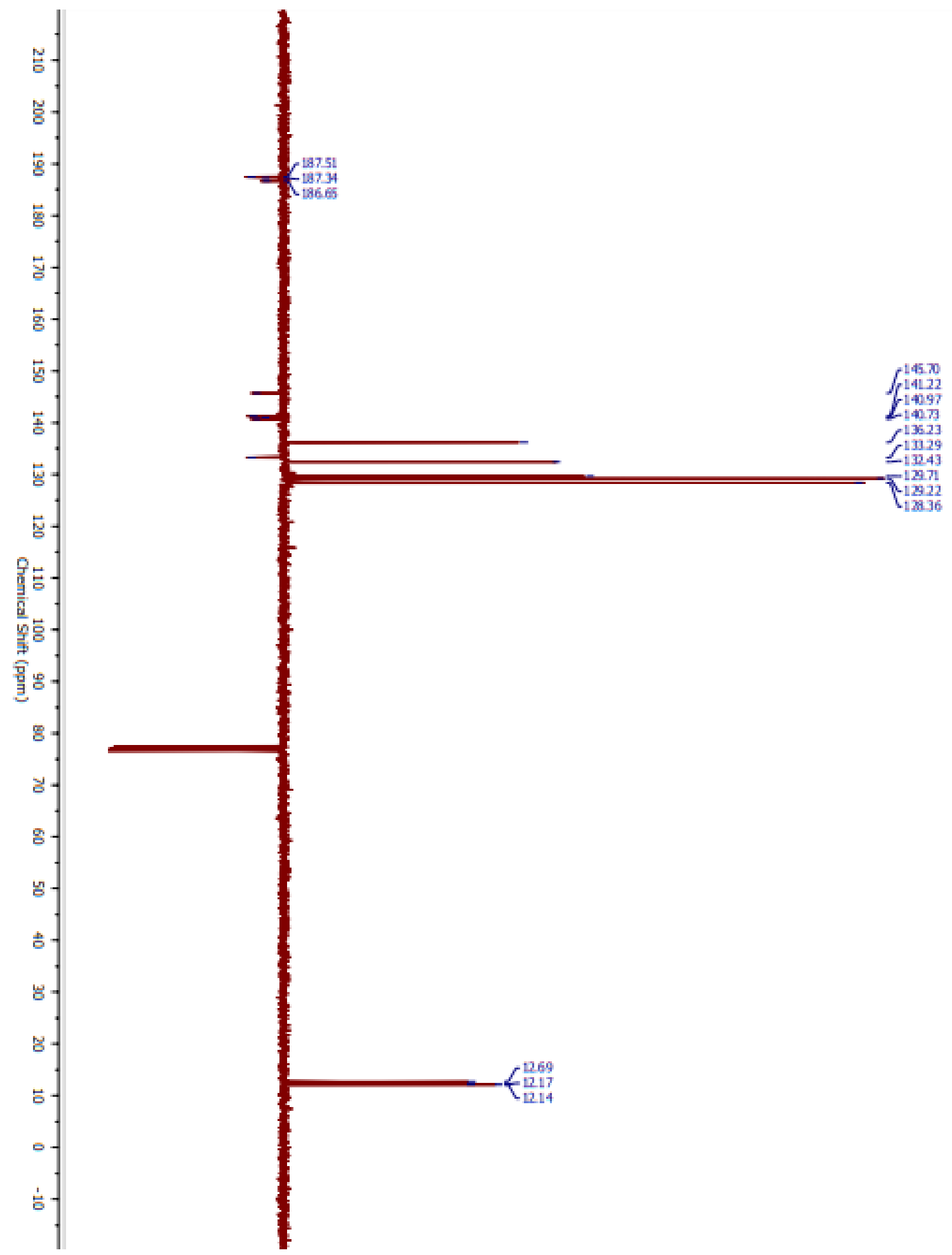


Figure A73 Gas chromatogram and mass spectrum of a mixture of 2,3-dimethyl-5-phenyl-1,4-benzoquinone and 2,3-dimethyl-1,4-benzoquinone

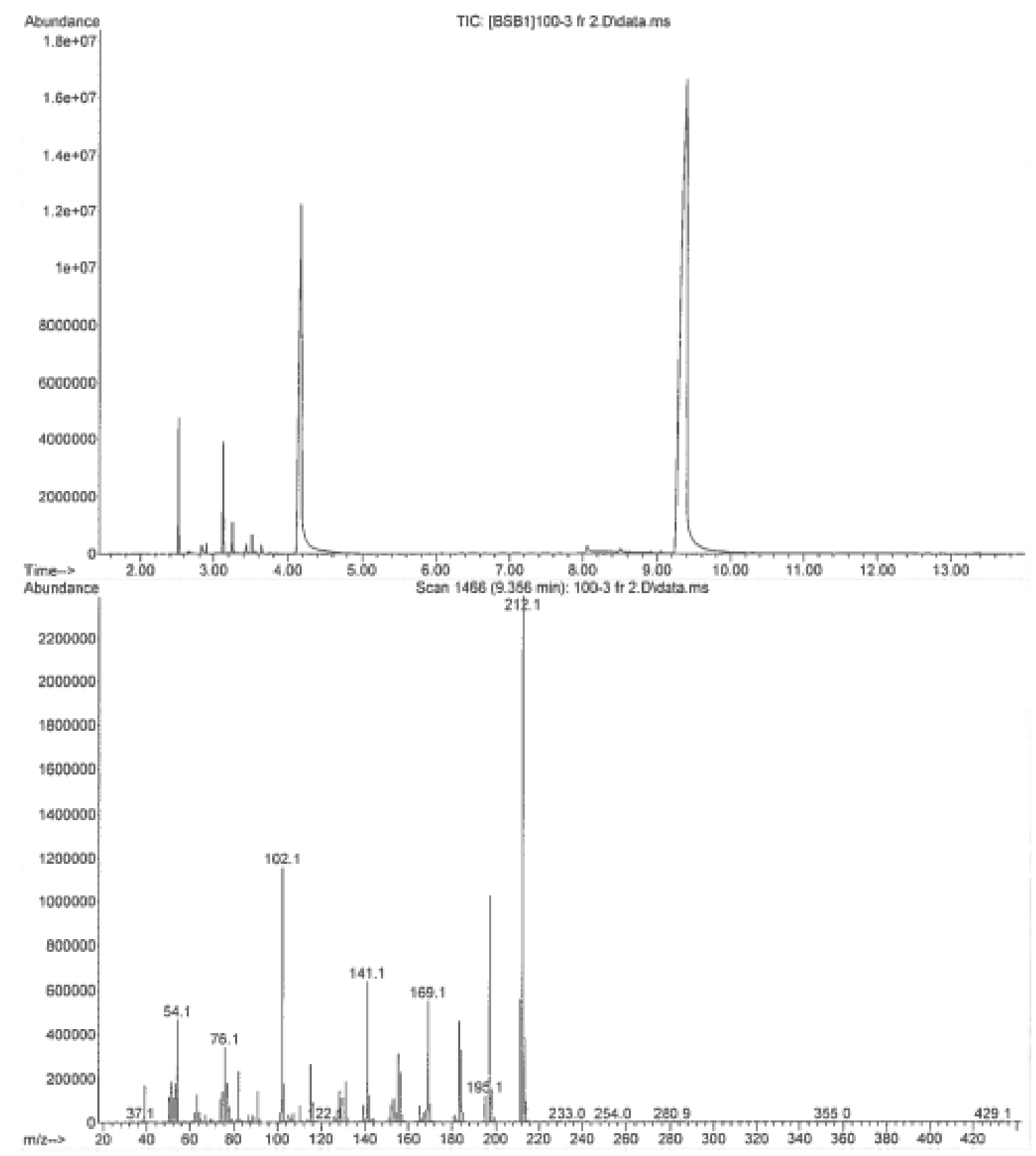


Figure A74 ${ }^{1} \mathrm{H}$ NMR spectrum of 2-phenyl-4-cyanopyridine

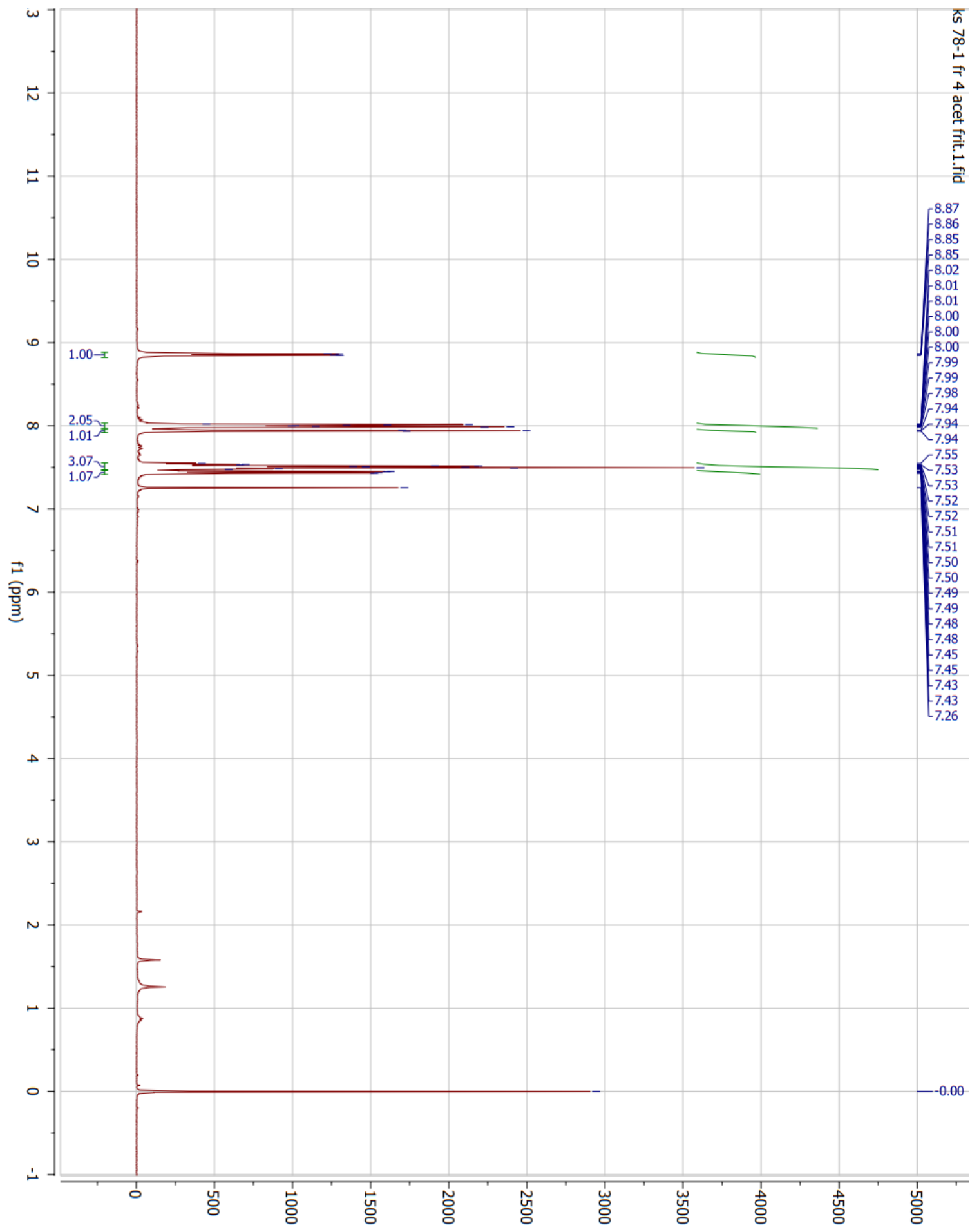


Figure A75 ${ }^{13} \mathrm{C}$ NMR spectrum of 2-phenyl-4-cyanopyridine

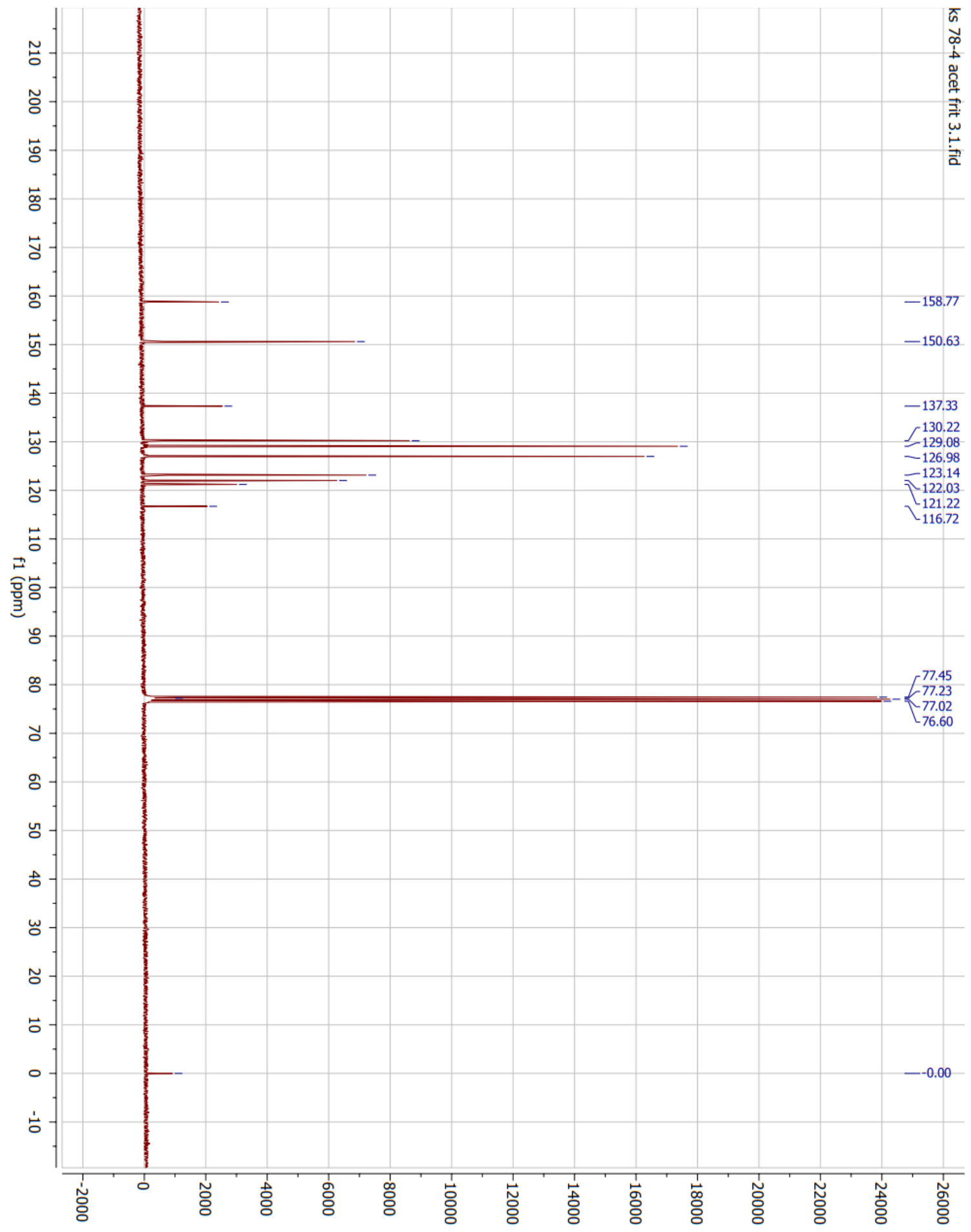


Figure A76 DEPT NMR spectrum of 2-phenyl-4-cyanopyridine

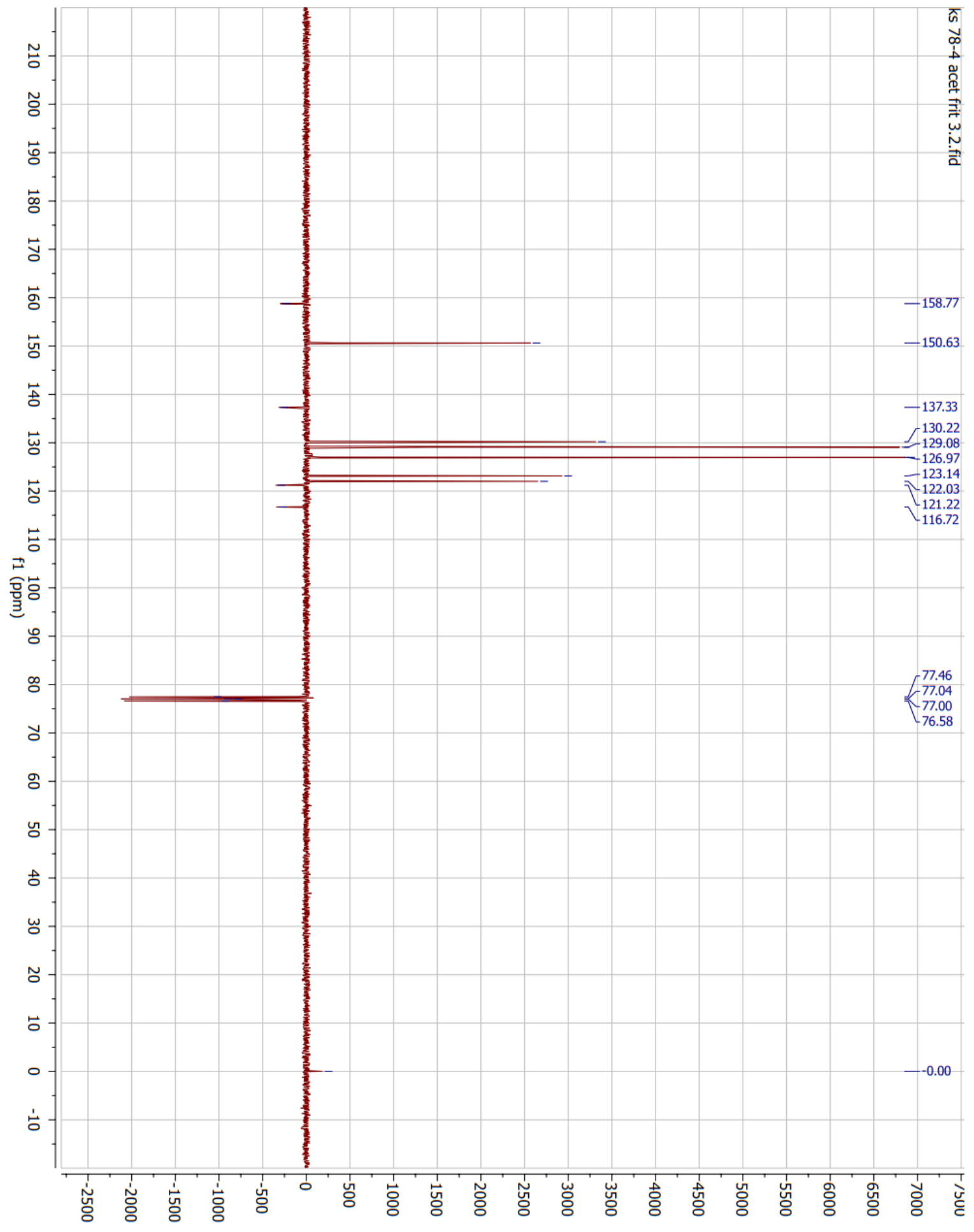


Figure A77 Gas chromatogram and mass spectrum of 2-phenyl-4-cyanopyridine

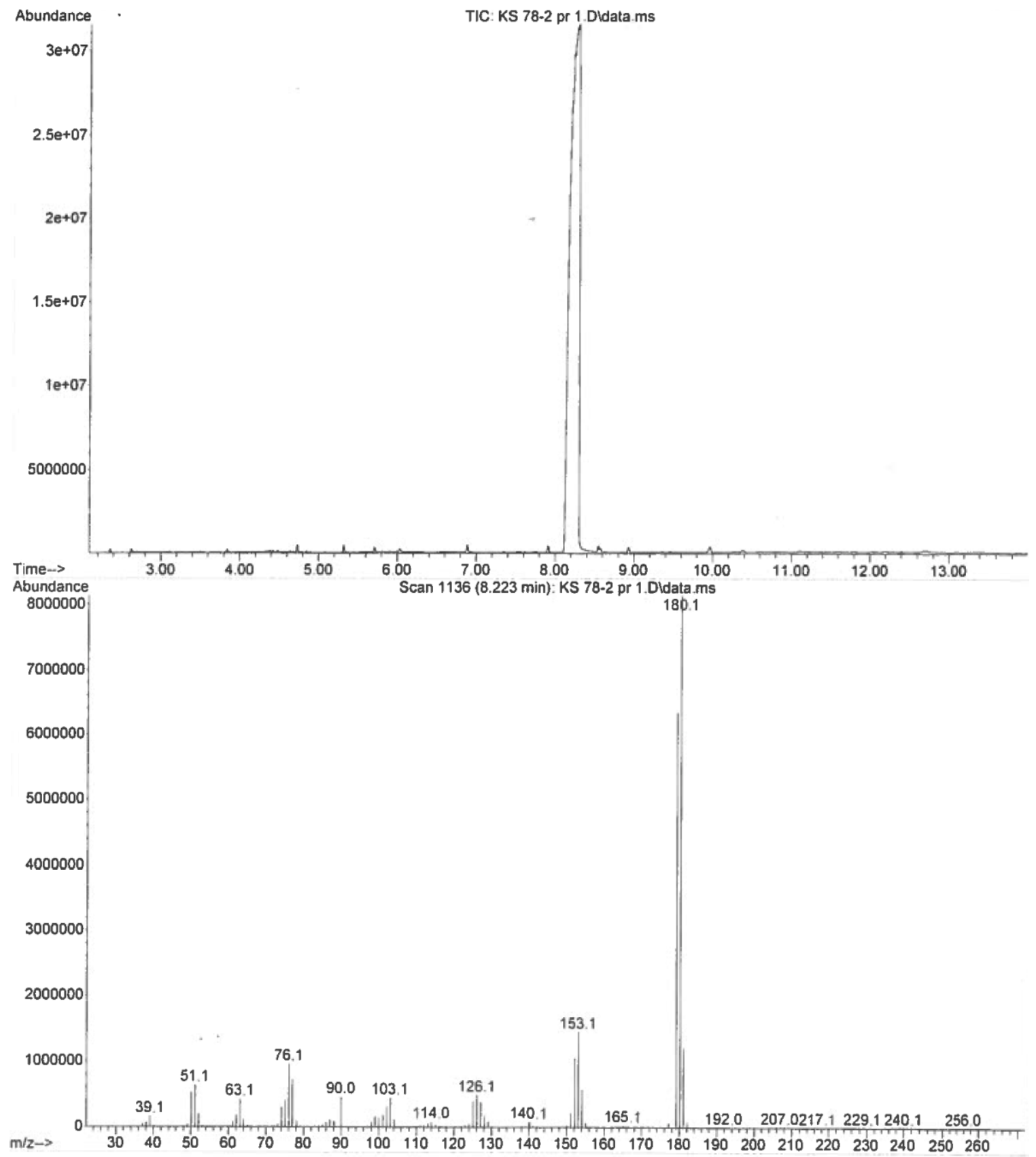


Figure A78 ${ }^{1} \mathrm{H}$ NMR spectrum of 3-phenyl-4-cyanopyridine

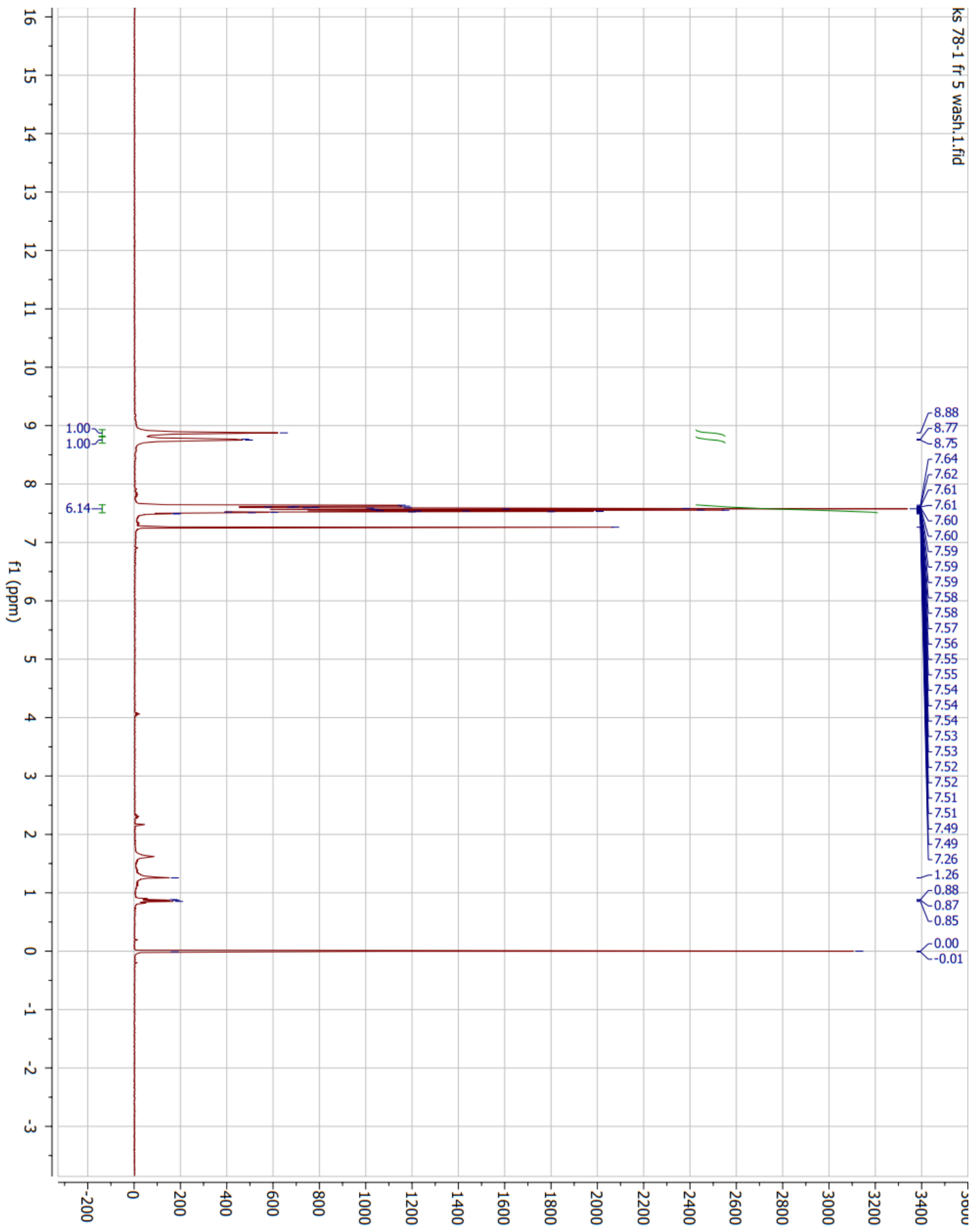


Figure A79 ${ }^{13} \mathrm{C}$ NMR spectrum of 3-phenyl-4-cyanopyridine

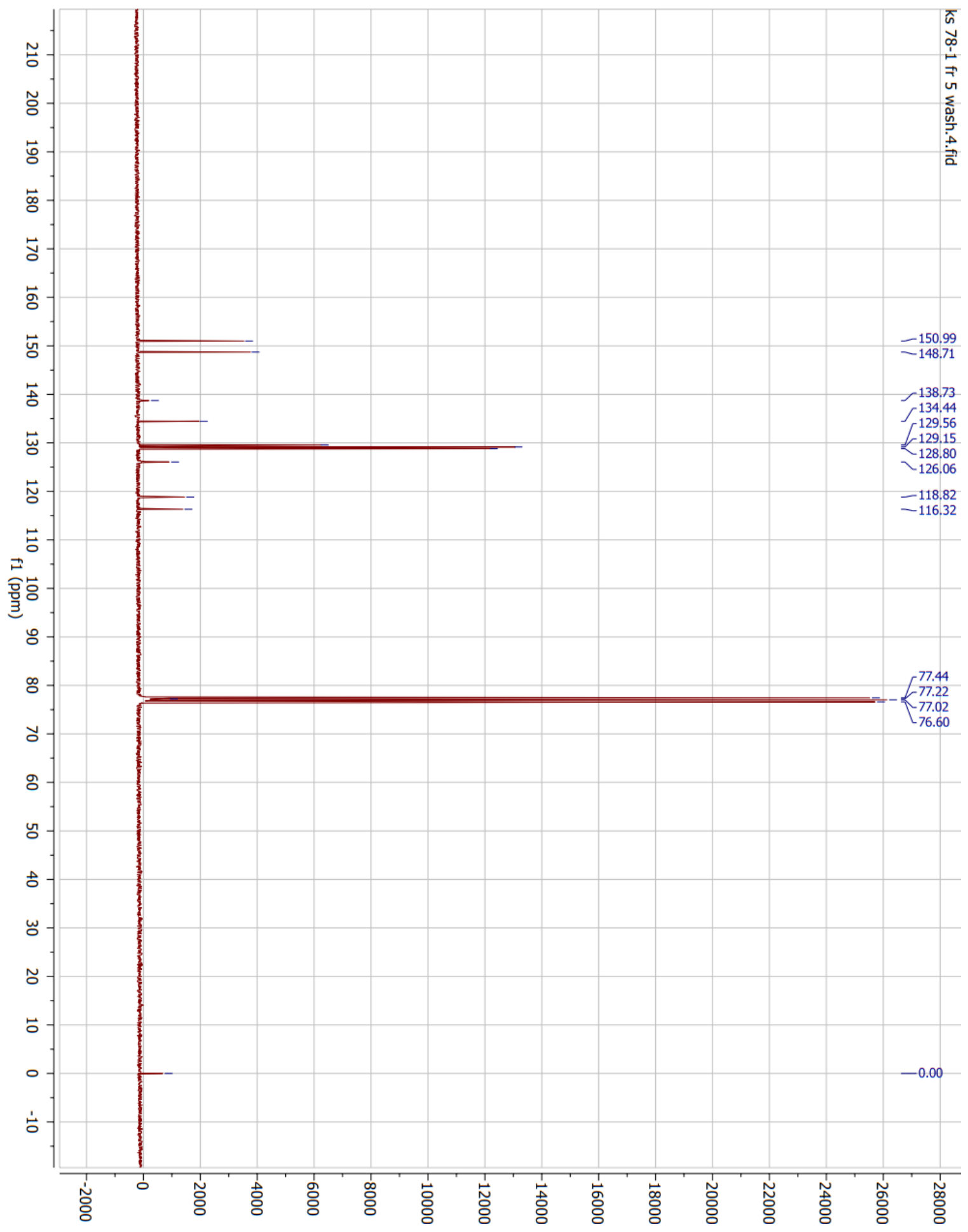


Figure A80 DEPT NMR spectrum of 3-phenyl-4-cyanopyridine

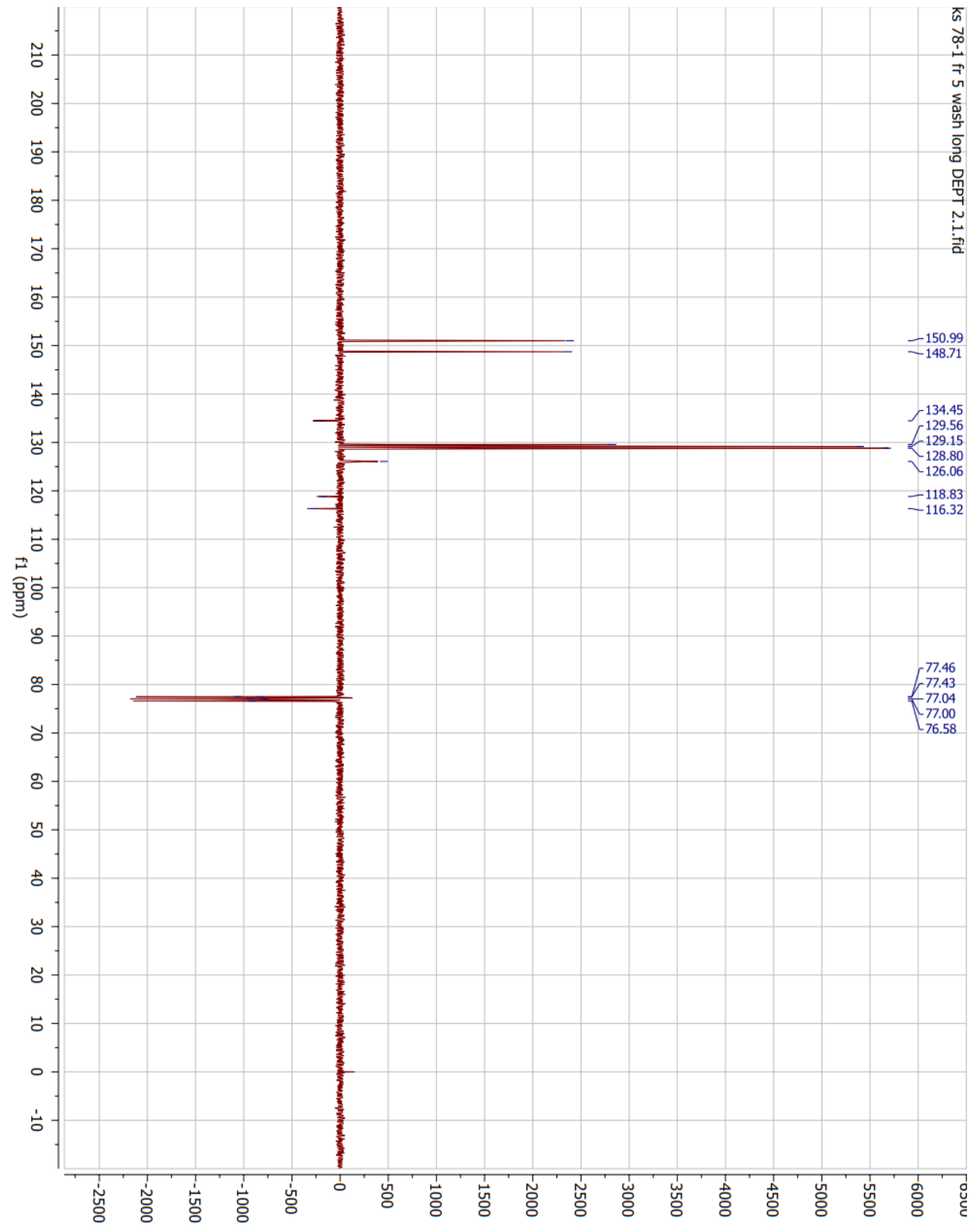


Figure A81 Gas chromatogram and mass spectrum of 3-phenyl-4-cyanopyridine

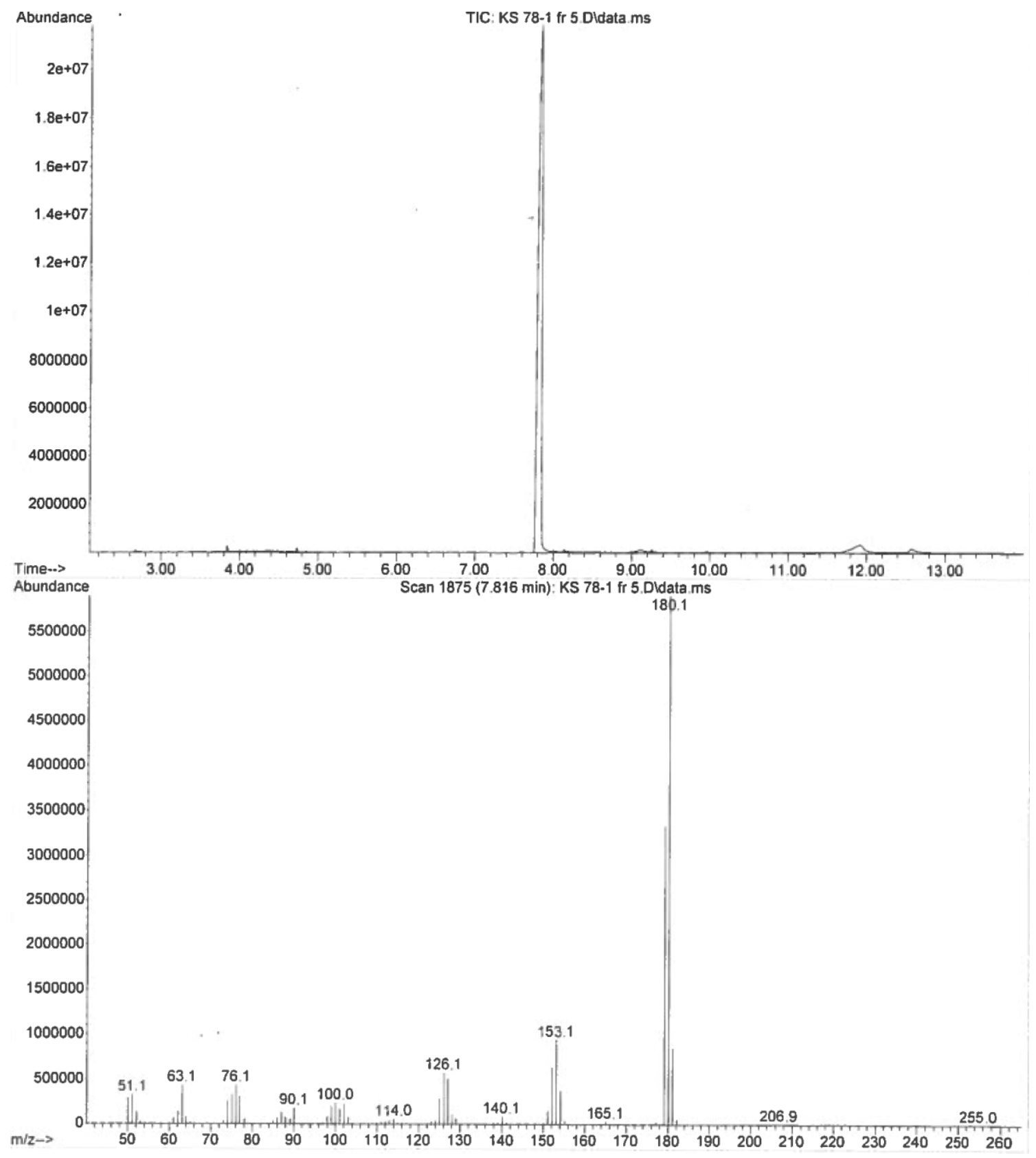


Figure A82 ${ }^{1} \mathrm{H}$ NMR spectrum of 3-phenylpyridazine

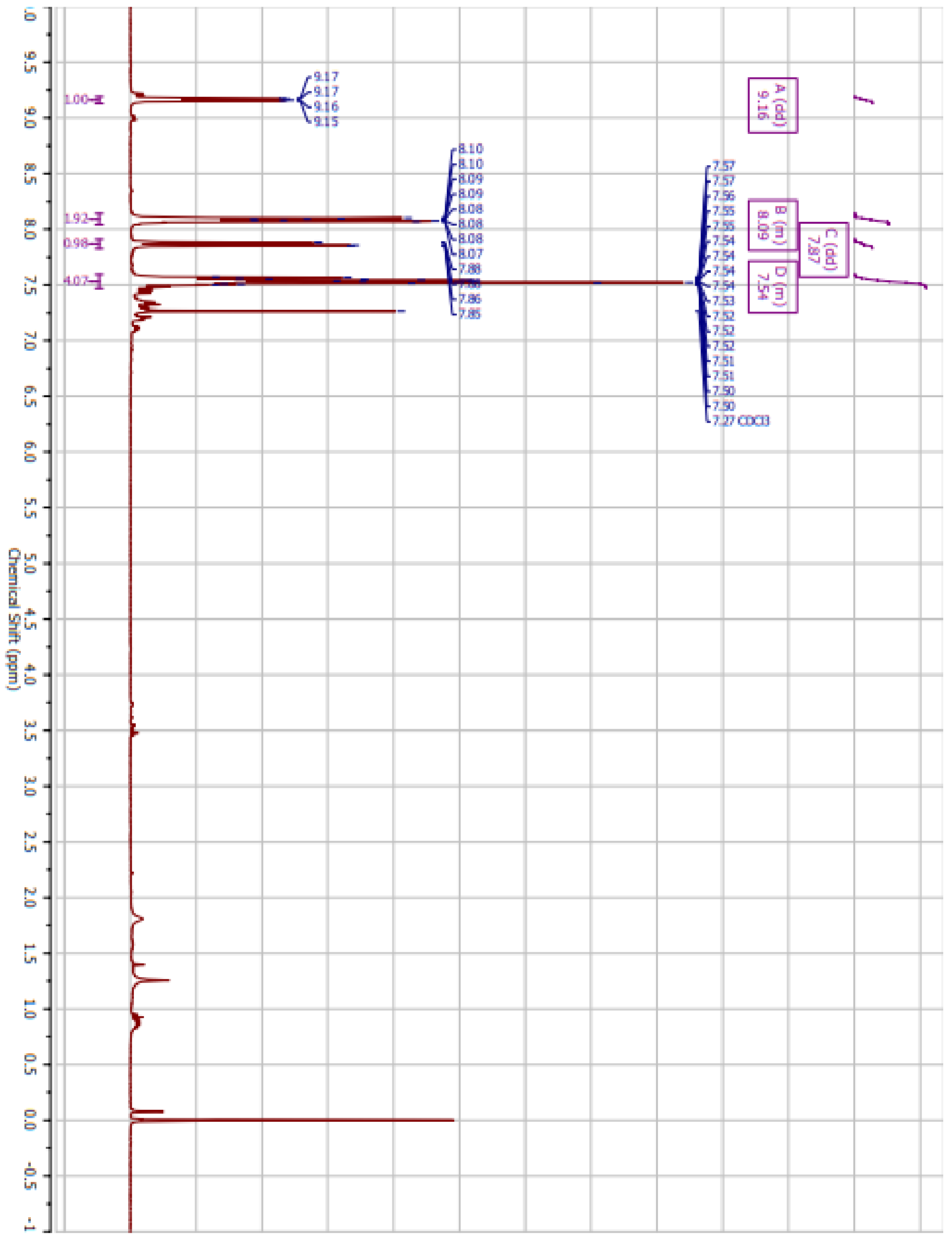


Figure $\mathbf{A 8 3}{ }^{13} \mathrm{C}$ NMR spectrum of 3-phenylpyridazine

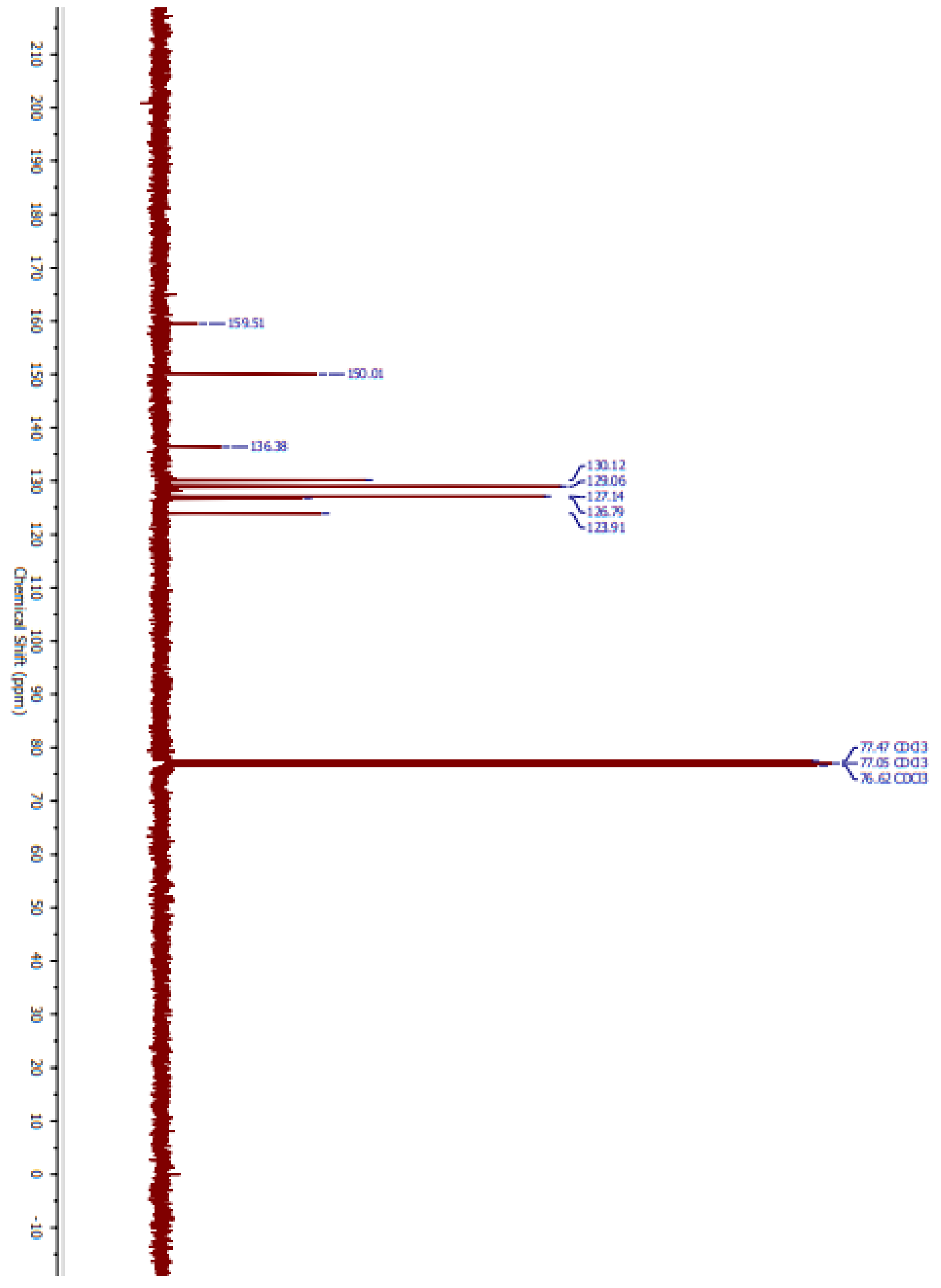


Figure A84 DEPT NMR spectrum of 3-phenylpyridazine

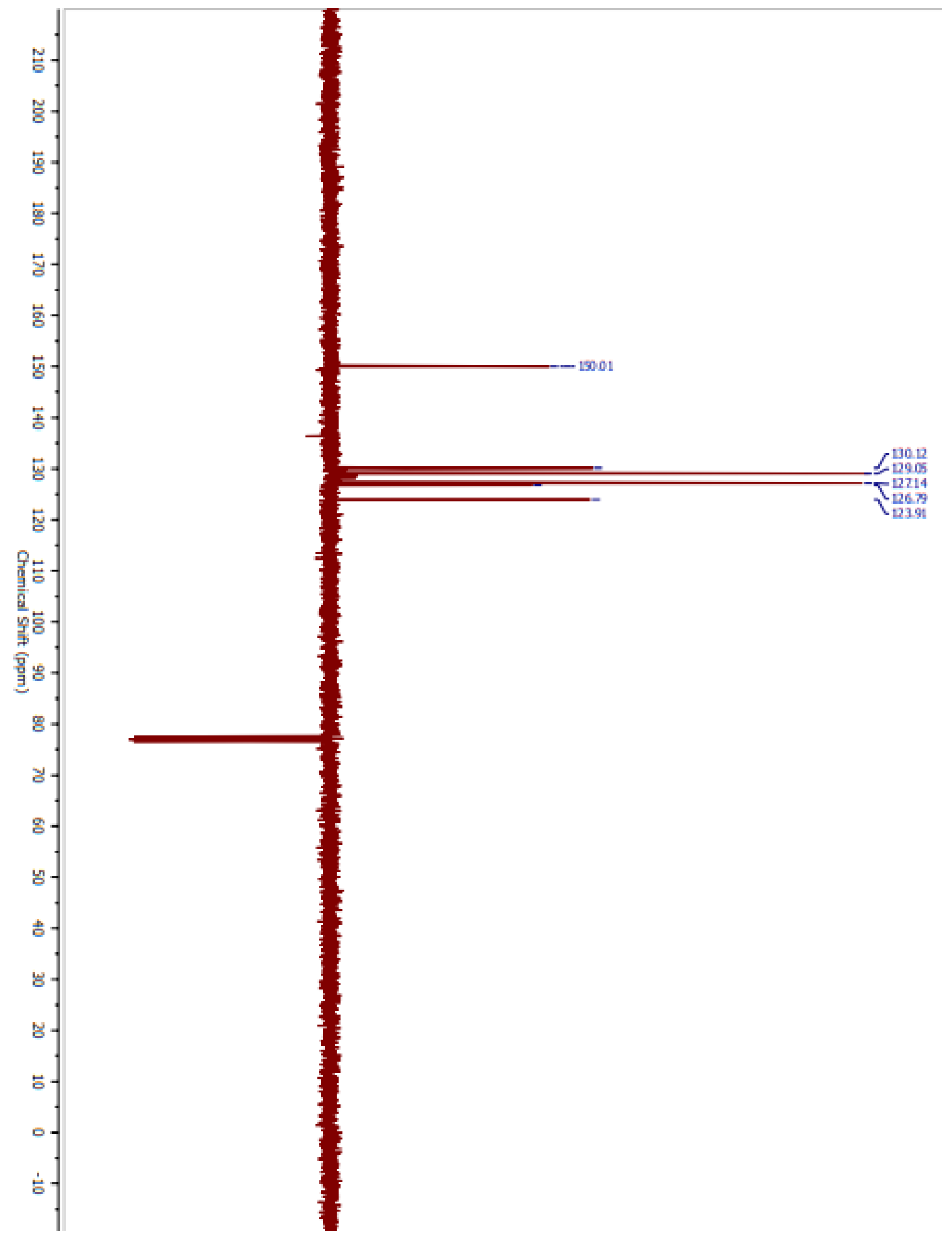


Figure A85 Gas chromatogram and mass spectrum of 3-phenylpyridazine

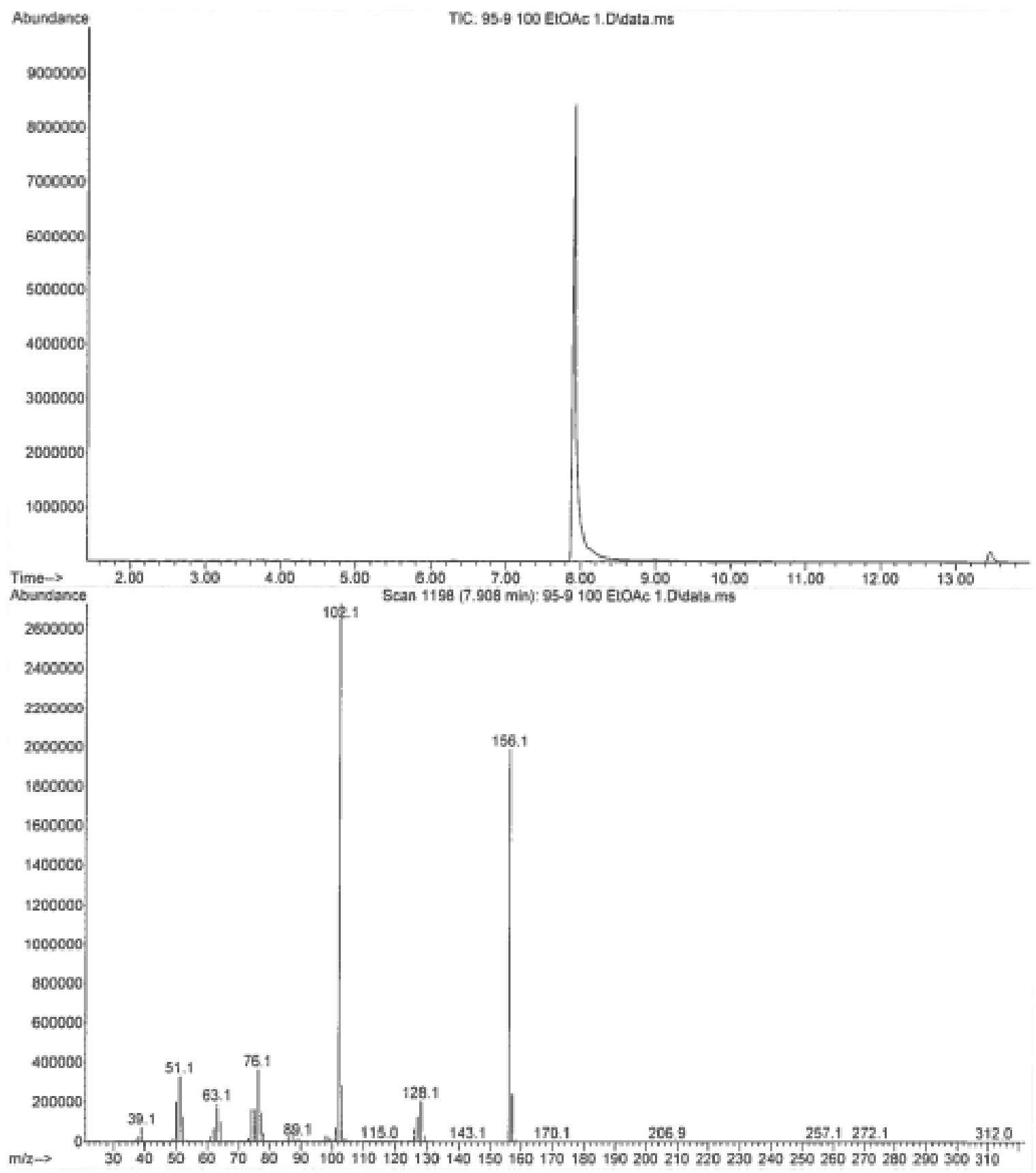


Figure A86 ${ }^{1} \mathrm{H}$ NMR spectrum of 4-phenylpyridazine

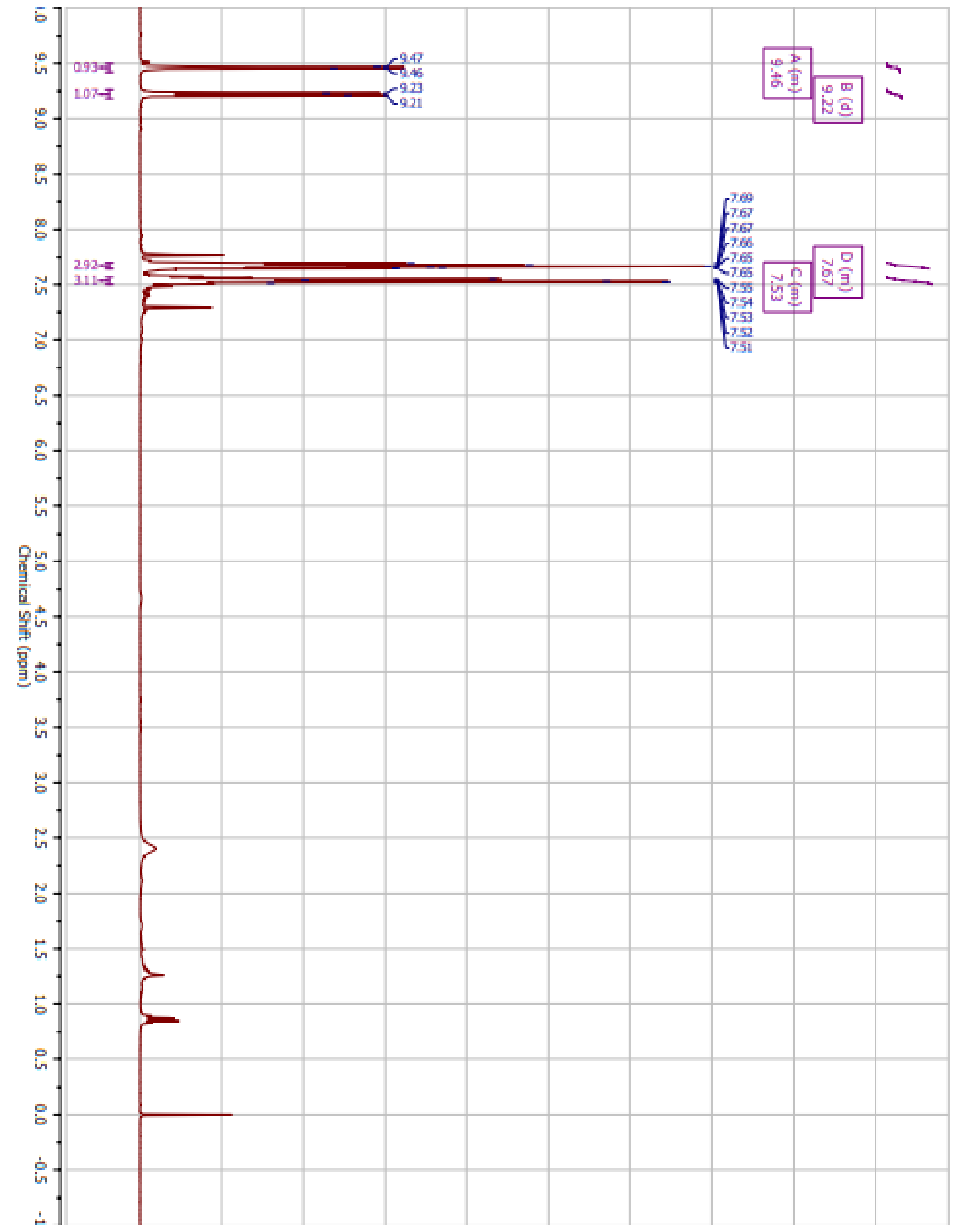


Figure $\mathbf{A 8 7}{ }^{13} \mathrm{C}$ NMR spectrum of 4-phenylpyridazine

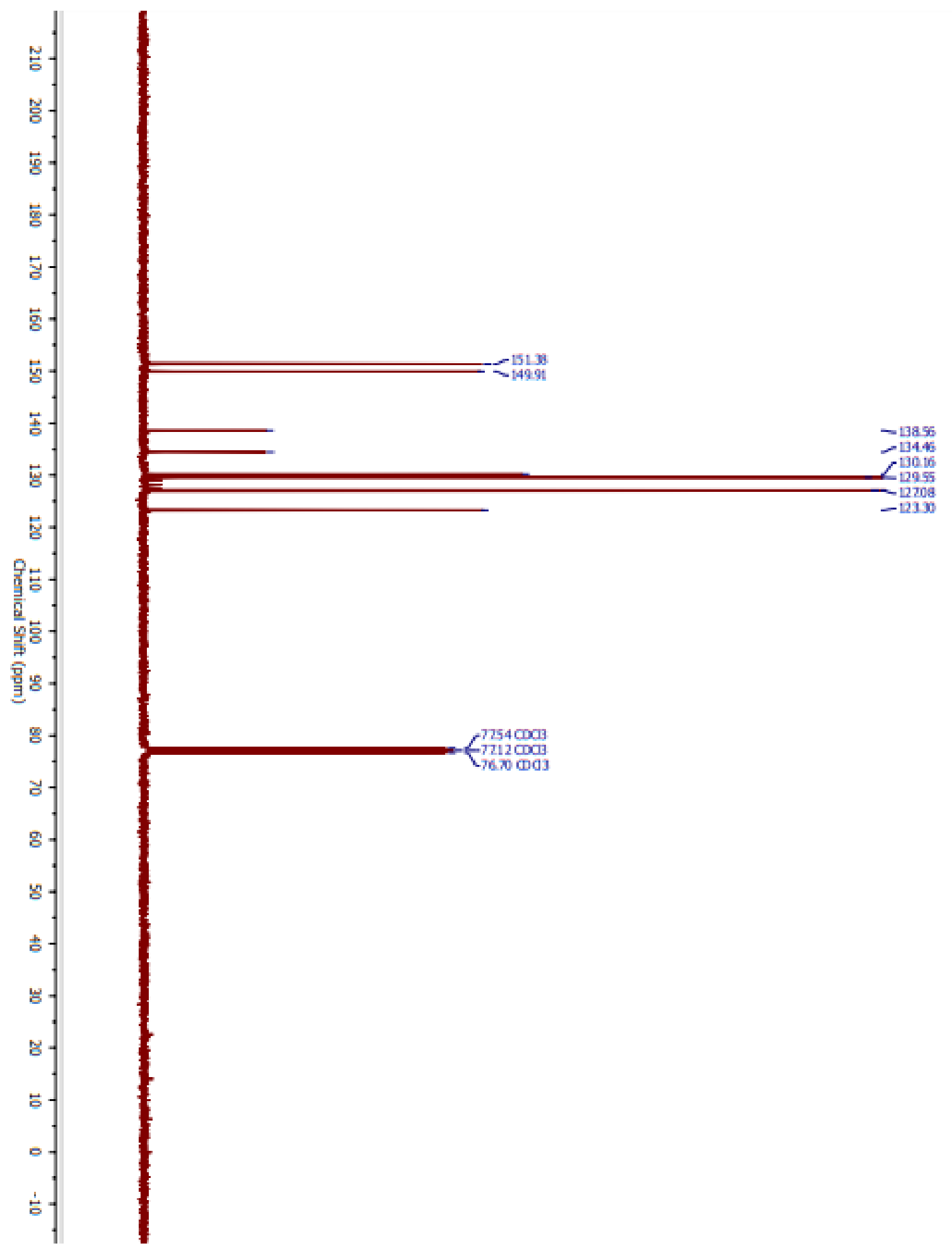


Figure A88 DEPT NMR spectrum of 4-phenylpyridazine

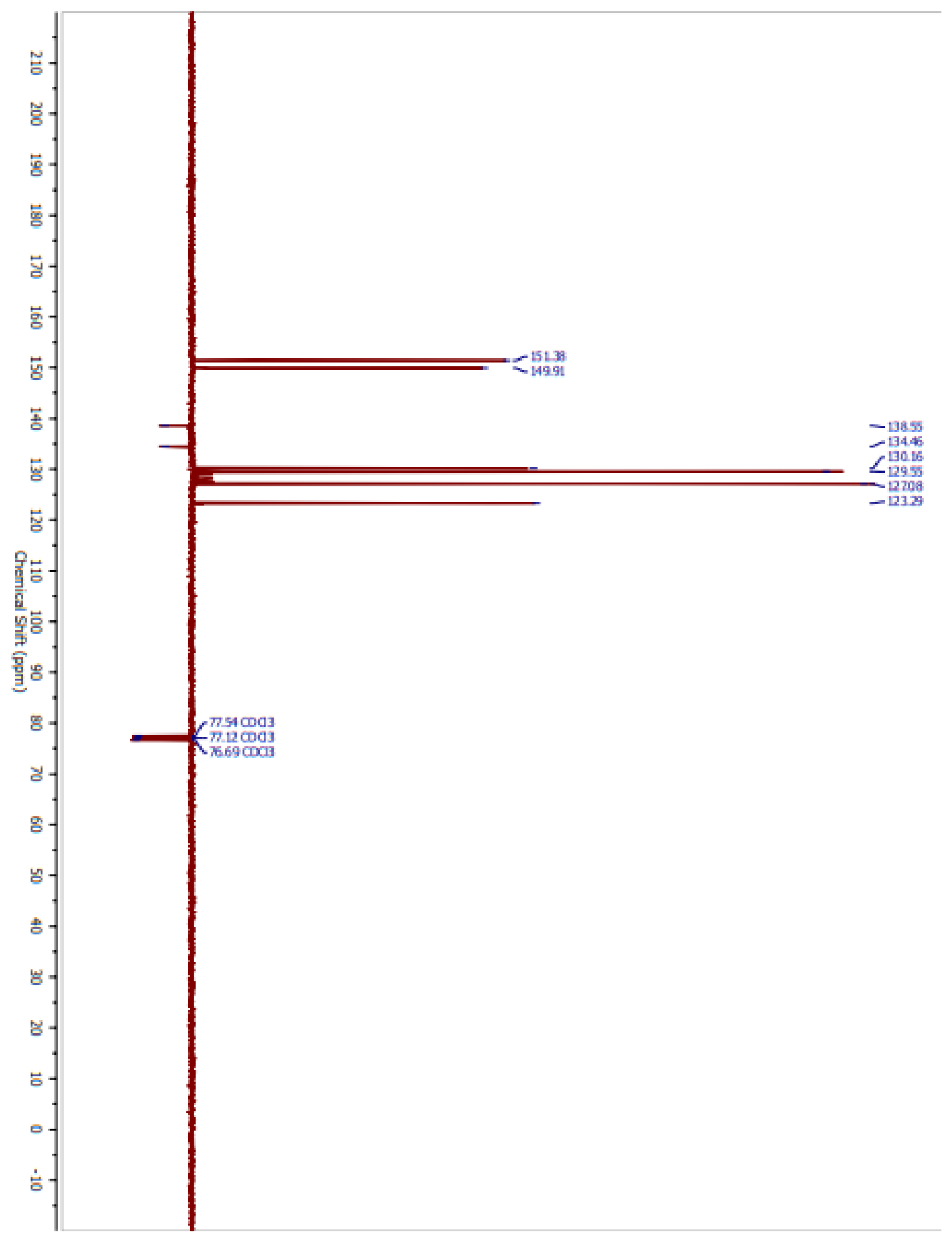


Figure A89 Gas chromatogram and mass spectrum of 4-phenylpyridazine

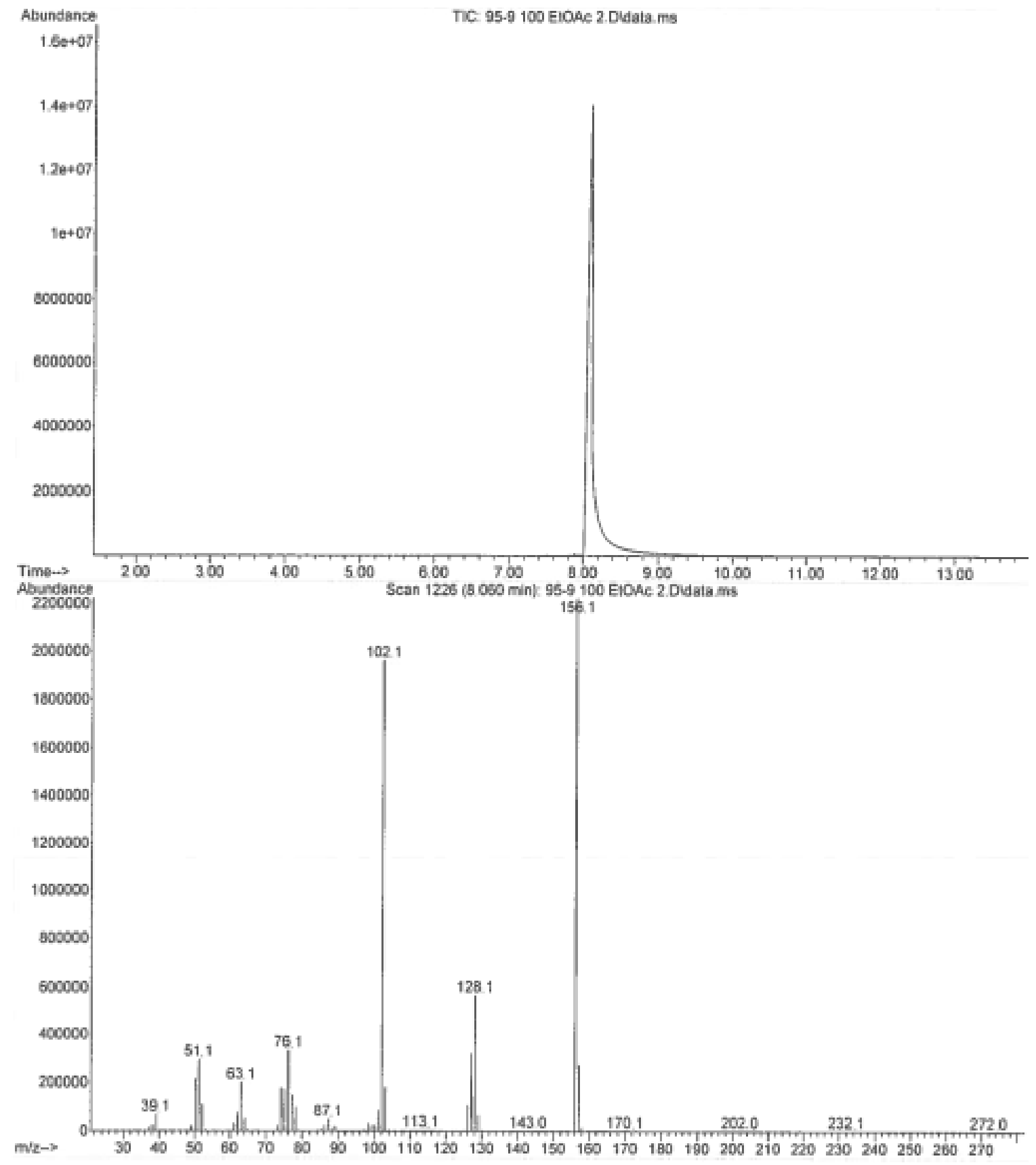


Figure A90 ${ }^{1} \mathrm{H}$ NMR spectrum of 2-phenylquinoline

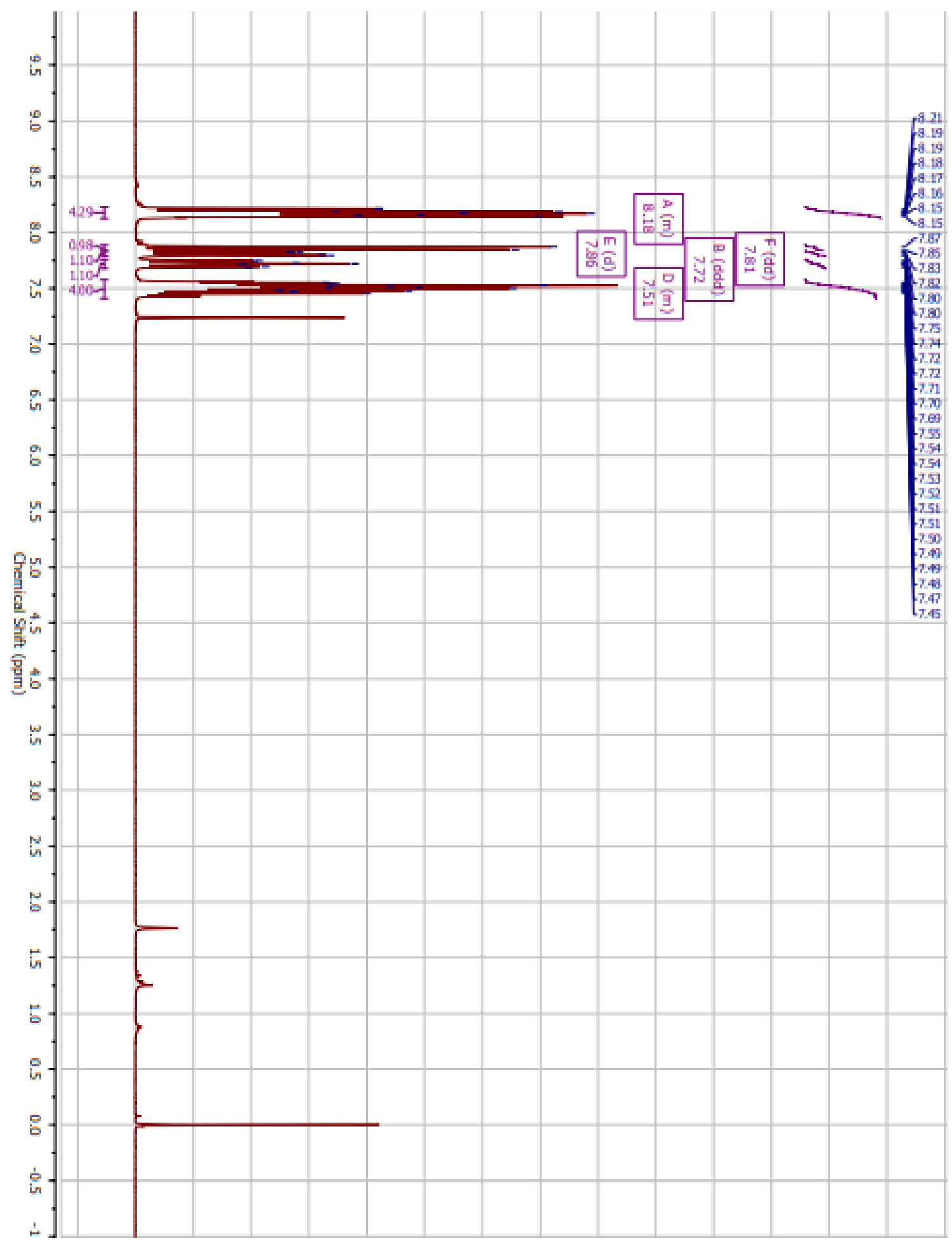


Figure A91 ${ }^{13} \mathrm{C}$ NMR spectrum of 2-phenylquinoline

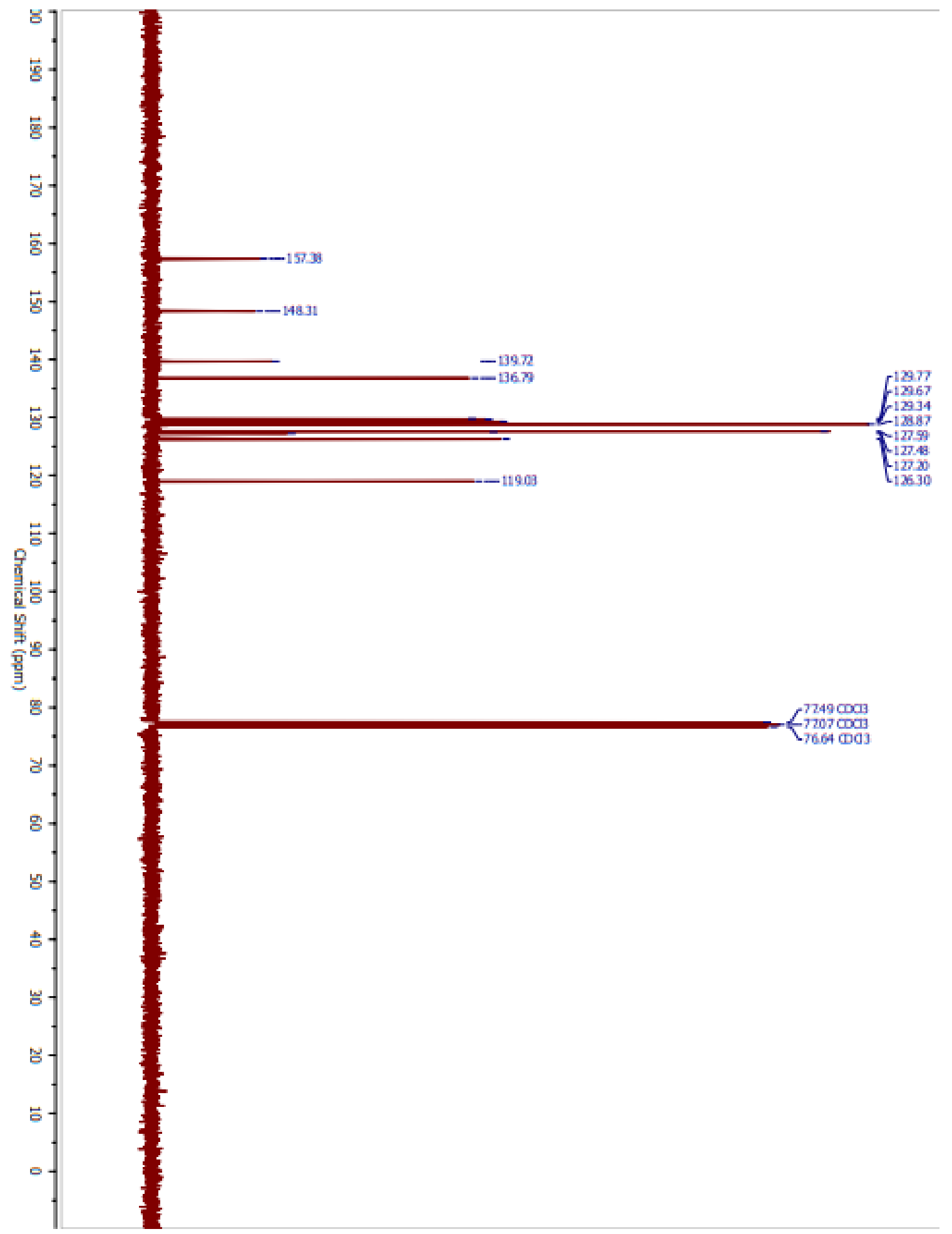


Figure A92 DEPT NMR 2-phenylquinoline

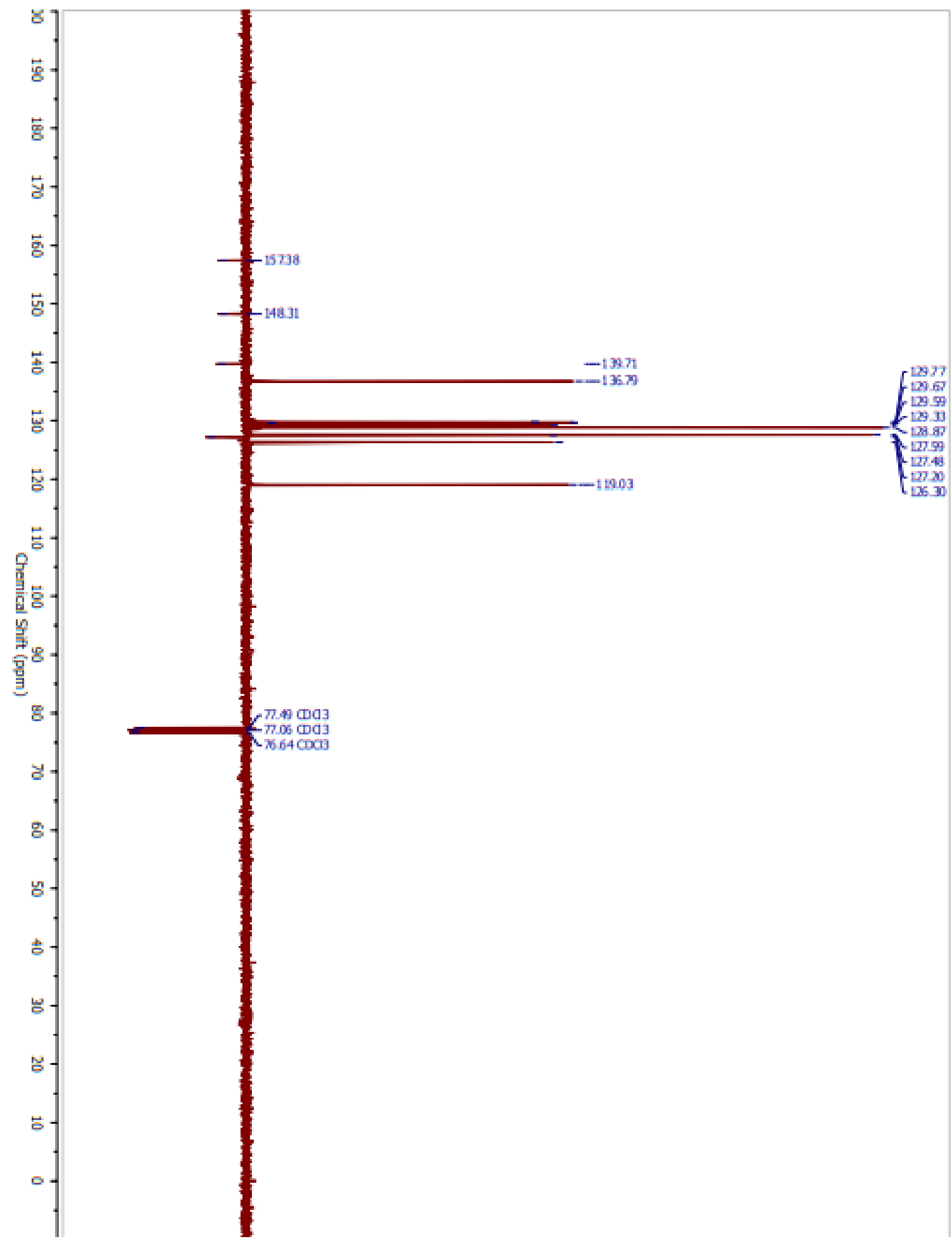


Figure A93 Gas chromatogram and mass spectrum of 2-phenylquinoline

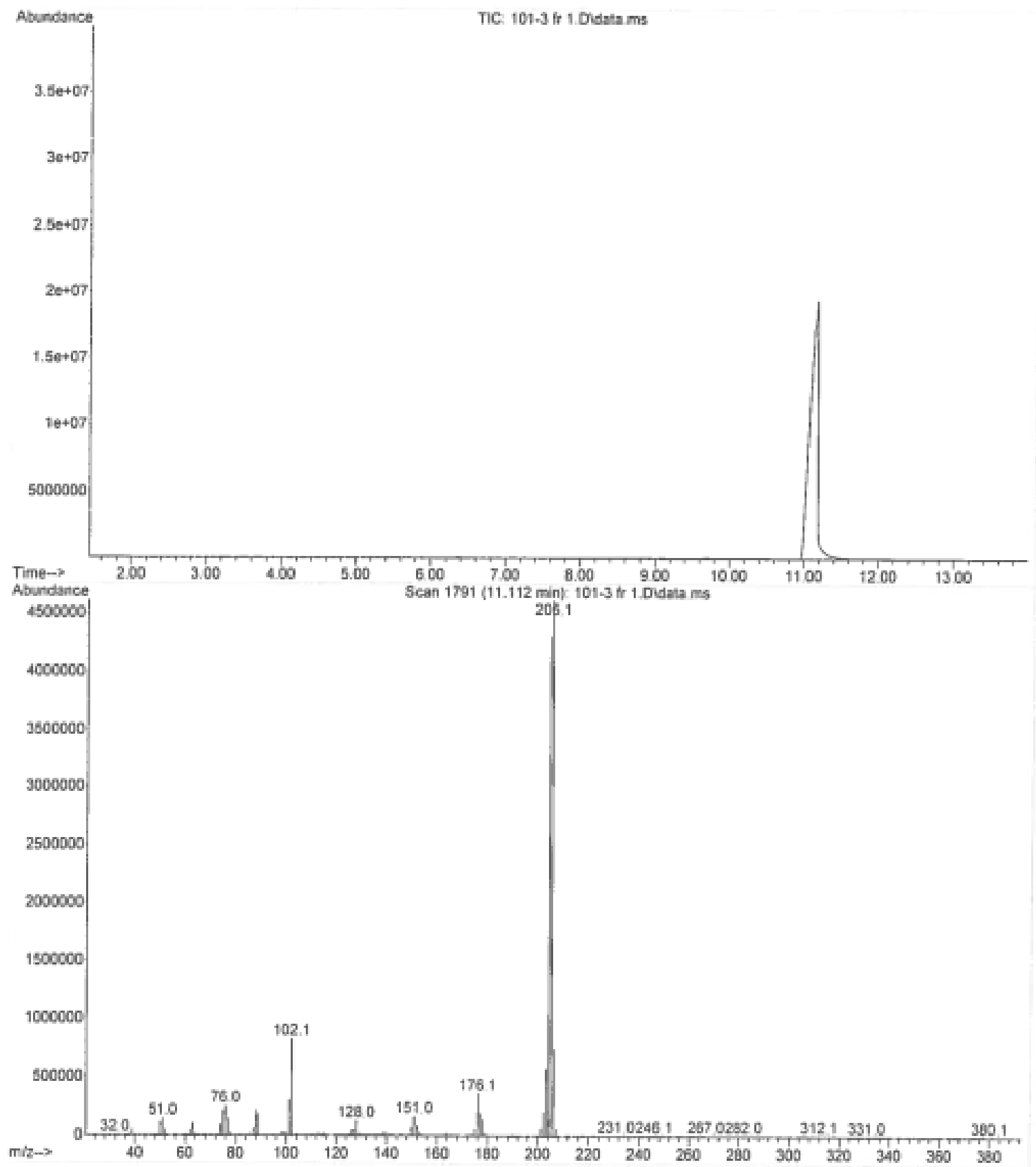


Figure A94 ${ }^{1} \mathrm{H}$ NMR spectrum of 4-phenylquinoline

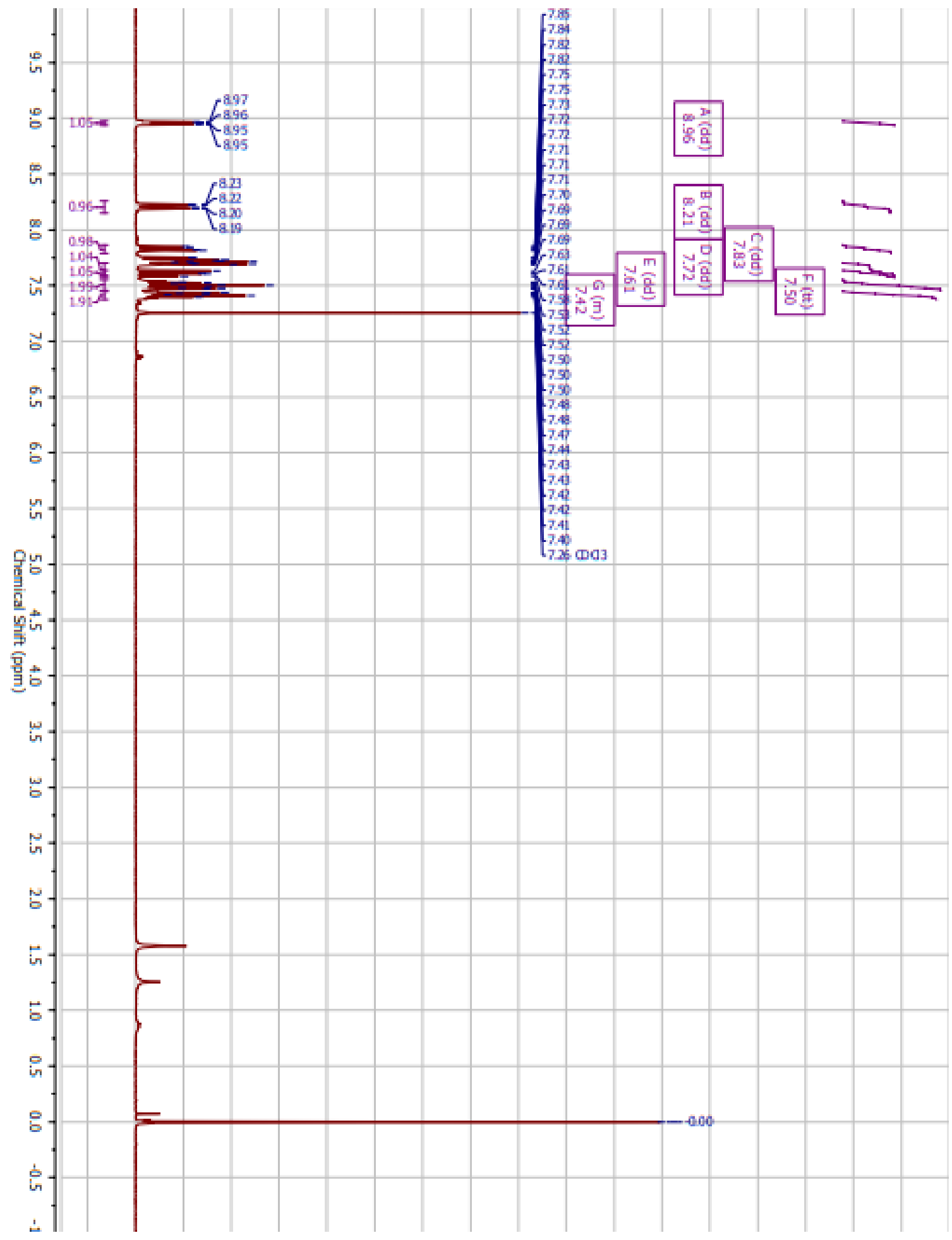


Figure A95 ${ }^{13} \mathrm{C}$ NMR spectrum of 4-phenylquinoline

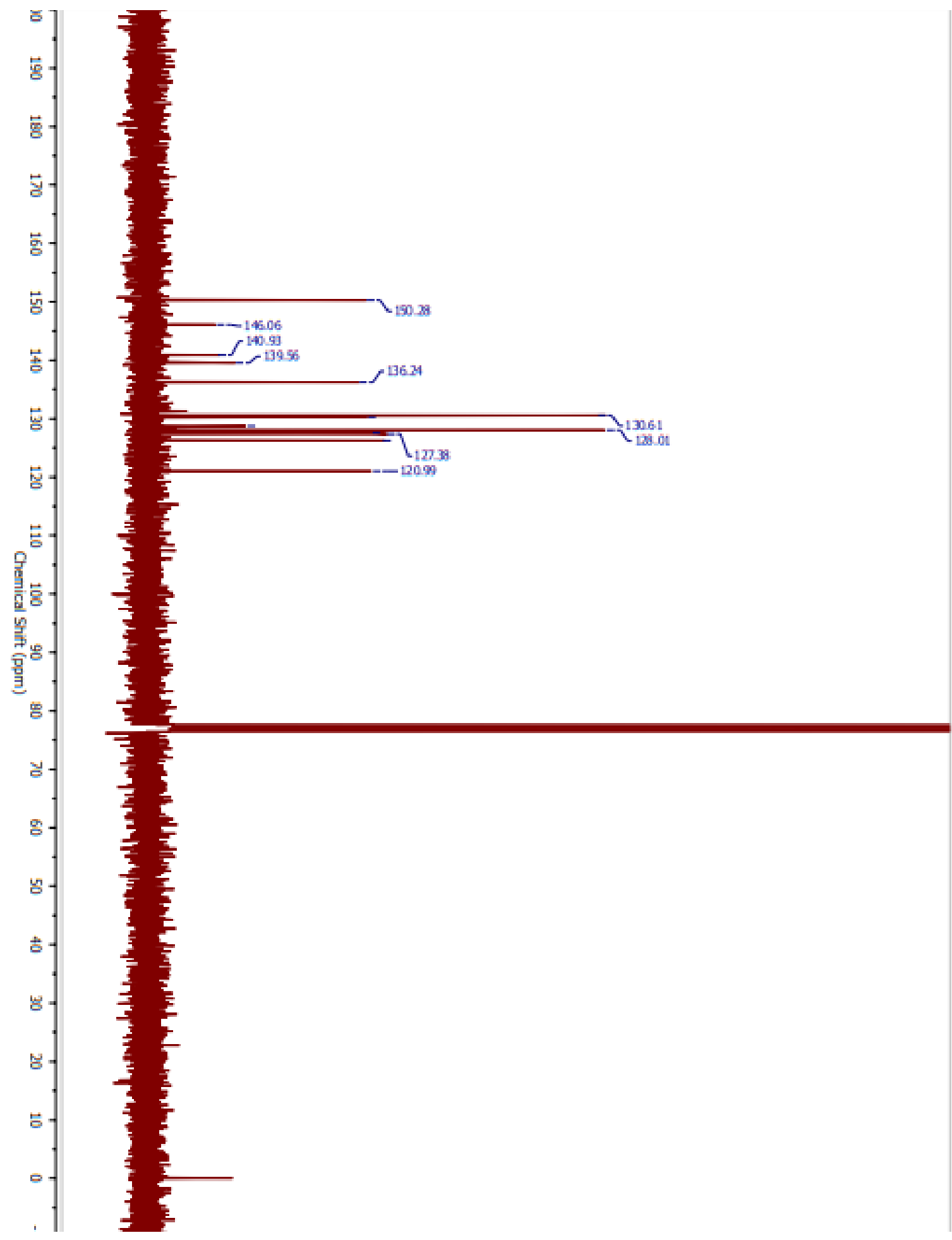


Figure A96 DEPT NMR spectrum of 4-phenylquinoline

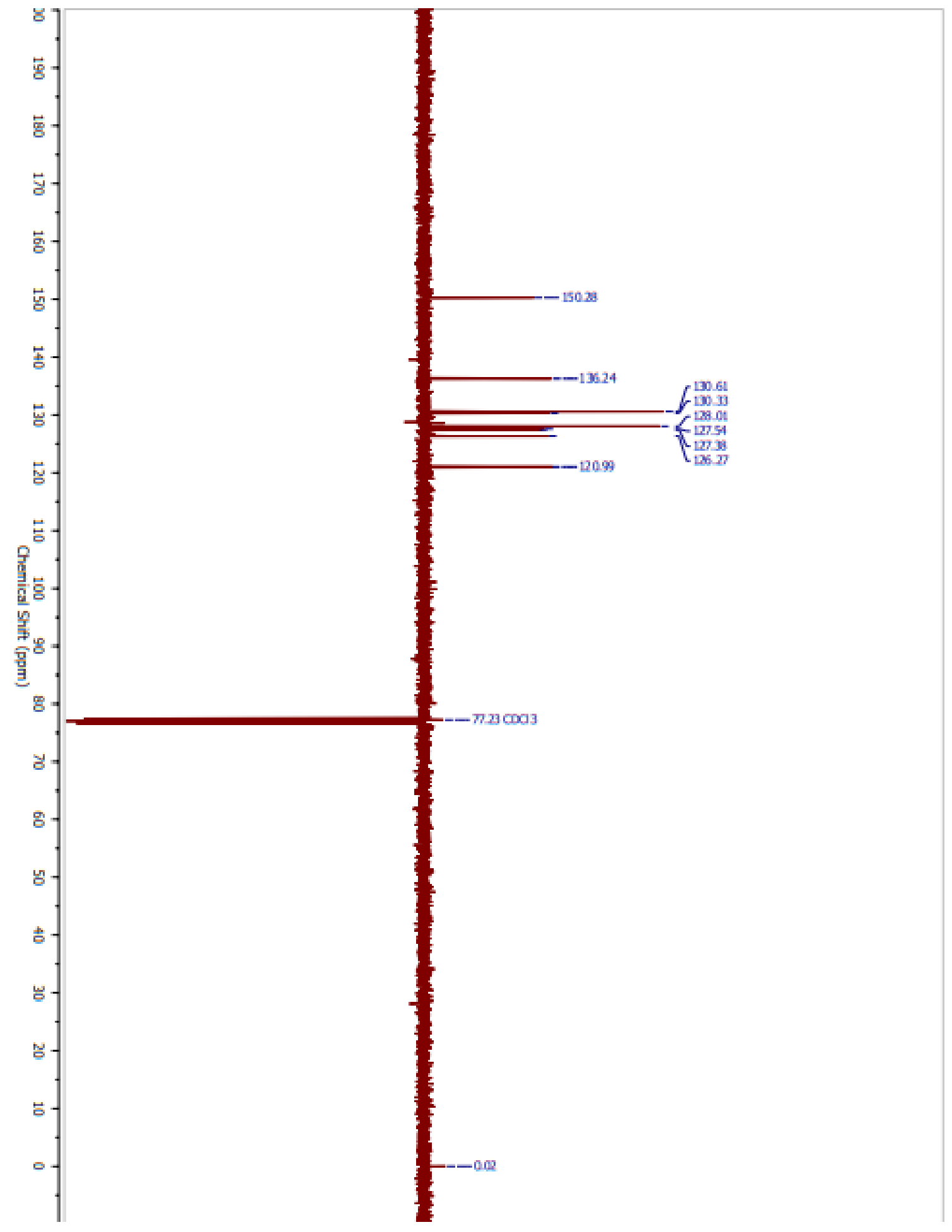


Figure A97 Gas chromatogram and mass spectrum of 4-phenylquinoline

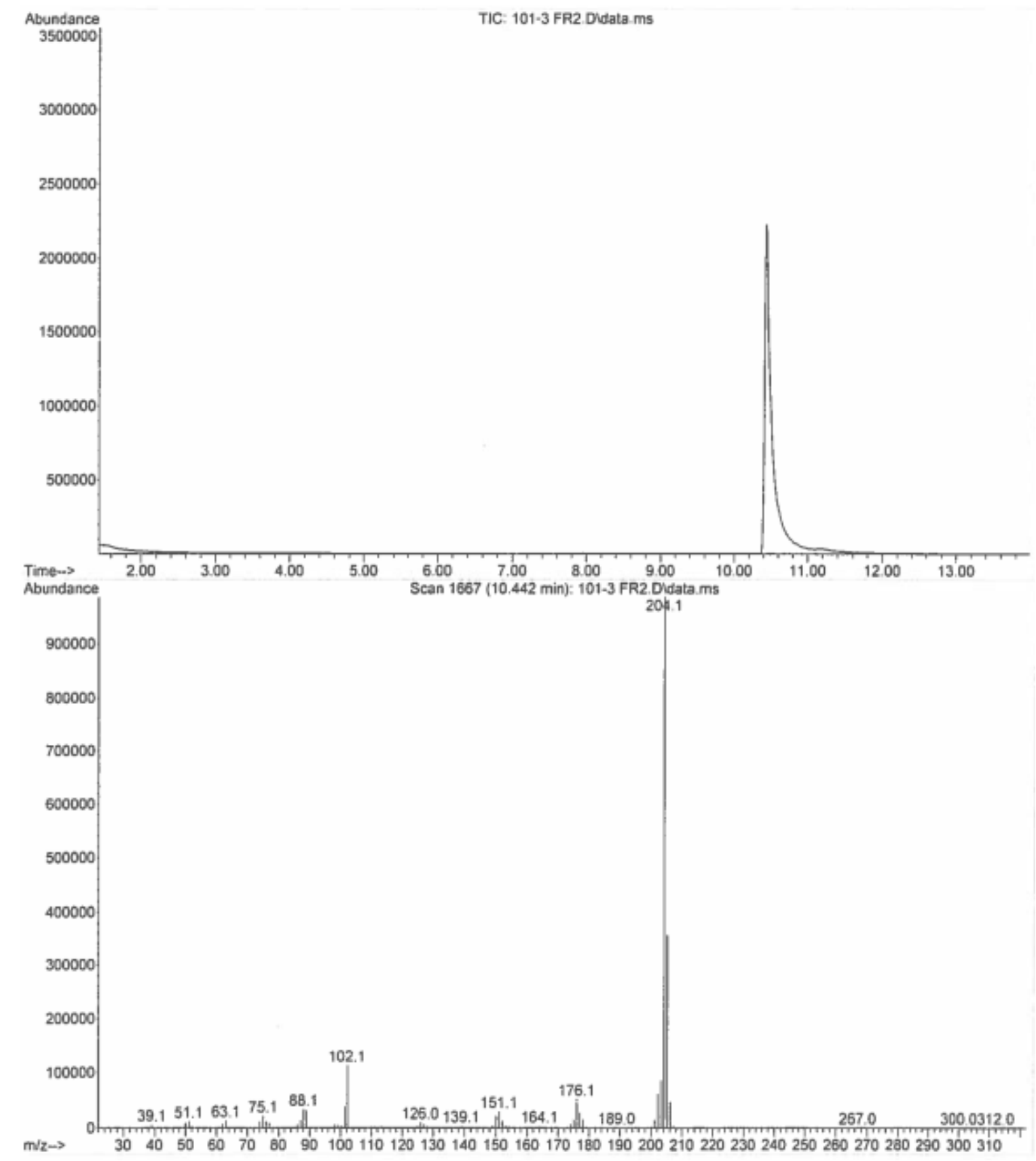


Figure A98 ${ }^{1} \mathrm{H}$ NMR spectrum of 3-phenyllutidine

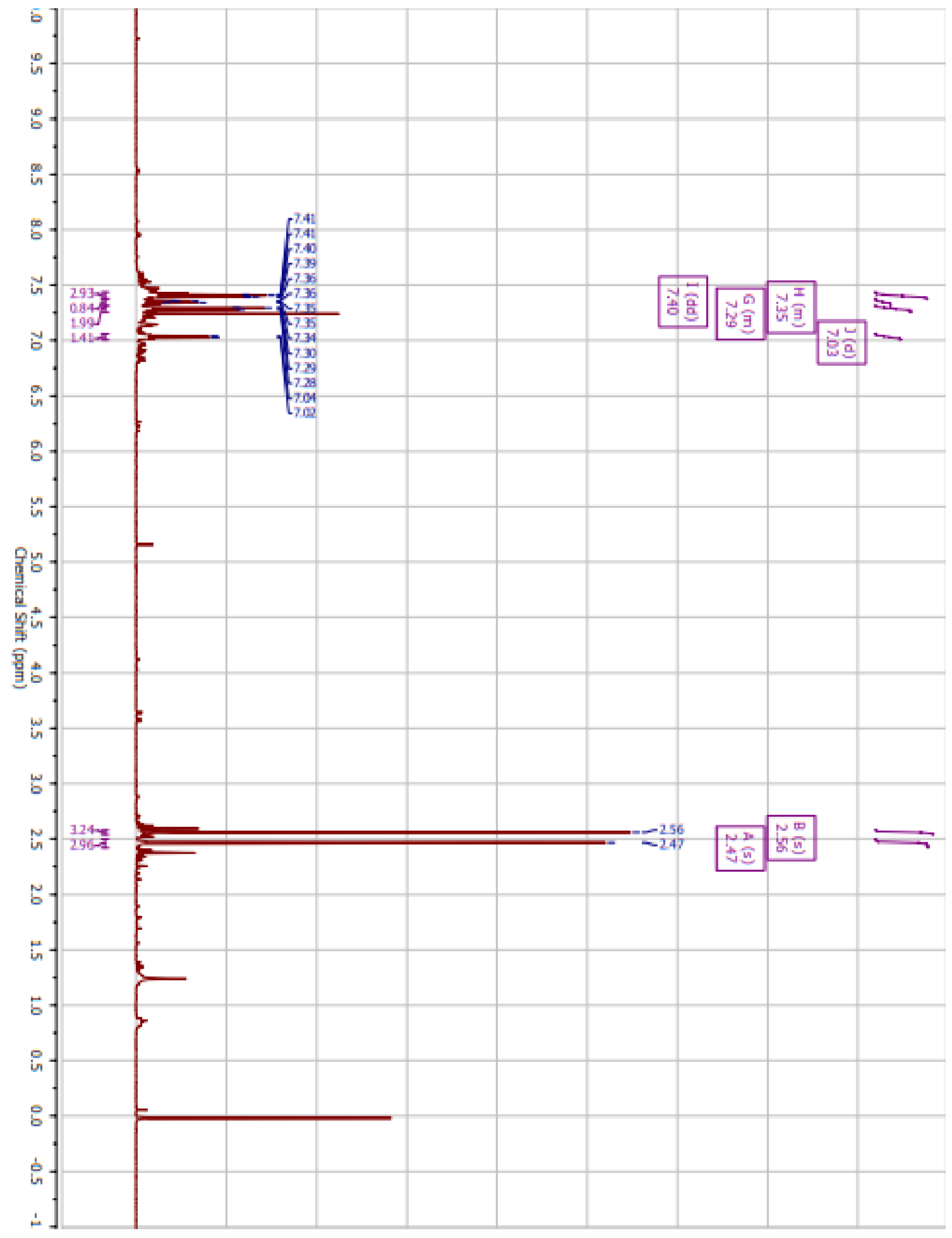


Figure A99 ${ }^{13} \mathrm{C}$ NMR spectrum of 3-phenyllutidine

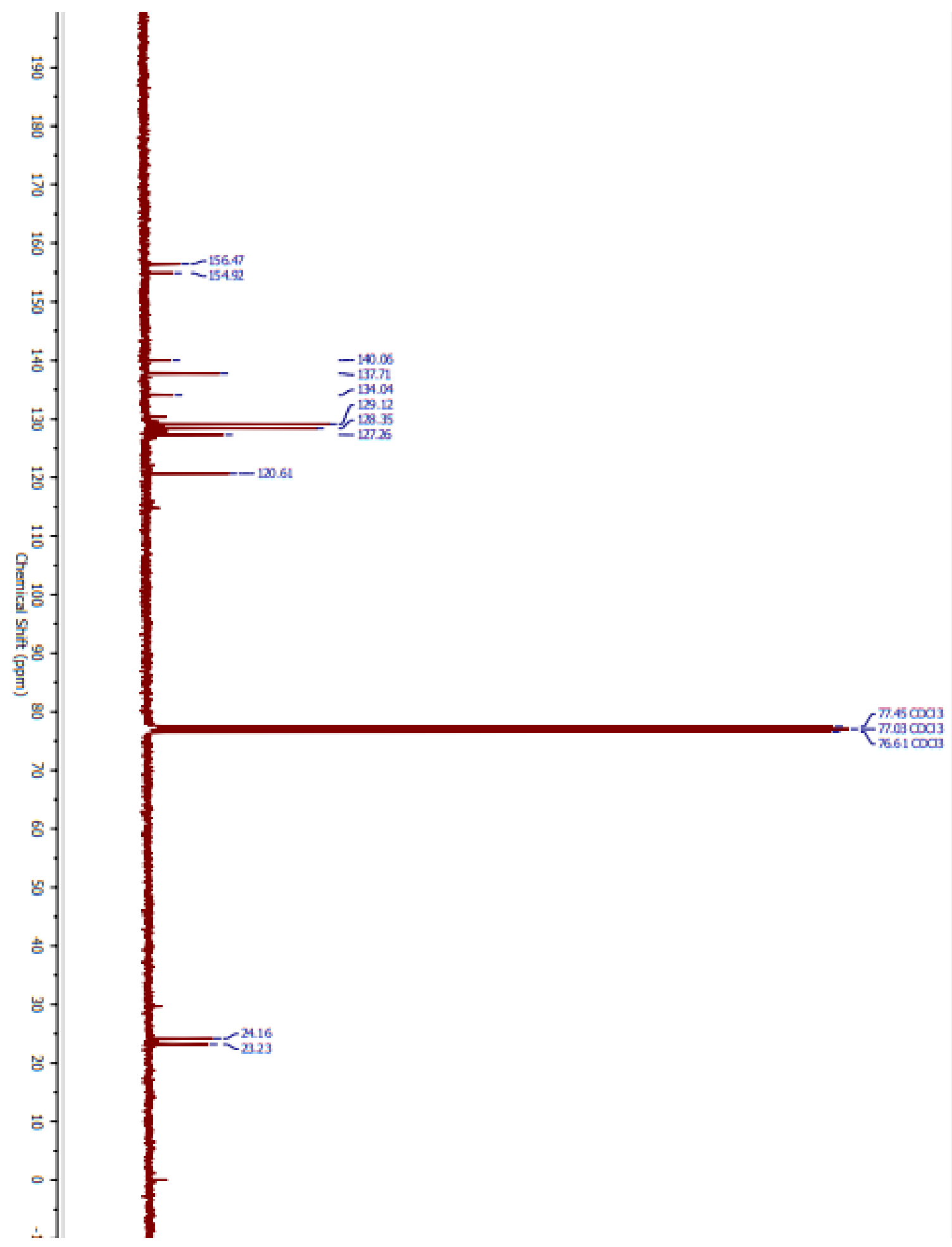


Figure A100 DEPT NMR spectrum of 3-phenyllutidine

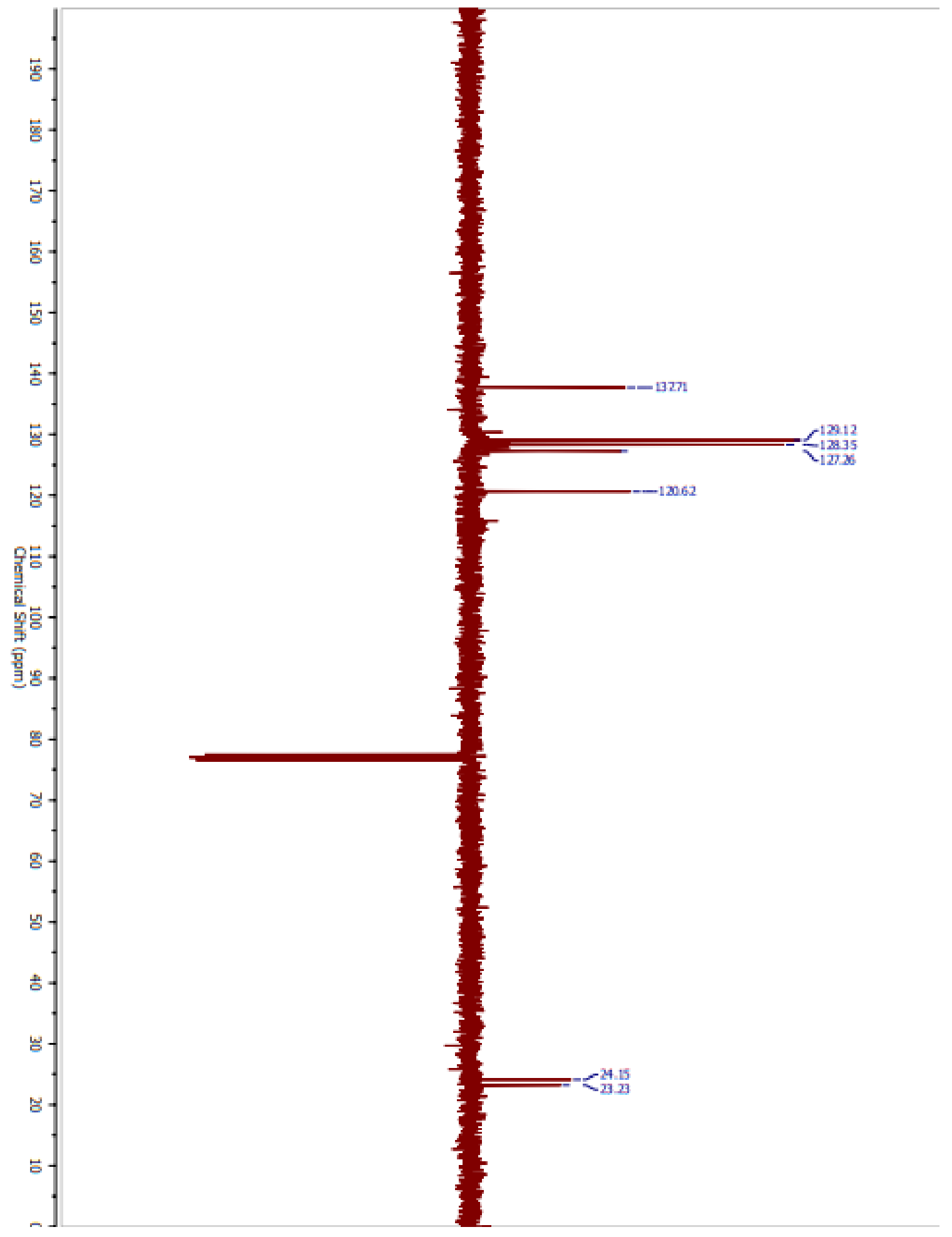


Figure A101 Gas chromatogram and mass spectrum of 3-phenyllutidine

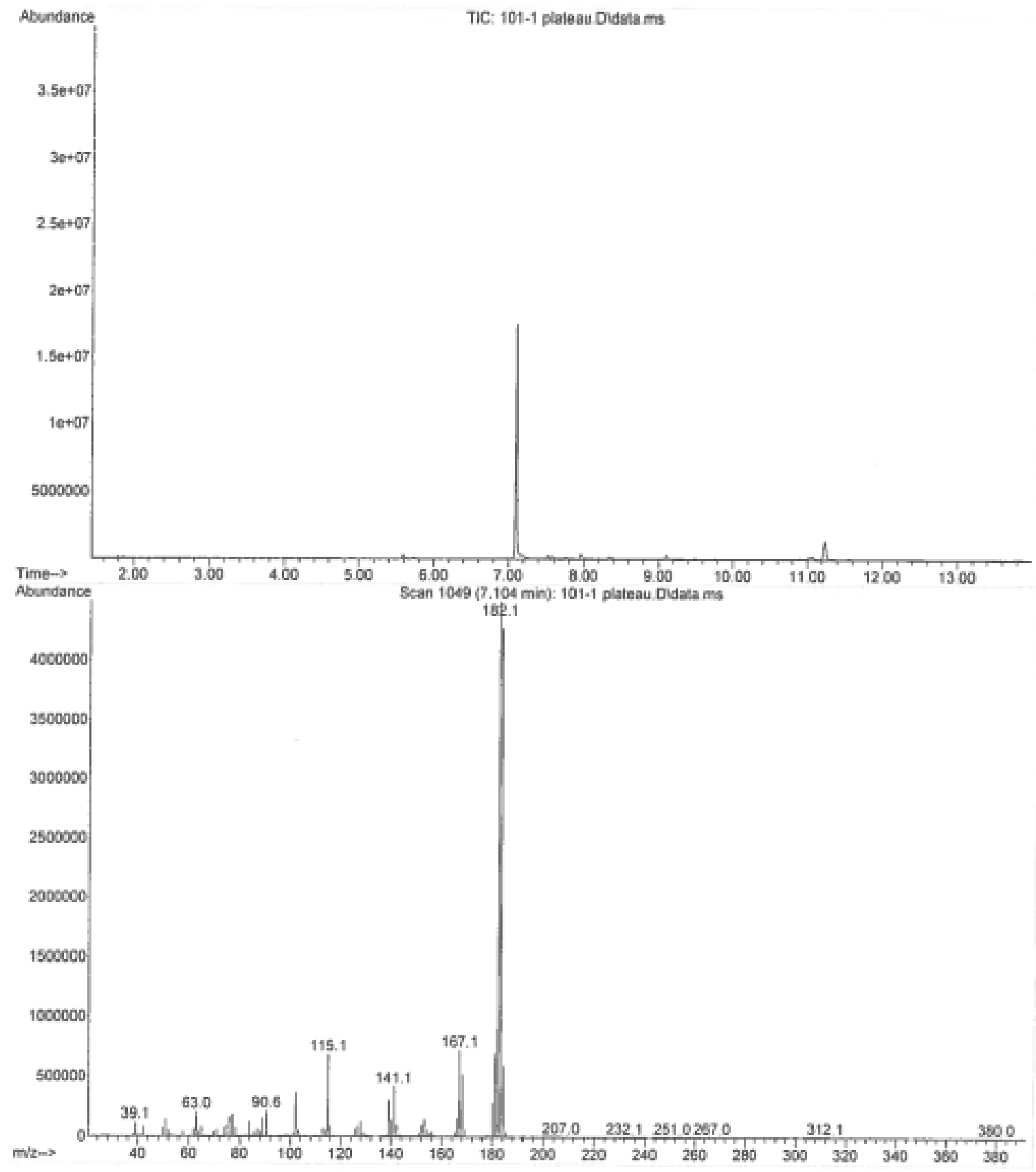


Figure A102 ${ }^{1} \mathrm{H}$ NMR spectrum of 4-phenyllutidine

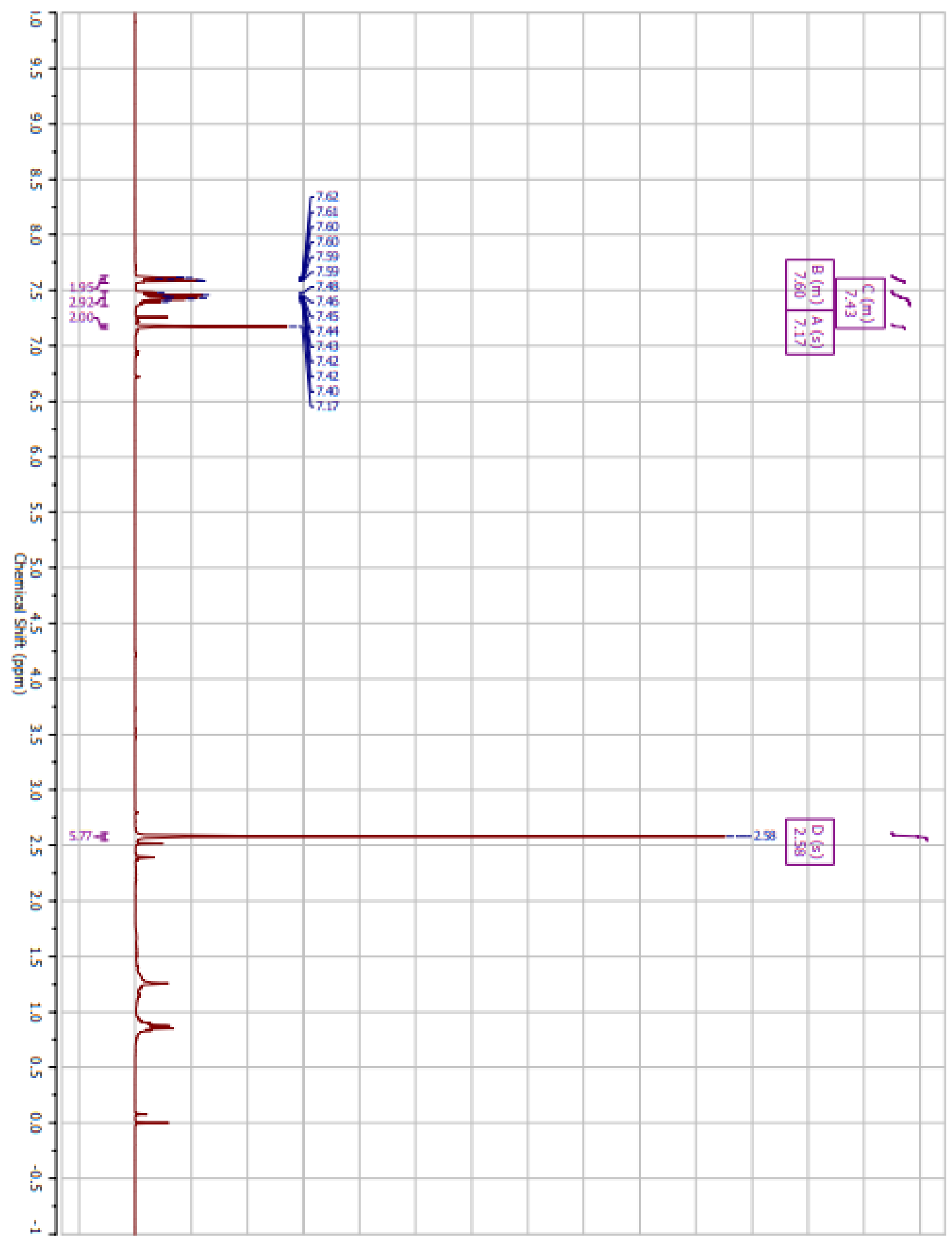


Figure $\mathbf{A 1 0 3}{ }^{13} \mathrm{C}$ NMR spectrum of 4-phenyllutidine

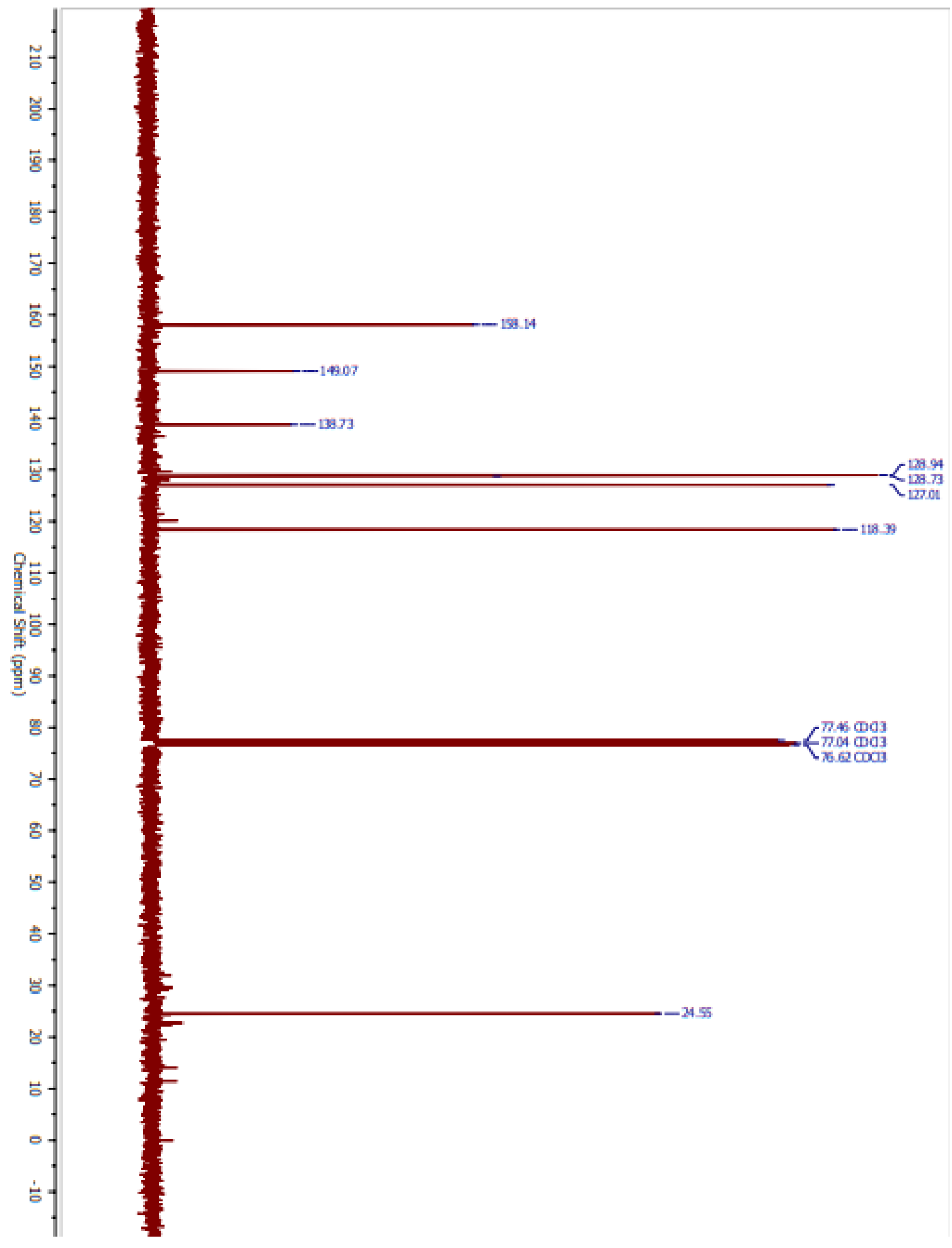


Figure A104 DEPT NMR spectrum of 4-phenyllutidine

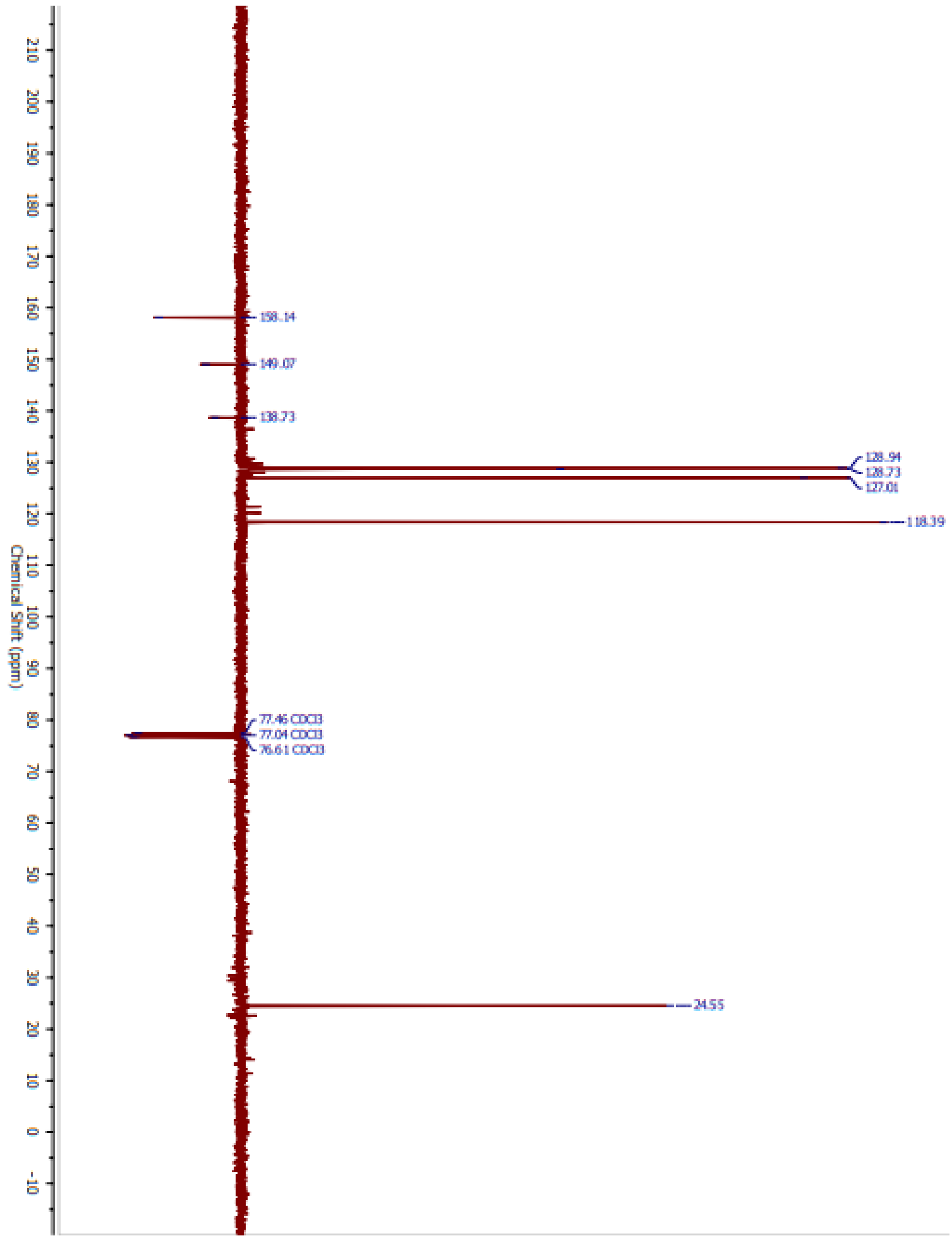


Figure A105 Gas chromatogram and mass spectrum of 4-phenyllutidine

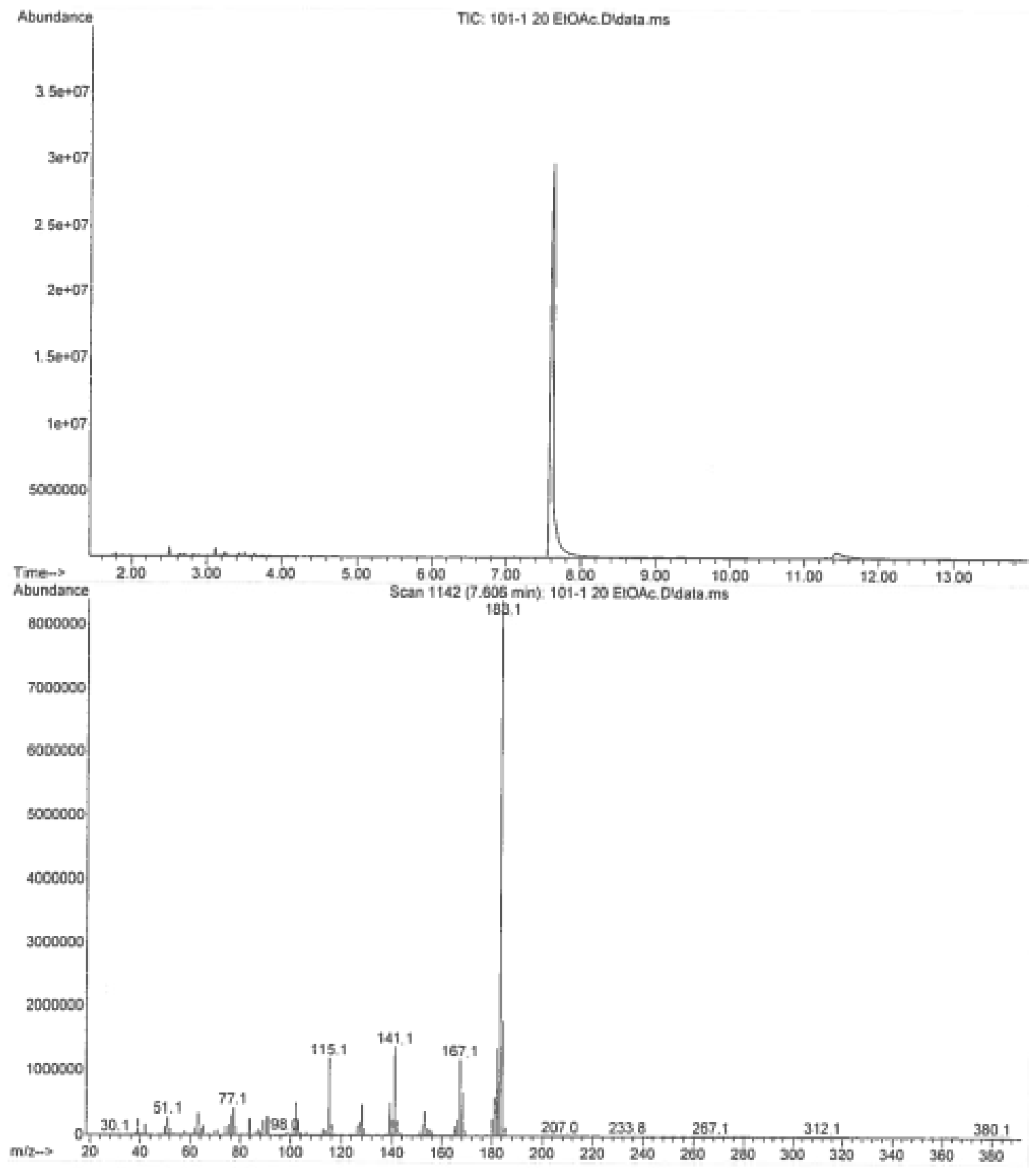


Figure A106 ${ }^{1} \mathrm{H}$ NMR spectrum of 2-phenylpyridine

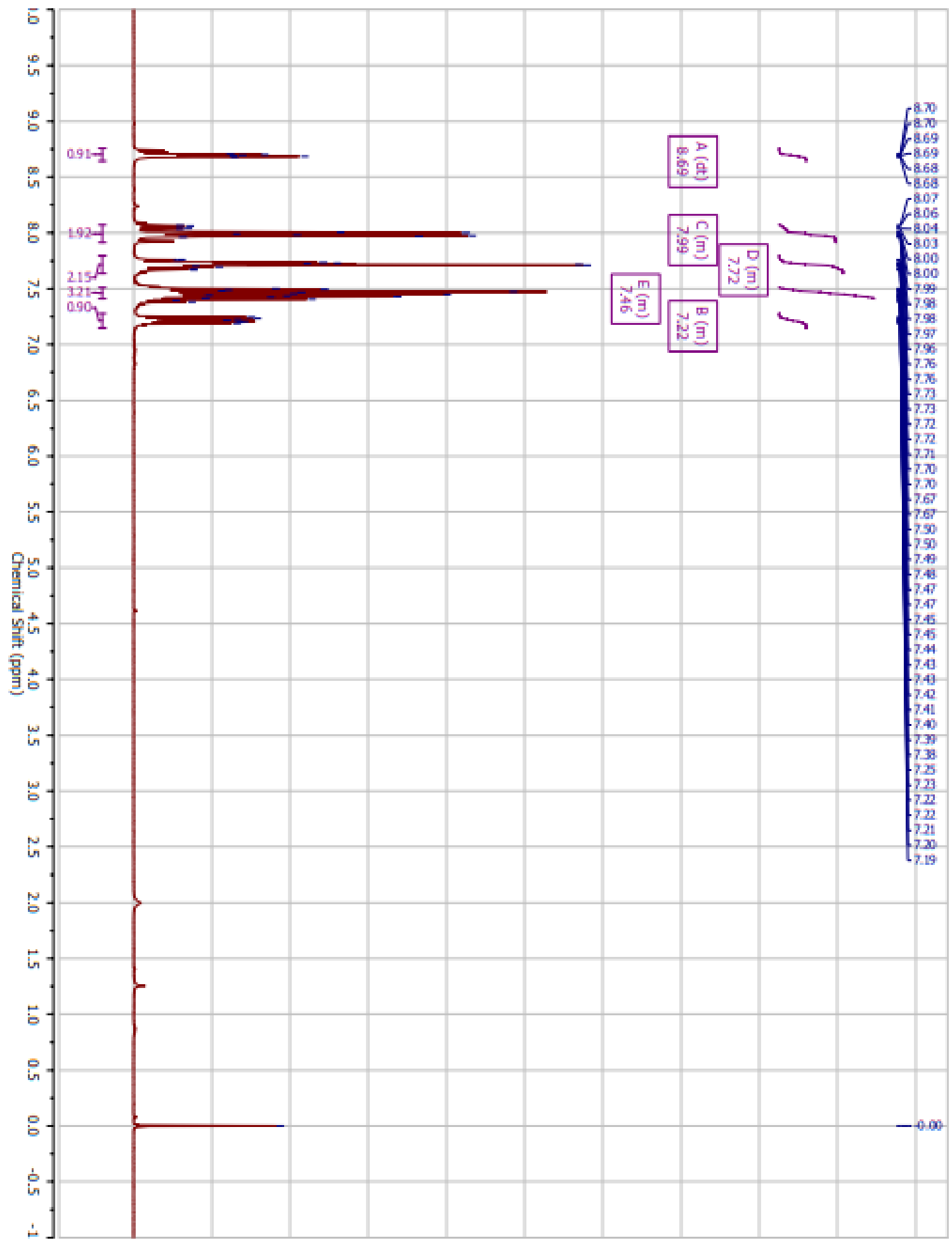


Figure A107 ${ }^{13} \mathrm{C}$ NMR spectrum of 2-phenylpyridine

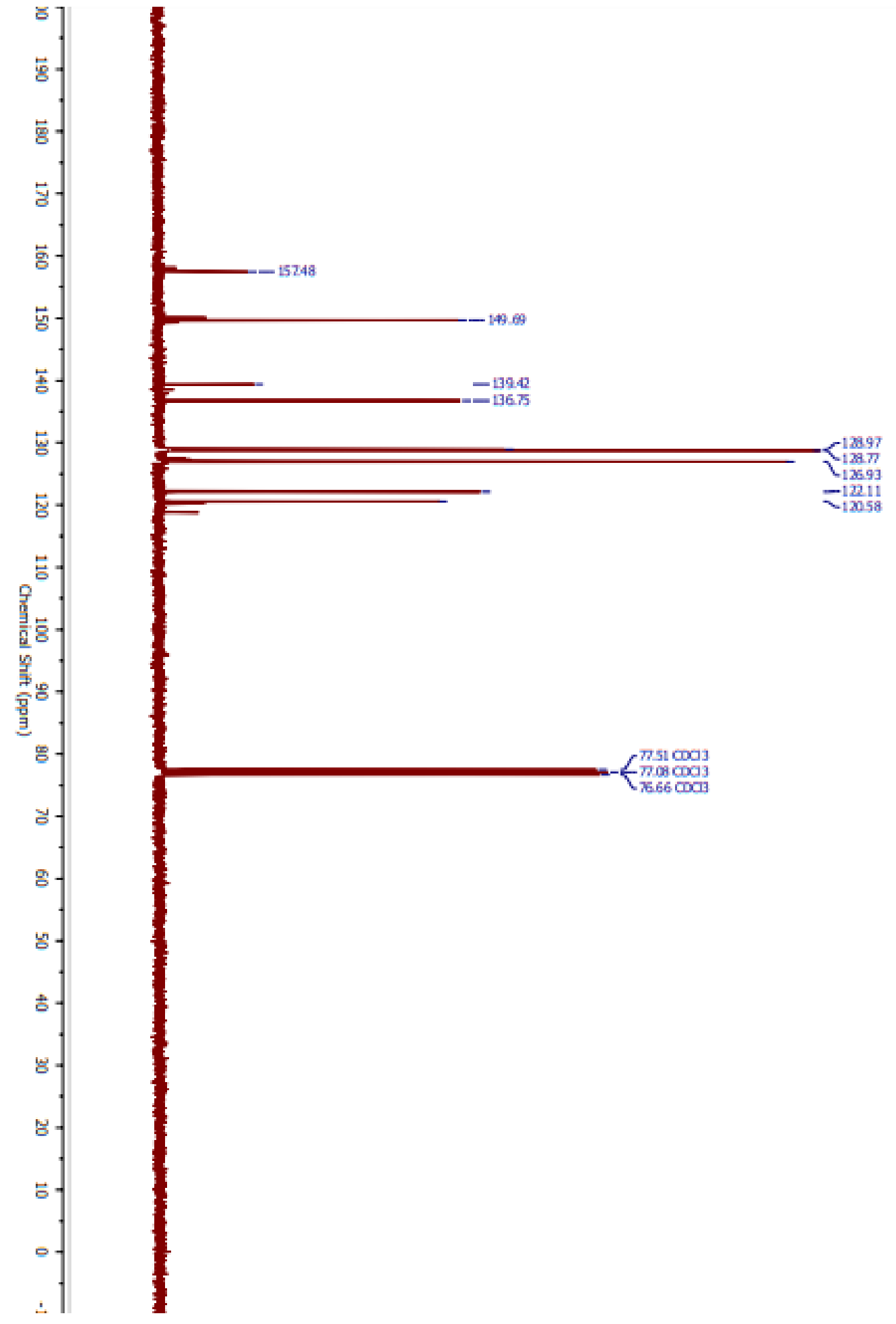


Figure A108 DEPT NMR spectrum of 2-phenylpyridine

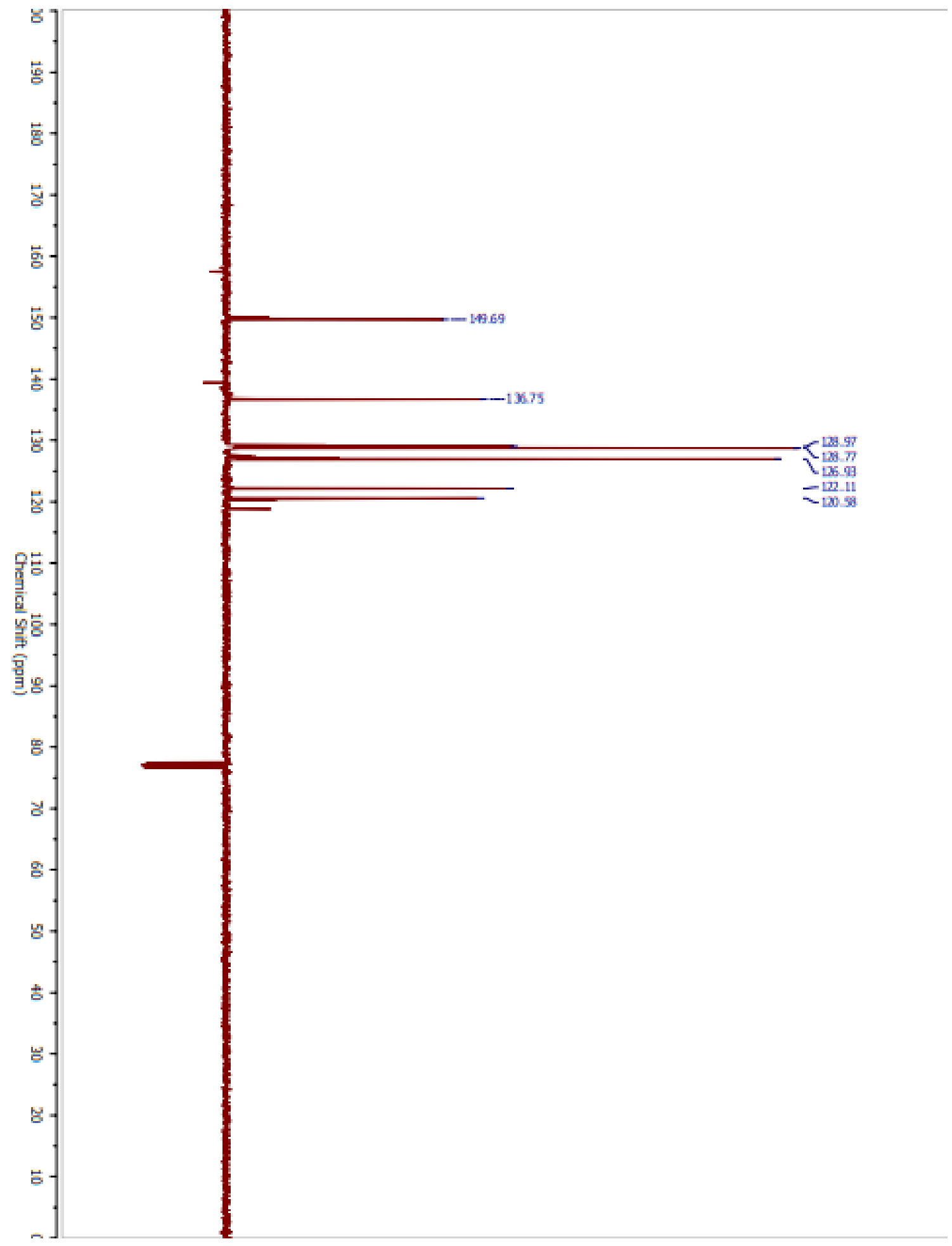


Figure A109 Gas chromatogram and mass spectrum of 2-phenylpyridine

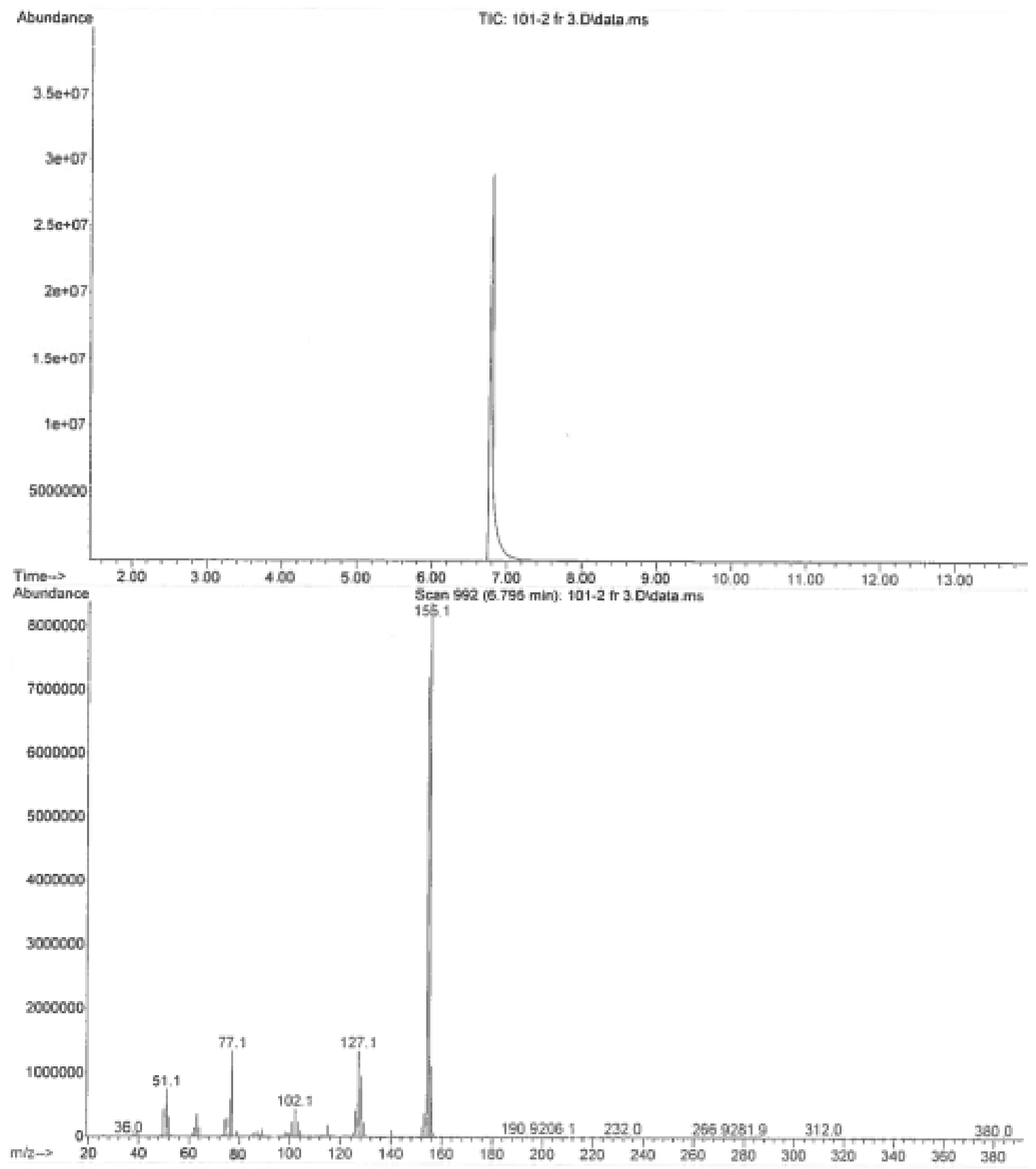


Figure A110 ${ }^{1} \mathrm{H}$ NMR spectrum of 4-phenylpyridine

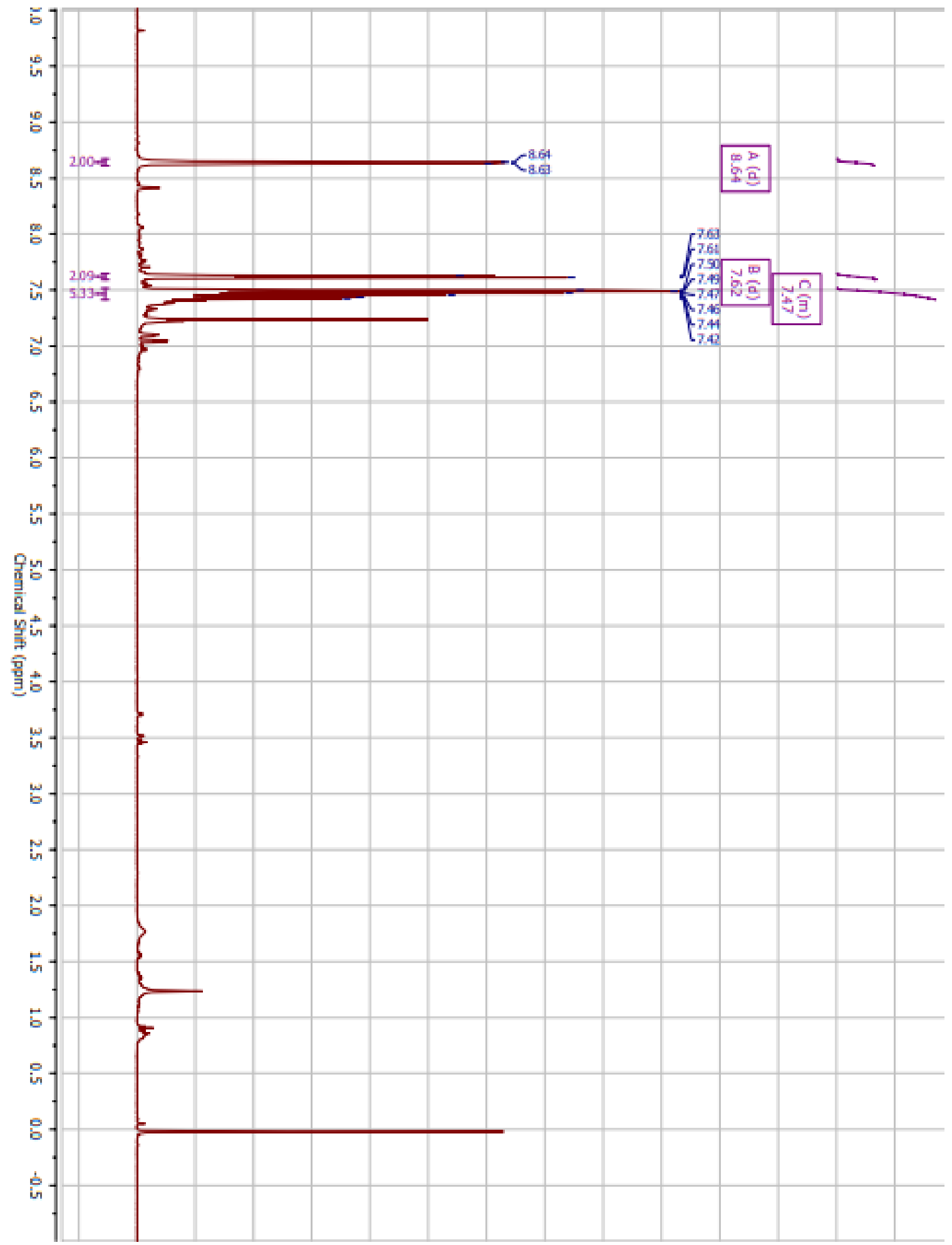


Figure A111 ${ }^{13} \mathrm{C}$ NMR spectrum of 4-phenylpyridine

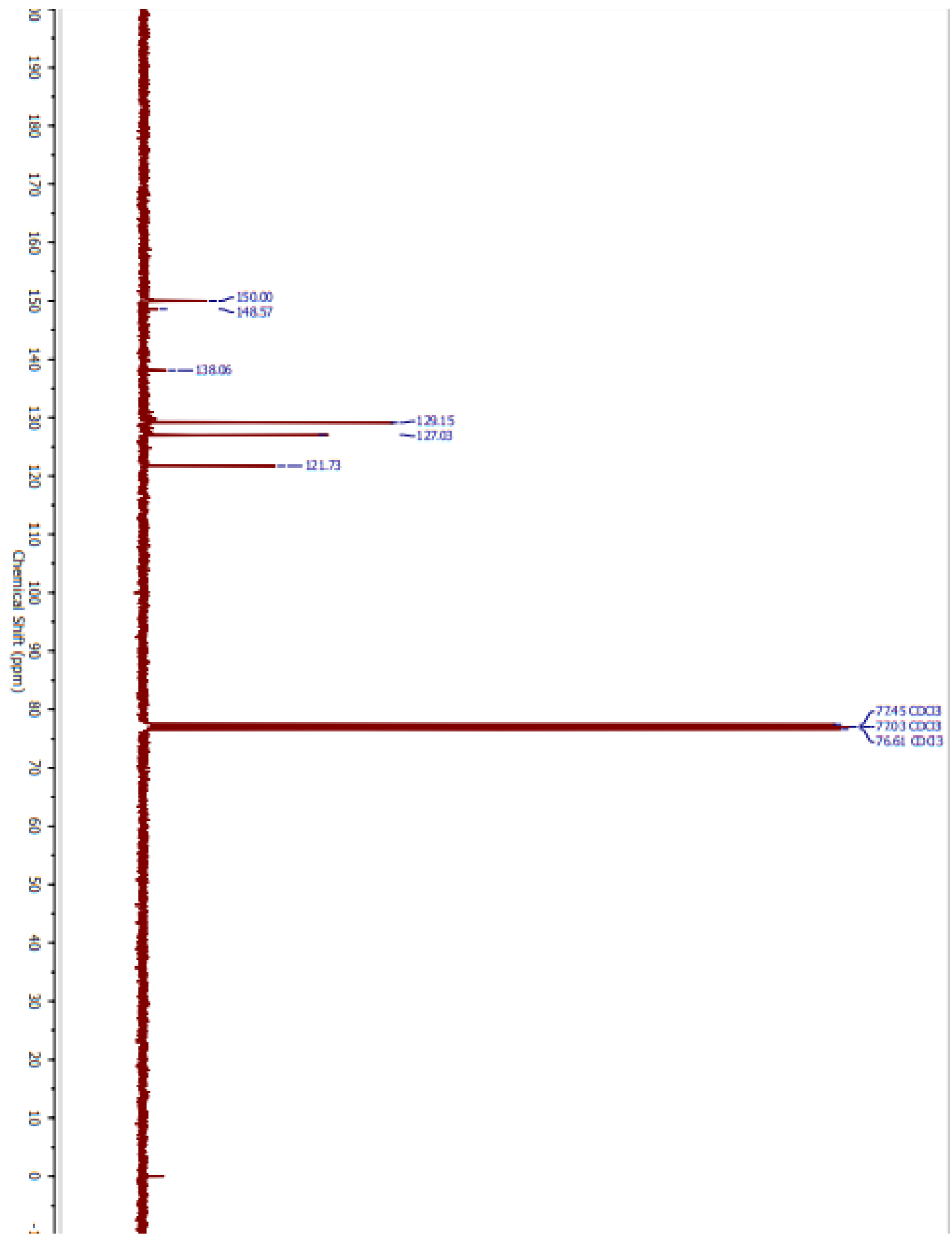


Figure A112 DEPT NMR spectrum of 4-phenylpyridine

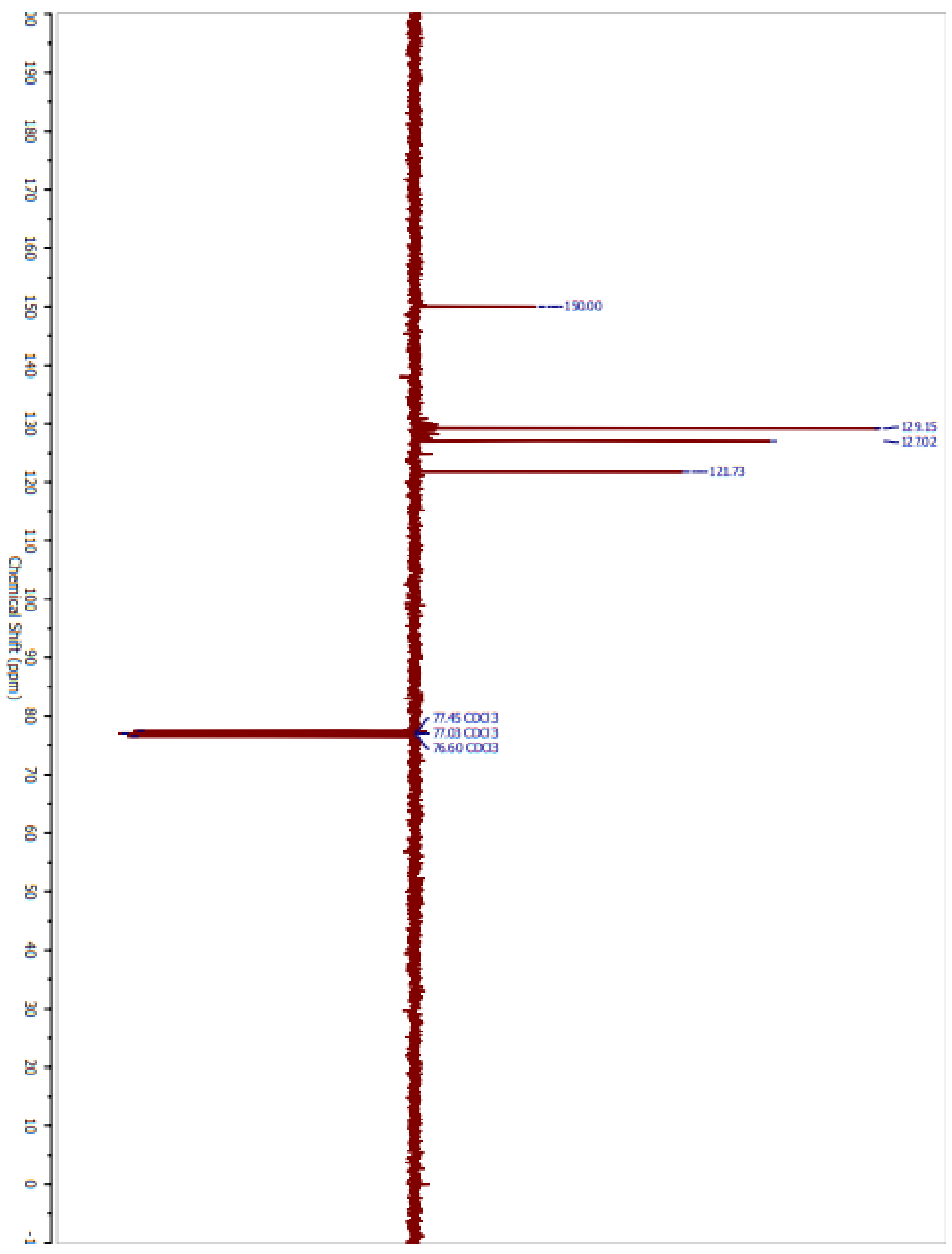

290 
Figure A113 Gas chromatogram and mass spectrum of 4-phenylpyridine

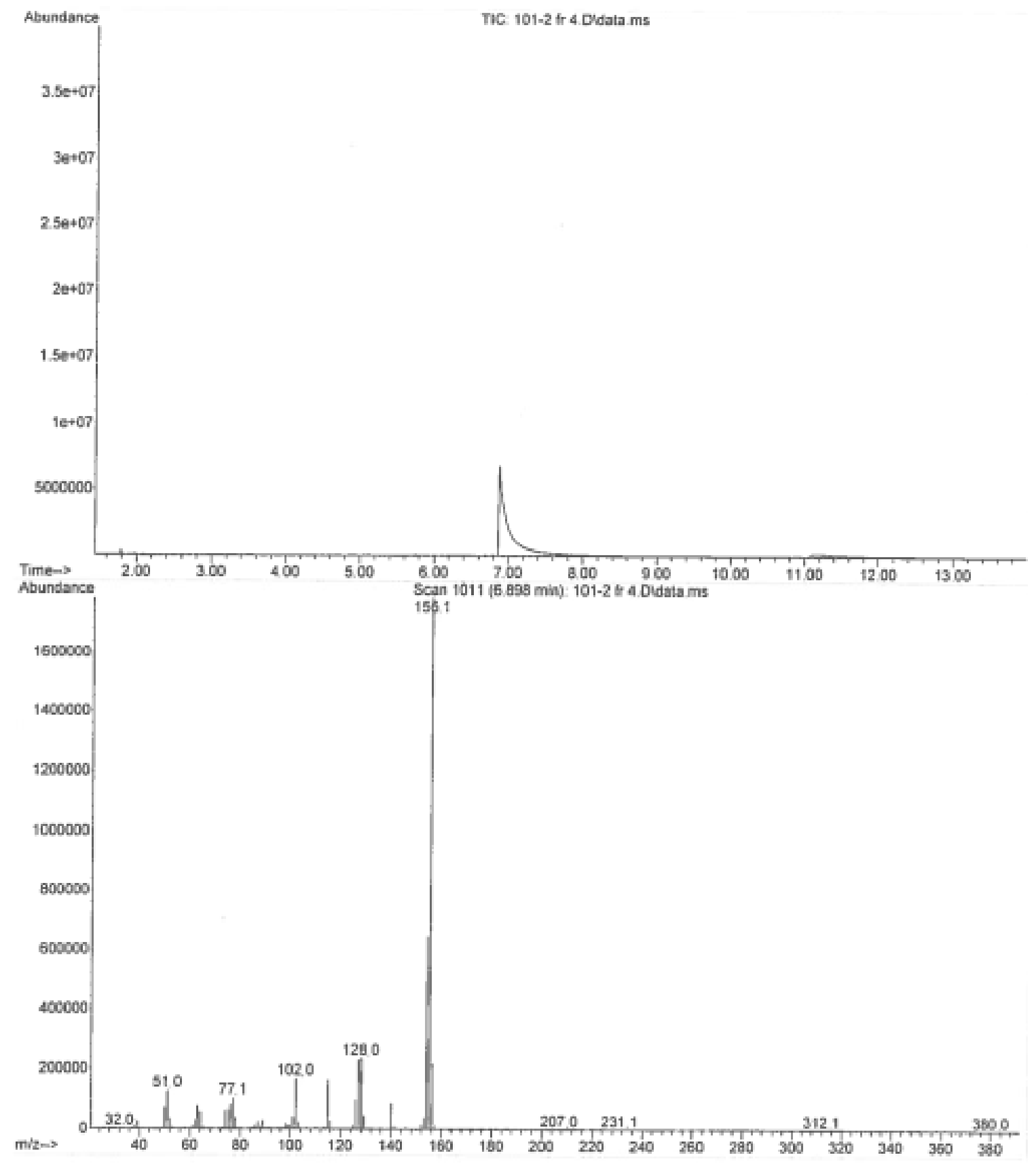


Figure A114 ${ }^{1} \mathrm{H}$ NMR spectrum of 1-methyl-2-phenylimidazole

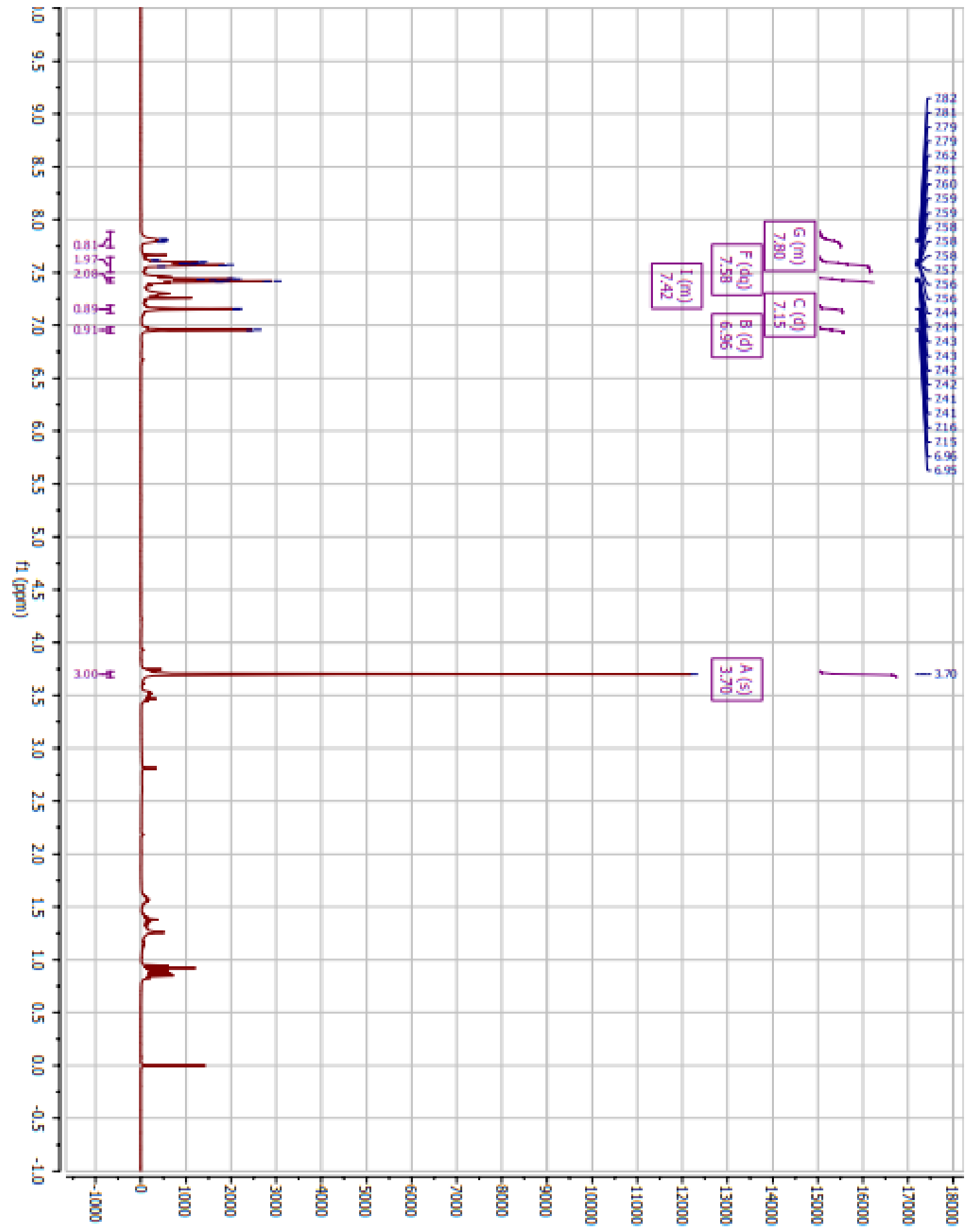


Figure A115 ${ }^{13} \mathrm{C}$ NMR spectrum of 1-methyl-2-phenylimidazole

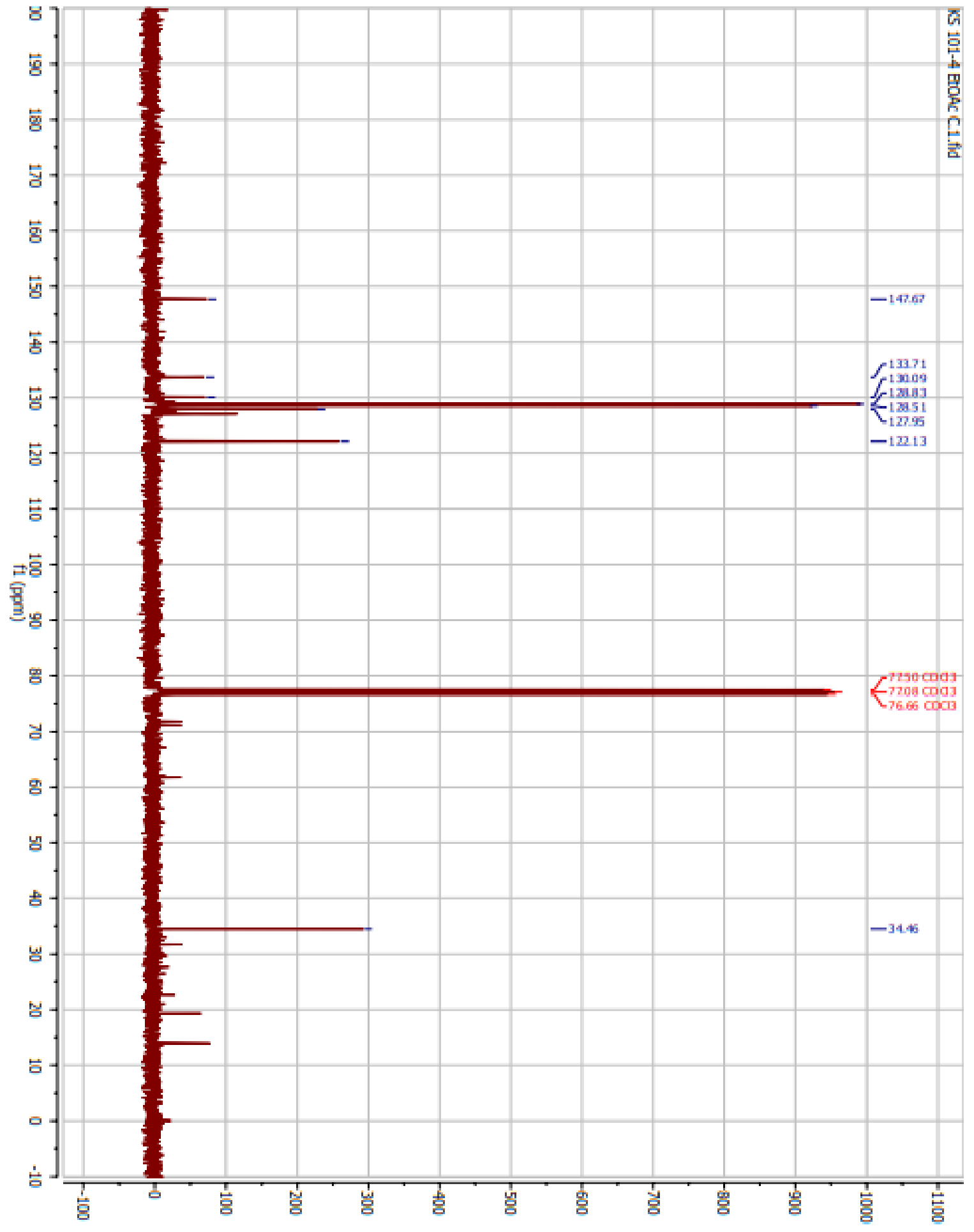


Figure A116 DEPT NMR spectrum of 1-methyl-2-phenylimidazole

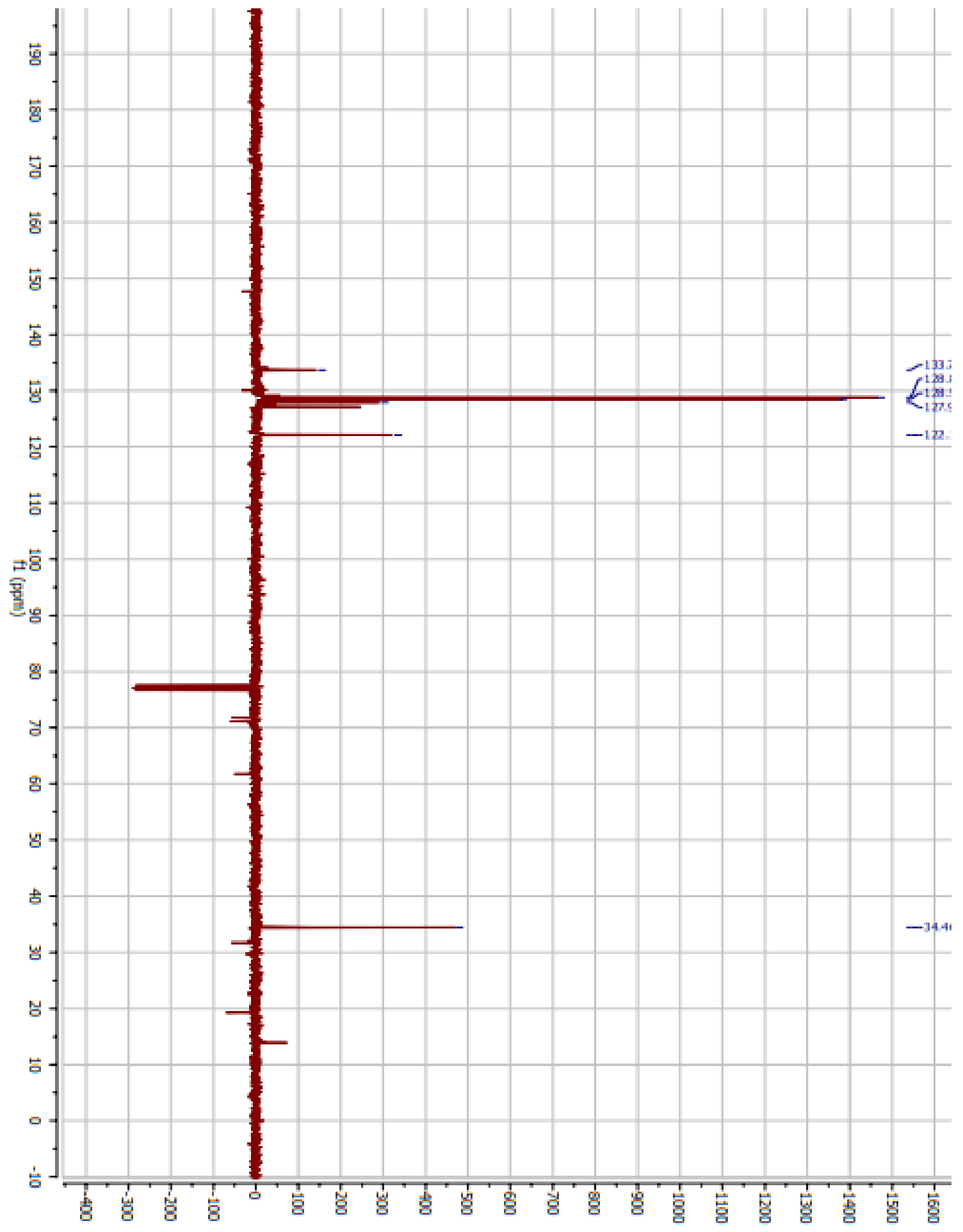


Figure A117 Gas chromatogram and mass spectrum of 1-methyl-2-phenylimidazole

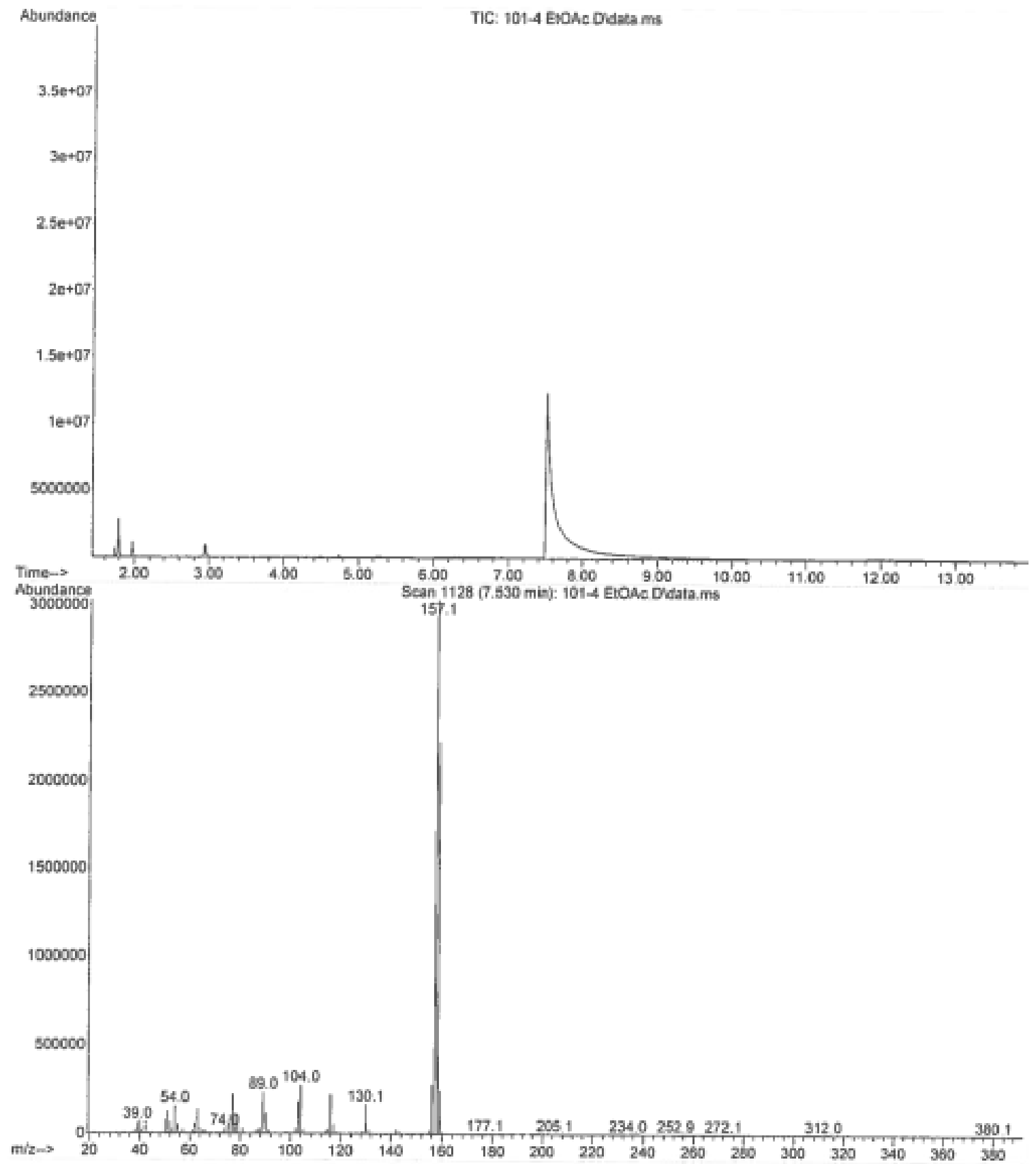


Figure A118 Gas chromatogram of the raw reaction mixture of the reaction of 2-bromopyridine and phenylboronic acid (top). Mass spectrum of 2-bromophenylpyridine, isomer unknown (bottom).

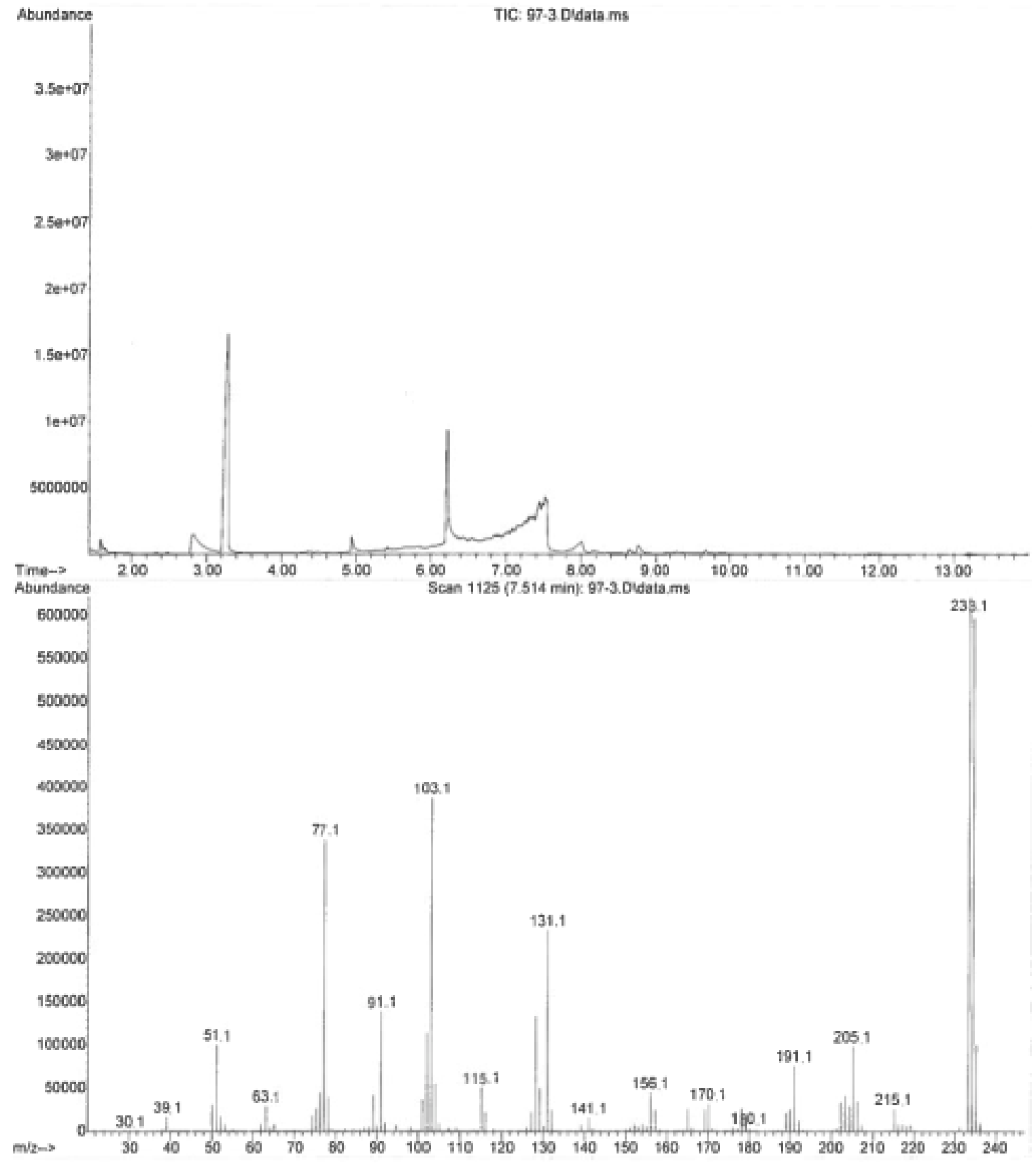

2-bromopyridine: $\mathrm{R}_{\mathrm{t}}=3.26 \mathrm{~min} . \mathrm{m} / \mathrm{z}: 159.0\left(\mathrm{M}^{+}\right), 157.0\left(\mathrm{M}^{+}-2\right), 78.1\left(\mathrm{M}^{+}-81\right), 51.1\left(\mathrm{M}^{+}-\right.$ 108). 
Biphenyl: $\mathrm{R}_{\mathrm{t}}=6.21 \mathrm{~min} . \mathrm{m} / \mathrm{z}: 154.1\left(\mathrm{M}^{+}\right), 139.1\left(\mathrm{M}^{+}-15\right), 128.1\left(\mathrm{M}^{+}-26\right), 115.1\left(\mathrm{M}^{+}-39\right)$, $76.1\left(\mathrm{M}^{+}-78\right)$.

2-bromophenylpyridine, isomer unknown: $\mathrm{R}_{\mathrm{t}}=6.53-7.55 \mathrm{~m} / \mathrm{z}: 234.1\left(\mathrm{M}^{+}\right), 233.1\left(\mathrm{M}^{+}-\right.$ 1), $205.1\left(\mathrm{M}^{+}-29\right), 191.1\left(\mathrm{M}^{+}-43\right), 131.1\left(\mathrm{M}^{+}-97\right), 128.1\left(\mathrm{M}^{+}-106\right), 103.1\left(\mathrm{M}^{+}-131\right), 91.1$ $\left(\mathrm{M}^{+}-143\right), 77.1\left(\mathrm{M}^{+}-157\right), 51.1\left(\mathrm{M}^{+}-183\right)$. 
Figure A119 Gas chromatogram of the crude reaction mixture of the reaction with 4-methlphenylboronic acid and pyrazine using toluene and water as solvent

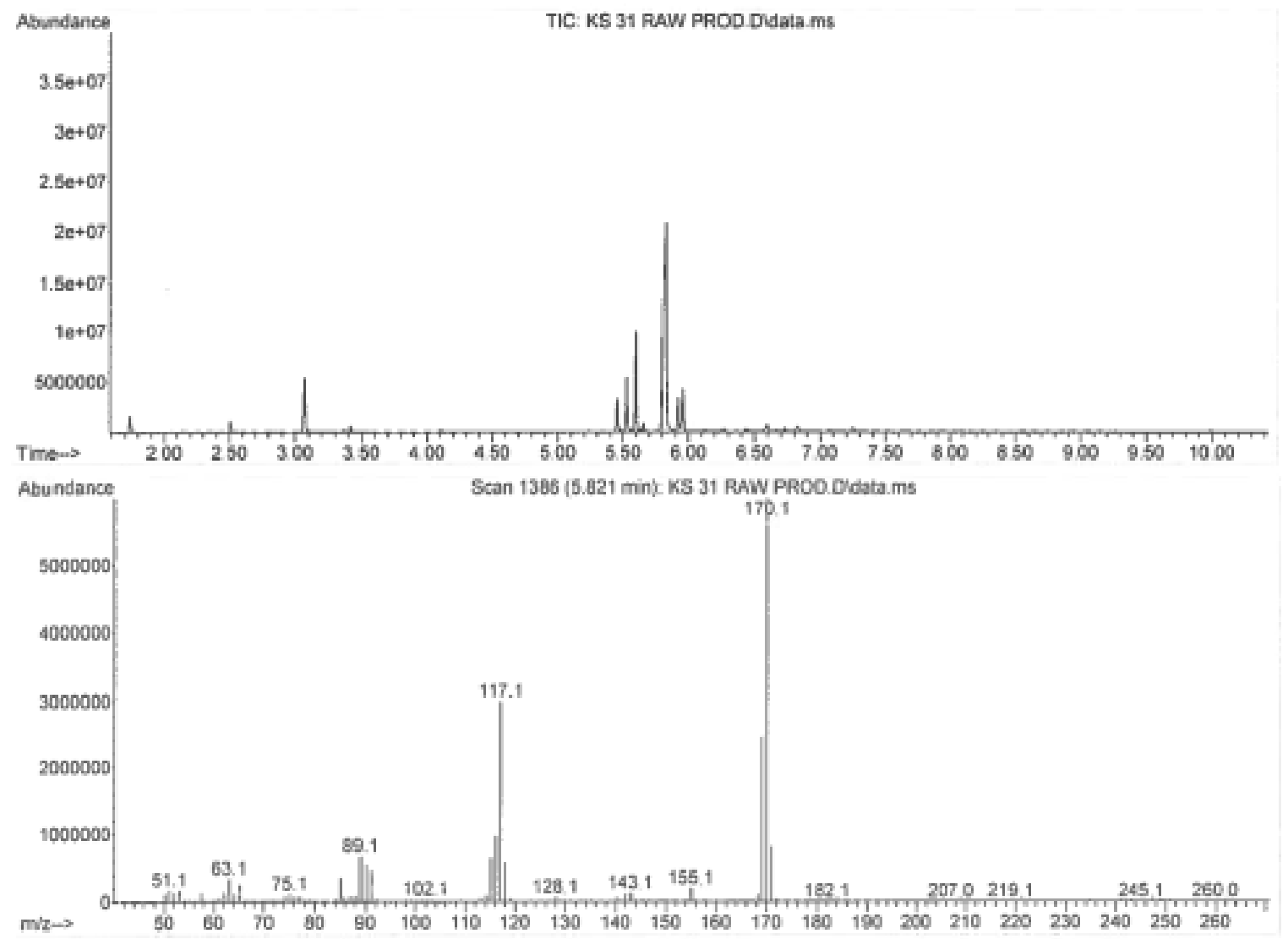

Benzyl alcohol: $\mathrm{R}_{\mathrm{t}}=3.07 \mathrm{~min} . \mathrm{m} / \mathrm{z}=108.1\left(\mathrm{M}^{+}\right), 107.1\left(\mathrm{M}^{+}-1\right), 90.1\left(\mathrm{M}^{+}-18\right), 79.1\left(\mathrm{M}^{+}-\right.$ 29), $77.1\left(\mathrm{M}^{+}-31\right)$.

2-benzylpyrazine: $R_{t}=5.45 \mathrm{~min} . \mathrm{m} / \mathrm{z}=170.1\left(\mathrm{M}^{+}\right), 169.1\left(\mathrm{M}^{+}-1\right), 115.1\left(\mathrm{M}^{+}-55\right), 91.1$ $\left(\mathrm{M}^{+}-79\right), 65.1\left(\mathrm{M}^{+}-104\right)$.

Dimethylbiphenyl isomer: $\mathrm{R}_{\mathrm{t}}=5.52 \mathrm{~min} . \mathrm{m} / \mathrm{z}=182.1\left(\mathrm{M}^{+}\right), 167.1\left(\mathrm{M}^{+}-15\right), 152.1\left(\mathrm{M}^{+}-\right.$ 30), $141.1\left(\mathrm{M}^{+}-41\right), 128.1\left(\mathrm{M}^{+}-54\right), 115.1\left(\mathrm{M}^{+}-67\right), 89.1\left(\mathrm{M}^{+}-93\right)$.

Bibenzyl: $\mathrm{R}_{\mathrm{t}}=5.59$ min. $\mathrm{m} / \mathrm{z}: 182.1\left(\mathrm{M}^{+}\right), 104.1\left(\mathrm{M}^{+}-78\right), 91.1\left(\mathrm{M}^{+}-91\right), 77.1\left(\mathrm{M}^{+}-105\right)$. $65.1\left(\mathrm{M}^{+}-117\right)$. 
Dimethylbiphenyl isomer: $\mathrm{R}_{\mathrm{t}}=5.92 \mathrm{~min} . \mathrm{m} / \mathrm{z}=182.1\left(\mathrm{M}^{+}\right), 167.1\left(\mathrm{M}^{+}-15\right), 152.1\left(\mathrm{M}^{+}-\right.$ 30), $139.1\left(\mathrm{M}^{+}-43\right), 128.1\left(\mathrm{M}^{+}-54\right), 115.1\left(\mathrm{M}^{+}-67\right), 89.1\left(\mathrm{M}^{+}-93\right)$.

Dimethylbiphenyl isomer: $R_{t}=5.96 \mathrm{~min} . \mathrm{m} / \mathrm{z}=182.1\left(\mathrm{M}^{+}\right), 167.1\left(\mathrm{M}^{+}-15\right), 152.1\left(\mathrm{M}^{+}-\right.$ 30), $139.1\left(\mathrm{M}^{+}-43\right), 128.1\left(\mathrm{M}^{+}-54\right), 115.1\left(\mathrm{M}^{+}-67\right), 89.1\left(\mathrm{M}^{+}-93\right)$. 
Figure A120 Gas chromatogram of the crude reaction mixture of the reaction with 4-methylphenylboronic acid and pyrazine under optimized conditions.

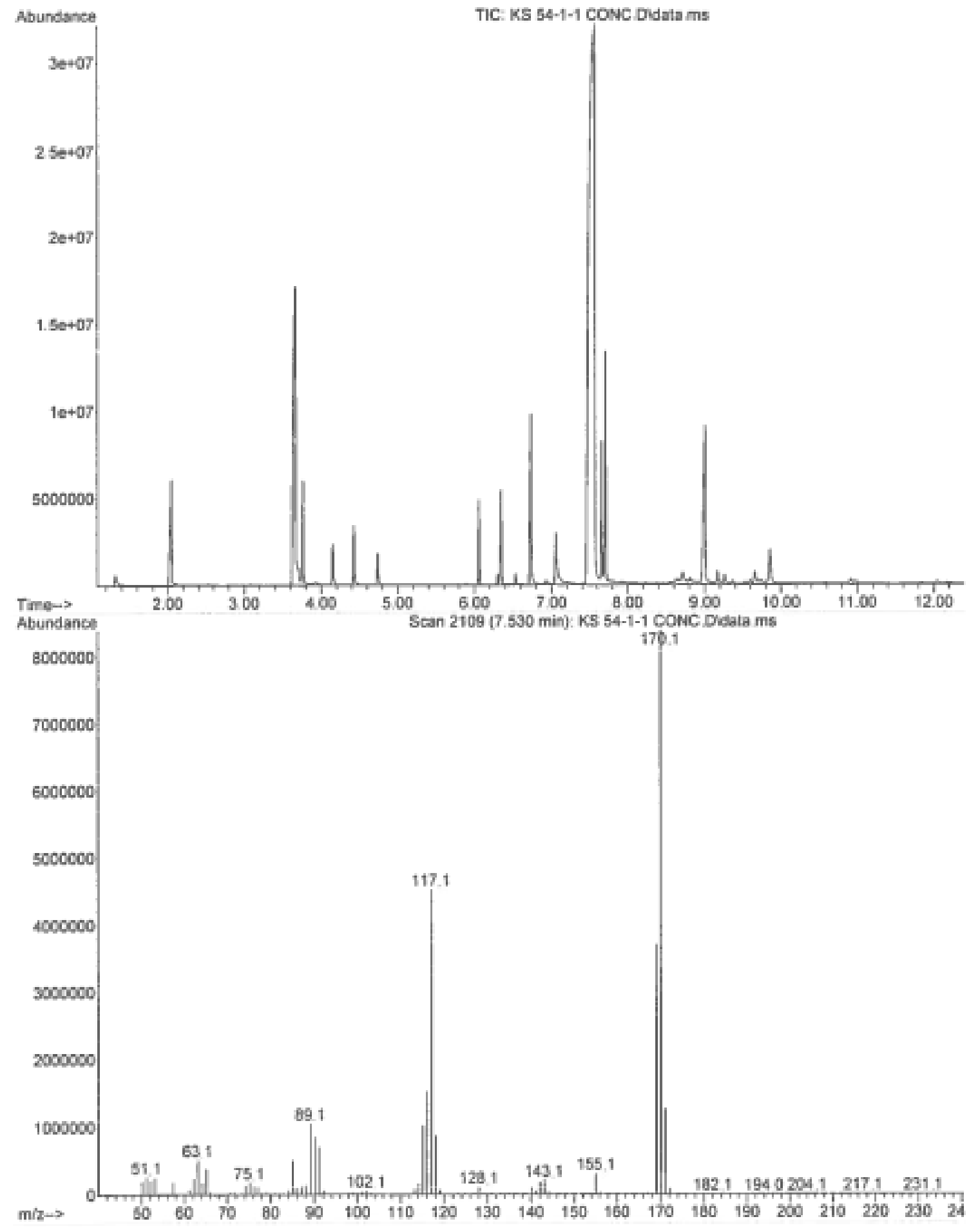


Unknown: $\mathrm{R}_{\mathrm{t}}=2.03 \mathrm{~min} . \mathrm{m} / \mathrm{z}=100.1\left(\mathrm{M}^{+}\right), 87.1\left(\mathrm{M}^{+}-13\right), 75.1\left(\mathrm{M}^{+}-25\right), 57.1\left(\mathrm{M}^{+}-43\right)$.

Benzyl alcohol: $\mathrm{R}_{\mathrm{t}}=3.66 \mathrm{~min} . \mathrm{m} / \mathrm{z}=108.1\left(\mathrm{M}^{+}\right), 107.1\left(\mathrm{M}^{+}-1\right), 90.1\left(\mathrm{M}^{+}-18\right), 79.1\left(\mathrm{M}^{+}-\right.$ 29), $77.1\left(\mathrm{M}^{+}-31\right)$.

Methylbenzaldehyde, isomer unknown: $R_{t}=3.76 \mathrm{~min} . \mathrm{m} / \mathrm{z}=120.1\left(\mathrm{M}^{+}\right), 119.1\left(\mathrm{M}^{+}-1\right)$, 107.1 ( $\left.\mathrm{M}^{+}-13\right), 91.1\left(\mathrm{M}^{+}-29\right), 65.1\left(\mathrm{M}^{+}-55\right)$.

2-dichloromethylpyrazine: $\mathrm{R}_{\mathrm{t}}=4.15 \mathrm{~min} . \mathrm{m} / \mathrm{z}=162.0\left(\mathrm{M}^{+}\right), 164.1\left(\mathrm{M}^{+}+2\right), 129.1\left(\mathrm{M}^{+}-\right.$ 33), $127.1\left(\mathrm{M}^{+}-35\right), 100.0\left(\mathrm{M}^{+}-62\right), 73.0\left(\mathrm{M}^{+}-89\right)$.

4-methylphenylboronic acid: $\mathrm{R}_{\mathrm{t}}=4.43 \mathrm{~min} . \mathrm{m} / \mathrm{z}=136.1\left(\mathrm{M}^{+}\right), 135.1\left(\mathrm{M}^{+}-1\right), 118.1\left(\mathrm{M}^{+}-\right.$ 16), $107.1\left(\mathrm{M}^{+}-19\right) .90 .1\left(\mathrm{M}^{+}-46\right), 77.1\left(\mathrm{M}^{+}-59\right)$.

Methylbenzaldehyde, isomer unknown: $\mathrm{R}_{\mathrm{t}}=4.74 \mathrm{~min} . \mathrm{m} / \mathrm{z}=119.1\left(\mathrm{M}^{+}\right), 91.1\left(\mathrm{M}^{+}-28\right)$, $65.1\left(\mathrm{M}^{+}-54\right)$.

Unknown: $\mathrm{R}_{\mathrm{t}}=6.06 \mathrm{~min} . \mathrm{m} / \mathrm{z}=176.1\left(\mathrm{M}^{+}\right), 161.1\left(\mathrm{M}^{+}-15\right), 132.1\left(\mathrm{M}^{+}-44\right), 117.1\left(\mathrm{M}^{+}-\right.$ 59), $105.1\left(\mathrm{M}^{+}-71\right), 91.1\left(\mathrm{M}^{+}-85\right)$.

Dichloromethylbenzyl alcohol, isomer unknown: $\mathrm{R}_{\mathrm{t}}=6.34 \mathrm{~min} . \mathrm{m} / \mathrm{z}=190.1\left(\mathrm{M}^{+}\right), 192.1$ $\left(\mathrm{M}^{+}+2\right), 157.1\left(\mathrm{M}^{+}-33\right), 155.1\left(\mathrm{M}^{+}-35\right) .127 .0\left(\mathrm{M}^{+}-63\right), 107.1\left(\mathrm{M}^{+}-83\right), 91.1\left(\mathrm{M}^{+}-99\right), 79.1$ $\left(\mathrm{M}^{+}-111\right), 77.1\left(\mathrm{M}^{+}-113\right), 65.1\left(\mathrm{M}^{+}-125\right), 51.1\left(\mathrm{M}^{+}-139\right)$.

Bipyrazine: $\mathrm{R}_{\mathrm{t}}=6.72 \mathrm{~min} . \mathrm{m} / \mathrm{z}=158.1\left(\mathrm{M}^{+}\right), 131.1\left(\mathrm{M}^{+}-27\right), 106.1\left(\mathrm{M}^{+}-52\right), 80.1\left(\mathrm{M}^{+}-\right.$ 78).

Dimethylbiphenyl, isomer unknown: $R_{t}=7.06 \mathrm{~min} . \mathrm{m} / \mathrm{z}=182.1\left(\mathrm{M}^{+}\right), 167.1\left(\mathrm{M}^{+}-15\right)$, $152.1\left(\mathrm{M}^{+}-30\right), 141.1\left(\mathrm{M}^{+}-41\right), 128.1\left(\mathrm{M}^{+}-54\right), 115.1\left(\mathrm{M}^{+}-67\right), 89.1\left(\mathrm{M}^{+}-93\right)$. 
2-(4-methylphenyl)pyrazine: $\mathrm{R}_{\mathrm{t}}=7.47 \mathrm{~min} . \mathrm{m} / \mathrm{z}: 170.1\left(\mathrm{M}^{+}\right), 155.1\left(\mathrm{M}^{+}-15\right), 117.1\left(\mathrm{M}^{+}-\right.$ 53), $89.1\left(\mathrm{M}^{+}-81\right)$.

Dimethylbiphenyl, isomer unknown: $R_{t}=7.66 \mathrm{~min} . \mathrm{m} / \mathrm{z}=182.1\left(\mathrm{M}^{+}\right) .167 .1\left(\mathrm{M}^{+}-15\right)$, $152.1\left(\mathrm{M}^{+}-30\right), 139.1\left(\mathrm{M}^{+}-43\right), 128.1\left(\mathrm{M}^{+}-54\right), 115.1\left(\mathrm{M}^{+}-67\right), 89.1\left(\mathrm{M}^{+}-93\right)$.

Dimethylbiphenyl, isomer unknown: $\mathrm{R}_{\mathrm{t}}=7.71 \mathrm{~min} . \mathrm{m} / \mathrm{z}=182.1\left(\mathrm{M}^{+}\right) .167 .1\left(\mathrm{M}^{+}-15\right)$, $152.1\left(\mathrm{M}^{+}-30\right), 139.1\left(\mathrm{M}^{+}-43\right), 128.1\left(\mathrm{M}^{+}-54\right), 115.1\left(\mathrm{M}^{+}-67\right), 89.1\left(\mathrm{M}^{+}-93\right)$.

Biphenyldimethanol, isomer unknown: $R_{t}=9.00 \mathrm{~min} . \mathrm{m} / \mathrm{z}=214.1\left(\mathrm{M}^{+}\right) .199 .1\left(\mathrm{M}^{+}-15\right)$, 185.1 ( $\left.\mathrm{M}^{+}-29\right), 171.1\left(\mathrm{M}^{+}-43\right), 159.1\left(\mathrm{M}^{+}-55\right), 145.1\left(\mathrm{M}^{+}-69\right), 128.1\left(\mathrm{M}^{+}-86\right), 115.1\left(\mathrm{M}^{+}-\right.$ 99), $91.1\left(\mathrm{M}^{+}-123\right), 77.1\left(\mathrm{M}^{+}-137\right)$. 
Figure A121 Gas chromatogram of the crude reaction mixture of the reaction with 4-chlorophenylboronic acid and pyrazine under optimized conditions.

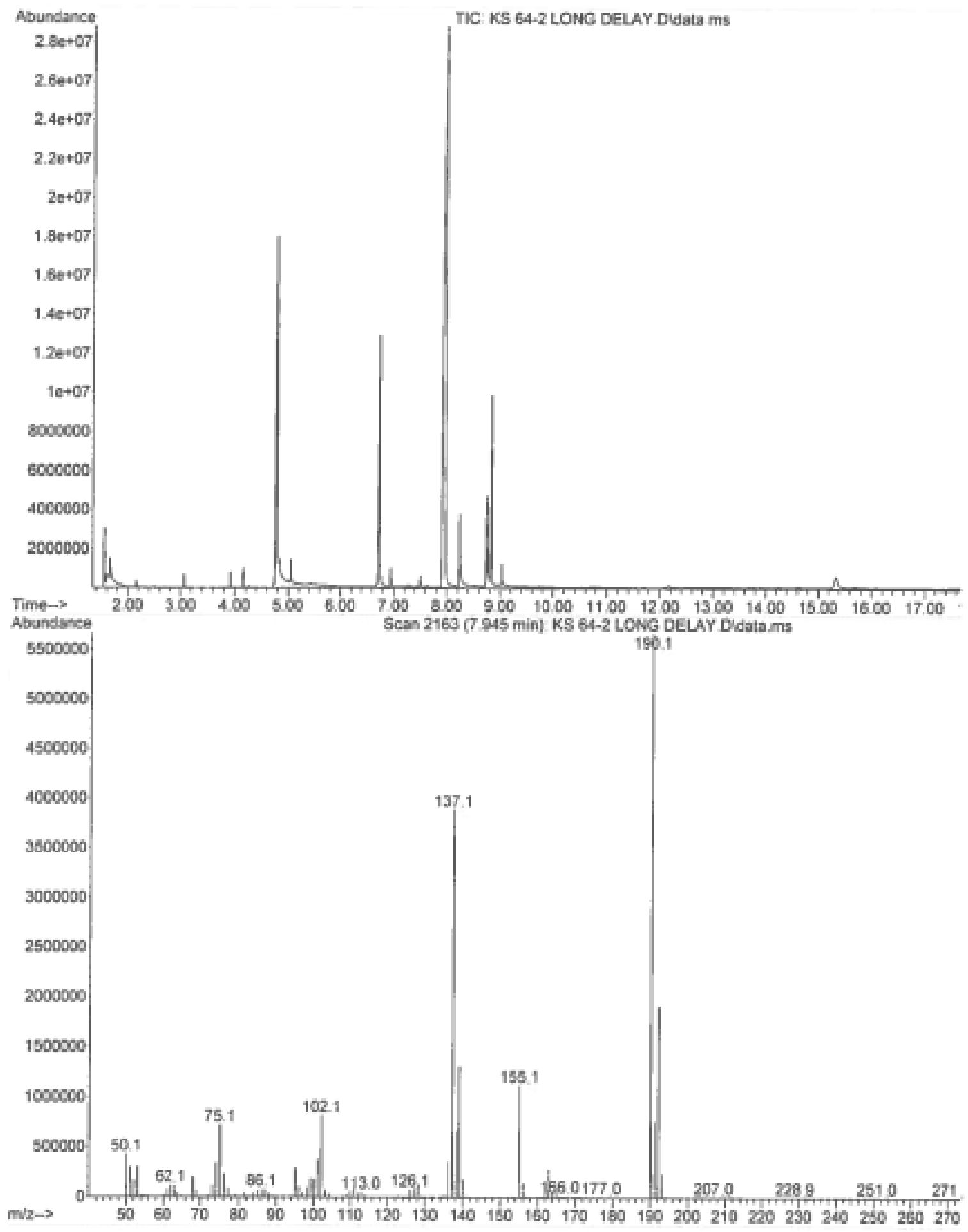


4-chlorophenol: $\mathrm{R}_{\mathrm{t}}=4.78 \mathrm{~min} . \mathrm{m} / \mathrm{z}: 128.1\left(\mathrm{M}^{+}\right), 130.1\left(\mathrm{M}^{+}+2\right), 100.0\left(\mathrm{M}^{+}-28\right), 93.1\left(\mathrm{M}^{+}-\right.$ 35), $65.1\left(\mathrm{M}^{+}-63\right)$.

Bipyrazine: $\mathrm{R}_{\mathrm{t}}=6.72 \mathrm{~min} . \mathrm{m} / \mathrm{z}=158.1\left(\mathrm{M}^{+}\right), 131.1\left(\mathrm{M}^{+}-27\right), 106.1\left(\mathrm{M}^{+}-52\right), 80.1\left(\mathrm{M}^{+}-\right.$ 78).

2-(4-chlorophenyl)pyrazine: $\mathrm{R}_{\mathrm{t}}=8.00 \mathrm{~min} . \mathrm{m} / \mathrm{z}: 190.1\left(\mathrm{M}^{+}\right), 192.1\left(\mathrm{M}^{+}+2\right), 155.1\left(\mathrm{M}^{+}-\right.$ 35), $137.1\left(\mathrm{M}^{+}-53\right), 102.1\left(\mathrm{M}^{+}-88\right)$.

Isomers of $\mathbf{x}, \mathbf{x}^{\prime}$-dichlorobiphenyl. Isomer 1: $R_{t}=8.24 \mathrm{~min} . \mathrm{m} / \mathrm{z}: 222.0\left(\mathrm{M}^{+}\right), 224.0$ $\left(\mathrm{M}^{+}+2\right), 226.0\left(\mathrm{M}^{+}+4\right), 188.0\left(\mathrm{M}^{+}-34\right), 187.1\left(\mathrm{M}^{+}-35\right), 186.1\left(\mathrm{M}^{+}-36\right), 152.1\left(\mathrm{M}^{+}-35\right)$. Isomer 2: $\mathrm{R}_{\mathrm{t}}=8.75 \mathrm{~min} . \mathrm{m} / \mathrm{z}: 222.0\left(\mathrm{M}^{+}\right), 224.0\left(\mathrm{M}^{+}+2\right), 226.0\left(\mathrm{M}^{+}+4\right), 188.0\left(\mathrm{M}^{+}-34\right)$, 187.1 ( $\left.\mathrm{M}^{+}-35\right), 186.1\left(\mathrm{M}^{+}-36\right), 152.1\left(\mathrm{M}^{+}-35\right)$. Isomer 3: $\mathrm{R}_{\mathrm{t}}=8.84 \mathrm{~min} . \mathrm{m} / \mathrm{z}: 222.0\left(\mathrm{M}^{+}\right)$, $224.0\left(\mathrm{M}^{+}+2\right), 226.0\left(\mathrm{M}^{+}+4\right), 188.0\left(\mathrm{M}^{+}-34\right), 187.1\left(\mathrm{M}^{+}-35\right), 186.1\left(\mathrm{M}^{+}-36\right), 152.1\left(\mathrm{M}^{+}-\right.$ 35). 
Figure A122 Gas chromatogram of the crude reaction mixture of the reaction with 4-trifluoromethylphenylboronic acid and pyrazine under optimized conditions.

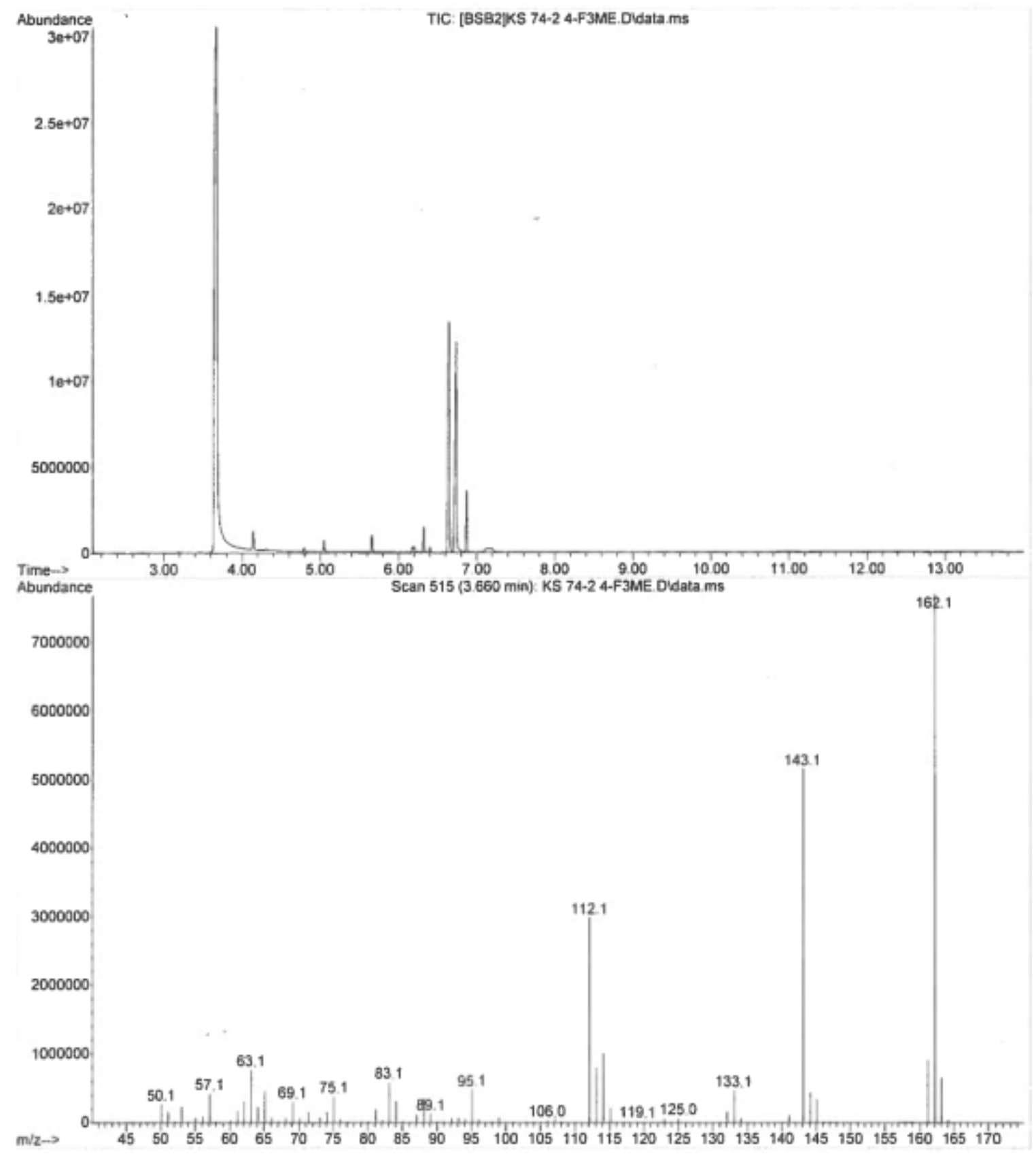

4-trifluorophenol: $\mathrm{R}_{\mathrm{t}}=3.67 \mathrm{~min} . \mathrm{m} / \mathrm{z}: 162.1\left(\mathrm{M}^{+}\right), 143.1\left(\mathrm{M}^{+}-19\right), 133.1\left(\mathrm{M}^{+}-29\right), 114.1$ $\left(\mathrm{M}^{+}-48\right), 112.1\left(\mathrm{M}^{+}-50\right)$. 
2-(4-trifluoromethylphenyl)pyrazine: $\mathrm{R}_{\mathrm{t}}=6.63 \mathrm{~min} . \mathrm{m} / \mathrm{z}: 224.1\left(\mathrm{M}^{+}\right), 205.1\left(\mathrm{M}^{+}-19\right)$, $171.0\left(\mathrm{M}^{+}-53\right)$.

Bipyrazine: $\mathrm{R}_{\mathrm{t}}=6.72 \mathrm{~min} . \mathrm{m} / \mathrm{z}=158.1\left(\mathrm{M}^{+}\right), 131.1\left(\mathrm{M}^{+}-27\right), 106.1\left(\mathrm{M}^{+}-52\right), 80.1\left(\mathrm{M}^{+}-\right.$ 78). 
Figure A123 Gas chromatogram of the crude reaction mixture of the reaction with phenylboronic acid and quinone under optimized conditions (top). Mass spectrum of 2-phenylquinone (bottom).

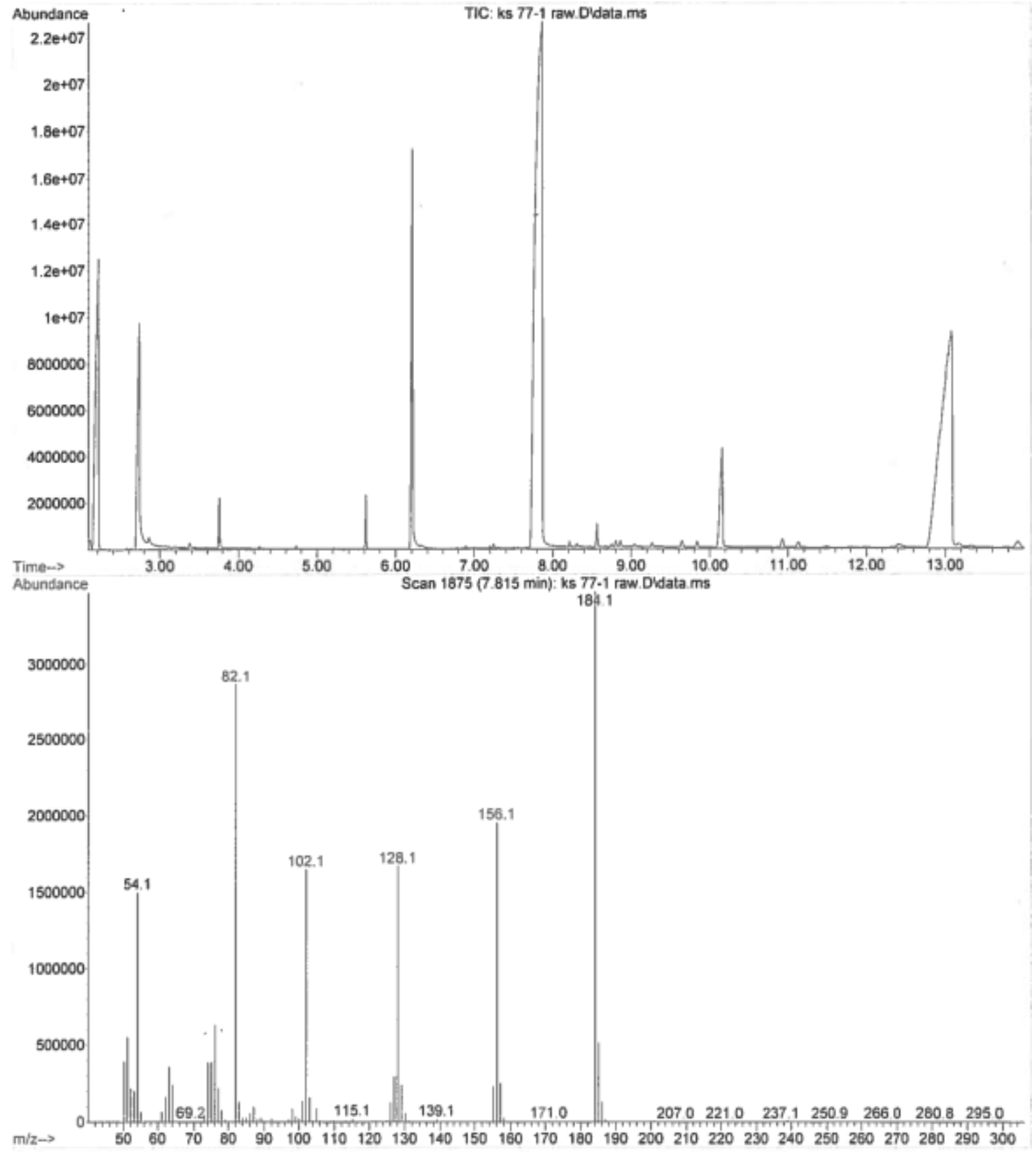

Quinone: $\mathrm{R}_{\mathrm{t}}=2.22 \mathrm{~min} . \mathrm{m} / \mathrm{z}: 108.0\left(\mathrm{M}^{+}\right), 82.0\left(\mathrm{M}^{+}-26\right), 80.0\left(\mathrm{M}^{+}-28\right), 54.0\left(\mathrm{M}^{+}-54\right)$.

Phenol: $\mathrm{R}_{\mathrm{t}}=2.73 \mathrm{~min} . \mathrm{m} / \mathrm{z}: 94.1\left(\mathrm{M}^{+}\right), 66.1\left(\mathrm{M}^{+}-28\right), 55.1\left(\mathrm{M}^{+}-39\right)$. 
2-chloroquinone: $\mathrm{R}_{\mathrm{t}}=3.75 \mathrm{~min} . \mathrm{m} / \mathrm{z}: 142.0\left(\mathrm{M}^{+}\right), 144.0\left(\mathrm{M}^{+}+2\right), 116.0\left(\mathrm{M}^{+}-26\right), 114.0$ $\left(\mathrm{M}^{+}-28\right), 107.0\left(\mathrm{M}^{+}-35\right), 88.0\left(\mathrm{M}^{+}-54\right), 82.0\left(\mathrm{M}^{+}-60\right), 60.0\left(\mathrm{M}^{+}-82\right)$.

2-dichloromethylquinone: $\mathrm{R}_{\mathrm{t}}=5.67 \mathrm{~min} . \mathrm{m} / \mathrm{z}: 190.0\left(\mathrm{M}^{+}\right), 192.0\left(\mathrm{M}^{+}+2\right), 194.0\left(\mathrm{M}^{+}+4\right)$, $166.0\left(\mathrm{M}^{+}-24\right), 164.0\left(\mathrm{M}^{+}-26\right), 162.1\left(\mathrm{M}^{+}-28\right), 155.1\left(\mathrm{M}^{+}-35\right), 128.0\left(\mathrm{M}^{+}-62\right), 126.0\left(\mathrm{M}^{+}-\right.$ 64), $101.0\left(\mathrm{M}^{+}-89\right), 99.0\left(\mathrm{M}^{+}-91\right)$.

Biphenyl: $\mathrm{R}_{\mathrm{t}}=6.21 \mathrm{~min} . \mathrm{m} / \mathrm{z}: 154.1\left(\mathrm{M}^{+}\right), 139.1\left(\mathrm{M}^{+}-15\right), 128.1\left(\mathrm{M}^{+}-26\right), 115.1\left(\mathrm{M}^{+}-39\right)$, $76.1\left(\mathrm{M}^{+}-78\right)$.

2-phenyl-1,4-benzoquinone: $\mathrm{R}_{t}=7.82 \mathrm{~min} . \mathrm{m} / \mathrm{z}: 184.1\left(\mathrm{M}^{+}\right), 156.1\left(\mathrm{M}^{+}-28\right), 128.1\left(\mathrm{M}^{+}-\right.$ 56), $102.1\left(\mathrm{M}^{+}-82\right), 82.1\left(\mathrm{M}^{+}-102\right)$.

Terphenyl (unknown isomer): $R_{t}=10.16-13.09$ min. $\mathrm{m} / \mathrm{z}: 230.0\left(\mathrm{M}^{+}\right), 203.0\left(\mathrm{M}^{+}-27\right)$, $195.0\left(\mathrm{M}^{+}-35\right), 175.0\left(\mathrm{M}^{+}-55\right), 168.1\left(\mathrm{M}^{+}-62\right), 149.1\left(\mathrm{M}^{+}-81\right), 139.1\left(\mathrm{M}^{+}-91\right), 114.1\left(\mathrm{M}^{+}-\right.$ 116), $82.0\left(\mathrm{M}^{+}-148\right), 63.0\left(\mathrm{M}^{+}-167\right), 54.0\left(\mathrm{M}^{+}-176\right)$.

2,5-diphenylquinone or 2,6-diphenylquinone: $R_{t}=12.82-13.09$ min. $\mathrm{m} / \mathrm{z}: 260.1\left(\mathrm{M}^{+}\right)$, $231.1\left(\mathrm{M}^{+}-29\right), 215.1\left(\mathrm{M}^{+}-45\right), 203.1\left(\mathrm{M}^{+}-57\right), 178.1\left(\mathrm{M}^{+}-82\right), 176.1\left(\mathrm{M}^{+}-84\right), 152.1\left(\mathrm{M}^{+}-\right.$ $108), 102.1\left(\mathrm{M}^{+}-158\right)$. 
Figure A124 Gas chromatogram of the crude reaction mixture of the reaction with 4-trifluoromethlphenylboronic acid and quinone under optimized conditions (top).

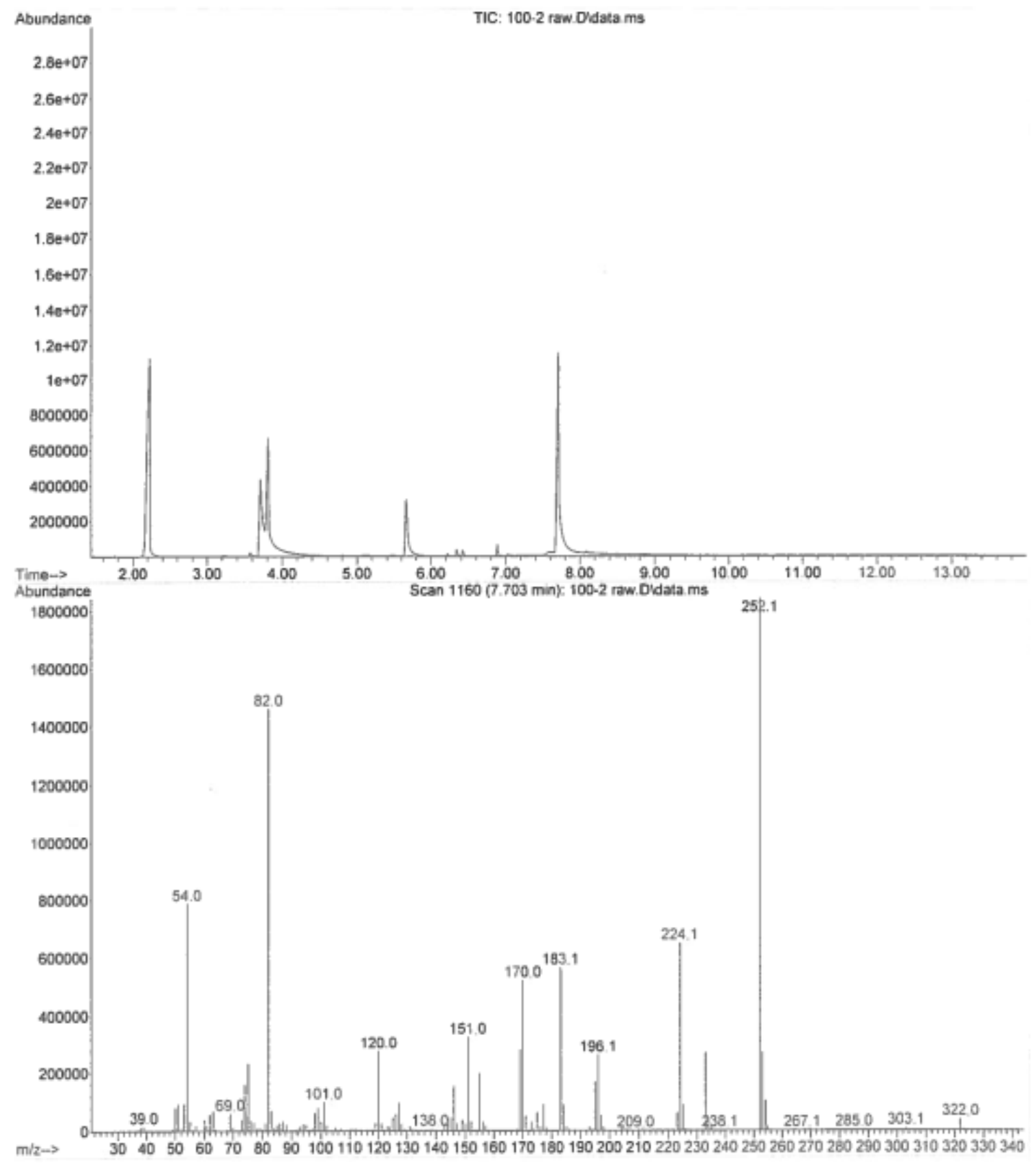

Quinone: $\mathrm{R}_{\mathrm{t}}=2.22 \mathrm{~min} . \mathrm{m} / \mathrm{z}: 108.0\left(\mathrm{M}^{+}\right), 82.0\left(\mathrm{M}^{+}-26\right), 80.0\left(\mathrm{M}^{+}-28\right), 54.0\left(\mathrm{M}^{+}-54\right)$.

4-trifluorophenol: $\mathrm{R}_{\mathrm{t}}=3.67 \mathrm{~min} . \mathrm{m} / \mathrm{z}: 162.1\left(\mathrm{M}^{+}\right), 143.1\left(\mathrm{M}^{+}-19\right), 133.1\left(\mathrm{M}^{+}-29\right), 114.1$ $\left(\mathrm{M}^{+}-48\right), 112.1\left(\mathrm{M}^{+}-50\right)$. 
Trifluorophenol, isomer unknown: $\mathrm{R}_{\mathrm{t}}=3.82 \mathrm{~min} . \mathrm{m} / \mathrm{z}: 162.1\left(\mathrm{M}^{+}\right), 144.0\left(\mathrm{M}^{+}-18\right), 142.0$ $\left(\mathrm{M}^{+}-20\right), 116.0\left(\mathrm{M}^{+}-46\right), 114.0\left(\mathrm{M}^{+}-48\right), 88.0\left(\mathrm{M}^{+}-74\right), 82.0\left(\mathrm{M}^{+}-80\right), 60.0\left(\mathrm{M}^{+}-98\right)$.

2-dichloromethylquinone: $\mathrm{R}_{\mathrm{t}}=5.67 \mathrm{~min} . \mathrm{m} / \mathrm{z}: 190.0\left(\mathrm{M}^{+}\right), 192.0\left(\mathrm{M}^{+}+2\right), 194.0\left(\mathrm{M}^{+}+4\right)$, $166.0\left(\mathrm{M}^{+}-24\right), 164.0\left(\mathrm{M}^{+}-26\right), 162.1\left(\mathrm{M}^{+}-28\right), 155.1\left(\mathrm{M}^{+}-35\right), 128.0\left(\mathrm{M}^{+}-62\right), 126.0\left(\mathrm{M}^{+}-\right.$ 64), $101.0\left(\mathrm{M}^{+}-89\right), 99.0\left(\mathrm{M}^{+}-91\right)$.

2-(4-trifluoromethylphenyl)-1,4-benzoquinone: $\mathrm{R}_{\mathrm{t}}=7.70 \mathrm{~min} . \mathrm{m} / \mathrm{z}: 252.1\left(\mathrm{M}^{+}\right), 233.0$ ( $\left.\mathrm{M}^{+}-19\right), 224.1\left(\mathrm{M}^{+}-28\right), 196.1\left(\mathrm{M}^{+}-56\right), 183.1\left(\mathrm{M}^{+}-69\right), 170.0\left(\mathrm{M}^{+}-82\right), 151.0\left(\mathrm{M}^{+}-99\right)$, $120.0\left(\mathrm{M}^{+}-132\right), 82.0\left(\mathrm{M}^{+}-170\right)$. 
Figure A125 Gas chromatogram of the crude reaction mixture of the reaction of quinone and toluene (top). Mass spectrum of the desired product, 2-benzyl-1,4-benzoquinone (bottom).

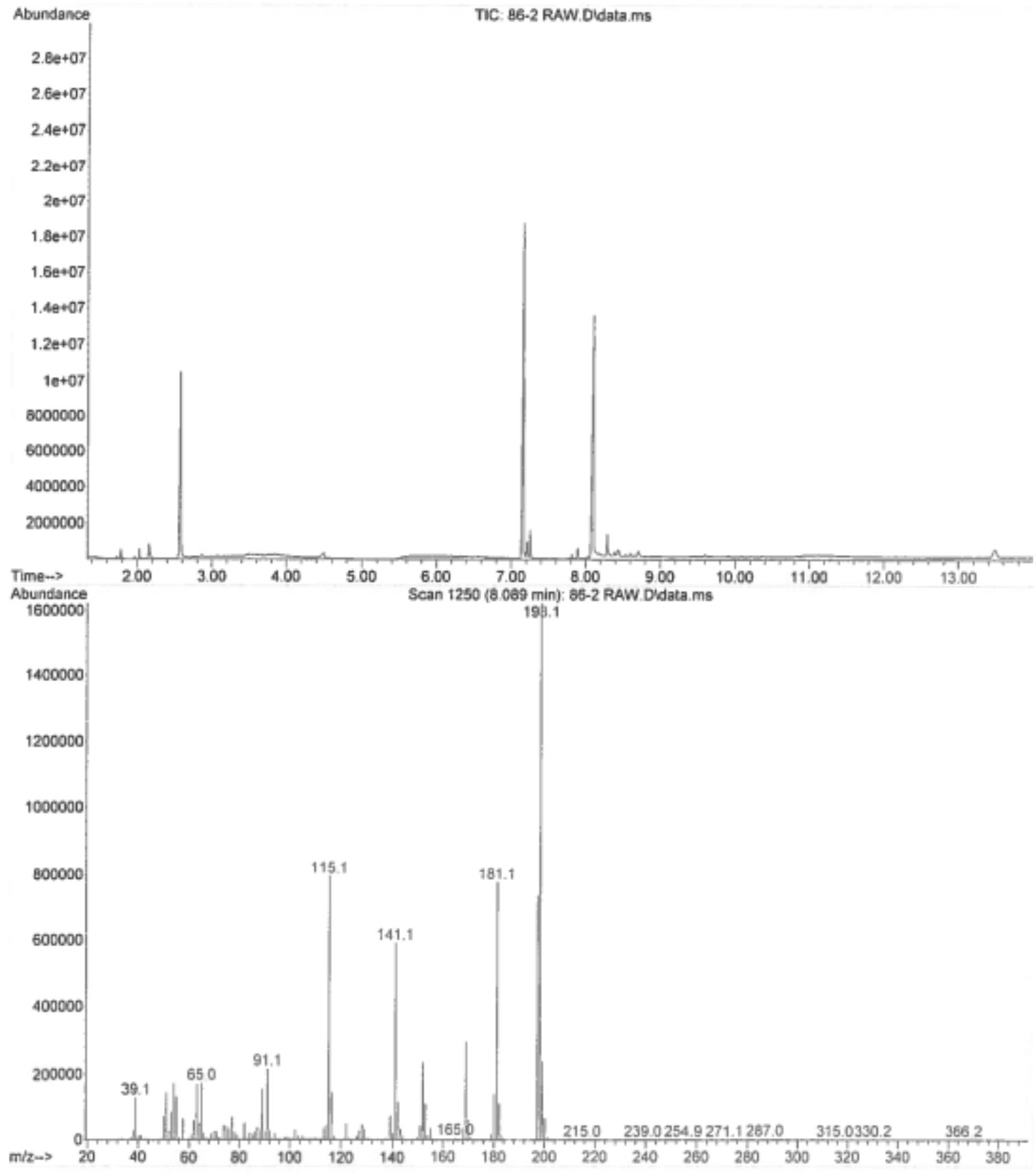

Benzaldehyde: $\mathrm{R}_{\mathrm{t}}=2.57 \mathrm{~min} . \mathrm{m} / \mathrm{z}: 106.0\left(\mathrm{M}^{+}\right), 105.0\left(\mathrm{M}^{+}-1\right), 77.1\left(\mathrm{M}^{+}-29\right), 51.0\left(\mathrm{M}^{+}-55\right)$. 
Bibenzyl: $\mathrm{R}_{\mathrm{t}}=7.16$ min. m/z: $182.1\left(\mathrm{M}^{+}\right), 104.1\left(\mathrm{M}^{+}-78\right), 91.1\left(\mathrm{M}^{+}-91\right), 77.1\left(\mathrm{M}^{+}-105\right)$. $65.1\left(\mathrm{M}^{+}-117\right)$.

2-benzyl-1,4-benzoquinone: $R_{t}=8.07$ min. m/z: $198.1\left(\mathrm{M}^{+}\right), 181.1\left(\mathrm{M}^{+}-17\right), 169.1\left(\mathrm{M}^{+}-\right.$ 29), $152.1\left(\mathrm{M}^{+}-46\right), 141.1\left(\mathrm{M}^{+}-57\right), 115.1\left(\mathrm{M}^{+}-83\right), 91.1\left(\mathrm{M}^{+}-107\right) 77.1\left(\mathrm{M}^{+}-121\right)$. 
Figure A126 Gas chromatogram of the crude reaction mixture of the reaction of 4-cyanopyridine and toluene (top).

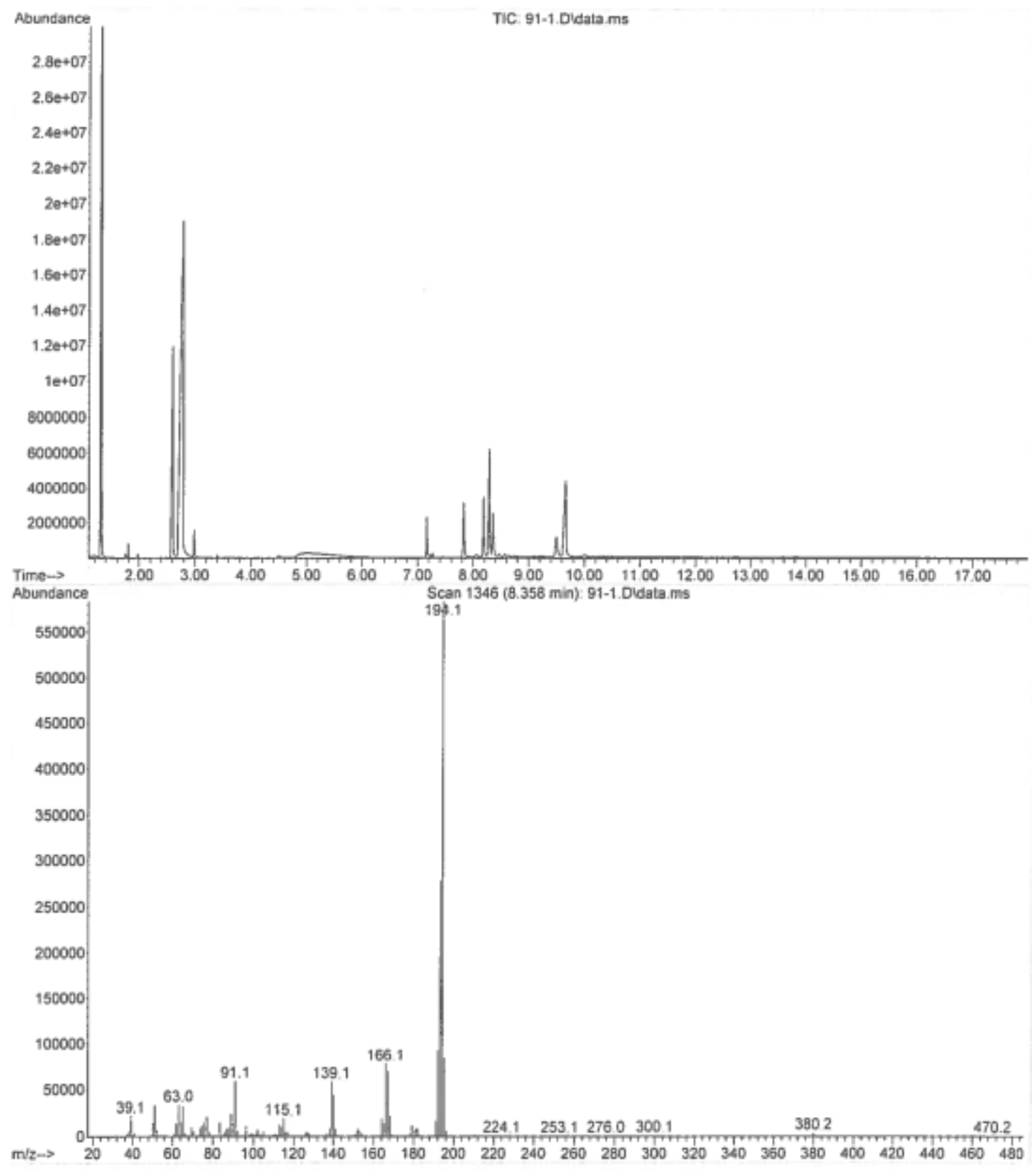

Toluene: $\mathrm{R}_{\mathrm{t}}=1.30 \mathrm{~min} . \mathrm{m} / \mathrm{z}: 92.1\left(\mathrm{M}^{+}\right), 91.1\left(\mathrm{M}^{+}-1\right), 77.1\left(\mathrm{M}^{+}-15\right), 65.1\left(\mathrm{M}^{+}-27\right) 51.1$ $\left(\mathrm{M}^{+}-40\right)$. 
Benzaldehyde: $\mathrm{R}_{\mathrm{t}}=2.57 \mathrm{~min} . \mathrm{m} / \mathrm{z}: 106.0\left(\mathrm{M}^{+}\right), 105.0\left(\mathrm{M}^{+}-1\right), 77.1\left(\mathrm{M}^{+}-29\right), 51.0\left(\mathrm{M}^{+}-55\right)$.

4-Cyanopyridine: $\mathrm{R}_{\mathrm{t}}=2.76 \mathrm{~min} . \mathrm{m} / \mathrm{z}: 104.1\left(\mathrm{M}^{+}\right), 77.1\left(\mathrm{M}^{+}-27\right), 64.1\left(\mathrm{M}^{+}-40\right), 50.1\left(\mathrm{M}^{+}-\right.$ $54)$.

Bibenzyl: $\mathrm{R}_{\mathrm{t}}=7.16 \mathrm{~min} . \mathrm{m} / \mathrm{z}: 182.1\left(\mathrm{M}^{+}\right), 91.1\left(\mathrm{M}^{+}-1\right), 104.1\left(\mathrm{M}^{+}-78\right), 91.1\left(\mathrm{M}^{+}-91\right), 77.1$ $\left(\mathrm{M}^{+}-115\right) .65 .1\left(\mathrm{M}^{+}-117\right)$.

3-phenyl-4-cyanopyridine: $\mathrm{R}_{\mathrm{t}}=7.82 \mathrm{~min} . \mathrm{m} / \mathrm{z}: 180.1\left(\mathrm{M}^{+}\right), 153.1\left(\mathrm{M}^{+}-27\right), 126.1\left(\mathrm{M}^{+}-54\right)$, $103.1\left(\mathrm{M}^{+}-77\right), 76.1\left(\mathrm{M}^{+}-104\right)$.

2-phenyl-4-cyanopyridine: $\mathrm{R}_{\mathrm{t}}=8.22 \mathrm{~min} . \mathrm{m} / \mathrm{z}: 180.1\left(\mathrm{M}^{+}\right), 153.1\left(\mathrm{M}^{+}-27\right), 126.1\left(\mathrm{M}^{+}-54\right)$, $103.1\left(\mathrm{M}^{+}-77\right), 76.1\left(\mathrm{M}^{+}-104\right)$.

benzyl-4-cyanopyridine, isomer unknown: $\mathrm{R}_{\mathrm{t}}=8.29 \mathrm{~min} . \mathrm{m} / \mathrm{z}: 194.1\left(\mathrm{M}^{+}\right), 193.1\left(\mathrm{M}^{+}-\right.$ 15), $168.1\left(\mathrm{M}^{+}-26\right), 139.1\left(\mathrm{M}^{+}-55\right), 115.1\left(\mathrm{M}^{+}-79\right), 91.1\left(\mathrm{M}^{+}-103\right)$.

methylphenyl-4-cyanopyridine, isomer unknown: $R_{t}=8.35 \mathrm{~min} . \mathrm{m} / \mathrm{z}: 194.1\left(\mathrm{M}^{+}\right), 179.1$ $\left(\mathrm{M}^{+}-15\right), 166.1\left(\mathrm{M}^{+}-28\right), 139.1\left(\mathrm{M}^{+}-55\right), 115.1\left(\mathrm{M}^{+}-79\right), 91.1\left(\mathrm{M}^{+}-103\right)$.

benzoyl-4-cyanopyridine, isomer unknown: $R_{t}=9.50 \mathrm{~min} . \mathrm{m} / \mathrm{z}: 208.1\left(\mathrm{M}^{+}\right), 181.1\left(\mathrm{M}^{+}-\right.$ 27), $131.0\left(\mathrm{M}^{+}-77\right), 105.1\left(\mathrm{M}^{+}-103\right), 103.0\left(\mathrm{M}^{+}-105\right), 77.1\left(\mathrm{M}^{+}-131\right)$.

(4-formylphenyl)-4-cyanopyridine, isomer unknown: $R_{t}=9.65 \mathrm{~min} . \mathrm{m} / \mathrm{z}: 208.1\left(\mathrm{M}^{+}\right)$, $207.1\left(\mathrm{M}^{+}-1\right), 180.1\left(\mathrm{M}^{+}-28\right), 105.1\left(\mathrm{M}^{+}-103\right), 77.1\left(\mathrm{M}^{+}-131\right)$. 